TECHNICAL DOCUMENT 3214

February 2007

\title{
Advanced Propagation Model (APM) Version 2.1.04 Computer Software Configuration Item (CSCI) Documents
}

\author{
A. E. Barrios \\ W. L. Patterson \\ R. A. Sprague
}
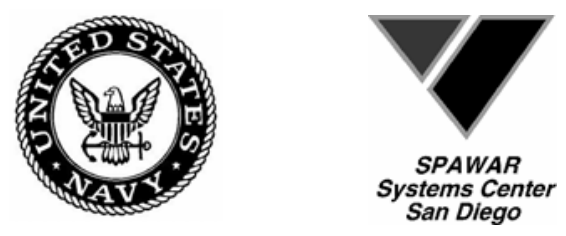

SSC San Diego

San Diego, CA 92152-5001 


\section{Report Documentation Page}

Form Approved

OMB No. 0704-0188

Public reporting burden for the collection of information is estimated to average 1 hour per response, including the time for reviewing instructions, searching existing data sources, gathering and maintaining the data needed, and completing and reviewing the collection of information. Send comments regarding this burden estimate or any other aspect of this collection of information,

including suggestions for reducing this burden, to Washington Headquarters Services, Directorate for Information Operations and Reports, 1215 Jefferson Davis Highway, Suite 1204, Arlington

VA 22202-4302. Respondents should be aware that notwithstanding any other provision of law, no person shall be subject to a penalty for failing to comply with a collection of information if it

does not display a currently valid OMB control number.

1. REPORT DATE

FEB 2007

4. TITLE AND SUBTITLE

Advanced Propagation Model (APM) Version 2.1.04 Computer Software Configuration Item (CSCI) Documents

6. $\operatorname{AUTHOR}(\mathrm{S})$

7. PERFORMING ORGANIZATION NAME(S) AND ADDRESS(ES)

Space and Naval Warfare Systems Center,53560 Hull Street,San Diego,CA,92152-5001

9. SPONSORING/MONITORING AGENCY NAME(S) AND ADDRESS(ES)
3. DATES COVERED

00-00-2007 to 00-00-2007

5a. CONTRACT NUMBER

5b. GRANT NUMBER

5c. PROGRAM ELEMENT NUMBER

5d. PROJECT NUMBER

5e. TASK NUMBER

5f. WORK UNIT NUMBER

8. PERFORMING ORGANIZATION REPORT NUMBER

10. SPONSOR/MONITOR'S ACRONYM(S)

11. SPONSOR/MONITOR'S REPORT NUMBER(S)

12. DISTRIBUTION/AVAILABILITY STATEMENT

Approved for public release; distribution unlimited

13. SUPPLEMENTARY NOTES

14. ABSTRACT

15. SUBJECT TERMS

16. SECURITY CLASSIFICATION OF:

a. REPORT

b. ABSTRACT

unclassified

unclassified

c. THIS PAGE

17. LIMITATION OF ABSTRACT
18. NUMBER

OF PAGES

438 19a. NAME OF

RESPONSIBLE PERSON 


\section{PREFACE}

This document provides Version 2.1.04 Computer Software Configuration Item documents for the Advanced Propagation Model (APM). The APM calculates range-dependent electromagnetic system propagation loss and propagation factor within a heterogeneous atmospheric medium over variable terrain, where the radio-frequency index of refraction is allowed to vary vertically and horizontally.

The first document specifies the functional requirements that are to be met by the APM CSCI. A discussion of the input software requirements is presented together with a general description of the internal structure of the APM CSCI as it relates to the CSCI's capability.

The second document describes the design of the APM CSCI. An overview of the input software requirements is presented together with an overview of the CSCI design architecture and a detailed design description of each CSCI component.

The third document specifies the test cases and test procedures necessary to perform APM CSCI qualification testing. A discussion of precise input values of each input variable required to perform the test together with final expected test results is presented. 


\section{SOFTWARE REQUIREMENTS SPECIFICATION FOR THE ADVANCED PROPAGATION MODEL CSCI (Version 2.1.04)}

20 December 2006 


\section{TABLE OF CONTENTS}

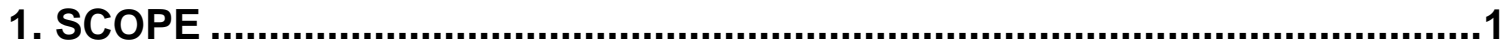

1.1 IDENTIFICATION

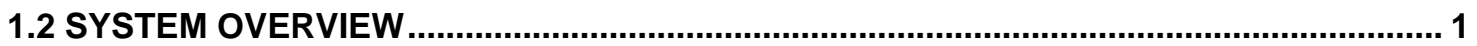

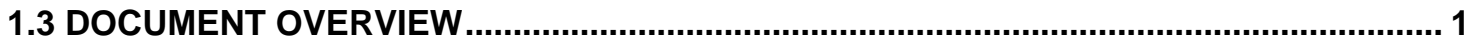

2. REFERENCED DOCUMENTS

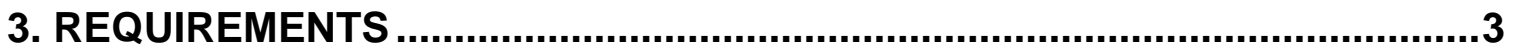

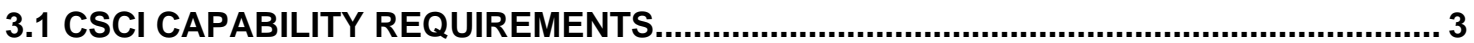

3.1.1 Advanced Propagation Model Initialization (APMINIT) CSC ………………………... 9

3.1.1.1 Allocate Arrays APM (ALLARRAY_APM) SU ………................................. 10

3.1.1.2 Allocate Array PE (ALLARRAY_PE) SU ………………................................ 10

3.1.1.3 Allocate Array RO (ALLARRAY_RO) SU ……………………………….... 10

3.1.1.4 Allocate Array XORUF (ALLARRAY_XORUF) SU …………………………... 10

3.1.1.5 Alpha Impedance Initialization (ALN_INIT) SU …………................................. 11

3.1.1.6 Antenna Pattern (ANTPAT) SU ……………………....................................... 11

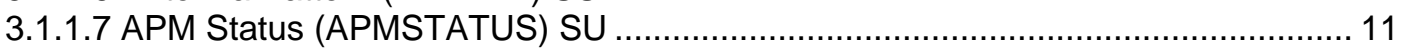

3.1.1.8 Dielectric Initialization (DIEINIT) SU.......................................................... 11

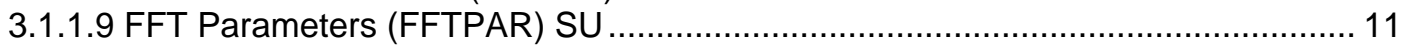

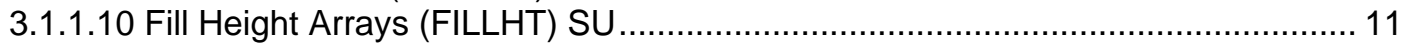

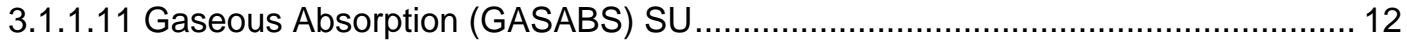

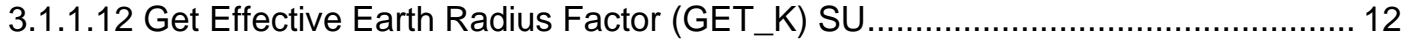

3.1.1.13 Get Alpha Impedance (GETALN) SU ........................................................... 12

3.1.1.14 Get Angles (GETANGLES) SU ………………................................... 12

3.1.1.15 Get Maximum Angle (GETTHMAX) SU ………………………………...... 12

3.1.1.16 Grazing Angle Interpolation (GRAZE_INT) SU …………………………..... 13

3.1.1.17 Height Check (HTCHECK) SU .................................................................. 13

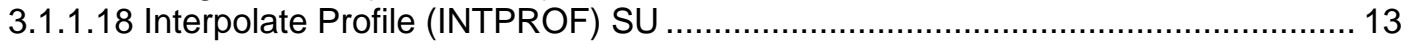

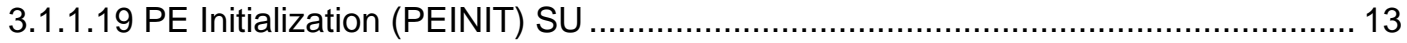

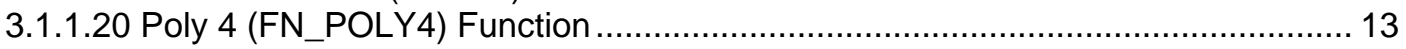

3.1.1.21 Poly 5 (FN_POLY5) Function ................................................................. 13

3.1.1.22 Profile Reference (PROFREF) SU .......................................................... 13

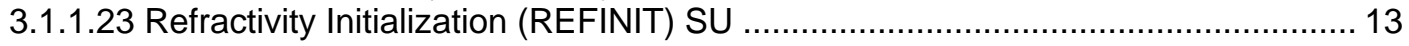

3.1.1.24 Remove Duplicate Refractivity Levels (REMDUP) SU...................................... 14

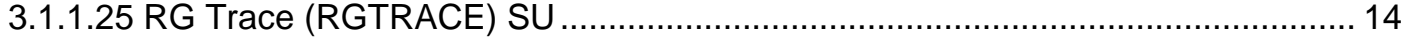

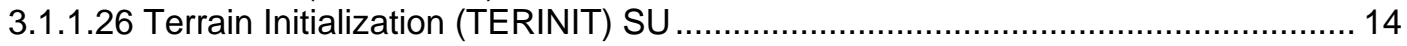

3.1.1.27 Trace to Output Range (TRACE_ROUT) SU ……………………………..... 14

3.1.1.28 Trace to next Step (TRACE_STEP) SU ................................................... 14

3.1.1.29 Troposcatter Initialization (TROPOINIT) SU …………................................. 15

3.1.1.30 Starter Field Initialization (XYINIT) SU …………..................................... 15

3.1.2 Advanced Propagation Model Step (APMSTEP) CSC ………………………….... 15

3.1.2.1 Calculate Propagation Loss (CALCLOS) SU ………....................................... 16

3.1.2.2 Current Wind (FN_CURWIND) Function ........................................................... 16

3.1.2.3 Dielectric Constant (FN_DIECON) Function ...................................................... 16

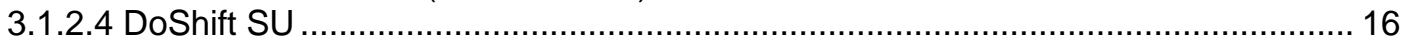

3.1.2.5 Discrete Sine/Cosine Fast-Fourier Transform (DRST) SU ................................. 17

3.1.2.6 Flat-Earth Direct Ray (FEDR) SU ………………….................................... 17

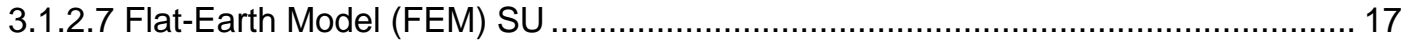

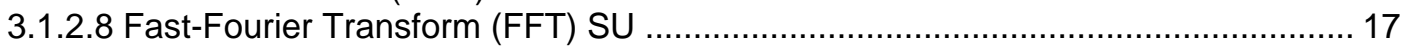

3.1.2.9 Free Space Range Step (FRSTP) SU …………….................................. 18

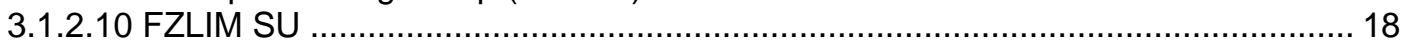

3.1.2.11 Get Propagation Factor (FN_GETPFAC) Function .......................................... 18

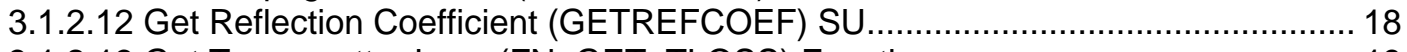

3.1.2.13 Get Troposcatter Loss (FN_GET_TLOSS) Function ........................................ 19 


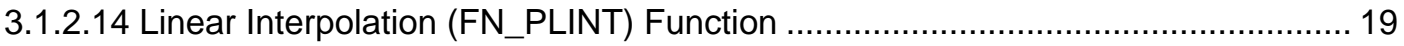

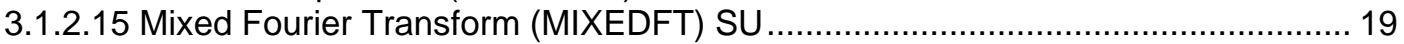

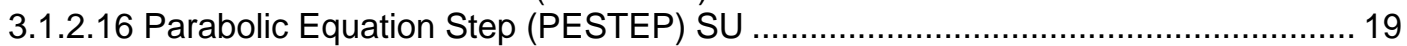

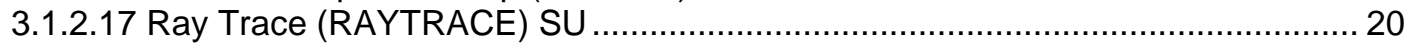

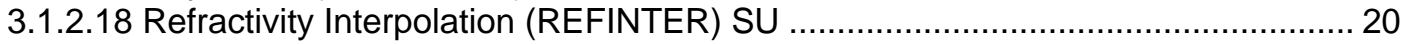

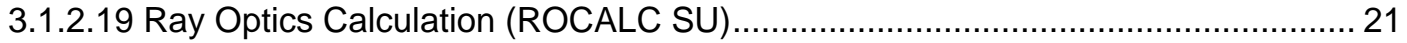

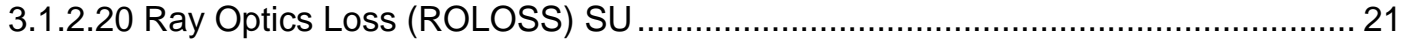

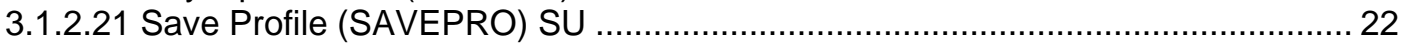

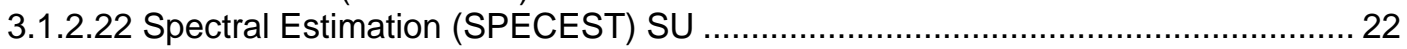

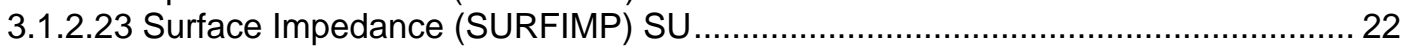

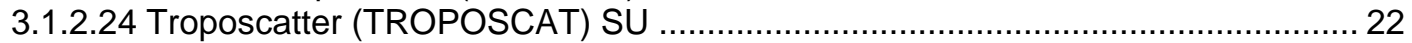

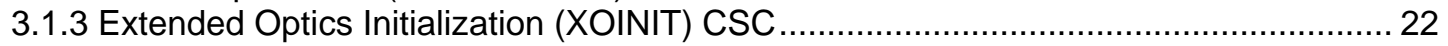

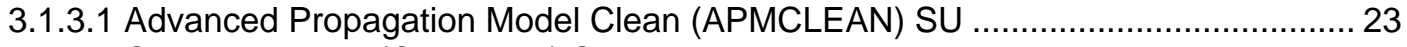

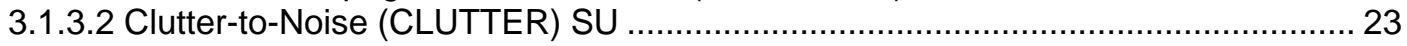

3.1.3.3 Diffraction LoSS (FN_DLOSS) Function ....................................................... 23

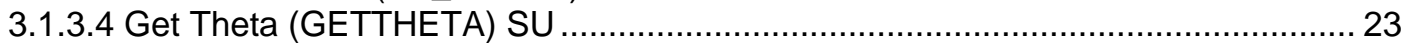

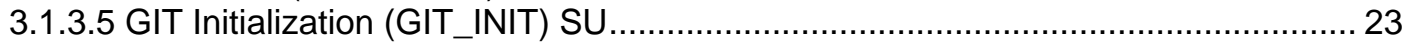

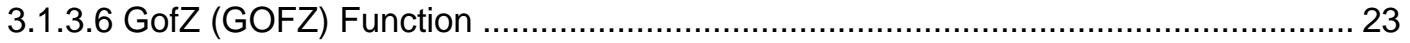

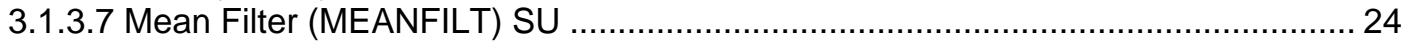

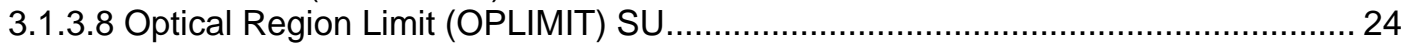

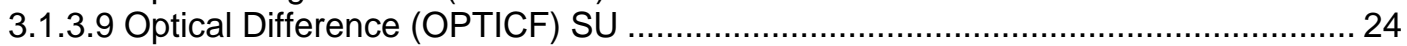

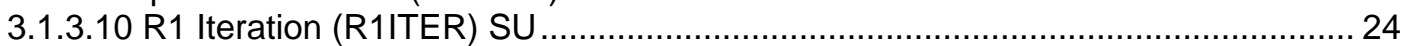

3.1.3.11 Standard Propagation Model Initialization (SPM_INIT) SU.............................. 24

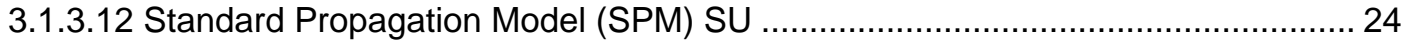

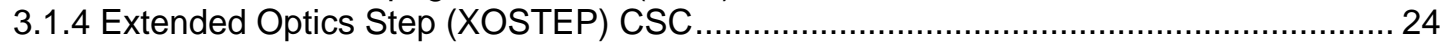

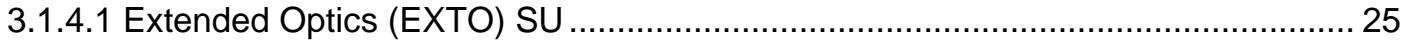

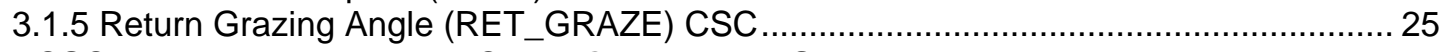

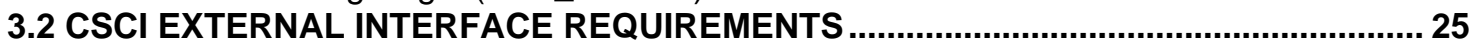

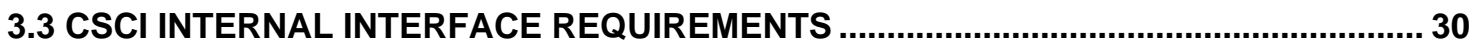

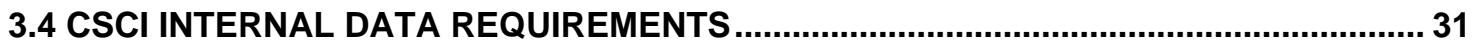

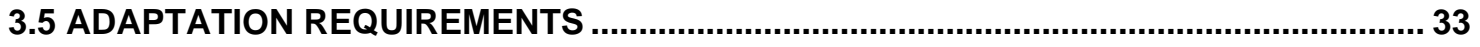

3.5.1 Environmental Radio Refractivity Field Data Elements ............................................. 33

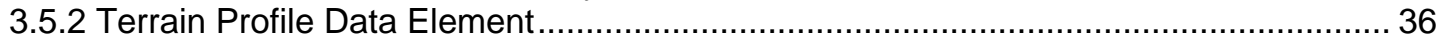

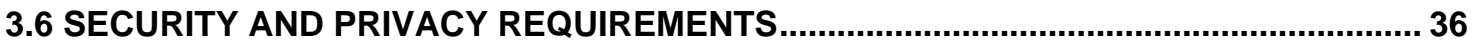

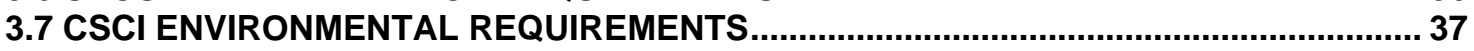

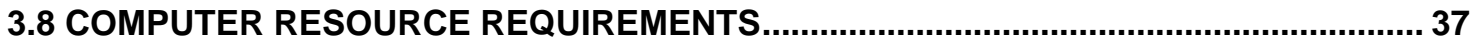

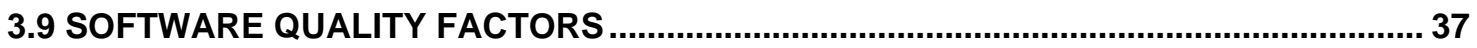

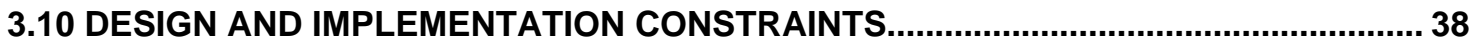

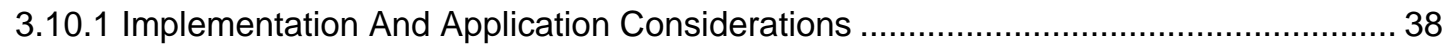

3.10.2 Programming Language And Source Implementation .......................................... 39

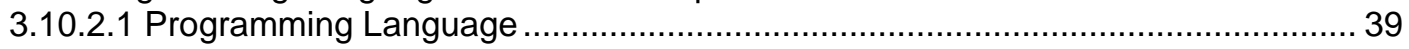

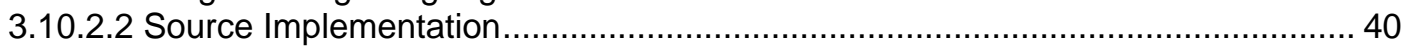

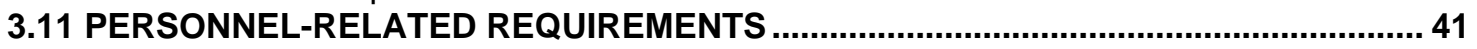

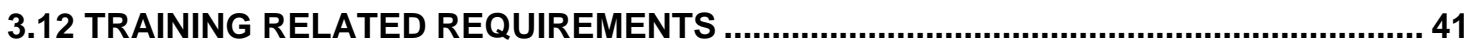

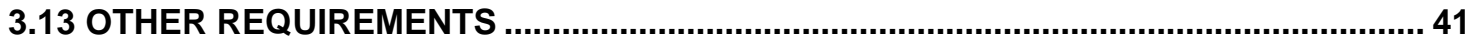

3.14 PRECEDENCE AND CRITICALITY OF REQUIREMENTS ......................................... 41

4. QUALIFICATION PROVISIONS..............................................................42

5. REQUIREMENTS TRACEABILITY ...................................................42

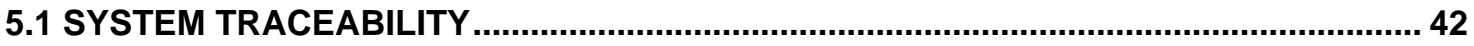

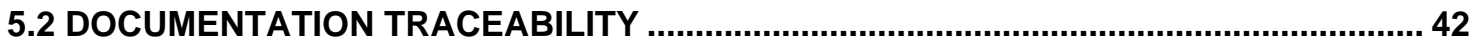

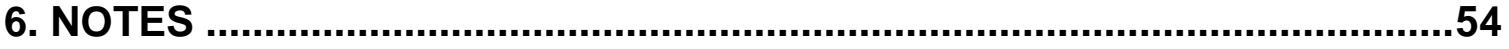




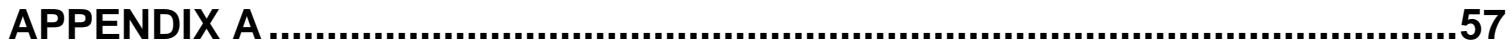

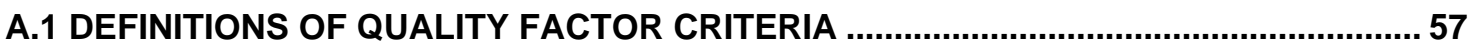

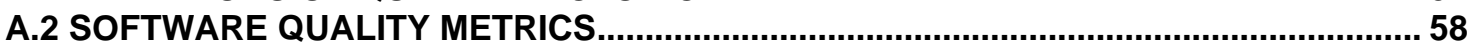

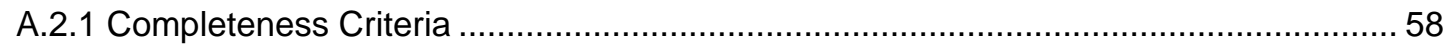

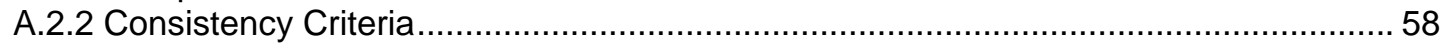

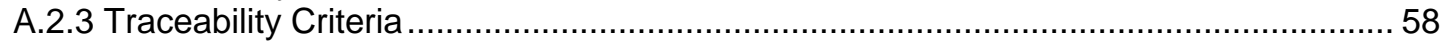

\section{FIGURES}

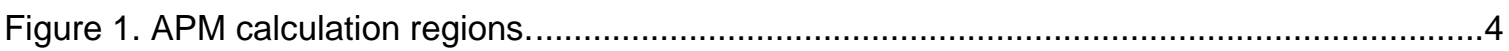

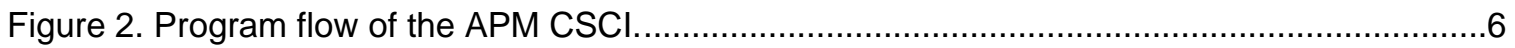

Figure 3. APMINIT CSC program flow.

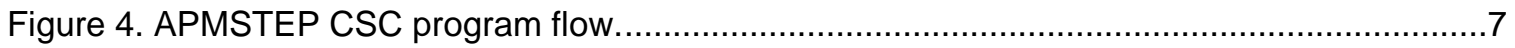

Figure 5. XOINIT CSC program flow.

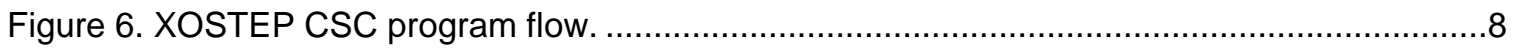

Figure 7. Idealized M-unit profiles (solid) and lines of interpolation (dashed)................................35

\section{TABLES}

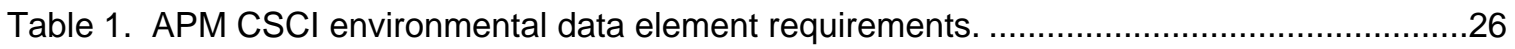

Table 2. APM CSCl external EM system data element requirements. ......................................27

Table 3. APM CSCl external implementation data element requirements...................................28

Table 4. APM CSCl external terrain data element requirements.............................................29

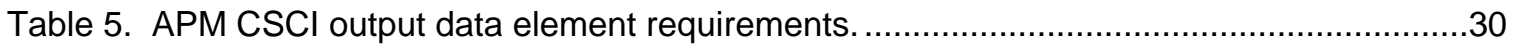

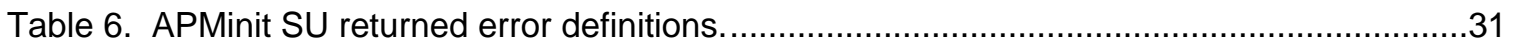

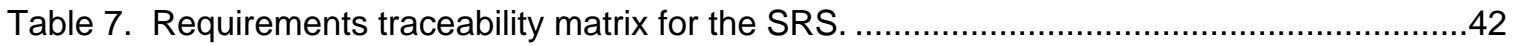

Table 8. Acronyms and abbreviations.

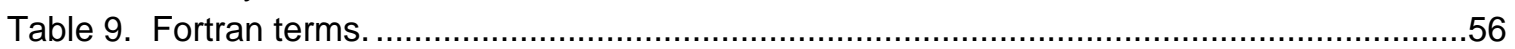




\section{SCOPE}

\subsection{IDENTIFICATION}

The Advanced Propagation Model (APM) Version 2.1.04 computer software configuration item (CSCI) calculates range-dependent electromagnetic (EM) system propagation loss within a heterogeneous atmospheric medium over variable terrain, where the radio-frequency index of refraction is allowed to vary vertically and horizontally, also accounting for terrain effects along the path of propagation.

\subsection{SYSTEM OVERVIEW}

The APM CSCI model calculates propagation loss values as EM energy propagates through a laterally heterogeneous atmospheric medium where the index of refraction is allowed to vary vertically and horizontally, also accounting for terrain effects along the propagation path. Numerous external applications require EM-system propagation loss values. The APM model described by this document may be applied to two external applications, one that displays propagation loss on a range versus height scale (commonly referred to as a coverage diagram) and one that displays propagation loss on a propagation loss versus range/height scale (commonly referred to as a loss diagram).

\subsection{DOCUMENT OVERVIEW}

This document specifies the functional requirements that are to be met by the APM CSCI. A discussion of the input software requirements is presented together with a general description of the internal structure of the APM CSCI as it relates to the CSCI's capability.

\section{REFERENCED DOCUMENTS}

American National Standards Institute (ANSI), "Program Language - Fortran Extended”, 1992.

Barrick, D.E. 1971a. "Theory of HF and VHF Propagation Across the Rough Sea, 1. The Effective Surface Impedance for a Slightly Rough Highly Conducting Medium at Grazing Incidence,” Radio Sci. (May), V6, no. 5, pp.571-526.

Barrick, D.E. 1971b. "Theory of HF and VHF Propagation Across the Rough Sea, 2. Application to HF and VHF Propagation Above the Sea," Radio Sci. (May), V6, no. 5, pp. 527-533. 
Barrios, A. E., "Terrain and Refractivity Effects on Non-Optical Paths," AGARD Conference Proceedings 543, Multiple Mechanism Propagation Paths (MMPPs): Their Characteristics and Influence on System Design, pp. 10-1 to 10-9, October 1993.

Barrios, A. E., "A Terrain Parabolic Equation Model for Propagation in the Troposphere,” IEEE Trans. Antennas Propagat., Vol. 42, pp. 90-98, January 1994.

Barrios, A. E., “Terrain Parabolic Equation Model (TPEM) Version 1.5 User’s Manual,” Naval Command, Control and Ocean Surveillance Center, RDT\&E Division, San Diego, CA, NRaD TD 2898, February 1996.

Barrios, A.E., Patterson, W.L., "Advanced Propagation Model (APM) Ver. 1.3.1 Computer Software Configuration Item (CSCI) Documents”, SSC-SD TD 3145, Aug. 2002.

Bergland, G. D., "A Radix-eight Fast Fourier Transform Subroutine for Real-valued Series,” IEEE Trans. Audio and Electro-acoust., Vol. AU-17, pp. 138-144, 1969.

Commander-In-Chief, Pacific Fleet Meteorological Requirement (PAC MET) 87-04, "Range Dependent Electromagnetic Propagation Models,” 1987.

Cooley, J. W., P. A. W. Lewis and P. D. Welsh, "The Fast Fourier Transform Algorithm: Programming Considerations in the Calculation of Sine, Cosine and Laplace Transforms,” J. Sound Vib., Vol. 12, pp. 315-337, 1970.

Dockery, G. D., "Modeling Electromagnetic Wave Propagation in the Troposphere Using the Parabolic Equation”, IEEE Trans. Antennas Propagat., Vol. 36, No. 10, pp. 14641470, October 1988.

Dockery, G.D, Kuttler, J.R., "An Improved Impedance-Boundary Algorithm for Fourier Split-Step Solutions of the Parabolic Wave Equation," IEEE Trans. On Antennas and Propagat., Vol. 44, No. 12, December 1996, pp. 1592-1599.

Hitney, H.V., Hattan, C.P., Paulus, R.A., Anderson, K.D., "Electromagnetic Propagation Functions Program Performance Specification (PPS) For the Tactical Environmental Support System (TESS)”, NOSC TD 1015, Feb. 1984.

Horst, M.M., Dyer, F.B., Tuley, M.T., "Radar Sea Clutter Model,”, IEEE International Conference on Antennas and Propagation.

International Radio Consulting Committee (CCIR) XVth Plenary Assembly Dubrovnik, 1986, "Propagation in Non-Ionized Media," Recommendations and Reports of the CCIR, 1986, Vol. V, International Telecommunications Union, Geneva, 1986. 
Kuttler, J. R. and G. D. Dockery., "Theoretical Description of the Parabolic Approximation/Fourier Split-Step Method of Representing Electromagnetic Propagation in the Troposphere," Radio Sci., Vol. 26, pp. 381-393, 1991.

Miller, A.R., R.M. Brown, E. Vegh,"New Derivation for the Rough-Surface Reflection Coefficient and for the Distribution of Sea-Wave Elevations" Proc. IEEE, Vol. 131, Part H, 2, 1984, pp 114-116.

Naval Oceanographic Office, "Software Documentation Standards for Environmental System Product Development,” February 1996.

Patterson, W.L., Hitney, H.V., "Radio Physical Optics CSCI Software Documents", Naval Command, Control and Ocean Surveillance Center, RDT\&E Division, San Diego, CA, NRaD TD 2403, December 1992.

Sailors, D.B. 1997. "Excess System Power Available for Short-Range High-Frequency Communication Systems," NRaD TD-2978 (August), Naval Command, Control and Ocean Surveillance Center RDT\&E Division, San Diego, CA.

Sailors, D.B., Barrios, A.E., "Terrain Parabolic Equation Model (TPEM) Computer Software Configuration Item (CSCI) Documents”, Naval Command, Control and Ocean Surveillance Center, RDT\&E Division, San Diego, CA, NRaD TD 2963, May 1997.

Sailors, D.B. Barrios, A.E., Patterson, W.L., Hitney, H.V., "Advanced Propagation Model [Ver. 1.0] (APM) Computer Software Configuration Item (CSCI) Documents," SPAWARSYSCEN-SD TD 3033, August 1998.

Tappert, F. D., "The Parabolic Approximation Method," Wave Propagation and Underwater Acoustics, J. B. Keller and J. S. Papadakis, Eds., New York, SpringerVerlag, pp. 224-285, 1977.

\section{REQUIREMENTS}

\subsection{CSCI CAPABILITY REQUIREMENTS}

The required APM CSCI propagation model is a range-dependent true hybrid model that uses the complimentary strengths of Ray Optics (RO) and Parabolic Equation $\mathrm{PE}$ ) techniques to calculate propagation loss both in range and altitude.

The atmospheric volume is divided into regions that lend themselves to the application of the various propagation loss calculation methods. Figure 1 illustrates these regions. 


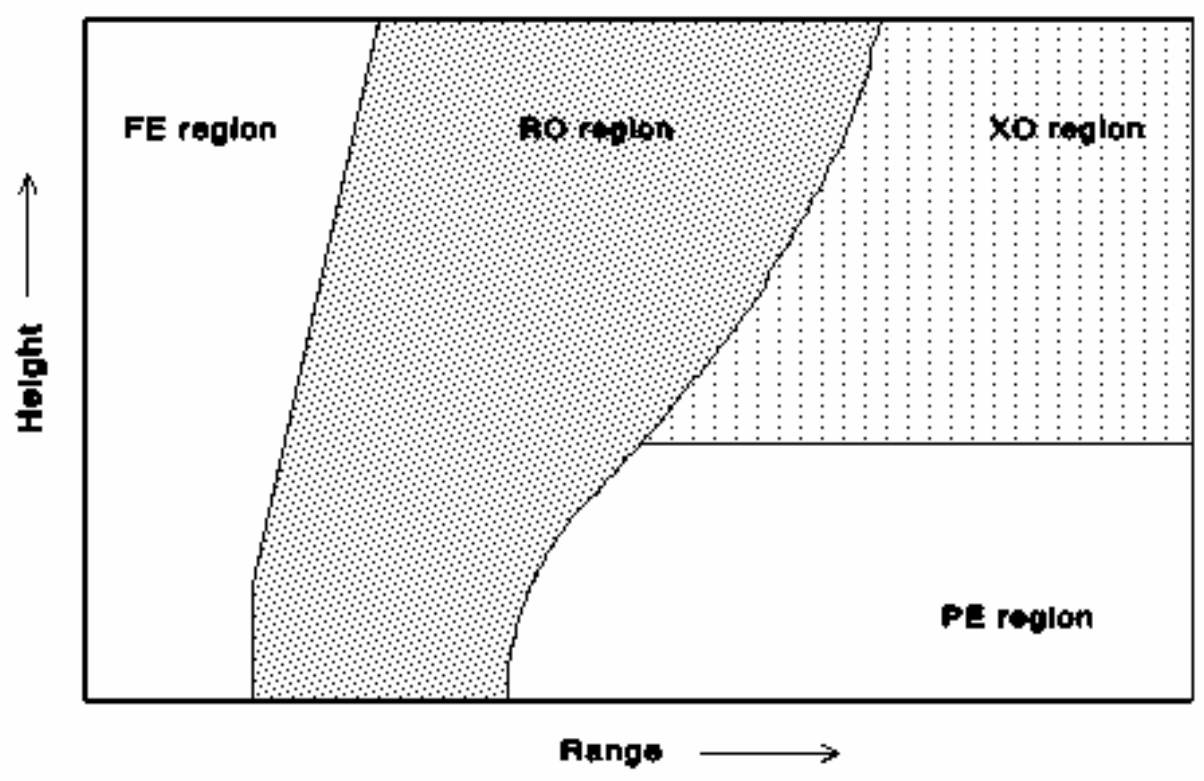

Figure 1. APM calculation regions.

For antenna elevation angles above 5 degrees or for ranges less than approximately 2.5 kilometers $(\mathrm{km})$, a flat-earth (FE) ray-optics model is used. In this region, only receiver height is corrected for average refraction and earth curvature.

Within the RO region (as defined by a limiting ray), propagation loss is calculated from the mutual interference between the direct-path and surface-reflected ray components using the refractivity profile at zero range. Full account is given to focusing or de-focusing along direct and reflected ray paths and to the integrated optical path length difference between the two ray paths, to give precise phase difference, and, hence, accurate coherent sums for the computation of propagation loss.

For the low-altitude region beyond the RO region, a $\mathrm{PE}$ approximation to the Helmholtz full wave equation is employed. The PE model allows for range-dependent refractivity profiles and variable terrain along the propagation path and uses a split-step Fourier method for the solution of the PE. The PE model is run in the minimum region required to contain all terrain and trapping layer heights.

For the area beyond the RO region but above the PE region, an extended optics region (XO) is defined. Within the $\mathrm{XO}$ region, ray-optics methods that are initialized by the PE solution from below, are used.

APM will run in three "execution" modes depending on environmental inputs. APM will use the FE, RO, $\mathrm{XO}$, and PE models if the terrain profile is flat for the first $2.5 \mathrm{~km}$ and if the antenna height is less than or equal to $100 \mathrm{~m}$. It will use only the XO and PE models if the terrain profile is not flat for the first $2.5 \mathrm{~km}$ and if the antenna 
height is less than or equal to $100 \mathrm{~m}$. For applications in which the antenna height is greater than 100 meters, a combination of FE and PE methods are used. The FE model is used for all propagation angles greater than $\pm 5^{\circ}$ from the source and the PE model is used for angles within $\pm 5^{\circ}$. By default, APM will automatically choose which mode of operation it will use for a specified set of inputs. However, the ability to run only the PE model for any case is allowed by setting a logical flag upon input. APM will automatically run only the PE algorithm for frequencies less than $50 \mathrm{MHz}$, regardless of the logical flag set by the user.

The APM CSCI allows for horizontal and vertical antenna polarization, finite conductivity based on user-specified ground composition and dielectric parameters, and the complete range of EM system parameters and most antenna patterns required by various external applications. APM also allows for gaseous absorption effects in all submodels and computes troposcatter losses within the diffraction region and beyond.

The program flow of the required APM CSCI is illustrated Figure 2. Note that the APM CSCI is shown within the context of a calling CSCI application such as one that generates a coverage or loss diagram. The efficient implementation of the APM CSCI will have far reaching consequences on the design of an application CSCI beyond those mentioned in Section 3.10. For example, Figure 2 shows checking for the existence of a previously created APM output file prior to the access of the APM CSCI. The application CSCI will have to consider if the atmospheric or terrain environment has changed since the APM output file was created or if any new height or range requirement is accommodated within the existing APM CSCI output file. Because these and many more considerations are beyond the scope of this document to describe, an application CSCI designer should work closely with the APM CSCI development agency in the implementation of the APM CSCI. Figure 3 through Figure 6 illustrate the program flow for the main compute software components (CSC), APMINIT CSC, APMSTEP CSC, XOINIT CSC, and XOSTEP CSC, respectively. 


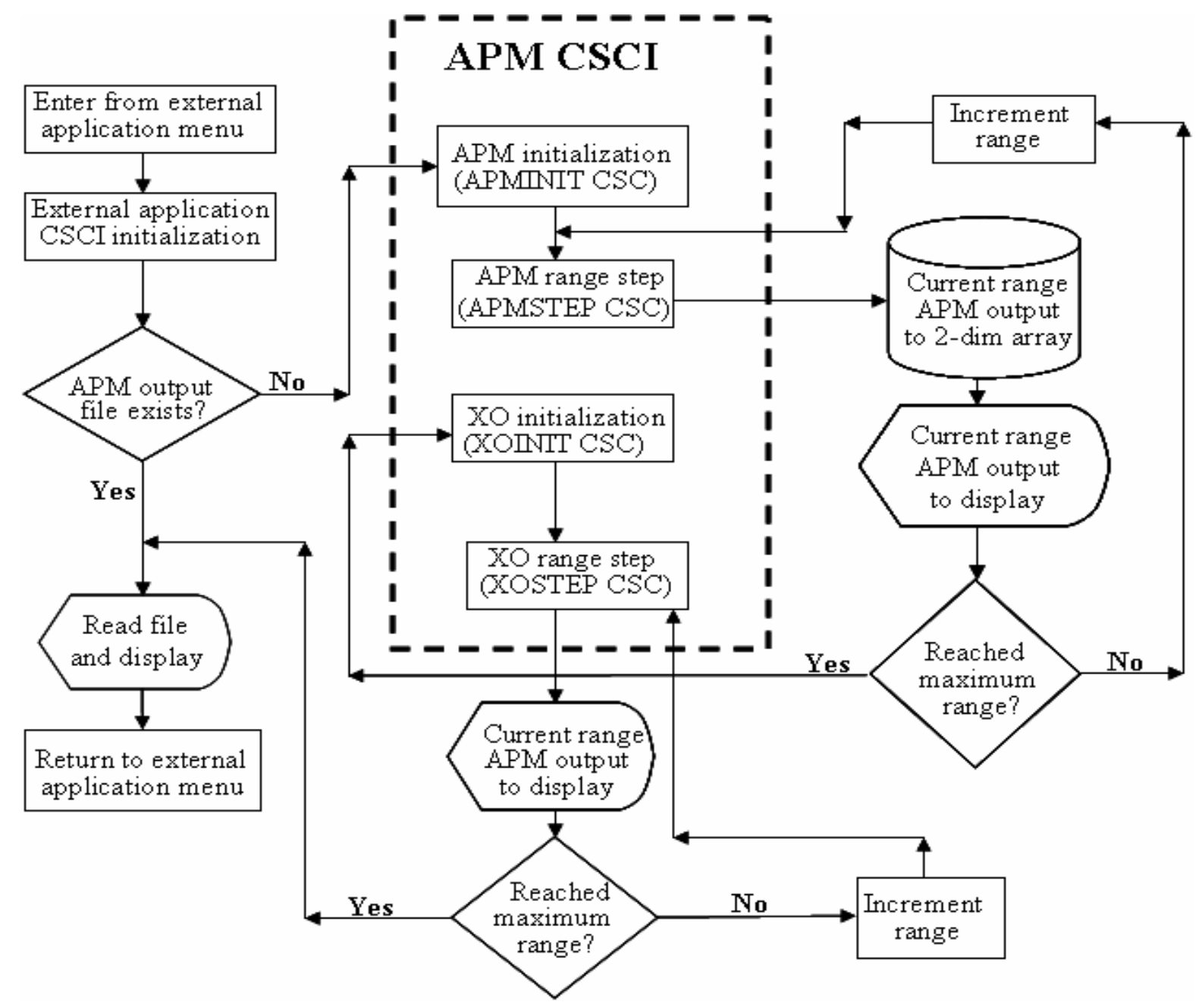

Figure 2. Program flow of the APM CSCI. 

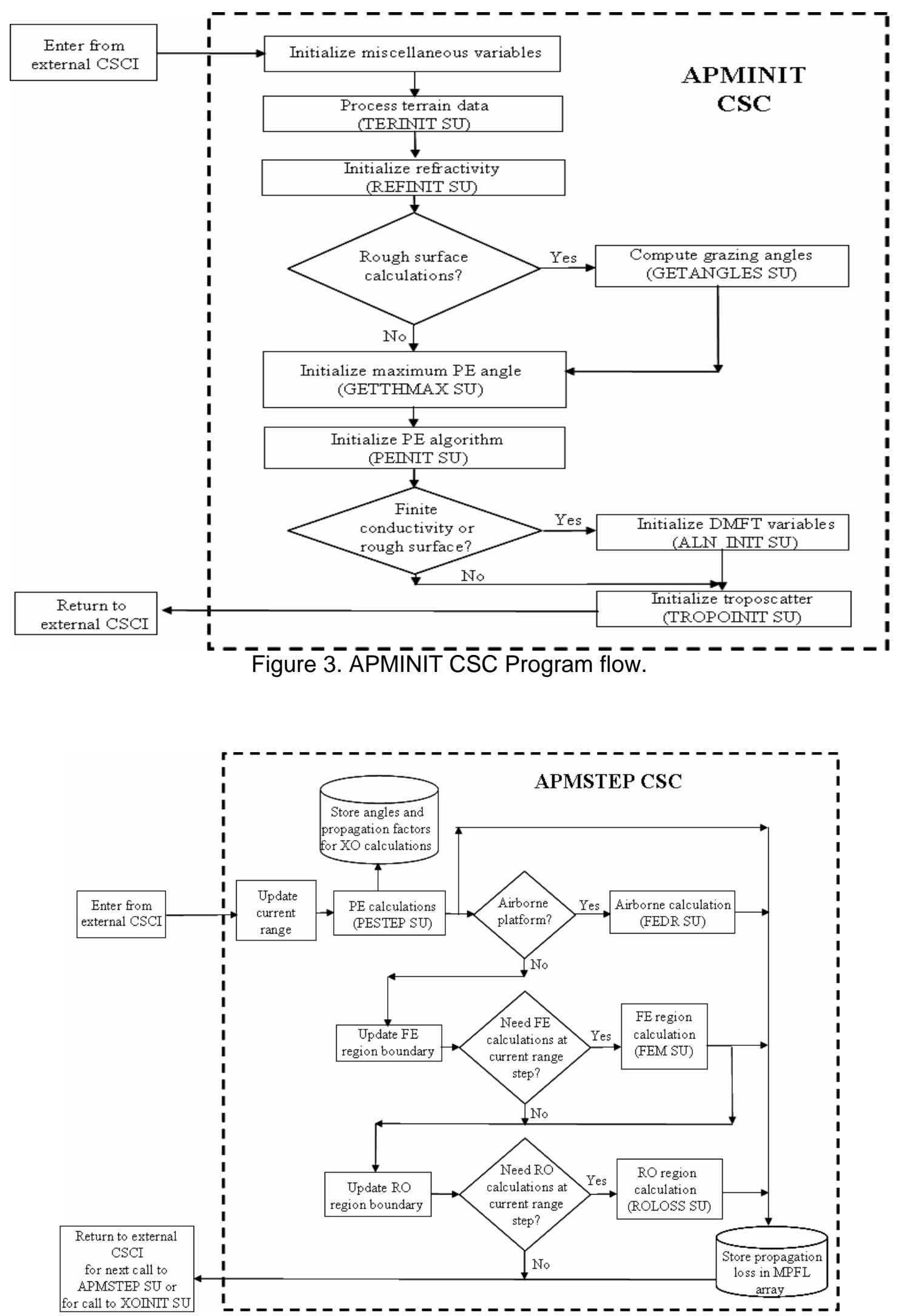

Figure 4. APMSTEP CSC Program flow. 

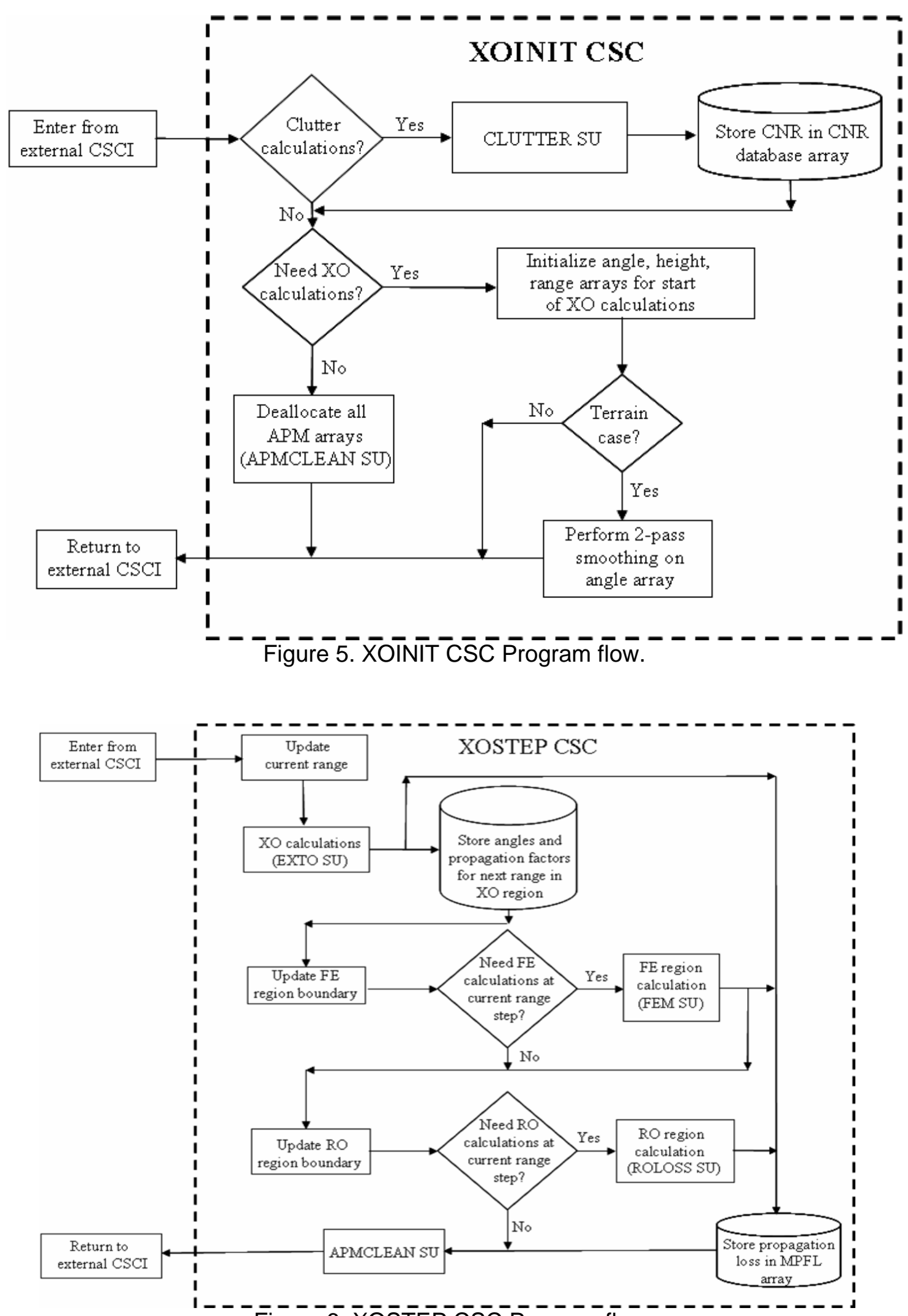

Figure $\overline{6} . \bar{X} \overline{\mathrm{S}} \overline{\mathrm{T}} \overline{\mathrm{A}} \overline{\mathrm{CSC}} \mathrm{C}$ Program flow. 
The APM CSCI is divided into 5 main computer software components (CSC) and 67 additional software units (SU). The name, purpose, and a general description of processing required for each SU follows.

\subsubsection{Advanced Propagation Model Initialization (APMINIT) CSC}

The APMINIT CSC interfaces with various SUs for the complete initialization of the APM CSCI.

The atmospheric volume must be "covered" or resolved with a mesh of calculation points that will normally exceed the height/range resolution requirements of the particular application of the APM CSCI. Upon entering the APMINIT CSC, a range and height grid size per the APM CSCI output point is calculated from the number of APM outputs and the maximum CSCI range and height.

A TERINIT SU is referenced to examine the terrain profile. The minimum terrain height is determined, and then the entire terrain profile is adjusted by this height so that all internal calculations are referenced to this height. This is done in order to minimize the required PE transform size.

User-specified environmental and system parameters are examined to determine if the APM CSCI will execute in a full hybrid mode, a partial hybrid mode, or PE-only mode.

A REFINIT SU is referenced to initialize the external CSCI specified modified refractivity and also to test for valid environment profiles. A PROFREF SU adjusts the environment profiles by the internal reference height, and a INTPROF SU defines the modified refractivity at all $\mathrm{PE}$ vertical mesh points.

If rough surface calculations are required (i.e., a non-zero wind speed is specified) a GETANGLES SU is referenced to determine the grazing angles for the given refractivity profile. The grazing angles are then sorted and a GRAZE_INT SU is referenced to combine grazing angles computed by both methods (if necessary) and interpolate for subsequent use in rough surface calculations.

To automatically determine the maximum PE calculation angle, a GETTHMAX

$\mathrm{SU}$ is referenced. This determines, via ray tracing, the minimum angle for which adequate coverage can be given with the specified terrain and environment profile. A FFTPAR SU is referenced to determine the fast Fourier transform (FFT) size for the calculated angle and to initialize data elements within the PE region which are dependent on the size of the FFT. The minimum size for the FFT is determined from the Nyquist criterion. An option also exists to activate only the PE algorithm within the APM CSCI, regardless of inputs. If this option is enabled, the PE maximum calculation angle is supplied by the calling CSCI. 
If vertical polarization is specified, then additional calculations are performed in the starter solution using Kuttler and Dockery's mixed transform method. In this case a DIEINIT SU is used to initialize dielectric ground constants. For general ground types, the permittivity and conductivity are calculated as a function of frequency from curve fits to the permittivity and conductivity graphs shown in recommendations and reports of the International Radio Consulting Committee.

A PE initialization SU (PEINIT) is referenced to initialize all variables and arrays associated with PE calculations. A XYINIT SU and an antenna pattern factor SU (ANTPAT) are referenced to generate a first solution to the PE. A FFT SU is referenced for data elements required in obtaining the PE's starting solution.

If rough surface calculations are required, or if one is performing a finiteconducting boundary case, then a ALN_INIT SU is referenced to initialize field variables used in the mixed transform algorithm.

If running in a full hybrid mode, a FILLHT SU is referenced to determine the heights at each output range separating the $\mathrm{FE}, \mathrm{RO}$, and $\mathrm{PE}$ calculation regions. If running in a partial hybrid or PE-only mode, then the heights at each output range are determined, below which, propagation loss/factor solutions are valid. No propagation loss/factor solutions are provided above these heights for those execution modes.

A TROPOINIT SU is referenced to initialize all variables and arrays associated with troposcatter calculations and is referenced only if the user has enabled the $T_{\text {ropo }}$ flag.

Finally, a GASABS SU is referenced to initialize the attenuation parameter due to oxygen and water vapor absorption.

\subsubsection{Allocate Arrays APM (ALLARRAY_APM) SU}

The ALLARRAY_APM SU allocates and initializes all dynamically dimensioned arrays associated with APM terrain, refractivity, troposcatter, and general variable arrays.

\subsubsection{Allocate Array PE (ALLARRAY_PE) SU}

The ALLARRAY_PE SU allocates and initializes all dynamically dimensioned arrays associated with PE calculations.

\subsubsection{Allocate Array RO (ALLARRAY_RO) SU}

The ALLARRAY_RO SU allocates and initializes all dynamically dimensioned arrays associated with RO calculations.

\subsubsection{Allocate Array XORUF (ALLARRAY_XORUF) SU}

The ALLARRAY_XORUF SU allocates and initializes all dynamically dimensioned arrays associated with $\mathrm{XO}$ and rough surface calculations. 


\subsubsection{Alpha Impedance Initialization (ALN_INIT) SU}

The ALN_INIT SU initializes variables used in the discrete mixed Fourier transform (DMFT) algorithm for finite conductivity and/or rough surface calculations.

\subsubsection{Antenna Pattern (ANTPAT) SU}

The ANTPAT SU calculates a normalized antenna gain (antenna pattern factor) for a specified antenna elevation angle.

From the antenna beam width, elevation angle (an angle for which the antenna pattern factor is desired), and the antenna radiation pattern type; an antenna factor is calculated.

\subsubsection{APM Status (APMSTATUS) SU}

The APMSTATUS SU accesses the current status of APM calculations of the grazing angle. The SU is declared as an external subroutine within the main driver program.

\subsubsection{Dielectric Initialization (DIEINIT) SU}

The DIEINIT SU determines the conductivity and relative permittivity as functions of frequency in $\mathrm{MHz}$ based on general ground composition types.

\subsubsection{FFT Parameters (FFTPAR) SU}

The FFTPAR SU determines the required transform size based on the maximum $\mathrm{PE}$ propagation angle and the maximum height needed. If running in full or partial hybrid modes, the maximum height needed is the height necessary to encompass at least $20 \%$ above the maximum terrain peak along the path or the highest trapping layer specified in the environment profiles, whichever is greater. If running in a PE-only mode, the maximum height needed is the specified maximum output height. An error is returned if the computed transform size reaches $2^{30}$.

For computational efficiency reasons, an artificial upper boundary must be established for the PE solution. To prevent upward propagating energy from being "reflected" downward from this boundary and contaminating the PE solution, the PE solution field strength should be attenuated or "filtered" above a certain height to ensure that the field strength just below this boundary is reduced to zero.

The total number of vertical points for which a transformation will be computed is determined. This term is also referred to as the FFT size. The filtering boundary height is also determined.

\subsubsection{Fill Height Arrays (FILLHT) SU}

The FILLHT SU calculates the effective earth radius for an initial launch angle of

$5^{\circ}$ and to fill an array with height values at each output range of the limiting sub-model, depending on which mode is being used. If running in a full hybrid mode, the array 
contains height values at each output range separating the FE from the RO region. If running in other modes, the array contains those height values at each output range at which the initial launch angle has been traced to the ground or surface. For an execution mode in which PE and XO models are used, these height values represent the separating region where, above that height, valid loss is computed, and below that height, no loss is computed. This is done so only loss values that fall within a valid calculation region are output. For airborne applications in which a combination of PE and FE models are used, the array contains the height values at each range separating the FE and PE regions.

\subsubsection{Gaseous Absorption (GASABS) SU}

The GASABS SU computes the specific attenuation based on air temperature and absolute humidity. This SU is based on CCIR (International Telecommunication Union, International Radio Consultative Committee, now the ITU-R) Recommendation 676-1, “Attenuation by Atmospheric Gases in the Frequency Range 1-350 GHz.”

\subsubsection{Get Effective Earth Radius Factor (GET_K) SU}

The GET_K SU computes the effective earth radius factor and the effective earth radius. The computation is made for a launch angle of $5^{\circ}$ if the $\mathrm{SU}$ is called from the APMINIT CSC. If called from the TROPOINIT SU, then the computation is made for a launch angle equal to the critical angle.

\subsubsection{Get Alpha Impedance (GETALN) SU}

The GETALN SU computes the impedance term in the Leontovich boundary condition, and the complex index of refraction for finite conductivity, vertical polarization, and rough sea surface calculations. These formulas follow Kuttler and Dockery 's method.

\subsubsection{Get Angles (GETANGLES) SU}

The GETANGLES SU computes the grazing angles at each PE range step for subsequent use in rough sea surface calculations, and also computes the propagation angles within the PE region for subsequent output via the APMSTEP CSC if so desired. Two methods are used to compute the grazing angle. The first method uses ray trace by referencing a RGTRACE SU to compute grazing angles. The second is done by spectral estimation of the near-surface PE field. The near-surface PE field is computed by performing a "PE run" for a smooth-surface, perfect-conducting, horizontal polarization case at a fixed frequency $(10 \mathrm{GHz})$ and fixed $\mathrm{PE}$ maximum calculation angle $\left(4^{\circ}\right)$.

\subsubsection{Get Maximum Angle (GETTHMAX) SU}

The GETTHMAX SU performs an iterative ray trace to determine the minimum angle required (based on the reflected ray) in obtaining a PE solution. The determination of this angle will depend on the particular mode of execution. For the full and partial hybrid modes, a ray is traced up to a height that exceeds at least $20 \%$ above the maximum terrain peak along the path or the highest trapping layer specified in the environment profiles, whichever is greater. The maximum PE propagation angle is then determined from the local maximum angle of the traced ray. 


\subsubsection{Grazing Angle Interpolation (GRAZE_INT) SU}

The GRAZE_INT SU interpolates grazing angles at each PE range step based on angles computed from ray trace (takes precedence) and those computed from spectral estimation. These are used for subsequent rough surface calculations.

\subsubsection{Height Check (HTCHECK) SU}

The HTCHECK SU determines if the current traced height is below the current ground height. If so, it will calculate the reflection point and return with the modified angle, range, and height of reflection.

\subsubsection{Interpolate Profile (INTPROF) SU}

The INTPROF SU performs a linear interpolation vertically with height on the refractivity profile. Interpolation is performed at each PE mesh height point.

\subsubsection{PE Initialization (PEINIT) SU}

The PEINIT SU initializes all variables used in the PE model for subsequent calls to the PESTEP SU. The PE calculation height mesh and range step size are determined. The free-space propagator term is computed at each PE angle, or p-space, mesh point using the wide-angle propagator. A filter, or attenuation function (frequently called "window"), is applied to the upper one-quarter of the array corresponding to the highest one-quarter of the maximum propagation angle. Next, the environmental phase term is computed at each PE height, or z-space, mesh point (if performing a smooth surface, refractive homogeneous case). A filter, or attenuation function (frequently called "window"), is applied to the upper one-quarter of the mesh points corresponding to the highest one-quarter of the calculation height domain. Finally, a XYINIT SU is referenced to initialize the PE starting field.

\subsubsection{Poly 4 (FN_POLY4) Function}

The FN_POLY4 function evaluates a fourth degree polynomial.

\subsubsection{Poly 5 (FN_POLY5) Function}

The FN_POLY5 function evaluates a fifth degree polynomial.

\subsubsection{Profile Reference (PROFREF) SU}

The PROFREF SU adjusts the current refractivity profile so that it is relative to a reference height. The reference height is initially the minimum height of the terrain profile. Upon subsequent calls from the PESTEP SU, the refractivity profile is adjusted by the local ground height at each PE range step.

\subsubsection{Refractivity Initialization (REFINIT) SU}

The REFINIT SU checks for valid environmental profile inputs and initializes all refractivity arrays. 
The environmental data are checked for a range-dependent profile and tested to determine if the range of the last profile entered is less than the maximum output range specified. If so, an integer error flag is returned and the SU exited, depending on the values of logical error flags set in the external calling CSCI itself.

The REFINIT SU also tests for valid refractivity level entries for each profile. If the last gradient in any profile is negative, it returns an integer error flag and the SU is exited. If no errors are detected, the REFINIT SU then extrapolates the environmental profiles vertically to $1000 \mathrm{~km}$ in height. Extrapolation is not performed horizontally from the last provided profile; rather, the last provided environment profile is duplicated at

$10^{7} \mathrm{~km}$ in range. This duplication of profiles is performed by the REFINTER SU. Finally, the REFINIT SU checks if an evaporation duct profile has been specified.

\subsubsection{Remove Duplicate Refractivity Levels (REMDUP) SU}

The REMDUP SU removes any duplicate refractivity levels in the currently interpolated profile.

\subsubsection{RG Trace (RGTRACE) SU}

The RGTRACE SU performs ray traces of many rays launched within an angle of $\pm 4^{\circ}$. All angles from rays striking the surface are then sorted and stored for subsequent interpolation in the GRAZE_INT SU.

\subsubsection{Terrain Initialization (TERINIT) SU}

The TERINIT SU examines and initializes terrain arrays for subsequent use in PE calculations. It tests for and determines a range increment if it is found that range/height points are provided in fixed range increments. The minimum terrain height is determined and the entire terrain profile is adjusted so all internal calculations are referenced to this height. This is done in order to minimize the PE transform calculation volume.

\subsubsection{Trace to Output Range (TRACE_ROUT) SU}

The TRACE_ROUT SU traces a single ray, whose launch angle is specified by the calling routine, to each output range. The height of this ray is stored at each output range for subsequent proper indexing and accessing of the appropriate sub-models.

\subsubsection{Trace to next Step (TRACE_STEP) SU}

The TRACE_STEP SU performs one ray trace step for a given starting angle, range, and height. When passed a starting angle, range, and height for a single ray, it will trace to the first boundary that occurs, whether that is a refractivity level or the surface. It then passes back the ending angle, range and height and a flag indicating if the ray has hit the surface. 


\subsubsection{Troposcatter Initialization (TROPOINIT) SU}

The TROPOINIT SU initializes all variables and arrays needed for subsequent troposcatter calculations. The tangent range and tangent angle are determined from the source and from all receiver heights and stored in arrays.

\subsubsection{Starter Field Initialization (XYINIT) SU \\ The XYINIT SU calculates the complex PE solution at range zero.}

Several constant terms that will be employed over the entire PE mesh are calculated. These terms are the angle difference between mesh points in p-space and a height-gain value at the source (transmitter).

For each point in the PE p-space mesh, the antenna pattern ANTPAT SU is referenced to obtain an antenna pattern factor for a direct-path ray and a surface-reflected ray. The complex portions of the PE solution are then determined from the antenna pattern factors, elevation angle, and antenna height. The initial field assumes the source is over a perfectly conducting ground.

\subsubsection{Advanced Propagation Model Step (APMSTEP) CSC}

The APMSTEP CSC advances the entire APM CSCI algorithm one output range step, referencing various SUs to calculate the propagation loss at the current output range. At this current range, APM calculations will be made within the vertical (up to the maximum PE height region) by accessing the appropriate region's SUs.

The current output range is determined. The PESTEP SU is referenced to obtain the PE portion of the propagation loss at this new range.

For an airborne application, the FEDR SU is referenced to obtain the FE portion of the propagation loss above and below the PE maximum propagation angle.

If running in full hybrid mode, then based upon a height array index used within the FE region, a determination is made for the necessity to include FE propagation calculations. If so, the FEM SU is referenced to obtain the FE portion of the propagation loss. If a FE calculation is made, the maximum height index for the RO region is adjusted (with the minimum height index corresponding to the maximum height index of the $\mathrm{PE}$ region), and the ROLOSS SU is referenced to obtain the RO portion of the propagation loss at the current range. FE and RO propagation loss will be computed only up to the range at which $\mathrm{XO}$ calculations will be performed.

If running in a partial hybrid (PE + XO) mode, then only the PESTEP SU will be referenced to obtain the $\mathrm{PE}$ portion of the propagation loss at this new range. 
Finally, absorption loss is computed for the current range and added to the propagation loss at all heights.

\subsubsection{Calculate Propagation Loss (CALCLOS) SU}

The CALCLOS SU determines the propagation loss from the complex PE field at each output height point at the current output range.

The local ground height at the current output range is determined. All propagation loss/factor values at output height points up to the local ground height are then set to -32766. The first valid loss point is determined corresponding to the first output height point above the ground height. Next, the last valid loss point is determined based on the smaller of the maximum output height or the height traced along the maximum $\mathrm{PE}$ propagation angle to the current output range.

From the height of the first valid loss point to the height of the last valid loss point, the GETPFAC SU is referenced to obtain the propagation factor in $\mathrm{dB}$ (field strength relative to free space) at all corresponding output heights at the previous and current PE ranges. Then, for each valid output height, horizontal interpolation in range is performed to obtain the propagation factor at the current output range. From the propagation factor and the free-space loss, the propagation loss at each valid output height is determined, with the propagation loss/factor set to -32767 for all output height points above the last valid output height but less than the maximum output height.

If running in full or partial hybrid modes, the propagation factor at the top of the $\mathrm{PE}$ region is determined at every output range and stored in an array for future reference in XO calculations. If troposcatter calculations are desired, the TROPOSCAT SU is referenced with the results added to the propagation loss/factor array. Absorption loss is computed for the current range and added to the propagation loss at all heights. All loss/factor values returned to the external CSCI at this point are in centibels $(10 \mathrm{cB}=1 \mathrm{~dB})$.

\subsubsection{Current Wind (FN_CURWIND) Function}

The FN_CURWIND function performs a linear interpolation in range to get the current wind speed at the specified range.

\subsubsection{Dielectric Constant (FN_DIECON) Function}

The FN_DIECON function extracts the complex dielectric constant at a particular range.

\subsubsection{DoShift SU}

The DOSHIFT SU shifts the complex PE field by the number of bins, or PE mesh heights corresponding to the local ground height. 
Upon entering the number of bins to be shifted are determined. The PE solution is then shifted downward if the local ground is currently at a positive slope, and upward if the local ground is at a negative slope.

\subsubsection{Discrete Sine/Cosine Fast-Fourier Transform (DRST) SU}

A function with a common period, such as a solution to the wave equation, may be represented by a series consisting of sines and cosines. This representation is known as a Fourier series. An analytical transformation of this function, known as a Fourier transform, may be used to obtain a solution for the function.

The solution to the PE approximation to Maxwell's wave equation is to be obtained by using such a Fourier transformation function. The APM CSCI requires the real-valued sine and cosine transformations in which the real and imaginary parts of the PE equation are transformed separately. A Fourier transformation for possible use with the APM CSCI is described by Bergland (1969) and Cooley, Lewis, and Welsh (1970).

\subsubsection{Flat-Earth Direct Ray (FEDR) SU}

The FEDR SU determines propagation loss based on FE calculations for the direct ray path only for regions above and below the PE maximum propagation angle.

\subsubsection{Flat-Earth Model (FEM) SU}

The FEM SU computes propagation loss at a specified range based upon FE approximations. Receiver heights are corrected for earth curvature and average refraction based on twice the effective earth radius computed in the GET_K SU. The following steps are performed for each APM output height.

1. The path lengths and elevation angles for the direct-path and surface-reflected path, along with the grazing angle, are computed from simple right-triangle calculations. Using the two elevation angles, the ANTPAT SU is referenced to obtain an antenna pattern factor for each angle. Using the grazing angle, the GETREFCOEF SU is referenced to obtain the magnitude and phase lag of the surface reflection coefficient.

2. From the path length difference, the phase lag of the surface reflected ray, and the wave number, a total phase lag is determined. Using the total phase lag, the magnitude of the surface reflection coefficient and the two antenna pattern factors, the two ray components are coherently summed to obtain a propagation factor. The propagation factor, together with the free-space propagation loss and path length difference of the direct-path ray, are used to compute the propagation loss.

\subsubsection{Fast-Fourier Transform (FFT) SU}

The FFT SU separates the real and imaginary components of the complex PE field into two real arrays and then reference the DRST SU that transforms each portion of the PE solution. 


\subsubsection{Free Space Range Step (FRSTP) SU}

The FRSTP SU propagates the complex PE solution field in free space by one range step.

The PE field is transformed to p-space and then multiplied by the free-space propagator. Before exiting, the PE field is transformed back to z-space. Both transforms are performed using a FFT SU.

\subsubsection{FZLIM SU}

The FZLIM SU determines the propagation factor (in $\mathrm{dB}$ ) and the outgoing propagation angle at the top of the PE calculation region. These values, along with the corresponding PE range, are stored for future reference by the XOINIT SU.

The GETPFAC SU is referenced to determine the propagation factor at the last height mesh point in the valid part of the PE region. The propagation factor, along with the range and the local ray angle (determined from the ray traced separating the RO and PE regions), is stored if this is the first call to the FZLIM SU. The SPECEST SU is then referenced to determine the outgoing propagation angle. Depending on the change of angles from one range step to the next, the calculated outgoing angle will be limited. The storage array counter is incremented and the outgoing angle stored.

Before exiting, the SAVEPRO SU is referenced to store the refractivity profiles from the top of the PE region to the maximum specified coverage height.

\subsubsection{Get Propagation Factor (FN_GETPFAC) Function}

The FN_GETPFAC function determines the propagation factor at the specified height in decibels.

A vertical interpolation with height on the PE solution field is performed to obtain the magnitude of the field at the desired output height point. An additional calculation is made and the propagation factor is then returned in decibels.

\subsubsection{Get Reflection Coefficient (GETREFCOEF) SU}

The GETREFCOEF SU calculates the complex surface reflection coefficient, along with the magnitude and phase angle.

The complex reflection coefficient is computed from a specified grazing angle and is based on the Fresnel reflection coefficient equations for vertical and horizontal polarization. The magnitude and phase angle are determined from the complex reflection coefficient. If rough surface calculations are required, the smooth surface reflection coefficient is then modified by the Miller-Brown rough surface reduction factor, which is a function of wind speed and grazing angle. 


\subsubsection{Get Troposcatter Loss (FN_GET_TLOSS) Function}

The FN_GET_TLOSS function determines the loss due to troposcatter and computes the appropriate loss from troposcatter and diffraction for a specific transmitter and receiver point over land and water. For each transmitter/receiver pair, the following steps are performed.

1. If the current output range is less than the minimum diffraction field range for a particular receiver height, then the SU is exited and no troposcatter loss is computed.

2. The tangent angle from the receiver height is determined.

3. The common volume scattering angle is determined and calculations are performed to obtain the loss due to troposcatter.

Troposcatter loss is compared to propagation loss. If the difference between the propagation loss and troposcatter loss is less than $18 \mathrm{~dB}$, then the corresponding power levels of the two loss values are added. If the difference is greater than $18 \mathrm{~dB}$, then the lesser of the two losses is used.

\subsubsection{Linear Interpolation (FN_PLINT) Function}

The FN_PLINT function performs a linear interpolation on two input parameters passed to the function.

\subsubsection{Mixed Fourier Transform (MIXEDFT) SU}

The MIXEDFT SU propagates the PE field in free space one PE range step, applying the Leontovich boundary condition, using the mixed Fourier transform as outlined by Kuttler and Dockery (1991). For finite conducting boundaries (i.e., if vertical polarization is specified or rough surface calculations are required) and the frequency is less than $400 \mathrm{MHz}$, the central difference form of the DMFT is used. If the frequency is greater than $400 \mathrm{MHz}$, the backward difference form of the DMFT is used.

\subsubsection{Parabolic Equation Step (PESTEP) SU}

The PESTEP SU advances the PE solution one output range step, referencing various SUs to calculate the propagation loss at the current output range.

The next output range is determined and an iterative loop begun to advance the $\mathrm{PE}$ solution such that for the current PE range, a PE solution is calculated from the solution at the previous PE range. This procedure is repeated until the output range is reached.

At each PE range step, the local ground height is determined and the PE field is "shifted" by the number of bins or PE mesh height points corresponding to the local ground height. This is performed in the DOSHIFT SU. 
If the APM CSCI is used in a range-dependent mode, that is, more than one profile has been input; or a terrain profile is specified, the REFINTER SU is referenced to compute a new modified refractive index profile adjusted by the local ground height at the current range. A new environmental phase term is computed using this new refractivity profile.

If using vertical polarization and the current ground type has changed from the previous one, or rough surface calculations are required, a GETALN SU is referenced to determine the impedance term and all associated variables used for the mixed transform calculations.

If rough surface calculations are required, or if using vertical polarization, then a MIXEDFT SU is referenced, otherwise, a FRSTP SU is referenced to advance the PE solution one range step in free space. The environmental phase term is then applied to obtain the new final PE solution at the current range. Once all calculations are made to determine the PE field at the current PE range, the FZLIM SU is referenced to determine and store the outgoing propagation factor and propagation angle at the top of the PE region. The FZLIM SU is only referenced if running in full or partial hybrid modes. Finally, a CALCLOS SU is referenced to obtain the propagation loss/factor at the desired output heights at the current output range.

\subsubsection{Ray Trace (RAYTRACE) SU}

Using standard ray trace techniques, a ray is traced from a starting height and range with a specified starting elevation angle to a termination range. As the ray is being traced, an optical path length difference and a derivative of range with respect to elevation angle are being continuously computed. If the ray should reflect from the surface, a grazing angle is determined. Upon reaching the termination range, a terminal elevation angle is determined along with a termination height.

\subsubsection{Refractivity Interpolation (REFINTER) SU}

The REFINTER SU interpolates horizontally and vertically on the modified refractivity profiles. Profiles are then adjusted so they are relative to the local ground height.

If range-dependent refractive profiles have been specified, horizontal interpolation to the current $\mathrm{PE}$ range is performed between the two neighboring profiles. A REMDUP SU is referenced to remove duplicate refractivity levels, and the PROFREF $\mathrm{SU}$ is then referenced to adjust the new profile relative to the internal reference height corresponding to the minimum height of the terrain profile. The PROFREF SU is referenced once more to adjust the profile relative to the local ground height, and upon exit from the PROFREF SU, the INTPROF SU is referenced to interpolate vertically on the refractivity profile at each $\mathrm{PE}$ mesh height point. 


\subsubsection{Ray Optics Calculation (ROCALC SU)}

The ROCALC SU computes the RO components needed in the calculation of propagation loss at a specified range and height within the RO region. These components are the amplitudes for a direct-path and surface-reflected ray, and the total phase lag angle between the direct-path and surface-reflected rays.

A test is made to determine if this is the first RO calculation. If an initial calculation is needed, the height, range, and elevation angle array indices are set to initial conditions. If not, the array indices are incremented from the previous RO calculation.

The following steps are performed for each series of vertical grid points, in a manner that ensures that RO calculations have been performed at ranges that span the current range of interest. The vertical grid points are taken in order, beginning with the one with the greatest height.

1. Using a Newton iteration method with a varying elevation angle, the RAYTRACE SU is referenced to find a direct-path ray and a surface-reflected ray originating at the transmitter height and terminating at the same grid point. Should a direct or reflected ray not be found to satisfy the condition, or should the computed grazing angle exceed the grazing angle limit, the height array index is adjusted to redefine the lower boundary of the RO region. Should the ray trace conditions be satisfied, the RAYTRACE SU will provide a terminal elevation angle, a derivative of range with respect to elevation angle, a path length, and for the surface-reflected ray, a grazing angle.

2. Using the final direct-path ray and surface-reflected ray elevation angles obtained from the Newton iteration method, the ANTPAT SU is referenced to obtain an antenna pattern factor for each angle. The GETREFCOEF SU is referenced to obtain the amplitude and phase lag angle of the surface reflection coefficient.

3. Using the antenna pattern factors, path length differences, and surface-reflection coefficients, the necessary RO components defined in the first paragraph above are calculated.

\subsubsection{Ray Optics Loss (ROLOSS) SU}

The ROLOSS SU calculates propagation factor and loss values at all valid RO heights at a specified range based based upon the components of magnitude for a directpath and surface-reflected ray and the total phase lag angle between the two rays as determined by the ROCALC SU.

For purposes of computational efficiency, an interpolation from the magnitude and total phase lag arrays, established by the ROCALC SU, is made to obtain these three quantities at each APM vertical output mesh point within the RO region. 
From the interpolated phase lag and ray amplitudes, a propagation factor is calculated that is used, in turn, with the free-space loss to obtain a propagation loss at each vertical APM output point. Absorption loss is computed for the current range and added to the propagation loss at all heights.

\subsubsection{Save Profile (SAVEPRO) SU}

The SAVEPRO SU stores refractivity profiles at each PE range step from the top of the PE region to the maximum user-specified height. This is only done if running in full or partial hybrid modes.

The refractivity height level just exceeding the PE region height limit is determined. From this level upward, all heights, M-units, and gradients are stored.

\subsubsection{Spectral Estimation (SPECEST) SU}

The SPECEST SU determines the outward propagation angle at the top of the PE calculation region or the grazing angle at the lower part of the PE region based on spectral estimation. The outward propagation angle is used for XO calculations and the grazing angle is used for rough surface calculations.

The upper 8 (if running smooth surface case) or 16 (if running terrain case) bins of the complex PE field at the current $\mathrm{PE}$ range are separated into their real and imaginary components. The upper $1 / 4$ of this portion of the field is then filtered and zeropadded to 256 points. It is then transformed to its spectral components via a reference to the DRST SU. The amplitudes of the spectral field are then determined and a 3-point average is performed. The peak of the 256-point field is found and the outgoing propagation angle is determined from the peak value.

\subsubsection{Surface Impedance (SURFIMP) SU}

The SURFIMP SU computes the normalized average surface impedance for surface wave propagation by vertically polarized waves along the sea surface. This is done for frequencies less than $50 \mathrm{MHz}$.

\subsubsection{Troposcatter (TROPOSCAT) SU}

The TROPOSCAT SU determines the loss due to troposcatter and to compute the appropriate loss from troposcatter and propagation loss for large receiver ranges.

The current output range is updated and the tangent angle from the source to the current output range is initialized. For all output receiver heights at the current output range, the following procedure is performed.

\subsubsection{Extended Optics Initialization (XOINIT) CSC}

The XOINIT SU initializes the range, height, and angle arrays in preparation for the XOSTEP CSC. 
Upon entering, if $\mathrm{XO}$ calculations are not required, the APMCLEAN SU is referenced to deallocate all arrays used for the current application, then the CSC is exited.

If XO calculations are required, all arrays used for XO calculations are allocated and initialized to 0 . The ranges and angles previously stored from referencing the FZLIM SU are now used to initialize the range and angle arrays for XO calculations. A 10-point smoothing average on the angle array is performed twice via reference to the MEANFILT SU. Upon exiting, the height array and initial height index for start of XO calculations are initialized.

\subsubsection{Advanced Propagation Model Clean (APMCLEAN) SU}

The APMCLEAN CSC deallocates all dynamically dimensioned arrays used in one complete run of APM calculations.

\subsubsection{Clutter-to-Noise (CLUTTER) SU}

The CLUTTER SU computes the clutter-to-noise ratio in $\mathrm{dB}$ at each output range based on the radar range equation over land or water.

The clutter is computed over water based on a modification to the Georgia Institute of Technology (GIT) reflectivity model, which is valid for frequencies greater than $1 \mathrm{GHz}$.

For those portions of the path over land the reflectivity is determined based on the "constant-gamma" model, where a parameter describing the backscattering effectiveness of the surface must be provided.

\subsubsection{Diffraction Loss (FN_DLOSS) Function}

The FN_DLOSS function computes loss in the diffraction region based on the CCIR model for standard atmosphere.

\subsubsection{Get Theta (GETTHETA) SU}

The GETTHETA SU calculates the optical phase-lag difference angle from the reflection range found in the R1ITER SU.

\subsubsection{GIT Initialization (GIT_INIT) SU}

The GIT_INIT SU initializes all variables used in the calculation of the reflectivity based on a modified version of the GIT model.

\subsubsection{GofZ (GOFZ) Function}

The GOFZ function calculates the diffraction region height-gain in $\mathrm{dB}$ from the CCIR diffraction region model for standard atmosphere. 


\subsubsection{Mean Filter (MEANFILT) SU}

The MEANFILT SU performs an n-point average smoothing on any array passed to it.

\subsubsection{Optical Region Limit (OPLIMIT) SU}

The OPLIMIT SU calculates the maximum range in the optical interference region and the corresponding loss at that range.

\subsubsection{Optical Difference (OPTICF) SU}

The OPTICF SU calculates the optical path-length difference angle by solving a cubic equation for the reflection point range.

\subsubsection{R1 Iteration (R1ITER) SU}

The R1ITER SU finds the range of the reflection point corresponding to a particular launch angle.

\subsubsection{Standard Propagation Model Initialization (SPM_INIT) SU}

The SPM_INIT SU initializes many of the variables used throughout the SPM SU.

\subsubsection{Standard Propagation Model (SPM) SU}

The SPM SU computes the propagation factor for a standard atmosphere only, with the assumption of omni-directional antenna patterns.

\subsubsection{Extended Optics Step (XOSTEP) CSC}

The XOSTEP CSC advances the APM CSCI algorithm one output range step from the top of the PE calculation region to the maximum output height specified, referencing various SUs to calculate the propagation loss and factor at the current output range.

Upon entering the XOSTEP CSC, the current output range is determined. The EXTO SU is referenced to obtain the XO portion of the propagation loss and factor at this new range.

If running in full hybrid mode, based on a height array index used within the FE region, determine if it is necessary to include FE propagation calculations. If necessary, the FEM SU is referenced to obtain the FE portion of the propagation loss and factor. If an FE calculation is made, the maximum height index for the RO region is adjusted (with the minimum height index corresponding to the maximum height index of the PE region), and the ROLOSS SU is referenced to obtain the RO portion of the propagation loss and factor at the current range. 
If running in partial hybrid mode, then only the EXTO SU is referenced to obtain the $\mathrm{XO}$ portion of the propagation loss and factor at this new range. The maximum height will correspond to the maximum user-specified coverage height.

Finally, the APMCLEAN SU is referenced to deallocate all allocated arrays used throughout the run.

\subsubsection{Extended Optics (EXTO) SU}

The EXTO SU calculates propagation loss and factor, based on extended optics techniques, at the current output range.

Upon entering, array indices for the current range, height, and angle arrays are initialized. A ray trace is then performed for all rays from the last output range to the current output range. The current heights are then sorted, along with their corresponding propagation factors. The propagation loss is then determined at each output receiver height by interpolation on the terminal heights of the traced rays.

Upon exiting, a reference to the TROPOSCAT SU provides any troposcatter losses and is added to the loss array. Absorption loss is also added to the propagation loss at all heights.

\subsubsection{Return Grazing Angle (RET_GRAZE) CSC}

The RET_GRAZE CSC interpolates the grazing angle to every output range step, and if necessary, will interpolate the propagation angles in height at every output range.

\subsection{CSCI EXTERNAL INTERFACE REQUIREMENTS}

The APM CSCI is accessed through the APMINIT CSC by a subroutine call from the external CSCI, which should provide, as global data elements, the values specified in Table 1 through Table 4.

The APM CSCI external data elements, i.e., those data that must be provided by the calling external CSCI prior to the APM CSCI execution may be divided into four classifications. The first classification is external data related to the atmospheric environment (Table 1), the second is data related to the EM system (Table 2), the third is data related to the implementation of the APM CSCI by the external CSCI (Table 3), and the fourth is data related to the terrain information (Table 4). Each table lists the type, units, and bounds of each data element. Table 5 specifies the output data of the APM CSCI model. 
Table 1. APM CSCl environmental data element requirements.

\begin{tabular}{|c|c|c|c|c|}
\hline Name & Description & Type & Units & Bounds \\
\hline refmsl & $\begin{array}{l}\text { Modified refractivity profile (dynamically } \\
\text { allocated) array referenced to mean sea level }\end{array}$ & real & M & $\geq 0.0^{\mathrm{a}}$ \\
\hline hmsl & Profile height (dynamically allocated) array & real & meters & See note b \\
\hline$n_{\text {prof }}$ & Number of refractivity profiles & integer & N/A & $\geq 1$ \\
\hline lvlp & Number of profile levels & integer & N/A & $\geq 2$ \\
\hline rngprof & $\begin{array}{l}\text { Dynamically allocated array of ranges to each } \\
\text { profile }\end{array}$ & real & meters & $\geq 0.0$ \\
\hline$a b s_{\text {hum }}$ & Surface absolute humidity & real & $\mathrm{g} / \mathrm{m}^{3}$ & 0 to $50^{\mathrm{c}}$ \\
\hline$t_{\text {air }}$ & Surface air temperature & real & ${ }^{\circ} \mathrm{C}$ & -20 to $40^{c}$ \\
\hline$\gamma_{a}$ & Surface specific attenuation & real & $\mathrm{dB} / \mathrm{km}$ & $\geq 0.0$ \\
\hline$i_{\text {extra }}$ & $\begin{array}{l}\text { Extrapolation flag for refractivity profiles } \\
\text { entered in combination with terrain below } \\
\text { mean sea level }\end{array}$ & integer & N/A & 0 or 1 \\
\hline$n_{w}$ & $\begin{array}{l}\text { Number of wind speeds and corresponding } \\
\text { ranges }\end{array}$ & integer & N/A & $\geq 0.0$ \\
\hline rngwind & $\begin{array}{l}\text { Dynamically allocated array of ranges } \\
\text { specified for each wind speed in wind(). }\end{array}$ & real & meters & $\geq 0.0$ \\
\hline wind & Dynamically allocated array of wind speeds. & real & $\begin{array}{l}\text { meters/ } \\
\text { second }\end{array}$ & 0.0 to $20.0^{\mathrm{d}}$ \\
\hline wind $_{\text {dir }}$ & $\begin{array}{l}\text { Angle between antenna boresight and upwind } \\
\text { direction }\end{array}$ & real & degrees & 0.0 to 360.0 \\
\hline
\end{tabular}

${ }^{\mathrm{a}}$ Couplets of height and modified refractivity associated with that height are referred to within this document as a refractivity profile.

${ }^{\mathrm{b}}$ All heights in the refractivity profile must be steadily increasing.

${ }^{\mathrm{C}}$ The CCIR gaseous absorption model implemented within APM provides a $\pm 15 \%$ accuracy for absolute humidity and surface air temperature within these bounds. While values beyond these limits are allowed within APM, note that this may result in less accurate attenuation rates being calculated.

${ }^{d}$ The maximum wind speed will vary depending on frequency. For frequencies less than $10 \mathrm{GHz}$, the maximum that can be specified is $20 \mathrm{~m} / \mathrm{s}$. Above $10 \mathrm{GHz}$, the maximum wind speed that can be specified will decrease to an absolute maximum of $15 \mathrm{~m} / \mathrm{s}$ at $20 \mathrm{GHz}$ and above. 
Table 2. APM CSCI external EM system data element requirements.

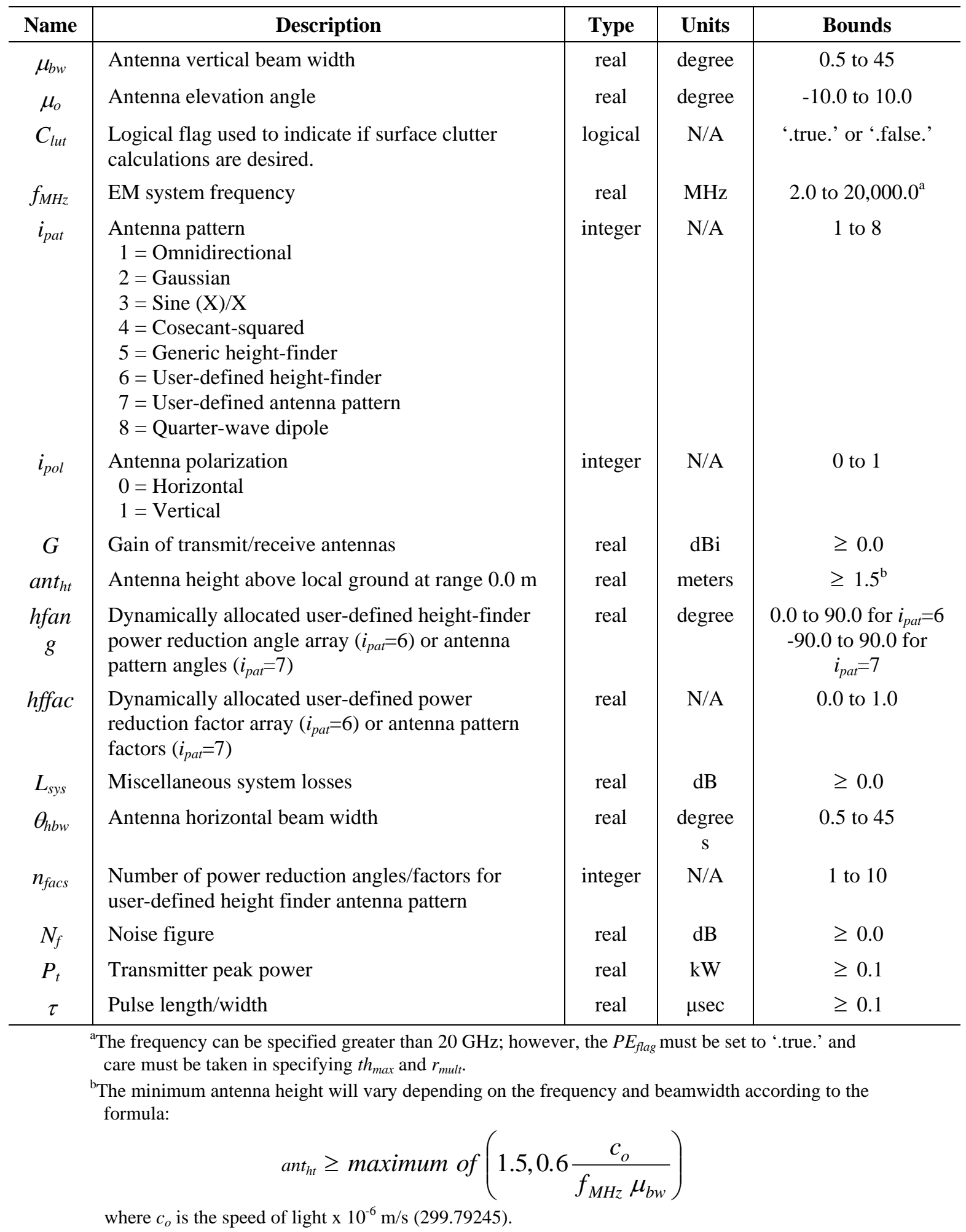


Table 3. APM CSCI external implementation data element requirements.

\begin{tabular}{|c|c|c|c|c|}
\hline Name & Description & Type & Units & Bounds \\
\hline$h_{\max }$ & $\begin{array}{l}\text { Maximum height output for a particular application of } \\
\text { APM }\end{array}$ & real & meters & $\geq 100.0^{\mathrm{a}}$ \\
\hline$h_{\min }$ & $\begin{array}{l}\text { Minimum height output for a particular application of } \\
\text { APM }\end{array}$ & real & meters & $\geq 0.0^{\mathrm{a}}$ \\
\hline lang & $\begin{array}{l}\text { Propagation angle and factor output flag } \\
\text { ‘.true.'= Output propagation angle and propagation } \\
\text { factor for direct and reflected ray (where applicable). } \\
\text { ‘.false.' = Do not output propagation angles and } \\
\text { factors }\end{array}$ & logical & N/A & $\begin{array}{l}\text { ‘.true.’ or } \\
\text { ‘.false.'b }\end{array}$ \\
\hline lerr6 & Logical flag to allow for error -6 to be bypassed & logical & N/A & $\begin{array}{l}\text { ‘.true.’ or } \\
\text { ‘.false. }{ }^{\mathrm{c}}\end{array}$ \\
\hline lerr12 & Logical flag to allow for error -12 to be bypassed & logical & N/A & $\begin{array}{l}\text { ‘.true.’ or } \\
\text { ‘ffalse.'c }\end{array}$ \\
\hline$n_{\text {rout }}$ & $\begin{array}{l}\text { Number of range output points for a particular } \\
\text { application of APM }\end{array}$ & $\begin{array}{l}\text { intege } \\
\mathrm{r}\end{array}$ & N/A & $\geq 1$ \\
\hline$n_{\text {zout }}$ & $\begin{array}{l}\text { Number of height output points for a particular } \\
\text { application of APM }\end{array}$ & $\underset{r}{\text { intege }}$ & N/A & $\geq 1$ \\
\hline$n_{\text {zout_rtg }}$ & $\begin{array}{l}\text { Number of height output points for receiver heights } \\
\text { relative to the local ground elevation. }\end{array}$ & $\underset{r}{\text { intege }}$ & N/A & $\geq 0$ \\
\hline$P E_{\text {flag }}$ & $\begin{array}{l}\text { Flag to indicate use of PE algorithm only: } \\
\text { ‘.true.' = only use PE sub-model } \\
\text { ‘.false.' = use automatic hybrid model }\end{array}$ & logical & N/A & $\begin{array}{l}\text { ‘.true.' or } \\
\text { '.false.' }\end{array}$ \\
\hline$r_{\max }$ & $\begin{array}{l}\text { Maximum range output for a particular application of } \\
\text { APM }\end{array}$ & real & meters & $\geq 5000.0^{\mathrm{c}}$ \\
\hline$r_{\text {mult }}$ & PE-range step multiplier & real & N/A & $>0.0^{\mathrm{c}}$ \\
\hline$t h_{\max }$ & Visible portion of PE maximum calculation angle & real & $\underset{\mathrm{s}}{\operatorname{degree}}$ & $>0.0^{\mathrm{c}}$ \\
\hline$T_{\text {ropo }}$ & Logical flag to include troposcatter calculations. & $\begin{array}{l}\text { intege } \\
\mathrm{r}\end{array}$ & N/A & $\begin{array}{l}\text { ‘.true.’ or } \\
\text { ‘ffalse.' }\end{array}$ \\
\hline zout_rtg & $\begin{array}{l}\text { Dynamically allocated array of receiver heights } \\
\text { specified relative to the local ground height. }\end{array}$ & real & meters & $\geq 0.0$ \\
\hline
\end{tabular}

a Refer to section 7.2 for a complete description.

${ }^{\mathrm{b}}$ This flag should not be enabled when any portion of the propagation path is over land.

' Refer to section 4.3 .4 for a complete description. 
Table 4. APM CSCI external terrain data element requirements.

\begin{tabular}{|c|c|c|c|c|}
\hline Name & Description & Type & Units & Bounds \\
\hline terx & Dynamically allocated terrain profile range array & real & meters & $\geq 0.0^{\mathrm{a}}$ \\
\hline tery & Dynamically allocated terrain profile height array & real & meters & $\geq 0.0^{\mathrm{a}}$ \\
\hline$\gamma c$ & $\begin{array}{l}\text { Dynamically allocated array of constants describing } \\
\text { the backscattering effectiveness of the surface }\end{array}$ & real & $\mathrm{dB}$ & $\begin{array}{l}-100.0 \leq \gamma C \\
\leq 100.0\end{array}$ \\
\hline$\gamma r n g$ & $\begin{array}{l}\text { Dynamically allocated array of ranges corresponding } \\
\text { to the values in } \gamma c\end{array}$ & real & meters & $\geq 0.0$ \\
\hline$i_{g c}$ & $\begin{array}{l}\text { Number of } \gamma c \text { values for a particular application of } \\
\text { APM }\end{array}$ & $\underset{r}{\text { intege }}$ & N/A & $\geq 0$ \\
\hline$i_{t p}$ & $\begin{array}{l}\text { Number of terrain profile points for a particular } \\
\text { application of APM }\end{array}$ & $\begin{array}{l}\text { intege } \\
\mathrm{r}\end{array}$ & N/A & $\geq 2$ \\
\hline$i_{g r}$ & $\begin{array}{l}\text { Number of ground types for a particular application } \\
\text { of APM }\end{array}$ & $\begin{array}{l}\text { intege } \\
\mathrm{r}\end{array}$ & N/A & $\geq 0^{\mathrm{a}}$ \\
\hline igrnd & $\begin{array}{l}\text { Array of ground composition types for a particular } \\
\text { application of APM } \\
0=\text { Sea water } \\
1=\text { Fresh water } \\
2 \text { = Wet ground } \\
3=\text { Medium dry ground } \\
4 \text { = Very dry ground } \\
5=\text { Ice at }-1^{\circ} \mathrm{C} \\
6 \text { = Ice at }-10^{\circ} \mathrm{C} \\
7 \text { = User-defined }\end{array}$ & $\begin{array}{l}\text { intege } \\
\mathrm{r}\end{array}$ & $\mathrm{N} / 0 \mathrm{~A}$ & $\begin{array}{c}0 \leq \text { igrnd } \leq \\
7^{\mathrm{a}}\end{array}$ \\
\hline rgrnd & $\begin{array}{l}\text { Dynamically allocated array of ranges for which } \\
\text { ground types are applied for a particular application } \\
\text { of APM }\end{array}$ & real & meters & $\geq 0.0^{\mathrm{a}}$ \\
\hline dielec & $\begin{array}{l}\text { Dynamically allocated two-dimensional array of } \\
\text { relative permittivity }\left(\varepsilon_{r}\right) \text { and conductivity }(\sigma) \text { for a } \\
\text { particular application of APM }\end{array}$ & real & $\begin{array}{c}\varepsilon_{r}-\mathrm{N} / \mathrm{A} \\
\sigma- \\
\text { Siemens/ } \\
\text { meter }\end{array}$ & $>0^{\mathrm{a}}$ \\
\hline
\end{tabular}

arefer to section 7.3 for a complete description 
Table 5. APM CSCl output data element requirements.

\begin{tabular}{|c|c|c|c|c|}
\hline Name & Description & Type & Units & Source \\
\hline CNR & Clutter-to-Noise ratio array & real & $\mathrm{dB}$ & XOINIT CSC \\
\hline$\Psi_{\text {rout }}$ & $\begin{array}{l}\text { Array of grazing angles at each output range } \\
r_{\text {out }}\end{array}$ & real & radians & RET_GRAZE SU \\
\hline$i_{\text {error }}$ & $\begin{array}{l}\text { Integer value that is returned if an error } \\
\text { occurs in called routine }\end{array}$ & integer & N/A & $\begin{array}{l}\text { APMINIT CSC } \\
\text { RET_GRAZE SU } \\
\text { XOINIT CSC }\end{array}$ \\
\hline$i_{\text {xostp }}$ & $\begin{array}{l}\text { Index of output range step at which } \mathrm{XO} \\
\text { model is to be applied }\end{array}$ & integer & N/A & APMINIT CSC \\
\hline$j_{\text {end }}$ & $\begin{array}{l}\text { Output height index at which valid } \\
\text { propagation loss values end }\end{array}$ & integer & N/A & APMSTEP CSC \\
\hline$j_{\text {start }}$ & $\begin{array}{l}\text { Output height index at which valid } \\
\text { propagation loss values begin }\end{array}$ & integer & N/A & APMSTEP CSC \\
\hline$j_{x e n d}$ & $\begin{array}{l}\text { Output height index at which valid XO } \\
\text { propagation loss values end }\end{array}$ & integer & N/A & XOSTEP CSC \\
\hline$j_{\text {xstart }}$ & $\begin{array}{l}\text { Output height index at which valid XO } \\
\text { propagation loss values begin }\end{array}$ & integer & N/A & XOINIT CSC \\
\hline$l_{\text {graze }}$ & $\begin{array}{l}\text { Logical flag indicating if grazing angles } \\
\text { were computed for a particular application } \\
\text { of APM }\end{array}$ & logical & N/A & APMINIT CSC \\
\hline$m p f l$ & Propagation loss and factor array & integer & $\mathrm{cB}$ & $\begin{array}{l}\text { APMSTEP CSC } \\
\text { XOSTEP CSC }\end{array}$ \\
\hline mpfl_rtg & $\begin{array}{l}\text { Propagation loss and factor at receiver } \\
\text { heights specified in the zout_rtg array }\end{array}$ & integer & $\mathrm{cB}$ & APMSTEP CSC \\
\hline propaf & $\begin{array}{l}\text { Two-dimensional array, containing the } \\
\text { propagation angles and factors for the direct } \\
\text { and reflected rays (where applicable) for all } \\
\text { output height/range points }\end{array}$ & real & radians, $\mathrm{dB}$ & $\begin{array}{l}\text { APMSTEP CSC } \\
\text { XOSTEP CSC }\end{array}$ \\
\hline$r_{\text {out }}$ & Current output range & real & meters & $\begin{array}{l}\text { APMSTEP CSC } \\
\text { XOSTEP CSC }\end{array}$ \\
\hline
\end{tabular}

\subsection{CSCI INTERNAL INTERFACE REQUIREMENTS}

Section 3.1 shows the relationship between the APM CSCI and its four CSCs APMINIT, APMSTEP, XOINIT, and XOSTEP. The required internal interface between these four CSCs and the APM CSCI is left to the designer. However, Table 7 should be used as a guide to the required internal interfaces in the CSCI. 


\subsection{CSCI INTERNAL DATA REQUIREMENTS}

The APM CSCI takes full advantage of Fortran 95 features, utilizing allocatable arrays for all internal and input arrays. The external CSCI designer must correctly allocate and initialize all arrays necessary for input to the APM CSCI.

Due to the computational intensity of the APM CSCI, it may not be necessary or desirable to use the extreme capability of the APM CSCI for all applications. The variables $n_{\text {rout }}$ and $n_{\text {zout }}$ refer to the desired number of range and height output points for any one particular application, and will be specified when the APMINIT CSC is called.

One of the parameters returned to the external application from the APMINIT CSC is $i_{\text {error }}$, which allows for greater flexibility in how input data are handled within the external application.

Table 6 lists all possible errors that can be returned.

Table 6. APMINIT SU returned error definitions.

\begin{tabular}{|c|c|}
\hline$i_{\text {error }}$ & Definition \\
\hline-5 & Frequency input must be greater than or equal to $2 \mathrm{MHz}$. \\
\hline-6 & $\begin{array}{l}\text { Last range in terrain profile is less than } r_{\text {max. }} \text { Will only return this error if lerr6 set to } \\
\text { '.true.’. }\end{array}$ \\
\hline-7 & $\begin{array}{l}\text { Specified cut-back angles (for user-defined height finder antenna pattern) are not } \\
\text { increasing. }\end{array}$ \\
\hline-8 & $h_{\max }$ is less than maximum height of terrain profile. \\
\hline-9 & Antenna height with respect to mean sea level is greater than maximum height $h_{\max }$. \\
\hline-10 & Beamwidth is less than or equal to zero for directional antenna pattern. \\
\hline-11 & $\begin{array}{l}\text { Number of antenna pattern or power reduction factors and angles is less than or equal } \\
\text { to } 1 \text {. For } i_{p a t}=6, n_{\text {facs }} \text { must be at least } 1 \text {; for } i_{p a t}=7, n_{\text {facs }} \text { must be at least } 2 \text {. }\end{array}$ \\
\hline-12 & $\begin{array}{l}\text { Range of last environment profile given (for range-dependent case) is less than } r_{\text {max }} \text {. } \\
\text { Will only return this error if lerr12 set to ‘.true.’. }\end{array}$ \\
\hline-13 & $\begin{array}{l}\text { Height of first level in any user-specified refractivity profile is greater than } 0 \text {. First } \\
\text { height must be at mean sea level }(0.0) \text { or }<0.0 \text { if below mean sea level. }\end{array}$ \\
\hline-14 & Last gradient in any environment profile is negative. \\
\hline-17 & Range points of terrain profile are not increasing. \\
\hline-18 & First range value in terrain profile is not 0. \\
\hline-21 & Clutter calculations are specified but no transmitter power has been provided. \\
\hline-22 & Clutter calculations are specified but no pulse length has been provided. \\
\hline-23 & Clutter calculations are specified, but no horizontal beamwidth has been provided. \\
\hline-24 & $\begin{array}{l}\text { Clutter calculations are desired over terrain or for frequencies less than } 1 \mathrm{GHz} \text {, but no } \\
\gamma c \text { values have been specified. }\end{array}$ \\
\hline
\end{tabular}


Table 6. APMinit SU returned error definitions. (continued)

\begin{tabular}{c|l}
\hline $\boldsymbol{i}_{\text {error }}$ & \multicolumn{1}{c}{ Definition } \\
\hline-25 & $\begin{array}{l}\text { Specified only the PE model to be used but did not specify maximum propagation } \\
\text { angle } h_{\max } \text {. }\end{array}$ \\
-26 & $\begin{array}{l}\text { Clutter calculations are specified with the propagation path partly or entirely, over } \\
\text { water but did not specify a wind speed. } \\
\text { Transmitter height is less than } 1.5 \text { meters. } \\
-41\end{array}$ \\
-42 & $\begin{array}{l}\text { Minimum height input by user, } h_{\text {min }} \text {, is greater than maximum height, } h_{\text {max }} . \\
\text { Transform size is greater than } 2^{30} .\end{array}$ \\
-44 & $\begin{array}{l}\text { Combination of frequency and antenna beamwidth results in antenna physically below } \\
\text { the surface. Increase frequency or beamwidth for valid combination. } \\
\text { Wind speed specified is greater than the maximum allowed for the specified } \\
\text { frequency. } \\
\text { Error in terrain ray trace (contact the APM CSCI developers if this occurs) } \\
\text { *WARNING*: Antenna height with respect to mean sea level is greater than the last } \\
\text { height in the refractivity profile at the source. }\end{array}$ \\
\hline
\end{tabular}

The logical variables lerr6 and lerr12, when set to '.false.', allow the external application to bypass their associated errors, as these are not critical to the operation of the APM CSCI.

The APM CSCI provides propagation loss and propagation factor for all heights and ranges when running in a full hybrid mode. When running in a partial hybrid mode, it provides propagation loss and factor for all heights, but not necessarily for all angles. Refer to Section 3.1 for environmental conditions under which each execution mode is automatically selected.

Absorption by atmospheric gases (oxygen and water vapor) may be important to some applications of the APM CSCI and is controlled by specifying a non-zero value for the absolute humidity, $a b s_{\text {hum }}$, and the surface air temperature, $t_{\text {air }}$, or likewise, by specifying a non-zero value for the gaseous absorption attenuation rate, $\gamma_{a}$.

A particular application of the APM CSCI may or may not require the consideration of troposcatter effects within the propagation loss/factor calculations. For example, a radar evaluation most likely would not be influenced by troposcatter; while an ESM evaluation would. APM has the feature of including or not including the troposcatter calculation by setting a logical flag called $T_{\text {ropo. }}$ Setting this flag to '.false.' would omit the calculation. Setting this flag to '.true.' would include the calculation. For the APM CSCI implementation within the external coverage and loss diagram applications, $T_{\text {ropo }}$ must be set to '.true.' so as to include the calculation. 
APM also has the added capability to account for rough sea surface effects. Specifying a wind speed and a corresponding range will produce forward scatter results based on the Philips ocean-wave model for the root-mean-squared (rms) wave height and the Miller-Brown reflection coefficient reduction factor. The capability also exists to allow variable wind speeds with range.

APM, by default, will run in an "automatic" mode in which, depending upon user-specified inputs, will choose the appropriate sub-models to use for a particular application. However, by setting the logical flag $P E_{\text {flag }}$ to '.true.' APM will be forced to use only the PE sub-model for a particular external application. By default, this flag is set to '.false.'. If this flag is '.true.' then the visible portion of the maximum PE propagation angle, $t h_{\max }$ (i.e., the maximum propagation angle the $\mathrm{PE}$ algorithm will accommodate in the field calculations), and the parameter, $r_{\text {mult }}$, must be specified. By default, $r_{\text {mult }}$ is equal to 1 ; however, $t h_{\max }$ does not have a default value and must be explicitly defined. The parameter $r_{\text {mult }}$ is a range step multiplier, allowing the user to vary the PE range step from the default calculated.

Use this option with caution, as you must have some basic knowledge of PE algorithms and how they work to input proper combinations of maximum calculation angles and range steps for a given frequency. When using this option, most error checking is bypassed and parameter limits can be over-ridden. Erroneous field values may result if a poorly chosen combination of th $h_{\max }$ and $r_{\text {mult }}$ are used.

APM Ver. 2.1.04 can determine and provide direct and reflected propagation angles, as well as the propagation factor from direct and reflected rays, to the main calling program. Note that these quantities are obtained only from the FE and RO submodels in APM. It does not compute the angles and propagation factors for the separate rays within the split-step $\mathrm{PE}$ and $\mathrm{XO}$ sub-models, but does provide the resultant propagation angle and factor within these regions. This information is returned if the logical flag lang is set to '.true.', however, do not enable this feature if any portion of the propagation path is over land. The computation is valid only when the propagation path is entirely over water.

\subsection{ADAPTATION REQUIREMENTS}

\subsubsection{Environmental Radio Refractivity Field Data Elements}

The radio-refractivity field, i.e., the profiles of modified refractivity (M-units) versus height, must consist of vertical piece-wise linear profiles specified by couplets of height in meters with respect to mean sea level and M-units at multiple arbitrary ranges. All vertical profiles must contain the same number of vertical data points and be specified such that each numbered data point corresponds to like-numbered points (i.e., features) in the other profiles. The first numbered data point of each profile must correspond to a height of zero mean sea level and the last numbered data point must 
correspond to a height such that the modified refractivity for all greater heights is well represented by extrapolation using the two highest profile points specified.

With the inclusion of terrain and allowing the terrain profile to fall below mean sea level, refractivity profiles can also be provided in which the first level is less than 0 (or below mean sea level). For a terrain profile that falls below mean sea level at some point, the assumption is that the minimum height may be less than the first height in any refractivity profile specified. Therefore, an extrapolation flag, $i_{\text {extra }}$, must be specified to indicate how the APM CSCI should extrapolate from the first refractivity level to the minimum height along the terrain profile. Setting $i_{\text {extra }}$ to 0 will cause the APM CSCI to extrapolate to the minimum height using a standard atmosphere gradient; setting $i_{\text {extra }}$ to 1 will cause the APM CSCI to extrapolate to the minimum height using the gradient determined from the first two levels of the refractivity profile.

Within each profile, each numbered data point must correspond to a height greater than or equal to the height of the previous data point. Note that this requirement allows for a profile containing redundant data points. Note also that all significant features of the refractivity profiles must be specified, even if they are above the maximum output height specified for a particular application of APM.

The external CSCI application designer and the external operator share responsibility for determining appropriate environmental inputs. For example, a loss diagram may be used to consider a surface-to-surface radar detection problem. Since the operator is interested in surface-to-surface, he may truncate the profile assuming that effects from elevated ducting conditions are negligible. It may be however, that the elevated duct does indeed produce a significant effect. The operator should insure therefore, that the maximum height of the profile allows for the inclusion of all significant refractive features.

This specification allows a complicated refractivity field to be described with a minimum of data points. For example, a field in which a single trapping layer linearly descends with increasing range can be described with just two profiles containing only four data points each, frame (a) of Figure 7. In the same manner, other evolutions of refractive layers may be described. Frames (b) and (c) of Figure 7 show two possible scenarios for the development of a trapping layer. The scenario of choice is the one that is consistent with the true thermodynamical and hydrological layering of the atmosphere. 

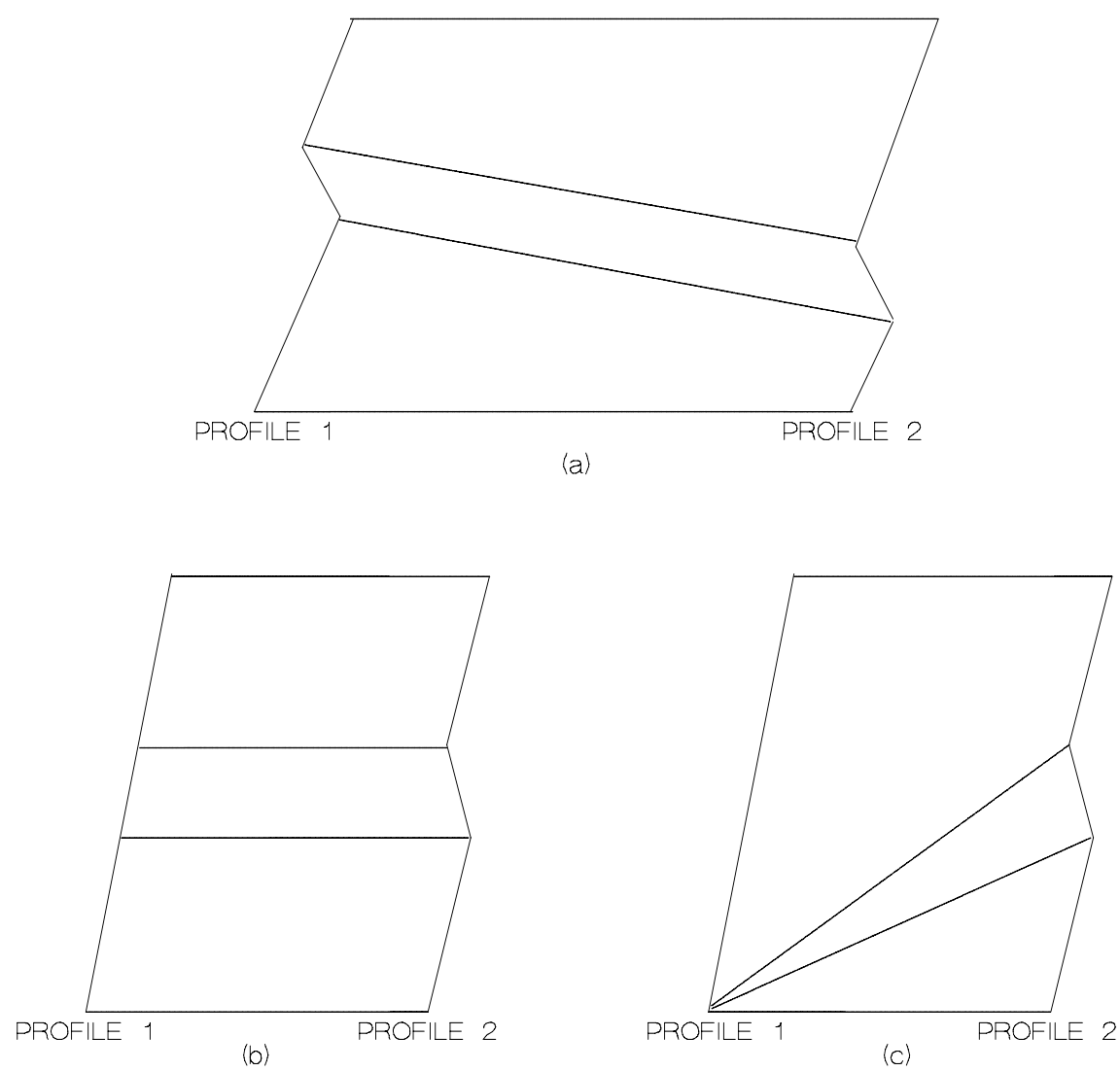

Figure 7. Idealized M-unit profiles (solid) and lines of interpolation (dashed).

Two external implementation data variables applicable to the external application operator and the calling application designer are $r_{\max }$, the maximum APM CSCI output range, and $h_{\max }$, the maximum APM CSCI output height. These two parameters are required by the APM CSCI to determine the horizontal and vertical resolution, respectively, for internal range and height calculations based on the current values of $n_{\text {rout }}$ and $n_{\text {zout }}$. Any value of $r_{\max }$ and $h_{\max }$ is allowed for the convenience of the external application operator and the calling application designer, provided $r_{\max } \geq 5 \mathrm{~km}$, and $h_{\max }$ $\geq 100 \mathrm{~m}$. For example, the external application operator may desire a coverage diagram that extends to a range of $500 \mathrm{~km}$. In addition to accommodating the desires of the operator, specification of such a convenient maximum range eases the burden for the application designer in determining incremental tick marks for the horizontal axis of the display.

Provided the value of the parameter lerr12 is set to '.false.', if the furthest environment profile range is less than $r_{\max }$, the APM CSCI will automatically create an environment profile at $r_{\max }$ equal to the last profile specified, making the environment homogeneous from the range of the last profile specified to $r_{\max }$. For example, a profile is input with an accompanying range of $450 \mathrm{~km}$. If the external application operator 
chooses an $r_{\max }$ of $500 \mathrm{~km}$, the APM CSCI will continue propagation loss/factor calculations to $500 \mathrm{~km}$, keeping the refractivity environment homogeneous from 450 to $500 \mathrm{~km}$.

If lerr12 is set to '.true.' and the furthest environment profile range is less than $r_{\max }$, then an error will be returned in $i_{\text {error }}$ from the APMINIT CSC, which allows the external CSCI application designer greater flexibility in how environment data are handled.

\subsubsection{Terrain Profile Data Element}

The terrain profile must consist of linear piece-wise segments specified as range/height pairs. All range values must be increasing, and the first terrain height value must be at range zero. General ground composition types can be specified (Table 4), along with corresponding ranges over which the ground type is to be applied. If ground type "User Defined" is specified ( igrnd $_{i}=7$ ), then numeric values of relative permittivity and conductivity must be given. If horizontal antenna polarization is specified, and if running a smooth surface case, the APM CSCI will assume perfect conductivity for the entire terrain profile and will ignore any information regarding ground composition. If vertical antenna polarization is specified, or if performing rough surface calculations, then information regarding ground composition must also be specified. If wind speed has been provided, then rough surface calculations will also be performed.

The maximum height, $h_{\max }$, must always be greater than the minimum height, $h_{\min }$, by at least $100 \mathrm{~m}$. Also, a value of $h_{\max }$ must be given such that it is larger than the maximum elevation height along a specified terrain profile.

If lerr6 is set to 'false.' and the furthest range point in the terrain profile is less than $r_{\max }$, the APM CSCI will automatically create a height/range pair as part of the terrain profile at $r_{\max }$ with elevation height equal to the last height specified in the profile, making the terrain profile flat from the range of the last profile point specified to $r_{\max }$. For example, a terrain profile is input where the last height/range pair is $50 \mathrm{~m}$ high with an accompanying range of $95 \mathrm{~km}$. If the external application operator chooses an $r_{\max }$ of $100 \mathrm{~km}$, the APM CSCI will continue propagation loss/factor calculations to $100 \mathrm{~km}$, keeping the terrain profile flat from 95 to $100 \mathrm{~km}$ with an elevation height of $50 \mathrm{~m}$.

If lerr6 is set to '.true.' and the furthest range point is less than $r_{\max }$, then an error is returned in $i_{\text {error }}$ from the APMINIT SU, which allows the external CSCI application designer greater flexibility in how terrain data is handled.

\subsection{SECURITY AND PRIVACY REQUIREMENTS}

The security and privacy requirements are the same as those required by the target employing the external CSCI. 


\subsection{CSCI ENVIRONMENTAL REQUIREMENTS}

The APM CSCI must operate in the same hardware and software environments that the target employing the external CSCI operates.

\subsection{COMPUTER RESOURCE REQUIREMENTS}

Section 3.1.2.5 describes requirements for a Discrete Sine/Cosine Fast-Fourier Transform (DRST) SU. However, other sine FFT routines are available in the commercial market, and such a sine FFT may already be available within another external CSCI. The selection of which FFT ultimately used by APM CSCI is left to the application designer, as every sine FFT will have hardware and/or software performance impacts.

\subsection{SOFTWARE QUALITY FACTORS}

The primary required quality factors can be divided into the three categories: design, performance, and adaptation.

The quality factors for the design category should include correctness, maintainability, and verifiability. Correctness describes the extent to which the APM CSCI conforms to its requirements and is determined from the criteria of completeness, consistency, and/or traceability. Maintainability specifies the effort required to locate and fix an error in the APM CSCI. Maintainability is determined from the criteria of consistency, modularity, self-descriptiveness (self-documentation), and/or simplicity. Verifiability characterizes the effort required to test the APM CSCI to ensure that it performs its intended function. Verifiability is determined from the criteria of modularity, self-descriptiveness, and/or simplicity.

The quality factor for the performance category is reliability, which depicts the confidence that can be placed in the APM CSCI calculations. Reliability is determined from the criteria of accuracy, anomaly management, auditability, consistency, and/or simplicity.

The quality factors for the adaptation category are portability and reusability. Portability determines how easy it is to transport the APM CSCI from one hardware and/or software environment to another. Portability is determined from the criteria of application independence, modularity, and/or self-descriptiveness. Reusability illustrates how easy it is to convert the APM CSCI (or parts of the CSCI) for use in another application. Reusability is determined from the criteria of application independence, document accessibility, functional scope, generality, hardware independence, modularity, simplicity, self-descriptiveness, and/or system clarity. 
Section A.A.1 defines the software quality criteria.

Only the software quality criteria of completeness, consistency, and traceability can be analyzed. Their calculation is described in Section A.2. The other criteria must be determined by demonstration, test, or inspection.

\subsection{DESIGN AND IMPLEMENTATION CONSTRAINTS}

\subsubsection{Implementation And Application Considerations}

The calling external CSCI application will determine the employment of the APM CSCI. However, the intensive computational nature of the APM CSCI must be considered when designing an efficient calling application. For this reason, the APM CSCI should be designed with flexibility for various hardware suites and computer resource management considerations. As stated in Section 1, this APM CSCI applies only to a coverage and loss diagram application. The following highly recommended guidelines will aid in the design of a coverage or loss diagram application that will most efficiently employ the APM CSCI.

The APM CSCI propagation loss calculations are independent of any target or receiver considerations; therefore, for any EM emitter, one execution of the APM CSCI may be used to create both a coverage diagram and a loss diagram. Since both execution time and computer memory allocation should be a consideration when employing this model, it is most efficient and appropriate to execute the APM CSCI for a particular EM system/environmental/terrain combination before executing any application. The output of the APM CSCI would be stored in a file that would be accessed by multiple applications.

For example, the external application operator may desire a coverage diagram for one particular radar system. At the beginning of the coverage diagram application, a check would be made for the existence of a previously created APM CSCI output file appropriate for the EM system and environmental and terrain conditions. If such a file exists, the propagation loss values would be read from the file and used to create the coverage diagram. If the file does not exist, the APM CSCI would be executed to create one. As the APM CSCI is executing, its output could be routed simultaneously to a graphics display device and a file. This file could then be used in the loss diagram application, should the operator also choose it. Two distinct applications, therefore, are achieved with only one execution of the APM CSCI. Additionally, should the operator desire an individual coverage diagram for each of multiple targets, or a single coverage diagram illustrating radar detection of a low-flying missile superimposed on a coverage diagram illustrating his/her own radar's vulnerability as defined by the missile's ESM receiver, only a single execution of the APM CSCI would be required, thereby saving valuable computer resources. 


\subsubsection{Programming Language And Source Implementation}

\subsubsection{Programming Language}

The ANSI Fortran 95 program language standard must be used in the development of the APM CSCI. This standard consists of the specifications of the language Fortran. With certain limitations, the syntax and semantics of the old International Standard commonly known as "FORTRAN 77" are contained entirely within this new International Standard. Therefore, any standard-conforming FORTRAN 77 program is standard, conforming under the Fortran 95 Standard. Note that the name of this language, Fortran, differs from that in FORTRAN 77 in that only the first letter is capitalized. The Overview section of the International Standard describes the major additions to FORTRAN 77 in this International Standard. Section 1.3 of the International Standard specifies the bounds of the Fortran language by identifying those items included and those items excluded. Section 1.4.1 describes the FORTRAN 77 compatibility of the International Standard with emphasis on four FORTRAN 77 features having different interpolations in the new International Standard. The International Standard provides facilities that encourage the design and the use of modular and reusable software.

Section 8.2 of the International Standard describes nine obsolescent features of FORTRAN 77 that are redundant and for which better methods are available in FORTRAN 77 itself. These nine obsolescent features should not be used. These obsolescent features are as follows:

1. Arithmetic IF - use the IF statement.

2. Real and double precision DO control variables and DO loop control expressions use integer.

3. Shared DO termination and termination on a statement other than END DO or CONTINUE - use an END DO or a CONTINUE statement for each DO statement.

4. Branching to an END IF statement from outside its IF block - branch to the statement following the END IF.

5. Alternate return.

6. PAUSE statement.

7. ASSIGN and assigned GO TO statements.

8. Assigned FORMAT specifiers.

9. $\mathrm{cH}(\mathrm{nH})$ edit descriptor. 
Remedies for the last five obsolescent features are described in Section 8.2 of the International standard.

\subsubsection{Source Implementation}

The Standards document by the Naval Oceanographic Office establishes a uniform standard for all software submitted by all contributors to them. It is recommended that the coding requirements set forth in Section 4 of that document be followed. Among these recommendations are:

1. Special non-ANSI features shall be avoided. Non-ANSI practices that are necessary must be documented in the code itself.

2. Maximum use should be made of existing commercially available FORTRAN callable libraries.

3. Programs shall be designed and coded using only five basic control structures sequence of operations (assignment, add, ...), IF THEN ELSE, DO WHILE, DO UNTIL, and CASE.

4. Procedures or routines that make up a module shall not exceed an average of 100 executable statements per procedure or routine and shall not exceed a maximum of 200 executable statements in any procedure or routine.

5. Branching statements (GO TOs) shall only pass control to a statement that is in the same procedure or routine. Each GO TO must pass control only forward of its point of occurrence.

6. Naming conventions shall be uniform throughout the software. Program, subprogram, module, procedure, and data names shall be uniquely chosen to identify the applicable function performed. The naming convention for COMMON shall be consistent across the entire program.

7. Constants shall be defined not calculated (e.g., do no use $H A L F=1 / 2$, use $H A L F=$ 0.5)

8. Mixed-mode numerical operations should be avoided whenever possible. When determined to be necessary, the use shall be explicit (FLOAT, FIX, or in assignment statement) and completely described in comments.

9. Each component of the software shall have a prologue containing the name of the program, subprogram, or function and any version number; purpose; inputs; outputs; list of routines that call this routine; complete list of routines called including intrinsic functions such as $A B S$ and FLOAT; glossary; and method. 
10. To facilitate program comprehension, comment statements shall be used throughout the program code.

11. The use of the EQUIVALENCE statement shall be restricted to those where it either improves the readability of the code or the efficiency of the program. If the EQUIVALENCE statement is used, it must be fully documented in the prologue and inline comment statements.

12. No machine-dependent techniques are allowed, unless there is no other way of performing the task.

13. Initialize every variable before use.

14. Do not depend on the values of "local" variables computed on a previous call to a routine.

15. Program structural indentation shall be used to improve readability and clarity.

\subsection{PERSONNEL-RELATED REQUIREMENTS}

N/A.

\subsection{TRAINING RELATED REQUIREMENTS}

The employing target software personnel implementing this CSCI into the external CSCI will require training to become familiar with APM. This requirement should be met by this document and the companion Software Design Description (SDD) and Software Test Description (STD) documents.

\subsection{OTHER REQUIREMENTS}

None.

\subsection{PRECEDENCE AND CRITICALITY OF REQUIREMENTS}

The requirements presented in Sections 3.1 through 3.5 and Sections 3.8 through 3.10 have precedence over Sections 3.6, 3.7, 3.11, 3.12, and 3.13 and should be given equal weight. 


\section{QUALIFICATION PROVISIONS}

N/A

\section{REQUIREMENTS TRACEABILITY}

\subsection{SYSTEM TRACEABILITY}

This section provides traceability of requirements between the APM CSCI and the external CSCI.

1. The APM CSCI environmental data requirements should be obtained from the environmental application or database within the external CSCI. The APM CSCI terrain data element requirements should be obtained from any desired terrain database within the external CSCI, however, it is up to the external CSCI to extract the terrain in the proper format for inputting to APM. The radar/communication system data element requirements should be obtained from the EM system database within the external CSCI.

2. The external CSCI requirement of propagation loss vs. range and height should be obtained from the APM CSCI.

\subsection{DOCUMENTATION TRACEABILITY}

This section provides the following types of traceability between the Software Requirements Specification (SRS), the Software Design Description (SDD), and the Software Test Description (STD):

1. Traceability between levels of requirements

2. Traceability between the software requirements and software design

3. Traceability between the software requirements and qualification test information obtained from the software testing 
This traceability of the Advanced Propagation Model is presented in two tables. The first table, Table 7, presents the traceability between levels of SRS requirements. The second table (Table 137 in the SDD) presents the traceability between the software requirements and software design.

Table 7. Requirements traceability matrix for the SRS.

\begin{tabular}{|c|c|c|c|}
\hline \multicolumn{2}{|c|}{ Software Requirements Specification } & \multicolumn{2}{|c|}{ Software Requirements Specification } \\
\hline SRS Requirement Name & $\begin{array}{c}\text { SRS } \\
\text { Paragraph } \\
\text { Number }\end{array}$ & SRS Requirement Name & $\begin{array}{c}\text { SRS } \\
\text { Paragraph } \\
\text { Number }\end{array}$ \\
\hline CSCI Capability Requirements & 3.1 & $\begin{array}{l}\text { Advance Propagation Initialization } \\
\text { (APMINIT) CSC }\end{array}$ & 3.1 .1 \\
\hline $\begin{array}{l}\text { Advance Propagation } \\
\text { Initialization (APMINIT) CSC }\end{array}$ & 3.1 .1 & $\begin{array}{l}\text { Allocate Arrays APM } \\
\text { (ALLARRAY_APM) SU }\end{array}$ & 3.1.1.1 \\
\hline $\begin{array}{l}\text { Advance Propagation } \\
\text { Initialization (APMINIT) CSC }\end{array}$ & 3.1 .1 & $\begin{array}{l}\text { Allocate Array RO } \\
\text { (ALLARRAY_RO) SU }\end{array}$ & 3.1.1.3 \\
\hline $\begin{array}{l}\text { Advance Propagation } \\
\text { Initialization (APMINIT) CSC }\end{array}$ & 3.1 .1 & $\begin{array}{l}\text { Allocate Array XORUF (XORUF) } \\
\text { SU }\end{array}$ & 3.1.1.4 \\
\hline $\begin{array}{l}\text { Advance Propagation } \\
\text { Initialization (APMINIT) CSC }\end{array}$ & 3.1 .1 & $\begin{array}{l}\text { Alpha Impedance Initialization } \\
\text { (ALN_INIT) SU }\end{array}$ & 3.1 .1 .5 \\
\hline $\begin{array}{l}\text { Alpha Impedance Initialization } \\
\text { (ALN_INIT) SU }\end{array}$ & 3.1.1.5 & $\begin{array}{l}\text { Get Alpha Impedance (GETALN) } \\
\text { SU }\end{array}$ & 3.1.1.13 \\
\hline $\begin{array}{l}\text { Get Alpha } \\
\text { Impedance(GETALN) SU }\end{array}$ & 3.1.1.13 & $\begin{array}{l}\text { Current Wind (FN_CURWIND) } \\
\text { Function }\end{array}$ & 3.1.2.2 \\
\hline $\begin{array}{l}\text { Get Alpha } \\
\text { Impedance(GETALN) SU }\end{array}$ & 3.1.1.13 & $\begin{array}{l}\text { Get Reflection Coefficient } \\
\text { (GETREFCOEF) SU }\end{array}$ & 3.1.2.12 \\
\hline $\begin{array}{l}\text { Get Reflection Coefficient } \\
\text { (GETREFCOEF) SU }\end{array}$ & 3.1.2.12 & $\begin{array}{l}\text { Current Wind (FN_CURWIND) } \\
\text { Function }\end{array}$ & 3.1.2.2 \\
\hline $\begin{array}{l}\text { Get Reflection Coefficient } \\
\text { (GETREFCOEF) SU }\end{array}$ & 3.1.2.12 & $\begin{array}{l}\text { Dielectric Constant (FN_DIECON) } \\
\text { Function }\end{array}$ & 3.1.2.3 \\
\hline $\begin{array}{l}\text { Get Alpha } \\
\text { Impedance(GETALN) SU }\end{array}$ & 3.1.1.13 & $\begin{array}{l}\text { Surface Impedance (SURFIMP) } \\
\text { SU }\end{array}$ & 3.1.2.23 \\
\hline $\begin{array}{l}\text { Surface Impedance } \\
\text { (SURFIMP) SU }\end{array}$ & 3.1.2.23 & Poly 4 (FN_POLY4) Function & 3.1.1.20 \\
\hline $\begin{array}{l}\text { Surface Impedance } \\
\text { (SURFIMP) SU }\end{array}$ & 3.1.2.23 & Poly 5 (FN_POLY5) Function & 3.1.1.21 \\
\hline $\begin{array}{l}\text { Advance Propagation } \\
\text { Initialization (APMINIT) CSC }\end{array}$ & 3.1 .1 & $\begin{array}{l}\text { Dielectric Initialization (DIEINIT) } \\
\text { SU }\end{array}$ & 3.1.1.8 \\
\hline $\begin{array}{l}\text { Advance Propagation } \\
\text { Initialization (APMINIT) CSC }\end{array}$ & 3.1 .1 & FFT Parameters (FFTPAR) SU & 3.1.1.9 \\
\hline
\end{tabular}


Table 7. Requirements traceability matrix for the SRS. (continued)

\begin{tabular}{|c|c|c|c|}
\hline \multicolumn{2}{|c|}{ Software Requirements Specification } & \multicolumn{2}{|c|}{ Software Requirements Specification } \\
\hline SRS Requirement Name & $\begin{array}{c}\text { SRS } \\
\text { Paragraph } \\
\text { Number }\end{array}$ & SRS Requirement Name & $\begin{array}{c}\text { SRS } \\
\text { Paragraph } \\
\text { Number }\end{array}$ \\
\hline $\begin{array}{l}\text { Advance Propagation } \\
\text { Initialization (APMINIT) CSC }\end{array}$ & 3.1 .1 & Fill Height Arrays (FILLHT) SU & 3.1.1.10 \\
\hline Fill Height Arrays (FILLHT) SU & 3.1.1.10 & $\begin{array}{l}\text { Trace to Output Range } \\
\text { (TRACE_ROUT) SU }\end{array}$ & 3.1.1.27 \\
\hline Fill Height Arrays (FILLHT) SU & 3.1.1.10 & $\begin{array}{l}\text { Trace to Next Step (TRACE_STEP) } \\
\text { SU }\end{array}$ & 3.1.1.28 \\
\hline $\begin{array}{l}\text { Trace to Next Step } \\
\text { (TRACE_STEP) SU }\end{array}$ & 3.1.1.28 & Height Check (HTCHECK) SU & 3.1.1.17 \\
\hline $\begin{array}{l}\text { Advance Propagation } \\
\text { Initialization (APMINIT) CSC }\end{array}$ & 3.1 .1 & Gaseous Absorption (GASABS) SU & 3.1.1.11 \\
\hline $\begin{array}{l}\text { Advance Propagation } \\
\text { Initialization (APMINIT) CSC }\end{array}$ & 3.1 .1 & $\begin{array}{l}\text { Get Effective Earth Radius Factor } \\
\text { (GET_K) SU }\end{array}$ & 3.1.1.12 \\
\hline $\begin{array}{l}\text { Advance Propagation } \\
\text { Initialization (APMINIT) CSC }\end{array}$ & 3.1 .1 & Get Angles (GETANGLES) SU & 3.1.1.14 \\
\hline Get Angles (GETANGLES) SU & 3.1.1.14 & APM Status (APMSTATUS) SU & 3.1.1.7 \\
\hline Get Angles (GETANGLES) SU & 3.1.1.14 & DOSHIFT SU & 3.1.2.4 \\
\hline Get Angles (GETANGLES) SU & 3.1.1.14 & Free Space Range Step (FRSTP) SU & 3.1.1.24 \\
\hline $\begin{array}{l}\text { Free Space Range Step } \\
\text { (FRSTP) SU }\end{array}$ & 3.1.2.9 & Fast Fourier Transform (FFT) SU & 3.1.2.8 \\
\hline $\begin{array}{l}\text { Fast Fourier Transform (FFT) } \\
\text { SU }\end{array}$ & 3.1.2.8 & $\begin{array}{l}\text { Discrete Sine/Cosine Transform } \\
\text { (DRST) SU }\end{array}$ & 3.1.2.5 \\
\hline Get Angles (GETANGLES) SU & 3.1.1.14 & $\begin{array}{l}\text { Refractivity Interpolation (REFINTER) } \\
\text { SU }\end{array}$ & 3.1.2.18 \\
\hline $\begin{array}{l}\text { Refractivity Interpolation } \\
\text { (REFINTER) SU }\end{array}$ & 3.1.2.18 & Interpolate Profile (INTPROF) SU & 3.1.1.18 \\
\hline $\begin{array}{l}\text { Interpolate Profile (INTPROF) } \\
\text { SU }\end{array}$ & 3.1.1.18 & $\begin{array}{l}\text { Linear Interpolation (FN_PLINT) } \\
\text { Function }\end{array}$ & 3.1.2.14 \\
\hline $\begin{array}{l}\text { Refractivity Interpolation } \\
\text { (REFINTER) SU }\end{array}$ & 3.1.2.18 & Profile Reference (PROFREF) SU & 3.1.1.22 \\
\hline $\begin{array}{l}\text { Refractivity Interpolation } \\
\text { (REFINTER) SU }\end{array}$ & 3.1.2.18 & $\begin{array}{l}\text { Remove Duplicate Refractivity Levels } \\
\text { (REMDUP) SU }\end{array}$ & 3.1.1.24 \\
\hline Get Angles (GETANGLES) SU & 3.1.1.14 & RG Trace (RGTRACE) SU & 3.1.1.25 \\
\hline RG Trace (RGTRACE) SU & 3.1.1.25 & $\begin{array}{l}\text { Trace to Next Step (TRACE_STEP) } \\
\text { SU }\end{array}$ & 3.1.1.28 \\
\hline
\end{tabular}


Table 7. Requirements traceability matrix for the SRS. (continued)

\begin{tabular}{|c|c|c|c|}
\hline \multicolumn{2}{|c|}{ Software Requirements Specification } & \multicolumn{2}{|l|}{ Software Requirements Specification } \\
\hline SRS Requirement Name & $\begin{array}{l}\text { SRS } \\
\text { Paragraph } \\
\text { Number }\end{array}$ & SRS Requirement Name & $\begin{array}{l}\text { SRS } \\
\text { Paragraph } \\
\text { Number }\end{array}$ \\
\hline $\begin{array}{l}\text { Trace to Next Step } \\
\text { (TRACE_STEP) SU }\end{array}$ & 3.1.1.28 & Height Check (HTCHECK) SU & 3.1.1.17 \\
\hline Get Angles (GETANGLES) SU & 3.1.1.14 & Spectral Estimation (SPECEST) SU & 3.1.2.22 \\
\hline $\begin{array}{l}\text { Spectral Estimation (SPECEST) } \\
\text { SU }\end{array}$ & 3.1.2.22 & $\begin{array}{l}\text { Discrete Sine/Cosine Transform } \\
\text { (DRST) SU }\end{array}$ & 3.1.2.5 \\
\hline Get Angles (GETANGLES) SU & 3.1.1.14 & $\begin{array}{l}\text { Trace to Output Range } \\
\text { (TRACE_ROUT) SU }\end{array}$ & 3.1.1.27 \\
\hline $\begin{array}{l}\text { Advance Propagation } \\
\text { Initialization (APMINIT) CSC }\end{array}$ & 3.1 .1 & $\begin{array}{l}\text { Get Maximum Angle (GETTHMAX) } \\
\text { SU }\end{array}$ & 3.1.1.15 \\
\hline $\begin{array}{l}\text { Get Maximum Angle } \\
\text { (GETTHMAX) SU }\end{array}$ & 3.1.1.15 & FFT Parameters (FFTPAR) SU & 3.1.1.9 \\
\hline $\begin{array}{l}\text { Get Maximum Angle } \\
(\text { GETTHMAX) SU }\end{array}$ & 3.1.1.15 & $\begin{array}{l}\text { Trace to Output Range } \\
\text { (TRACE_ROUT) SU }\end{array}$ & 3.1.1.27 \\
\hline $\begin{array}{l}\text { Advance Propagation } \\
\text { Initialization (APMinit) CSC }\end{array}$ & 3.1 .1 & $\begin{array}{l}\text { Grazing Angle Interpolation } \\
\text { (GRAZE_INT) SU }\end{array}$ & 3.1.1.16 \\
\hline $\begin{array}{l}\text { Grazing Angle Interpolation } \\
\text { (GRAZE_INT) SU }\end{array}$ & 3.1.1.16 & $\begin{array}{l}\text { Linear Interpolation (FN_PLINT) } \\
\text { Function }\end{array}$ & 3.1.2.14 \\
\hline $\begin{array}{l}\text { Advance Propagation } \\
\text { Initialization (APMinit) CSC }\end{array}$ & 3.1 .1 & PE Initialization (PEINIT) SU & 3.1.1.19 \\
\hline PE Initialization (PEINIT) SU & 3.1.1.19 & $\begin{array}{l}\text { Allocate Array PE (ALLARRAY_PE) } \\
\text { SU }\end{array}$ & 3.1.1.2 \\
\hline PE Initialization (PEINIT) SU & 3.1.1.19 & Interpolate Profile (INTPROF) SU & 3.1.1.18 \\
\hline PE Initialization (PEINIT) SU & 3.1.1.19 & $\begin{array}{l}\text { Starter Field Initialization (XYINIT) } \\
\text { SU }\end{array}$ & 3.1.1.30 \\
\hline $\begin{array}{l}\text { Starter Field Initialization } \\
(\text { XYINIT) SU }\end{array}$ & 3.1.1.30 & Antenna Pattern (ANTPAT) SU & 3.1.1.6 \\
\hline $\begin{array}{l}\text { Starter Field Initialization } \\
(\text { XYINIT) SU }\end{array}$ & 3.1.1.30 & $\begin{array}{l}\text { Discrete Sine/Cosine Transform } \\
\text { (DRST) SU }\end{array}$ & 3.1.2.5 \\
\hline $\begin{array}{l}\text { Advance Propagation } \\
\text { Initialization (APMINIT) CSC }\end{array}$ & 3.1 .1 & Profile Reference (PROFREF) SU & 3.1.1.22 \\
\hline $\begin{array}{l}\text { Advance Propagation } \\
\text { Initialization (APMINIT) CSC }\end{array}$ & 3.1 .1 & $\begin{array}{l}\text { Refractivity Initialization (REFINIT) } \\
\text { SU }\end{array}$ & 3.1 .1 .23 \\
\hline $\begin{array}{l}\text { Refractivity Initialization } \\
\text { (REFINIT) SU }\end{array}$ & 3.1.1.23 & Profile Reference (PROFREF) SU & 3.1.1.22 \\
\hline
\end{tabular}


Table 7. Requirements traceability matrix for the SRS. (continued)

\begin{tabular}{|c|c|c|c|}
\hline \multicolumn{2}{|c|}{ Software Requirements Specification } & \multicolumn{2}{|c|}{ Software Requirements Specification } \\
\hline SRS Requirement Name & $\begin{array}{c}\text { SRS } \\
\text { Paragraph } \\
\text { Number }\end{array}$ & SRS Requirement Name & $\begin{array}{c}\text { SRS } \\
\text { Paragraph } \\
\text { Number }\end{array}$ \\
\hline $\begin{array}{l}\text { Refractivity Initialization (REFINIT) } \\
\text { SU }\end{array}$ & 3.1.1.23 & $\begin{array}{l}\text { Remove Duplicate Refractivity } \\
\text { Levels (RemDup) SU }\end{array}$ & 3.1.1.24 \\
\hline $\begin{array}{l}\text { Advance Propagation Initialization } \\
\text { (APMINIT) CSC }\end{array}$ & 3.1 .1 & $\begin{array}{l}\text { Remove Duplicate Refractivity } \\
\text { Levels (RemDup) SU }\end{array}$ & 3.1.1.24 \\
\hline $\begin{array}{l}\text { Advance Propagation Initialization } \\
\text { (APMINIT) CSC }\end{array}$ & 3.1 .1 & $\begin{array}{l}\text { Terrain Initialization (TERINIT) } \\
\text { SU }\end{array}$ & 3.1.1.26 \\
\hline $\begin{array}{l}\text { Advance Propagation Initialization } \\
\text { (APMinit) CSC }\end{array}$ & 3.1 .1 & $\begin{array}{l}\text { Troposcatter Initialization } \\
\text { (TROPOINIT) SU }\end{array}$ & 3.1.1.29 \\
\hline $\begin{array}{l}\text { Troposcatter Initialization } \\
\text { (TROPOINIT) SU }\end{array}$ & 3.1.1.29 & Antenna Pattern (Antpat) SU & 3.1.1.6 \\
\hline $\begin{array}{l}\text { Troposcatter Initialization } \\
\text { (TROPOINIT) SU }\end{array}$ & 3.1.1.29 & $\begin{array}{l}\text { Get Effective Earth Radius Factor } \\
(\text { GET_K) SU }\end{array}$ & 3.1.1.12 \\
\hline CSCI Capability Requirements & 3.1 & $\begin{array}{l}\text { Advance Propagation Model Step } \\
\text { (APMSTEP) CSC }\end{array}$ & 3.1 .2 \\
\hline $\begin{array}{l}\text { Advance Propagation Model Step } \\
\text { (APMSTEP) CSC }\end{array}$ & 3.1 .2 & Flat-Earth Direct Ray (FEDR) SU & 3.1.2.6 \\
\hline Flat-Earth Direct Ray (FEDR) SU & 3.1.2.6 & Antenna Pattern (Antpat) SU & 3.1.1.6 \\
\hline $\begin{array}{l}\text { Advance Propagation Model Step } \\
\text { (APMSTEP) CSC }\end{array}$ & 3.1 .2 & Flat-Earth Model (FEM) SU & 3.1.2.7 \\
\hline Flat-Earth Model (FEM) SU & 3.1.2.7 & Antenna Pattern (Antpat) SU & 3.1.1.6 \\
\hline Flat-Earth Model (FEM) SU & 3.1.2.7 & $\begin{array}{l}\text { Get Reflection Coefficient } \\
\text { (GETREFCOEF) SU }\end{array}$ & 3.1.2.12 \\
\hline $\begin{array}{l}\text { Get Reflection Coefficient } \\
\text { (GETREFCOEF) SU }\end{array}$ & 3.1.2.16 & $\begin{array}{l}\text { Current Wind (FN_CURWIND) } \\
\text { Function }\end{array}$ & 3.1.2.2 \\
\hline $\begin{array}{l}\text { Get Reflection Coefficient } \\
\text { (GETREFCOEF) SU }\end{array}$ & 3.1.2.12 & $\begin{array}{l}\text { Dielectric Constant } \\
\text { (FN_DIECON) Function }\end{array}$ & 3.1.2.3 \\
\hline $\begin{array}{l}\text { Advance Propagation Model Step } \\
\text { (APMSTEP) CSC }\end{array}$ & 3.1.2.10 & $\begin{array}{l}\text { Parabolic Equation Step } \\
\text { (PESTEP) SU }\end{array}$ & 3.1.2.16 \\
\hline $\begin{array}{l}\text { Parabolic Equation Step } \\
\text { (PESTEP) SU }\end{array}$ & 3.1.2.16 & $\begin{array}{l}\text { Calculate Propagation Loss } \\
\text { (CALCLOS) SU }\end{array}$ & 3.1.2.1 \\
\hline $\begin{array}{l}\text { Calculate Propagation Loss } \\
\text { (CALCLOS) SU }\end{array}$ & 3.1.2.1 & $\begin{array}{l}\text { Get Propagation Factor } \\
\text { (FN_GETPFAC) Function }\end{array}$ & 3.1.2.11 \\
\hline $\begin{array}{l}\text { Calculate Propagation Loss } \\
\text { (CALCLOS) SU }\end{array}$ & 3.1.2.1 & $\begin{array}{l}\text { Linear Interpolation (FN_PLINT) } \\
\text { Function }\end{array}$ & 3.1.2.14 \\
\hline
\end{tabular}


Table 7. Requirements traceability matrix for the SRS. (continued)

\begin{tabular}{|c|c|c|c|}
\hline \multicolumn{2}{|c|}{ Software Requirements Specification } & \multicolumn{2}{|c|}{ Software Requirements Specification } \\
\hline SRS Requirement Name & $\begin{array}{l}\text { SRS } \\
\text { Paragraph } \\
\text { Number }\end{array}$ & SRS Requirement Name & $\begin{array}{l}\text { SRS } \\
\text { Paragraph } \\
\text { Number }\end{array}$ \\
\hline $\begin{array}{l}\text { Calculate Propagation Loss } \\
\text { (CALCLOS) SU }\end{array}$ & 3.1.2.1 & $\begin{array}{l}\text { Troposcatter (TROPOSCAT) } \\
\text { SU }\end{array}$ & 3.1.2.24 \\
\hline Troposcatter (TROPOSCAT) SU & 3.1.2.24 & $\begin{array}{l}\text { Get Troposcatter Loss } \\
\text { (FN_GET_TLOSS) Function }\end{array}$ & 3.1.2.13 \\
\hline $\begin{array}{l}\text { Get Troposcatter Loss } \\
\text { (FN_GET_TLOSS) Function }\end{array}$ & 3.1.2.13 & Antenna Pattern (ANTPAT) SU & 3.1.1.6 \\
\hline $\begin{array}{l}\text { Parabolic Equation Step } \\
\text { (PESTEP) SU }\end{array}$ & 3.1.2.16 & DOSHIFT SU & 3.1.2.4 \\
\hline $\begin{array}{l}\text { Parabolic Equation Step } \\
\text { (PESTEP) SU }\end{array}$ & 3.1.2.16 & $\begin{array}{l}\text { Free Space Range Step } \\
\text { (FRSTP) SU }\end{array}$ & 3.1.1.24 \\
\hline $\begin{array}{l}\text { Free Space Range Step (FRSTP) } \\
\text { SU }\end{array}$ & 3.1.1.24 & $\begin{array}{l}\text { Fast-Fourier Transform (FFT) } \\
\text { SU }\end{array}$ & 3.1.2.8 \\
\hline Fast-Fourier Transform (FFT) SU & 3.1.2.8 & $\begin{array}{l}\text { Discrete Sine/Cosine } \\
\text { Transform (DRST) SU }\end{array}$ & 3.1.2.5 \\
\hline $\begin{array}{l}\text { Parabolic Equation Step } \\
\text { (PESTEP) SU }\end{array}$ & 3.1.2.16 & FZLIM SU & 3.1.2.10 \\
\hline FZLIM SU & 3.1.2.10 & $\begin{array}{l}\text { Get Propagation Factor } \\
\text { (FN_GETPFAC) Function }\end{array}$ & 3.1.2.11 \\
\hline FZLIM SU & 3.1.2.10 & Save Profile (SAVEPRO) SU & 3.1.2.21 \\
\hline FZLIM SU & 3.1.2.10 & $\begin{array}{l}\text { Spectral Estimation } \\
\text { (SPECEST) SU }\end{array}$ & 3.1.2.22 \\
\hline $\begin{array}{l}\text { Spectral Estimation (SPECEST) } \\
\text { SU }\end{array}$ & 3.1.2.22 & $\begin{array}{l}\text { Discrete Sine/Cosine } \\
\text { Transform (DRST) SU }\end{array}$ & 3.1.2.5 \\
\hline $\begin{array}{l}\text { Parabolic Equation Step } \\
\text { (PESTEP) SU }\end{array}$ & 3.1.2.16 & $\begin{array}{l}\text { Get Alpha Impedance } \\
\text { (GETALN) SU }\end{array}$ & 3.1.1.13 \\
\hline $\begin{array}{l}\text { Get Alpha Impedance (GETALN) } \\
\text { SU }\end{array}$ & 3.1.1.13 & $\begin{array}{l}\text { Current Wind (FN_CURWIND) } \\
\text { Function }\end{array}$ & 3.1.2.2 \\
\hline $\begin{array}{l}\text { Get Alpha Impedance (GETALN) } \\
\text { SU }\end{array}$ & 3.1.1.13 & $\begin{array}{l}\text { Get Reflection Coefficient } \\
\text { (GETREFCOEF) SU }\end{array}$ & 3.1.2.12 \\
\hline $\begin{array}{l}\text { Get Reflection Coefficient } \\
\text { (GETREFCOEF) SU }\end{array}$ & 3.1.2.12 & $\begin{array}{l}\text { Current Wind (FN_CURWIND) } \\
\text { Function }\end{array}$ & 3.1.2.2 \\
\hline $\begin{array}{l}\text { Get Reflection Coefficient } \\
\text { (GETREFCOEF) SU }\end{array}$ & 3.1.2.12 & $\begin{array}{l}\text { Dielectric Constant } \\
\text { (FN_DIECON) Function }\end{array}$ & 3.1.2.3 \\
\hline $\begin{array}{l}\text { Get Alpha Impedance(GETALN) } \\
\text { SU }\end{array}$ & 3.1.1.13 & $\begin{array}{l}\text { Surface Impedance } \\
\text { (SURFIMP) SU }\end{array}$ & 3.1.2.23 \\
\hline
\end{tabular}


Table 7. Requirements traceability matrix for the SRS. (continued)

\begin{tabular}{|c|c|c|c|}
\hline \multicolumn{2}{|c|}{ Software Requirements Specification } & \multicolumn{2}{|c|}{ Software Requirements Specification } \\
\hline SRS Requirement Name & $\begin{array}{l}\text { SRS } \\
\text { Paragraph } \\
\text { Number }\end{array}$ & SRS Requirement Name & $\begin{array}{c}\text { SRS } \\
\text { Paragraph } \\
\text { Number }\end{array}$ \\
\hline $\begin{array}{l}\text { Surface Impedance (SURFIMP) } \\
\text { SU }\end{array}$ & 3.1.2.23 & Poly 4 (FN_POLY4) Function & 3.1.1.20 \\
\hline $\begin{array}{l}\text { Surface Impedance (SURFIMP) } \\
\text { SU }\end{array}$ & 3.1.2.23 & Poly 5 (FN_POLY5) Function & 3.1.1.21 \\
\hline $\begin{array}{l}\text { Parabolic Equation Step } \\
\text { (PESTEP) SU }\end{array}$ & 3.1.2.16 & $\begin{array}{l}\text { Mixed Fourier Transform } \\
\text { (MIXEDFT) SU }\end{array}$ & 3.1.2.15 \\
\hline $\begin{array}{l}\text { Mixed Fourier Transform } \\
\text { (MIXEDFT) SU }\end{array}$ & 3.1.2.15 & $\begin{array}{l}\text { Free Space Range Step (FRSTP) } \\
\text { SU }\end{array}$ & 3.1.1.24 \\
\hline $\begin{array}{l}\text { Free Space Range Step (FRSTP) } \\
\text { SU }\end{array}$ & 3.1.1.24 & Fast-Fourier Transform (FFT) SU & 3.1.2.8 \\
\hline Fast-Fourier Transform (FFT) SU & 3.1.2.8 & $\begin{array}{l}\text { Discrete Sine/Cosine Transform } \\
\text { (DRST) SU }\end{array}$ & 3.1.2.5 \\
\hline $\begin{array}{l}\text { Parabolic Equation Step } \\
\text { (PESTEP) SU }\end{array}$ & 3.1.2.16 & $\begin{array}{l}\text { Refractivity Interpolation } \\
\text { (REFINTER) SU }\end{array}$ & 3.1.2.18 \\
\hline $\begin{array}{l}\text { Refractivity Interpolation } \\
\text { (REFINTER) SU }\end{array}$ & 3.1.2.18 & Interpolate Profile (INTPROF) SU & 3.1.1.18 \\
\hline Interpolate Profile (INTPROF) SU & 3.1.1.18 & $\begin{array}{l}\text { Linear Interpolation (FN_PLINT) } \\
\text { Function }\end{array}$ & 3.1.2.14 \\
\hline $\begin{array}{l}\text { Refractivity Interpolation } \\
\text { (REFINTER) SU }\end{array}$ & 3.1.2.18 & Profile Reference (PROFREF) SU & 3.1.1.22 \\
\hline $\begin{array}{l}\text { Refractivity Interpolation } \\
\text { (REFINTER) SU }\end{array}$ & 3.1.2.18 & $\begin{array}{l}\text { Remove Duplicate Refractivity } \\
\text { Levels (REMDUP) SU }\end{array}$ & 3.1.1.24 \\
\hline $\begin{array}{l}\text { Advance Propagation Model Step } \\
\text { (APMSTEP) CSC }\end{array}$ & 3.1 .2 & Ray Optics Loss (ROLOSS) SU & 3.1.2.20 \\
\hline Ray Optics Loss (ROLOSS) SU & 3.1.2.20 & $\begin{array}{l}\text { Ray Optics Calculation (ROCALC) } \\
\text { SU }\end{array}$ & 3.1.2.19 \\
\hline $\begin{array}{l}\text { Ray Optics Calculation } \\
\text { (ROCALC) SU }\end{array}$ & 3.1.2.19 & Antenna Pattern (ANTPAT) SU & 3.1.1.6 \\
\hline $\begin{array}{l}\text { Ray Optics Calculation } \\
\text { (ROCALC) SU }\end{array}$ & 3.1.2.19 & $\begin{array}{l}\text { Get Reflection Coefficient } \\
\text { (GETREFCOEF) SU }\end{array}$ & 3.1.2.12 \\
\hline $\begin{array}{l}\text { Get Reflection Coefficient } \\
\text { (GETREFCOEF) SU }\end{array}$ & 3.1.2.12 & $\begin{array}{l}\text { Current Wind (FN_CURWIND) } \\
\text { Function }\end{array}$ & 3.1.2.2 \\
\hline $\begin{array}{l}\text { Get Reflection Coefficient } \\
\text { (GETREFCOEF) SU }\end{array}$ & 3.1.2.12 & $\begin{array}{l}\text { Dielectric Constant (FN_DIECON) } \\
\text { Function }\end{array}$ & 3.1.2.3 \\
\hline
\end{tabular}


Table 7. Requirements traceability matrix for the SRS. (continued)

\begin{tabular}{|c|c|c|c|}
\hline \multicolumn{2}{|c|}{ Software Requirements Specification } & \multicolumn{2}{|c|}{ Software Requirements Specification } \\
\hline SRS Requirement Name & $\begin{array}{l}\text { SRS } \\
\text { Paragraph } \\
\text { Number }\end{array}$ & SRS Requirement Name & $\begin{array}{l}\text { SRS } \\
\text { Paragraph } \\
\text { Number }\end{array}$ \\
\hline $\begin{array}{l}\text { Ray Optics Calculation } \\
\text { (ROCALC) SU }\end{array}$ & 3.1.2.19 & Ray Trace (RAYTRACE) SU & 3.1.2.17 \\
\hline CSCI Capability Requirements & 3.1 & $\begin{array}{l}\text { Extended Optics Initialization } \\
\text { (XOINIT) CSC }\end{array}$ & 3.1 .3 \\
\hline $\begin{array}{l}\text { Extended Optics Initialization } \\
\text { (XOINIT) CSC }\end{array}$ & 3.1 .3 & APM Clean (APMCLEAN) SU & 3.1.3.1 \\
\hline APM Clean (APMCLEAN) SU & 3.1.3.1 & Discrete Sine/Cosine (DRST) SU & 3.1.3.1 \\
\hline $\begin{array}{l}\text { Extended Optics Initialization } \\
\text { (XOINIT) CSC }\end{array}$ & 3.1 .3 & Clutter-to-Noise (CLUTTER) SU & 3.1.3.2 \\
\hline Clutter-to-Noise (CLUTTER) SU & 3.1.3.2 & GIT Initialization (GIT_INIT) SU & 3.1.3.5 \\
\hline GIT Initialization (GIT_INIT) SU & 3.1.3.5 & $\begin{array}{l}\text { Current Wind (FN_CURWIND) } \\
\text { Function }\end{array}$ & 3.1.2.2 \\
\hline Clutter-to-Noise (CLUTTER) SU & 3.1.3.2 & $\begin{array}{l}\text { Standard Propagation Model } \\
\text { Initialization (SPM_INIT) SU }\end{array}$ & 3.1.3.11 \\
\hline Clutter-to-Noise (CLUTTER) SU & 3.1.3.2 & $\begin{array}{l}\text { Standard Propagation Model (SPM) } \\
\text { SU }\end{array}$ & 3.1.3.12 \\
\hline $\begin{array}{l}\text { Standard Propagation Model } \\
\text { (SPM) SU }\end{array}$ & 3.1.3.12 & $\begin{array}{l}\text { Diffraction Loss (FN_DLOSS) } \\
\text { Function }\end{array}$ & 3.1.3.3 \\
\hline $\begin{array}{l}\text { Standard Propagation Model } \\
\text { (SPM) SU }\end{array}$ & 3.1.3.12 & GofZ (FN_GOFZ) Function & 3.1.3.6 \\
\hline $\begin{array}{l}\text { Standard Propagation Model } \\
\text { (SPM) SU }\end{array}$ & 3.1.3.12 & Optical Region Limit (OPLIMIT) SU & 3.1.3.8 \\
\hline $\begin{array}{l}\text { Optical Region Limit (OPLIMIT) } \\
\text { SU }\end{array}$ & 3.1.3.8 & Get Theta (GETTHETA) SU & 3.1.3.4 \\
\hline Get Theta (GETTHETA) SU & 3.1.3.8 & $\begin{array}{l}\text { Get Reflection Coefficient } \\
\text { (GETREFCOEF) SU }\end{array}$ & 3.1.2.12 \\
\hline $\begin{array}{l}\text { Get Reflection Coefficient } \\
\text { (GETREFCOEF) SU }\end{array}$ & 3.1.2.12 & $\begin{array}{l}\text { Current Wind (FN_CURWIND) } \\
\text { Function }\end{array}$ & 3.1.2.2 \\
\hline $\begin{array}{l}\text { Get Reflection Coefficient } \\
\text { (GETREFCOEF) SU }\end{array}$ & 3.1.2.12 & $\begin{array}{l}\text { Dielectric Constant (FN_DIECON) } \\
\text { Function }\end{array}$ & 3.1.2.3 \\
\hline $\begin{array}{l}\text { Optical Region Limit (OPLIMIT) } \\
\text { SU }\end{array}$ & 3.1.3.8 & R1 Iteration (R1ITER) SU & 3.1.2.3 \\
\hline R1 Iteration (R1ITER) SU & 3.1.2.3 & Get Theta (GETTHETA) SU & 3.1.3.4 \\
\hline
\end{tabular}


Table 7. Requirements traceability matrix for the SRS. (continued)

\begin{tabular}{|c|c|c|c|}
\hline \multicolumn{2}{|c|}{ Software Requirements Specification } & \multicolumn{2}{|c|}{ Software Requirements Specification } \\
\hline SRS Requirement Name & $\begin{array}{c}\text { SRS } \\
\text { Paragraph } \\
\text { Number }\end{array}$ & SRS Requirement Name & $\begin{array}{c}\text { SRS } \\
\text { Paragraph } \\
\text { Number }\end{array}$ \\
\hline Get Theta (GETTHETA) SU & 3.1.3.4 & $\begin{array}{l}\text { Get Reflection Coefficient } \\
\text { (GETREFCOEF) SU }\end{array}$ & 3.1.2.12 \\
\hline $\begin{array}{l}\text { Get Reflection Coefficient } \\
\text { (GETREFCOEF) SU }\end{array}$ & 3.1.2.12 & $\begin{array}{l}\text { Current Wind (FN_CURWIND) } \\
\text { Function }\end{array}$ & 3.1.2.2 \\
\hline $\begin{array}{l}\text { Get Reflection Coefficient } \\
\text { (GETREFCOEF) SU }\end{array}$ & 3.1.2.12 & $\begin{array}{l}\text { Dielectric Constant (FN_DIECON) } \\
\text { Function }\end{array}$ & 3.1.2.3 \\
\hline $\begin{array}{l}\text { Standard Propagation Model } \\
\text { (SPM) SU }\end{array}$ & 3.1.3.12 & Optical Difference (OPTICF) SU & 3.1.3.9 \\
\hline Optical Difference (OPTICF) SU & 3.1.3.9 & $\begin{array}{l}\text { Get Reflection Coefficient } \\
\text { (GETREFCOEF) SU }\end{array}$ & 3.1.2.12 \\
\hline $\begin{array}{l}\text { Get Reflection Coefficient } \\
\text { (GETREFCOEF) SU }\end{array}$ & 3.1.2.12 & $\begin{array}{l}\text { Current Wind (FN_CURWIND) } \\
\text { Function }\end{array}$ & 3.1.2.2 \\
\hline $\begin{array}{l}\text { Get Reflection Coefficient } \\
\text { (GETREFCOEF) SU }\end{array}$ & 3.1.2.12 & $\begin{array}{l}\text { Dielectric Constant (FN_DIECON) } \\
\text { Function }\end{array}$ & 3.1.2.3 \\
\hline $\begin{array}{l}\text { Extended Optics Initialization } \\
\text { (XOINIT) CSC }\end{array}$ & 3.1 .3 & Mean Filter (MEANFILT) SU & 3.1.3.7 \\
\hline CSCI Capability Requirements & 3.1 & $\begin{array}{l}\text { Extended Optics Step (XOSTEP) } \\
\text { CSC }\end{array}$ & 3.1 .4 \\
\hline $\begin{array}{l}\text { Extended Optics Step (XOSTEP) } \\
\text { CSC }\end{array}$ & 3.1 .4 & APM Clean (APMCLEAN) SU & 3.1.3.1 \\
\hline APM Clean (APMCLEAN) SU & 3.1.3.1 & $\begin{array}{l}\text { Discrete Sine/Cosine Transform } \\
\text { (DRST) SU }\end{array}$ & 3.1.3.1 \\
\hline $\begin{array}{l}\text { Extended Optics Step (XOSTEP) } \\
\text { CSC }\end{array}$ & 3.1 .4 & Extended Optics (EXTO) SU & 3.1 .4 \\
\hline Extended Optics (EXTO) SU & 3.1 .4 & $\begin{array}{l}\text { Linear Interpolation (FN_PLINT) } \\
\text { Function }\end{array}$ & 3.1.2.14 \\
\hline Extended Optics (EXTO) SU & 3.1 .4 & Troposcatter (TROPOSCAT) SU & 3.1.2.24 \\
\hline Troposcatter (TROPOSCAT) SU & 3.1.2.24 & $\begin{array}{l}\text { Get Troposcatter Loss } \\
\text { (FN_GET_TLOSS) Function }\end{array}$ & 3.1.2.13 \\
\hline $\begin{array}{l}\text { Get Troposcatter Loss } \\
\text { (FN_GET_TLOSS) Function }\end{array}$ & 3.1.2.13 & Antenna Pattern (ANTPAT) SU & 3.1.1.6 \\
\hline $\begin{array}{l}\text { Extended Optics Step (XOSTEP) } \\
\text { CSC }\end{array}$ & 3.1 .4 & Flat-Earth Model (FEM) SU & 3.1.2.7 \\
\hline Flat-Earth Model (FEM) SU & 3.1.2.7 & Antenna Pattern (ANTPAT) SU & 3.1.1.6 \\
\hline
\end{tabular}


Table 7. Requirements traceability matrix for the SRS. (continued)

\begin{tabular}{|c|c|c|c|}
\hline \multicolumn{2}{|c|}{ Software Requirements Specification } & \multicolumn{2}{|c|}{ Software Requirements Specification } \\
\hline SRS Requirement Name & $\begin{array}{c}\text { SRS } \\
\text { Paragraph } \\
\text { Number }\end{array}$ & SRS Requirement Name & $\begin{array}{c}\text { SRS } \\
\text { Paragraph } \\
\text { Number }\end{array}$ \\
\hline Flat-Earth Model (FEM) SU & 3.1.2.7 & $\begin{array}{l}\text { Get Reflection Coefficient } \\
\text { (GETREFCOEF) SU }\end{array}$ & 3.1.2.12 \\
\hline $\begin{array}{l}\text { Get Reflection Coefficient } \\
\text { (GETREFCOEF) SU }\end{array}$ & 3.1.2.12 & $\begin{array}{l}\text { Current Wind (FN_CURWIND) } \\
\text { Function }\end{array}$ & 3.1.2.2 \\
\hline $\begin{array}{l}\text { Get Reflection Coefficient } \\
\text { (GETREFCOEF) SU }\end{array}$ & 3.1.2.12 & $\begin{array}{l}\text { Dielectric Constant (FN_DIECON) } \\
\text { Function }\end{array}$ & 3.1.2.3 \\
\hline $\begin{array}{l}\text { Extended Optics Step (XOSTEP) } \\
\text { CSC }\end{array}$ & 3.1 .4 & Ray Optics Loss (ROLOSS) SU & 3.1.2.20 \\
\hline Ray Optics Loss (ROLOSS) SU & 3.1 .2 .20 & $\begin{array}{l}\text { Ray Optics Calculation (ROCALC) } \\
\text { SU }\end{array}$ & 3.1.2.19 \\
\hline $\begin{array}{l}\text { Ray Optics Calculation } \\
\text { (ROCALC) SU }\end{array}$ & 3.1.2.19 & Antenna Pattern (ANTPAT) SU & 3.1.1.6 \\
\hline $\begin{array}{l}\text { Ray Optics Calculation } \\
\text { (ROCALC) SU }\end{array}$ & 3.1.2.19 & $\begin{array}{l}\text { Get Reflection Coefficient } \\
\text { (GETREFCOEF) SU }\end{array}$ & 3.1.2.12 \\
\hline $\begin{array}{l}\text { Get Reflection Coefficient } \\
\text { (GETREFCOEF) SU }\end{array}$ & 3.1.2.12 & $\begin{array}{l}\text { Current Wind (FN_CURWIND) } \\
\text { Function }\end{array}$ & 3.1.2.2 \\
\hline $\begin{array}{l}\text { Get Reflection Coefficient } \\
\text { (GETREFCOEF) SU }\end{array}$ & 3.1.2.12 & $\begin{array}{l}\text { Dielectric Constant (FN_DIECON) } \\
\text { Function }\end{array}$ & 3.1.2.3 \\
\hline $\begin{array}{l}\text { Ray Optics Calculation } \\
\text { (ROCALC) SU }\end{array}$ & 3.1.2.19 & Ray Trace (RAYTRACE) SU & 3.1.2.17 \\
\hline CSCI Capability Requirements & 3.1 & $\begin{array}{l}\text { Return Grazing Angle } \\
\text { (RET_GRAZE) CSC }\end{array}$ & 3.1 .5 \\
\hline CSCI Capability Requirements & 3.1 & $\begin{array}{l}\text { CSCI External Interface } \\
\text { Requirements }\end{array}$ & 3.2 \\
\hline CSCI Capability Requirements & 3.1 & $\begin{array}{l}\mathrm{CSCI} \text { Internal Interface } \\
\text { Requirements }\end{array}$ & 3.3 \\
\hline CSCI Capability Requirements & 3.1 & CSCI Internal Data Requirements & 3.4 \\
\hline CSCI Capability Requirements & 3.1 & Adaptation Requirements & 3.5 \\
\hline CSCI Capability Requirements & 3.1 & $\begin{array}{l}\text { Security and Privacy } \\
\text { Requirements }\end{array}$ & 3.6 \\
\hline CSCI Capability Requirements & 3.1 & $\begin{array}{l}\text { CSCI Environmental } \\
\text { Requirements }\end{array}$ & 3.7 \\
\hline CSCI Capability Requirements & 3.1 & $\begin{array}{l}\text { Computer Resource } \\
\text { Requirements }\end{array}$ & 3.8 \\
\hline
\end{tabular}


Table 7. Requirements traceability matrix for the SRS. (continued)

\begin{tabular}{l|c|l|c}
\hline \multicolumn{2}{c|}{ Software Requirements Specification } & \multicolumn{2}{c}{ Software Requirements Specification } \\
\multicolumn{1}{c|}{ SRS Requirement Name } & $\begin{array}{c}\text { SRS } \\
\text { Paragraph } \\
\text { Number }\end{array}$ & \multicolumn{1}{c}{ SRS Requirement Name } & $\begin{array}{c}\text { SRS } \\
\text { Paragraph } \\
\text { Number }\end{array}$ \\
\hline CSCI Capability Requirements & 3.1 & Software Quality Factors & 3.9 \\
CSCI Capability Requirements & 3.1 & $\begin{array}{l}\text { Design And Implementation } \\
\text { Constraints }\end{array}$ & 3.10 \\
$\begin{array}{l}\text { Design And Implementation } \\
\text { Constraints }\end{array}$ & 3.10 & $\begin{array}{l}\text { Implementation and Application } \\
\text { Considerations }\end{array}$ & 3.10 .1 \\
$\begin{array}{l}\text { Design And Implementation } \\
\text { Constraints }\end{array}$ & 3.10 & $\begin{array}{l}\text { Programming Language And } \\
\text { Source Code Implementation }\end{array}$ & 3.10 .2 \\
$\begin{array}{l}\text { Programming Language And } \\
\text { Source Code Implementation }\end{array}$ & 3.10 .2 & Programming Language & 3.10 .2 .1 \\
$\begin{array}{l}\text { Programming Language And } \\
\text { Source Code Implementation }\end{array}$ & 3.10 .2 & Source Implementation & 3.10 .2 .2 \\
CSCI Capability Requirements & 3.1 & Personnel-Related Requirements & 3.11 \\
CSCI Capability Requirements & 3.1 & Training Related Requirements & 3.12 \\
CSCI Capability Requirements & 3.1 & Other Requirements & 3.13 \\
CSCI Capability Requirements & 3.1 & $\begin{array}{l}\text { Precedence and Criticality of } \\
\text { Requirements }\end{array}$ & 3.14 \\
\hline
\end{tabular}

\section{NOTES}

Table 8 is a glossary of acronyms and abbreviations used within this document. Table 9 is a glossary of Fortran terms used within this document. 
Table 8. Acronyms and abbreviations.

\begin{tabular}{c|l}
\hline Term & \multicolumn{1}{c}{ Definition } \\
\hline ANSI & American National Standards Institute \\
CB & Advanced Propagation Model \\
CSC & Contibel \\
CSCI & Computer Software Component \\
dB & decibel \\
EM & Electromagnetic \\
FFT & Fast-Fourier Transform \\
Fortran & Formula Translation \\
km & kilometers \\
m & meters \\
M & modified refractivity units \\
MHz & megahertz \\
N/A & not applicable \\
PE & Parabolic Equation \\
p-space & phase (angle) space \\
rad & radians \\
SDD & Software Design Description \\
SRS & Software Requirements Specification \\
STD & Software Test Description \\
SU & Software Unit \\
\hline Space & height space \\
\hline
\end{tabular}


Table 9. Fortran terms.

\begin{tabular}{|c|c|}
\hline Term & Action or Definitions \\
\hline ABS & Absolute value function \\
\hline Arithmetic IF & $\begin{array}{l}\text { Transfers control to one of three statement labels, depending on the } \\
\text { vallue of expression }\end{array}$ \\
\hline ASSIGN & Assigns the value of a format or statement label to an integer variable \\
\hline CASE & $\begin{array}{l}\text { Marks the beginning of a block of statements executed if an item in a } \\
\text { list of expressions matches the test expressions }\end{array}$ \\
\hline COMMON & $\begin{array}{l}\text { Allows two or more program units to directly share variables without } \\
\text { having to pass them as arguments }\end{array}$ \\
\hline CONTINUE & Does not have any effect \\
\hline DO & $\begin{array}{l}\text { Repeatedly executes the statements following the DO statement } \\
\text { through the statement which marks the end of the loop }\end{array}$ \\
\hline DO WHILE & $\begin{array}{l}\text { Executes a block of statements repeatedly while a logical condition } \\
\text { remains true }\end{array}$ \\
\hline END DO & Terminates a DO or DO WHILE loop \\
\hline END IF & Terminates a block of IF statements \\
\hline EQUIVALENCE & $\begin{array}{l}\text { Causes two or more variables or arrays to occupy the same memory } \\
\text { location }\end{array}$ \\
\hline FIX & Data type conversion function \\
\hline FLOAT & Data type conversion function \\
\hline FORMAT & Sets the format in which data are written to or read from a file \\
\hline GO TO & Transfers execution to the statement label assigned to variable \\
\hline IF & $\begin{array}{l}\text { If expression is true, statement is executed; if expression is false, } \\
\text { program execution continues with the next executable statement }\end{array}$ \\
\hline IF THEN ELSE & $\begin{array}{l}\text { If expression is true, statements in the IF block are executed; if } \\
\text { expression is false, control is transferred to the next ELSE, ELSE IF, } \\
\text { or END IF statement at the same IF level }\end{array}$ \\
\hline PAUSE & $\begin{array}{l}\text { Temporarily suspends program execution and allows you to execute } \\
\text { operating system commands during the suspension }\end{array}$ \\
\hline
\end{tabular}




\section{APPENDIX A}

\section{A.1 DEFINITIONS OF QUALITY FACTOR CRITERIA}

The criteria for judging the quality factors of Section 3.9 have the following definitions:

1. Accuracy. The precision of computations and control

2. Anomaly management. The degree to which the program detects failure in order to maintain consistency

3. Application independence. The degree to which the program is independent of nonstandard programming language features, operating system characteristics, and other environmental constraints

4. Auditability. The ease with which conformance to standards can be checked

5. Completeness. The degree to which full implementation of required function has been achieved

6. Consistency. The use of uniform design and documentation techniques throughout the software development project

7. Document accessibility. The availability of documents describing the program components

8. Functional scope. The generality of the feature set and capabilities of the program

9. Generality. The breadth of potential application of program components

10. Hardware independence. The degree to which the software is decoupled from the hardware on which it operates

11. Modularity. The functional independence of program components

12. Self-descriptiveness. The degree to which the source code provides meaningful documentation

13. Simplicity. The degree to which a program can be understood without difficulty 
14. System clarity. The ease for which the feature set and capabilities of the system can be determined

15. Traceability. The ability to trace a design representation or actual program component back to requirements

\section{A.2 SOFTWARE QUALITY METRICS}

\section{A.2.1 Completeness Criteria}

The criteria completeness can be determined from the metric:

1. no ambiguous references (input, function, output)

2. all data references defined

3. all referenced functions defined

4. all defined functions used

5. all conditions and processing defined for each decision point

6. all defined and referenced calling sequences parameters agree

7. all problem reports resolved

8. design agrees with requirements

9. code agrees with design

10. (score 0 for any untrue statement; 1 otherwise)

11. metric value $=\mathrm{SUM}($ scores $) / 9$

\section{A.2.2 Consistency Criteria}

The criteria consistency can be determined from the metric: number of modules violating the design standard divided by the number of modules.

\section{A.2.3 Traceability Criteria}

The criteria traceability can be determined from the metric: number of itemized requirements traced divided by the total number of requirements. 
SOFTWARE DESIGN DESCRIPTION FOR THE ADVANCED PROPAGATION MODEL CSCI (Version 2.1.04)

20 December 2006 


\section{TABLE OF CONTENTS}

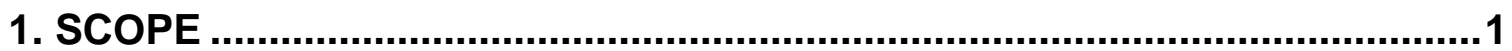

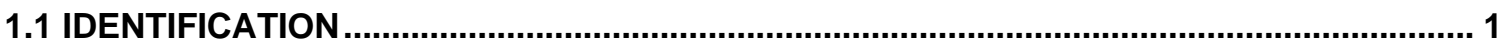

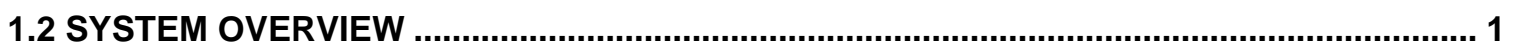

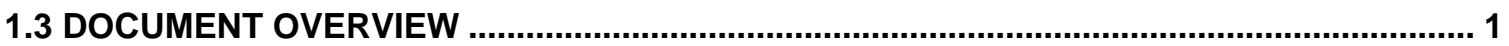

2. REFERENCED DOCUMENTS ....................................................................

3. CSCI-WIDE DESIGN DECISIONS ............................................................

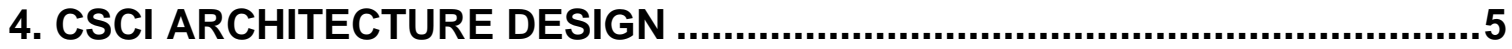

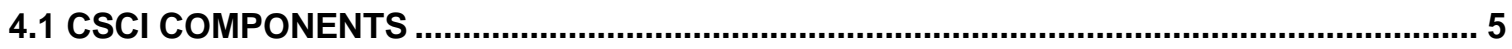

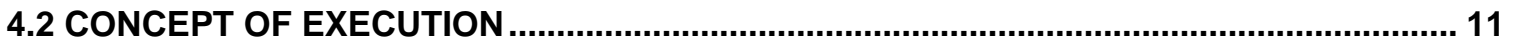

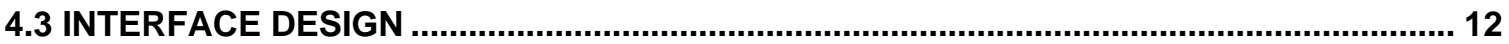

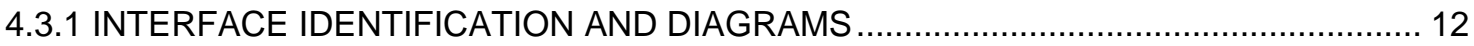

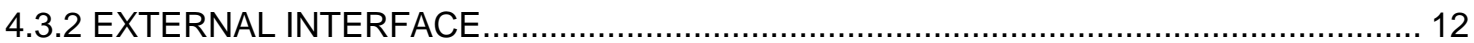

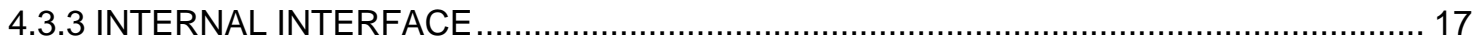

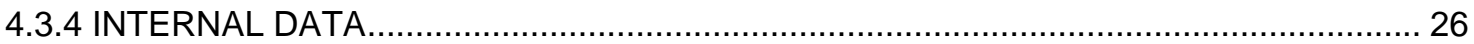

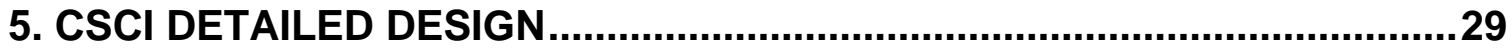

5.1 ADVANCE PROPAGATION MODEL INITIALIZATION (APMINIT) CSC ............................. 29

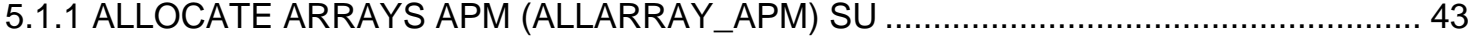

5.1.2 ALLOCATE ARRAY PE (ALLARRAY_PE) SU....................................................... 47

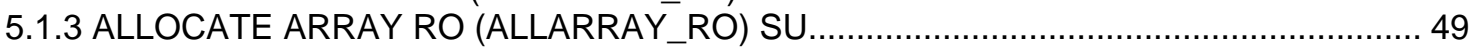

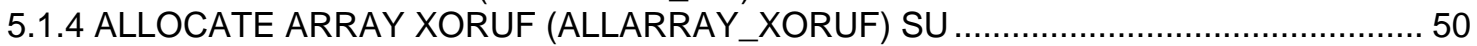

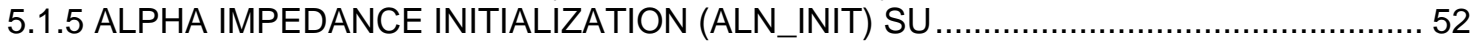

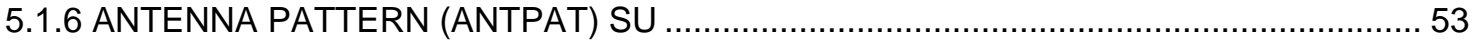

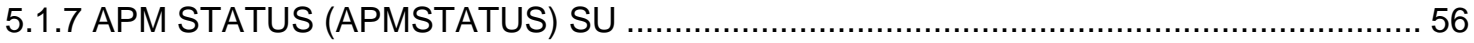

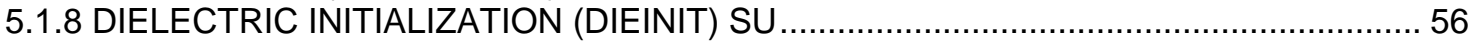

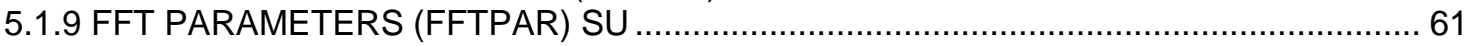

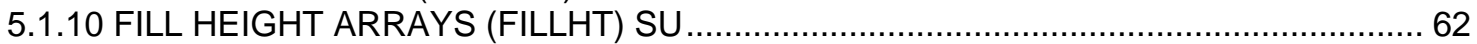

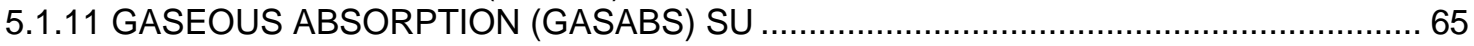

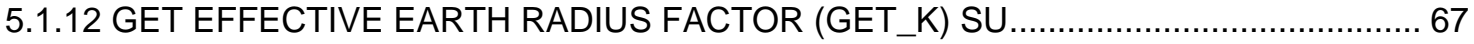

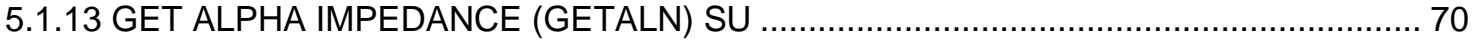

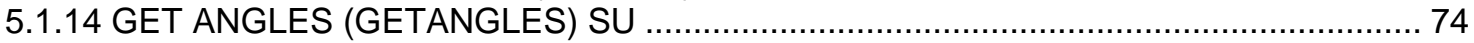

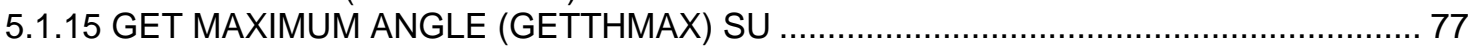

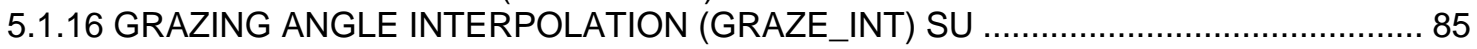

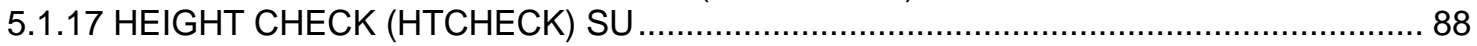

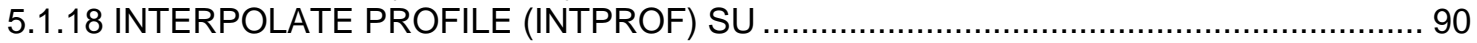

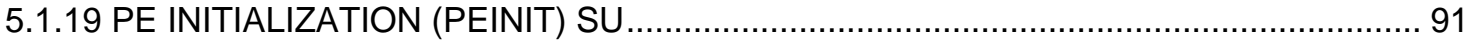

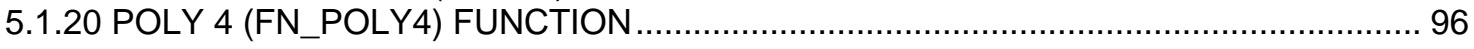




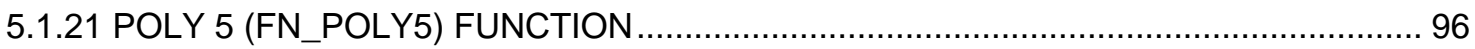

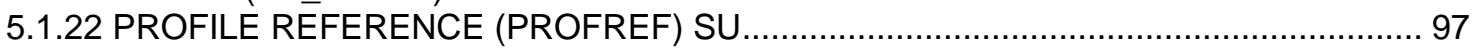

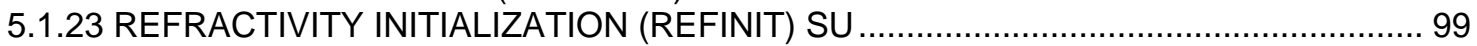

5.1.24 REMOVE DUPLICATE REFRACTIVITY LEVELS (REMDUP) SU …......................... 105

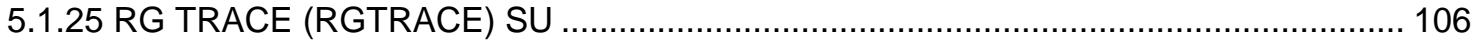

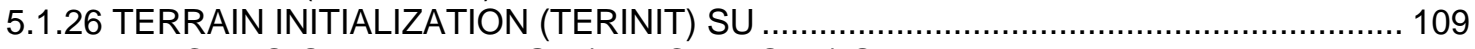

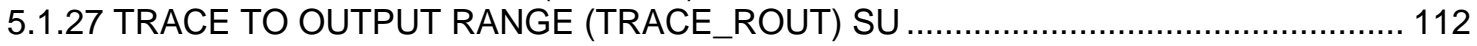

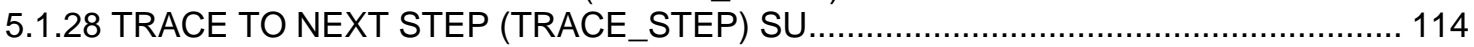

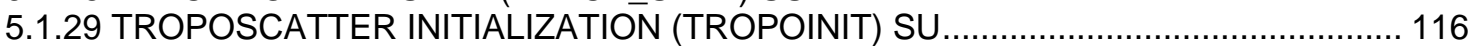

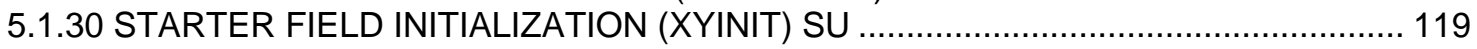

5.2 ADVANCED PROPAGATION MODEL STEP (APMSTEP) CSC …................................... 121

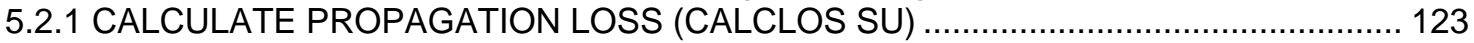

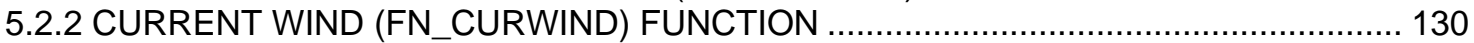

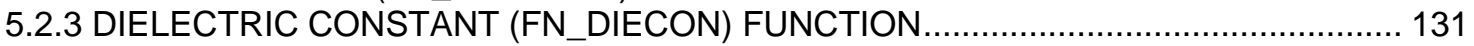

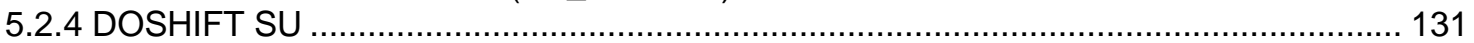

5.2.5 DISCRETE SINE/COSINE FAST-FOURIER TRANSFORM (DRST) SU ..................... 132

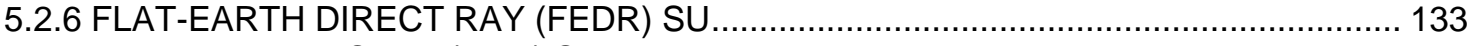

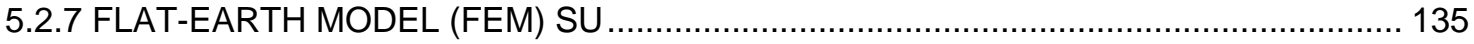

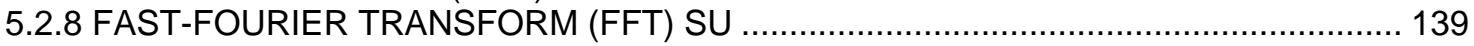

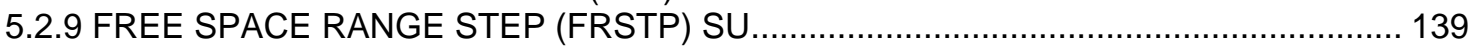

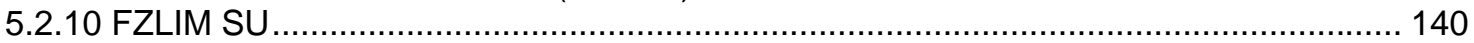

5.2.11 GET PROPAGATION FACTOR (FN_GETPFAC) SU ......................................... 142

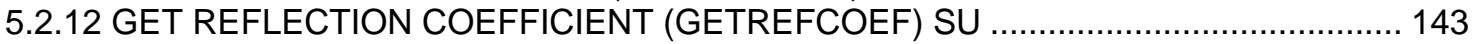

5.2.13 GET TROPOSCATTER LOSS (FN_GET_TLOSS) FUNCTION ............................. 145

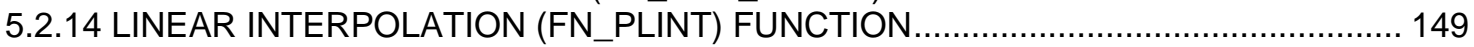

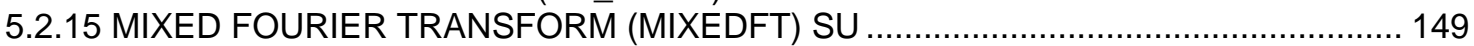

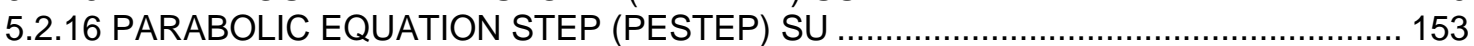

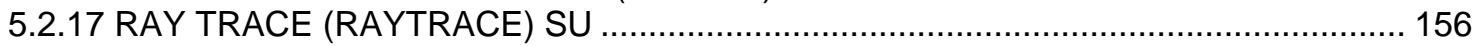

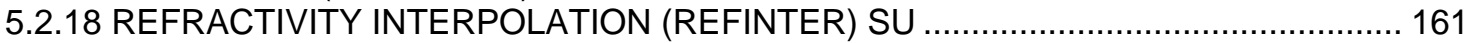

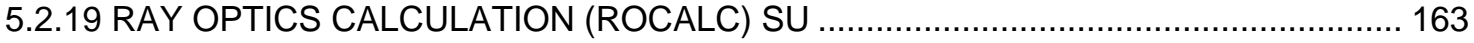

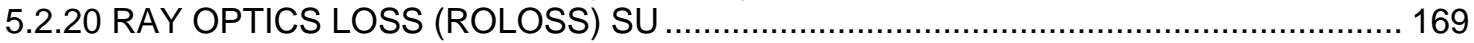

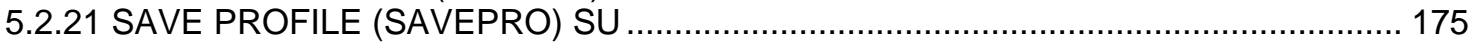

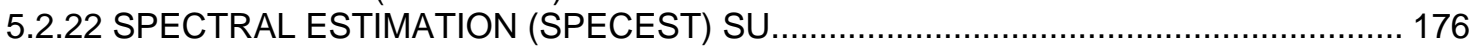

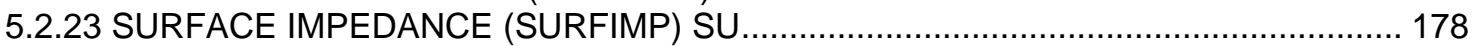

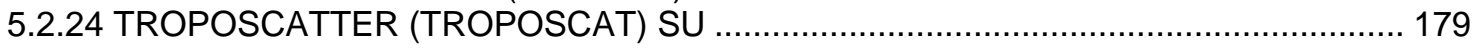

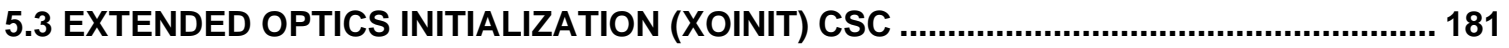

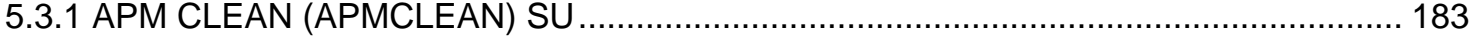

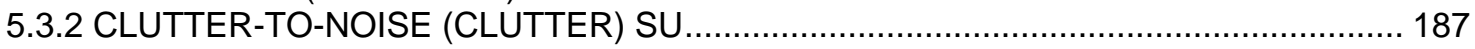

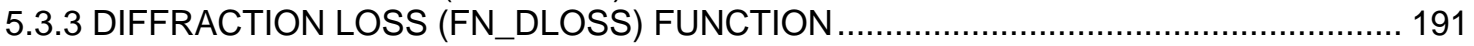

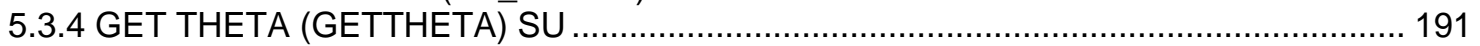

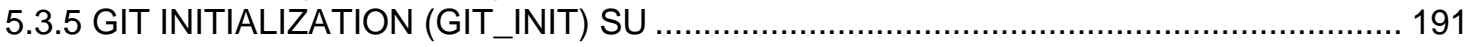

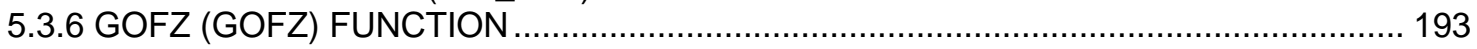

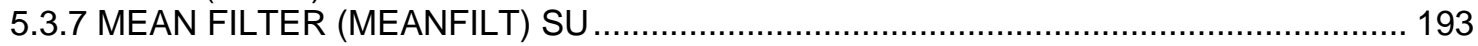

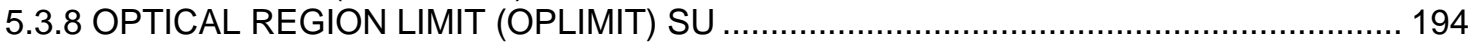

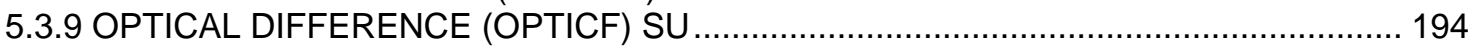

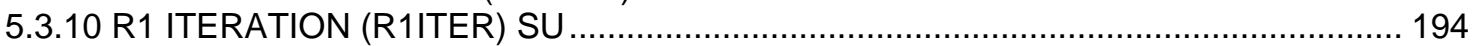

5.3.11 STANDARD PROPAGATION MODEL INITIALIZATION (SPM_INIT) SU .................... 194

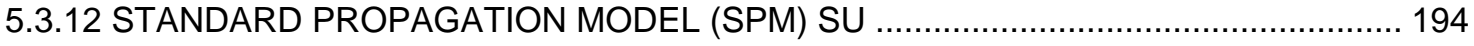

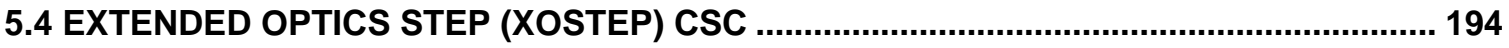

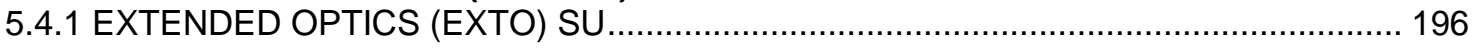

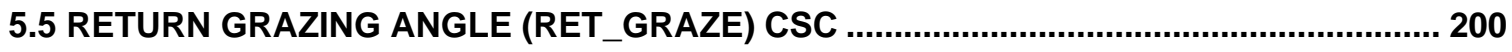


7. NOTES

7.1 APM CSCI IMPLEMENTATION AND APPLICATION CONSIDERATIONS

7.2 ENVIRONMENTAL RADIO REFRACTIVITY FIELD DATA ELEMENTS 207

7.3 TERRAIN PROFILE DATA ELEMENT 210

7.4 ACRONYM AND ABBREVIATIONS 210

7.5 SDD VARIABLE NAME, FORTRAN VARIABLE NAME CROSS REFERENCE 212

\section{Figures}

Figure 1. APM calculation regions. 4

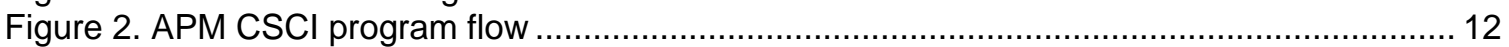

Figure 3. Idealized M-unit profiles (solid) and lines of interpolation (dashed) .......................... 208

Tables

Table 1. APM CSCl environmental data element requirements ............................................ 13

Table 2. APM CSCI external EM system data element requirements ..................................... 14

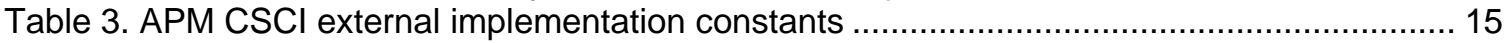

Table 4. APM CSCI external terrain data element requirements ............................................. 16

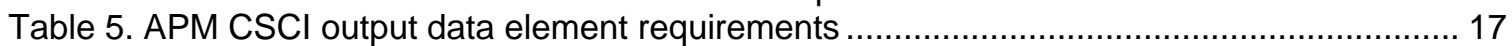

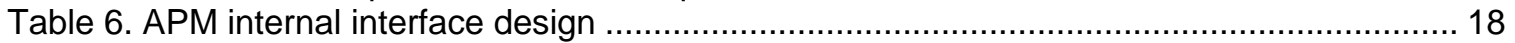

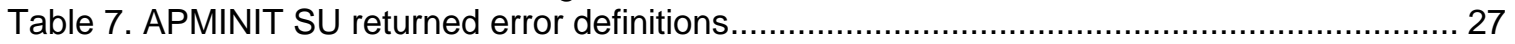

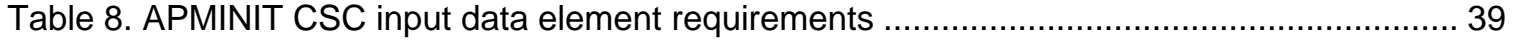

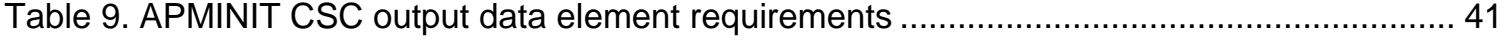

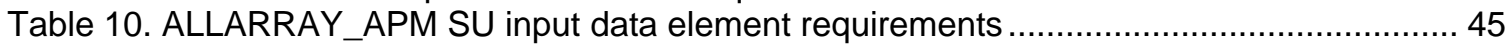

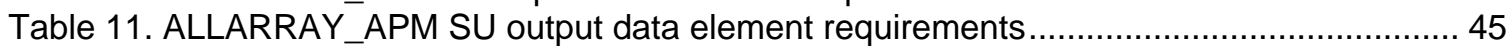

Table 12. ALLARRAY_PE SU input data element requirements ............................................ 48

Table 13. ALLARRAY_PE SU output data element requirements ............................................ 48

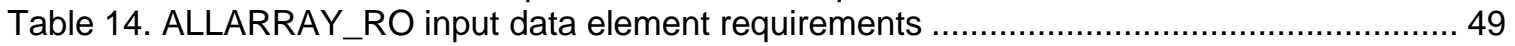

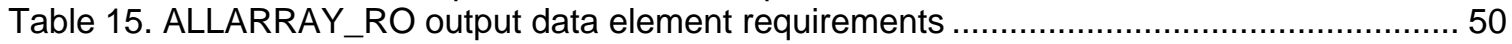

Table 16. ALLARRAY_XORUF SU input data element requirements ......................................... 51

Table 17. ALLARRAY_XORUF SU output data element requirements ..................................... 51

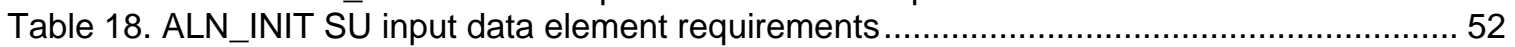

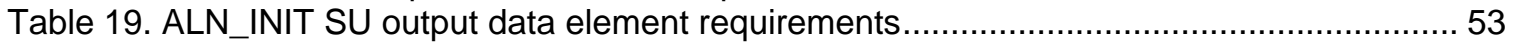

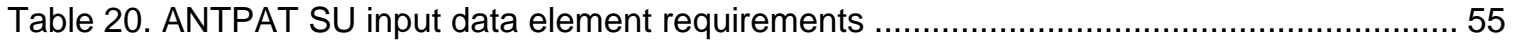

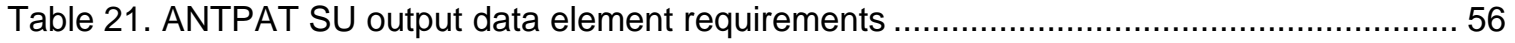

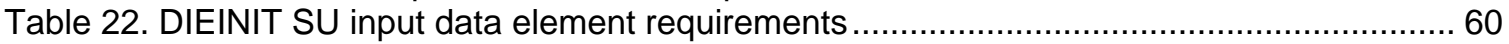

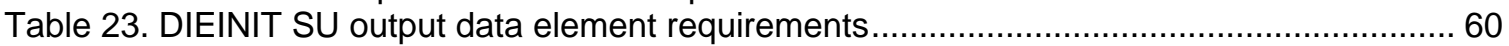

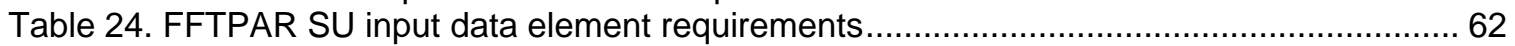

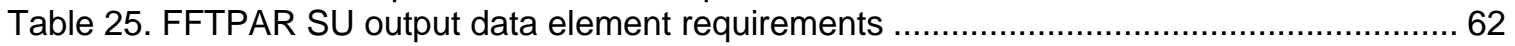

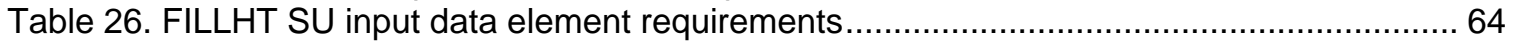

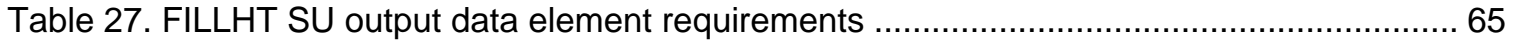

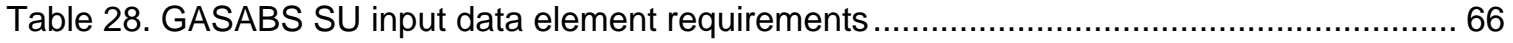

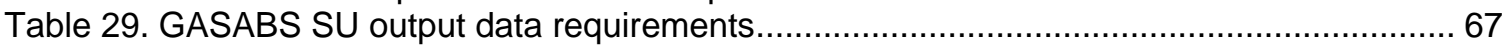

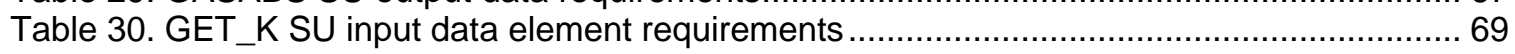

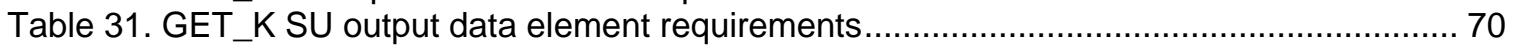




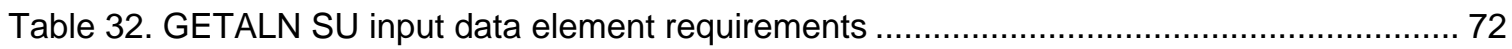

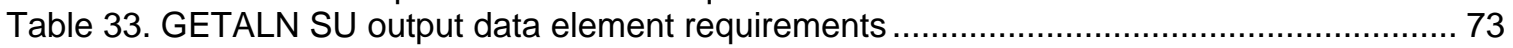

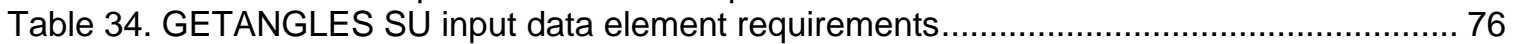

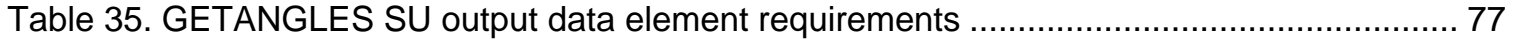

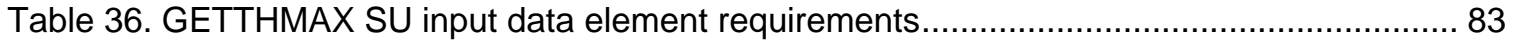

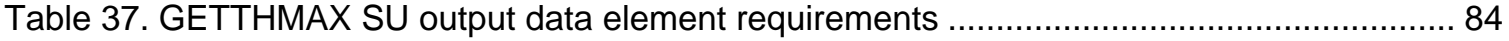

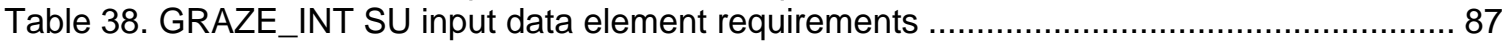

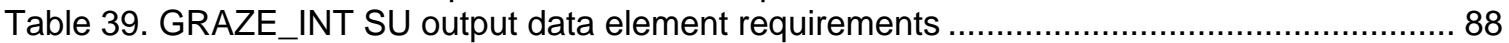

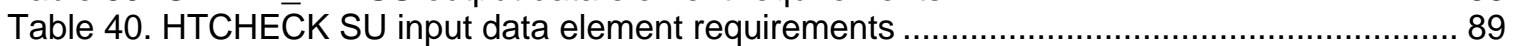

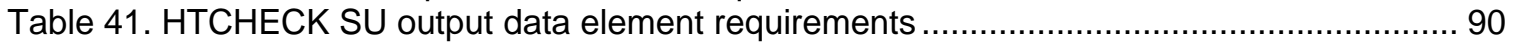

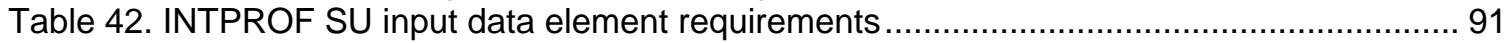

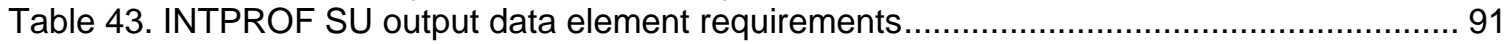

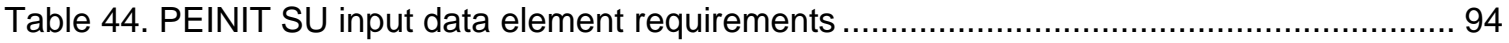

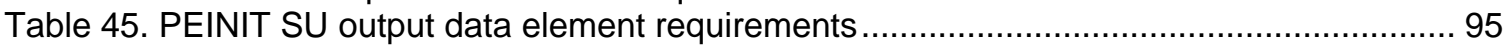

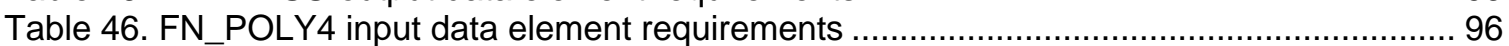

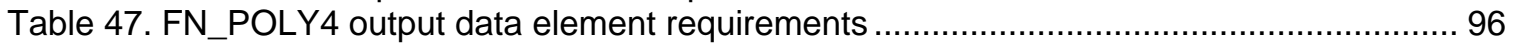

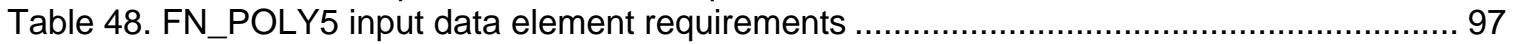

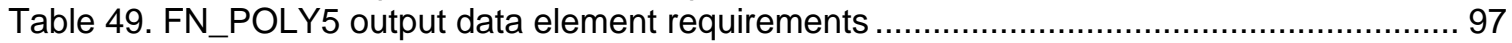

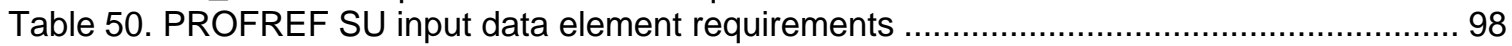

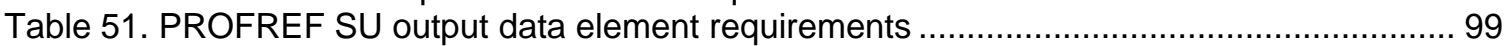

Table 52. REFINIT SU input data element requirements................................................... 103

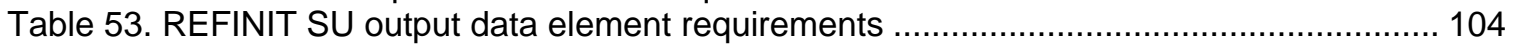

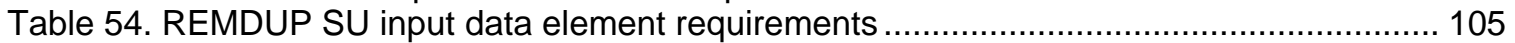

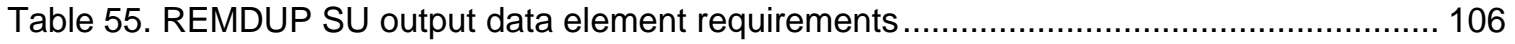

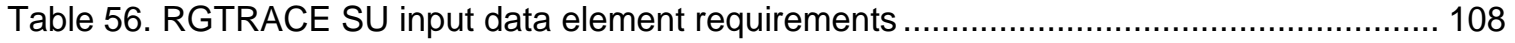

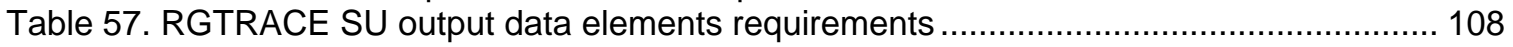

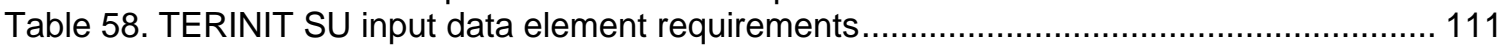

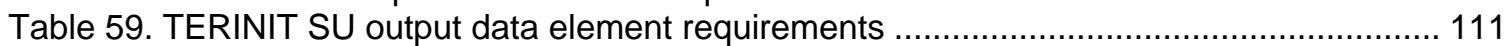

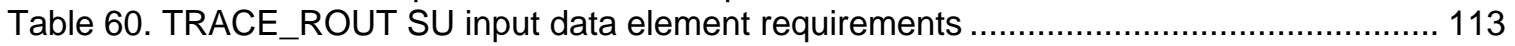

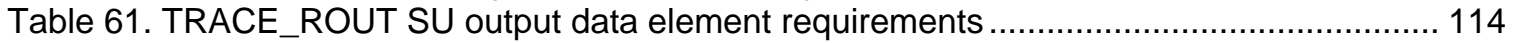

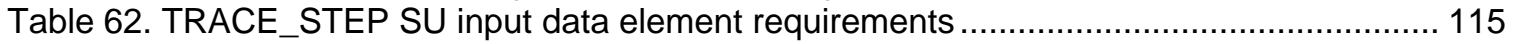

Table 63. TRACE_STEP SU output data element requirements .......................................... 116

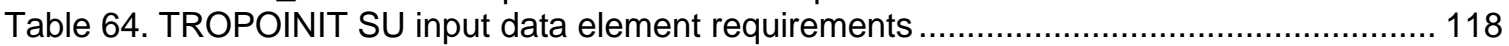

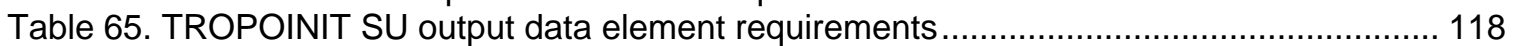

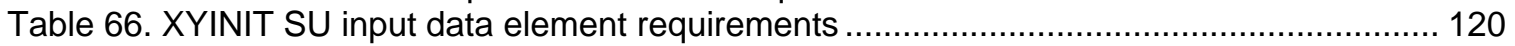

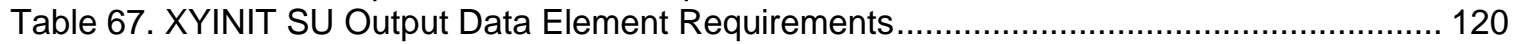

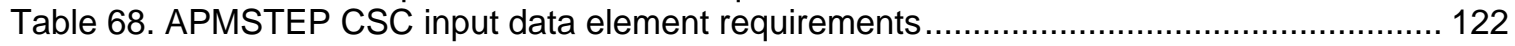

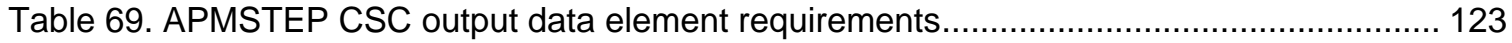

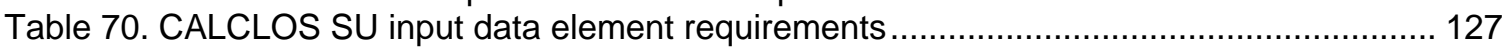

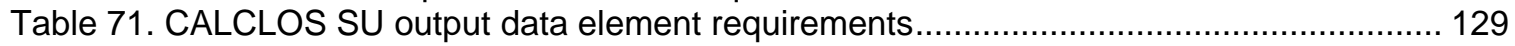

Table 72. FN_CURWIND function input data element requirements ..................................... 130

Table 73. FN_CURWIND function output data element requirements..................................... 130

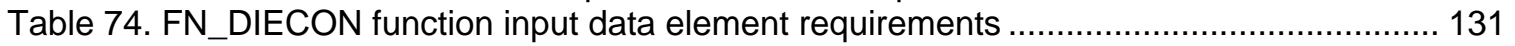

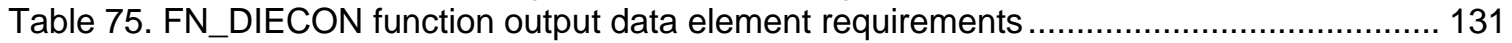

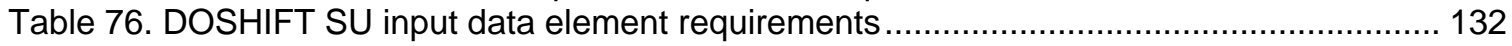

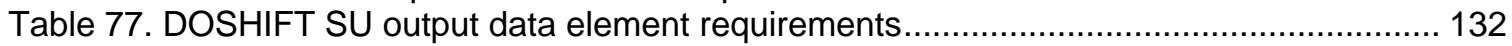

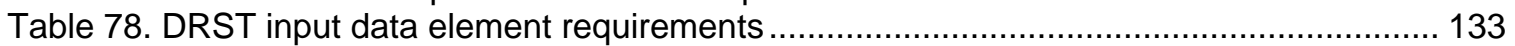

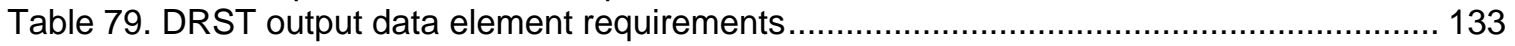

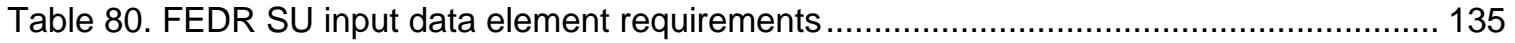

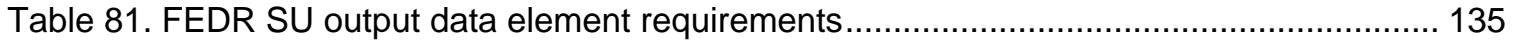

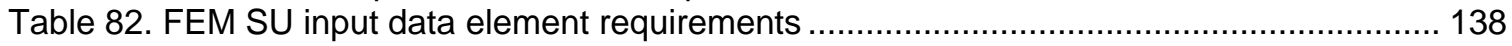

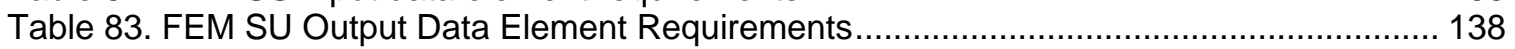

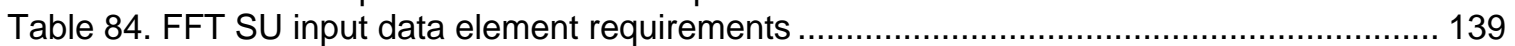

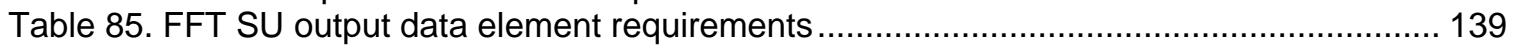

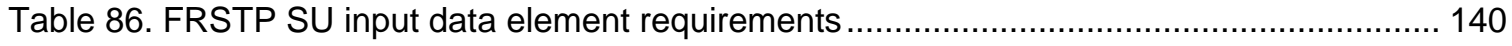

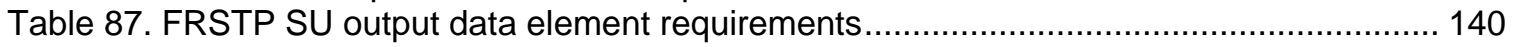




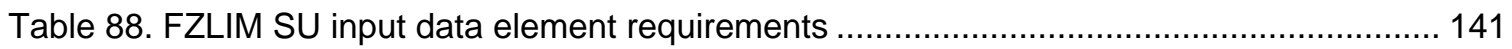

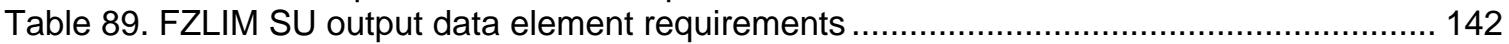

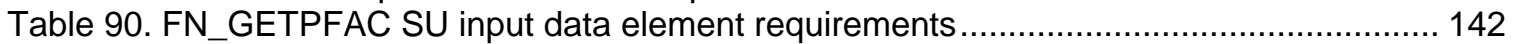

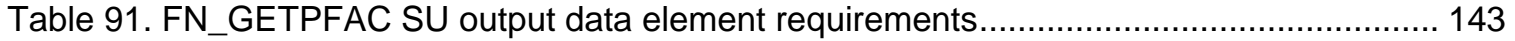

Table 92. GETREFCOEF SU input data element requirements .......................................... 144

Table 93. GETREFCOEF SU output data element requirements .......................................... 145

Table 94. FN_GET_TLOSS function input data element requirements .................................... 148

Table 95. FN_GET_TLOSS function output data element requirements................................. 148

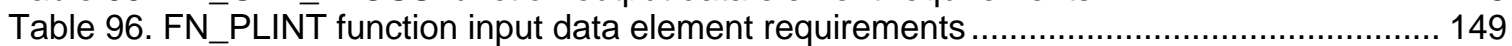

Table 97. FN_PLINT function output data element requirements .......................................... 149

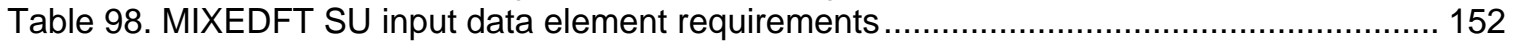

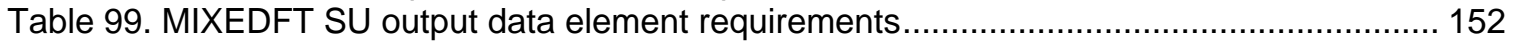

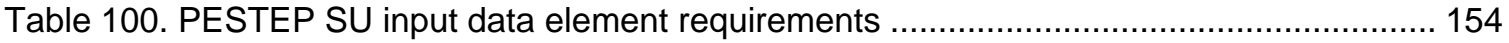

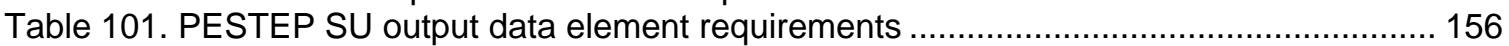

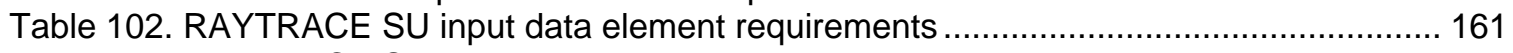

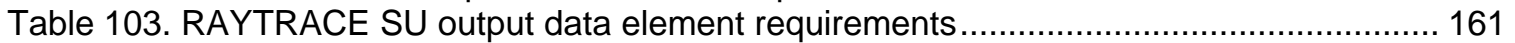

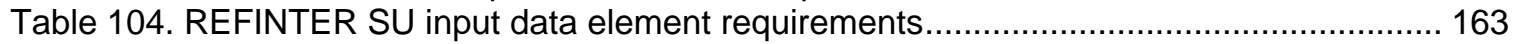

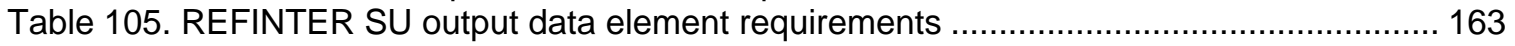

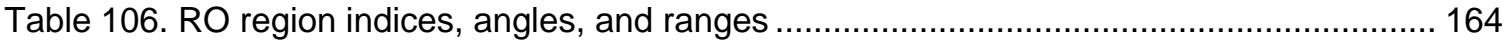

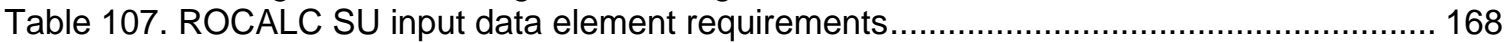

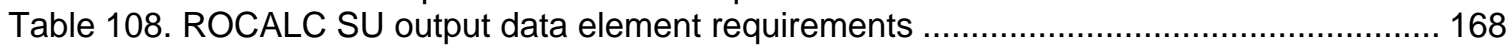

Table 109. ROLOSS SU input data element requirements.................................................. 173

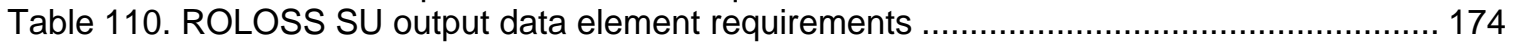

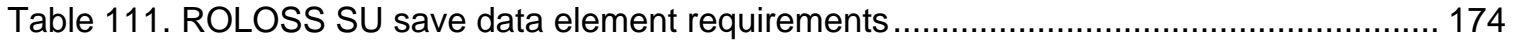

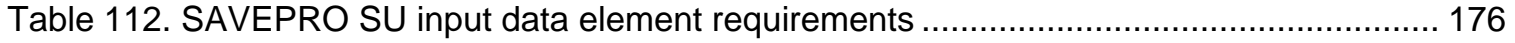

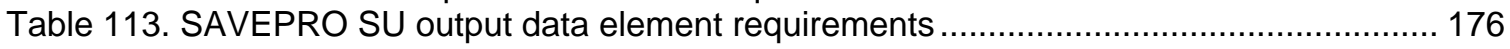

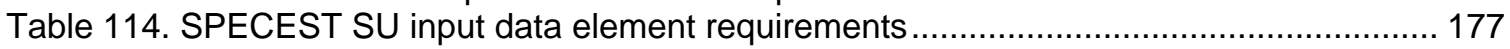

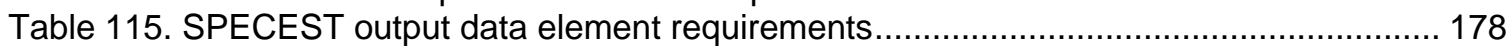

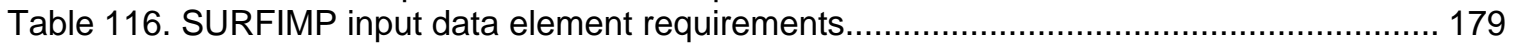

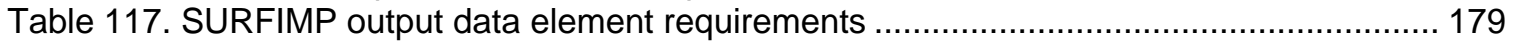

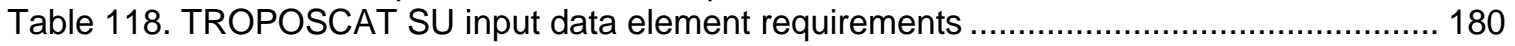

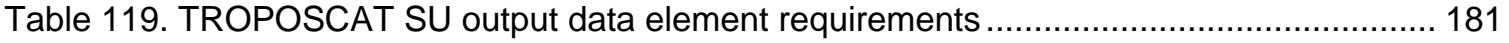

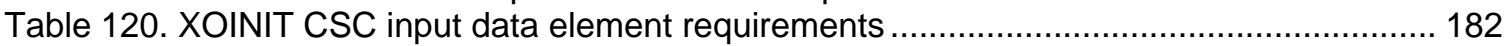

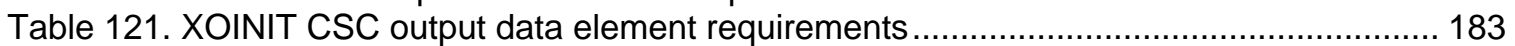

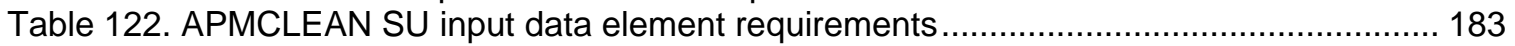

Table 123. APMCLEAN CSC output data element requirements ......................................... 186

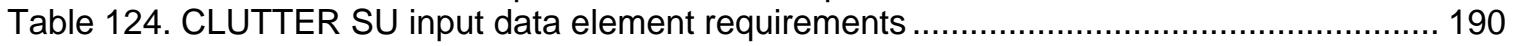

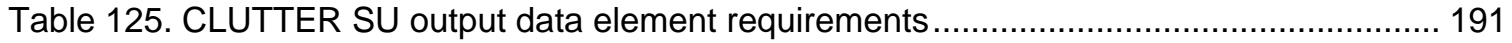

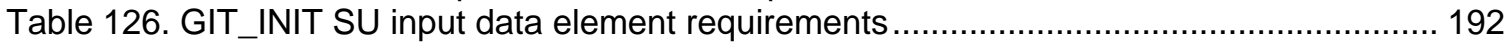

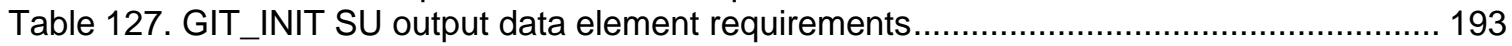

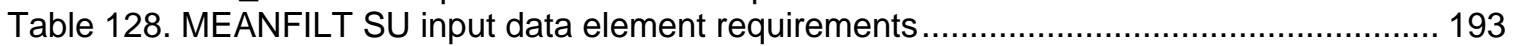

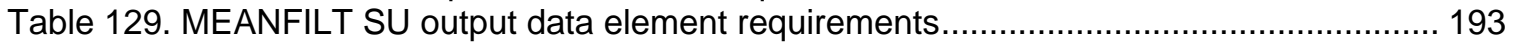

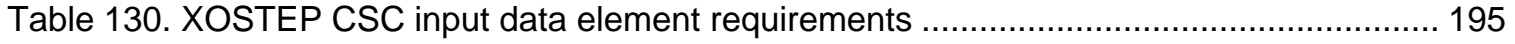

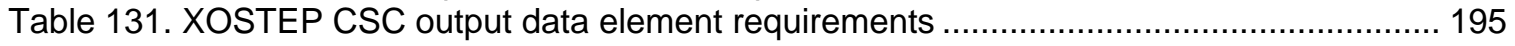

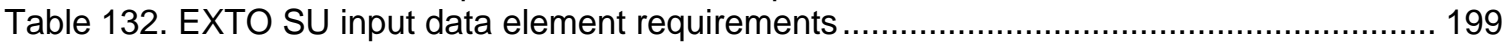

Table 133. EXTO SU output data element requirements ..................................................... 200

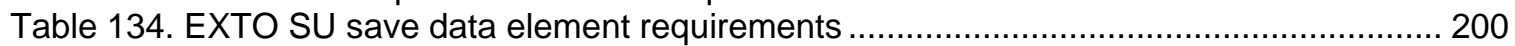

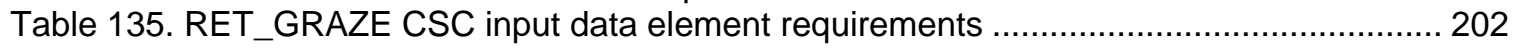

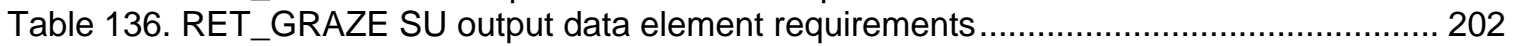

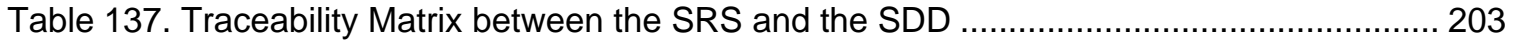

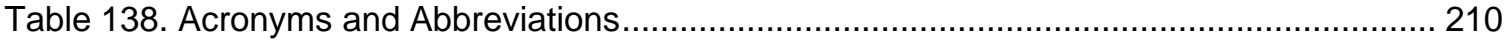

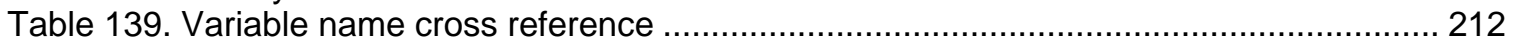




\section{SCOPE}

\subsection{IDENTIFICATION}

The Advanced Propagation Model (APM) Version 2.1.04 computer software configuration item (CSCI) calculates range-dependent electromagnetic (EM) system propagation loss within a heterogeneous atmospheric medium over variable terrain, where the radio-frequency index of refraction is allowed to vary vertically and horizontally, also accounting for terrain effects along the path of propagation.

\subsection{SYSTEM OVERVIEW}

The APM CSCI model calculates propagation loss values as EM energy propagates through a laterally heterogeneous atmospheric medium where the index of refraction is allowed to vary both vertically and horizontally, also accounting for terrain effects along the path of propagation. Numerous external applications require EM-system propagation loss values. The APM model described by this document may be applied to two external applications, one which displays propagation loss on a range versus height scale (commonly referred to as a coverage diagram) and one which displays propagation loss on a propagation loss versus range/height scale (commonly referred to as a loss diagram).

\subsection{DOCUMENT OVERVIEW}

This document describes the design of the APM CSCI. An overview of the input software requirements is presented together with an overview of the CSCI design architecture and a detailed design description of each component of the CSCI.

\section{REFERENCED DOCUMENTS}

American National Standards Institute (ANSI), "Program Language - Fortran Extended”, 1992.

Barrick, D.E. 1971a. "Theory of HF and VHF Propagation Across the Rough Sea, 1. The Effective Surface Impedance for a Slightly Rough Highly Conducting Medium at Grazing Incidence,” Radio Sci. (May), V6, no. 5, pp.571-526.

Barrick, D.E. 1971b. "Theory of HF and VHF Propagation Across the Rough Sea, 2. Application to HF and VHF Propagation Above the Sea,” Radio Sci. (May), V6, no. 5, pp. 527-533. 
Barrios, A. E., "Terrain and Refractivity Effects on Non-Optical Paths," AGARD Conference Proceedings 543, Multiple Mechanism Propagation Paths (MMPPs): Their Characteristics and Influence on System Design, pp. 10-1 to 10-9, October 1993.

Barrios, A. E., "A Terrain Parabolic Equation Model for Propagation in the Troposphere,” IEEE Trans. Antennas Propagat., Vol. 42, pp. 90-98, January 1994.

Barrios, A. E., "Terrain Parabolic Equation Model (TPEM) Version 1.5 User’s Manual,” Naval Command, Control and Ocean Surveillance Center, RDT\&E Division, San Diego, CA, NRaD TD 2898, February 1996.

Barrios, A.E., Patterson, W.L., “Advanced Propagation Model (APM) Ver. 1.3.1 Computer Software Configuration Item (CSCI) Documents”, SSC-SD TD 3145, Aug. 2002.

Bergland, G. D., "A Radix-eight Fast Fourier Transform Subroutine for Real-valued Series,” IEEE Trans. Audio and Electro-acoust., Vol. AU-17, pp. 138-144, 1969.

Commander-In-Chief, Pacific Fleet Meteorological Requirement (PAC MET) 87-04, "Range Dependent Electromagnetic Propagation Models,” 1987.

Cooley, J. W., P. A. W. Lewis and P. D. Welsh, “The Fast Fourier Transform Algorithm: Programming Considerations in the Calculation of Sine, Cosine and Laplace Transforms,” J. Sound Vib., Vol. 12, pp. 315-337, 1970.

Dockery, G. D., "Modeling Electromagnetic Wave Propagation in the Troposphere Using the Parabolic Equation”, IEEE Trans. Antennas Propagat., Vol. 36, No. 10, pp. 14641470, October 1988.

Dockery, G.D, Kuttler, J.R., "An Improved Impedance-Boundary Algorithm for Fourier Split-Step Solutions of the Parabolic Wave Equation," IEEE Trans. On Antennas and Propagat., Vol. 44, No. 12, December 1996, pp. 1592-1599.

Hitney, H.V., Hattan, C.P., Paulus, R.A., Anderson, K.D., "Electromagnetic Propagation Functions Program Performance Specification (PPS) For the Tactical Environmental Support System (TESS)”, NOSC TD 1015, Feb. 1984.

Horst, M.M., Dyer, F.B., Tuley, M.T., "Radar Sea Clutter Model,”, IEEE International Conference on Antennas and Propagation.

International Radio Consulting Committee (CCIR) XVth Plenary Assembly Dubrovnik, 1986, "Propagation in Non-Ionized Media," Recommendations and Reports of the CCIR, 1986, Vol. V, International Telecommunications Union, Geneva, 1986. 
Kuttler, J. R. and G. D. Dockery., "Theoretical Description of the Parabolic Approximation/Fourier Split-Step Method of Representing Electromagnetic Propagation in the Troposphere," Radio Sci., Vol. 26, pp. 381-393, 1991.

Miller, A.R., R.M. Brown, E. Vegh,”New Derivation for the Rough-Surface Reflection Coefficient and for the Distribution of Sea-Wave Elevations" Proc. IEEE, Vol. 131, Part H, 2, 1984, pp 114-116.

Naval Oceanographic Office, "Software Documentation Standards for Environmental System Product Development,” February 1996.

Patterson, W.L., Hitney, H.V., "Radio Physical Optics CSCI Software Documents", Naval Command, Control and Ocean Surveillance Center, RDT\&E Division, San Diego, CA, NRaD TD 2403, December 1992.

Sailors, D.B. 1997. "Excess System Power Available for Short-Range High-Frequency Communication Systems," NRaD TD-2978 (August), Naval Command, Control and Ocean Surveillance Center RDT\&E Division, San Diego, CA.

Sailors, D.B., Barrios, A.E., "Terrain Parabolic Equation Model (TPEM) Computer Software Configuration Item (CSCI) Documents”, Naval Command, Control and Ocean Surveillance Center, RDT\&E Division, San Diego, CA, NRaD TD 2963, May 1997.

Sailors, D.B. Barrios, A.E., Patterson, W.L., Hitney, H.V., "Advanced Propagation Model [Ver. 1.0] (APM) Computer Software Configuration Item (CSCI) Documents," SPAWARSYSCEN-SD TD 3033, August 1998.

Tappert, F. D., "The Parabolic Approximation Method," Wave Propagation and Underwater Acoustics, J. B. Keller and J. S. Papadakis, Eds., New York, SpringerVerlag, pp. 224-285, 1977.

\section{CSCI-WIDE DESIGN DECISIONS}

The required APM CSCI propagation model is a range-dependent hybrid model that uses the complimentary strengths of Ray Optics (RO) and Parabolic Equation (PE) techniques to calculate propagation loss in range and altitude.

The atmospheric volume is divided into regions that lend themselves to the application of the various propagation loss calculation methods. Figure 1 illustrates these regions. 


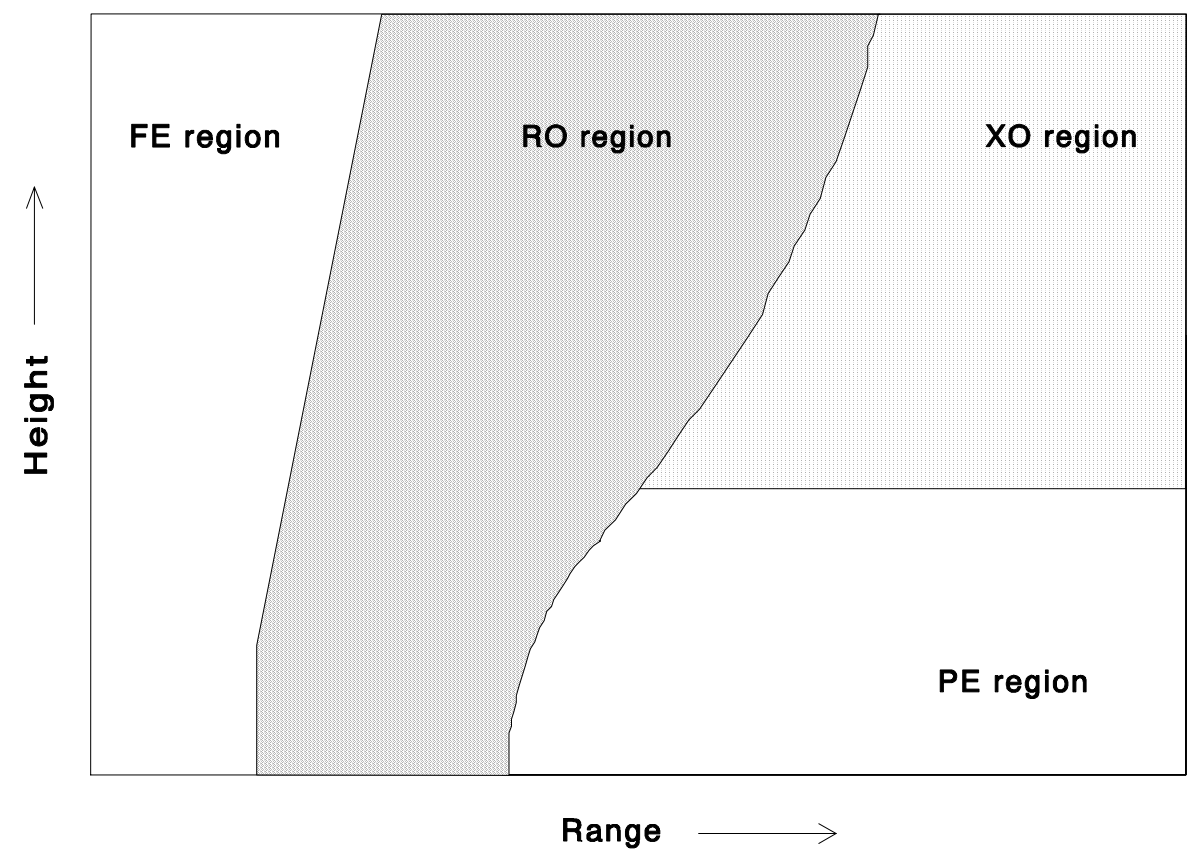

Figure 1. APM calculation regions.

For antenna elevation angles above $5^{\circ}$ or for ranges less than approximately 2.5 $\mathrm{km}$, a flat-earth (FE) (RO) model is used. In this region, only receiver height is corrected for average refraction and earth curvature.

Within the RO region (as defined by a limiting ray), propagation loss is calculated from the mutual interference between the direct-path and surface-reflected ray components using the refractivity profile at zero range. Full account is given to focusing or de-focusing along direct and reflected ray paths and to the integrated optical path length difference between the two ray paths, to give precise phase difference, and, hence, accurate coherent sums for the computation of propagation loss.

For the low-altitude region beyond the RO region, a PE approximation to the Helmholtz full-wave equation is employed. The PE model allows for range-dependent refractivity profiles and variable terrain along the propagation path and uses a split step Fourier method for the solution of the PE. The PE model is run in the minimum region required to contain all terrain and trapping layer heights.

For the area beyond the $\mathrm{RO}$ region but above the PE region, an extended optics region $(\mathrm{XO})$ is defined. Within the $\mathrm{XO}$ region, $\mathrm{RO}$ methods that are initialized by the $\mathrm{PE}$ solution from below, are used.

APM will run in three "execution" modes, depending on environmental inputs. APM will use the FE, RO, XO, and PE models if the terrain profile is flat for the first 2.5 $\mathrm{km}$ and if the antenna height is less than or equal to $100 \mathrm{~m}$. It will use only the $\mathrm{XO}$ and $\mathrm{PE}$ models if the terrain profile is not flat for the first $2.5 \mathrm{~km}$ and if the antenna height is 
less than or equal to $100 \mathrm{~m}$. For applications in which the antenna height is greater than 100 meters, a combination of FE and PE methods are used. The FE model is used for all propagation angles greater than $\pm 5^{\circ}$ from the source and the PE model is used for angles within $\pm 5^{\circ}$. By default, APM will automatically choose which mode of operation it will use for a specified set of inputs. However, the ability to run only the PE model for any case is allowed by setting a logical flag upon input. APM will automatically run only the $\mathrm{PE}$ algorithm for frequencies less than $50 \mathrm{MHz}$, regardless of the logical flag set by the user.

The APM CSCI allows for horizontal and vertical antenna polarization, finite conductivity based on user-specified ground composition and dielectric parameters, and the complete range of EM system parameters and most antenna patterns required by various external applications. APM also allows for gaseous absorption effects in all submodels and computes troposcatter losses within the diffraction region and beyond.

The APM CSCI is divided into 5 main computer software components (CSC) and 67 additional software units (SU). The first CSC, the APMINIT CSC, interfaces with various SUs for the complete initialization of the APM CSCI. The second CSC, the APMSTEP CSC, advances the entire APM CSCI algorithm one output range step, referencing various SUs to calculate the propagation loss at the current output range. The Extended Optics Initilization (XOINIT) CSC initializes the range, height, and angle arrays in preparation for the Extended Optics Step (XOSTEP) CSC, and also computes and returns the surface clutter values if requested. The fourth CSC (XOSTEP) advances the APM CSCI algorithm one output step from the top of the PE calculation region to the maximum output height specified, referencing various SUs to calculate the propagation output range. Lastly, the RET_GRAZE CSC is used to return the grazing angles for use in other applications outside the APM CSCI for a specified set of environmental inputs and system parameters.

\section{CSCI ARCHITECTURE DESIGN}

\subsection{CSCI COMPONENTS}

The APM CSCI is accessed by a subroutine call which provides, as global data elements, the values specified in Table 1 through Table 4. The source code for the APM CSCI is listed in Appendix A. The name and purpose for each CSC and SU are listed below.

The Advance Propagation Initialization (APMINIT) CSC interfaces with various SUs for the complete initialization of the APM CSCI. 
The APMINIT CSC component SUs include the following:

1. Allocate Arrays APM (ALLARRAY_APM) SU. Allocates and initializes all dynamically dimensioned arrays associated with APM terrain, refractivity, troposcatter, and general variable arrays.

2. Allocate Array PE (ALLARRAY_PE) SU. Allocates and initializes all dynamically dimensioned arrays associated with PE calculations.

3. Allocate Array RO (ALLARRAY_RO) SU. Allocates and initializes all dynamically dimensioned arrays associated with RO calculations.

4. Allocate Array XO (ALLARRAY_XORUF) SU. Allocates and initializes all dynamically dimensioned arrays associated with $\mathrm{XO}$ and rough surface calculations.

5. Alpha Impedance Initialization (ALN_INIT) SU. Initializes variables used in the Discrete Mixed Fourier Transform (DMFT) algorithm for finite conductivity and/or rough surface calculations.

6. Antenna Pattern (ANTPAT) SU. Calculates a normalized antenna gain (antenna pattern factor) for a specified antenna elevation angle.

7. APM Status (APMSTATUS) SU. Declared as an external subroutine within the main driver program. Used only for accessing status of grazing angle routine.

8. Dielectric Initialization (DIEINIT) SU. Determines the conductivity and relative permittivity as a function of frequency $(\mathrm{MHz})$ based on general ground composition types.

9. FFT Parameters (FFTPAR) SU. Determines the required transform size based on the maximum PE propagation angle and the maximum height needed.

10. Fill Height Arrays (FILLHT) SU. Calculates the effective earth radius for an initial launch angle of $5^{\circ}$ and fills an array with height values at each output range of the limiting sub-model, depending on which mode is used.

11. Gaseous Absorption (GASABS) SU. Computes the specific attenuation based on air temperature and absolute humidity.

12. Get Effective Earth Radius Factor (GET_K) SU. Computes the effective earth radius factor and the effective earth radius. 
13. Get Alpha Impedance (GETALN) SU. Computes the impedance term in the Leontovich boundary condition and the complex index of refraction for finite conductivity and vertical polarization calculations.

14. Get Angles (GETANGLES) SU. Computes grazing angles for use in subsequent rough surface calculations, and if necessary, also the propagation angles for output via APMSTEP.

15. Get Maximum Angle (GETTHMAX) SU. Performs an iterative ray trace to determine the minimum angle required (based on the reflected ray) in obtaining a PE solution.

16. Grazing Angle Interpolation (GRAZE_INT) SU. Interpolates grazing angles at each $\mathrm{PE}$ range step based on angles computed from ray trace (takes precedence) and those computed from spectral estimation.

17. Height Check (HTCHECK) SU. Checks if the current traced ray height is below the current ground height.

18. Interpolate Profile (INTPROF) SU. Performs a linear interpolation vertically with height on the refractivity profile.

19. PE Initialization (PEINIT) SU. Initializes all variables used in the PE model for subsequent calls to the PESTEP SU

20. Poly 4 (FN_POLY4) Function. Evaluates a fourth degree polynomial.

21. Poly 5 (FN_POLY5) Function. Evaluates a fifth degree polynomial.

22. Profile Reference (PROFREF) SU. Adjusts the current refractivity profile so that it is relative to a reference height.

23. Refractivity Initialization (REFINIT) SU. Checks for valid environmental profile inputs and initializes refractivity arrays.

24. Remove Duplicate Refractivity Levels (REMDUP) SU. Removes any duplicate refractivity levels in the currently interpolated profile.

25. RG Trace (RGTRACE) SU. Performs ray trace over terrain of many rays launched within an angle of $\pm 1.5^{\circ}$, storing grazing angles from these rays.

26. Terrain Initialization (TERINIT) SU. Examines and initializes terrain arrays for subsequent use in PE calculations. 
27. Trace to Output Range (TRACE_ROUT) SU. Traces a single ray, whose launch angle is specified by the calling routine, to each output range.

28. Trace to next Step (TRACE_STEP) SU. This routine performs one ray trace step. When passed a starting angle, range, and height for a single ray, it will trace to the first boundary that occurs (refractivity level or surface). It then passes back the ending angle, range and height, and a flag indicating if the ray has hit the surface.

29. Troposcatter Initialization (TROPOINIT) SU. Initializes all variables and arrays needed for subsequent troposcatter calculations.

30. Starter Field Initialization (XYINIT) SU. Calculates the complex PE solution at range zero.

The Advanced Propagation Model Step (APMSTEP) CSC advances the entire APM CSCI algorithm one output range step, referencing various SUs to calculate the propagation loss at the current output range. The APMSTEP CSC component SUs include the following:

1. Calculate Propagation Loss (CALCLOS) SU. Determines propagation loss from the complex PE field at each output height point at the current output range.

2. Current Wind (FN_CURWIND) Function. Performs a linear interpolation in range to get the current wind speed at the specified range.

3. Dielectric Constant (FN_DIECON) Function. Extracts the stored complex dielectric constant at a particular range.

4. DOSHIFT SU. Shifts the field by the number of bins, or PE mesh heights corresponding to the local ground height.

5. Discrete Sine/Cosine Fast-Fourier Transform (DRST) SU. Performs a sine or cosine transform, depending on the value of an integer flag provided by the calling $\mathrm{SU}$, on both the real and imaginary components of the PE field, which are passed separately.

6. Flat Earth Direct Ray (FEDR) SU. Determines the propagation loss based on FE calculations for the direct ray only, for all output heights specified at each output range.

7. Flat Earth Model (FEM) SU. Computes propagation loss at a specified range based on FE approximations. 
8. Fast-Fourier Transform (FFT) SU. Separates the real and imaginary components of the complex PE field into two real arrays and then references the DRST SU.

9. Free Space Range Step (FRSTP) SU. Propagates the complex PE solution field in free space by one range step.

10. FZLIM SU. Determines the propagation factor (in $\mathrm{dB}$ ) and the outgoing propagation angle at the top of the PE calculation region.

11. Get Propagation Factor (FN_GETPFAC) Function. Determines the propagation factor at the specified height in decibels.

12. Get Reflection Coefficient (GETREFCOEF) SU. Calculates the complex surface reflection coefficient, along with the Miller-Brown rough surface reduction factor.

13. Get Troposcatter Loss (FN_GET_TLOSS) Function. Determines the loss due to troposcatter and computes the appropriate loss from troposcatter and diffraction for a specific transmitter and receiver point over land and water.

14. Linear Interpolation (FN_PLINT) Function. Performs linear interpolation on two input parameters passed to the function.

15. Mixed Fourier Transform (MIXEDFT) SU. Propagates the PE field in free space one $\mathrm{PE}$ range step, applying the Leontovich boundary condition, using the mixed Fourier transform as outlined by Kuttler and Dockery (1991).

16. Parabolic Equation Step (PESTEP) SU. Determines the next output range and begins an iterative loop to advance the PE solution such that for the current PE range, a PE solution is calculated from the solution at the previous PE range. This procedure is to be repeated until the output range is reached.

17. Ray Trace (RAYTRACE) SU. Traces a ray from a starting height and range with a specified starting elevation angle to a termination range.

18. Refractivity Interpolation (REFINTER) SU. Interpolates horizontally and vertically on the modified refractivity profiles.

19. Ray Optics Calculation (ROCALC SU). Computes the RO components that will be needed in the calculation of propagation loss at a specified range and height within the $\mathrm{RO}$ region. 
20. Ray Optics Loss (ROLOSS) SU. Calculates the propagation loss and propagation factor values at a specified range and height based upon the components of magnitude for a direct-path and surface-reflected ray and the total phase lag angle between the two rays as determined by the ROCALC SU.

21. Save Profile (SAVEPRO) SU. Stores the refractivity profiles at each PE range step from the top of the PE region to the maximum user-specified height.

22. Spectral Estimation (SPECEST) SU. Determines, via spectral estimation, the outward propagation angle at the top of the PE calculation region.

23. Surface Impedance (SURFIMP) SU. Computes the normalized average surface impedance for surface wave propagation by vertically polarized waves along the sea surface for frequencies less than $50 \mathrm{MHz}$.

24. Troposcatter (TROPOSCAT) SU. Determines the loss due to troposcatter and computes the appropriate loss from troposcatter and diffraction beyond the radio horizon for an array of receiver heights.

The XOINIT CSC initializes the range, height, and angle arrays in preparation for the XOSTEP CSC. It also accesses the surface clutter computation SUs and returns the surface clutter, if specified by the user. The XOINIT CSC component SUs include the following:

1. Advanced Propagation Model Clean (APMCLEAN) SU. Deallocates all dynamically dimensioned arrays used in one complete run of APM calculations.

2. Clutter-to-Noise (CLUTTER) SU. Calculates returned clutter-to-noise ratio at each output range.

3. Diffraction Loss (FN_DLOSS) Function. Computes loss in the diffraction region based on the CCIR model.

4. Get Theta (GETTHETA) SU. Calculates the optical phase-lag difference angle from the reflection range found in the R1ITER SU.

5. GIT Initialization (GIT_INIT) SU. Initializes all variables used in the calculation of the reflectivity based on a modified version of the GIT model.

6. GofZ (GOFZ) Function. Calculates the diffraction region height-gain in decibels from the CCIR diffraction region model. 
7. Mean Filter (MEANFILT) SU. Performs an n-point average smoothing on any array passed to it.

8. Optical Region Limit (OPLIMIT) SU. Calculates the maximum range in the optical interference region and the corresponding loss at that range.

9. Optical Difference (OPTICF) SU. Calculates the optical path-length difference angle by solving a cubic equation for the reflection point range.

10. R1 Iteration (R1ITER) SU. Finds the range of the reflection point corresponding to a particular launch angle.

11. Standard Propagation Model Initialization (SPM_INIT) SU. Initializes much of the variables used throughout the SPM SU.

12. Standard Propagation Model (SPM) SU. Computes the propagation factor for a standard atmosphere only, with the assumption of omni-directional antenna patterns.

The XOSTEP CSC advances the APM CSCI algorithm one output range step from the top of the PE calculation region to the maximum output height specified, referencing various SUs to calculate the propagation loss at the current output range. The XOSTEP CSC component SUs include the following:

1. Extended Optics (EXTO) SU. Calculates propagation loss and propagation factor, based on extended optics techniques, at the current output range.

The Return Grazing Angles (RET_GRAZE) CSC interpolates grazing angles to every output range step, and if necessary, will interpolate the propagation angles in height at every output range.

\subsection{CONCEPT OF EXECUTION}

The program flow of the APM CSCI is illustrated in Figure 2. Note that the APM CSCI is shown within the context of a calling CSCI application such as one that generates a coverage or loss diagram. The efficient implementation of the APM CSCI will have far reaching consequences on the design of an application CSCI beyond those mentioned in Section 7.1. For example, Figure 2 shows checking for the existence of a previously created APM output file prior to the access of the APM CSCI. The application CSCI must consider if the atmospheric or terrain environment has changed since the APM output file was created or if any new height or range requirement is accommodated within the existing APM CSCI output file. Because these and many more considerations are beyond the scope of this document, an application CSCI designer should work closely with the APM CSCI development agency in the implementation of the APM CSCI. 


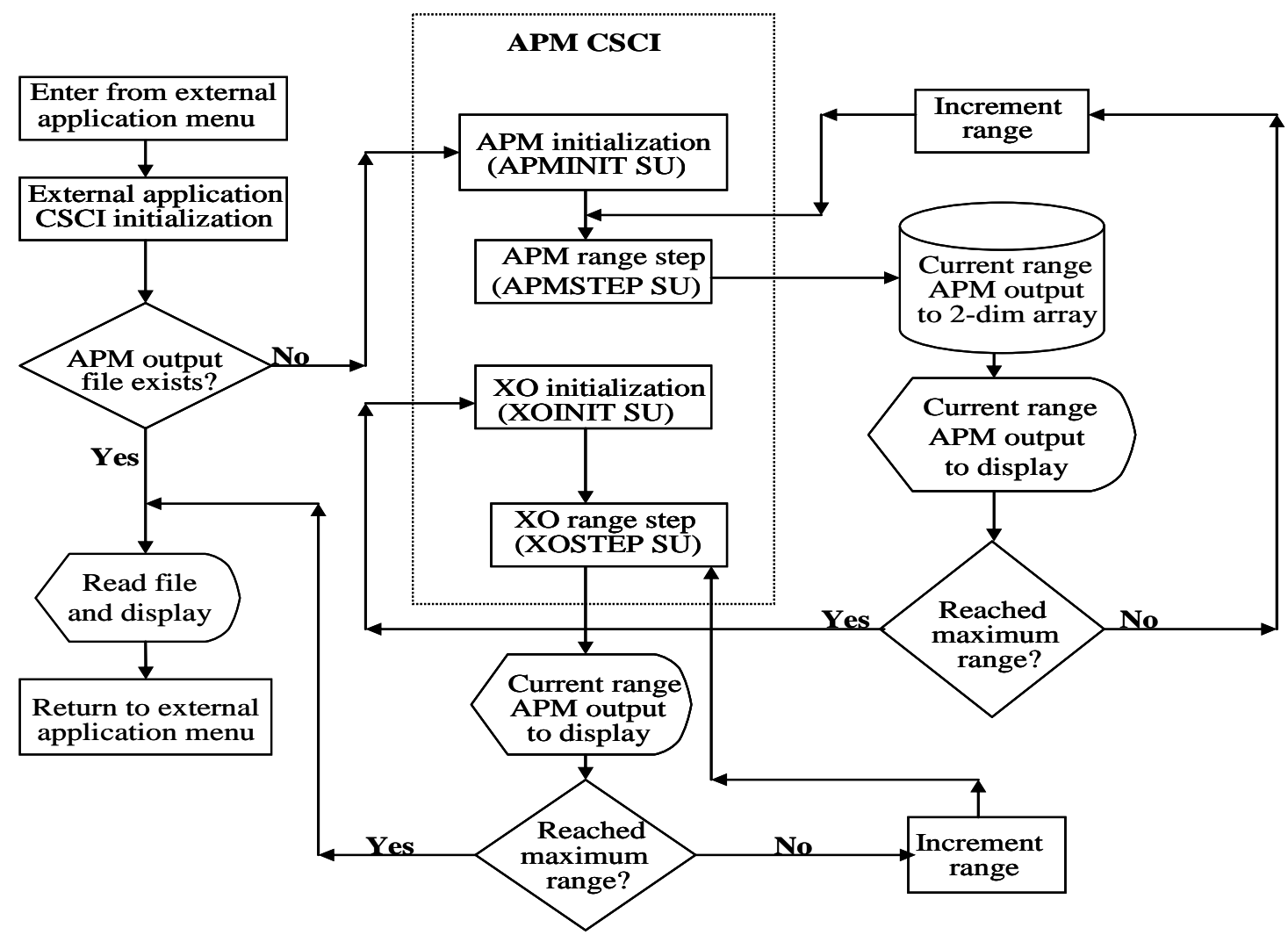

Figure 2. APM CSCI program flow.

\subsection{INTERFACE DESIGN}

\subsubsection{Interface Identification and Diagrams}

The APM CSCI interface design consists of one FORTRAN MODULE file for the external and internal data interface, FORTRAN CALL statements for output data and internal interfacing, and several FORTRAN COMMON blocks for the internal interface. The MODULE file is called APM_MOD. This MODULE's statements provide several constants, COMMON blocks, and the dynamically allocated array names. The COMMON block names are (1) APM_VAR, (2) ERRORFLAG, (3) INPUTVAR, (4) REFRACTIVITY, (5) SYSTEMVAR, and (6) TERRAIN.

\subsubsection{External Interface}

The APM CSCI is accessed, through the APMINIT CSC, by a subroutine call from the external CSCI, which should provide, as global data elements, the values specified in Table 1 through Table 4.

The APM CSCI external data elements, i.e. those data which must be provided by the calling CSCI in the MODULE file prior to the APM CSCI execution can be divided into four classifications. The first classification is external data related to the atmospheric environment (Table 1), the second is data related to the EM system (Table 2), the third is 
data related to the implementation of the APM CSCI by the external CSCI (Table 3), and the fourth is data related to the terrain information (Table 4). Each table lists the type, units, and bounds of each data element. Table 5 specifies the output data of the APM CSCI model passed back to the calling CSCI via the FORTRAN CALL statements.

Table 1. APM CSCI environmental data element requirements.

\begin{tabular}{|c|c|c|c|c|}
\hline Name & Description & Type & Units & Bounds \\
\hline refmsl & $\begin{array}{l}\text { Modified refractivity profile (dynamically } \\
\text { allocated) array referenced to mean sea level }\end{array}$ & real & M & $\geq 0.0^{\mathrm{a}}$ \\
\hline hmsl & Profile height (dynamically allocated) array & real & meters & See note $b$ \\
\hline$n_{\text {prof }}$ & Number of refractivity profiles & integer & N/A & $\geq 1$ \\
\hline lvlp & Number of profile levels & integer & N/A & $\geq 2$ \\
\hline rngprof & $\begin{array}{l}\text { Dynamically allocated array of ranges to each } \\
\text { profile }\end{array}$ & real & meters & $\geq 0.0$ \\
\hline$a b s_{h u m}$ & Surface absolute humidity & real & $\mathrm{g} / \mathrm{m}^{3}$ & 0 to $50^{c}$ \\
\hline$t_{\text {air }}$ & Surface air temperature & real & ${ }^{\circ} \mathrm{C}$ & -20 to $40^{c}$ \\
\hline$\gamma_{a}$ & Surface specific attenuation & real & $\mathrm{dB} / \mathrm{km}$ & $\geq 0.0$ \\
\hline$i_{\text {extra }}$ & $\begin{array}{l}\text { Extrapolation flag for refractivity profiles } \\
\text { entered in combination with terrain below } \\
\text { mean sea level }\end{array}$ & integer & N/A & 0 or 1 \\
\hline$n_{w}$ & $\begin{array}{l}\text { Number of wind speeds and corresponding } \\
\text { ranges }\end{array}$ & integer & N/A & $\geq 0.0$ \\
\hline rngwind & $\begin{array}{l}\text { Dynamically allocated array of ranges } \\
\text { specified for each wind speed in wind(). }\end{array}$ & real & meters & $\geq 0.0$ \\
\hline wind & Dynamically allocated array of wind speeds. & real & $\begin{array}{l}\text { meters/ } \\
\text { second }\end{array}$ & 0.0 to $20.0^{\mathrm{d}}$ \\
\hline wind $_{\text {dir }}$ & $\begin{array}{l}\text { Angle between antenna boresight and upwind } \\
\text { direction }\end{array}$ & real & degrees & 0.0 to 360.0 \\
\hline
\end{tabular}

${ }^{\mathrm{a}}$ Couplets of height and modified refractivity associated with that height are referred to in this document as a refractivity profile.

${ }^{\mathrm{b}}$ All heights in the refractivity profile must be steadily increasing.

${ }^{\mathrm{C}}$ The CCIR gaseous absorption model implemented within APM provides a $\pm 15 \%$ accuracy for absolute humidity and surface air temperature within these bounds. While values beyond these limits are allowed within APM, Note that this may result in less accurate attenuation rates calculated.

${ }^{\mathrm{d}}$ The maximum wind speed will vary depending on frequency. For frequencies less than $10 \mathrm{GHz}$, the maximum that can be specified is $20 \mathrm{~m} / \mathrm{s}$. Above $10 \mathrm{GHz}$, the maximum wind speed that can be specified will decrease to an absolute maximum of $15 \mathrm{~m} / \mathrm{s}$ at $20 \mathrm{GHz}$ and above. 
Table 2. APM CSCI external EM system data element requirements.

\begin{tabular}{|c|c|c|c|c|}
\hline Name & Description & Type & Units & Bounds \\
\hline$\mu_{b w}$ & Antenna vertical beam width & real & degree & 0.5 to 45 \\
\hline$\mu_{o}$ & Antenna elevation angle & real & degree & -10.0 to 10.0 \\
\hline$C_{\text {lut }}$ & $\begin{array}{l}\text { Logical flag used to indicate if surface clutter } \\
\text { calculations are desired. }\end{array}$ & logical & N/A & ‘.true.' or '.false.' \\
\hline$f_{\mathrm{MHz}}$ & EM system frequency & real & $\mathrm{MHz}$ & 2.0 to $20,000.0^{\mathrm{a}}$ \\
\hline$i_{p a t}$ & $\begin{array}{l}\text { Antenna pattern } \\
1=\text { Omnidirectional } \\
2=\text { Gaussian } \\
3=\text { Sine }(\mathrm{X}) / \mathrm{X} \\
4=\text { Cosecant-squared } \\
5=\text { Generic height-finder } \\
6=\text { User-defined height-finder } \\
7=\text { User-defined antenna pattern } \\
8=\text { Quarter-wave dipole }\end{array}$ & integer & N/A & 1 to 8 \\
\hline$i_{p o l}$ & $\begin{array}{l}\text { Antenna polarization } \\
0=\text { Horizontal } \\
1 \text { = Vertical }\end{array}$ & integer & N/A & 0 to 1 \\
\hline$G$ & Gain of transmit/receive antennas & real & $\mathrm{dBi}$ & $\geq 0.0$ \\
\hline$a n t_{h t}$ & Antenna height above local ground at range $0.0 \mathrm{~m}$ & real & meters & $\geq 1.5^{\mathrm{b}}$ \\
\hline hfang & $\begin{array}{l}\text { Dynamically allocated user-defined height-finder } \\
\text { power reduction angle array }\left(i_{p a t}=6\right) \text { or antenna } \\
\text { pattern angles }\left(i_{p a t}=7\right)\end{array}$ & real & degree & $\begin{array}{c}0.0 \text { to } 90.0 \text { for } i_{p a t}=6 \\
-90.0 \text { to } 90.0 \text { for } \\
i_{p a t}=7\end{array}$ \\
\hline hffac & $\begin{array}{l}\text { Dynamically allocated user-defined power } \\
\text { reduction factor array }\left(i_{p a t}=6\right) \text { or antenna pattern } \\
\text { factors }\left(i_{p a t}=7\right)\end{array}$ & real & N/A & 0.0 to 1.0 \\
\hline$L_{s y s}$ & Miscellaneous system losses & real & $\mathrm{dB}$ & $\geq 0.0$ \\
\hline$\theta_{h b w}$ & Antenna horizontal beam width & real & degrees & 0.5 to 45 \\
\hline$n_{\text {facs }}$ & $\begin{array}{l}\text { Number of power reduction angles/factors for user- } \\
\text { defined height finder antenna pattern }\end{array}$ & integer & N/A & 1 to 10 \\
\hline$N_{f}$ & Noise figure & real & $\mathrm{dB}$ & $\geq 0.0$ \\
\hline$P_{t}$ & Transmitter peak power & real & $\mathrm{kW}$ & $\geq 0.1$ \\
\hline$\tau$ & Pulse length/width & real & $\mu$ sec & $\geq 0.1$ \\
\hline
\end{tabular}

${ }^{\text {a}}$ The frequency can be specified greater than $20 \mathrm{GHz}$; however, the $P E_{\text {flag }}$ must be set to '.true.' and care must be taken in specifying $t h_{\max }$ and $r_{\text {mult }}$.

${ }^{\mathrm{b}}$ The minimum antenna height will vary, depending on the frequency and beamwidth according to the formula:

$$
a_{h t} \geq \text { maximum of }\left(1.5,0.6 \frac{c_{o}}{f_{M H z} \mu_{b w}}\right)
$$

where $c_{o}$ is the speed of light $\times 10^{-6} \mathrm{~m} / \mathrm{s}$ (299.79245). 
Table 3. APM CSCI external implementation constants.

\begin{tabular}{|c|c|c|c|c|}
\hline Name & Description & Type & Units & Bounds \\
\hline$h_{\max }$ & $\begin{array}{l}\text { Maximum height output for a particular application of } \\
\text { APM }\end{array}$ & real & meters & $\geq 100.0^{\mathrm{a}}$ \\
\hline$h_{\min }$ & $\begin{array}{l}\text { Minimum height output for a particular application of } \\
\text { APM }\end{array}$ & real & meters & $\geq 0.0^{\mathrm{a}}$ \\
\hline lang & $\begin{array}{l}\text { Propagation angle and factor output flag } \\
\text { ‘.true.'= Output propagation angle and propagation } \\
\text { factor for direct and reflected ray (where applicable). } \\
\text { ‘.false.' = Do not output propagation angles and } \\
\text { factors }\end{array}$ & logical & N/A & $\begin{array}{l}\text { ‘.true.’ or } \\
\text { ‘ffalse.'b }\end{array}$ \\
\hline lerr6 & Logical flag to allow for error -6 to be bypassed & logical & N/A & $\begin{array}{l}\text { ‘.true.’ or } \\
\text { ‘ffalse. }{ }^{\text {c }}\end{array}$ \\
\hline lerr12 & Logical flag to allow for error -12 to be bypassed & logical & N/A & $\begin{array}{l}\text { ‘.true.’ or } \\
\text { ‘.false.'c }\end{array}$ \\
\hline$n_{\text {rout }}$ & $\begin{array}{l}\text { Number of range output points for a particular } \\
\text { application of APM }\end{array}$ & integer & N/A & $\geq 1$ \\
\hline$n_{\text {zout }}$ & $\begin{array}{l}\text { Number of height output points for a particular } \\
\text { application of APM }\end{array}$ & integer & N/A & $\geq 1$ \\
\hline$n_{\text {zout_rtg }}$ & $\begin{array}{l}\text { Number of height output points for receiver heights } \\
\text { relative to the local ground elevation. }\end{array}$ & integer & N/A & $\geq 0$ \\
\hline$P E_{\text {flag }}$ & $\begin{array}{l}\text { Flag to indicate use of PE algorithm only: } \\
\text { '.true.' = only use PE sub-model } \\
\text { '.false.' = use automatic hybrid model }\end{array}$ & logical & $\mathrm{N} / \mathrm{A}$ & $\begin{array}{l}\text { ‘.true.' or } \\
\text { ‘false.' }\end{array}$ \\
\hline$r_{\max }$ & $\begin{array}{l}\text { Maximum range output for a particular application of } \\
\text { APM }\end{array}$ & real & meters & $\geq 5000.0^{c}$ \\
\hline$r_{\text {mult }}$ & PE-range step multiplier & real & N/A & $>0.0^{c}$ \\
\hline$t h_{\max }$ & Visible portion of PE maximum calculation angle & real & degrees & $>0.0^{\mathrm{c}}$ \\
\hline$T_{\text {ropo }}$ & Logical flag to include troposcatter calculations. & integer & N/A & $\begin{array}{l}\text { '.true.’ or } \\
\text { '.false.’ }\end{array}$ \\
\hline zout_rtg & $\begin{array}{l}\text { Dynamically allocated array of receiver heights } \\
\text { specified relative to the local ground height. }\end{array}$ & real & meters & $\geq 0.0$ \\
\hline
\end{tabular}

${ }^{\mathrm{a}}$ Refer to section 7.2 for a complete description.

${ }^{\mathrm{b}}$ This flag should not be enabled when any portion of the propagation path is over land.

' Refer to section 4.3.4 for a complete description. 
Table 4. APM CSCI external terrain data element requirements.

\begin{tabular}{|c|c|c|c|c|}
\hline Name & Description & Type & Units & Bounds \\
\hline ter $x$ & Dynamically allocated terrain profile range array & real & meters & $\geq 0.0^{\mathrm{a}}$ \\
\hline tery & Dynamically allocated terrain profile height array & real & meters & $\geq 0.0^{\mathrm{a}}$ \\
\hline$\gamma C$ & $\begin{array}{l}\text { Dynamically allocated array of constants describing } \\
\text { the backscattering effectiveness of the surface }\end{array}$ & real & $\mathrm{dB}$ & $\begin{aligned}-100.0 & \leq \gamma C \\
& \leq 100.0\end{aligned}$ \\
\hline yrng & $\begin{array}{l}\text { Dynamically allocated array of ranges corresponding } \\
\text { to the values in } \gamma c\end{array}$ & real & meters & $\geq 0.0$ \\
\hline$i_{g c}$ & $\begin{array}{l}\text { Number of } \gamma c \text { values for a particular application of } \\
\text { APM }\end{array}$ & integer & N/A & $\geq 0$ \\
\hline$i_{t p}$ & $\begin{array}{l}\text { Number of terrain profile points for a particular } \\
\text { application of APM }\end{array}$ & integer & N/A & $\geq 2$ \\
\hline$i_{g r}$ & $\begin{array}{l}\text { Number of ground types for a particular application } \\
\text { of APM }\end{array}$ & integer & N/A & $\geq 0^{\mathrm{a}}$ \\
\hline igrnd & $\begin{array}{l}\text { Array of ground composition types for a particular } \\
\text { application of APM } \\
0=\text { Sea water } \\
1=\text { Fresh water } \\
2=\text { Wet ground } \\
3=\text { Medium dry ground } \\
4=\text { Very dry ground } \\
5=\text { Ice at }-1^{\circ} \mathrm{C} \\
6=\text { Ice at }-10^{\circ} \mathrm{C} \\
7=\text { User-defined }\end{array}$ & integer & $\mathrm{N} / 0 \mathrm{~A}$ & $\begin{array}{l}0 \leq \text { igrnd } \leq \\
7^{\mathrm{a}}\end{array}$ \\
\hline rgrnd & $\begin{array}{l}\text { Dynamically allocated array of ranges for which } \\
\text { ground types are applied for a particular application } \\
\text { of APM }\end{array}$ & real & meters & $\geq 0.0^{\mathrm{a}}$ \\
\hline dielec & $\begin{array}{l}\text { Dynamically allocated two-dimensional array of } \\
\text { relative permittivity }\left(\varepsilon_{r}\right) \text { and conductivity }(\sigma) \text { for a } \\
\text { particular application of APM }\end{array}$ & real & $\begin{array}{l}\varepsilon_{r}-\mathrm{N} / \mathrm{A} \\
\sigma- \\
\text { Siemens/ } \\
\text { meter }\end{array}$ & $>0^{\mathrm{a}}$ \\
\hline
\end{tabular}

${ }^{a}$ refer to section 7.3 for a complete description 
Table 5. APM CSCI output data element requirements.

\begin{tabular}{|c|c|c|c|c|}
\hline Name & Description & Type & Units & Source \\
\hline CNR & Clutter-to-Noise ratio array & real & $\mathrm{dB}$ & XOINIT CSC \\
\hline$\Psi_{\text {rout }}$ & $\begin{array}{l}\text { Array of grazing angles at each output } \\
\text { range } r_{\text {out }}\end{array}$ & real & radians & RET_GRAZE SU \\
\hline$i_{\text {error }}$ & $\begin{array}{l}\text { Integer value that is returned if an error } \\
\text { occurs in called routine }\end{array}$ & integer & N/A & $\begin{array}{l}\text { APMINIT CSC } \\
\text { RET_GRAZE SU } \\
\text { XOINIT CSC }\end{array}$ \\
\hline$i_{x o s t p}$ & $\begin{array}{l}\text { Index of output range step at which } \mathrm{XO} \\
\text { model is to be applied }\end{array}$ & integer & N/A & APMINIT CSC \\
\hline$j_{\text {end }}$ & $\begin{array}{l}\text { Output height index at which valid } \\
\text { propagation loss values end }\end{array}$ & integer & N/A & APMSTEP CSC \\
\hline$j_{\text {start }}$ & $\begin{array}{l}\text { Output height index at which valid } \\
\text { propagation loss values begin }\end{array}$ & integer & N/A & APMSTEP CSC \\
\hline$j_{x e n d}$ & $\begin{array}{l}\text { Output height index at which valid XO } \\
\text { propagation loss values end }\end{array}$ & integer & N/A & XOSTEP CSC \\
\hline$j_{\text {xstart }}$ & $\begin{array}{l}\text { Output height index at which valid XO } \\
\text { propagation loss values begin }\end{array}$ & integer & N/A & XOINIT CSC \\
\hline$l_{\text {graze }}$ & $\begin{array}{l}\text { Logical flag indicating if grazing angles } \\
\text { were computed for a particular } \\
\text { application of APM }\end{array}$ & logical & N/A & APMINIT CSC \\
\hline$m p f l$ & Propagation loss and factor array & integer & cB & $\begin{array}{l}\text { APMSTEP CSC } \\
\text { XOSTEP CSC }\end{array}$ \\
\hline mpfl_rtg & $\begin{array}{l}\text { Propagation loss and factor at receiver } \\
\text { heights specified in the zout_rtg array }\end{array}$ & integer & cB & APMSTEP CSC \\
\hline propaf & $\begin{array}{l}\text { Two-dimensional array, containing the } \\
\text { propagation angles and factors for the } \\
\text { direct and reflected rays (where } \\
\text { applicable) for all output height/range } \\
\text { points }\end{array}$ & real & radians, $\mathrm{dB}$ & $\begin{array}{l}\text { APMSTEP CSC } \\
\text { XOSTEP CSC }\end{array}$ \\
\hline$r_{\text {out }}$ & Current output range & real & meters & $\begin{array}{l}\text { APMSTEP CSC } \\
\text { XOSTEP CSC }\end{array}$ \\
\hline
\end{tabular}

\subsubsection{Internal Interface}

Section 4.2 shows the relationship between the APM CSCI and its five main CSCs: APMINIT, AMPSTEP, RET_GRAZE, XOINIT, and XOSTEP. This relationship is illustrated in Figure 2. The internal interface between these five CSCs and the APM CSCI is left to the design. However, the internal structure of the APM CSCI and its CSCs and SUs is shown in Table 6. The left two columns show the calling subroutines, and the right two columns the subroutines called. Columns 2 and 4 in Table 6 give the section number in Section 5 where more details about the various CSCs and SUs of the APM CSCI can be found. 
Table 6. APM internal interface design.

\begin{tabular}{|c|c|c|c|}
\hline \multicolumn{2}{|c|}{ Software Design Description } & \multicolumn{2}{|c|}{ Software Design Description } \\
\hline $\begin{array}{c}\text { Software Design Description } \\
\text { Name }\end{array}$ & $\begin{array}{c}\text { SDD } \\
\text { Paragraph } \\
\text { Number }\end{array}$ & $\begin{array}{c}\text { Software Design Description } \\
\text { Name }\end{array}$ & $\begin{array}{c}\text { SDD } \\
\text { Paragraph } \\
\text { Number }\end{array}$ \\
\hline CSCI Detailed Design & 5 & $\begin{array}{l}\text { Advance Propagation } \\
\text { Initialization (APMINIT) CSC }\end{array}$ & 5.1 \\
\hline $\begin{array}{l}\text { Advance Propagation } \\
\text { Initialization (APMINIT) CSC }\end{array}$ & 5.1 & $\begin{array}{l}\text { Allocate Arrays APM } \\
\text { (ALLARRAY_APM) SU }\end{array}$ & 5.1 .1 \\
\hline $\begin{array}{l}\text { Advance Propagation } \\
\text { Initialization (APMINIT) CSC }\end{array}$ & 5.1 & $\begin{array}{l}\text { Allocate Array RO } \\
\text { (ALLARRAY_RO) SU }\end{array}$ & 5.1 .3 \\
\hline $\begin{array}{l}\text { Advance Propagation } \\
\text { Initialization (APMINIT) CSC }\end{array}$ & 5.1 & $\begin{array}{l}\text { Allocate Array XORUF (XORUF) } \\
\text { SU }\end{array}$ & 5.1 .4 \\
\hline $\begin{array}{l}\text { Advance Propagation } \\
\text { Initialization (APMINIT) CSC }\end{array}$ & 5.1 & $\begin{array}{l}\text { Alpha Impedance Initialization } \\
\text { (ALN_INIT) SU }\end{array}$ & 5.1 .5 \\
\hline $\begin{array}{l}\text { Alpha Impedance Initialization } \\
\text { (ALN_INIT) SU }\end{array}$ & 5.1 .5 & $\begin{array}{l}\text { Get Alpha Impedance (GETALN) } \\
\text { SU }\end{array}$ & 5.1 .13 \\
\hline $\begin{array}{l}\text { Get Alpha } \\
\text { Impedance(GETALN) SU }\end{array}$ & 5.1 .13 & $\begin{array}{l}\text { Current Wind (FN_CURWIND) } \\
\text { Function }\end{array}$ & 5.2 .2 \\
\hline $\begin{array}{l}\text { Get Alpha } \\
\text { Impedance(GETALN) SU }\end{array}$ & 5.1 .13 & $\begin{array}{l}\text { Get Reflection Coefficient } \\
\text { (GETREFCOEF) SU }\end{array}$ & 5.2 .12 \\
\hline $\begin{array}{l}\text { Get Reflection Coefficient } \\
\text { (GETREFCOEF) SU }\end{array}$ & 5.2 .12 & $\begin{array}{l}\text { Current Wind (FN_CURWIND) } \\
\text { Function }\end{array}$ & 5.2 .2 \\
\hline $\begin{array}{l}\text { Get Reflection Coefficient } \\
\text { (GETREFCOEF) SU }\end{array}$ & 5.2 .12 & $\begin{array}{l}\text { Dielectric Constant } \\
\text { (FN_DIECON) Function }\end{array}$ & 5.2 .3 \\
\hline $\begin{array}{l}\text { Get Alpha } \\
\text { Impedance(GETALN) SU }\end{array}$ & 5.1 .13 & $\begin{array}{l}\text { Surface Impedance (SURFIMP) } \\
\text { SU }\end{array}$ & 5.2 .23 \\
\hline $\begin{array}{l}\text { Surface Impedance } \\
\text { (SURFIMP) SU }\end{array}$ & 5.2 .23 & Poly 4 (FN_POLY4) Function & 5.1 .20 \\
\hline $\begin{array}{l}\text { Surface Impedance } \\
\text { (SURFIMP) SU }\end{array}$ & 5.2 .23 & Poly 5 (FN_POLY5) Function & 5.1 .21 \\
\hline $\begin{array}{l}\text { Advance Propagation } \\
\text { Initialization (APMINIT) CSC }\end{array}$ & 5.1 & $\begin{array}{l}\text { Dielectric Initialization (DIEINIT) } \\
\text { SU }\end{array}$ & 5.1 .8 \\
\hline $\begin{array}{l}\text { Advance Propagation } \\
\text { Initialization (APMINIT) CSC }\end{array}$ & 5.1 & FFT Parameters (FFTPAR) SU & 5.1 .9 \\
\hline $\begin{array}{l}\text { Advance Propagation } \\
\text { Initialization (APMINIT) CSC }\end{array}$ & 5.1 & Fill Height Arrays (FILLHT) SU & 5.1 .10 \\
\hline Fill Height Arrays (FILLHT) SU & 5.1 .10 & $\begin{array}{l}\text { Trace to Output Range } \\
\text { (TRACE_ROUT) SU }\end{array}$ & 5.1 .27 \\
\hline
\end{tabular}


Table 6. APM internal interface design. (continued)

\begin{tabular}{|c|c|c|c|}
\hline \multicolumn{2}{|c|}{ Software Design Description } & \multicolumn{2}{|c|}{ Software Design Description } \\
\hline $\begin{array}{l}\text { Software Design Description } \\
\text { Name }\end{array}$ & $\begin{array}{l}\text { SDD } \\
\text { Paragraph } \\
\text { Number }\end{array}$ & $\begin{array}{l}\text { Software Design Description } \\
\text { Name }\end{array}$ & $\begin{array}{l}\text { SDD } \\
\text { Paragraph } \\
\text { Number }\end{array}$ \\
\hline $\begin{array}{l}\text { Fill Height Arrays (FILLHT) } \\
\text { SU }\end{array}$ & 5.1 .10 & $\begin{array}{l}\text { Trace to Next Step } \\
\text { (TRACE_STEP) SU }\end{array}$ & 5.1 .28 \\
\hline $\begin{array}{l}\text { Trace to Next Step } \\
\text { (TRACE_STEP) SU }\end{array}$ & 5.1 .28 & $\begin{array}{l}\text { Height Check (HTCHECK) } \\
\text { SU }\end{array}$ & 5.1 .17 \\
\hline $\begin{array}{l}\text { Advance Propagation } \\
\text { Initialization (APMINIT) CSC }\end{array}$ & 5.1 & $\begin{array}{l}\text { Gaseous Absorption } \\
\text { (GASABS) SU }\end{array}$ & 5.1 .11 \\
\hline $\begin{array}{l}\text { Advance Propagation } \\
\text { Initialization (APMINIT) CSC }\end{array}$ & 5.1 & $\begin{array}{l}\text { Get Effective Earth Radius } \\
\text { Factor (GET_K) SU }\end{array}$ & 5.1 .12 \\
\hline $\begin{array}{l}\text { Advance Propagation } \\
\text { Initialization (APMINIT) CSC }\end{array}$ & 5.1 & $\begin{array}{l}\text { Get Angles (GETANGLES) } \\
\text { SU }\end{array}$ & 5.1 .14 \\
\hline $\begin{array}{l}\text { Get Angles (GETANGLES) } \\
\text { SU }\end{array}$ & 5.1 .14 & $\begin{array}{l}\text { APM Status (APMSTATUS) } \\
\text { SU }\end{array}$ & 5.1 .7 \\
\hline $\begin{array}{l}\text { Get Angles (GETANGLES) } \\
\text { SU }\end{array}$ & 5.1 .14 & DOSHIFT SU & 5.2 .2 \\
\hline $\begin{array}{l}\text { Get Angles (GETANGLES) } \\
\text { SU }\end{array}$ & 5.1 .14 & $\begin{array}{l}\text { Free Space Range Step } \\
\text { (FRSTP) SU }\end{array}$ & 5.2 .9 \\
\hline $\begin{array}{l}\text { Free Space Range Step } \\
\text { (FRSTP) SU }\end{array}$ & 5.2 .9 & $\begin{array}{l}\text { Fast Fourier Transform (FFT) } \\
\text { SU }\end{array}$ & 5.2 .8 \\
\hline $\begin{array}{l}\text { Fast Fourier Transform } \\
\text { (FFT) SU }\end{array}$ & 5.2 .8 & $\begin{array}{l}\text { Discrete Sine/Cosine } \\
\text { Transform (DRST) SU }\end{array}$ & 5.2 .5 \\
\hline $\begin{array}{l}\text { Get Angles (GETANGLES) } \\
\text { SU }\end{array}$ & 5.1 .14 & $\begin{array}{l}\text { Refractivity Interpolation } \\
\text { (REFINTER) SU }\end{array}$ & 5.2 .18 \\
\hline $\begin{array}{l}\text { Refractivity Interpolation } \\
\text { (REFINTER) SU }\end{array}$ & 5.2 .18 & $\begin{array}{l}\text { Interpolate Profile (INTPROF) } \\
\text { SU }\end{array}$ & 5.1 .18 \\
\hline $\begin{array}{l}\text { Interpolate Profile } \\
\text { (INTPROF) SU }\end{array}$ & 5.1 .18 & $\begin{array}{l}\text { Linear Interpolation } \\
\text { (FN_PLINT) Function }\end{array}$ & 5.2 .14 \\
\hline $\begin{array}{l}\text { Refractivity Interpolation } \\
\text { (REFINTER) SU }\end{array}$ & 5.2 .18 & $\begin{array}{l}\text { Profile Reference } \\
\text { (PROFREF) SU }\end{array}$ & 5.1 .22 \\
\hline $\begin{array}{l}\text { Refractivity Interpolation } \\
\text { (REFINTER) SU }\end{array}$ & 5.2 .18 & $\begin{array}{l}\text { Remove Duplicate } \\
\text { Refractivity Levels } \\
\text { (REMDUP) SU }\end{array}$ & 5.1 .24 \\
\hline $\begin{array}{l}\text { Get Angles (GETANGLES) } \\
\text { SU }\end{array}$ & 5.1 .14 & RG Trace (RGTRACE) SU & 5.1 .25 \\
\hline RG Trace (RGTRACE) SU & 5.1 .25 & $\begin{array}{l}\text { Trace to Next Step } \\
\text { (TRACE_STEP) SU }\end{array}$ & 5.1 .28 \\
\hline
\end{tabular}


Table 6. APM internal interface design. (continued)

\begin{tabular}{|c|c|c|c|}
\hline \multicolumn{2}{|c|}{ Software Design Description } & \multicolumn{2}{|c|}{ Software Design Description } \\
\hline $\begin{array}{c}\text { Software Design Description } \\
\text { Name }\end{array}$ & $\begin{array}{l}\text { SDD Paragraph } \\
\text { Number }\end{array}$ & $\begin{array}{c}\text { Software Design Description } \\
\text { Name }\end{array}$ & $\begin{array}{l}\text { SDD Paragraph } \\
\text { Number }\end{array}$ \\
\hline $\begin{array}{l}\text { Trace to Next Step } \\
\text { (TRACE_STEP) SU }\end{array}$ & 5.1 .28 & $\begin{array}{l}\text { Height Check (HTCHECK) } \\
\text { SU }\end{array}$ & 5.1 .17 \\
\hline $\begin{array}{l}\text { Get Angles (GETANGLES) } \\
\text { SU }\end{array}$ & 5.1 .14 & $\begin{array}{l}\text { Spectral Estimation } \\
\text { (SPECEST) SU }\end{array}$ & 5.2 .22 \\
\hline $\begin{array}{l}\text { Spectral Estimation } \\
\text { (SPECEST) SU }\end{array}$ & 5.2 .22 & $\begin{array}{l}\text { Discrete Sine/Cosine } \\
\text { Transform (DRST) SU }\end{array}$ & 5.2 .5 \\
\hline $\begin{array}{l}\text { Get Angles (GETANGLES) } \\
\text { SU }\end{array}$ & 5.1 .14 & $\begin{array}{l}\text { Trace to Output Range } \\
\text { (TRACE_ROUT) SU }\end{array}$ & 5.1 .27 \\
\hline $\begin{array}{l}\text { Advance Propagation } \\
\text { Initialization (APMINIT) CSC }\end{array}$ & 5.1 & $\begin{array}{l}\text { Get Maximum Angle } \\
\text { (GETTHMAX) SU }\end{array}$ & 5.1 .15 \\
\hline $\begin{array}{l}\text { Get Maximum Angle } \\
\text { (GETTHMAX) SU }\end{array}$ & 5.1 .15 & $\begin{array}{l}\text { FFT Parameters (FFTPAR) } \\
\text { SU }\end{array}$ & 5.1 .9 \\
\hline $\begin{array}{l}\text { Get Maximum Angle } \\
\text { (GETTHMAX) SU }\end{array}$ & 5.1 .15 & $\begin{array}{l}\text { Trace to Output Range } \\
\text { (TRACE_ROUT) SU }\end{array}$ & 5.1 .27 \\
\hline $\begin{array}{l}\text { Advance Propagation } \\
\text { Initialization (APMinit) CSC }\end{array}$ & 5.1 & $\begin{array}{l}\text { Grazing Angle Interpolation } \\
\text { (GRAZE_INT) SU }\end{array}$ & 5.1 .16 \\
\hline $\begin{array}{l}\text { Grazing Angle Interpolation } \\
\text { (GRAZE_INT) SU }\end{array}$ & 5.1 .16 & $\begin{array}{l}\text { Linear Interpolation } \\
\text { (FN_PLINT) Function }\end{array}$ & 5.2 .14 \\
\hline $\begin{array}{l}\text { Advance Propagation } \\
\text { Initialization (APMinit) CSC }\end{array}$ & 5.1 & $\begin{array}{l}\text { PE Initialization (PEINIT) } \\
\text { SU }\end{array}$ & 5.1 .19 \\
\hline PE Initialization (PEINIT) SU & 5.1 .19 & $\begin{array}{l}\text { Allocate Array PE } \\
\text { (ALLARRAY_PE) SU }\end{array}$ & 5.1 .2 \\
\hline PE Initialization (PEINIT) SU & 5.1 .19 & $\begin{array}{l}\text { Interpolate Profile } \\
\text { (INTPROF) SU }\end{array}$ & 5.1 .18 \\
\hline PE Initialization (PEINIT) SU & 5.1 .19 & $\begin{array}{l}\text { Starter Field Initialization } \\
(\text { XYINIT) SU }\end{array}$ & 5.1 .30 \\
\hline $\begin{array}{l}\text { Starter Field Initialization } \\
\text { (XYINIT) SU }\end{array}$ & 5.1 .30 & $\begin{array}{l}\text { Antenna Pattern (ANTPAT) } \\
\text { SU }\end{array}$ & 5.1 .6 \\
\hline $\begin{array}{l}\text { Starter Field Initialization } \\
\text { (XYINIT) SU }\end{array}$ & 5.1 .30 & $\begin{array}{l}\text { Discrete Sine/Cosine } \\
\text { Transform (DRST) SU }\end{array}$ & 5.2 .5 \\
\hline $\begin{array}{l}\text { Advance Propagation } \\
\text { Initialization (APMINIT) CSC }\end{array}$ & 5.1 & $\begin{array}{l}\text { Profile Reference } \\
\text { (PROFREF) SU }\end{array}$ & 5.1 .22 \\
\hline $\begin{array}{l}\text { Advance Propagation } \\
\text { Initialization (APMINIT) CSC }\end{array}$ & 5.1 & $\begin{array}{l}\text { Refractivity Initialization } \\
\text { (REFINIT) SU }\end{array}$ & 5.1 .23 \\
\hline
\end{tabular}


Table 6. APM internal interface design. (continued)

\begin{tabular}{|c|c|c|c|}
\hline \multicolumn{2}{|c|}{ Software Design Description } & \multicolumn{2}{|c|}{ Software Design Description } \\
\hline $\begin{array}{l}\text { Software Design Description } \\
\text { Name }\end{array}$ & $\begin{array}{l}\text { SDD } \\
\text { Paragraph } \\
\text { Number }\end{array}$ & $\begin{array}{c}\text { Software Design Description } \\
\text { Name }\end{array}$ & $\begin{array}{l}\text { SDD } \\
\text { Paragraph } \\
\text { Number }\end{array}$ \\
\hline $\begin{array}{l}\text { Refractivity Initialization } \\
\text { (REFINIT) SU }\end{array}$ & 5.1 .23 & $\begin{array}{l}\text { Profile Reference } \\
\text { (PROFREF) SU }\end{array}$ & 5.1 .22 \\
\hline $\begin{array}{l}\text { Refractivity Initialization } \\
\text { (REFINIT) SU }\end{array}$ & 5.1 .23 & $\begin{array}{l}\text { Remove Duplicate } \\
\text { Refractivity Levels (RemDup) } \\
\text { SU }\end{array}$ & 5.1 .24 \\
\hline $\begin{array}{l}\text { Advance Propagation } \\
\text { Initialization (APMINIT) CSC }\end{array}$ & 5.1 & $\begin{array}{l}\text { Remove Duplicate } \\
\text { Refractivity Levels (RemDup) } \\
\text { SU }\end{array}$ & 5.1 .24 \\
\hline $\begin{array}{l}\text { Advance Propagation } \\
\text { Initialization (APMINIT) CSC }\end{array}$ & 5.1 & $\begin{array}{l}\text { Terrain Initialization } \\
\text { (TERINIT) SU }\end{array}$ & 5.1 .26 \\
\hline $\begin{array}{l}\text { Advance Propagation } \\
\text { Initialization (APMINIT) CSC }\end{array}$ & 5.1 & $\begin{array}{l}\text { Troposcatter Initialization } \\
\text { (TROPOINIT) SU }\end{array}$ & 5.1 .28 \\
\hline $\begin{array}{l}\text { Troposcatter Initialization } \\
\text { (TROPOINIT) SU }\end{array}$ & 5.1 .28 & Antenna Pattern(Antpat) SU & 5.1 .6 \\
\hline $\begin{array}{l}\text { Troposcatter Initialization } \\
\text { (TROPOINIT) SU }\end{array}$ & 5.1 .28 & $\begin{array}{l}\text { Get Effective Earth Radius } \\
\text { Factor (GET_K) SU }\end{array}$ & 5.1 .12 \\
\hline CSCI Detailed Design & 5 & $\begin{array}{l}\text { Advance Propagation Model } \\
\text { Step (APMSTEP) CSC }\end{array}$ & 5.2 \\
\hline $\begin{array}{l}\text { Advance Propagation Model } \\
\text { Step (APMSTEP) CSC }\end{array}$ & 5.2 & $\begin{array}{l}\text { Flat Earth Direct Ray (FEDR) } \\
\text { SU }\end{array}$ & 5.2 .6 \\
\hline $\begin{array}{l}\text { Flat Earth Direct Ray (FEDR) } \\
\text { SU }\end{array}$ & 5.2 .6 & Antenna Pattern(Antpat) SU & 5.1 .6 \\
\hline $\begin{array}{l}\text { Advance Propagation Model } \\
\text { Step (APMSTEP) CSC }\end{array}$ & 5.2 & Flat Earth Model (FEM) SU & 5.2 .7 \\
\hline Flat Earth Model (FEM) SU & 5.2 .7 & Antenna Pattern (Antpat) SU & 5.1 .6 \\
\hline Flat Earth Model (FEM) SU & 5.2 .7 & $\begin{array}{l}\text { Get Reflection Coefficient } \\
\text { (GETREFCOEF) SU }\end{array}$ & 5.2 .12 \\
\hline $\begin{array}{l}\text { Get Reflection Coefficient } \\
\text { (GETREFCOEF) SU }\end{array}$ & 5.2 .12 & $\begin{array}{l}\text { Current Wind } \\
\text { (FN_CURWIND) Function }\end{array}$ & 5.2 .2 \\
\hline $\begin{array}{l}\text { Get Reflection Coefficient } \\
\text { (GETREFCOEF) SU }\end{array}$ & 5.2 .12 & $\begin{array}{l}\text { Dielectric Constant } \\
\text { (FN_DIECON) Function }\end{array}$ & 5.2 .3 \\
\hline $\begin{array}{l}\text { Advance Propagation Model } \\
\text { Step (APMSTEP) CSC }\end{array}$ & 5.2 & $\begin{array}{l}\text { Parabolic Equation Step } \\
\text { (PESTEP) SU }\end{array}$ & 5.2 .16 \\
\hline $\begin{array}{l}\text { Parabolic Equation Step } \\
\text { (PESTEP) SU }\end{array}$ & 5.2 .16 & $\begin{array}{l}\text { Calculate Propagation Loss } \\
\text { (CALCLOS) SU }\end{array}$ & 5.2 .1 \\
\hline
\end{tabular}


Table 6. APM internal interface design. (continued)

\begin{tabular}{|c|c|c|c|}
\hline \multicolumn{2}{|c|}{ Software Design Description } & \multicolumn{2}{|c|}{ Software Design Description } \\
\hline $\begin{array}{l}\text { Software Design Description } \\
\text { Name }\end{array}$ & $\begin{array}{l}\text { SDD } \\
\text { Paragraph } \\
\text { Number }\end{array}$ & $\begin{array}{c}\text { Software Design Description } \\
\text { Name }\end{array}$ & $\begin{array}{l}\text { SDD } \\
\text { Paragraph } \\
\text { Number }\end{array}$ \\
\hline $\begin{array}{l}\text { Calculate Propagation Loss } \\
\text { (CALCLOS) SU }\end{array}$ & 5.2 .1 & $\begin{array}{l}\text { Get Propagation Factor } \\
\text { (FN_GETPFAC) Function }\end{array}$ & 5.2 .11 \\
\hline $\begin{array}{l}\text { Calculate Propagation Loss } \\
\text { (CALCLOS) SU }\end{array}$ & 5.2 .1 & $\begin{array}{l}\text { Linear Interpolation } \\
\text { (FN_PLINT) Function }\end{array}$ & 5.2 .14 \\
\hline $\begin{array}{l}\text { Calculate Propagation Loss } \\
\text { (CALCLOS) SU }\end{array}$ & 5.2 .1 & $\begin{array}{l}\text { Troposcatter (TROPOSCAT) } \\
\text { SU }\end{array}$ & 5.2 .24 \\
\hline $\begin{array}{l}\text { Troposcatter (TROPOSCAT) } \\
\text { SU }\end{array}$ & 5.2 .24 & $\begin{array}{l}\text { Get Troposcatter Loss } \\
\text { (FN_GET_TLOSS) Function }\end{array}$ & 5.2 .13 \\
\hline $\begin{array}{l}\text { Get Troposcatter Loss } \\
\text { (FN_GET_TLOSS) Function }\end{array}$ & 5.2 .13 & $\begin{array}{l}\text { Antenna Pattern (ANTPAT) } \\
\text { SU }\end{array}$ & 5.1 .6 \\
\hline $\begin{array}{l}\text { Parabolic Equation Step } \\
\text { (PESTEP) SU }\end{array}$ & 5.2 .16 & DOSHIFT SU & 5.2 .4 \\
\hline $\begin{array}{l}\text { Parabolic Equation Step } \\
\text { (PESTEP) SU }\end{array}$ & 5.2 .16 & $\begin{array}{l}\text { Free Space Range Step } \\
\text { (FRSTP) SU }\end{array}$ & 5.2 .9 \\
\hline $\begin{array}{l}\text { Free Space Range Step } \\
\text { (FRSTP) SU }\end{array}$ & 5.2 .9 & $\begin{array}{l}\text { Fast-Fourier Transform (FFT) } \\
\text { SU }\end{array}$ & 5.2 .8 \\
\hline $\begin{array}{l}\text { Fast-Fourier Transform (FFT) } \\
\text { SU }\end{array}$ & 5.2 .8 & $\begin{array}{l}\text { Discrete Sine/Cosine } \\
\text { Transform (DRST) SU }\end{array}$ & 5.2 .5 \\
\hline $\begin{array}{l}\text { Parabolic Equation Step } \\
\text { (PESTEP) SU }\end{array}$ & 5.2 .16 & FZLIM SU & 5.2 .10 \\
\hline FZLIM SU & 5.2 .10 & $\begin{array}{l}\text { Get Propagation Factor } \\
\text { (FN_GETPFAC) Function }\end{array}$ & 5.2 .11 \\
\hline FZLIM SU & 5.2 .10 & Save Profile (SAVEPRO) SU & 5.2 .21 \\
\hline FZLIM SU & 5.2 .10 & $\begin{array}{l}\text { Spectral Estimation } \\
\text { (SPECEST) SU }\end{array}$ & 5.2 .22 \\
\hline $\begin{array}{l}\text { Spectral Estimation } \\
\text { (SPECEST) SU }\end{array}$ & 5.2 .22 & $\begin{array}{l}\text { Discrete Sine/Cosine } \\
\text { Transform (DRST) SU }\end{array}$ & 5.2 .5 \\
\hline $\begin{array}{l}\text { Parabolic Equation Step } \\
\text { (PESTEP) SU }\end{array}$ & 5.2 .16 & $\begin{array}{l}\text { Get Alpha Impedance } \\
\text { (GETALN) SU }\end{array}$ & 5.1 .13 \\
\hline $\begin{array}{l}\text { Get Alpha Impedance } \\
\text { (GETALN) SU }\end{array}$ & 5.1 .13 & $\begin{array}{l}\text { Current Wind } \\
\text { (FN_CURWIND) Function }\end{array}$ & 5.2 .2 \\
\hline $\begin{array}{l}\text { Get Alpha Impedance } \\
\text { (GETALN) SU }\end{array}$ & 5.1 .13 & $\begin{array}{l}\text { Get Reflection Coefficient } \\
\text { (GETREFCOEF) SU }\end{array}$ & 5.2 .12 \\
\hline $\begin{array}{l}\text { Get Reflection Coefficient } \\
\text { (GETREFCOEF) SU }\end{array}$ & 5.2 .12 & $\begin{array}{l}\text { Current Wind } \\
\text { (FN_CURWIND) Function }\end{array}$ & 5.2 .2 \\
\hline
\end{tabular}


Table 6. APM internal interface design. (continued)

\begin{tabular}{|c|c|c|c|}
\hline \multicolumn{2}{|c|}{ Software Design Description } & \multicolumn{2}{|c|}{ Software Design Description } \\
\hline $\begin{array}{c}\text { Software Design Description } \\
\text { Name }\end{array}$ & $\begin{array}{l}\text { SDD } \\
\text { Paragraph } \\
\text { Number }\end{array}$ & $\begin{array}{c}\text { Software Design Description } \\
\text { Name }\end{array}$ & $\begin{array}{l}\text { SDD } \\
\text { Paragraph } \\
\text { Number }\end{array}$ \\
\hline $\begin{array}{l}\text { Get Reflection Coefficient } \\
\text { (GETREFCOEF) SU }\end{array}$ & 5.2 .12 & $\begin{array}{l}\text { Dielectric Constant } \\
\text { (FN_DIECON) Function }\end{array}$ & 5.2 .3 \\
\hline $\begin{array}{l}\text { Get Alpha } \\
\text { Impedance(GETALN) SU }\end{array}$ & 5.1 .13 & $\begin{array}{l}\text { Surface Impedance } \\
\text { (SURFIMP) SU }\end{array}$ & 5.2 .23 \\
\hline $\begin{array}{l}\text { Surface Impedance } \\
\text { (SURFIMP) SU }\end{array}$ & 5.2 .23 & $\begin{array}{l}\text { Poly } 4 \text { (FN_POLY4) } \\
\text { Function }\end{array}$ & 5.1 .20 \\
\hline $\begin{array}{l}\text { Surface Impedance } \\
\text { (SURFIMP) SU }\end{array}$ & 5.2 .23 & $\begin{array}{l}\text { Poly } 5 \text { (FN_POLY5) } \\
\text { Function }\end{array}$ & 5.1 .21 \\
\hline $\begin{array}{l}\text { Parabolic Equation Step } \\
\text { (PESTEP) SU }\end{array}$ & 5.2 .16 & $\begin{array}{l}\text { Mixed Fourier Transform } \\
\text { (MIXEDFT) SU }\end{array}$ & 5.2 .15 \\
\hline $\begin{array}{l}\text { Mixed Fourier Transform } \\
\text { (MIXEDFT) SU }\end{array}$ & 5.2 .15 & $\begin{array}{l}\text { Free Space Range Step } \\
\text { (FRSTP) SU }\end{array}$ & 5.2 .9 \\
\hline $\begin{array}{l}\text { Free Space Range Step } \\
\text { (FRSTP) SU }\end{array}$ & 5.2 .9 & $\begin{array}{l}\text { Fast-Fourier Transform } \\
\text { (FFT) SU }\end{array}$ & 5.2 .8 \\
\hline $\begin{array}{l}\text { Fast-Fourier Transform (FFT) } \\
\text { SU }\end{array}$ & 5.2 .8 & $\begin{array}{l}\text { Discrete Sine/Cosine } \\
\text { Transform (DRST) SU }\end{array}$ & 5.2 .5 \\
\hline $\begin{array}{l}\text { Parabolic Equation Step } \\
\text { (PESTEP) SU }\end{array}$ & 5.2 .16 & $\begin{array}{l}\text { Refractivity Interpolation } \\
\text { (REFINTER) SU }\end{array}$ & 5.2 .18 \\
\hline $\begin{array}{l}\text { Refractivity Interpolation } \\
\text { (REFINTER) SU }\end{array}$ & 5.2 .18 & $\begin{array}{l}\text { Interpolate Profile } \\
\text { (INTPROF) SU }\end{array}$ & 5.1 .18 \\
\hline $\begin{array}{l}\text { Interpolate Profile (INTPROF) } \\
\text { SU }\end{array}$ & 5.1 .18 & $\begin{array}{l}\text { Linear Interpolation } \\
\text { (FN_PLINT) Function }\end{array}$ & 5.2 .14 \\
\hline $\begin{array}{l}\text { Refractivity Interpolation } \\
\text { (REFINTER) SU }\end{array}$ & 5.2 .18 & $\begin{array}{l}\text { Profile Reference } \\
\text { (PROFREF) SU }\end{array}$ & 5.1 .22 \\
\hline $\begin{array}{l}\text { Refractivity Interpolation } \\
\text { (REFINTER) SU }\end{array}$ & 5.2 .18 & $\begin{array}{l}\text { Remove Duplicate } \\
\text { Refractivity Levels } \\
\text { (REMDUP) SU }\end{array}$ & 5.1 .24 \\
\hline $\begin{array}{l}\text { Advance Propagation Model } \\
\text { Step (APMSTEP) CSC }\end{array}$ & 5.2 & $\begin{array}{l}\text { Ray Optics Loss (ROLOSS) } \\
\text { SU }\end{array}$ & 5.2 .20 \\
\hline $\begin{array}{l}\text { Ray Optics Loss (ROLOSS) } \\
\text { SU }\end{array}$ & 5.2 .20 & $\begin{array}{l}\text { Ray Optics Calculation } \\
\text { (ROCALC) SU }\end{array}$ & 5.2 .19 \\
\hline $\begin{array}{l}\text { Ray Optics Calculation } \\
\text { (ROCALC) SU }\end{array}$ & 5.2 .19 & $\begin{array}{l}\text { Antenna Pattern (ANTPAT) } \\
\text { SU }\end{array}$ & 5.1 .6 \\
\hline $\begin{array}{l}\text { Ray Optics Calculation } \\
\text { (ROCALC) SU }\end{array}$ & 5.2 .19 & $\begin{array}{l}\text { Get Reflection Coefficient } \\
\text { (GETREFCOEF) SU }\end{array}$ & 5.2 .12 \\
\hline
\end{tabular}


Table 6. APM internal interface design. (continued)

\begin{tabular}{|c|c|c|c|}
\hline \multicolumn{2}{|c|}{ Software Design Description } & \multicolumn{2}{|c|}{ Software Design Description } \\
\hline $\begin{array}{l}\text { Software Design } \\
\text { Description Name }\end{array}$ & $\begin{array}{l}\text { SDD } \\
\text { Paragraph } \\
\text { Number }\end{array}$ & $\begin{array}{l}\text { Software Design Description } \\
\text { Name }\end{array}$ & $\begin{array}{l}\text { SDD } \\
\text { Paragraph } \\
\text { Number }\end{array}$ \\
\hline $\begin{array}{l}\text { Get Reflection Coefficient } \\
\text { (GETREFCOEF) SU }\end{array}$ & 5.2 .12 & $\begin{array}{l}\text { Current Wind (FN_CURWIND) } \\
\text { Function }\end{array}$ & 5.2 .2 \\
\hline $\begin{array}{l}\text { Get Reflection Coefficient } \\
\text { (GETREFCOEF) SU }\end{array}$ & 5.2 .12 & $\begin{array}{l}\text { Dielectric Constant } \\
\text { (FN_DIECON) Function }\end{array}$ & 5.2 .3 \\
\hline $\begin{array}{l}\text { Ray Optics Calculation } \\
\text { (ROCALC) SU }\end{array}$ & 5.2 .19 & Ray Trace (RAYTRACE) SU & 5.2 .17 \\
\hline CSCI Detailed Design & 5 & $\begin{array}{l}\text { Extended Optics Initialization } \\
\text { (XOINIT) CSC }\end{array}$ & 5.3 \\
\hline $\begin{array}{l}\text { Extended Optics } \\
\text { Initialization (XOINIT) CSC }\end{array}$ & 5.3 & APM Clean (APMCLEAN) SU & 5.3 .1 \\
\hline $\begin{array}{l}\text { APM Clean (APMCLEAN) } \\
\text { SU }\end{array}$ & 5.3 .1 & $\begin{array}{l}\text { Discrete Sine/Cosine (DRST) } \\
\text { SU }\end{array}$ & 5.2 .5 \\
\hline $\begin{array}{l}\text { Extended Optics } \\
\text { Initialization (XOINIT) CSC }\end{array}$ & 5.3 & $\begin{array}{l}\text { Clutter-to-Noise (CLUTTER) } \\
\text { SU }\end{array}$ & 5.3 .2 \\
\hline $\begin{array}{l}\text { Clutter-to-Noise } \\
\text { (CLUTTER) SU }\end{array}$ & 5.3 .2 & GIT Initialization (GIT_INIT) SU & 5.3 .5 \\
\hline $\begin{array}{l}\text { GIT Initialization (GIT_INIT) } \\
\text { SU }\end{array}$ & 5.3 .5 & $\begin{array}{l}\text { Current Wind (FN_CURWIND) } \\
\text { Function }\end{array}$ & 5.2 .2 \\
\hline $\begin{array}{l}\text { Clutter-to-Noise } \\
\text { (CLUTTER) SU }\end{array}$ & 5.3 .2 & $\begin{array}{l}\text { Standard Propagation Model } \\
\text { Initialization (SPM_INIT) SU }\end{array}$ & 5.3 .11 \\
\hline $\begin{array}{l}\text { Clutter-to-Noise } \\
\text { (CLUTTER) SU }\end{array}$ & 5.3 .2 & $\begin{array}{l}\text { Standard Propagation Model } \\
\text { (SPM) SU }\end{array}$ & 5.3.12 \\
\hline $\begin{array}{l}\text { Standard Propagation } \\
\text { Model (SPM) SU }\end{array}$ & 5.3 .12 & $\begin{array}{l}\text { Diffraction Loss (FN_DLOSS) } \\
\text { Function }\end{array}$ & 5.3 .3 \\
\hline $\begin{array}{l}\text { Standard Propagation } \\
\text { Model (SPM) SU }\end{array}$ & 5.3 .12 & GofZ (FN_GOFZ) Function & 5.3 .6 \\
\hline $\begin{array}{l}\text { Standard Propagation } \\
\text { Model (SPM) SU }\end{array}$ & 5.3 .12 & $\begin{array}{l}\text { Optical Region Limit (OPLIMIT) } \\
\text { SU }\end{array}$ & 5.3 .8 \\
\hline $\begin{array}{l}\text { Optical Region Limit } \\
\text { (OPLIMIT) SU }\end{array}$ & 5.3 .8 & Get Theta (GETTHETA) SU & 5.3 .4 \\
\hline $\begin{array}{l}\text { Get Theta (GETTHETA) } \\
\text { SU }\end{array}$ & 5.3 .4 & $\begin{array}{l}\text { Get Reflection Coefficient } \\
\text { (GETREFCOEF) SU }\end{array}$ & 5.2 .12 \\
\hline $\begin{array}{l}\text { Get Reflection Coefficient } \\
\text { (GETREFCOEF) SU }\end{array}$ & 5.2 .12 & $\begin{array}{l}\text { Current Wind (FN_CURWIND) } \\
\text { Function }\end{array}$ & 5.2 .2 \\
\hline
\end{tabular}


Table 6. APM internal interface design. (continued)

\begin{tabular}{|c|c|c|c|}
\hline \multicolumn{2}{|c|}{ Software Design Description } & \multicolumn{2}{|c|}{ Software Design Description } \\
\hline $\begin{array}{l}\text { Software Design } \\
\text { Description Name }\end{array}$ & $\begin{array}{c}\text { SDD } \\
\text { Paragraph } \\
\text { Number }\end{array}$ & $\begin{array}{c}\text { Software Design Description } \\
\text { Name }\end{array}$ & $\begin{array}{c}\text { SDD } \\
\text { Paragraph } \\
\text { Number }\end{array}$ \\
\hline $\begin{array}{l}\text { Get Reflection Coefficient } \\
\text { (GETREFCOEF) SU }\end{array}$ & 5.2 .12 & $\begin{array}{l}\text { Dielectric Constant } \\
\text { (FN_DIECON) Function }\end{array}$ & 5.2 .3 \\
\hline $\begin{array}{l}\text { Optical Region Limit } \\
\text { (OPLIMIT) SU }\end{array}$ & 5.3 .8 & R1 Iteration (R1ITER) SU & 5.3 .10 \\
\hline R1 Iteration (R1ITER) SU & 5.3 .10 & Get Theta (GETTHETA) SU & 5.3 .4 \\
\hline $\begin{array}{l}\text { Get Theta (GETTHETA) } \\
\text { SU }\end{array}$ & 5.3 .4 & $\begin{array}{l}\text { Get Reflection Coefficient } \\
\text { (GETREFCOEF) SU }\end{array}$ & 5.2 .12 \\
\hline $\begin{array}{l}\text { Get Reflection Coefficient } \\
\text { (GETREFCOEF) SU }\end{array}$ & 5.2 .12 & $\begin{array}{l}\text { Current Wind (FN_CURWIND) } \\
\text { Function }\end{array}$ & 5.2 .2 \\
\hline $\begin{array}{l}\text { Get Reflection Coefficient } \\
\text { (GETREFCOEF) SU }\end{array}$ & 5.2 .12 & $\begin{array}{l}\text { Dielectric Constant } \\
\text { (FN_DIECON) Function }\end{array}$ & 5.2 .3 \\
\hline $\begin{array}{l}\text { Standard Propagation } \\
\text { Model (SPM) SU }\end{array}$ & 5.3.12 & $\begin{array}{l}\text { Optical Difference (OPTICF) } \\
\text { SU }\end{array}$ & 5.3 .9 \\
\hline $\begin{array}{l}\text { Optical Difference } \\
\text { (OPTICF) SU }\end{array}$ & 5.3 .9 & $\begin{array}{l}\text { Get Reflection Coefficient } \\
\text { (GETREFCOEF) SU }\end{array}$ & 5.2 .12 \\
\hline $\begin{array}{l}\text { Get Reflection Coefficient } \\
\text { (GETREFCOEF) SU }\end{array}$ & 5.2 .12 & $\begin{array}{l}\text { Current Wind (FN_CURWIND) } \\
\text { Function }\end{array}$ & 5.2 .2 \\
\hline $\begin{array}{l}\text { Get Reflection Coefficient } \\
\text { (GETREFCOEF) SU }\end{array}$ & 5.2 .12 & $\begin{array}{l}\text { Dielectric Constant } \\
\text { (FN_DIECON) Function }\end{array}$ & 5.2 .3 \\
\hline $\begin{array}{l}\text { Extended Optics } \\
\text { Initialization (XOINIT) CSC }\end{array}$ & 5.3 & Mean Filter (MEANFILT) SU & 5.3 .7 \\
\hline CSCI Detailed Design & 5 & $\begin{array}{l}\text { Extended Optics Step } \\
\text { (XOSTEP) CSC }\end{array}$ & 5.4 \\
\hline $\begin{array}{l}\text { Extended Optics Step } \\
\text { (XOSTEP) CSC }\end{array}$ & 5.4 & APM Clean (APMCLEAN) SU & 5.3 .1 \\
\hline $\begin{array}{l}\text { APM Clean (APMCLEAN) } \\
\text { SU }\end{array}$ & 5.3 .1 & $\begin{array}{l}\text { Discrete Sine/Cosine } \\
\text { Transform (DRST) SU }\end{array}$ & 5.2 .5 \\
\hline $\begin{array}{l}\text { Extended Optics Step } \\
\text { (XOSTEP) CSC }\end{array}$ & 5.4 & Extended Optics (EXTO) SU & 5.4 .1 \\
\hline $\begin{array}{l}\text { Extended Optics (EXTO) } \\
\text { SU }\end{array}$ & 5.4 .1 & $\begin{array}{l}\text { Linear Interpolation } \\
\text { (FN_PLINT) Function }\end{array}$ & 5.2 .14 \\
\hline $\begin{array}{l}\text { Extended Optics (EXTO) } \\
\text { SU }\end{array}$ & 5.4 .1 & $\begin{array}{l}\text { Troposcatter (TROPOSCAT) } \\
\text { SU }\end{array}$ & 5.2 .24 \\
\hline $\begin{array}{l}\text { Troposcatter } \\
\text { (TROPOSCAT) SU }\end{array}$ & 5.2 .24 & $\begin{array}{l}\text { Get Troposcatter Loss } \\
\text { (FN_GET_TLOSS) Function }\end{array}$ & 5.2 .13 \\
\hline
\end{tabular}


Table 6. APM internal interface design. (continued)

\begin{tabular}{|c|c|c|c|}
\hline \multicolumn{2}{|c|}{ Software Design Description } & \multicolumn{2}{|c|}{ Software Design Description } \\
\hline $\begin{array}{c}\text { Software Design Description } \\
\text { Name }\end{array}$ & $\begin{array}{c}\text { SDD } \\
\text { Paragraph } \\
\text { Number }\end{array}$ & $\begin{array}{c}\text { Software Design Description } \\
\text { Name }\end{array}$ & $\begin{array}{l}\text { SDD } \\
\text { Paragraph } \\
\text { Number }\end{array}$ \\
\hline $\begin{array}{l}\text { Get Troposcatter Loss } \\
\text { (FN_GET_TLOSS) Function }\end{array}$ & 5.2 .13 & Antenna Pattern (ANTPAT) SU & 5.1 .6 \\
\hline $\begin{array}{l}\text { Extended Optics Step } \\
\text { (XOSTEP) CSC }\end{array}$ & 5.4 & Flat Earth Model (FEM) SU & 5.2 .7 \\
\hline Flat Earth Model (FEM) SU & 5.2 .7 & Antenna Pattern (ANTPAT) SU & 5.1 .6 \\
\hline Flat Earth Model (FEM) SU & 5.2 .7 & $\begin{array}{l}\text { Get Reflection Coefficient } \\
\text { (GETREFCOEF) SU }\end{array}$ & 5.2 .12 \\
\hline $\begin{array}{l}\text { Get Reflection Coefficient } \\
\text { (GETREFCOEF) SU }\end{array}$ & 5.2 .12 & $\begin{array}{l}\text { Current Wind (FN_CURWIND) } \\
\text { Function }\end{array}$ & 5.2 .2 \\
\hline $\begin{array}{l}\text { Get Reflection Coefficient } \\
\text { (GETREFCOEF) SU }\end{array}$ & 5.2 .12 & $\begin{array}{l}\text { Dielectric Constant } \\
\text { (FN_DIECON) Function }\end{array}$ & 5.2 .3 \\
\hline $\begin{array}{l}\text { Extended Optics Step } \\
\text { (XOSTEP) CSC }\end{array}$ & 5.4 & Ray Optics Loss (ROLOSS) SU & 5.2 .20 \\
\hline Ray Optics Loss (ROLOSS) SU & 5.2 .20 & $\begin{array}{l}\text { Ray Optics Calculation } \\
\text { (ROCALC) SU }\end{array}$ & 5.2 .19 \\
\hline $\begin{array}{l}\text { Ray Optics Calculation } \\
\text { (ROCALC) SU }\end{array}$ & 5.2 .19 & Antenna Pattern (ANTPAT) SU & 5.1 .6 \\
\hline $\begin{array}{l}\text { Ray Optics Calculation } \\
\text { (ROCALC) SU }\end{array}$ & 5.2 .19 & $\begin{array}{l}\text { Get Reflection Coefficient } \\
\text { (GETREFCOEF) SU }\end{array}$ & 5.2 .12 \\
\hline $\begin{array}{l}\text { Get Reflection Coefficient } \\
\text { (GETREFCOEF) SU }\end{array}$ & 5.2 .12 & $\begin{array}{l}\text { Current Wind (FN_CURWIND) } \\
\text { Function }\end{array}$ & 5.2 .2 \\
\hline $\begin{array}{l}\text { Get Reflection Coefficient } \\
\text { (GETREFCOEF) SU }\end{array}$ & 5.2 .12 & $\begin{array}{l}\text { Dielectric Constant } \\
\text { (FN_DIECON) Function }\end{array}$ & 5.2 .3 \\
\hline $\begin{array}{l}\text { Ray Optics Calculation } \\
\text { (ROCALC) SU }\end{array}$ & 5.2 .19 & Ray Trace (RAYTRACE) SU & 5.2 .17 \\
\hline CSCI Detailed Design & 5 & $\begin{array}{l}\text { Return Grazing Angle } \\
\text { (RET_GRAZE) CSC }\end{array}$ & 5.5 \\
\hline
\end{tabular}

\subsubsection{Internal Data}

The APM CSCI takes full advantage of Fortran 95 features, utilizing allocatable arrays for all internal and input arrays. The external CSCI designer must correctly allocate and initialize all arrays necessary for input to the APM CSCI. 
Due to the computational intensity of the APM CSCI, it may not be necessary or desirable to use the extreme capability of the APM CSCI for all applications. The variables $n_{\text {rout }}$ and $n_{\text {zout }}$ refer to the desired number of range and height output points for any one particular application, and will be specified when the APMINIT CSC is called.

One of the parameters returned to the external application from the APMINIT CSC is $i_{\text {error }}$, which allows for greater flexibility in how input data are handled in the external application. Table 7 lists all possible errors that can be returned.

The logical variables lerr6 and lerr12, when set to '.false.', allow the external application to bypass their associated errors, as these are not critical to the operation of the APM CSCI.

Table 7. APMINIT SU returned error definitions.

\begin{tabular}{|c|c|}
\hline$i_{\text {error }}$ & Definition \\
\hline-5 & Frequency input must be greater than or equal to $2 \mathrm{MHz}$. \\
\hline-6 & $\begin{array}{l}\text { Last range in terrain profile is less than } r_{\max } \text {. Will only return this error if lerr6 set to } \\
\text { ‘.true.'. }\end{array}$ \\
\hline-7 & $\begin{array}{l}\text { Specified cut-back angles (for user-defined height finder antenna pattern) are not } \\
\text { increasing. }\end{array}$ \\
\hline-8 & $h_{\max }$ is less than maximum height of terrain profile. \\
\hline-9 & Antenna height with respect to mean sea level is greater than maximum height $h_{\max }$. \\
\hline-10 & Beamwidth is less than or equal to zero for directional antenna pattern. \\
\hline-11 & $\begin{array}{l}\text { Number of antenna pattern or power reduction factors and angles is less than or equal } \\
\text { to } 1 \text {. For } i_{\text {pat }}=6, n_{\text {facs }} \text { must be at least } 1 \text {; for } i_{\text {pat }}=7, n_{\text {facs }} \text { must be at least } 2 \text {. }\end{array}$ \\
\hline-12 & $\begin{array}{l}\text { Range of last environment profile given (for range-dependent case) is less than } r_{\max } \text {. } \\
\text { Will only return this error if lerr12 set to '.true.'. }\end{array}$ \\
\hline-13 & $\begin{array}{l}\text { Height of first level in any user-specified refractivity profile is greater than } 0 \text {. First } \\
\text { height must be at mean sea level }(0.0) \text { or }<0.0 \text { if below mean sea level. }\end{array}$ \\
\hline-14 & Last gradient in any environment profile is negative. \\
\hline-17 & Range points of terrain profile are not increasing. \\
\hline-18 & First range value in terrain profile is not 0. \\
\hline-21 & Clutter calculations are specified but no transmitter power has been provided. \\
\hline-22 & Clutter calculations are specified but no pulse length has been provided. \\
\hline-23 & Clutter calculations are specified, but no horizontal beamwidth has been provided. \\
\hline-24 & $\begin{array}{l}\text { Clutter calculations are desired over terrain or for frequencies less than } 1 \mathrm{GHz} \text {, but no } \\
\gamma \mathrm{c} \text { values have been specified. }\end{array}$ \\
\hline-25 & $\begin{array}{l}\text { Specified only the PE model to be used but did not specify maximum propagation } \\
\text { angle } t h_{\max } \text {. }\end{array}$ \\
\hline-26 & $\begin{array}{l}\text { Clutter calculations are specified with the propagation path partly or entirely, over } \\
\text { water but did not specify a wind speed. }\end{array}$ \\
\hline-41 & Transmitter height is less than 1.5 meters. \\
\hline-42 & Minimum height input by user, $h_{\min }$, is greater than maximum height, $h_{\max }$. \\
\hline
\end{tabular}


Table 7. APMINIT SU returned error definitions (continued).

\begin{tabular}{c|l}
\hline $\boldsymbol{i}_{\text {error }}$ & \multicolumn{1}{c}{ Definition } \\
\hline-43 & Transform size is greater than $2^{30}$. \\
\hline-44 & $\begin{array}{l}\text { Combination of frequency and antenna beamwidth results in antenna physically below the } \\
\text { surface. Increase frequency or beamwidth for valid combination. }\end{array}$ \\
\hline-45 & $\begin{array}{l}\text { Wind speed specified is greater than the maximum allowed for the specified frequency. } \\
-100\end{array}$ \\
$\begin{array}{l}\text { Error in terrain ray trace (contact the APM CSCI developers if this occurs) } \\
\text { *WARNING*: Antenna height with respect to mean sea level is greater than the last } \\
\text { height in the refractivity profile at the source. }\end{array}$ \\
\hline
\end{tabular}

The APM CSCI provides propagation loss and factor for all heights and ranges when running in a full hybrid mode. When running in a partial hybrid mode, it provides propagation loss and factor for limited heights and angles. Finally, it will also be limited in both height and angle coverage when running in a PE-only mode. Refer to Section 7.1 for environmental conditions under which each execution mode is automatically selected.

Absorption by atmospheric gases (oxygen and water vapor) may be important to some applications of the APM CSCI and is controlled by specifying a non-zero value for the absolute humidity, $a b s_{\text {hum }}$, and the surface air temperature, $t_{\text {air }}$; or likewise, specifying a non-zero value for the gaseous absorption attenuation rate, $\gamma_{a}$.

A particular application of the APM CSCI may or may not require the consideration of troposcatter effects within the propagation loss calculations. For example, a radar evaluation most likely would not be influenced by troposcatter; while an ESM evaluation would. APM has the feature of including or not including the troposcatter calculation by setting a logical flag called $T_{\text {ropo }}$. Setting this flag to '.false.' would omit the calculation. Setting this flag to '.true.' would include the calculation. For the APM CSCI implementation in external coverage and loss diagram applications, $T_{\text {ropo }}$ must be set equal to '.true.' so as to include the calculation.

APM, by default, will run in an "automatic" mode in which, depending upon userspecified inputs, will choose the appropriate sub-models to use for a particular application. However, by setting the logical flag $P E_{\text {flag }}$ to '.true.', APM will be forced to use only the PE sub-model for a particular external application. By default, this flag is set to '.false.'. If this flag is '.true.', then the visible portion of the maximum PE propagation angle, $t h_{\max }$ (i.e., the maximum propagation angle the PE algorithm will accommodate in the field calculations), and the parameter, $r_{\text {mult }}$, must be specified. By default, $r_{\text {mult }}$ is equal to 1 ; however, $t h_{\max }$ does not have a default value and must be explicitly defined. The parameter $r_{\text {mult }}$ is a range step multiplier that allows the user to vary the PE range step from the default calculated. 
Use this option with caution, as you must have some basic knowledge of PE algorithms and how they work to input proper combinations of maximum calculation angles and range steps for a given frequency. When using this option, most error checking is bypassed and parameter limits can be over-ridden. Erroneous field values may result if a poorly chosen combination of th $h_{\max }$ and $r_{\text {mult }}$ are used.

APM Ver. 2.1.04 has the capability of determining and providing to the main calling program direct and reflected propagation angles, as well as the propagation factor from both direct and reflected rays. Note that these quantities are obtained only from the FE and RO sub-models within APM. It does not compute the angles and propagation factors for the separate rays within the split-step PE and XO sub-models, but does provide the resultant propagation angle and factor within these regions. This information is returned if the logical flag lang is set to '.true.'; however, do not enable this feature if any portion of the propagation path is over land. The computation is valid only when the propagation path is entirely over water.

\section{CSCI DETAILED DESIGN}

A description of each component of the APM CSCI is provided in the following subsections.

\subsection{ADVANCE PROPAGATION MODEL INITIALIZATION (APMINIT) CSC}

The APMINIT CSC interfaces with various SUs for the complete initialization of the APM CSCI.

Upon entering the APMINIT CSC, several variables are initialized. All internal logical flags controlling certain environmental calculations and errors are initialized.

The wind speeds and ranges are checked to see if they fall within the specified bounds. If not, then an error is returned.

The variables and arrays necessary for rough surface and clutter calculations are initialized and checked for valid values.

Next, the absorption calculation flag, $k_{a b s}$, is set to 1 if the air temperature, $t_{a i r}$, or the absolute humidity, $a b s_{\text {hum }}$, are non-zero. If an attenuation rate is specified $\left(\gamma_{a} \neq 0\right)$, then $k_{a b s}$ is set to 2 . If the user specifies $t_{a i r}$ and $a b s_{\text {hum }}$ to be 0 , then $k_{a b s}$ is set equal to 0 , in which case, no absorption losses are computed. 
Next, if running the APM CSCI under the hybrid mode ( $P E_{\text {flag }}=$ '.false.'), then the antenna height, the maximum output range, $r_{\max }$, the maximum output height, $h_{\max }$; and the minimum output height, $h_{\min }$, are checked for valid numerical values. If the antenna height is below $1.5 \mathrm{~m}$ then $i_{\text {error }}$ is set to -41 and the APMINIT CSC is exited. $r_{\max }$ is set to the value specified from the calling CSCI or $5000 \mathrm{~m}$, whichever is greater; and $h_{\max }$, is set to the value specified from the calling CSCI or $100 \mathrm{~m}$, whichever is greater. If $h_{\min }$ is greater than $h_{\max }$, then $i_{\text {error }}$ is set to -42 and the APMINIT CSC is exited. If the maximum output range and minimum and maximum output height values are valid, then the APMINIT CSC proceeds to the next step.

The atmospheric volume must be "covered" or resolved with a mesh of calculation points that will, as a matter of routine, exceed the height/range resolution requirements of the particular application of the APM CSCI. The height and range mesh size per APM CSCI output point, $\Delta z_{\text {out }}$ and $\Delta r_{\text {out }}$, respectively, are calculated from the number of APM output points and the maximum range and height as follows:

$$
\begin{gathered}
\Delta r_{\text {out }}=\frac{r_{\text {max }}}{n_{\text {rout }}}, \\
\Delta z_{\text {out }}=\frac{h_{\text {max }}-h_{\text {min }}}{n_{\text {zout }}} .
\end{gathered}
$$

The number of terrain range/height pairs, $i_{t p a}$, used for internal calculations is initialized to 1 plus the user-specified number of range/height pairs, $i_{t p}$. The ALLARRAY_APM SU is then referenced to dynamically allocate and initialize all arrays associated with terrain, refractivity, troposcatter, and general variable arrays. If an error has occurred while allocating memory, $i_{\text {error }}$ is returned with a non-zero value and the CSC is exited; otherwise, the CSC proceeds to the next step.

Next, the constants used to determine the antenna pattern factor are computed. First, if a user-defined height-finder antenna pattern has been specified ( $i_{\text {pat }}=6$ ), along with power cut-back angles and factors, then the angles are converted to radians and stored in array hfangr. If the cut-back angles are not steadily increasing, $i_{\text {error }}$ is set to -7 and the CSC is exited; otherwise, the CSC proceeds with the next step.

If a directional antenna pattern has been specified, the antenna vertical beamwidth in degrees, $\mu_{b w}$, is checked for extremely small beamwidth values. If the value is less than or equal to $10^{-4}, i_{\text {error }}$ is set to -10 and the CSC is exited; otherwise, the CSC proceeds with the next step.

The antenna beamwidth and elevation angles are converted to radians ( $\mu_{b w r}$ and $\mu_{o r}$, respectively) and the variables, $a t_{f a c}$ and $\mu_{\max }$, for use in the ANTPAT SU are determined as follows. 
If the antenna pattern is Gaussian $\left(i_{p a t}=2\right)$, then $a n t_{f a c}$ is given by

$$
a n t_{f a c}=\frac{.34657359}{\left[\operatorname{SIN}\left(\frac{\mu_{b w r}}{2}\right)\right]^{2}} .
$$

If the antenna pattern is $\operatorname{Sin}(\mathrm{X}) / \mathrm{X}\left(i_{p a t}=3\right)$, or a generic height finder $\left(i_{p a t}=5\right)$, then ant $_{\text {fac }}$ is given by

$$
\text { ant }_{f a c}=\frac{1.39157}{\operatorname{SIN}\left(\frac{\mu_{b w r}}{2}\right)}
$$

and $\mu_{\max }$ is given by

$$
\mu_{\max }=\mathbf{T A N}^{-1}\left(\frac{\pi}{a n t_{f a c} \sqrt{1-\left(\frac{\pi}{a n t_{f a c}}\right)^{2}}}\right) .
$$

If running the APM CSCI in hybrid mode, the antenna height is next checked for a valid height based on the combination of frequency and beamwidth specified. The antenna height must not be less than the radius of the antenna and is bounded by

$$
a n t_{h t} \geq \text { maximum of }\left(1.5, .6 \frac{c_{o}}{f_{m h z} \mu_{b w}}\right)
$$

where $c_{o}$ is the speed of light $\left(299.79 \times 10^{6} \mathrm{~m} / \mathrm{s}\right)$.

Next, the TERINIT SU is referenced to initialize all terrain profile and associated arrays. If an error has occurred while in the TERINIT SU, $i_{\text {error }}$ is returned with a nonzero value and the CSC is exited; otherwise, the CSC proceeds with the next step.

If vertical polarization and/or rough surface calculations are required (i.e., a nonzero wind speed is specified) then the flag, $i_{a l g}$, indicating which DMFT algorithm to use, is set to 1 (central difference) for frequencies less than $400 \mathrm{MHz}$, and is set to 2 (backward difference) for frequencies greater than $400 \mathrm{MHz}$. The default value for $i_{a l g}$ is 0 , in which case, no DMFT algorithm is used for the particular APM application. 
Arrays containing all output ranges, rngout, 20 times the logarithm of all output ranges, rlogo, and the square of the output ranges, rsqrd, are initialized according to

$$
\begin{aligned}
\text { rngout }_{i} & =i \Delta r_{\text {out }}, \\
\operatorname{rlogo}_{i} & =20 \mathbf{L O G}_{10}\left(i \Delta r_{\text {out }}\right), \quad i=1,2, \ldots . n_{\text {rout }} \\
\operatorname{rsqrd}_{i} & =\left(i \Delta r_{\text {out }}\right)^{2} .
\end{aligned}
$$

The minimum power of 2 transform, $l n_{\min }$, is next initialized to 10 . If the PE-only option is not activated ( $P E_{\text {flag }}=$ '.false.'), then the execution mode in which the APM CSCI will operate is determined. Based on inputs, it determines whether to use the airborne hybrid mode $\left(i_{\text {hybrid }}=0\right)$, full hybrid mode $\left(i_{\text {hybrid }}=1\right)$, or partial hybrid mode $\left(i_{\text {hybrid }}=2\right)$. For antenna heights greater than $100 \mathrm{~m}$ above the local ground height, $i_{\text {hybrid }}$ is set equal to 0 . For antenna heights less than $100 \mathrm{~m}$, $i_{\text {hybrid }}$ is initialized to 1 . If performing a terrain case ( $f_{\text {ter }}=$ '.true.') and the first $2500 \mathrm{~m}$ of the terrain profile is not flat, then $i_{\text {hybrid }}$ is set equal to 2 . If running in full hybrid mode $\left(i_{\text {hybrid }}=1\right)$ then the ALLARRAY_RO SU is referenced to allocate and initialize all arrays associated with RO calculations.

The variable $y_{\text {fref }}$ is initialized to 0 . If a terrain profile has been specified $\left(f_{\text {ter }}=\right.$ '.true.') then $y_{\text {fref }}$ is set equal to $t y_{1}$. Next, the output height arrays zout and zro are initialized as follows:

$$
\begin{aligned}
\text { zout }_{i} & =h m_{\text {ref }}+i \Delta z_{\text {out }} ; \quad i=1,2, \ldots . . . n_{\text {zout }}, \\
\text { zro }_{i} & =\text { zout }_{i}-y_{\text {fref }}
\end{aligned}
$$

where the variables $t y_{1}$ and $h m_{r e f}$ are obtained from the TERINIT SU.

Next, the REFINIT SU is referenced to initialize all refractivity associated arrays. If an error has occurred while in the REFINIT SU, $i_{\text {error }}$ is returned with a non-zero value and the CSC is exited; otherwise, the CSC proceeds to the next step.

If the PE-only option is activated ( $P E_{\text {flag }}=$ '.true.'), then the minimum height for the PE calculation region $z_{\text {test }}$ is set equal to $h t_{\text {lim }}$. If the PE-only option is not activated $\left(P E_{\text {flag }}=\right.$ '.false.' $)$, then output height arrays used in FE calculations are computed:

$$
\begin{aligned}
& \text { zoutma }_{i}=\text { zout }_{i}-\text { ant }_{\text {ref }} \\
& \text { zoutpa }_{i}=\text { zout }_{i}-y_{\text {fref }}+\text { ant }_{\text {ref }}
\end{aligned} ; i=1,2, \ldots . . n_{\text {zout }} .
$$

The limiting grazing angle, $\psi_{\text {lim }}$, is computed as

$$
\psi_{\text {lim }}=\mathbf{M A X}\left(.002, \frac{.04443}{f_{M H z}^{.3333}}\right) \text {. }
$$


If more than one refractivity profile has been specified ( $\left.n_{\text {prof }}>1\right)$, then $\psi_{\text {lim }}$ is multiplied by 2 . It is then adjusted for trapping effects by

$$
\psi_{\text {lim }}=\psi_{\text {lim }}+\sqrt{\left|2\left(r m_{\max }-r m_{\min }\right)\right|},
$$

where $r m_{\max }$ and $r m_{\min }$ are determined in the REFINIT SU. The RO elevation angle limit, $\alpha_{\text {lim }}$, is given by

$$
\alpha_{\text {lim }}=\sqrt{\mid \psi_{\text {lim }}^{2}+2\left(r m_{t x}-\text { refdum }_{0}\right) \mid}
$$

where the variable $r m_{t x}$ and array refdum are determined in the REFINIT SU. Next, the height tolerance, $z_{t o l}$ is initialized to 0.05 and the range and index variables for the RO region, $i_{R O p}$ and $x_{R O n}$, are initialized to -1 and 0 , respectively. The minimum height for the $\mathrm{PE}$ calculation region is determined next. The minimum height encompassing all trapping refractive layers is given by

$$
h_{\text {test }}=h_{\text {trap }}+h_{\text {thick }} \text {, }
$$

where $h_{\text {trap }}$ and $h_{\text {thick }}$ are determined in the REFINIT SU. If running in full hybrid mode $\left(i_{\text {hybrid }}=1\right)$, the minimum height for the $\mathrm{PE}$ calculation region is given by

$$
z_{\text {test }}=\mathbf{M A X}\left(h_{\text {test }}, 1.2 h_{\text {termax }}\right) \text {, }
$$

where $h_{\text {termax }}$ is determined in the TERINIT SU. If running in either the partial hybrid mode or PE-only mode, $z_{\text {test }}$ is then given by

$$
z_{\text {test }}=\mathbf{A M A X}\left(h t_{\text {lim }}, a n t_{r e f}\right) \text {. }
$$

The tangent angle, $a_{\text {test }}$, used for automatic calculation of the maximum propagation angle, is given by

$$
a_{\text {test }}=\mathbf{T A N}^{-1}\left(\frac{z_{\text {test }}+a n t_{\text {ref }}+\frac{r_{\text {max }}^{2}}{2 a_{e k s t}}}{r_{\max }}\right) \text {, }
$$


with $\alpha_{\text {lim }}$ is now set equal to the greater of $a_{\text {test }}$ or the previously determined $\alpha_{\text {lim }}$, and $a_{e k s t}$ is $4 / 3$ times the mean earth's radius. The GET_K SU is then referenced to determine the effective earth's radius factor.

If the grazing angles need to be computed for rough surface and clutter calculations then the wavelength, $\lambda$, and the free-space wavenumber, $k_{o}$, are initialized using a fixed frequency of $10 \mathrm{GHz}\left(f_{\text {rqg }}\right)$ :

$$
\lambda=\frac{c_{o}}{f_{\text {rqg }}}, k_{o}=\frac{2 \pi}{\lambda} .
$$

The maximum PE calculation angle is set equal to the greater of $4^{\circ}$ (thmxg) or the maximum terrain tangent angle, $\alpha_{u}$, determined in TERINIT SU. The FFTPAR SU is then referenced to determine $\mathrm{PE}$ grid variables and the transform size required. Next, the PEINIT SU is referenced to initialize all arrays and variables associated with the PE calculation algorithm. Variables needed for spectral estimation of the grazing angles are then initialized. The number of bins, $n_{p}$, considered in the near-surface PE region, is set equal to 16 . The power of 2 transform, $\ln _{p}$, is set equal to 9 . The following variables used in the spectral estimation calculations are given by

$$
\begin{aligned}
& n_{s}=2^{l n_{p}}, \\
& n_{p 4}=\frac{n_{p}}{4}, \\
& n_{p 34}=3 n_{p 4}, \\
& c n_{p 75}=\frac{\pi}{n_{p 4}} .
\end{aligned}
$$

The ALLARRAY_XORUF SU is then referenced to allocate and initialize all arrays associated with rough surface calculations. The filter array filtp is now determined by

$$
\text { filtp }_{i}=1 / 2+1 / 2 \operatorname{COS}\left(i c n_{p 75}\right) ; \quad i=0,1,2, \ldots n_{p 4} \text {, }
$$

and the variable $x o_{c o n}$ is given by

$$
x O_{\text {con }}=\frac{\lambda}{2 n_{s} \Delta z_{P E}} .
$$

The GETANGLES SU is next referenced to determine all grazing angles and/or propagation angles, if desired, for subsequent rough surface calculations. This involves computing a complete PE run out to the maximum range, $r_{\max }$. In doing this, the 
refractivity arrays initially set in the REFINIT SU must be re-initialized for the second, or "real”, PE run that includes rough surface effects.

If rough surface calculations are not required (ruf = '.false.') then the grazing angle array $\Psi$ is initialized to 0 , with the number of grazing angles, $i_{g r z}$, set to 1 .

Next, the wavelength and free-space wavenumber are recomputed for the specified frequency $f_{M H z}$ :

$$
\lambda=\frac{c_{o}}{f_{M H z}}, k_{o}=\frac{2 \pi}{\lambda} .
$$

The DIEINIT SU is then referenced to initialize all dielectric ground constants. If rough surface calculations are required, two terms used in the computation of the rough surface reflection coefficient are determined as follows:

$$
\begin{aligned}
& r u f_{f a c}=\frac{4 \pi(.0051)}{\lambda}, \\
& r u f_{h t}=r u f_{f a c} \text { wind }_{1}^{2}
\end{aligned}
$$

where wind $_{1}$ is the first wind speed in $\mathrm{m} / \mathrm{s}$ provided by the calling CSCI.

If the PE-only option is activated ( $P E_{\text {flag }}=$ '.true.') and the frequency specified is greater or equal to $50 \mathrm{MHz}$, then the maximum propagation calculation angle used in the PE region, $\Theta_{\max }$, is determined according to

$$
\Theta_{\max }=4 / 3 t h_{\max } .
$$

The minimum transform size is then set to $\ln _{\min }$ plus 1 for every $5^{\circ}$ in $\Theta_{\max }$. For frequencies less than $50 \mathrm{MHz}\left(H F_{\text {flag }}=\right.$ '.true.'), $\Theta_{75}$ and $\Theta_{\max }$ are defined as

$$
\begin{aligned}
& \Theta_{75}=\operatorname{MAX}\left(2 s l p_{\text {max }}, 40^{\circ}\right) \\
& \Theta_{\text {max }}=\operatorname{MIN}\left(4 / 3 \Theta_{75}, 80^{\circ}\right)
\end{aligned},
$$

where $s l p_{\max }$ is the maximum of the terrain slope along the path. If no terrain is specified, then $\Theta_{75}$ is set equal to $20^{\circ}$. The FFTPAR SU is then referenced to determine the PE grid variables and the maximum valid height within the $P E$ calculation region, $z_{\text {lim }}$, is set equal to the smaller of $z_{\text {test }}$ and $h t_{\text {lim. }}$. The PEINIT SU is then referenced to initialize all PE variables and arrays and, if required (i.e., $i_{a l g}>0$ ), the ALN_INIT SU is referenced to initialize all arrays and variables used in the surface impedance calculations. 
If the PE-only option is not activated ( $P E_{\text {flag }}=$ '.false.') and if using the airborne hybrid mode ( $i_{\text {hybrid }}=0$ ), then the maximum PE calculation angle is determined from $\Theta_{75}$, obtained from the GET_K SU, according to

$$
\Theta_{\max }=4 / 3 \Theta_{75},
$$

and the minimum transform size is set to $\ln _{\min }$ plus 1 for every $5^{\circ}$ in $\Theta_{\max }$. The FFTPAR $\mathrm{SU}$ is then referenced to determine the PE grid variables and the maximum valid height within the PE calculation region, $z_{\text {lim }}$, is set equal to $z_{\text {test }}$. If the airborne mode is not the mode of execution ( $i_{h y b r i d} \neq 0$ ), then the GETTHMAX SU is referenced to determine the minimum angle $\Theta_{75}$ to use within the PE calculation region. $z_{\text {lim }}$ is then set equal to the smaller of $z_{\text {lim }}$ and $h t_{\text {lim. }}$.

To determine if XO calculations are required, the following steps 1 through 6 are performed for $i_{\text {hybrid }}$ not equal to 0 .

1. If $z_{\text {lim }}$ is less than $h t_{l i m}-10^{-5}$, then the SU proceeds with steps 2 through 6 . Otherwise, these steps are skipped and the output range and index, $r_{\text {atz }}$ and $i_{\text {ratz }}$, respectively, are calculated as

$$
\begin{aligned}
& r_{\text {atz }}=2 r_{\text {max }}, \\
& i_{\text {ratz }}=n_{\text {rout }}+1
\end{aligned} .
$$

2. The bin number $j z_{\text {lim }}$, corresponding to $z_{\text {lim }}$, is given by

$$
j z_{l i m}=\mathbf{I N T}\left(\frac{z_{l i m}}{\Delta z_{P E}}\right),
$$

and $z_{\text {lim }}$ is recomputed such that it corresponds to an integer multiple of bins or mesh heights: $z_{\text {lim }}=j z_{\text {lim }} \Delta z_{P E}$.

3. Next, $r_{a t z}$ and $i_{\text {ratz }}$ are determined based on the height, angle, and range arrays htemp, raya, and rtemp, previously determined in the GETTHMAX SU. First, the index $j$ is initialized to $i_{a p}$ (previously determined in the GETTMAX SU) and the index id is initialized to 1 . Steps 3.a through 3.b are repeated until $j$ is greater than $i_{\text {rtemp }}$.

a. If htemp $p_{j}$ is greater than $z_{\text {lim }}$, then the iteration is ended and the SU proceeds with step 4. 
b. If htemp $p_{j}$ is greater than $z r t_{i d}$, then id is incremented by 1 . The index $j$ is now incremented by 1 . Steps $3 a$ through $3 b$ are then repeated.

4. The index ira is set equal to the greater of 1 or $j-1$; the index idg is set equal to id-1; and the gradient $g_{r d}$ is set equal to $g r_{i d g}$.

5. Next, the ray with initial launch angle $a_{\text {launch }}$ is traced from height htempira to $z_{\text {lim }}$. The square of the local ray angle, rad, at the end of the ray trace step is given by

$$
r a d=r a y a_{i r a}^{2}+2 g_{r d}\left(z_{\text {lim }}-h_{t e m p} \text { ira }\right) .
$$

The local ray angle, $a_{a t z}$, at height $z_{\text {lim }}$ is initialized to 0 . If rad is greater than 0 , then $a_{a t z}$ is given by

$$
a_{a t z}=\operatorname{SIGN}\left(1, \text { raya }_{i r a}\right) \sqrt{r a d},
$$

and the range $r_{a t z}$ is now given by

$$
r_{a t z}=r t e m p_{i r a}+\frac{a_{a t z}-r a y a_{i r a}}{g_{r d}} .
$$

6. If $r_{a t z}$ is less than $r_{\max }$ and $z_{\text {lim }}$ is less than $h t_{\text {lim }}$, then the index $k$ is determined such that rngout $_{k-1}<r_{\text {atz }}<$ rngout $_{k}$. Then $i_{\text {ratz }}$ is set equal to the smaller of $n_{\text {rout }}$ and $k$, and $i_{x o s t p}$ is set equal to $i_{\text {ratz. }}$. The number of XO calculations needed, $i_{x o}$, is then set equal to $i_{\text {xostp }}$.

The PEINIT SU is next referenced to initialize all variables and arrays necessary for PE calculations. All variables and arrays associated with XO calculations are now initialized, provided $i_{x o}$ is greater than 0 . The maximum number of points, $i z_{\max }$, allocated for arrays used in $\mathrm{XO}$ calculations, is determined by

$$
i z_{\text {max }}=\frac{\mathbf{N I N T}\left(\frac{r_{\text {max }}-r_{\text {atz }}}{\Delta r_{P E}}\right)}{i z_{\text {inc }}}+4 .
$$

Next, variables needed for spectral estimation calculations are initialized. The number of bins, $n_{p}$, considered in the upper PE region, is set equal to 8 if no terrain profile or wind speeds are specified, and 16, otherwise. The power of 2 transform, $\ln _{p}$, is set equal to 8 . 
The following variables are given by

$$
\begin{aligned}
& n_{s}=2^{\ln n_{p}}, \\
& n_{p 4}=\frac{n_{p}}{4}, \\
& n_{p 34}=3 n_{p 4}, \\
& c n_{p 75}=\frac{\pi}{n_{p 4}} .
\end{aligned}
$$

The ALLARRAY_XORUF SU is then referenced to allocate and initialize all arrays associated with XO calculations. The filter array filtp is now determined by

$$
\text { filtp }_{i}=1 / 2+1 / 2 \operatorname{COS}\left({\text { i } c n_{p 75}}_{1}\right) ; \quad i=0,1,2, \ldots n_{p 4}
$$

and the variable $x o_{c o n}$ is given by

$$
x O_{\text {con }}=\frac{\lambda}{2 n_{s} \Delta z_{P E}}
$$

The ALN_INIT SU is next referenced to initialize all arrays and variables used in the surface impedance calculations and the FILLHT SU is referenced to obtain the htfe array separating the FE from the RO region.

Several variables associated with the beginning, middle, and end of a PE range step, are initialized. If grazing angles were computed, the GRAZE_INT SU is referenced to interpolate the angles (as determined in the GETANGLES SU) at every output range step. If troposcatter calculations are required $\left(T_{\text {ropo }}=1\right)$, then the TROPOINIT SU is referenced to initialize all variables and arrays. An additive loss term $p l_{c n s t}$ and the free space loss $f s l$ are determined at each output range step by

$$
\begin{aligned}
& p l_{c n s t}=20 \mathbf{L O G}_{10}\left(2 k_{o}\right), \\
& f s l_{i}=r \log o_{i}+p l_{c n s t} ; \quad i=1,2, \ldots . . n_{\text {rout }} .
\end{aligned}
$$

Finally, if the absorption flag $k_{a b s}$ is equal to 1 , then the GASABS SU is referenced to determine the absorption attenuation rate, gas $a_{a t t}$. If $k_{a b s}$ is equal to 2, then gas $_{\text {att }}$ is determined by the calling CSCI-specified attenuation rate, $\gamma_{a}$, multiplied by $10^{-3}$ to convert $\gamma_{a}$ from $\mathrm{dB} / \mathrm{km}$ to $\mathrm{dB} / \mathrm{m}$. Several dynamically allocated terrain arrays used in the TERINIT SU are deallocated and the CSC is exited. Table 8 and Table 9 provide identification, description, unit of measure, and the computational source for each APMINIT CSC input and output data element. 
Table 8. APMINIT CSC input data element requirements.

\begin{tabular}{|c|c|c|c|}
\hline Name & Description & Units & Source \\
\hline$a b s_{\text {hum }}$ & Absolute humidity near the surface & g/meters ${ }^{3}$ & Calling CSCI \\
\hline$a_{e k s t}$ & $4 / 3$ times mean earth radius & meters & APM_MOD \\
\hline$G$ & Gain of transmit/receive antennas & $\mathrm{dBi}$ & Calling CSCI \\
\hline$a_{n t}$ & Transmitting antenna height above local ground & meters & Calling CSCI \\
\hline$c_{o}$ & Speed of light multiplied by $10^{-6}$ & meters/sec & APM_MOD \\
\hline$C_{\text {lut }}$ & $\begin{array}{l}\text { Clutter calculation flag: } \\
\text { '.false.' = do not compute surface clutter } \\
\text { '.true.' = compute surface clutter }\end{array}$ & N/A & Calling CSCI \\
\hline dielec & $\begin{array}{l}\text { Two-dimensional array containing the relative permittivity } \\
\text { and conductivity; } \text { dielec }_{1, i} \text { and } \text { dielec }_{2, i} \text {, respectively. }\end{array}$ & $\begin{array}{l}\mathrm{N} / \mathrm{A} \\
\mathrm{S} / \mathrm{m}\end{array}$ & Calling CSCI \\
\hline$f_{M H z}$ & Frequency & $\mathrm{MHz}$ & Calling CSCI \\
\hline$\gamma_{a}$ & Gaseous absorption attenuation rate & $\mathrm{dB} / \mathrm{km}$ & Calling CSCI \\
\hline$\gamma c$ & $\begin{array}{l}\text { Array of "backscattering effectiveness" values used in } \\
\text { computing the reflectivity over land }\end{array}$ & $\mathrm{dB}$ & Calling CSCI \\
\hline prng & Array of corresponding ranges for each $\gamma$ value & meters & Calling CSCI \\
\hline hfang & Cut-back angles & degrees & Calling CSCI \\
\hline hffac & Cut-back antenna pattern factors & N/A & Calling CSCI \\
\hline$h_{\max }$ & Maximum output height with respect to mean sea level & meters & Calling CSCI \\
\hline$h_{\min }$ & Minimum output height with respect to mean sea level & meters & Calling CSCI \\
\hline hmsl & $\begin{array}{l}\text { Two-dimensional array containing heights with respect to } \\
\text { mean sea level of each profile. Array format must be } h m s l_{i, j} \\
=\text { height of } i^{\text {th }} \text { level of } j^{\text {th }} \text { profile; } j=1 \text { for range-independent } \\
\text { cases }\end{array}$ & meters & Calling CSCI \\
\hline$i_{\text {extra }}$ & $\begin{array}{l}\text { Extrapolation flag for refractivity profiles entered in } \\
\text { combination with terrain below below mean sea level } \\
0=\text { extrapolate to minimum terrain height standard } \\
\text { atmosphere gradient } \\
1=\text { extrapolate to minimum terrain height using first } \\
\text { gradient in profile }\end{array}$ & N/A & Calling CSCI \\
\hline$i_{g c}$ & Number of $\gamma c$ values specified & N/A & Calling CSCI \\
\hline$i_{g r}$ & Number of different ground types specified & N/A & Calling CSCI \\
\hline igrnd & $\begin{array}{l}\text { Integer array containing ground type composition for given } \\
\text { terrain profile - can vary with range. Different ground types } \\
\text { are: } \\
\qquad \begin{aligned} & 0=\text { seawater } \\
& 1=\text { freshwater } \\
& 2=\text { wet ground } \\
& 3=\text { medium dry ground } \\
& 4=\text { very dry ground } \\
& 5=\text { ice at }-1 \text { degree } C \\
& 6=\text { ice at }-10 \text { degree } C \\
& 7=\text { user-defined (in which case, values of relative } \\
& \text { permittivity and conductivity must be given) }\end{aligned}\end{array}$ & N/A & Calling CSCI \\
\hline
\end{tabular}


Table 8. APMINIT CSC input data element requirements (continued).

\begin{tabular}{|c|c|c|c|}
\hline Name & Description & Units & Source \\
\hline$i_{p a t}$ & $\begin{array}{l}\text { Antenna pattern type } \\
\qquad \begin{aligned} 1 & =\text { Omnidirectional } \\
2 & =\text { Gaussian } \\
3 & =\text { Sine }(\mathrm{x}) / \mathrm{x} \\
4 & =\text { Cosecant-squared } \\
5 & =\text { Generic height-finder } \\
6 & =\text { User-defined height-finder } \\
7 & =\text { User-defined antenna patter }\end{aligned}\end{array}$ & N/A & Calling CSCI \\
\hline$i_{p o l}$ & $\begin{array}{l}\text { Polarization flag: } \\
\qquad \begin{array}{l}0=\text { horizontal polarization } \\
1 \text { = vertical polarization }\end{array}\end{array}$ & N/A & Calling CSCI \\
\hline$i_{\text {rtemp }}$ & Temporary number of range steps (used for ray tracing) & N/A & APM_MOD \\
\hline$i_{t p}$ & Number of height/range points in profile & N/A & Calling CSCI \\
\hline lang & $\begin{array}{l}\text { Propagation angle and factor output flag } \\
\text { '.true.'= Output propagation angle and propagation } \\
\text { factor for direct and reflected ray (where applicable). } \\
\text { '.false.' = Do not output propagation angles and } \\
\text { factors }\end{array}$ & N/A & Calling CSCI \\
\hline lerr6 & Logical flag to allow for error -6 to be bypassed & N/A & Calling CSCI \\
\hline lerr12 & Logical flag to allow for error -12 to be bypassed & N/A & Calling CSCI \\
\hline$L_{\text {sys }}$ & Miscellaneous system losses & $\mathrm{dB}$ & Calling CSCI \\
\hline lvlp & Number of levels in refractivity profile & N/A & Calling CSCI \\
\hline$N_{f}$ & Noise figure & $\mathrm{dB}$ & Calling CSCI \\
\hline$n_{\text {facs }}$ & $\begin{array}{l}\text { Number of user-defined cut-back angles and cut-back } \\
\text { antenna factors for user specified height-finder antenna type }\end{array}$ & N/A & Calling CSCI \\
\hline$n_{\text {prof }}$ & Number of refractivity profiles & N/A & Calling CSCI \\
\hline$n_{\text {rout }}$ & Number of output range points desired & N/A & Calling CSCI \\
\hline$n_{w}$ & Number of wind speeds & N/A & Calling CSCI \\
\hline$n_{\text {zout }}$ & Number of output height points desired & N/A & Calling CSCI \\
\hline$n_{\text {zout_rtg }}$ & $\begin{array}{l}\text { Number of output receiver heights specified relative to the } \\
\text { local surface elevation. }\end{array}$ & N/A & Calling CSCI \\
\hline$P E_{\text {flag }}$ & $\begin{array}{l}\text { Flag to indicate use of PE algorithm only: } \\
\text { '.true.' = only use PE sub-model } \\
\text { '.false.' = use automatic hybrid model }\end{array}$ & N/A & Calling CSCI \\
\hline pi & Constant equal to the value of $\pi$ & N/A & APM_MOD \\
\hline$P_{t}$ & Transmitter peak power & kW & Calling CSCI \\
\hline refmsl & $\begin{array}{l}\text { Two-dimensional array containing refractivity with respect } \\
\text { to mean sea level of each profile. Array format must be } \\
\text { refms } l_{i, j}=\mathrm{M} \text {-unit at } i^{\text {th }} \text { level of } j^{\text {th }} \text { profile; } j=1 \text { for range- } \\
\text { independent cases }\end{array}$ & M-units & Calling CSCI \\
\hline rgrnd & $\begin{array}{l}\text { Array containing ranges at which varying ground types } \\
\text { apply }\end{array}$ & meters & Calling CSCI \\
\hline
\end{tabular}


Table 8. APMINIT CSC input data element requirements (continued).

\begin{tabular}{|c|c|c|c|}
\hline Name & Description & Units & Source \\
\hline$r_{\max }$ & Maximum specified range & meters & Calling CSCI \\
\hline$r_{\text {mult }}$ & PE range step multiplication factor & N/A & Calling CSCI \\
\hline rngprof & Ranges of each profile: $r n g p r o f_{i}=$ range of $i^{\text {th }}$ profile & meters & Calling CSCI \\
\hline rngwind & $\begin{array}{l}\text { Ranges of wind speeds entered: } \text { rngwind }_{i}=\text { range of } i^{\text {th }} \\
\text { wind speed }\end{array}$ & meters & Calling CSCI \\
\hline$t_{\text {air }}$ & Air temperature near the surface & ${ }^{\circ} \mathrm{C}$ & Calling CSCI \\
\hline terx & Range points of terrain profile & meters & Calling CSCI \\
\hline tery & Height points of terrain profile & meters & Calling CSCI \\
\hline zout_rtg & $\begin{array}{l}\text { Array of output receiver heights specified relative to the } \\
\text { local surface elevation. }\end{array}$ & meters & Calling CSCI \\
\hline$t h_{\max }$ & Visible portion of maximum PE calculation angle & degrees & Calling CSCI \\
\hline$\theta_{\text {hbw }}$ & Horizontal beamwidth & degrees & Calling CSCI \\
\hline$\tau$ & Pulse length/width & $\mu$ sec & Calling CSCI \\
\hline$T_{\text {ropo }}$ & $\begin{array}{l}\text { Troposcatter calculation flag: } \\
\text { '.false.'= no troposcatter calcs } \\
\text { '.true.' = troposcatter calcs }\end{array}$ & N/A & Calling CSCI \\
\hline$\mu_{b w}$ & Antenna vertical beamwidth & degrees & Calling CSCI \\
\hline$\mu_{0}$ & Antenna elevation angle & degrees & Calling CSCI \\
\hline wind & Array of wind speeds & $\begin{array}{l}\text { meters/ } \\
\text { sec }\end{array}$ & Calling CSCI \\
\hline wind $_{\text {dir }}$ & Angle between antenna boresight and upwind direction & degrees & Calling CSCI \\
\hline
\end{tabular}

Table 9. APMINIT CSC output data element requirements.

\begin{tabular}{c|l|c}
\hline Name & \multicolumn{1}{|c|}{ Description } & Units \\
\hline$a_{a t z}$ & Local ray or propagation angle at height $z_{\text {lim }}$ and range $r_{\text {atz }}$ & radians \\
$a_{\text {launch }}$ & Launch angle used which, when traced, separates PE and XO regions from the & N/A \\
& RO region & \\
$\alpha_{\text {lim }}$ & Elevation angle of the RO limiting ray & radians \\
$a n t_{\text {fac }}$ & Antenna pattern parameter (depends on $i_{\text {pat }}$ and $\left.\mu_{b w}\right)$ & N/A \\
$\Delta r_{\text {out }}$ & Output range step & meters \\
$\Delta z_{\text {out }}$ & Output height increment & meters \\
$f i l t p$ & Array filter for spectral estimation calculations & N/A \\
$f s l$ & Free space loss array for output ranges & dB \\
$f_{\text {ter }}$ & Logical flag indicating if terrain profile has been specified: & N/A \\
& \multicolumn{1}{|c|}{ '.frue.' = terrain profile.' = terrain profile not specified } & \\
$g a s_{\text {att }}$ & Gaseous absorption attenuation rate & dB/m
\end{tabular}


Table 9. APMINIT CSC output data element requirements. (continued)

\begin{tabular}{|c|c|c|}
\hline Name & Description & Units \\
\hline hfangr & $\begin{array}{l}\text { Antenna pattern cut-back angles (for specific height finder, or user-defined } \\
\text { antenna patterns) }\end{array}$ & radians \\
\hline$H F_{\text {flag }}$ & HF computation flag indicating the frequency specified is less than $50 \mathrm{MHz}$ & N/A \\
\hline$i_{a l g}$ & $\begin{array}{l}\text { Integer flag indicating which DMFT algorithm is being used: } \\
\begin{aligned} 0 & =\text { no DMFT algorithm will be used } \\
1 & =\text { use central difference algorithm } \\
2 & =\text { use backward difference algorithm }\end{aligned}\end{array}$ & N/A \\
\hline$i_{\text {error }}$ & Error flag & N/A \\
\hline$i_{\text {hybrid }}$ & $\begin{array}{l}\text { Integer indicating which sub-models will be used: } \\
\begin{aligned} 0 & =\text { airborne hybrid model (FEDR + PE) } \\
1 & =\text { full hybrid model }(\mathrm{PE}+\mathrm{FE}+\mathrm{RO}+\mathrm{XO}) \\
2 & =\text { partial hybrid model }(\mathrm{PE}+\mathrm{XO})\end{aligned}\end{array}$ & N/A \\
\hline$i_{\text {ratz }}$ & $\begin{array}{l}\text { Index of output range step in which to begin storing propagation factor and } \\
\text { outgoing angle for XO region }\end{array}$ & N/A \\
\hline$i_{R O p}$ & Array index for previous range in RO region & N/A \\
\hline$i_{t p a}$ & Number of height/range points pairs in terrain profile arrays $t x$, ty & N/A \\
\hline$i_{x o}$ & Number of range steps in $\mathrm{XO}$ calculation region & N/A \\
\hline$i_{\text {Xostp }}$ & Current output range step index for XO calculations & N/A \\
\hline$i z_{\max }$ & $\begin{array}{l}\text { Maximum number of points allocated for arrays associated with XO } \\
\text { calculations }\end{array}$ & N/A \\
\hline$j z_{l i m}$ & PE bin \# corresponding to $z_{\text {lim }}$, i.e., $z_{\text {lim }}=j z_{l i m} \Delta z_{P E}$ & N/A \\
\hline$k_{a b s}$ & 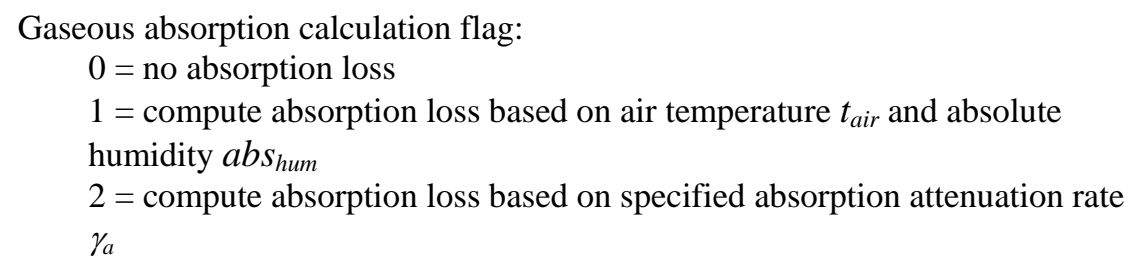 & N/A \\
\hline$k_{o}$ & Free-space wavenumber & meters $^{-1}$ \\
\hline$\lambda$ & Wavelength & meters \\
\hline $\ln _{\min }$ & Minimum power of 2 transform size & N/A \\
\hline $\ln _{p}$ & Power of 2 transform size used in spectral estimation calculations; i.e., $n_{p}=2^{\ln p}$ & N/A \\
\hline lrtg & $\begin{array}{l}\text { Logical flag indicating if loss relative to the local ground height needs to be } \\
\text { computed } \\
\text { 'true.' = Compute loss relative to ground at heights specified by array } \\
\text { zout_rtg } \\
\text { '.false.' = Do not compute loss }\end{array}$ & N/A \\
\hline$\mu_{o r}$ & Antenna pattern elevation angle & radians \\
\hline$\mu_{b w r}$ & Antenna vertical beamwidth in radians & radians \\
\hline$\mu_{\max }$ & Limiting angle for $\operatorname{SIN}(\mathrm{X}) / \mathrm{X}$ and generic height finder antenna pattern factors & N/A \\
\hline$n_{p}$ & $\begin{array}{l}\text { Number of bins in upper and near-surface PE region to consider for spectral } \\
\text { estimation }\end{array}$ & N/A \\
\hline$n_{p 34}$ & $3 / 4 n_{p}$ & N/A \\
\hline
\end{tabular}


Table 9. APMINIT CSC output data element requirements. (continued)

\begin{tabular}{|c|c|c|}
\hline Name & Description & Units \\
\hline$n_{p 4}$ & $1 / 4 n_{p}$ & N/A \\
\hline$n_{s}$ & Transform size for spectral estimation calculations & N/A \\
\hline$p l_{\text {cnst }}$ & Constant used in determining propagation loss $\left(p l_{c n s t}=20 \log _{10}\left(2 k_{o}\right)\right)$ & $\mathrm{dB} / \mathrm{m}$ \\
\hline$\psi_{\lim }$ & Grazing angle of limiting ray & radians \\
\hline$r_{a t z}$ & Range at which $z_{\text {lim }}$ is reached (used for hybrid model) & meters \\
\hline rlogo & Array containing 20 times the logarithm of all output ranges & N/A \\
\hline rngout & Array containing all desired output ranges & meters \\
\hline rsqrd & Array containing the square of all desired output ranges & meters $^{2}$ \\
\hline ruf & $\begin{array}{l}\text { Logical flag indicating if rough sea surface calculations are required } \\
\text { ‘.true.' = perform rough sea surface calculations } \\
\text { '.false.' = do not perform rough sea surface calculations }\end{array}$ & N/A \\
\hline$r u f_{f a c}$ & Factor used for wave height calculation & meters $^{-1}$ \\
\hline$r u f_{h t}$ & RMS sea surface wave height multiplied by $2 k_{o}$ & meters $^{-1}$ \\
\hline$\Theta_{\max }$ & Maximum propagation angle used in PE calculations & radians \\
\hline$\Theta_{75}$ & $75 \%$ of maximum propagation angle in PE calculations & radians \\
\hline$x O_{c o n}$ & Constant used in determining outgoing propagation angle $\vartheta_{\text {out }}$ & N/A \\
\hline$y_{\text {fref }}$ & Ground elevation height at source & meters \\
\hline$x R O n$ & Next range in $\mathrm{RO}$ region & meters \\
\hline$z_{l i m}$ & Height limit for PE calculation region & meters \\
\hline zout & Array containing all desired output heights referenced to $h_{\text {minter }}$ & meters \\
\hline zoutma & Array output heights relative to "real" ant $t_{r e f}$ & meters \\
\hline zoutpa & Array output heights relative to "image" ant $t_{r e f}$ & meters \\
\hline zro & Array of output heights in RO region & meters \\
\hline$Z_{\text {test }}$ & Height in PE region that must be reached for hybrid model & meters \\
\hline$Z_{\text {tol }}$ & Height tolerance for Newton's method & meters \\
\hline
\end{tabular}

\subsubsection{Allocate Arrays APM (ALLARRAY_APM) SU}

The ALLARRAY_APM SU allocates and initializes all dynamically dimensioned arrays associated with the APM terrain, refractivity, troposcatter, and general variable arrays.

The ALLARRAY_APM SU utilizes the FORTRAN ALLOCATE and DEALLOCATE statements to dynamically size arrays that have been previously declared with the ALLOCATABLE attribute in the APM_MOD MODULE or to free the array storage space previously reserved in an ALLOCATE statement. Each dimension of the ALLOCATABLE array is indicated by a colon in the APM_MOD module (e.g., array(:)). The ALLOCATE statement establishes the upper and lower bounds of each dimension and reserves sufficient memory. Because attempting to allocate a previously 
allocated array causes a run-time error, each ALLOCATE statement for an array is preceded by a test to determine if it has been allocated. If it has, it is deallocated before it is allocated.

Initially, the integer used to indicate an error, $i_{\text {error }}$, is set to zero. If, in attempting to allocate an array, a value of $i_{\text {error }}$ other than zero is returned by an ALLOCATE statement, the SU is exited. Note that each array that is dynamically allocated in this SU is initialized to zero.

Seven integers input to this SU are used to dynamically allocate the arrays. Unless otherwise indicated, these integers are used as the single dimension of the dynamically allocated array. The first, $i_{g c}$, is the number of $\gamma c$ values specified, describing the backscattering effectiveness of the surface. The second, $i_{g r}$, is the number of different ground types specified. The third, $i_{t p a}$, is the number of terrain points used internally in arrays $t x$ and ty. The fourth, lvlp, is the number of levels in the refractivity profile. The fifth, $n_{\text {facs }}$, is the number of user-defined cut-back antenna pattern factors for the userdefined height-finder antenna type. The sixth, $n_{\text {rout }}$, is the integer number of output range points desired. And finally, the seventh, $n_{\text {zout }}$, is the integer number of output height points desired.

The definitions of the following arrays allocated in this SU are given in Table 11. The only array that is allocated using the integer $n_{\text {facs }}$ is $h$ fangr. The arrays that are allocated using the integer $n_{\text {rout }}$ are ffat $1 \mathrm{~m}$, rsqrd, fsl, rlogo, hlim, htfe, zxo, and rngout. The arrays that are allocated using the integer $n_{\text {zout }}$ are zout, zoutma, zoutpa, rfac1, rfac2, and rloss. Only those arrays associated with the hybrid mode of execution will be allocated; i.e., if the PE-only mode is activated ( $P E_{\text {flag }}=$ '.false'), then arrays rsqrd, zoutma, zoutpa, hlim, and htfe will not be allocated.

The arrays associated with terrain information use the integers $i_{t p a}, i_{g c}$, or $i_{g r}$. The arrays that are allocated with the integer $i_{t p a}$ are $t x$, ty, and slp. The arrays allocated using the integer $i_{g r}$ are igrnd, rgrnd, and $n c^{2}$, and those arrays allocated with size $i_{g c}$ are $\gamma c$ and $\gamma r n g$. The array dielec is allocated using two as the first dimension and $i_{g r}$ as the second dimension. While arrays igrnd, rgrnd, and dielec are usually specified by the calling CSCI, it is not necessary when performing an over-water case. In this case, $i_{g r}$ can have a value of 0 and these arrays will be defaulted to the size of one element with a sea-surface ground type.

All refractivity arrays used in the PE algorithm are allocated using the integer Ivlp and include refdum, htdum, grdum, href, and refref.

The arrays associated with troposcatter calculations use either integers $n_{\text {zout }}$ and $n_{\text {rout }}$ and will be allocated only if troposcatter calculations are required for the particular APM CSCI application ( $T_{\text {ropo }}=$ '.true.'). The arrays allocated using the integer $n_{\text {zout }}$ include adif, $d 2 s, r d t$, and $\vartheta 2 s$, and the array allocated for size, $n_{\text {rout }}$, is $\vartheta$. 
Table 10 and Table 11 provide identification, description, units of measure, and the computational source for each ALLARRAY_APM SU input and output data element.

Table 10. ALLARRAY_APM SU input data element requirements.

\begin{tabular}{|c|c|c|c|}
\hline Name & Description & Units & Source \\
\hline$C_{\text {lut }}$ & $\begin{array}{l}\text { Clutter calculation flag: } \\
\text { '.false.' = do not compute surface clutter } \\
\text { '.true.' = compute surface clutter }\end{array}$ & N/A & Calling CSCI \\
\hline$i_{g c}$ & Number of $\gamma c$ values specified & N/A & Calling CSCI \\
\hline$i_{g r}$ & Number of different ground types specified & N/A & Calling CSCI \\
\hline$i_{\text {tpa }}$ & $\begin{array}{l}\text { Number of terrain points used internally in terrain profile } \\
\text { arrays } t x, \text { ty }\end{array}$ & N/A & APMINIT CSC \\
\hline lang & $\begin{array}{l}\text { Propagation angle and factor output flag } \\
\text { '.true.'= Output propagation angle and propagation factor } \\
\text { for direct and reflected ray (where applicable). } \\
\text { '.false.' = Do not output propagation angles and factors }\end{array}$ & N/A & Calling CSCI \\
\hline lvlp & Number of levels in refractivity profile & N/A & Calling CSCI \\
\hline$n_{\text {facs }}$ & $\begin{array}{l}\text { Number of user-defined cut-back angles and cut-back antenna } \\
\text { factors for user specified height-finder antenna type }\end{array}$ & N/A & Calling CSCI \\
\hline$n_{\text {rout }}$ & Number of output height points desired & N/A & Calling CSCI \\
\hline$n_{\text {zout }}$ & Number of output range points desired & N/A & Calling CSCI \\
\hline$P E_{\text {flag }}$ & $\begin{array}{l}\text { Flag to indicate use of PE algorithm only: } \\
\text { '.true.' = only use PE sub-model } \\
\text { '.false.' = use automatic hybrid mode }\end{array}$ & N/A & Calling CSCI \\
\hline$T_{\text {ropo }}$ & $\begin{array}{l}\text { Troposcatter calculation flag: } \\
\text { '.false.'= no troposcatter calcs } \\
\text { '.true.' = troposcatter calcs }\end{array}$ & N/A & Calling CSCI \\
\hline
\end{tabular}

Table 11. ALLARRAY_APM SU output data element requirements.

\begin{tabular}{c|l|c}
\hline Name & \multicolumn{1}{|c}{ Description } & Units \\
\hline adif & Height differences between ant $_{\text {ref }}$ and all output receiver heights & meters \\
$d 2 s$ & Array of tangent ranges for all output receiver heights over smooth surface & meters \\
dielec & Two-dimensional array containing the relative permittivity and conductivity; & $\mathrm{N} / \mathrm{A}$, \\
& dielec $_{1, i}$ and diele $_{2, i}$, respectively. & $\mathrm{S} / \mathrm{m}$ \\
$f f a t 1 m$ & Propagation factor array computed at $1 \mathrm{~m}$ above the surface. & $\mathrm{dB}$ \\
$f s l$ & Free space loss array for output ranges & $\mathrm{dB}$ \\
$\gamma c$ & Array of "backscattering effectiveness” values used in computing the & $\mathrm{dB}$ \\
& reflectivity over land & meters
\end{tabular}


Table 11. ALLARRAY_APM SU output data element requirements. (continued)

\begin{tabular}{|c|c|c|}
\hline Name & Description & Units \\
\hline grdum & Array of refractivity gradients defined by profile htdum and refdum & $\begin{array}{l}\text { M-units/ } \\
\text { meter }\end{array}$ \\
\hline hfangr & Cut-back angles & radians \\
\hline hlim & $\begin{array}{l}\text { Array containing height at each output range separating the RO region from } \\
\text { the PE (at close ranges) and XO (at far ranges) regions }\end{array}$ & meters \\
\hline href & Heights of refractivity profile with respect to $y_{\text {ref }}$ & meters \\
\hline htdum & Height array for current interpolated profile & meters \\
\hline htfe & $\begin{array}{l}\text { Array containing the height at each output range separating the FE region } \\
\text { from the RO region (full hybrid mode), or the FE region from the PE region } \\
\text { (partial hybrid mode) }\end{array}$ & meters \\
\hline$i_{\text {error }}$ & $\begin{array}{l}\text { Integer variable indicating error number for ALLOCATE and } \\
\text { DEALLOCATE statements }\end{array}$ & N/A \\
\hline igrnd & 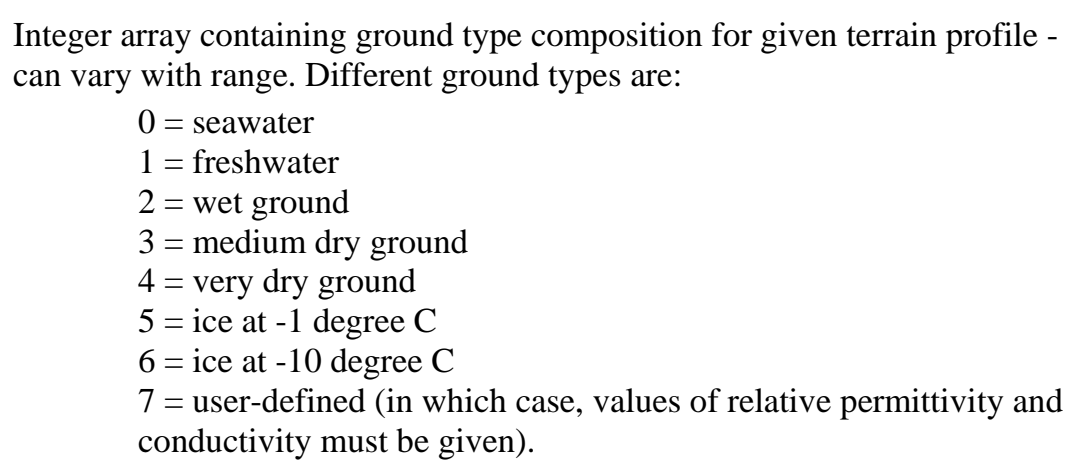 & N/A \\
\hline$n c^{2}$ & Array of complex dielectric constants & N/A \\
\hline$r d t$ & $\begin{array}{l}\text { Array of minimum ranges at which diffraction field solutions are applicable } \\
\text { (for smooth surface) for all output receiver heights. }\end{array}$ & meters \\
\hline refdum & M-unit array for current interpolated profile & M-units \\
\hline refref & Refractivity profile with respect to $y_{r e f}$ & M-units \\
\hline rfac1 & Propagation factor at valid output height points from PE field at range $r_{\text {last }}$. & $\mathrm{dB}$ \\
\hline rfac2 & Propagation factor at valid output height points from PE field at range $r$ & $\mathrm{~dB}$ \\
\hline rgrnd & Array containing ranges at which varying ground types apply & meters \\
\hline rlogo & Array used for storing $20 \mathrm{LOG}_{10}$ (output ranges) & N/A \\
\hline rloss & Propagation loss & $\mathrm{dB}$ \\
\hline rngout & Array containing all desired output ranges & meters \\
\hline rsqrd & Array containing the square of all desired output ranges & meters $^{2}$ \\
\hline slp & Slope of each segment of terrain & N/A \\
\hline$\vartheta 0$ & Array of angles used to determine common volume scattering angle & radians \\
\hline ९2s & $\begin{array}{l}\text { Array of tangent angles from all output receiver heights - used with smooth } \\
\text { surface }\end{array}$ & radians \\
\hline$t x$ & Range points of terrain profile & meters \\
\hline ty & Adjusted height points of terrain profile & meters \\
\hline zout & Array containing all desired output heights referenced to $h_{\text {minter }}$ & meters \\
\hline
\end{tabular}


Table 11. ALLARRAY_APM SU output data element requirements. (continued)

\begin{tabular}{c|l|c}
\hline Name & \multicolumn{1}{|c|}{ Description } & Units \\
\hline zoutma & Array output heights relative to "real" ant $t_{\text {ref }}$ & meters \\
zoutpa & Array output heights relative to "image" ant $t_{\text {ref }}$ & meters \\
zxo & Height of the ground at the current output range step & meters \\
\hline
\end{tabular}

\subsubsection{Allocate Array PE (ALLARRAY_PE) SU}

The ALLARRAY_PE SU allocates and initializes all dynamically dimensioned arrays associated with PE calculations.

The ALLARRAY_PE SU utilizes the FORTRAN ALLOCATE and DEALLOCATE statements to dynamically size arrays that have been previously declared with the ALLOCATABLE attribute in the APM_MOD module or to free the array storage space previously reserved in an ALLOCATE statement. Each dimension of the ALLOCATABLE array is indicated by a colon in the APM_MOD MODULE (e.g., array(:)). The ALLOCATE statement establishes the upper and lower bounds of each dimension and reserves sufficient memory. Because attempting to allocate a previously allocated array causes a run-time error, each ALLOCATE statement for an array is preceded by a test to determine if it has been allocated. If it has, it is deallocated before it is allocated.

Initially, the integer used to indicate an error, $i_{\text {error }}$, is set to zero. If in attempting to allocate an array, a value of $i_{\text {error }}$ other than zero is returned by an ALLOCATE statement, then the SU is exited. Note that each array that is dynamically allocated in this SU is initialized to zero.

Three integers input to this SU are used to dynamically allocate the arrays. Unless otherwise indicated, these integers are used as the single dimension of the dynamically allocated array. The first, $n_{f f t}$, is the transform size. The second, $n_{4}$, is the transform size $n_{\text {fft }}$ divided by four. The third, $n_{\text {zout_rtg }}$, is the number of receiver heights specified relative to the local surface elevation and is used to allocate the rloss_rtg array.

The definitions of the following arrays allocated in this SU are given in Table 13. The arrays that are allocated using the integer $n_{f f t}$ are envpr, frsp, $U$, Ulast, $h t$, profint, udum, $r n, w$, and $y m$. The only array allocated using the integer $n_{4}$ is filt. If rough sea surface or finite conductivity calculations are not required ( $r u f=$ '.false.' and $i_{p o l}=0$ ), then arrays $r n, w$, and $y m$ will not be allocated.

Table 12 and Table 13 provide identification, description, units of measure, and the computational source for each ALLARRAY_PE SU input and output data element. 
Table 12. ALLARRAY_PE SU input data element requirements.

\begin{tabular}{|c|c|c|c|}
\hline Name & Description & Units & Source \\
\hline$i_{a l g}$ & $\begin{array}{l}\text { Integer flag indicating which DMFT algorithm is being used: } \\
\begin{aligned} 0 & =\text { no DMFT algorithm will be used } \\
1 & =\text { use central difference algorithm } \\
2 & =\text { use backward difference algorithm }\end{aligned}\end{array}$ & N/A & APMINIT CSC \\
\hline$i_{p o l}$ & $\begin{array}{l}\text { Polarization flag: } \\
\qquad \begin{array}{l}0=\text { horizontal polarization } \\
1=\text { vertical polarization }\end{array}\end{array}$ & N/A & Calling CSCI \\
\hline lrtg & $\begin{array}{l}\text { Logical flag indicating if loss relative to the local ground } \\
\text { height needs to be computed. } \\
\text { '.true.' = Compute loss relative to ground at heights } \\
\text { specified by array zout_rtg. } \\
\text { '.false.' = Do not compute loss. }\end{array}$ & N/A & APMINIT CSC \\
\hline$n_{f f t}$ & Transform size & N/A & FFTPAR SU \\
\hline$n_{4}$ & $1 / 4 \mathrm{nfft}$ & N/A & APMINIT CSC \\
\hline$n_{\text {zout_rtg }}$ & $\begin{array}{l}\text { Number of output receiver heights specified relative to the } \\
\text { local surface elevation. }\end{array}$ & N/A & Calling CSCI \\
\hline ruf & $\begin{array}{l}\text { Logical flag indicating if rough sea surface calculations are } \\
\text { required } \\
\text { '.true.' = perform rough sea surface calculations } \\
\text { '.false.' = do not perform rough sea surface calculations }\end{array}$ & N/A & APMINIT CSC \\
\hline
\end{tabular}

Table 13. ALLARRAY_PE SU output data element requirements.

\begin{tabular}{|c|c|c|}
\hline Name & Description & Units \\
\hline envpr & Complex [refractivity] phase term array interpolated every $\Delta z_{P E}$ in height & N/A \\
\hline filt & Cosine-tapered (Tukey) filter array & N/A \\
\hline frsp & Complex free space propagator term array & N/A \\
\hline$h t$ & PE mesh height array of size $n_{f f t}$ & meters \\
\hline$i_{\text {error }}$ & $\begin{array}{l}\text { Integer variable indicating error number for ALLOCATE and DEALLOCATE } \\
\text { statements }\end{array}$ & N/A \\
\hline profint & Profile interpolated to every $\Delta z_{P E}$ in height & M-units \\
\hline rloss_rtg & $\begin{array}{l}\text { Propagation loss computed relative to the local ground height at heights } \\
\text { specified by zout_rtg }\end{array}$ & $\mathrm{dB}$ \\
\hline$r n$ & Array of $R_{T}$ to the $i^{\text {th }}$ power (e.g., $r n_{i}=R_{T}^{i}$ ) & N/A \\
\hline$U$ & Complex field at current PE range $r$ & $\mu \mathrm{V} / \mathrm{m}$ \\
\hline Udum & $\begin{array}{l}\text { Dummy array used for temporary storage of real or imaginary part of complex } \\
\text { PE field array } U\end{array}$ & $\mu \mathrm{V} / \mathrm{m}$ \\
\hline Ulast & Complex field at previous PE range $r_{\text {last }}$ & $\mu \mathrm{V} / \mathrm{m}$ \\
\hline$w$ & Difference equation of complex PE field & $\mu \mathrm{V} / \mathrm{m}$ \\
\hline$y m$ & Particular solution of difference equation & N/A \\
\hline
\end{tabular}




\subsubsection{Allocate Array RO (ALLARRAY_RO) SU}

The ALLARRAY_RO SU allocates and initializes all dynamically dimensioned arrays associated with $\mathrm{RO}$ calculations.

The ALLARRAY_RO SU utilizes the FORTRAN ALLOCATE and DEALLOCATE statements to dynamically size arrays that have been previously declared with the ALLOCATABLE attribute in the APM_MOD MODULE or to free the array storage space previously reserved in an ALLOCATE statement. Each dimension of the ALLOCATABLE array is indicated by a colon in the APM_MOD module (e.g., $\operatorname{array}(:))$. The ALLOCATE statement establishes the upper and lower bounds of each dimension and reserves sufficient memory. Because attempting to allocate a previously allocated array causes a run time error, each ALLOCATE statement for an array is preceded by a test to determine if it has been allocated. If it has, it is deallocated before it is allocated.

Initially, the integer used to indicate an error, $i_{\text {error }}$, is set to zero. If in attempting to allocate an array, a value of $i_{\text {error }}$ other than zero is returned by an ALLOCATE statement, then the SU is exited. Note that each array that is dynamically allocated in this $\mathrm{SU}$ is initialized to zero.

Two integers input to this SU are used to dynamically allocate the arrays. Unless otherwise indicated, these integers are used as the single dimension of the dynamically allocated array. The first of these is $l v l p$, the number of points in the refractivity profile, and the second is $n_{\text {zout }}$, which is the number of output height points desired.

The definitions of the following arrays allocated in this SU are given in Table 15. The array zro is allocated using the integer $n_{\text {zout }}$. The remainder of the arrays associated with refractivity information, $g r, q, r m$, and $z r t$, are allocated using the integer $l v l p t$, which is equal to lvlp plus one.

Table 14 and Table 15 provide identification, description, units of measure, and the computational source for each ALLARRAY_RO SU input and output data element.

Table 14. ALLARRAY_RO input data element requirements.

\begin{tabular}{c|c|c|c}
\hline Name & Description & Units & Source \\
\hline$l v l p$ & Number of levels in refractivity profile & N/A & Calling CSCI \\
$n_{\text {zout }}$ & Number of desired output height points & N/A & Calling CSCI \\
\hline
\end{tabular}


Table 15. ALLARRAY_RO output data element requirements.

\begin{tabular}{c|l|c}
\hline Name & \multicolumn{1}{|c}{ Description } & Units \\
\hline$g r$ & Intermediate M-unit gradient array & M-units/meter \\
$q$ & Intermediate M-unit difference array & M-units \\
$r m$ & Intermediate M-unit array & M-units \\
zro & Array of output heights & meters \\
$z r t$ & Intermediate height array & meters \\
\hline
\end{tabular}

\subsubsection{Allocate Array XORUF (ALLARRAY_XORUF) SU}

The ALLARRAY_XORUF SU allocates and initializes all dynamically dimensioned arrays associated with XO and rough sea surface calculations.

The ALLARRAY_XORUF SU utilizes the FORTRAN ALLOCATE and DEALLOCATE statements to dynamically size arrays that have been previously declared with the ALLOCATABLE attribute in the APM_MOD MODULE or to free the array storage space previously reserved in an ALLOCATE statement. Each dimension of the ALLOCATABLE array is indicated by a colon in the APM_MOD module (e.g., $\operatorname{array}(:))$. The ALLOCATE statement establishes the upper and lower bounds of each dimension and reserves sufficient memory. Because attempting to allocate a previously allocated array causes a run time error, each ALLOCATE statement for an array is preceded by a test to determine if it has been allocated. If it has, it is deallocated before it is allocated.

Initially, the integer used to indicate an error, $i_{\text {error }}$, is set to zero. If in attempting to allocate an array, a value of $i_{\text {error }}$ other than zero is returned by an ALLOCATE statement, then the SU is exited. Note that each array that is dynamically allocated in this $\mathrm{SU}$ is initialized to zero.

Six integers input to this SU are used to dynamically allocate the arrays. Unless otherwise indicated, these integers are used as the single dimension of the dynamically allocated array. The first of these is $i_{x o}$ and is the number of XO range step calculations required. The second is $i z_{\max }$, the maximum number of points allocated for arrays associated with $\mathrm{XO}$ calculations. The third is lvlp, the number of points in the refractivity profile. The fourth is $n_{p 4}, 1 / 4$ of the number of points, $n_{p}$, used in the top or bottom portion of the PE region for spectral estimation. The fifth is $n_{\text {rout }}$, the number of output range points desired, and the last is $n_{s}$, the transform size used in spectral estimation calculations (i.e., $n_{s}=2^{\ln p}$ ), where the integer $\ln _{p}$ is the power of 2 transform size.

The definitions of the following arrays allocated in this SU are given in Table 17. The array ffrout is allocated using the integer $n_{\text {rout }}$ as the first dimension and two as the second dimension. The integer $i z_{\max }$ is used as the first dimension limit, and the integer three is used as the second dimension in the allocation of the array ffacz. Both of the 
arrays, grad and htr are allocated with the first dimension given by lvlp and the second dimension given by $i z_{\max }$. The array $l v l$ is allocated using the integer $i z_{\max }$. The array filtp is allocated using the integer $n_{p 4}$. The three arrays $x p, y p$, and spectr are allocated using the integer $n_{s}$.

If no XO calculations are required $\left(i_{x o}=0\right)$ then the arrays ffrout, ffacz, grad, htr, and $l v l$ will not be allocated.

Table 16 and 17 provide identification, description units of measure, and the computational source for each ALLARRAY_XORUF SU input and output data element.

Table 16. ALLARRAY_XORUF SU input data element requirements.

\begin{tabular}{c|l|c|c}
\hline Name & \multicolumn{1}{|c|}{ Description } & Units & Source \\
\hline$i_{x o}$ & Number of range steps in XO calculation region & N/A & APMINIT CSC \\
$i z_{\max }$ & $\begin{array}{l}\text { Maximum number of points allocated for arrays associated } \\
\text { with XO calculations }\end{array}$ & N/A & APMINIT CSC \\
$l v l p$ & Number of height/refractivity levels in profiles & N/A & Calling CSCI \\
$n_{p 4}$ & $1 / 4 n_{p}$ & N/A & APMINIT CSC \\
$n_{\text {rout }}$ & Integer number of output range points desired & N/A & Calling CSCI \\
$n_{s}$ & Transform size for spectral estimation calculations & N/A & APMINIT CSC \\
\hline
\end{tabular}

Table 17. ALLARRAY_XORUF SU output data element requirements.

\begin{tabular}{|c|c|c|}
\hline Name & Description & Units \\
\hline ffacz & $\begin{array}{l}\text { Two-dimensional array containing propagation factor, range, and outgoing } \\
\text { propagation angle at } z_{\text {lim }}\end{array}$ & $\begin{array}{l}\mathrm{dB} \text {, meters, } \\
\text { radians }\end{array}$ \\
\hline ffrout & $\begin{array}{l}\text { Two-dimensional array of propagation factors at each output range beyond } \\
r_{a t z} \text { and at height } z_{\text {lim }}\end{array}$ & $\mathrm{dB}$ \\
\hline filtp & Array filter for spectral estimation calculations & N/A \\
\hline grad & $\begin{array}{l}\text { Two-dimensional array containing gradients of each profile used in XO } \\
\text { calculations }\end{array}$ & M-units/ meter \\
\hline$h t r$ & $\begin{array}{l}\text { Two-dimensional array containing heights of each profile used in XO } \\
\text { calculations }\end{array}$ & meters \\
\hline$i_{\text {error }}$ & $\begin{array}{l}\text { Integer variable indicating error number for ALLOCATE and } \\
\text { DEALLOCATE statements }\end{array}$ & N/A \\
\hline$l v l$ & Number of height levels in each profile used in XO calculations & N/A \\
\hline spectr & Spectral amplitude of field & $\mathrm{dB}$ \\
\hline$x p$ & Real part of spectral portion of PE field & $\mu \mathrm{V} / \mathrm{m}$ \\
\hline yp & Imaginary part of spectral portion field & $\mu \mathrm{V} / \mathrm{m}$ \\
\hline
\end{tabular}




\subsubsection{Alpha Impedance Initialization (ALN_INIT) SU}

The ALN_INIT SU initializes variables and arrays used for the backward or central difference form of the DMFT algorithm. The DMFT algorithm is used only for finite conductivity and/or rough sea surface calculations.

Upon entering, the GETALN SU is referenced to obtain the surface impedance, $\alpha_{s}$, the complex root $R_{T}$, the array $r n$ containing the powers of the complex root, and a coefficient $R_{k}$ used in the central difference algorithm. These variables are computed from the grazing angle $\psi$ and current range, which, for initialization purposes are set equal to $\pi / 2$ and 0 , respectively.

If the central difference algorithm is required $\left(i_{a l g}=1\right)$ for the particular APM CSCI application, then coefficients necessary for this form of the DMFT are computed as follows:

$$
\begin{aligned}
& c k_{1}=R_{k}\left[.5\left(U_{0}+U_{n_{\text {fft }}} r n_{n_{\text {fft }}}\right)+\sum_{i=1}^{n_{m 1}} U_{i} r n_{i}\right] \\
& c k_{2}=R_{k}\left[.5\left(U_{0} r n_{n_{f f t}}+U_{n_{f f t}}\right)+\sum_{i=1}^{n_{m 1}}(-1)^{i} U_{n_{\text {fft }}-i} r n_{i}\right]
\end{aligned}
$$

If the backward difference algorithm is required $\left(i_{a l g}=2\right)$ for the particular APM CSCI application, then the variable $\mathrm{cmft}$ is initialized using the starting PE field $U$ and the array $r n$ according to

$$
c m f t=\sum_{i=1}^{n_{m 1}} U_{i} r n_{i} .
$$

Table 18 and Table 19 provide identification, description, units of measure, and the computational source for each ALN_INIT SU input and output data element.

Table 18. ALN_INIT SU input data element requirements.

\begin{tabular}{c|l|c|c}
\hline Name & \multicolumn{1}{|c|}{ Description } & Units & Source \\
\hline$\psi$ & Grazing angle & radians & APMINIT CSC \\
$i_{a l g}$ & Integer flag indicating which DMFT algorithm is being used: & N/A & APMINIT CSC \\
& $0=$ no DMFT algorithm will be used & & \\
& $2=$ use central difference algorithm & & \\
$r$ & Current range & & \\
$n_{m 1}$ & $n_{f f t}-1$ & meters & APMINIT CSC \\
& & N/A & APMINIT CSC
\end{tabular}


Table 18. ALN_INIT SU input data element requirements. (continued)

\begin{tabular}{c|l|c|c}
\hline Name & \multicolumn{1}{|c|}{ Description } & Units & Source \\
\hline$R_{k}$ & $\begin{array}{l}\text { Constant used to compute coefficients in central difference } \\
\text { form of the DMFT }\end{array}$ & N/A & GETALN SU \\
$r n$ & Array of $R_{T}$ to the $i^{\text {th }}$ power (e.g., $\left.r n_{i}=R_{T}{ }^{i}\right)$ & N/A & GETALN SU \\
$U$ & Complex field at current PE range $r$ & $\mu \mathrm{V} / \mathrm{m}$ & XYINIT SU \\
\hline
\end{tabular}

Table 19. ALN_INIT SU output data element requirements.

\begin{tabular}{c|l|c}
\hline Name & \multicolumn{1}{|c}{ Description } & Units \\
\hline$c k_{1}$ & Coefficient used in central difference form of DMFT & N/A \\
$c k_{2}$ & Coefficient used in central difference form of DMFT & N/A \\
$c m f t$ & Coefficient used in backward difference form of DMFT & N/A \\
\hline
\end{tabular}

\subsubsection{Antenna Pattern (ANTPAT) SU}

The ANTPAT SU calculates an antenna pattern factor (normalized antenna gain), $f(\alpha)$, for a specified antenna elevation angle, $\alpha$. Currently, antenna pattern factors are included for seven types of antennas. These patterns include an omnidirectional $\left(i_{p a t}=1\right)$ type; a Gaussian $\left(i_{p a t}=2\right)$ type; a $\operatorname{Sin}(\mathrm{X}) / \mathrm{X}\left(i_{p a t}=3\right)$ type; a cosecant-squared $\left(i_{p a t}=4\right)$ type; a generic height-finder $\left(i_{p a t}=5\right)$ type; a user-defined height-finder type $\left(i_{p a t}=6\right)$, in which the calling CSCI must also specify an array of cut-back angles and pattern factors; a user-defined antenna type ( $i_{p a t}=7$ ), in which the calling CSCI must specify an array of antenna pattern factors and angles; and finally, a quarter-wave dipole antenna type $\left(i_{p a t}=8\right)$, suitable for HF applications.

From two antenna pattern quantities determined in the APMINIT CSC, $a_{\text {fac }}$ and $p_{\text {elev }}$; the antenna beam width, $\mu_{b w r}$, and elevation angle, $\mu_{o r}$; a specified angle, $\alpha$, for which the antenna pattern factor is desired; and the antenna radiation pattern type, $i_{\text {pat }}$, the antenna pattern factor is calculated as follows.

If the antenna pattern is omnidirectional, then $f(\alpha)=1$. If the antenna pattern is Gaussian, then

$$
f(\alpha)=e^{-a n t} f a c\left[\operatorname{SIN}(\alpha)-p_{e l e v}\right]^{2}
$$

If the antenna pattern is cosecant-squared, compute the elevation angle relative to the antenna elevation angle as

$$
\alpha_{p a t}=\alpha-\mu_{o r} .
$$


The antenna pattern is now given as

$$
\begin{gathered}
f(\alpha)=\frac{\operatorname{SIN}\left(\mu_{b w r}\right)}{\operatorname{SIN}\left(\alpha_{p a t}\right)}, \quad \text { for } \alpha_{p a t}>\mu_{b w r}, \\
f(\alpha)=\operatorname{MAX}\left(0.0,\left[1+\frac{\alpha_{p a t}}{\mu_{b w r}}\right]\right), \quad \text { for } \alpha_{p a t}<0, \\
f(\alpha)=1 \quad \text { otherwise. }
\end{gathered}
$$

If the antenna pattern is $\operatorname{Sin}(\mathrm{X}) / \mathrm{X}$, a generic height-finder, or a user-specified height-finder, the following calculations are made:

1. The elevation angle relative to the antenna elevation angle, $\alpha_{p a t}$, is determined as in the previous definition. If the antenna radiation pattern type is a generic or userspecified height-finder, the radiation pattern is simulated as a $\operatorname{Sin}(\mathrm{X}) / \mathrm{X}$ type with the elevation angle adjusted to account for the current pointing angle of the main beam, $\chi \cdot \chi$ is set equal to the antenna elevation angle $\mu_{o r}$. If the direct-path ray angle, $\alpha_{d}$, is greater than the antenna elevation angle, then $\alpha_{p a t}$ is computed as $\alpha_{p a t}=\alpha-\alpha_{d}$ and $\chi$ is set equal to $\alpha_{d}$.

2. The antenna pattern is now given as

$$
\begin{array}{cc}
f(\alpha)=1 & \text { for }\left|\alpha_{\text {pat }}\right| \leq 10^{-6} \\
f(\alpha)=\frac{\operatorname{SIN}\left[a n t_{f a c} \operatorname{SIN}\left(\alpha_{\text {pat }}\right)\right]}{\operatorname{ant}_{\text {fac }} \operatorname{SIN}\left(\alpha_{\text {pat }}\right)} ; & \text { for }\left|\alpha_{\text {pat }}\right|<\mu_{\text {max }}, \\
f(\alpha)=\text { side }_{\text {lim }} \text {, otherwise, } &
\end{array}
$$

where side $_{\text {lim }}$ is 0.03 for a $\operatorname{Sin}(\mathrm{x}) / \mathrm{x}$ antenna pattern, and 0.0 otherwise.

For a user-defined height-finder, the pattern factor is further adjusted by a power reduction factor, $h f f a c_{i}$, as

$$
f(\alpha)=f(\alpha) \text { hffac }_{i} \quad i=n_{f a c s}, \ldots .2,1
$$


where $i$ is an angle counter, decremented by one from the number of power reduction angles, $n_{\text {facs }}$, for each power reduction angle, $h$ fangr $_{i}$, which exceeds $\chi$.

For the user-defined antenna type, the antenna pattern factor is simply the array hffac provided by the calling CSCI at angles hfangr, where pattern factors are interpolated from $h f f a c$ for angles $\alpha$ within $h$ fangr.

Finally, for the quarter-wave dipole antenna pattern, the antenna pattern factor is defined as

$$
\begin{gathered}
s_{\alpha}=\operatorname{SIN}(|\alpha|), c_{\alpha}=\operatorname{COS}(|\alpha|) \\
s_{r}=n c_{1}^{2^{*}} s_{\alpha}, c_{r}=\sqrt{n c_{1}^{2^{*}}-c_{\alpha}^{2}} \\
\Gamma_{n}=s_{r}-c_{r}, \Gamma_{d}=s_{r}+c_{r} \\
t_{4}=\mathbf{T A N}^{-1} \frac{\mathfrak{I}\left(\Gamma_{n}\right)}{\mathfrak{R}\left(\Gamma_{n}\right)}, t_{5}=\mathbf{T A N}^{-1} \frac{\mathfrak{I}\left(\Gamma_{d}\right)}{\mathfrak{R}\left(\Gamma_{d}\right)} \\
\Gamma=\frac{\left|\Gamma_{n}\right|}{\left|\Gamma_{d}\right|}, e_{o}=\operatorname{COS}\left(\pi / 2 s_{\alpha}\right), t_{9}=t_{4}-t_{5}+\pi \mathrm{s}_{\alpha} \\
E_{r}=e_{o}-e_{o} \Gamma \operatorname{COS}\left(t_{9}\right), E_{j}=e_{o} \Gamma \operatorname{SIN}\left(t_{9}\right) \\
f(\alpha)=1 / 2 \sqrt{E_{r}^{2}+E_{j}^{2}}
\end{gathered}
$$

Table 20 and Table 21 provide identification, description, units of measure, and the computational source for each ANTPAT SU input and output data element.

Table 20. ANTPAT SU input data element requirements.

\begin{tabular}{c|l|c|c}
\hline Name & \multicolumn{1}{|c|}{ Description } & Units & Source \\
\hline$a n t_{f a c}$ & Antenna pattern parameter (depends on $i_{p a t}$ and $\mu_{b w r}$ ) & N/A & APMINIT CSC \\
$\alpha$ & Antenna elevation angle & radians & Calling SU \\
$\alpha_{d}$ & Direct ray elevation angle & radians & FEDR SU \\
& & & FEM SU \\
& & & ROCALC SU \\
& & & TROPOINIT SU \\
& & & TROPOSCAT SU \\
& & & XYINIT SU
\end{tabular}


Table 20. ANTPAT SU input data element requirements. (continued)

\begin{tabular}{|c|c|c|c|}
\hline Name & Description & Units & Source \\
\hline hfangr & $\begin{array}{l}\text { Cut-back angles if } i_{p a t}=6 ; \\
\text { Antenna pattern angles if } i_{p a t}=7\end{array}$ & radians & Calling CSCI \\
\hline hffac & $\begin{array}{l}\text { Cut-back antenna pattern factors if } i_{p a t}=6 \text {; } \\
\text { Antenna pattern factors if } i_{p a t}=7\end{array}$ & N/A & Calling CSCI \\
\hline$i_{p a t}$ & $\begin{array}{l}\text { Antenna pattern type } \\
\qquad \begin{aligned} 1 & =\text { Omnidirectional } \\
2 & =\text { Gaussian } \\
3 & =\text { Sine }(\mathrm{x}) / \mathrm{x} \\
4 & =\text { Cosecant-squared } \\
5 & =\text { Generic height-finder } \\
6 & =\text { User-defined height-finder } \\
7 & =\text { User-defined antenna pattern }\end{aligned}\end{array}$ & N/A & Calling CSCI \\
\hline$\mu_{o r}$ & Antenna pattern (pointing) elevation angle & radians & APMINIT CSC \\
\hline$\mu_{b w r}$ & Antenna vertical beam width & radians & APMINIT CSC \\
\hline$\mu_{\max }$ & $\begin{array}{l}\text { Limiting angle for } \operatorname{Sin}(\mathrm{X}) / \mathrm{X} \text { and generic height finder } \\
\text { antenna pattern factors }\end{array}$ & radians & APMINIT CSC \\
\hline$n c^{2}$ & Array of complex dielectric constants & N/A & DIEINIT SU \\
\hline$n_{\text {facs }}$ & $\begin{array}{l}\text { Number of user-defined cut-back angles and cut-back } \\
\text { pattern factors }\end{array}$ & N/A & Calling CSCI \\
\hline$p_{\text {elev }}$ & Sine of antenna elevation angle & N/A & APMINIT CSC \\
\hline
\end{tabular}

Table 21. ANTPAT SU output data element requirements.

\begin{tabular}{c|l|c}
\hline Name & Description & Units \\
\hline$f(\alpha)$ & Antenna pattern factor for elevation angle $\alpha$ & N/A \\
\hline
\end{tabular}

\subsubsection{APM Status (APMSTATUS) SU}

This SU is supplied with the APM CSCI and should be declared as an external subroutine. It is called from the GETANGLES SU as a means of checking the status of the algorithm for computing grazing angles, as this can be time-intensive before the actual propagation loss calculations are performed.

\subsubsection{Dielectric Initialization (DIEINIT) SU}

The DIEINIT SU determines the conductivity and relative permittivity as a function of frequency in megahertz, based on general ground composition types.

The DIEINIT SU supports the following general ground types: salt water, freshwater, wet ground, medium dry ground, very dry ground, ice at $-1{ }^{\circ} \mathrm{C}$, ice at $-10{ }^{\circ} \mathrm{C}$, and user-defined. For all ground types other than "user-defined," the permittivity and 
conductivity are calculated as functions of frequency from curve fits to the permittivity and conductivity graphs shown in the Recommendations and Reports of the International Radio Consultative Committee (CCIR, 1986). For the $i^{\text {th }}$ input ground type case, igrnd, the permittivity $\varepsilon_{r}$ and conductivity $\sigma$ are determined as follows:

For salt water igrnd $_{i}=0$ ), the relative permittivity is given by 70 for $f_{M H z} \leq 2253.5895$; and the conductivity is given by $5.0 \mathrm{~S} / \mathrm{m}$ for $f_{M H z} \leq 1106.207$. For $f_{M H z}>2253.5895$, the relative permittivity is given by

$$
\varepsilon_{r}=\left[\begin{array}{l}
1.4114535 \times 10^{-2}-5.2122497 \times 10^{-8} f_{M H z}+5.8547829 \times 10^{-11} f_{M H z}^{2} \\
-7.6717423 \times 10^{-16} f_{M H z}^{3}+2.9856318 \times 10^{-21} f_{M H z}^{4}
\end{array}\right]^{-1} .
$$

For $f_{M H z}>1106.207$, the conductivity $\sigma$ in $\mathrm{S} / \mathrm{m}$ is given by

$$
\sigma=\frac{3.8586749+9.1253873 \times 10^{-4} f_{M H z}+1.530992 \times 10^{-8} f_{M H z}^{2}}{1 .-2.1179295 \times 10^{-5} f_{M H z}+6.5727504 \times 10^{-10} f_{M H z}^{2}-1.9647664 \times 10^{-15} f_{M H z}^{3}}
$$

For freshwater igrnd $_{i}=1$ ), the relative permittivity $\varepsilon_{r}$ is given by 80 for $f_{M H z} \leq 6165.776$. For higher frequencies, $\varepsilon_{r}$ is given by

$$
\varepsilon_{r}=\frac{79.027635-3.5486605 \times 10^{-4} f_{M H z}+8.210184 \times 10^{-9} f_{M H z}^{2}}{1 .-2.2083308 \times 10^{-5} f_{M H z}+2.7067836 \times 10^{-9} f_{M H z}^{2}-1.0007669 \times 10^{-14} f_{M H z}^{3}} .
$$

For $f_{M H Z}>5776.157$, the conductivity $\sigma$ in $\mathrm{S} / \mathrm{m}$ is given by

$$
\sigma=\left(\frac{-.65750351+6.6113198 \times 10^{-4} f_{M H z}+1.4876952 \times 10^{-9} f_{M H z}^{2}}{1+5.5620223 \times 10^{-5} f_{M H z}+3.0140816 \times 10^{-10} f_{M H z}^{2}}\right)^{2} .
$$

For $f_{M H Z} \leq 5776.157$, the conductivity $\sigma$ in $\mathrm{S} / \mathrm{m}$ is given by

$$
\sigma=\left(\frac{201.97103+1.2197967 \times 10^{-2} f_{M H z}-1.728776 \times 10^{-6} f_{M H z}^{2}}{1 .-2.5539582 \times 10^{-3} f_{M H z}-3.7853169 \times 10^{5} f_{M H z}^{2}}\right)^{-1}
$$


For wet ground $\left(\right.$ igrnd $_{i}=2$ ), the relative permittivity $\varepsilon_{r}$ is given by 30 for $f_{M H z} \leq 1312.054$. For $1312.054<f_{M H z}<4228.11$, the relative permittivity $\varepsilon_{r}$ is given by

$$
\varepsilon_{r}=\sqrt{\frac{857.94335+5.5275278 \times 10^{-2} f_{M H z}}{1-8.9983662 \times 10^{-5} f_{M H z}+8.8247139 \times 10^{-8} f_{M H z}^{2}}} .
$$

For $f_{M H z} \geq 4228.11$, the relative permittivity $\varepsilon_{r}$ is given by

$$
\varepsilon_{r}=\sqrt{\frac{915.31026-4.0348211 \times 10^{-3} f_{M H z}+7.4342897 \times 10^{-7} f_{M H z}^{2}}{1-9.4530022 \times 10^{-6} f_{M H z}+4.892281 \times 10^{-8} f_{M H z}^{2}}} .
$$

For $f_{M H z}>15454.4$, the conductivity $\sigma$ in $\mathrm{S} / \mathrm{m}$ for wet ground is given by

$$
\begin{aligned}
\sigma= & 0.8756665+4.7236085 \times 10^{-5} f_{M H z}+2.6051966 \times 10^{-8} f_{M H z}^{2} \\
& -9.235936 \times 10^{-13} f_{M H z}^{3}+1.4560078 \times 10^{-17} f_{M H z}^{4} \\
& -1.1129348 \times 10^{-22} f_{M H z}^{5}+3.3253339 \times 10^{-28} f_{M H z}^{6} .
\end{aligned}
$$

For $f_{M H z} \leq 15454.4$, the conductivity $\sigma$ in $\mathrm{S} / \mathrm{m}$ for wet ground is given by

$$
\begin{aligned}
\sigma= & 5.5990969 \times 10^{-3}+8.7798277 \times 10^{-5} f_{M H z}+6.2451017 \times 10^{-8} f_{M H z}^{2} \\
& -7.1317207 \times 10^{-12} f_{M H z}^{3}+4.2515914 \times 10^{-16} f_{M H z}^{4} \\
& -1.240806 \times 10^{-20} f_{M H z}^{5}+1.3854354 \times 10^{-25} f_{M H z}^{6} .
\end{aligned}
$$

For medium dry ground $\left(\right.$ igrnd $_{i}=3$ ), the relative permittivity $\varepsilon_{r}$ is given by 15 for $f_{M H z} \leq 4841.945$. For $f_{M H z}>4841.945$, the relative permittivity $\varepsilon_{r}$ is given by

$$
\varepsilon_{r}=\sqrt{\frac{215.87521-2.6151055 \times 10^{-3} f_{M H z}+1.9484482 \times 10^{-7} f_{M H z}^{2}}{1 .-7.6649237 \times 10^{-5} f_{M H z}+1.2565999 \times 10^{-8} f_{M H z}^{2}}} .
$$

At $f_{M H z} \leq 4946.751$ for medium dry ground, the conductivity $\sigma$ in $\mathrm{S} / \mathrm{m}$ is given by

$$
\begin{aligned}
\sigma= & \left(2.4625032 \times 10^{-2}+1.8254018 \times 10^{-4} f_{M H z}-2.664754 \times 10^{-8} f_{M H z}^{2}\right. \\
& \left.+7.6508732 \times 10^{-12} f_{M H z}^{3}-7.4193268 \times 10^{-16} f_{M H z}^{4}\right)^{2} .
\end{aligned}
$$


For $f_{M H z}>4946.751$, for medium dry ground, the conductivity $\sigma$ in $\mathrm{S} / \mathrm{m}$ is given by

$$
\begin{aligned}
\sigma= & \left(0.17381269+1.2655183 \times 10^{-4} f_{M H z}-1.6790756 \times 10^{-9} f_{M H z}^{2}\right. \\
& \left.+1.1037608 \times 10^{-14} f_{M H z}^{3}-2.9223433 \times 10^{-20} f_{M H z}^{4}\right)^{2} .
\end{aligned}
$$

For very dry ground $\left(\right.$ igrnd $\left._{i}=4\right)$, the relative permittivity $\varepsilon_{r}$ is given by 3 and the conductivity $\sigma$ in $\mathrm{S} / \mathrm{m}$ is 0.0001 for $f_{M H z}<590.8924$. For $590.8924 \leq f_{M H z} \leq 7131.933$, the conductivity $\sigma$ in $\mathrm{S} / \mathrm{m}$ is given by

$$
\begin{aligned}
\sigma= & 2.2953743 \times 10^{-4}-8.1212741 \times 10^{-7} f_{M H z}+1.8045461 \times 10^{-9} f_{M H z}^{2} \\
& -1.960677 \times 10^{-12} f_{M H z}^{3}+1.256959 \times 10^{-15} f_{M H z}^{4}-4.46811 \times 10^{-19} f_{M H z}^{5} \\
& +9.4623158 \times 10^{-23} f_{M H z}^{6}-1.1787443 \times 10^{-26} f_{M H z}^{7}+7.9254217 \times 10^{-31} f_{M H z}^{8} \\
& -2.2088286 \times 10^{-35} f_{M H z}^{9} .
\end{aligned}
$$

For $f_{M H z}>7131.933 \mathrm{MHz}$, the conductivity $\sigma$ in $\mathrm{S} / \mathrm{m}$ is given by

$$
\begin{aligned}
\sigma= & \left(-4.9560275 \times 10^{-2}+2.9876572 \times 10^{-5} f_{M H z}-3.0561848 \times 10^{-10} f_{M H z}^{2}\right. \\
& \left.+1.1131828 \times 10^{-15} f_{M H z}^{3}\right)^{2} .
\end{aligned}
$$

For ice at $-1^{\circ} \mathrm{C}\left(\right.$ igrnd $\left._{i}=5\right)$, the relative permittivity $\varepsilon_{r}$ is 3 for all frequencies, and the conductivity $\sigma$, for $f_{M H z} \leq 300$, is given by

$$
\sigma=\frac{3.8814567 \times 10^{-5}+9.878241 \times 10^{-6} f_{M H z}+7.9902484 \times 10^{-8} f_{M H z}^{2}}{1+8.467523 \times 10^{-2} f_{M H z}-9.736703 \times 10^{-5} f_{M H z}^{2}+3.269059 \times 10^{-7} f_{M H z}^{3}},
$$

and for $f_{M H z}>300$ is given by

$$
\sigma=\frac{1.2434792 \times 10^{-4}+8.680839 \times 10^{-7} f_{M H z}+7.2701689 \times 10^{-11} f_{M H z}^{2}-2.6416983 \times 10^{-14} f_{M H z}^{3}+1.37552 \times 10^{-18} f_{M H z}^{4}}{1+2.824598 \times 10^{-4} f_{M H z}-6.755389 \times 10^{-8} f_{M H z}^{2}+2.8728975 \times 10^{-12} f_{M H z}^{3}-1.8795958 \times 10^{-18} f_{M H z}^{4}} .
$$

For ice at $-10^{\circ} \mathrm{C}\left(\right.$ igrnd $\left._{i}=6\right)$, the relative permittivity $\varepsilon_{r}$ is 3 for all frequencies, and the conductivity $\sigma$, for $f_{M H z} \leq 8753.398$, is given by

$$
\sigma=\frac{1+3.883854 \times 10^{-2} f_{M H z}+6.832108 \times 10^{-5} f_{M H z}^{2}}{51852.543+389.58894 f_{M H z}}
$$


and for $f_{M H z}>8753.398$, is given by

$$
\sigma=4.13105 \times 10^{-5}+2.03589 \times 10^{-7} f_{M H z}-3.1739 \times 10^{-12} f_{M H z}^{2}+4.52331 \times 10^{-17} f_{M H z}^{3} .
$$

For the user-defined ground type (igrnd ${ }_{i}=7$ ), the relative permittivity $\varepsilon_{r}$ and the conductivity $\sigma$ in $\mathrm{S} / \mathrm{m}$ are set equal to the input values dielec $c_{1, i}$ and dielec $_{2, i}$, respectively.

Finally, the complex dielectric constant is given by

$$
n c_{i}^{2}=\varepsilon_{r i}+60 \lambda \sigma_{i} ; \text { for } i=1,2,3, \ldots i_{g r} \text {. }
$$

Table 22 and Table 23 provide identification, description, units of measure, and the computational source for each DIEINIT SU input and output data element.

\begin{tabular}{|c|c|c|c|}
\hline Name & Description & Units & Source \\
\hline dielec & $\begin{array}{l}\text { Two-dimensional array containing the relative permittivity } \\
\text { and conductivity; dielec } c_{1, i} \text { and diele } c_{2, i} \text {, respectively. }\end{array}$ & $\begin{array}{l}\mathrm{N} / \mathrm{A} \\
\mathrm{S} / \mathrm{m}\end{array}$ & Calling CSCI \\
\hline$f_{M H z}$ & Frequency & $\mathrm{MHz}$ & APM_MOD \\
\hline$i_{g r}$ & Number of different ground types specified & N/A & Calling CSCI \\
\hline igrnd & 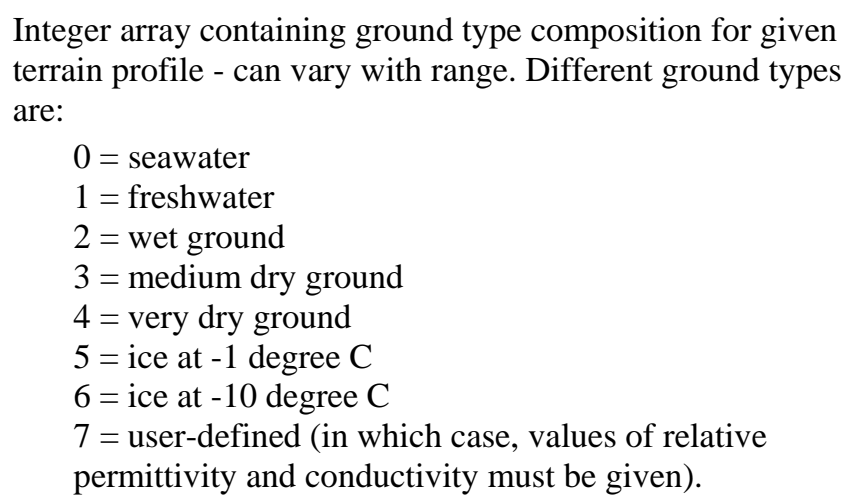 & N/A & Calling CSCI \\
\hline$\lambda$ & Wavelength & meters & APMINIT CSC \\
\hline rgrnd & $\begin{array}{l}\text { Array containing ranges at which varying ground types } \\
\text { apply. }\end{array}$ & meters & Calling CSCI \\
\hline
\end{tabular}

Table 22. DIEINIT SU input data element requirements.

Table 23. DIEINIT SU output data element requirements.

\begin{tabular}{c|c|c}
\hline Name & Description & Units \\
\hline$n c^{2}$ & Array of complex dielectric constants & N/A \\
\hline
\end{tabular}




\subsubsection{FFT Parameters (FFTPAR) SU}

The FFTPAR SU determines the required transform size based on the maximum PE propagation angle and the maximum height needed for desired coverage. If running in full or partial hybrid modes, the maximum height is the height necessary to encompass at least $20 \%$ above the maximum terrain peak along the path or the highest trapping layer specified in the environment profiles, whichever is greater. If running in a PE-only mode, the maximum height is the specified maximum output height.

For computational efficiency reasons, an artificial upper boundary is established for the PE solution. To prevent upward propagating energy from being "reflected" downward from this boundary and contaminating the PE solution, the PE solution field strength is attenuated or "filtered" above a certain height to ensure that the field strength just above this boundary is reduced to zero. The bin width in z-space $\Delta z_{P E}$ is found from

$$
\Delta Z_{P E}=\frac{\lambda}{2 \operatorname{SIN}\left(\Theta_{\max }\right)},
$$

where $\lambda$ is the wavelength in meters and $\Theta_{\max }$ is the maximum propagation angle in radians.

The flag, $i_{\text {flag }}$, is used to determine maximum FFT size based on a given $\Theta_{\max }$ and desired coverage height $z_{\text {lim }}$ or it will determine $z_{\text {lim }}$ based on a given $\Theta_{\max }$ and FFT size.

For $i_{\text {fla } g}=0$, the constants $l n_{f f t}, n_{f f t}$, and $z_{\max }$ are found from $l n_{\min }$ as follows,

$$
\begin{aligned}
\ln _{f f t} & =\ln _{\text {min }}, \\
n_{f f t} & =2^{l n_{f f t}}, \\
z_{\text {max }} & =n_{f f t} \Delta z_{P E},
\end{aligned}
$$

where $l n_{\min }$ is the minimum power of two transform size. $l n_{\min }$ is initialized to 10 and for every $5^{\circ}$ in $\Theta_{\max }$ is increased by 1 . Next, the transform size needed to perform calculations to a test height $z_{t}$ is determined. First, $z_{t}$ is set equal to $z_{l i m}$ minus a small height precision tolerance. Then a DO WHILE loop is executed as long as the condition $3 / 4 Z_{\max }<z_{t}$ is satisfied. Within this DO WHILE loop $z_{\max }$ is found from

$$
\begin{aligned}
\ln _{f f t} & =\ln _{f f t}+1, \\
n_{f f t} & =2^{l n_{f f t}}, \\
z_{m a z} & =n_{f f t} \Delta z_{P E} .
\end{aligned}
$$

If $\ln$ fft reaches the value of 30 , then the SU is exited with a non-zero error code. 
For the case where $i_{\text {flag }}=1$, no iteration needs to be performed. $z_{\text {lim }}$ is determined by a given $\ln _{\text {fft }}$ and $\Theta_{\max }$ from equations shown above.

Upon exiting, $z_{\text {lim }}$ is computed as $3 / 4 Z_{\max }$.

Table 24 and Table 25 provide identification, description, units of measure, and the computational source for each FFTPAR SU input and output data element.

Table 24. FFTPAR SU input data element requirements.

\begin{tabular}{c|l|c|c}
\hline Name & \multicolumn{1}{|c|}{ Description } & Units & Source \\
\hline$i_{f l a g}$ & $\begin{array}{l}\text { Flag indicating whether to determine maximum FFT size } \\
n_{\text {fft }} \text { based on given } \Theta_{\max } \text { and } z_{\text {lim }} \text { or determine } z_{\text {lim }} \text { based on } \\
\text { given } \Theta_{\max } \text { and FFT size } n_{f f \cdot}\end{array}$ & N/A & APMINIT CSC \\
$\lambda$ & Wavelength & meters & APMINIT SU \\
$l n_{\min }$ & Minimum power of 2 transform size & N/A & APMINIT SU \\
$\Theta_{\max }$ & Maximum propagation angle in PE calculations & radians & APMINIT CSC \\
$z_{\text {lim }}$ & Maximum height region where PE solution is valid & meters & GETTHMAX SU \\
& & & APMINIT CSC \\
\end{tabular}

Table 25. FFTPAR SU output data element requirements.

\begin{tabular}{c|l|c}
\hline Name & \multicolumn{1}{|c}{ Description } & Units \\
\hline$\Delta z_{P E}$ & Bin width in z space & meters \\
$i_{e r r}$ & Error code & N/A \\
$l_{f f t}$ & Power of 2 transform size, i.e. $n_{f f t}=2 n_{f f t}$ & N/A \\
$n_{f f t}$ & Transform size & N/A \\
$z_{l i m}$ & Maximum height region where PE solution is valid & meters \\
$z_{\max }$ & Total height of the FFT/PE calculation domain & meters \\
\hline
\end{tabular}

\subsubsection{Fill Height Arrays (FILLHT) SU}

The FILLHT SU calculates the effective earth radius for an initial launch angle of $5^{\circ}$ and to fill an array with height values at each output range of the limiting sub-model, depending on which mode is used. If running in a full hybrid mode, the array contains height values at each output range, separating the PE from the RO region. If running in partial hybrid or PE-only modes, the array contains those height values at each output range at which the initial launch angle has been traced to the ground or surface. These height values represent the separating region where, above that height, valid loss is computed, and below that height, no loss is computed so that only loss values that fall within a valid calculation region are output. 
For the case when $i_{\text {hybrid }}=1$ (full hybrid mode), all height values at each output range separating the $\mathrm{FE}$ region from the $\mathrm{RO}$ region are determined and stored in array $h t f e$. For ranges greater than $2.5 \mathrm{~km}$, the ray defined by a $5^{\circ}$ elevation angle is traced up to the maximum height $h t_{\text {lim }}$. The ray is "traced" by simple geometry at every output range and the height array htfe is determined as follows. The temporary variable $y_{a r}$ is found from

$$
y_{a r}=y_{\text {fref }}-a n t_{r e f},
$$

where the parameter $y_{\text {fref }}$ is the ground elevation height at the source, and $a n t_{\text {ref }}$ is the transmitting antenna height relative to the height $h_{\text {minter }}$. The values of $h t f e_{i}$ are then determined by

$$
\begin{aligned}
& h t f e_{i}=y_{\text {fref }} ; \quad \text { for } \text { rngout }_{i} \leq r_{\text {tst }} \\
& h t f e_{i}=\operatorname{MIN}\left(h t_{\text {lim }}, \operatorname{MAX}\left\{y_{\text {fref }}, y_{\text {ar }}+t_{5} \text { rngout }_{i}\right\}\right) ; \text { for } \text { rngout }_{i}>r_{\text {tst }}, i=1,2, \ldots n_{\text {rout }}
\end{aligned}
$$

where $r_{\text {tst }}$ is a constant range of $2.5 \mathrm{~km}, t_{5}$ is the tangent of $5^{\circ}$, and rngout $t_{i}$ is the output range at every $i^{\text {th }}$ range step.

For the airborne hybrid model $\left(i_{\text {hybrid }}=0\right)$, the TRACE_ROUT SU is referenced to determine the heights at every output range separating the upper FE region from the PE region. These heights are stored in array hlim. The heights separating the lower FE region from the PE region are stored in array htfe as outlined below.

For partial hybrid (PE plus XO) or airborne modes, the initial launch angle is traced until it hits the surface, storing heights traced at each output range.

First, several variables are initialized. The angle at the start of the trace, $a_{0}$, is set to $-a_{\text {launch }}$ (determined in the GETTHMAX SU); the initial range, $r_{0}$, is set equal to zero; and the height at the start of the ray trace step, $h_{0}$, is set equal to ant $t_{r e}$. The index, $l$, indicating the location of the source height in array htdum, is set equal to the index $i_{\text {start }}$. The terrain elevation at the current range, $t y_{r}$, is initialized to 0 , and the index $j$ is set equal to one.

The following steps (1 through 2 ) are performed until the ray has reached the surface or the ray has been traced to $r_{\max }$, whichever comes first.

1. The output range to trace to $r_{o}$ is initialized to rngout $t_{j}$, and $h t f e_{j}$ is initialized to 0 . 
2. The TRACE_STEP SU is accessed to trace the ray to the next range step, which is incremented by $\Delta r_{P E}$. The height at that range is then stored in array $h t f e$. The index $j$ is incremented by one, and if the ray has not reached the surface or $r_{\max }$, then steps 1 and 2 are repeated.

Once the ray trace is completed, the index $j$ is decremented by one and $h t f e_{j}$ is set equal to $h m_{\text {ref }}$ for all remaining output range steps $j$ through $n_{\text {rout }}$.

Table 26 and Table 27 provide identification, description, units of measure, and the computational source for each FILLHT SU input and output data element.

Table 26. FILLHT SU input data element requirements.

\begin{tabular}{|c|c|c|c|}
\hline Name & Description & Units & Source \\
\hline$a_{\text {launch }}$ & $\begin{array}{l}\text { Launch angle used which, when traced, separates the PE } \\
\text { and XO regions from the RO region }\end{array}$ & radians & GETTHMAX SU \\
\hline$a n t_{r e f}$ & $\begin{array}{l}\text { Transmitting antenna height relative to the reference } \\
\text { height } h_{\text {minter }}\end{array}$ & meters & TERINIT SU \\
\hline$\Delta r_{P E}$ & PE range step & meters & PEINIT SU \\
\hline$f_{\text {ter }}$ & $\begin{array}{l}\text { Logical flag indicating if terrain profile has been } \\
\text { specified: } \\
\text { '.true.' = terrain profile specified } \\
\text { '.false.' = terrain profile not specified }\end{array}$ & N/A & APMINIT CSC \\
\hline grdum & M-unit gradient array & $\begin{array}{l}\text { (M-unit/ } \\
\text { meter) }\end{array}$ & REFINIT SU \\
\hline hmref & Height relative to $h_{\text {minter }}$ & meters & TERINIT SU \\
\hline htdum & Height array for current interpolated profile & meters & REFINIT SU \\
\hline$h t_{l i m}$ & $\begin{array}{l}\text { User-supplied maximum height relative to } h_{\text {minter }} \text {, i.e., } \\
h t_{\text {lim }}=h_{\text {max }}-h_{\text {minter }}\end{array}$ & meters & TERINIT SU \\
\hline$i_{\text {hybrid }}$ & $\begin{array}{l}\text { Integer indicating which sub-models will be used: } \\
\begin{aligned} 0 & =(\text { FEDR }+ \text { PE }) \text { model } \\
1 & =\text { full hybrid model }(\mathrm{PE}+\mathrm{FE}+\mathrm{RO}+\mathrm{XO}) \\
2 & =\text { partial hybrid model }(\mathrm{PE}+\mathrm{XO})\end{aligned}\end{array}$ & N/A & APMINIT CSC \\
\hline$i_{\text {start1 }}$ & Refractivity level index within $h t d u m$ at $a n t_{r e f}$ & N/A & REFINIT SU \\
\hline lvlep & Number of refractivity levels in profile htdum, refdum & N/A & REFINIT SU \\
\hline$n_{\text {rout }}$ & Integer number of the output range points desired & N/A & Calling CSCI \\
\hline rngout & Array containing all output ranges & meters & APMINIT CSC \\
\hline$r_{t s t}$ & Range set at $2.5 \mathrm{~km}$ to begin calculation of RO values & meters & APM_MOD \\
\hline$\Theta_{75}$ & $75 \%$ of maximum propagation angle in PE calculations & radians & APMINIT CSC \\
\hline$t x$ & Range points of terrain profile & meters & TERINIT SU \\
\hline$y_{\text {fref }}$ & Ground elevation height at the source & meters & APMINIT CSC \\
\hline
\end{tabular}


Table 27. FILLHT SU output data element requirements.

\begin{tabular}{c|l|c}
\hline Name & \multicolumn{1}{|c|}{ Description } & Units \\
\hline hlim & $\begin{array}{l}\text { Array containing the height at each output range separating the RO region from the } \\
\text { PE (at close ranges) and XO (at far ranges) regions }\end{array}$ & meters \\
$h t f e$ & $\begin{array}{l}\text { Array of height values at each output range separating the PE region from the RO } \\
\text { region }\end{array}$ & meters \\
\hline
\end{tabular}

\subsubsection{Gaseous Absorption (GASABS) SU}

The GASABS SU computes the specific attenuation based on air temperature and absolute humidity. This SU is based on CCIR (International Telecommunication Union, International Radio Consultative Committee, now the ITU-R) Recommendation 676-1, “Attenuation by Atmospheric Gases in the Frequency Range 1-350 GHz.”

The oxygen absorption for $15^{\circ} \mathrm{C}$ air temperature is computed from

$$
\gamma_{o}=10^{-3}\left(t_{1}+t_{2}+0.00719\right)\left(\frac{f_{M H z}}{1000}\right)^{2}
$$

where $f_{M H z}$ is the frequency in $\mathrm{MHz}$ and the temporary variables $t_{1}$ and $t_{2}$ are given by

$$
\begin{aligned}
& t_{1}=\frac{6.09}{\left(\frac{f_{M H z}}{1000 .}\right)^{2}+0.227}, \\
& t_{2}=\frac{4.81}{\left(\frac{f_{M H z}}{1000 .}-57.0\right)^{2}+1.50} .
\end{aligned}
$$

A correction is made for the oxygen absorption for the actual air temperature, which is given by

$$
\gamma_{o}=\left(1.0+0.01\left\{t_{\text {air }}-15.0\right\}\right) \gamma_{o},
$$

where $t_{\text {air }}$ is the surface air temperature in degrees Centigrade. 
The water vapor absorption is computed from

$$
\gamma_{w}=\frac{\left(0.05+0.0021 a b s_{\text {hum }}+t_{1}+t_{2}+t_{3}\right)\left(\frac{f_{M H z}}{1000 .}\right)^{2} a b s_{\text {hum }}}{10000.0},
$$

where the temporary variables $t_{1}, t_{2}$, and $t_{3}$ are given respectively by

$$
\begin{gathered}
t_{1}=\frac{3.6}{\left(\frac{f_{M H z}}{1000}-22.2\right)^{2}+8.5}, \\
t_{2}=\frac{10.6}{\left(\frac{f_{M H z}}{1000}-183.3\right)^{2}+9.0},
\end{gathered}
$$

and

$$
t_{3}=\frac{8.9}{\left(\frac{f_{M H z}}{1000 .}-325.4\right)^{2}+26.3} .
$$

The total specific absorption for sea-level air in $\mathrm{dB} / \mathrm{km}$ multiplied by a conversion factor to convert to $\mathrm{dB} / \mathrm{m}$ is given by

$$
\operatorname{gas}_{a t t}=\left(\gamma_{o}+\gamma_{w}\right) 10^{-3} \text {. }
$$

Table 28 and Table 29 provide identification, description, units of measure, and the computational source for each GASABS SU input and output data element.

Table 28. GASABS SU input data element requirements.

\begin{tabular}{c|l|c|l}
\hline Name & \multicolumn{1}{|c|}{ Description } & Units & \multicolumn{1}{c}{ Source } \\
\hline$a b s_{\text {hum }}$ & Absolute humidity near the surface & $\mathrm{g} / \mathrm{meter}^{3}$ & Calling CSCI \\
$f_{\mathrm{MHz}}$ & Frequency & $\mathrm{MHz}$ & Calling CSCI \\
$t_{a i r}$ & Air temperature near the surface & ${ }^{\circ} \mathrm{C}$ & Calling CSCI \\
\hline
\end{tabular}


Table 29. GASABS SU output data requirements.

\begin{tabular}{c|l|l}
\hline Name & Description & Units \\
\hline gas $_{\text {att }}$ & Gaseous absorption & $\mathrm{dB} / \mathrm{m}$ \\
\hline
\end{tabular}

\subsubsection{Get Effective Earth Radius Factor (GET_K) SU}

The GET_K SU computes the effective earth radius factor and the effective earth radius. The computation is made for a launch angle of $5^{\circ}$ if the $\mathrm{SU}$ is called from the APMINIT CSC. If called from the TROPOINIT SU, then the computation is made for a launch angle equal to the critical angle.

Upon entering the SU, internal one-line ray trace functions are defined as

$$
\begin{aligned}
\operatorname{RADA} 1(a, b) & =a^{2}+2 g_{r d} b, \\
\operatorname{RP}(a, b) & =a+\frac{b}{g_{r d}},
\end{aligned}
$$

for general parameters $a, b$, and refractivity gradient $g_{r d}$.

The starting launch angle $a_{\text {start }}$ for tracing a ray to determine the effective earth radius is initialized to the critical angle $a_{\text {crit }}$.

If the SU is called from the APMINIT CSC $\left(i_{\text {org }}=0\right)$, then $a_{\text {start }}$ is re-initialized to $5^{\circ}$. If running the airborne model then the beamwidth and antenna elevation angle are taken into account and the starting angle is initialized according to

$$
a_{\text {start }}=\mathbf{M I N}\left[\mathbf{M A X}\left(a_{\text {start }}, \mu_{b w r}+\mu_{\text {lim }}\right), 10^{\circ}\right],
$$

where

$$
\mu_{\text {lim }}=\mathbf{M I N}\left(10^{\circ},\left|\mu_{o r}\right|\right)
$$

If a terrain profile has been specified ( $f_{\text {ter }}=$ '.true.'), then $a_{\text {start }}$ is set equal to

$$
a_{\text {start }}=\operatorname{MIN}\left(1.5 a_{\text {start }}, 10^{\circ}\right) \text {. }
$$

If using the airborne or full hybrid modes, or if calling from the TROPOINIT SU, then perform steps 1 through 4 to compute the effective earth radius from the antenna height up to height $h t_{\text {lim }}$. 
1. The propagation angle, range, and height at the start of the ray trace step are initialized to $a_{\text {start }}, 0$, and $a n t_{r e f}$, respectively. The current refractivity level $i$ is also initialized to $i_{\text {start } 1}$.

2. Steps 2a through 2c are performed for an upward ray until it has reached the last height in the refractivity level or $h t_{\text {lim }}$, whichever comes first.

a. The gradient $g_{r d}$ is set equal to $\operatorname{grdum}_{i}$. The propagation angle $a_{1}$ and range $r_{1}$ at the end of the trace step are computed as

$$
\begin{aligned}
a_{1} & =\sqrt{\mathbf{R A D A} 1\left(a_{0}, \text { htdum }_{i+1}-h_{0}\right)}, \\
r_{1} & =\mathbf{R P}\left(r_{0}, a_{1}-a_{0}\right) .
\end{aligned}
$$

b. $a_{0}, r_{0}$, and $h_{0}$ are now set equal to the values of $a_{1}, r_{1}$ and $h t d u m_{i+1}$, respectively. If $h_{0}$ is greater than $h t_{\text {lim }}$, then the integer flag $i_{\text {flag }}$ is set equal to 1 , and the propagation angle $a_{1}$ at $h t_{\text {lim }}$ is computed as

$$
a_{1}=\sqrt{\mathbf{R A D A} 1\left(a_{0}, h t_{\text {lim }}-h \text { ddum }_{i}\right)} .
$$

A temporary maximum propagation angle $\Theta_{75 a}$ is then set equal to $a_{1}$.

c. The current refractivity level $i$ is incremented by 1 . If one of the conditions in step 2 has been met, then the SU proceeds to step 3; otherwise, steps 2a through 2c are repeated.

3. If $h_{0}$ is less than $h t_{\text {lim }}$ and $i_{\text {flag }}$ is equal to 0 , then propagation angle $a_{1}$ at $h t_{\text {lim }}$ is recomputed as

$$
a_{1}=\sqrt{\operatorname{RADA} 1\left(a_{0}, h t_{l i m}-h_{0}\right)}
$$

and the variable $\Theta_{75 a}$ is then set equal to $a_{1}$.

4. The propagation angle and range $a_{1}$ and $r_{1}$ are now re-computed from

$$
\begin{aligned}
& a_{1}=\sqrt{\operatorname{RADA} 1\left(a_{0}, \text { htdum }_{\text {lvlep }}-h_{0}\right)}, \\
& r_{1}=\operatorname{RP}\left(r_{0}, a_{1}-a_{0}\right),
\end{aligned}
$$


and the effective earth radius $a_{e k}$, the effective earth radius factor $e_{k}$, and twice the effective earth radius, twoka are given by

$$
\begin{aligned}
& a_{e k}=\frac{r_{1}}{a_{1}-a_{\text {start }}}, \\
& \text { twoka }=2 a_{e k}, \\
& e_{k}=6.37 \times 10^{-6} a_{e k} .
\end{aligned}
$$

If using the airborne hybrid model and the calling SU is the APMINIT CSC, then twice the effective earth radius factor is computed for a downward ray where the initial launch angle is - $a_{\text {start. }}$ Steps 1 through $2 \mathrm{a}$ are repeated with $a_{1}$ negative, $i$ decremented by 1 , and htdum $_{i+1}$ replaced with tdum $_{i}$. Finally, the variable twoka $a_{\text {down }}$ is computed from

$$
\text { twok }_{\text {down }}=\frac{2 r_{1}}{a_{1}+a_{\text {start }}},
$$

and $\Theta_{75}$ is determined from

$$
\Theta_{75}=\mathbf{M A X}\left(\Theta_{75 a}, a_{\text {start }}\right)
$$

\begin{tabular}{|c|c|c|c|}
\hline Name & Description & Units & Source \\
\hline$a_{\text {crit }}$ & Critical angle (angle above which no rays are trapped) & radians & REFINIT SU \\
\hline$a_{\text {launch }}$ & $\begin{array}{l}\text { Launch angle used which, when traced, separates the PE } \\
\text { and XO regions from the RO region }\end{array}$ & radians & GETTHMAX SU \\
\hline$a^{a n t_{\text {ref }}}$ & $\begin{array}{l}\text { Transmitting antenna height relative to the reference } \\
\text { height } h_{\text {minter }}\end{array}$ & meters & TERINIT SU \\
\hline$\mu_{b w r}$ & Antenna vertical beam width & radians & APMINIT CSC \\
\hline$\mu_{o r}$ & Antenna pattern elevation angle & radians & APMINIT CSC \\
\hline$f_{\text {ter }}$ & $\begin{array}{l}\text { Logical flag indicating if terrain profile has been } \\
\text { specified: } \\
\text { ‘.true.' = terrain profile specified } \\
\text { '.false.' = terrain profile not specified }\end{array}$ & N/A & APMINIT CSC \\
\hline grdum & M-unit gradient array & $\begin{array}{c}\text { (M-unit/ } \\
\text { meter) }\end{array}$ & REFINIT SU \\
\hline hmref & Height relative to $h_{\text {minter }}$ & meters & TERINIT SU \\
\hline htdum & Height array for current interpolated profile & meters & REFINIT SU \\
\hline$h t_{l i m}$ & $\begin{array}{l}\text { User-supplied maximum height relative to } h_{\text {minter }} \text {, i.e., } \\
h t_{\text {lim }}=h_{\text {max }}-h_{\text {minter }}\end{array}$ & meters & TERINIT SU \\
\hline
\end{tabular}

Table 30 and Table 31 provide identification, description, units of measure, and show the computational source for each input and output data element, respectively, of the GET_K SU.

Table 30. GET_K SU input data element requirements. 
Table 30. GET_K SU input data element requirements. (continued)

\begin{tabular}{|c|c|c|c|}
\hline Name & Description & Units & Source \\
\hline$i_{\text {hybrid }}$ & $\begin{array}{l}\text { Integer indicating which sub-models will be used: } \\
\begin{aligned} 0 & =(\text { FEDR }+\mathrm{PE}) \text { model } \\
1 & =\text { full hybrid model }(\mathrm{PE}+\mathrm{FE}+\mathrm{RO}+\mathrm{XO}) \\
2 & =\text { partial hybrid model }(\mathrm{PE}+\mathrm{XO})\end{aligned}\end{array}$ & N/A & APMINIT CSC \\
\hline$i_{\text {org }}$ & $\begin{array}{l}\text { Integer flag indicating origin of calling SU } \\
0=\text { called from APMINIT CSC } \\
1=\text { called from TROPOINIT SU }\end{array}$ & N/A & $\begin{array}{l}\text { APMINIT CSC } \\
\text { TROPOINIT SU }\end{array}$ \\
\hline$i_{\text {start1 }}$ & Refractivity level index within $h t d u m$ at $a n t_{r e f}$ & N/A & REFINIT SU \\
\hline lvlep & Number of refractivity levels in profile htdum, refdum & N/A & REFINIT SU \\
\hline$y_{\text {fref }}$ & Ground elevation height at the source & meters & APMINIT CSC \\
\hline
\end{tabular}

Table 31. GET_K SU output data element requirements.

\begin{tabular}{c|l|c}
\hline Name & \multicolumn{1}{|c|}{ Description } & Units \\
\hline$a_{e k}$ & Effective earth radius & meters \\
$e_{k}$ & Effective earth radius factor & N/A \\
$\Theta_{75}$ & $75 \%$ of maximum propagation angle in PE calculations & radians \\
$t w o k a$ & Twice the effective earth radius & meters \\
$t w o k a_{\text {down }}$ & Twice the effective earth radius for downward path & meters \\
\hline
\end{tabular}

\subsubsection{Get Alpha Impedance (GETALN) SU}

The GETALN SU computes the surface impedance term in the Leontovich boundary condition and the complex index of refraction for finite conductivity. The implementation of these impedance formulas follow Kuttler and Dockery's method (1991).

Upon entering the $\mathrm{SU}$, the smooth surface impedance term $\alpha$ is computed from the complex dielectric constant $n c^{2}$ and free space wavenumber $k_{o}$, for both vertical and horizontal polarization, by

$$
\begin{aligned}
& \alpha_{h}=i k_{o} \sqrt{n c_{i_{g}}^{2}-1}, \\
& \alpha_{v}=i k_{o} \frac{\sqrt{n c_{i_{g}}^{2}-1}}{n c_{i_{g}}^{2}}
\end{aligned}
$$

where $I$ is the imaginary number $\sqrt{-1}$. 
If a frequency less than $50 \mathrm{MHz}$ has been specified ( $H F_{\text {flag }}=$ '.true.'), then the SURFIMP SU is referenced to compute the effective surface impedance, $\zeta$, over seawater. The surface impedance term for vertical polarization is then computed as

$$
\alpha_{v}=i k_{o} \zeta
$$

over seawater, and for those portions of the path over land, it is computed as

$$
\begin{aligned}
& \alpha_{h, v}^{\prime}=i k_{o} \operatorname{SIN} \psi \frac{1-\Gamma_{h, v}}{1+\Gamma_{h, v}} \\
& \alpha_{h, v}=\operatorname{COS}(\beta)\left(\alpha_{h, v}^{\prime}+\operatorname{slp}\left(1 / \operatorname{Cos}(\beta)^{-\operatorname{COS} \psi))}\right.\right.
\end{aligned}
$$

where

$$
\beta=\mathbf{T A N}^{-1}(\operatorname{slp})
$$

and slp is the slope of the terrain segment at the current range. For a non-zero grazing angle the rough surface reflection coefficient, $\Gamma$, is determined from referencing the GETREFCOEF SU.

For frequencies above $50 \mathrm{MHz}$, and if rough surface calculations are required (ruf $=$ '.true.'), then if a non-zero grazing angle $\psi$ exists for the current range step, the surface impedance is computed as

$$
\alpha_{h, v}=i k_{o} \operatorname{SIN} \psi \frac{1-\Gamma_{h, v}}{1+\Gamma_{h, v}}
$$

where the subscripts $h, v$ indicate horizontal and vertical polarization quantities, respectively.

If using the central difference algorithm $\left(i_{a l g}=1\right)$, follow steps 1 through 2 to compute constants and variables for subsequent use in the MIXEDFT SU.

1. The determination of the complex root, $R_{T}$, of the quadratic equation for the mixed transform method is based on Kuttler's formulation:

$$
\begin{aligned}
& R_{T}=-\sqrt{1.0+\left(\alpha_{h} \Delta z_{P E}\right)^{2}}-\alpha_{h} \Delta z_{P E} \text { for horizontal polarization, } \\
& R_{T}=\sqrt{1.0+\left(\alpha_{v} \Delta z_{P E}\right)^{2}}-\alpha_{v} \Delta z_{P E} \text { for vertical polarization }
\end{aligned}
$$


2. Next, the array $r n$ is determined according to

$$
r n_{i}=R_{T}^{i} . \quad I=1,2, \ldots n_{f f t}
$$

Several parameters used in the central difference algorithm are now computed:

$$
\begin{gathered}
R_{k}=\frac{2\left(1-r n_{2}\right)}{\left(1+r n_{2}\right)\left(1-r n_{n_{f f t}}^{2}\right)}, \\
C_{1 x}=e^{i \Delta r_{P E}\left(\sqrt{k_{o}^{2}+\left(\frac{\mathbf{L N}\left(R_{T}\right)}{\Delta z_{P E}}\right)^{2}}-k_{o}\right)}, \\
C_{2 x}=e^{i \Delta r_{P E}\left(\sqrt{k_{o}^{2}+\left(\frac{\mathbf{L N}\left(R_{T}\right)-i \pi}{\Delta z_{P E}}\right)^{2}}-k_{o}\right)} .
\end{gathered}
$$

If using the backward difference algorithm $\left(i_{a l g}=2\right), R_{T}$ is computed as

$$
R_{T}=\frac{1}{\left(1+\alpha_{h, v} \Delta z_{P E}\right)},
$$

the array $r n$ is computed as in step 2 above, and the parameter $c m f t_{x}$ is computed using the same equation for $C_{1 x}$ in step 2 above.

Table 32 and Table 33 provide identification, description, units of measure, and the computational source for each GETALN SU input and output data element.

Table 32. GETALN SU input data element requirements.

\begin{tabular}{c|l|c|c}
\hline Name & \multicolumn{1}{|c|}{ Description } & Units & \multicolumn{1}{|c}{ Source } \\
\hline$\Delta r_{P E}$ & PE range step & meters & PEINIT SU \\
$\Delta z_{P E}$ & Bin width in z space & meters & FFTPAR SU \\
$f_{M H z}$ & Frequency & $\mathrm{MHz}$ & APM_MOD \\
$f t e r$ & Logical flag indicating if terrain profile has been & N/A & APMINIT CSC \\
& specified: & & \\
& '.true.' = terrain profile specified & & \\
& '.false.' = terrain profile not specified & & \\
HFflag & HF computation flag indicating the frequency specified & N/A & APMINIT CSC \\
& is less than 50 MHz & &
\end{tabular}


Table 32. GETALN SU input data element requirements. (continued)

\begin{tabular}{|c|c|c|c|}
\hline Name & Description & Units & Source \\
\hline$i_{\text {alg }}$ & $\begin{array}{l}\text { Integer flag indicating which DMFT algorithm is being } \\
\text { used: } \\
\qquad \begin{array}{l}0=\text { no DMFT algorithm will be used } \\
1=\text { use central difference algorithm } \\
2=\text { use backward difference algorithm }\end{array}\end{array}$ & N/A & APMINIT CSC \\
\hline$i_{g}$ & Counter indicating current ground type being modeled & N/A & $\begin{array}{c}\text { APMINIT CSC } \\
\text { PESTEP SU }\end{array}$ \\
\hline$i_{p o l}$ & 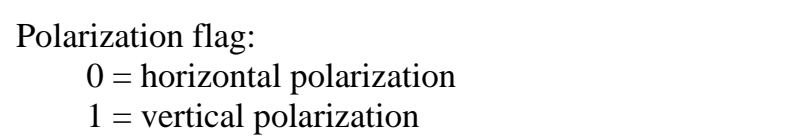 & N/A & Calling CSCI \\
\hline$k_{o}$ & Free-space wavenumber & meters $^{-1}$ & APMINIT CSC \\
\hline$n c^{2}$ & Array of complex dielectric constants & N/A & DIEINIT SU \\
\hline$n_{f f t}$ & Transform size & N/A & FFTPAR SU \\
\hline$\psi$ & Grazing angle & radians & Calling SU \\
\hline$r$ & Current calculation range & meters & Calling SU \\
\hline rngwind & $\begin{array}{l}\text { Ranges of wind speeds entered: } r n g w i n d_{i}=\text { range of } \\
i^{\text {th }} \text { wind speed }\end{array}$ & meters & Calling CSCI \\
\hline ruf & $\begin{array}{l}\text { Logical flag indicating if rough sea surface } \\
\text { calculations are required } \\
\text { 'true.' = perform rough sea surface calculations } \\
\text { '.false.' = do not perform rough sea surface } \\
\text { calculations }\end{array}$ & N/A & APMINIT CSC \\
\hline$s l p$ & Slope of the terrain segment at the current range. & N/A & TERINIT SU \\
\hline wind & Array of wind speeds & $\begin{array}{l}\text { meters/ } \\
\text { sec }\end{array}$ & Calling CSCI \\
\hline
\end{tabular}

Table 33. GETALN SU output data element requirements.

\begin{tabular}{c|l|c}
\hline Name & \multicolumn{1}{|c}{ Description } & Units \\
\hline$\alpha_{h, v}$ & Surface impedance term for horizontal and vertical polarization & N/A \\
$C_{1 x}$ & Constant used to propagate $c_{k 1}$ by one range step in central difference algorithm & N/A \\
$C_{2 x}$ & Constant used to propagate $c_{k 2}$ by one range step in central difference algorithm & N/A \\
$c m f t_{x}$ & Constant used to propagate $c m f t$ by one range step in backward difference & N/A \\
& algorithm & \\
$R_{k}$ & Coefficient used in $c_{k 1}$ and $c_{k 2}$ calculations. & N/A \\
$r n$ & Array of $R_{T}$ to the $i^{\text {th }}$ power (e.g., $r n_{i}=R_{T}^{i}$ ) & N/A \\
$R T$ & Complex root of quadratic equation for mixed transform method based on & N/A \\
& Kuttler's formulation & \\
\hline
\end{tabular}




\subsubsection{Get Angles (GETANGLES) SU}

The GETANGLES SU computes grazing angles at each PE range step via ray trace and spectral estimation for subsequent use in rough sea surface calculations. This SU is referenced only if $l_{\text {graze }}$ is '.true.'. This flag is set to '.true.' for any number of conditions that require grazing angle calculations, such as rough surface, HF frequency has been specified, clutter calculations are desired, and if lang is specified as '.true.'.

Upon entering the SU, the RGTRACE SU is referenced to determine the grazing angles $\psi_{\text {ray }}$ from ray trace.

If a terrain profile has been specified ( $f_{\text {ter }}=$ '.true.') and a surface-based duct has been specified ( $l_{\text {duct }}=$ '.true.') then the grazing angles $\psi_{P E}$ are computed from spectral estimation of the near-surface PE field by running the PE algorithm out to the maximum range $r_{\max }$, assuming horizontal polarization and smooth surface conditions (i.e., no wind speed).

First, the array $\psi_{P E}$ is allocated for size of $i_{P E}$ - equal to the number of PE range steps required to propagate the field out to $r_{\max }$. The array is initialized to zero, with the first element initialized to $\pi / 2$ radians. If the propagation angles and factors are to be computed (lang = '.true.') then the appropriate arrays are allocated and initialized. The TRACE_ROUT SU is then referenced to trace one ray at the maximum calculation angle. The current PE range $r$ and PE integer step $i_{P E s t p}$ are then set equal to zero, with the terrain height $y_{\text {last }}$ at the previous range step set equal to $t y h_{0}$ if a terrain profile has been specified, or 0 otherwise. An iterative DO WHILE loop is then begun to advance the PE solution such that for the current PE range, a PE solution is calculated from the solution at the previous PE range. This iterative procedure is repeated in the DO WHILE loop until $r$ is greater than $r_{\max }$. The following steps (1 through 5) are performed for each PE range step within the DO WHILE loop.

1. The current PE calculation range $r$ is incremented by one PE range step, $\Delta r_{P E}$ and the PE range step counter $i_{P E s t p}$ is incremented by 1 . The range at which interpolation for range-dependent refractivity profiles is performed, $r_{\text {mid }}$, is also incremented by onehalf the PE range step.

2. If performing a terrain case ( $f_{\text {ter }}=$ '.true.'), the ground heights, $y_{\text {cur }}$ and $y_{\text {curm }}$, at range $r$ and $r_{\text {mid }}$, respectively, are determined according to

$$
\begin{aligned}
& y_{\text {cur }}=t y h_{i_{\text {DESp }}}, \\
& y_{\text {curm }}=1 / 2\left(t y h_{i_{\text {PESp }}-1}+y_{\text {cur }}\right) .
\end{aligned}
$$

If $y_{\text {cur }}$ is less than $y_{\text {curm }}$, the DOSHIFT SU is referenced to adjust the PE field relative to the terrain height. 
The PE field array $U$ is now propagated in free space one range step by referencing the FRSTP SU.

If the APM CSCI is used in a range-dependent mode (i.e., the number of profiles $n_{\text {prof }}$ is greater than 1), or a terrain profile is specified, the REFINTER SU is referenced to compute a new modified refractive index profile, profint, adjusted by the local ground height $y_{\text {curm }}$ at range $r_{\text {mid. }}$. A new environmental phase array, envpr, based on this new refractivity profile is then computed from

$$
\begin{gathered}
\text { envpr }_{j}=e^{i \Delta r_{P E} \text { profint }_{j}} ; j=1,2, \ldots n_{f f t} \\
\text { envpr }_{j}=\text { filt }_{j-n_{34}} \text { envpr }_{j} ; j=n_{34}, n_{34}+1, n_{34}+2, \ldots n_{f f t}
\end{gathered}
$$

3. The complex field $U$ is now multiplied by the environmental phase array for all bins from 0 through $n_{\text {fft }}-1$.

4. Next, if a terrain profile has been specified and the terrain slop is positive ( $y_{c u r}>$ $y_{\text {curm }}$ ), the DOSHIFT SU is referenced to adjust the PE field relative to the terrain height.

5. The SPECEST SU is then referenced to determine the grazing angle $\vartheta_{\text {out }}$ and this angle is stored in array $\psi_{P E}$. If no terrain has been specified and the range is greater than the horizon range $\mathrm{r}_{\mathrm{hor}}$, then the grazing angle stored is the smaller of the tangent angle $a_{\text {cut }}$ or $\vartheta_{\text {out }}$.

6. This step is performed only if lang is '.true.'. The propagation angle at select height points, as long as they are less than the height defined by the ray traced at the maximum calculation angle, are computed by referencing the SPECEST SU. These are then stored in array $\Theta_{p}$.

Finally, $i_{g P E}$, the number of grazing angles computed, is initialized to $i_{P E}$ and the SU is exited. 
Table 34 and Table 35 provide identification, description, units of measure, and the computational source for each GETANGLES SU input and output data element.

Table 34. GETANGLES SU input data element requirements.

\begin{tabular}{|c|c|c|c|}
\hline Name & Description & Units & Source \\
\hline$a_{\text {cut }}$ & Tangent angle from antenna height to radio horizon & radians & PEINIT SU \\
\hline ant $_{\text {ref }}$ & $\begin{array}{l}\text { Transmitting antenna height relative to the reference height } \\
h_{\text {minter }}\end{array}$ & meters & TERINIT SU \\
\hline$\Delta r_{P E}$ & PE range step & meters & PEINIT SU \\
\hline$\Delta r_{P E 2}$ & 1/2 PE range step & meters & PEINIT SU \\
\hline filt & Cosine-tapered (Tukey) filter array & N/A & PEINIT SU \\
\hline$f_{\text {ter }}$ & $\begin{array}{l}\text { Logical flag indicating if terrain profile has been specified: } \\
\text { '.true.' = terrain profile specified } \\
\text { '.false.' = terrain profile not specified }\end{array}$ & N/A & APMINIT CSC \\
\hline$h t_{l i m}$ & $\begin{array}{l}\text { User-supplied maximum height relative to } h_{\text {minter }} \text {, i.e., } h t_{\text {lim }} \\
=h_{\text {max }}-h_{\text {minter }}\end{array}$ & meters & TERINIT SU \\
\hline$h_{\text {trap }}$ & $\begin{array}{l}\text { Height of the highest trapping layer from all refractivity } \\
\text { profiles }\end{array}$ & meters & REFINIT SU \\
\hline$i_{\text {hybrid }}$ & $\begin{array}{l}\text { Integer indicating which sub-models will be used: } \\
\begin{aligned} 0 & =\text { pure } \mathrm{PE} \text { model } \\
1 & =\text { full hybrid model }(\mathrm{PE}+\mathrm{FE}+\mathrm{RO}+\mathrm{XO}) \\
2 & =\text { partial hybrid model }(\mathrm{PE}+\mathrm{XO})\end{aligned}\end{array}$ & N/A & APMINIT CSC \\
\hline$i_{P E}$ & Number of PE range steps & N/A & PEINIT SU \\
\hline lang & $\begin{array}{l}\text { Propagation angle and factor output flag } \\
\text { '.true.'= Output propagation angle and propagation } \\
\text { factor for direct and reflected ray (where applicable). } \\
\text { '.false.' = Do not output propagation angles and factors }\end{array}$ & N/A & Calling CSCI \\
\hline$l_{\text {duct }}$ & $\begin{array}{l}\text { Logical flag indicating if surface-based duct profile has been } \\
\text { specified } \\
\text { '.true’. = surface-based duct exists } \\
\text { '.false.' = no surface-based duct exists }\end{array}$ & N/A & REFINIT SU \\
\hline$l_{\text {evap }}$ & $\begin{array}{l}\text { Logical flag indicating if evaporation duct profile has been } \\
\text { specified } \\
\text { '.true'. = evaporation duct exists } \\
\text { '.false.' = no evaporation duct exists }\end{array}$ & N/A & REFINIT SU \\
\hline$n_{f f t}$ & Transform size & N/A & FFTPAR SU \\
\hline$n_{34}$ & $3 / 4 n_{f f t}$ & N/A & PEINIT SU \\
\hline$n_{f 4}$ & $1 / 4 n_{f f t}$ & N/A & PEINIT SU \\
\hline$n_{\text {prof }}$ & Number of refractivity profiles & N/A & Calling CSCI \\
\hline$r_{\text {hor }}$ & Radio horizon range & meters & PEINIT SU \\
\hline$r_{\max }$ & Maximum specified range & meters & Calling CSCI \\
\hline$\vartheta_{m \times g}$ & $\begin{array}{l}\text { Maximum PE calculation angle for spectral estimation of } \\
\text { grazing angles }\end{array}$ & radians & APMINIT CSC \\
\hline
\end{tabular}


Table 34. GETANGLES SU input data element requirements. (continued)

\begin{tabular}{c|l|c|c}
\hline Name & \multicolumn{1}{|c|}{ Description } & Units & Source \\
\hline tyh & $\begin{array}{l}\text { Adjusted height points of sampled terrain profile at every PE } \\
\text { range step }\end{array}$ & meters & TERINIT SU \\
\hline
\end{tabular}

Table 35. GETANGLES SU output data element requirements.

\begin{tabular}{|c|c|c|}
\hline Name & Description & Units \\
\hline$\Delta z_{\text {spec }}$ & $\begin{array}{l}\text { Height increment at which the propagation angles are computed from spectral } \\
\text { estimation }\end{array}$ & meters \\
\hline$l_{\text {spec }}$ & $\begin{array}{l}\text { Logical flag indicating if grazing angles need to be computed via spectral } \\
\text { estimation. } \\
\text { '.true.' = compute grazing angles by spectral estimation } \\
\text { '.false.' = do not compute grazing angles by spectral estimation }\end{array}$ & N/A \\
\hline$n_{\text {ang }}$ & $\begin{array}{l}\text { Number of points in the vertical at which to spectrally estimate propagation } \\
\text { angles. }\end{array}$ & N/A \\
\hline$\psi_{P E}$ & Array containing grazing angles computed from spectral estimation of PE field & radians \\
\hline$\psi_{\text {ray }}$ & $\begin{array}{l}\text { Two-dimensional array containing grazing angles and corresponding ranges } \\
\text { computed from ray trace }\end{array}$ & $\begin{array}{c}\text { radians, } \\
\text { meters }\end{array}$ \\
\hline$i_{\text {error }}$ & $\begin{array}{l}\text { Integer variable indicating error number for ALLOCATE and DEALLOCATE } \\
\text { statements }\end{array}$ & N/A \\
\hline$i_{g P E}$ & Number of grazing angles computed from spectral estimation & N/A \\
\hline$i_{g r z}$ & Number of grazing angles computed from ray trace & N/A \\
\hline$\Theta_{p}$ & $\begin{array}{l}\text { Two-dimensional array containing the propagation angle estimated from PE at } \\
n_{\text {ang }} \text { height points and at every PE calculation range step during the initialization } \\
\text { routine. Format is } \Theta_{p}(i, j)=\text { propagation angle at the } i^{\text {th }} \text { height point ( } i=1 \text { to } \\
n_{\text {ang }} \text { ) and } j^{\text {th }} \text { PE range step }\left(j=0 \text { to } i_{g P E}\right) \text {. }\end{array}$ & radians \\
\hline
\end{tabular}

\subsubsection{Get Maximum Angle (GETTHMAX) SU}

The GETTMAX SU performs an iterative ray trace to determine the minimum angle required (based on the reflected ray) in obtaining a PE solution. The determination of this angle depends on the particular mode of execution. For full and partial hybrid modes, a ray is traced up to a height that exceeds at least $20 \%$ above the maximum terrain peak along the path or the highest trapping layer specified in the environment profiles, whichever is greater. Heights and angles of this ray are stored at each output range. The maximum PE propagation angle, $\Theta_{\max }$, is then determined from the local maximum angle of the traced ray. For the full hybrid mode, the minimum PE propagation angle is required to meet the following criteria: (1) the top of the PE region must contain all trapping layers for all refractivity profiles, (2) the top of the PE region must be at least $20 \%$ higher than the highest peak along the terrain profile, and (3) the minimum PE propagation angle must be at least as large as the grazing angle of the limiting ray $\psi_{\text {lim. }}$. 
First, four in-line ray trace functions are defined for general parameters $a, b, c$, and $g_{r d}$ :

$$
\begin{gathered}
\operatorname{RADA1}(a, b)=a^{2}+2 g_{r d} b, \\
\mathbf{R P}(a, b)=a+\frac{b}{g_{r d}}, \\
\operatorname{AP}(a, b)=a+b g_{r d}, \\
\mathbf{H P}(a, b, c)=a+\frac{b^{2}-c^{2}}{2 g_{r d}} .
\end{gathered}
$$

The first parameter to be determined is the minimum PE angle limit $a_{m l i m}$. The parameter to be determined later, $\Theta_{\max }$, must be at least this value. The initial estimate of $a_{m l i m}$ is given by

$$
a_{m \lim }=\pi / 90\left(.37541+4.331 e^{\frac{-f_{M H z}}{248.4}}+1.42 e^{\frac{-f_{M H z}}{2867}}+.4091 e^{\frac{-f_{M H z}}{2495}}\right) .
$$

If the DMFT algorithm is not required based on the specified inputs, the propagation path is entirely over water and no rough surface calculations are required, then $a_{m l i m}$ is decreased by $1 / 2$.

For a specific height-finder antenna pattern $\left(i_{\text {pat }}=6\right)$, the PE angle limit is recomputed as

$$
\begin{array}{ll}
a_{\text {mlim }}=\pi / 180\left(4.36985-1.02784 f_{G H z}+0.0786 f_{G H z}^{2}\right) & \text { for } f_{G H z}<6.0 \\
a_{\text {mlim }}=\pi / 180\left(2.3-0.1 f_{G H z}\right) & \text { for } f_{G H z} \geq 6.0
\end{array} \text {, }
$$

where $f_{G H z}$ is the frequency in gigahertz. If rough surface calculations are to be performed and if a surface-based duct has been specified, then $a_{m l i m}$ is doubled.

If the backward difference DMFT algorithm will be used, then $a_{m l i m}$ is adjusted to accommodate low antenna heights according to

$$
a_{m l i m}=\mathbf{M A X}\left(a_{m l i m}, \mathbf{S I N}^{-1}\left(\frac{\lambda}{2 a n t_{h t}}\right)\right) .
$$

A multiplicative height factor $h_{m t}$ is determined to ensure clearance of the ray path for low antenna heights over large terrain elevations: 


$$
h_{m t}=\mathbf{M I N}\left(.2, \frac{a n t_{h t}}{\operatorname{MAX}\left(t y_{1}, 1\right)}\right)
$$

If $h_{m t}$ is less than 0.1 , then it is set equal to 0 . It is then increased by 1 .

Several constants needed in subsequent steps in this SU are determined. An initial estimate of the launch angle $a_{\text {launch }}$, is initialized to $\alpha_{\text {lim }}$, the elevation angle of the RO limiting ray. If using the full hybrid mode, then $a_{\text {launch }}$ is set equal to the negative of $a_{\text {launch. }}$. The maximum height to trace to, $z_{\text {limt }}$, is set equal to $h t_{\text {lim }}-10^{-5}$, and the range step, $\Delta r_{\text {temp }}$, for subsequent ray tracing is given by $r_{\max } / 200$. The terrain elevation height at the source, $y_{n t}$, is initialized to $t y_{1}$, provided APM is running in a full hybrid mode and $t y_{1}$ is greater than zero; otherwise, $y_{n t}$ is initialized to 0 .

An iterative ray trace to determine the launch angle $a_{\text {launch }}$ and subsequently $\Theta_{\max }$ is then begun. Steps 1 through 3 are performed until a ray has been safely traced from height $a n t_{r e f}$ to $z_{\text {limt }}$.

1. At the start of the ray trace, the current local angle $\left(a_{0}\right)$, range $\left(r_{0}\right)$, height $\left(h_{0}\right)$, and refractive gradient index $(j)$ are initialized to $a_{\text {launch }}, 0$, ant $t_{r e f}$, and $i_{\text {start } 1}$, respectively. The counter index, $k t$, for the terrain profile arrays $t x$ and ty is initialized to one. The variable $r_{\mathrm{o}}$, the current output range to trace to, is set equal to zero. Steps 1.a through 1.d are then performed for each ray trace step from 1 to $i_{\text {rtemp }}$.

a. First, $r_{\mathrm{o}}$ is incremented by $\Delta r_{\text {temp. }}$. Now steps i through vii are performed until $r_{0}$ reaches $r_{0}$.

i. The range at the end of the ray trace step, $r_{1}$, is set equal to $r_{o}$, and the current refractive gradient $g_{r d}$ is set equal to $\operatorname{grdum}_{j}$

ii. The angle at the end of the trace, $a_{1}$, is then given by

$$
a_{1}=\operatorname{AP}\left(a_{0}, r_{1}-r_{0}\right)
$$

iii. If $a_{1}$ is of the opposite sign of $a_{0}$, then $a_{1}$ is set to zero and $r_{1}$ is given by

$$
r_{1}=\mathrm{RP}\left(r_{0}, a_{1}-a_{0}\right)
$$

iv. The height at the end of the ray trace $h_{1}$ is given by

$$
h_{1}=\mathbf{H P}\left(h_{0}, a_{1}, a_{0}\right) .
$$


v. If $a_{1}$ is positive and $h_{1}$ has reached or surpassed the next height level, then $a_{1}$, $r_{1}, j$, and $h_{1}$ are found as follows. First, $h_{1}$ is set equal to $h t d u m_{j+1}$, and $a_{1}$ and $r_{1}$ are given by

$$
\begin{aligned}
& a_{1}=\sqrt{\mathbf{R A D A} \mathbf{1}\left(a_{0}, h_{1}-h_{0}\right)} \\
& r_{1}=\mathbf{R P}\left(r_{0}, a_{1}-a_{0}\right)
\end{aligned}
$$

The index $j$ is incremented by one, and the height, $h_{1}$, at the end of the ray trace step is given by the smaller of $h t_{l i m}$ or $h t d u m_{j}$.

vi. However, if either of the conditions for $a_{1}$ and $h_{1}$ in step $\mathrm{v}$ are not met, and $a_{1}$ is less than or equal to 0 , then $h_{1}$ is set equal to $y_{n t}$ if the calculated value in step iv is less than $y_{n t}$. If the calculated value of $h_{1}$ in step iv is less than $h t d u m_{j}$, then $h_{1}$ is set equal to $h_{t d u m_{j}}$, and $j$ is set equal to the maximum of 0 or $j-1$. The variables $a_{1}$ and $r_{1}$ are then determined from

$$
\begin{aligned}
& a_{1}=-\sqrt{\mathbf{R A D A} 1\left(a_{0}, h_{1}-h_{0}\right)} . \\
& r_{1}=\mathbf{R P}\left(r_{0}, a_{1}-a_{0}\right)
\end{aligned}
$$

vii. If the ray has hit the surface and is reflected, which would be the condition for which $h_{1}$ is set equal to $y_{n t}$ in step vi, then $a_{1}$ is set equal to minus $a_{1}, \psi_{\text {lim }}$ is set equal to $a_{1}$, the range, $r_{\text {pest }}$ (at which loss values from the PE model will start being calculated) is set equal to $r_{1}$, and the height $h_{\text {start }}$ is set equal to $y_{n t}$. The variable $h_{\text {start }}$ is used for subsequent initialization of ray tracing to fill in array $h_{\text {lim. }}$. In preparation for the next ray trace step; $h_{0}$ is set equal to $h_{1}, r_{0}$ is set equal to $r_{1}$, and $a_{0}$ is set equal to $a_{1}$. If the range $r_{1}$ is greater than $r_{\text {flat }}$, then the current iteration is exited and the SU proceeds to step b; otherwise, steps i through vii are repeated until $r_{0}$ reaches $r_{0}$.

b. If running a terrain case ( $f_{\text {ter }}=$ '.true.'), at the end of the ray trace for the current step, a check is made to see that the current height of the ray is at least $20 \%$ higher than the current terrain height. The counter $k t$ is determined such that $r_{0}>$ $t x_{k t+1}$ and $k t<i_{\text {tpa }}$. If using the partial hybrid mode and range $r_{0}$ is less than $5 \mathrm{~km}$, then the clearance height of the terrain, $y_{n}$, at the current range for the traced ray, is given by

$$
y_{n}=h_{m t}\left[t y_{k t}+\operatorname{slp}_{k t}\left(r_{0}-t x_{k t}\right)\right] .
$$

If the previous conditions are not met, then $h_{m t}$ in the above equation is replaced with the constant 1.2. 
c. The ending angle, range, and height for each ray trace step is now stored in arrays raya, rtemp, and htemp, respectively.

d. Now, if running a full hybrid case ( $i_{\text {hybrid }}=1$ ), a test is made to determine if both $h_{0}$ is less than $y_{n}$ and if $r_{0}$ is greater than $r_{\text {flat }}$. If these conditions are true, then the flag $i_{\text {quit }}$ is set equal to 1 . If the case is not a full hybrid case and if $h_{0}$ is less than $y_{n}$, then $i_{\text {quit }}$ is set equal to 1 . Finally, if $h_{0}$ is greater than or equal to $z_{\text {limt }}$; or $i_{\text {quit }}$ equals 1, then the current iteration is exited and the SU proceeds to step 2; otherwise, steps 1.a through 1.d are repeated.

2. If the iteration defined by steps 1.a through $1 . \mathrm{d}$ has been prematurely terminated ( $i_{\text {quit }}=1$ ), then the initial elevation angle $a_{\text {launch }}$ is decreased by $10^{-3}$ radians for the full hybrid case $\left(i_{\text {hybrid }}=1\right)$, and is increased by $10^{-3}$, otherwise. If the previous iteration has not been prematurely terminated $\left(i_{\text {quit }}=0\right)$, the $\mathrm{SU}$ continues with step 3 .

3. If height $z_{\text {limt }}$ is reached, then an initial launch angle (i.e., ray) has been found with all traced heights, ranges, and angles stored. The integer flag to continue ray tracing, $i_{\text {ray }}$, is set to equal 1 to terminate the iterative loop, and the index $i_{\text {hmax }}$, indicating the range step at which $z_{\text {limt }}$ is reached, is set equal to the range step index $i$ (the range step index counter in the iterative loop defined by steps 1 through 3 .

The remaining elements from $i_{\text {hmax }}$ to $i_{\text {rtemp }}$ in arrays htemp, rtemp, and raya are filled with the values $h_{0}, r_{\max }$, and $a_{0}$, respectively. Next, the index $i_{\text {hmax }}$ is set equal to the minimum of $i_{\text {hmax }}$ or $i_{\text {rtemp }}$.

The variable $\Theta_{\max }$ is found for the PE region based on the local ray angles just determined for the particular ray traced. First, the index $i_{a p}$ at which the local ray angle becomes positive (i.e., raya $i_{a p}$ ) is determined. If $i_{a p}$ equals $i_{\text {rtemp }}$ this indicates that no PE calculations are required for the specific geometry, in which case the flag $n_{P E}$ is set equal to $1, \Theta_{75}$ is set equal to $a_{m l i m}, r_{p e s t}$ is set equal to $r_{\max }, z_{\text {lim }}$ is set equal to $h t_{\text {lim }}$, and the SU is exited . Otherwise, several variables are next initialized. The local indices, $i_{o k}$ and $i_{\text {flag }}$, plus the variables $z_{\text {lim }}$ and $a_{m x c u r}$, are each set equal to zero. The variable $a_{m x c u r}$ is the maximum local angle along the traced ray up to height $z_{\text {lim }}$ with a minimum limit of $a_{\text {mlim }}$.

The variable $\Theta_{\max }$ is then found from an iteration performed on the local angle and height at which the local maximum angle is reached. The following steps 1 through 6 are performed while the flag $i_{o k}$ is 0 .

1. The height in the PE region that must be reached for the hybrid model is $z_{\text {test }}$. The first occurrence of $h t e m p_{j}$ that is greater than $z_{\text {test }}$ is found and the index $i_{s t}$ is then set to the smaller of the index $j$ where this occurs or $i_{\text {hmax }}$. 
2. The angle $a_{m x c u r}$ is now initialized to raya $a_{1}$. The maximum angle in raya is then found looking only at elements from raya $a_{2}$ to raya $i_{s t}$ and $a_{m x c u r}$ is set equal to this angle.

3. $a_{m x c u r}$ is now set equal to the maximum of $a_{m l i m}$ and $a_{m x c u r}$. The variable $a_{\text {temp }}$ is now set to $a_{m x c u r}$ divided by 0.75 . If using the partial hybrid mode ( $\left.i_{h y b r i d}=2\right), z_{\text {test }}$ is given by

$$
z_{\text {test }}=\operatorname{MAX}\left(\text { ant }_{\text {ref }}, h_{\text {test }}, 1.2 h_{\text {termax }}, 10^{3}\right) .
$$

4. A reference is then made to the FFTPAR SU to determine new values for $z_{\text {test }}, z_{\max }$, $\Delta z_{P E}, l_{f f t}$, and $n_{f f t}$ using the inputs: $\ln _{\min }, \lambda, a_{\text {temp }}$, and $i_{\text {flag }}$.

5. After the reference to the FFTPAR SU is made, if $i_{\text {flag }}=0$ it is set equal to 1 . In addition, if not running a full hybrid case, $i_{o k}$ is set equal to 1 . However, if after the reference to the FFTPAR SU is made, $i_{f l a g}$ is equal to one and if the case is not a partial hybrid case; the iterative height tolerance tol is given by

$$
\text { tol }=\frac{\left|z_{\text {test }}-z_{\text {lim }}\right|}{z_{\text {test }}} .
$$

A test is then made to determine whether this value of tol is less than or equal to $z_{\text {tol }}$, the height tolerance for Newton's method. If it is, then the index $i_{o k}$ is set equal to one.

6. Now $z_{\text {lim }}$ is set equal to $z_{\text {test }}$ and if $i_{o k}$ is 0 , steps 1 through 6 are repeated. Otherwise, the SU proceeds to the next step.

The variable $\Theta_{75}$ is now set equal to $a_{m x c u r}$, and $\Theta_{\max }$ is set equal to $a_{\text {temp. }}$. The variable $\ln _{f t t}$ is then adjusted such that for every $5^{\circ}$ in $\Theta_{75}$, it is increased by 1 .

Next, the TRACE_ROUT SU is referenced to trace the ray to each output range step, $\Delta r_{\text {out }}$, storing heights in array hlim. If running in a full hybrid mode, the ray is traced from starting angle, range, and height equal to $\psi_{\text {lim }}, r_{\text {pest}}$, and $h_{\text {start, }}$ respectively. Otherwise, the starting angle, range, and height are equal to $a_{\text {launch, }}$ 0, and antref, respectively.

Before exiting, all elements of hlim corresponding to ranges less than $r_{\text {pest }}$ are set equal to $y_{\text {fref }}$. 
Table 36 and Table 37 provide identification, description, units of measure, and the computational source for each GETTHMAX SU input and output data element.

Table 36. GETTHMAX SU input data element requirements.

\begin{tabular}{|c|c|c|c|}
\hline Name & Description & Units & Source \\
\hline$\alpha_{\lim }$ & Elevation angle of the RO limiting ray & radians & Calling SU \\
\hline ant $_{h t}$ & Transmitting antenna height above local ground & meters & Calling CSCI \\
\hline ant $_{\text {ref }}$ & Transmitting antenna height relative to $h_{\text {minter }}$ & meters & TERINIT SU \\
\hline$f_{M H z}$ & Frequency & $\mathrm{MHz}$ & Calling CSCI \\
\hline$f_{\text {ter }}$ & $\begin{array}{l}\text { Logical flag indicating if terrain profile has been } \\
\text { specified: } \\
\text { '.true.' = terrain profile specified } \\
\text { '.false.' = terrain profile not specified }\end{array}$ & N/A & APMINIT CSC \\
\hline grdum & M-unit gradient array & $\begin{array}{l}\text { (M-unit/ } \\
\text { meter) }\end{array}$ & REFINIT SU \\
\hline htdum & Height array for current interpolated profile & meters & REFINIT SU \\
\hline$h_{\text {termax }}$ & Maximum terrain height along profile path & meters & Calling SU \\
\hline$h_{\text {test }}$ & $\begin{array}{l}\text { Minimum height at which all trapping refractivity } \\
\text { features are below }\end{array}$ & meters & Calling SU \\
\hline htlim & User specified maximum height relative to $h_{\text {minter }}$ & meters & TERINIT SU \\
\hline ihybrid & $\begin{array}{l}\text { Integer indicating which sub-models will be used: } \\
\begin{aligned} 0 & =\text { FEDR+PE models } \\
1 & =\text { full hybrid model }(\mathrm{PE}+\mathrm{FE}+\mathrm{RO}+\mathrm{XO}) \\
2 & =\text { partial hybrid model }(\mathrm{PE}+\mathrm{XO})\end{aligned}\end{array}$ & N/A & APMINIT CSC \\
\hline$i_{a l g}$ & $\begin{array}{l}\text { Integer flag indicating which DMFT algorithm is being } \\
\text { used: } \\
\qquad \begin{array}{l}0=\text { no DMFT algorithm will be used } \\
1=\text { use central difference algorithm } \\
2=\text { use backward difference algorithm }\end{array}\end{array}$ & N/A & APMINIT CSC \\
\hline$i_{\text {rtemp }}$ & Temporary number of range steps (used for ray tracing) & N/A & APM_MOD \\
\hline$i_{\text {start } 1}$ & Refractivity level index within $h t d u m$ at $a n t_{r e f}$ & N/A & REFINIT SU \\
\hline$i_{t p a}$ & $\begin{array}{l}\text { Number of terrain points in used internally in arrays } t x \\
\text { and ty }\end{array}$ & N/A & APMINIT CSC \\
\hline$\lambda$ & Wavelength & meters & APMINIT CSC \\
\hline Inmin & Minimum power of 2 transform size & N/A & APMINIT CSC \\
\hline$n_{\text {rout }}$ & Integer number of output range points desired & N/A & Calling CSCI \\
\hline$r_{\text {flat }}$ & $\begin{array}{l}\text { Maximum range at which the terrain profile remains flat } \\
\text { from the source }\end{array}$ & meters & Calling SU \\
\hline$r_{\max }$ & Maximum output range & meters & Calling CSCI \\
\hline rngout & Array containing all desired output ranges & meters & APMINIT CSC \\
\hline
\end{tabular}


Table 36. GETTHMAX SU input data element requirements. (continued)

\begin{tabular}{|c|c|c|c|}
\hline Name & Description & Units & Source \\
\hline ruf & $\begin{array}{l}\text { Logical flag indicating if rough sea surface calculations } \\
\text { are required } \\
\text { ‘.true.' = perform rough sea surface calculations } \\
\text { ‘.false.' = do not perform rough sea surface } \\
\text { calculations }\end{array}$ & N/A & APMINIT CSC \\
\hline $\operatorname{slp}$ & Slope of each segment of terrain & N/A & TERINIT SU \\
\hline$t x$ & Range points of terrain profile & meters & TERINIT SU \\
\hline ty & Adjusted height points of terrain profile & meters & TERINIT SU \\
\hline$y_{\text {fref }}$ & Ground elevation height at source & meters & APMINIT CSC \\
\hline$Z_{\text {lim }}$ & Height limit for PE calculation region & meters & APMINIT CSC \\
\hline$Z_{\text {test }}$ & $\begin{array}{l}\text { Height in PE region that must be reached for hybrid } \\
\text { model }\end{array}$ & meters & Calling SU \\
\hline$z_{\text {tol }}$ & Height tolerance for Newton’s method & meters & APMINIT CSC \\
\hline
\end{tabular}

Table 37. GETTHMAX SU output data element requirements.

\begin{tabular}{|c|c|c|}
\hline Name & Description & Units \\
\hline$a_{\text {launch }}$ & $\begin{array}{l}\text { Launch angle used which, when traced, separates PE and XO regions from the } \\
\text { RO region }\end{array}$ & radians \\
\hline hlim & $\begin{array}{l}\text { Array containing height at each output range separating the } \mathrm{RO} \text { region from the } \\
\mathrm{PE} \text { (at close ranges) and XO (at far ranges) regions }\end{array}$ & meters \\
\hline htemp & Heights at which ray is traced to every range in rtemp & meters \\
\hline$i_{a p}$ & Index indicating when the local ray angle becomes positive in array raya & N/A \\
\hline$i_{\text {err }}$ & Return error code & N/A \\
\hline $\ln _{\text {fft }}$ & Power of 2 transform size, i.e. $n_{f f t}=2^{l n} f f t$ & N/A \\
\hline$n o_{P E}$ & $\begin{array}{l}\text { Integer flag indicating if PE calculations are needed: } \\
\begin{array}{l}0=\mathrm{PE} \text { calculations needed } \\
1=\text { no PE calculations needed }\end{array}\end{array}$ & N/A \\
\hline$\psi_{\text {lim }}$ & Grazing angle of limiting ray & radians \\
\hline raya & Array containing all local angles of traced ray alaunch at each $i_{\text {rtemp }}$ range & radians \\
\hline$r_{\text {pest }}$ & Range at which loss values from the PE model will start being calculated & meters \\
\hline rtemp & Range steps for tracing to determine maximum PE angle & meters \\
\hline$\Theta_{\max }$ & Maximum propagation angle in PE calculations & radians \\
\hline$\Theta_{75}$ & $75 \%$ of maximum propagation angle in $\mathrm{PE}$ calculations & radians \\
\hline$Z_{\max }$ & Maximum height in PE calculation region & meters \\
\hline
\end{tabular}




\subsubsection{Grazing Angle Interpolation (GRAZE_INT) SU}

The GRAZE_INT SU interpolates, for each PE range step, grazing angles computed from both ray trace and spectral estimation. Those angles from ray trace take precedence.

Upon entering the SU, the grazing angle array $\Psi$ is allocated for size $i_{P E}$ and initialized to 0 , with the first element initialized to $1 / 2 \pi$ radians. Several variables are next initialized. The variable $r$ which is the range to interpolate to, is initialized to $\Delta r_{P E}$. The range $r_{g r z}$ at which the spectrally estimated angles were computed in the GETANGLES $\mathrm{SU}$ is initialized to $\Delta r_{\text {grz }}$.

If a surface-based duct has been specified, no evaporation duct exists, and the range $r_{\text {flat }}$ is greater than the horizon range $r_{\text {hor }}$, then a check is made for the possible existence of a skip zone produced by a surface-based duct. If one exists, then the spectrally estimated grazing angles will be included in the interpolation algorithm for those ranges beyond the start of the skip zone. The check for a skip zone is done by performing an iterative loop on the ranges $r_{\text {ray }}$ corresponding to the grazing angles in $\psi_{\text {ray }}$. For those ranges beyond $r_{h o r}$ the maximum difference between successive ranges in $r_{\text {ray }}$ is determined according to

$$
r_{\text {skip }}=\text { maximum of }\left(r_{r a y_{j+1}}-r_{r a y_{j}}\right) ; \text { for } j=k-1, k, \ldots i_{g r z}-1 \text {, }
$$

where $k$ is the first element in $r_{\text {ray }}$ corresponding to the first range beyond $r_{\text {hor }}$. If $r_{\text {skip }}$ is greater than $5 \mathrm{~km}$, then a skip zone is assumed to exist and the range per at which spectrally estimated grazing will be included in the interpolation algorithm is set equal to the minimum of $r_{\text {flat }}$ or $r_{\text {end }}$, where $r_{\text {end }}$ is the first range point in $r_{\text {ray }}$ just beyond the skip zone. If no skip zone exists, then per is set equal to $r_{\max }$. Next, the following steps 1 through 3 are performed for each PE range step $i$, indexed from 1 to $i_{P E}$.

1. For range $r$ less than $r_{\text {hor }}$ steps 1.a through 1.c are performed; otherwise, the SU proceeds to step 2 .

a. An iterative loop is performed to find $k$, the element in $r_{\text {ray }}$ corresponding to the first range point beyond $r_{\text {hor }}$.

b. For $k$ equal to 1 the grazing angle is determined as

$$
\begin{aligned}
& \Psi_{i}=\left|\mathbf{T A N}^{-1}\left(\frac{s}{r}\right)\right|, \\
& s=h_{\text {mref }}-y_{\text {fref }}+a n t_{h t}-\frac{r^{2}}{2 a_{e k s t}} .
\end{aligned}
$$


c. For all other values of $k$ the grazing angle is determined as

$$
\begin{aligned}
& \Psi_{i}=\mathbf{M A X}(0, \psi), \\
& \psi=\psi_{\text {ray }_{k-1}}+\psi_{\text {ray }_{k}}\left[\frac{r-r_{\text {ray }_{k-1}}}{r_{\text {ray }_{k}}-r_{\text {ray }_{k-1}}}\right] .
\end{aligned}
$$

2. For range $r$ greater than $r_{\text {hor }}$ an iterative loop is performed to determine the number of elements icr within array $r_{\text {ray }}$ satisfying the condition $r m d<r_{\text {ray }}<r m d+\Delta r_{P E}$, where $r m d$ is the range at mid-PE range step. The following steps a through $b$ are then performed.

a. If icr is non-zero, then the indices $j r 1$ and $j r 2$ are initialized such that ranges $r_{r a y_{j r}}$ through $r_{r a y}$ satisfies the condition in step 2 and jr2-jr1 equals icr. If $r_{r a y}$ is greater than range $r$, then the grazing angle $\Psi_{i}$ is interpolated as

$$
\Psi_{i}=\psi_{r a y_{j}}+\psi_{r a_{j+1}}\left[\frac{r-r_{r a y_{j}}}{r_{r a y_{j+1}}-r_{r a y_{j}}}\right]
$$

where the index $j$ lies between $j r 1$ and $j r 2$ and is defined such that $r_{\text {ray }}$ is the nearest range point less than $r$ and $r_{r a y_{j+1}}$ is the nearest range point greater than $r$. If $r_{r_{j} y_{j 2}}$ is less than $r$, then the grazing angle $\Psi_{i}$ is averaged according to

$$
\Psi_{i}=\left(\frac{1}{i c r}\right) \sum_{j=j r 1}^{j r 2} \psi_{j}
$$

b. If icr is equal to 0 and $r$ is greater than per, then grazing angle $\Psi_{i}$ is computed from interpolation of angles $\psi_{P E}$ determined from spectral estimation. If no spectrally estimated angles exist, then $\Psi_{i}$ is set equal to 0 . If an evaporation duct profile has been specified, then $\Psi_{i}$ is set equal to $\psi_{r^{\prime a y}}{ }_{g r z}$.

3. Both $r m d$ and $r$ are then incremented by $\Delta r_{P E}$.

Once all grazing angles $\Psi$ have been determined, the arrays $\psi_{\text {ray }}$ and $\psi_{P E}$ are deallocated and the SU is exited.

Table 38 and Table 39 provides identification, description, units of measure, and the computational source for each GRAZE_INT SU input and output data element. 
Table 38. GRAZE_INT SU input data element requirements.

\begin{tabular}{|c|c|c|c|}
\hline Name & Description & Units & Source \\
\hline$a_{\text {cut }}$ & Tangent angle from antenna height to radio horizon & radians & PEINIT SU \\
\hline$a_{e k s t}$ & $4 / 3$ times mean earth radius & meters & APM_MOD \\
\hline$a_{n t}$ & Transmitting antenna height above local ground & meters & Calling CSCI \\
\hline$\Delta r_{g r z}$ & PE range step used for calculation of grazing angles & meters & APMINIT CSC \\
\hline$\Delta r_{P E}$ & PE range step & meters & PEINIT SU \\
\hline$\Delta r_{P E 2}$ & $1 / 2 \mathrm{PE}$ range step & meters & PEINIT SU \\
\hline$f_{\text {ter }}$ & $\begin{array}{l}\text { Logical flag indicating if terrain profile has been } \\
\text { specified: } \\
\text { '.true.' = terrain profile specified } \\
\text { '.false.' = terrain profile not specified }\end{array}$ & N/A & APMINIT CSC \\
\hline hmref & Height relative to $h_{\text {minter }}$ & meters & TERINIT SU \\
\hline ihybrid & $\begin{array}{l}\text { Integer indicating which sub-models will be used: } \\
\begin{aligned} 0 & =\text { FEDR }+ \text { PE model } \\
1 & =\text { full hybrid model }(\mathrm{PE}+\mathrm{FE}+\mathrm{RO}+\mathrm{XO}) \\
2 & =\text { partial hybrid model }(\mathrm{PE}+\mathrm{XO})\end{aligned}\end{array}$ & N/A & APMINIT CSC \\
\hline$i_{g P E}$ & $\begin{array}{l}\text { Number of grazing angles computed from spectral } \\
\text { estimation }\end{array}$ & N/A & GETANGLES SU \\
\hline$i_{g r z}$ & Number of grazing angles computed from ray trace & N/A & GETANGLES SU \\
\hline$i_{P E}$ & Number of PE range steps & N/A & PEINIT SU \\
\hline$l_{\text {duct }}$ & $\begin{array}{l}\text { Logical flag indicating if surface-based duct profile has } \\
\text { been specified } \\
\text { '.true'. = surface-based duct exists } \\
\text { '.false.' = no surface-based duct exists }\end{array}$ & N/A & REFINIT SU \\
\hline$l_{\text {evap }}$ & $\begin{array}{l}\text { Logical flag indicating if evaporation duct profile has } \\
\text { been specified } \\
\text { '.true'. = evaporation duct exists } \\
\text { '.false.' = no evaporation duct exists }\end{array}$ & N/A & REFINIT SU \\
\hline$l_{\text {spec }}$ & $\begin{array}{l}\text { Logical flag indicating if grazing angles need to be } \\
\text { computed via spectral estimation. } \\
\text { '.true.' = compute grazing angles by spectral } \\
\text { estimation } \\
\text { '.false.' = do not compute grazing angles by spectral } \\
\text { estimation }\end{array}$ & N/A & GETANGLES SU \\
\hline$\psi_{P E}$ & $\begin{array}{l}\text { Array containing grazing angles computed from spectral } \\
\text { estimation of PE field }\end{array}$ & radians & GETANGLES SU \\
\hline$\psi_{\text {ray }}$ & $\begin{array}{l}\text { Two-dimensional array containing grazing angles and } \\
\text { corresponding ranges } r_{\text {ray }} \text { computed from ray trace }\end{array}$ & $\begin{array}{l}\text { radians, } \\
\text { meters }\end{array}$ & GETANGLES SU \\
\hline$r_{\text {flat }}$ & $\begin{array}{l}\text { Maximum range at which the terrain profile remains flat } \\
\text { from the source }\end{array}$ & meters & Calling SU \\
\hline$r_{\text {hor }}$ & Radio horizon range & meters & PEINIT SU \\
\hline$r_{\max }$ & Maximum output range & meters & Calling CSCI \\
\hline$y_{\text {fref }}$ & Ground elevation height at the source & meters & APMINIT CSC \\
\hline
\end{tabular}


Table 39. GRAZE_INT SU output data element requirements.

\begin{tabular}{c|l|c}
\hline Name & \multicolumn{1}{|c|}{ Description } & Units \\
\hline$i_{\text {error }}$ & $\begin{array}{l}\text { Integer variable indicating error number for ALLOCATE and DEALLOCATE } \\
\text { statements }\end{array}$ & N/A \\
$\Psi$ & Array of interpolated grazing angles at each PE range step & radians \\
\hline
\end{tabular}

\subsubsection{Height Check (HTCHECK) SU}

The HTCHECK SU checks to see if the current traced height is below the current ground height. If so, the SU will calculate the reflection point and return with the modified angle, range, and height of the reflection point.

Upon entering the SU the following in-line functions are defined:

$$
\begin{gathered}
\mathbf{A P}(a, b)=a+b g_{r d} \\
\mathbf{H P}(a, b, c)=a+\frac{b^{2}-c^{2}}{2 g_{r d}} .
\end{gathered}
$$

Next, the current terrain height is determined according to

$$
h_{\text {ter }}=t y_{i}+\left(r-t x_{i}\right) s l p_{i}
$$

where $i$ is the terrain segment at the current range. If the current traced ray height, $h_{1}$, is greater than $h_{t e r}$, then nothing is done and the SU is exited. Otherwise, the SU proceeds with the following steps.

a. If the current angle $\left(a_{1}\right)$ is less than zero, and both the current traced ray height $\left(h_{1}\right)$ and $h_{t e r}$ are less than $h t_{l v l}$, then nothing is done and the SU is exited.

b. At this point $h_{1}$ is less than $h_{\text {ter }}$ and appears to have a valid reflection point. Therefore, the range at which this occurs is then computed based on solving a quadratic.

$$
\begin{gathered}
r a=b^{2}-4 g_{r d} c, \\
b=2\left(a_{0}-s \operatorname{lp}_{i}-g_{r d} r_{0}\right), \\
c=g_{r d} r_{0}^{2}-2 a_{0} r_{0}-2\left(t y_{i}-h_{0}\right)+2 \operatorname{slp} t x_{i} .
\end{gathered}
$$

If $r a$ is greater than 0 , then two possible range values are determined in step 3 . Otherwise, the SU proceeds to step 4. 
c. The two range points are determined according to

$$
r x_{1}=\frac{-b+\sqrt{r a}}{2 g_{r d}} ; r x_{2}=\frac{-b-\sqrt{r a}}{2 g_{r d}} \text {. }
$$

The two range values are then compared with the previous and current range values $r_{0}$ and $r_{1}$ for valid ranges between these two points. One valid range is then selected and $r_{1}$ is set to this value. If no valid range is determined, then the SU exits with an $i_{\text {error }}$ value of -100 . If this occurs, please see the APM developers.

d. A new angle and height of the traced ray is then determined as

$$
\begin{array}{ll}
a_{1}=\mathbf{A P}\left(a_{0}, r_{1}-r_{0}\right) ; & \text { for } a_{0}<10^{\circ} \\
h_{1}=\mathbf{H P}\left(h_{0}, a_{1}, a_{0}\right), &
\end{array}
$$

or

$$
\begin{aligned}
& a_{1}=a_{0} \\
& h_{1}=h_{0}+\left(r_{1}-r_{0}\right) \mathbf{T A N}^{-1}\left(a_{1}\right)-\frac{\left(r_{1}-r_{0}\right)^{2}}{t w o k a}, \text { for } a_{0} \geq 10^{\circ},
\end{aligned}
$$

and the SU is then exited.

Table 40 and Table 41 provide identification, description, units of measure, and the computational source for each HTCHECK SU input and output data element.

Table 40. HTCHECK SU input data element requirements.

\begin{tabular}{c|l|c|c}
\hline Name & \multicolumn{1}{|c|}{ Description } & Units & Source \\
\hline$a_{0}$ & Starting angle of ray trace step & radians & RGTRACE SU \\
$a_{1}$ & Ending angle of ray trace step & meters & RGTRACE SU \\
$g_{r d}$ & Gradient at current ray trace step & M-units/ & meter \\
& & RGTRACE SU \\
$h_{0}$ & Starting height of ray trace step & meters & RGTRACE SU \\
$h_{1}$ & Ending height of ray trace step & RGTRACE SU \\
$h t_{l v l}$ & Height of the upper refractivity level within the current & meters & RGTRACE SU \\
& layer & & \\
$r_{0}$ & Index of the current terrain segment in arrays $t x$ and ty & N/A & RGTRACE SU \\
& Starting range of ray trace step & meters & RGTRACE SU
\end{tabular}


Table 40. HTCHECK SU input data element requirements. (continued)

\begin{tabular}{c|l|c|c}
\hline Name & \multicolumn{1}{|c|}{ Description } & Units & Source \\
\hline slp & Slope of the terrain segment at the current range. & N/A & TERINIT SU \\
twoka & Twice the effective earth radius & meters & GET_K SU \\
$t x$ & Range points of terrain profile & meters & TERINIT SU \\
$t y$ & Height points of terrain profile & meters & TERINIT SU \\
\hline
\end{tabular}

Table 41. HTCHECK SU output data element requirements.

\begin{tabular}{c|l|c}
\hline Name & \multicolumn{1}{|c|}{ Description } & Units \\
\hline$i_{\text {error }}$ & Integer variable indicating error number for ALLOCATE and DEALLOCATE & N/A \\
& statements & \\
$a_{1}$ & Ending angle of ray trace step & meters \\
$h_{1}$ & Ending height of ray trace step & meters \\
$i h i t$ & Integer flag indicating if ray has hit surface: & N/A \\
& $\quad$ ihit =0; ray has not hit the surface & \\
$r_{1}$ & Enit =1; ray has hit the surface & meters \\
\hline
\end{tabular}

\subsubsection{Interpolate Profile (INTPROF) SU}

The INTPROF SU performs a linear interpolation vertically with height on the refractivity profile, refref. Interpolation is performed at each PE mesh height point.

To interpolate vertically at each $\mathrm{PE}$ mesh height, the following iteration is performed. The index $j$ is determined such that for every $i^{\text {th }} \mathrm{PE}$ bin, $h t_{i}$ is just greater than $h r e f_{j}$ and $j<n l v l$. The interpolated profile profint is then determined from

$$
\text { profint }_{i}=\text { refref }_{j-1}+\text { con }\left(\text { refref }_{j}-\text { refref }_{j-1}\right) \frac{h t_{i}-\text { href }_{j-1}}{h r e f_{j}-h r e f_{j-1}} ; \quad i=1,2,3, . . . n_{f f t} \text {, }
$$

where the array $h t$ and constant con have been determined in the APMINIT CSC.

Table 42 and Table 43 provide identification, description, units of measure, and the computational source for each INTPROF SU input and output data element. 
Table 42. INTPROF SU input data element requirements.

\begin{tabular}{c|l|c|c}
\hline Name & \multicolumn{1}{|c|}{ Description } & Units & Source \\
\hline con & $10^{-6} k_{o}$ & meters & \\
$h r e f$ & Heights of refractivity profile with respect to local ground & APMINIT CSC \\
& height & & PROFREF SU \\
$h t$ & PE mesh height array of size $n_{f f t}$ & meters & PEINIT SU \\
$n_{f f t}$ & Transform size & N/A & FFTPAR SU \\
$n l v l$ & Number of levels in new profile & N/A & PROFREF SU \\
$r e f r e f$ & Refractivity array & M-units & PROFREF SU \\
\hline
\end{tabular}

Table 43. INTPROF SU output data element requirements.

\begin{tabular}{c|c|c}
\hline Name & Description & Units \\
\hline profint & Profile interpolated to every $\Delta z_{P E}$ in height & M-units \\
\hline
\end{tabular}

\subsubsection{PE Initialization (PEINIT) SU}

The PEINIT SU initializes all variables used in the PE model for subsequent calls to the PESTEP SU.

Upon entering the SU several variables are initialized. The following PE transform variables are computed - the angle (or p-space) mesh size, $\Delta p$; the Fourier transform normalization constant, $f_{\text {norm }}$; the angle bin width, $\Delta \theta$, and various transform size factors:

$$
\begin{aligned}
& \Delta p=\frac{\pi}{z_{\text {max }}}, f_{\text {norm }}=\frac{2}{n_{f f t}}, n_{34}=3 / 4 n_{f f t}, \\
& \Delta \theta=\frac{\Delta p}{k_{o}}, \quad n_{m 1}=n_{f f t}-1, n_{4}=1 / 4 n_{f f t},
\end{aligned}
$$

The ALLARRAY_PE SU is then referenced to allocate and initialize all arrays associated with PE calculations.

Next, the horizon range, $r_{\text {hor }}$, for 0 receiver height and the tangent angle, $a_{\text {cut }}$, to the radio horizon are computed:

$$
\begin{gathered}
r_{\text {hor }}=\sqrt{\text { twoka } * a n t_{h t}} \\
a_{c u t}=\mathbf{T A N}^{-1}\left(\frac{a n t_{h t}}{r_{h o r}}\right) .
\end{gathered}
$$


A temporary range step variable is computed as

$$
\Delta r t=55.67485+3.52969 \times 10^{-3} r_{\max }-.01122 \times 10^{-6} r_{\max }^{2}
$$

Due to numerical constraints, limits will be imposed on the PE range step as follows. If performing a terrain case, then the PE range step is computed from

$$
\Delta r_{P E}=\operatorname{MAX}\left(\mathbf{M I N}\left(2 k_{o} \Delta z_{P E}^{2}, 700 .\right), \Delta r t\right)
$$

If $r_{\text {fix }}$ (previously determined in the TERINIT SU) is greater than 0 , then the temporary range step variable $r_{d}$ is given by $r_{d}=\frac{r_{f i x}}{\Delta r_{P E}}$ and $\Delta r_{P E}$ is recomputed according to

$$
\begin{aligned}
& \Delta r_{P E}=\mathbf{N I N T}\left(\frac{1}{r_{d}}\right) r_{f i x} ; \text { for } r_{d}<1, \\
& \Delta r_{P E}=\frac{r_{f i x}}{\operatorname{NINT}\left(r_{d}\right)} ; \text { for } r_{d} \geq 1 .
\end{aligned}
$$

The variable $i z_{i n c}$ is then initialized to 1 .

If no terrain profile is specified, then $\Delta r_{P E}$ is given by

$$
\Delta r_{P E}=\mathbf{M A X}\left(2 k_{o} \Delta z_{P E}^{2}, \Delta r t, 100 .\right)
$$

with the variable $i z_{\text {inc }}$ initialized to 1 for frequencies greater than $10 \mathrm{GHz}, 2$ for frequencies greater than $5 \mathrm{GHz}$, and 3, otherwise.

If the PEINIT SU has been referenced from the GET SU, then the PE range step (which in this case will be used for calculation of the grazing angle by spectral estimation) is further modified:

$$
\Delta r_{P E}=\mathbf{M A X}\left(\Delta r_{P E}, 150 .\right)-1
$$

Otherwise, the range step is multiplied by the range step modifier $r_{\text {mult }}$.

The number of PE range steps is then computed: 


$$
i_{P E}=\mathbf{N I N T}\left(\frac{r_{\max }}{\Delta r_{P E}}\right)+1
$$

If a terrain profile has been specified, the terrain elevations at each PE range step are now interpolated from the user-specified profile and stored in array tyh. The array is allocated for size $i_{P E}$, the range $r$ is initialized to 0 , and the elements in tyh are determined according to

$$
t y h_{i}=t y_{k}+\operatorname{slp}_{k}\left(r-t x_{k}\right) ; \quad i=1,2, \ldots, i_{P E}
$$

where $r$ is $i \Delta r_{P E}$ and the index $k$ is determined such that $t x_{k}<r \leq t x_{k+1}$. The tangent of each angle determined from the terrain slopes are stored in array tang.

The filter array, filt, for subsequent filtering of the PE field, is given by

$$
\text { filt }_{i}=1 / 2+1 / 2 \operatorname{Cos}\left(i \frac{\pi}{n_{4}}\right) ; \quad i=1,2, \ldots ., n_{4}
$$

The PE mesh height array ht is next given by

$$
h t_{i}=i \Delta z_{P E} ; \quad i=1,2, \ldots, n_{f f t},
$$

Next, the free-space propagator array frsp is computed for subsequent use in the PESTEP SU. The propagator term is computed at each PE angle, or p-space, mesh point using the wide-angle propagator. A filter, or attenuation function (frequently called "window"), is then applied to the upper one-quarter of the array corresponding to the highest one-quarter of the maximum propagation angle.

The complex free-space propagator phase array frsp is given by

$$
\operatorname{frsp}_{j}=f_{\text {norm }} e^{i \Delta r_{P E}\left(\sqrt{k_{o}^{2}-(j \Delta p)^{2}}-k_{o}\right)} ; \quad j=0,1,2, \ldots, n_{f f t},
$$

where $I$ is the imaginary number $\sqrt{-1}$. The upper one-quarter of the free-space propagator array is filtered by a cosine-tapered (Tukey) filter array, filt, according to

$$
\text { frsp }_{j}=\text { filt }_{j-n_{34}} \text { frsp }_{j} ; \quad j=n_{34}, n_{34}+1, n_{34}+2, \ldots, n_{f f t} .
$$


If a simple environmental case has been specified with no terrain and a rangeindependent refractivity profile, then the INTPROF SU is referenced for interpolation of the refractivity at every PE mesh point. The z-space propagator array envpr is then computed from

$$
\text { envpr }_{j}=e^{i \Delta r_{P E} \text { profint }_{j}} ; \quad j=0,1,2, \ldots, n_{f f t},
$$

where $I$ is the imaginary number $\sqrt{-1}$ and profint is the sampled profile obtained from the INTPROF SU. The upper $1 / 4$ of envpr is filtered by a cosine-tapered (Tukey) filter array, filt, according to

$$
\text { envpr }_{j}=\text { filt }_{j-n_{34}} \text { envpr }_{j} ; \text { for } j=n_{34}, n_{34}+1, n_{34}+2, \ldots, n_{f f t} \text {. }
$$

Finally, the XYINIT SU is referenced to determine the initial PE solution and the SU is exited.

Table 44 and Table 45 provide identification, description, units of measure, and the computational source for each PEINIT SU input and output data element.

\begin{tabular}{|c|c|c|c|}
\hline Name & Description & Units & Source \\
\hline ant ${ }_{h t}$ & Transmitting antenna height above local ground & meters & Calling CSCI \\
\hline$\Delta z_{P E}$ & Bin width in z-space & meters & FFTPAR SU \\
\hline$f_{M H z}$ & Frequency & $\mathrm{MHz}$ & Calling CSCI \\
\hline$f_{\text {ter }}$ & $\begin{array}{l}\text { Logical flag indicating if terrain profile has been specified: } \\
\text { '.true.' = terrain profile specified } \\
\text { '.false.' = terrain profile not specified }\end{array}$ & N/A & APMINIT CSC \\
\hline$i_{\text {flag }}$ & $\begin{array}{l}\text { Integer flag indicating where in the APMINIT CSC the } \\
\text { PEINIT SU is being referenced } \\
0=\text { called before reference to GETANGLES SU } \\
1=\text { called for "real” PE run }\end{array}$ & N/A & Calling SU \\
\hline$i_{p l}$ & $\begin{array}{l}\text { Polarization flag } \\
\qquad \begin{array}{l}0=\text { horizontal } \\
1\end{array}=\text { vertical }\end{array}$ & N/A & Calling SU \\
\hline$i_{\text {tpa }}$ & $\begin{array}{l}\text { Number of height/range points pairs in terrain profile arrays } \\
t x, t y\end{array}$ & N/A & TERINIT SU \\
\hline$k_{o}$ & Free-space wavenumber & meters $^{-1}$ & APMINIT CSC \\
\hline$n_{f f t}$ & Transform size & N/A & FFTPAR SU \\
\hline$n_{\text {prof }}$ & Number of refractivity profiles & N/A & Calling CSCI \\
\hline
\end{tabular}

Table 44. PEINIT SU input data element requirements. 
Table 44. PEINIT SU input data element requirements. (continued)

\begin{tabular}{c|l|c|c}
\hline Name & \multicolumn{1}{|c|}{ Description } & Units & Source \\
\hline$P E_{\text {flag }}$ & Flag to indicate use of PE algorithm only: & N/A & Calling CSCI \\
& \multicolumn{1}{|c}{ '.false.' = unly use PE sub-model } \\
$r_{\text {fix }}$ & Fixed range increment of terrain profile & & \\
$r_{\max }$ & Maximum specified range & meters & Calling SU \\
$r_{\operatorname{mult}}$ & PE range step multiplication factor & meters & Calling CSCI \\
$s l p$ & Slope of each segment of terrain & N/A & Calling CSCI \\
$t w o k a$ & Twice the effective earth radius & N/A & TERINIT SU \\
$t x$ & Range points of terrain profile & meters & GET_K SU \\
$t y$ & Adjusted height points of terrain profile & meters & TERINIT SU \\
$z_{\max }$ & Total height of the FFT/PE calculation domain & meters & TERINIT SU \\
\hline
\end{tabular}

Table 45. PEINIT SU output data element requirements.

\begin{tabular}{|c|c|c|}
\hline Name & Description & Units \\
\hline$a_{\text {cut }}$ & Tangent angle from antenna height to radio horizon & radians \\
\hline$\Delta r_{P E}$ & PE range step & meters \\
\hline$\Delta p$ & P-space mesh size & $\begin{array}{r}\text { radians/ } \\
\text { meters }\end{array}$ \\
\hline$\Delta \theta$ & Angle bin width & radians \\
\hline envpr & Complex [refractivity] phase term array interpolated every $\Delta z_{P E}$ in height & N/A \\
\hline filt & Cosine-tapered (Tukey) filter array & N/A \\
\hline$f_{\text {norm }}$ & Normalization factor & N/A \\
\hline frsp & Complex free space propagator term array & N/A \\
\hline$h t$ & PE mesh height array of size $n_{f f t}$ & meters \\
\hline$i_{\text {error }}$ & $\begin{array}{l}\text { Integer variable indicating error number for ALLOCATE and DEALLOCATE } \\
\text { statements }\end{array}$ & N/A \\
\hline$i_{P E}$ & Number of PE range steps & N/A \\
\hline$i z_{i n c}$ & $\begin{array}{l}\text { Integer increment for storing points at top of PE region (i.e., points are stored at } \\
\text { every } i z_{i n c} \text { range step) }\end{array}$ & N/A \\
\hline$n_{34}$ & $3 / 4 n_{f f t}$ & N/A \\
\hline$n_{4}$ & $1 / 4 n_{f f t}$ & N/A \\
\hline$n_{m 1}$ & $n_{f f t}-1$ & N/A \\
\hline$r_{\text {hor }}$ & Radio horizon range & meters \\
\hline tang & Tangent of angle array from terrain slopes. & radians \\
\hline tyh & Adjusted height points of terrain profile at every PE range step. & meters \\
\hline$U$ & Complex PE field & $\mu \mathrm{V} / \mathrm{m}$ \\
\hline
\end{tabular}




\subsubsection{Poly 4 (FN_POLY4) Function}

The real, double precision function FN_POLY4 evaluates a $4^{\text {th }}$ order polynomial in the independent variable, $X$, for the SURFIMP SU. It returns the real (imaginary) part of the complex normalized surface impedance to the SURFIMP SU. The expression evaluated is

$$
f x=\text { const }_{5} X^{4}+\text { const }_{4} X^{3}+\text { const }_{3} X^{2}+\text { const }_{2} X+\text { const }_{1} .
$$

Variables passed to the POLY4 function by the SURFIMP SU are the frequency in megahertz and the array of five coefficients needed to evaluate the polynomial.

Table 46 and Table 47 provide identification, description, units of measure, and the computational source for each FN_POLY4 function input and output data element.

Table 46. FN_POLY4 input data element requirements.

\begin{tabular}{c|l|c|c}
\hline Name & \multicolumn{1}{|c|}{ Description } & Units & Source \\
\hline const4 & Polynomial coefficients array & See a & Calling SU \\
$X$ & Independent variable (frequency) & $\mathrm{MHz}$ & Calling SU \\
\hline${ }^{\mathrm{a}} 1 /(\mathrm{MHz})^{\mathrm{n}-1}$ where $\mathrm{n}=1,2,3,4,5$
\end{tabular}

Table 47. FN_POLY4 output data element requirements.

\begin{tabular}{c|c|c}
\hline Name & Description & Units \\
\hline$f x$ & Real (imaginary) part of normalized surface impedance & N/A \\
\hline
\end{tabular}

\subsubsection{Poly 5 (FN_POLY5) Function}

The real, double-precision function FN_POLY5 evaluates a 5th order polynomial in the independent variable, $X$, for the SURFIMP SU. Each call of the routine returns one element of the array const4, which is used in the FN_POLY4 SU. The expression evaluated is

$$
f x=\text { const } 5_{6} X^{5}+\text { const } 5_{5} X^{4}+\text { const } 5_{4} X^{3}+\text { const } 5_{3} X^{2}+\text { const } 5_{2} X+\text { const } 5_{1} \text {. }
$$

Variables passed to the FN_POLY5 function by the SURFIMP SU are the wind speed in knots and the set of six polynomial coefficients needed to evaluate the polynomial.

Table 48 and Table 49 provide identification, description, units of measure, and the computational source for each FN_POLY5 function input and output data element. 
Table 48. FN_POLY5 input data element requirements.

\begin{tabular}{c|l|c|c}
\hline Name & Description & Units & Source \\
\hline const5 & Polynomial coefficients array & See a & Calling SU \\
$X$ & Independent variable (wind speed) & knots & Calling SU \\
\hline${ }^{\mathrm{a}} 1 /(\mathrm{MHz})^{\mathrm{n}-1}(\mathrm{knots})^{\mathrm{n}-1}$ where $\mathrm{n}=1,2,3,4,5,6$
\end{tabular}

Table 49. FN_POLY5 output data element requirements.

\begin{tabular}{c|c|c}
\hline Name & Description & Units \\
\hline$f x$ & Element of the array const4 & See a \\
\hline${ }^{\mathrm{a}} 1 /(\mathrm{MHz})^{\mathrm{n}-1}$ where $\mathrm{n}=1,2,3,4$ or 5
\end{tabular}

\subsubsection{Profile Reference (PROFREF) SU}

The PROFREF SU adjusts the current refractivity profile so that it is relative to a reference height, $y_{\text {ref. }}$ The reference height is initially the minimum height of the terrain profile. Upon subsequent calls from the PESTEP SU, the refractivity profile is adjusted by the local ground height at each PE range step.

The reference height $y_{\text {ref }}$, depending on the value of $i_{\text {flag }}$, can be $h_{\text {minter }}$ or the local ground height above $h_{\text {minter }}$. If $i_{\text {flag }}$ is 0 , the profile arrays refref and href will be relative to $h_{\text {minter }}$ and will also be used to initialize refdum and htdum . If $\square$ is 1 , then the profile arrays refref and href will be referenced to the local ground height. The parameter $h_{\text {minter }}$ is the reference height for internal calculations in the APM CSCI of the complex field $U$. Arrays refdum and htdum are dummy arrays containing refractivity values and height values, respectively, for the currently interpolated profile.

Determining refref and href proceeds as follows. First, the index nlvl is initialized to the number of refractivity levels, lvlep, in refdum and htdum; and refref and href are initialized to zero. Next, a test is made to determine whether the absolute value of the reference height $y_{\text {ref }}$ is greater than $10^{-3}$ (i.e., is $y_{\text {ref }}$ greater than approximately 0 ). If $y_{\text {ref }}$ is approximately zero, the elements of refref are set equal to the corresponding M-unit values of refdum, and the elements of href are set equal to the corresponding height values of htdum and the SU is exited.

For the case when $y_{r e f}$ is not zero, the following calculations are made. First, the flag $i_{b m s l}$ and the index $j_{s}$ are set equal to zero and minus one, respectively. Then, $y_{r e f}$ is tested to determine if it is below mean sea level. If so, $i_{b m s l}$ and $j$ are set equal to one and zero, respectively. If $y_{\text {ref }}$ is not below mean sea level, then the refractivity profile level at which $y_{\text {ref }}$ is just above is determined. The index $j$ is determined such that htdum $_{j}<y_{\text {ref }} \leq$ htdum $_{j+1}$. 
The refractivity at $y_{r e f}$ is now computed from

$$
r m u=\text { refdum }_{j}+\left(\text { refdum }_{j+1}-\text { refdum }_{j}\right) \frac{y_{\text {ref }}-\text { htdum }}{\text { htdum }_{j+1}-\text { htdum }_{j}} .
$$

If $y_{\text {ref }}$ falls below mean sea level and the extrapolation flag $i_{e x t r a}$ is zero, then $r m u$ is given by

$$
r m u=\text { refdum }_{j}+0.118 \frac{y_{\text {ref }}-\text { htdum }_{j}}{\text { htdum }_{j+1}-\text { htdum }_{j}} .
$$

The first element in refref and href is now set equal to rmu and 0, respectively. The number of refractivity levels in the arrays is now $l_{\text {new }}=n l v l-j$ and the remainder of the current refractivity profile is adjusted in height and stored in refref and href according to

$$
\begin{aligned}
\text { refref }_{i} & =\text { refdum }_{k} \\
\text { href }_{i} & =\text { htdum }_{k}-y_{\text {ref }} ; i=1,2,3, \ldots, l_{\text {new }},
\end{aligned}
$$

where the index $k$ is initialized to $j+1$ and is incremented by one with each iteration of $i$. The variable $n l v l$, indicating the number of levels in the newly created profile, is now set to $l_{\text {new. }}$.

Finaly, if $i_{\text {flag }}$ equals zero, then lvlep is set equal to $n l v l$ and refref and href are used to initialize refdum and htdum, respectively, before exiting.

Table 50 and Table 51 provide identification, description, units of measure, and the computational source for each PROFREF SU input and output data element.

Table 50. PROFREF SU input data element requirements.

\begin{tabular}{c|l|c|c}
\hline Name & \multicolumn{1}{|c|}{ Description } & Units & Source \\
\hline htdum & Height array for current interpolated profile & meters & REFINTER SU \\
$i_{\text {extra }}$ & $\begin{array}{l}\text { Extrapolation flag for refractivity profiles entered below } \\
\text { mean sea level } \\
0=\text { extrapolate to minimum terrain height standard } \\
\text { atmosphere gradient } \\
1=\text { extrapolate to minimum terrain height using first } \\
\text { gradient in profile }\end{array}$ & & \\
& $\begin{array}{l}\text { Calling CSCI } \\
\end{array}$ & &
\end{tabular}


Table 50. PROFREF SU input data element requirements. (continued)

\begin{tabular}{c|l|c|c}
\hline Name & \multicolumn{1}{|c|}{ Description } & Units & Source \\
\hline$i_{\text {flag }}$ & $\begin{array}{l}\text { Integer flag indicating height at which to reference the } \\
\text { refractivity profile } \\
0=\text { adjust profile relative to } h_{\text {minter }} \\
1=\text { adjust profile relative to local ground height above } \\
h_{\text {minter }}\end{array}$ & N/A & Calling SU \\
& $\begin{array}{c}\text { Number of height/refractivity levels in profile refdum and } \\
\text { lvlep }\end{array}$ & N/A & Calling CSCI \\
refdum & $\begin{array}{l}\text { M-unit array for current interpolated profile } \\
y_{\text {ref }}\end{array}$ & Ground elevation height at current range & M-units \\
\hline
\end{tabular}

Table 51. PROFREF SU output data element requirements.

\begin{tabular}{c|l|c}
\hline Name & \multicolumn{1}{|c|}{ Description } & Units \\
\hline href & Height array for current interpolated profile & meters \\
htdum & Dummy array containing height values for current (horizontally interpolated) & meters \\
& profile & \\
lvlep & Number of height/refractivity levels in profile & N/A \\
$n l v l$ & Number of levels in new profile & N/A \\
refdum & M-unit array for current interpolated profile & M-units \\
refref & Refractivity array & M-units \\
\hline
\end{tabular}

\subsubsection{Refractivity Initialization (REFINIT) SU}

The REFINIT SU checks for valid environmental profile inputs and initializes all refractivity arrays used within one application of APM.

Upon entering, the maximum height $h_{\text {large }}$ at which the refractivity profile is extrapolated is set to $10^{6}$ meters in a DATA statement. In addition, $i_{\text {error }}$ is initialized to zero.

The environmental data are checked to determine if range-dependent profiles have been specified $\left(n_{\text {prof }}>1\right)$. If so, the range of the last profile entered, rngprof $f_{n_{\text {prof }}}$ is checked, and if it is less than the maximum output range specified, $r_{\max }$, an error message is returned (i.e., $i_{\text {error }}$ is set equal to -12) depending on the value of the error flag, lerr12, set in the calling CSCI application itself. The SU is then exited; otherwise, if no error occurs, the SU proceeds to the next step. 
Next, the REFINIT SU tests for valid refractivity level entries for each profile. Every user-specified profile is tested to make sure the first level in the profile begins with a value of zero height (or less than zero, if the first level is below mean sea level). If it does not, $i_{\text {error }}$ is set to -13 and the SU is exited; otherwise, the SU proceeds to the next step.

A test is then made to determine if the last gradient in each profile is negative. If the last gradient in any profile is negative, $i_{\text {error }}$ is set to -14 and the SU is exited; otherwise, an additional refractivity level is extrapolated to height $h_{\text {large }}$ and added to each profile. The additional level is added according to

$$
\begin{aligned}
h m s l_{l v l p, i} & =h_{\text {large }}, \\
r e f m s l_{l v l p, i} & =r e f m s l_{l v l p-1, i}+\operatorname{grd}\left[h_{\text {large }}-h m s l_{l v l p-1, i}\right] \quad i=1,2,3, \ldots n_{p r o f},
\end{aligned}
$$

where

$$
g r d=\frac{r e f m s l_{l v l p-1, i}-r e f m s l_{l v l p-2, i}}{h m s l_{l v l p-1, i}-h m s l_{l v l p-2, i}} .
$$

The counter for the current profile, $i_{s}$, is now initialized to 1 and the range of the next refractivity profile, $r v_{2}$, is initialized to $r n g p r o f_{i_{s}}$. Next, the results of the extrapolation of the first environmental profile (i.e., the profile at range 0 ) are transferred to dummy arrays, htdum and refdum, respectively. The index lvlep is now set equal to lvlp. Duplicate levels in the first profile are removed by a reference to the REMDUP SU, and refdum and htdum are adjusted to the minimum terrain height by a reference to the PROFREF SU. The parameter nlvl, returned from the PROFREF SU, is now the number of height/refractivity levels in the adjusted htdum and refdum arrays.

If troposcatter calculations have been specified $\left(T_{\text {ropo }}=\right.$ '.true.'), then the surface refractivity at the transmitter snref $f_{t x}$ is determined by referencing the PROFREF SU to adjust the profile relative to $y_{f r e f}$ and initializing snref $f_{t x}$ to refrefo.

Next, the height and thickness of the highest trapping layer (if one exists), $h_{\text {trap }}$ and $h_{\text {thick }}$, respectively, are found relative to $h_{\text {minter }}$. First, $h_{\text {trap }}$ and $h_{\text {thick }}$ are initialized to zero. Then, steps 1 through 2 are performed for each $i^{\text {th }}$ profile and for each $j^{\text {th }}$ refractivity level.

1. The gradient of the current height/refractivity level grd and its height relative to $h_{\text {minter }}, h_{p 1}$, are found from

$$
\begin{aligned}
& g r d=\text { refmsl }_{j+1, i}-\text { refmsl } \\
& j, i \\
& h_{p 1}=\text { hmsl }_{j+1, i}-h_{\text {minter }}
\end{aligned} .
$$


2. If $g r d$ is negative and $h_{p 1}$ is greater than $h_{\text {trap }}$, then $h_{\text {trap }}$ is set equal to $h_{p 1}$, and $h_{p 0}$ and $h_{\text {thick }}$ are determined from

$$
\begin{aligned}
& h_{p 0}=h_{m s} l_{j, i}-h_{\text {minter }} \\
& h_{\text {thick }}=h_{p 1}-h_{p 0}
\end{aligned}
$$

Next, the index level $i_{\text {start } 1}$ within the refractivity profile of the antenna height $a n t_{r e f}$ is determined and the gradient array grdum is computed as

$$
\operatorname{grdum}_{i}=10^{-6}\left(\frac{\text { refdum }_{i+1}-\text { refdum }_{i}}{\text { htdum }_{i+1}-\text { htdum }_{i}}\right) ; \quad i=0,1,2, \ldots . ., \text { lvlep }-1 .
$$

If using the full hybrid mode $\left(i_{\text {hybrid }}=1\right.$ ), then follow steps 1 through 4 to build arrays associated with RO calculations. Otherwise, the M-unit value $r m_{t x}$ at the antenna height is determined from

$$
r m_{t x}=10^{-6}\left[\operatorname{refdum}_{i_{\text {start } 1}}+\operatorname{grdum}_{i_{\text {start } 1}} 10^{6}\left(\text { ant }_{r e f}-\text { htdum }_{i_{\text {start } 1}}\right)\right]
$$

and the SU continues with the procedures after step 4.

1. First, the refractivity and height arrays $r m$ and zrt are built. All elements in zrt are set equal to all elements in htdum. An additional height level, equal to $a n t_{r e f}$, is included in $z r t$ and the index $i_{\text {start }}$ is initialized to that height level that corresponds to ant $t_{r e f}$. Array $r m$ is given by

$$
r m_{i}=10^{-6} \text { refdum }_{i}, \quad i=1,2,3, \ldots . ., n l v l,
$$

with the refractivity level at height $a n t_{r e f}$ interpolated according to

$$
r m_{i_{\text {start }}}=r m_{i_{\text {start }}+1}+\left(a n t_{r e f}-z r t_{i_{\text {start }}-1}\right)\left(\frac{r m_{i_{\text {start }}+1}-r m_{i_{\text {start }}-1}}{z r t_{i_{\text {start }}+1}-z r t_{i_{\text {start }}-1}}\right) .
$$

The total number of levels levels in zrt is reduced by 1 since the highest level is not needed.

2. For the special case when the terrain profile is initially flat but at non-zero height, perform steps 2.a through 2.d to adjust the refractivity arrays $\mathrm{rm}$ and zrt associated with RO calculations. First, the index nlevel is initialized to the number of refractivity 
levels, levels; $y_{\text {ref }}$ is initialized to ty $y_{1}$; refref and href are initialized to zero; and the index $j s$ is initialized to -1 .

a. Next, js is determined such that $z r t_{j s}<y_{r e f} \leq z r t_{j s+1}$. If a value for js is not found such that this condition holds true (i.e., js remains at -1 ), then the SU proceeds with step 2.d.

b. The refractivity at $y_{\text {ref }}$ is now computed from

$$
\begin{gathered}
f_{r a c}=\frac{y_{r e f}-z r t_{j s}}{z r t_{j s+1}-z r t_{j s}}, \\
r m u=r m_{j s}+f_{r a c}\left(r m_{j s+1}-r m_{j s}\right) .
\end{gathered}
$$

If $\operatorname{INT}\left(f_{\text {rac }}\right)$ is equal to 1 , then $j s$ is set equal to $j s+1$. The temporary counter $l_{\text {new }}$ is initialized to nlevel-js.

c. The first element in refref and href is now set equal to $r m u$ and 0 , respectively. The remainder of the current refractivity profile is adjusted in height and stored in refref and href according to

$$
\begin{aligned}
\text { refref }_{j} & =r m_{k} \\
\text { href }_{j} & =\mathrm{zrt}_{k}-y_{\text {ref }} ; \quad j=1,2,3, \ldots l_{\text {new }},
\end{aligned}
$$

where the index $k$ is initialized to $j s+1$ at the start and is incremented by one with each iteration of $j$. The variable levels, indicating the number of levels in the newly created profile, is now set to $l_{\text {new }}$. refref and href are now used to initialize $r m$ and zrt.

d. The variable $i_{\text {start }}$ is now reduced by the amount $j s$.

3. The arrays $g r$ and $q$, used in $\mathrm{RO}$ and ray-tracing calculations, are determined next. The gradient array $g r$ is given by

$$
g r_{i}=\frac{r m_{i+1}-r m_{i}}{z r t_{i+1}-z r t_{i}} ; i=0,1,2, \ldots \text { levels }
$$

The array $q$ is given by

$$
q_{i}=2\left(r m_{i+1}-r m_{i}\right) ; \quad i=0,1,2, \ldots, \text { levels } .
$$


4. The M-unit value $r m_{t x}$ at the antenna height is now set equal to $r m_{i_{\text {start }}}$.

Next, the minimum M-unit value $r_{\min }$ of the refractivity at range 0 is determined by searching for the minimum numerical value in arrray refdum and assigning $\mathrm{rm}_{\min }$ this value. The maximum M-unit value $r m_{\max }$ at or below the antenna height is then determined from

$$
r m_{\max }=\mathbf{M A X}\left(10^{6} \mathrm{rm}_{t x}, \text { refdum }_{i}\right), \quad i=0,1,2, \ldots, i_{\text {start } 1} .
$$

Both $\mathrm{rm}_{\min }$ and $\mathrm{rm}_{\max }$ are then multiplied by $10^{-6}$. If the antenna is within a duct, the flag $l_{\text {duct }}$ is set to '.true.' and the critical angle $a_{\text {crit }}$ is computed as

$$
a_{c r i t}=\sqrt{2\left(r m_{t x}-r_{c r i t}\right)}+10^{-6} \text {, }
$$

where $r_{\text {crit }}$ is the minimum M-unit value in the profile for levels above the height $a n t_{r e f}$.

Finally, a check is made to determine if an evaporation profile exists. This check is performed only if a range-independent profile has been specified $\left(n_{\text {prof }}=1\right)$ and if $h_{\text {trap }}$ is greater than 0 . An evaporation duct is assumed to exist if the height at which $r m_{\min }$ occurs is less than $50 \mathrm{~m}$ and if the second derivative of the profile is greater than an arbitrarily set $100 \mathrm{M}$-units $/ \mathrm{m}^{2}$. If these two conditions occur, then the flag $l_{\text {evap }}$ is set to ‘.true.’.

Table 52 and Table 53 provide identification, description, units of measure, and the computational source for each REFINIT SU input and output data element.

\begin{tabular}{|c|c|c|c|}
\hline Name & Description & Units & Source \\
\hline ant $_{\text {ref }}$ & $\begin{array}{l}\text { Transmitting antenna height relative to the reference } \\
\text { height } h_{\text {minter }}\end{array}$ & meters & TERINIT SU \\
\hline$f_{\text {ter }}$ & $\begin{array}{l}\text { Logical flag indicating if terrain profile has been } \\
\text { specified: } \\
\text { '.true.' = terrain profile specified } \\
\text { '.false.' = terrain profile not specified }\end{array}$ & N/A & APMINIT CSC \\
\hline$h_{\text {minter }}$ & Minimum height of terrain profile & meters & TERINIT SU \\
\hline hmsl & $\begin{array}{l}\text { Two-dimensional array containing heights with respect } \\
\text { to mean sea level of each profile. Array format must be } \\
h m s l_{i, j}=\text { height of } i^{\text {th }} \text { level of } j^{\text {th }} \text { profile; } j=1 \text { for range- } \\
\text { independent cases }\end{array}$ & meters & Calling CSCI \\
\hline
\end{tabular}

Table 52. REFINIT SU input data element requirements. 
Table 52. REFINIT SU input data element requirements. (continued)

\begin{tabular}{|c|c|c|c|}
\hline Name & Description & Units & Source \\
\hline$i_{\text {hybrid }}$ & $\begin{array}{l}\text { Integer indicating which sub-models will be used: } \\
\begin{aligned} 0 & =\text { FEDR }+ \text { PE model } \\
1 & =\text { full hybrid model }(\mathrm{PE}+\mathrm{FE}+\mathrm{RO}+\mathrm{XO}) \\
2 & =\text { partial hybrid model }(\mathrm{PE}+\mathrm{XO})\end{aligned}\end{array}$ & N/A & APMINIT CSC \\
\hline lerr12 & $\begin{array}{l}\text { User-provided error flag that will trap on certain errors if } \\
\text { set to '.true.' }\end{array}$ & N/A & Calling CSCI \\
\hline lvlp & Number of height/refractivity levels in profiles & N/A & Calling CSCI \\
\hline nprof & Number of refractivity profiles & N/A & Calling CSCI \\
\hline refmsl & $\begin{array}{l}\text { Two-dimensional array containing refractivity with } \\
\text { respect to mean sea level of each profile. Array format } \\
\text { must be } r e f m s l_{i, j}=\text { M-unit at } i^{\text {th }} \text { level of } j^{\text {th }} \text { profile; } j=1 \\
\text { for range-independent cases }\end{array}$ & M-unit & Calling CSCI \\
\hline$r_{\max }$ & Maximum range & meters & Calling CSCI \\
\hline rngprof & Ranges of each profile. $r n g p r o f_{i}=$ range of $i^{\text {th }}$ profile & meters & Calling CSCI \\
\hline tery & Dynamically allocated terrain profile height array & meters & Calling CSCI \\
\hline ty & Adjusted height points of terrain profile & meters & TERINIT SU \\
\hline
\end{tabular}

Table 53. REFINIT SU output data element requirements.

\begin{tabular}{|c|c|c|}
\hline Name & Description & Units \\
\hline$a_{\text {crit }}$ & Critical angle & radians \\
\hline$g r$ & Intermediate M-unit gradient array, RO region & (M-unit/m) $10^{-6}$ \\
\hline grdum & Array of refractivity gradients defined by profile htdum and refdum & M-units/meter \\
\hline hmsl & $\begin{array}{l}\text { Two-dimensional array containing heights with respect to mean sea level } \\
\text { of each profile. Array format must be } h m s l_{i, j}=\text { height of } i^{\text {th }} \text { level of } j^{\text {th }} \\
\text { profile; } j=1 \text { for range-independent cases }\end{array}$ & meters \\
\hline htdum & Height array for current interpolated profile & meters \\
\hline$h_{\text {thick }}$ & Thickness of highest trapping layer from all refractivity profiles & meters \\
\hline$h_{\text {trap }}$ & Height of highest trapping layer from all refractivity profiles & meters \\
\hline$i_{\text {error }}$ & Integer value that is returned if any errors exist in input data & N/A \\
\hline$i_{s}$ & Counter for current profile & N/A \\
\hline$i_{\text {start }}$ & RO height index at antenna height & N/A \\
\hline$i_{\text {start1 }}$ & Refractivity level index within $h t d u m$ at $a n t_{r e f}$ & N/A \\
\hline$l_{\text {duct }}$ & $\begin{array}{l}\text { Logical flag indicating if surface-based duct profile has been specified } \\
\text { 'true'. = surface-based duct exists } \\
\text { '.false.' = no surface-based duct exists }\end{array}$ & N/A \\
\hline$l_{\text {evap }}$ & $\begin{array}{l}\text { Logical flag indicating if evaporation duct profile has been specified } \\
\text { '.true'. = evaporation duct exists } \\
\text { '.false.' = no evaporation duct exists }\end{array}$ & N/A \\
\hline levels & Number of levels defined in $z r t, r m, q$, and $g r$ arrays & N/A \\
\hline
\end{tabular}


Table 53. REFINIT SU output data element requirements. (continued)

\begin{tabular}{|c|c|c|}
\hline Name & Description & Units \\
\hline lvlep & Number of height/refractivity levels in profile htdum, refdum & N/A \\
\hline lvlp & $\begin{array}{l}\text { Number of user-specified levels in refractivity profile (for range dependent } \\
\text { case all profiles must have same number of levels) }\end{array}$ & N/A \\
\hline$n l v l$ & Number of height/refractivity levels in profile refref, href & N/A \\
\hline$q$ & Intermediate M-unit difference array, RO region & M-unit $10^{-6}$ \\
\hline refdum & M-unit array for current profile & M-unit \\
\hline refmsl & $\begin{array}{l}\text { Two-dimensional array containing refractivity with respect to mean sea } \\
\text { level of each profile. Array format must be refmsl } l_{i, j}=\text { M-unit at } i^{\text {th }} \text { level of } \\
j^{\text {th }} \text { profile; } j=1 \text { for range-independent cases }\end{array}$ & M-unit \\
\hline$r m$ & Intermediate M-unit array, RO region & M-unit $10^{-6}$ \\
\hline$r m_{\max }$ & Maximum M-unit value of refractivity profile at range 0 & meters \\
\hline$r m_{\min }$ & Minimum M-unit value of refractivity profile at range 0 & meters \\
\hline$r m_{t x}$ & M-unit value at height $a n t_{r e f}$ & meters \\
\hline$r v 2$ & Range of the next refractivity profile & meters \\
\hline snref $f_{t x}$ & Surface refractivity at transmitter & M-unit \\
\hline snref $f_{0}$ & $\begin{array}{l}\text { Surface refractivity taken from of the reference profile with respect to } \\
\text { mean sea level }\end{array}$ & M-unit \\
\hline$z r t$ & Intermediate height array, RO region & meters \\
\hline
\end{tabular}

\subsubsection{Remove Duplicate Refractivity Levels (REMDUP) SU}

The REMDUP SU is to remove any duplicate refractivity levels in the current interpolated profile. Adjoining profile levels are checked to see if the heights are within $0.001 \mathrm{~m}$. If they are, the duplicate level in the profile is removed. This process continues until all profile levels (Ivlep) have been checked.

Table 54 and Table 55 provide identification, description, units of measure, and the computational source for each REMDUP SU input and output data element.

Table 54. REMDUP SU input data element requirements.

\begin{tabular}{c|l|c|c}
\hline Name & Description & Units & Source \\
\hline htdum & Height array for current interpolated profile & meters & REFINIT SU \\
REFINTER SU \\
RElep & Number of height/refractivity levels in profile & N/A & REFIN SU \\
REFINTER SU \\
refdum & M-unit array for current interpolated profile & M-unit & REFINIT SU \\
& & & REFINTER SU \\
\hline
\end{tabular}


Table 55. REMDUP SU output data element requirements.

\begin{tabular}{c|l|c}
\hline Name & \multicolumn{1}{|c|}{ Description } & Units \\
\hline htdum & Height array for current interpolated profile & meters \\
lvlep & Number of height/refractivity levels in profile & N/A \\
refdum & M-unit array for current interpolated profile & M-unit \\
\hline
\end{tabular}

\subsubsection{RG Trace (RGTRACE) SU}

The RGTRACE SU performs ray traces of many rays launched within an angle of $\pm 4^{\circ}$. All angles from rays striking the surface are then sorted and stored for subsequent interpolation in the GRAZE_INT SU.

Upon entering the SU, two in-line ray trace functions are defined for general parameters $a, b$, and $g_{r d}$ : RADA1 and RP. These function definitions are identical to those given in Section 5.1.15.

Rays are traced with different angular increments at varying intervals. Angular increments are determined such that 1500 rays will be traced between the angular interval $\pm 0.5^{\circ}$, 1000 rays will be traced with launch angles between $\left|0.5^{\circ}\right|$ and $\left|\theta_{t}\right|$, and 500 rays will be traced for angles between $\left|\theta_{t}\right|$ and $\left|\vartheta_{m x g}\right|$. The angular increments are computed as

$$
\begin{aligned}
& \text { ainc }_{1}=\frac{\theta_{t}}{1500}, \\
& \text { ainc }_{2}=\frac{\theta_{t}}{500}, \\
& \text { ainc }_{3}=\frac{\vartheta_{m x g}-\theta_{t}}{250},
\end{aligned}
$$

where $\theta_{t}$ is $1^{\circ}$ for $i_{\text {hybrid }}$ equal to 1 and $1.5^{\circ}$, otherwise. The maximum number of rays to trace, $n_{\text {ray }}$, is then initialized to a large value of 10 times the amount specifed above.

Next, the grazing angle array $\psi_{\text {ray }}$ is allocated and initialized with the first element set equal to $1 / \pi$. The height of the terrain $y_{t}$ at the current traced range step is initialized to 0 , or $t y h_{1}$ if a terrain profile has been specified ( $f_{\text {ter }}=$ '.true.'). The number of grazing angles $i_{\text {grz }}$ and the launch angle $a_{\text {launch }}$ are initialized to 0 and $-\vartheta_{m x g}$, respectively. A DO loop is now implemented where the following steps 1 through 4 are performed an $n_{\text {ray }}$ number of times.

1. At the start of the ray trace, the current local angle $\left(a_{0}\right)$, range $\left(r_{0}\right)$, height $\left(h_{0}\right)$, and refractive gradient index $(j)$ are initialized to $a_{\text {launch }}, 0$, ant $t_{r e f}$, and $i_{\text {start } 1}$, respectively. The index, it, of the terrain segment at the current traced range is also initialized to 1. 
2. If the antenna height antref is at an inflection point in the refractivity profile and $a_{0}$ is less than 0 , the index $j$ is decremented by 1 . The current range to trace to, $r o$, is initialized to 0 .

3. A loop is now begun to trace a ray starting with launch angle $a_{\text {launch }}$ to every PE range step. The current range to trace to, ro, is incremented by $\Delta r_{P E}$ and steps 0 through 3.e are performed until one of the following conditions are met: $r_{0}$ reaches $r o, h_{0}$ reaches $h t_{l i m}$, or the difference in reflection range between consecutive grazing angles is less than $10^{-3}$. All references to the index $i$ in the steps below refer to the index in this loop varying from 1 to the number of $P E$ range steps, $i_{P E}$.

a. The TRACE_STEP SU is referenced to determine the new angle $\left(a_{1}\right)$, height $\left(h_{1}\right)$, and range $\left(r_{1}\right)$ at the end of the traced step.

b. Once $r_{1}, h_{1}$, and $a_{1}$ have been computed, it must be determined if $h_{1}$ is above the height maximum $h t_{\text {lim }}$. If so, then $h_{1}$ is set equal to $h t_{l i m}$ and a new $a_{1}$ and $r_{1}$ are computed:

$$
\begin{aligned}
& a_{1}=\sqrt{\operatorname{RADA} 1\left(a_{0}, h_{1}-h_{0}\right)} \\
& r_{1}=\operatorname{RP}\left(r_{0}, a_{1}-a_{0}\right) .
\end{aligned}
$$

c. If the ray has hit the surface and is reflected, which would be the condition for which the index $j$ is equal to 0 , then the grazing angle and the angle of reflection with respect to the horizontal are computed as

$$
\begin{aligned}
\psi & =\mathrm{TAN}^{-1}\left(s l p_{i t}\right)-a_{1} \\
a_{r e f} & =2 \operatorname{TAN}^{-1}\left(\operatorname{slp}_{i t}\right)-a_{1} .
\end{aligned}
$$

The range $r_{1}$ and grazing angle are then stored for later use in the GRAZE_INT SU.

d. In preparation for the next ray trace step, $h_{0}$ is set equal to $h_{1}, r_{0}$ is set equal to $r_{1}$, and $a_{0}$ is set equal to $a_{r e f}$. If $a_{0}$ is greater than $1 / \pi$, or if $r_{1}$ has reached $r o$, then the current iteration is exited and the SU proceeds to step 3.e; otherwise, steps 0 through 3.d are repeated until $r_{0}$ reaches ro.

e. The range ro [to trace to] for the next step is incremented by $\Delta r_{P E}$ and step 3 is repeated for all range steps.

4. Once a ray has been traced through the entire $i_{P E}$ number of range steps, $a_{\text {launch }}$ is increased. If $\left|a_{\text {launch }}\right|$ is less than $0.5^{\circ}$, the launch angle is increased by ainc 1 ; 
otherwise, if $\left|a_{\text {launch }}\right|$ is less than $\theta_{t}$, then it is increased by ainc 2 . If neither of these conditions are met, then $a_{\text {launch }}$ is increased by ainc 3 . Steps 1 through 4 are repeated until an $n_{\text {ray }}$ number of rays have been traced.

Finally, the grazing angles are sorted by range and stored in array $\psi_{\text {ray }}$ and the SU is exited.

Table 56 and Table 57 provide identification, description, units of measure, and the computational source for each RGTRACE SU input and output data element.

Table 56. RGTRACE SU input data element requirements.

\begin{tabular}{|c|c|c|c|}
\hline Name & Description & Units & Source \\
\hline$a^{a n t_{\text {ref }}}$ & $\begin{array}{l}\text { Transmitting antenna height relative to the reference height } \\
h_{\text {minter }}\end{array}$ & meters & TERINIT SU \\
\hline$\Delta r_{P E}$ & PE range step & meters & PEINIT SU \\
\hline$f_{\text {ter }}$ & $\begin{array}{l}\text { Logical flag indicating if terrain profile has been specified: } \\
\text { '.true.' = terrain profile specified } \\
\text { '.false.' = terrain profile not specified }\end{array}$ & N/A & APMINIT CSC \\
\hline grdum & $\begin{array}{l}\text { Array of refractivity gradients defined by profile htdum and } \\
\text { refdum }\end{array}$ & $\begin{array}{l}\text { M-units/ } \\
\text { meter }\end{array}$ & REFINTER SU \\
\hline$h_{\max }$ & Maximum output height with respect to mean sea level & meters & Calling CSCI \\
\hline htdum & Height array for current interpolated profile & meters & REFINTER SU \\
\hline$h t_{l i m}$ & $\begin{array}{l}\text { User-supplied maximum height relative to } h_{\text {minter }} \text {, i.e., } h t_{\text {lim }} \\
=h_{\text {max }}-h_{\text {minter }}\end{array}$ & meters & TERINIT SU \\
\hline$i_{\text {hybrid }}$ & $\begin{array}{l}\text { Integer indicating which sub-models will be used: } \\
\begin{aligned} 0 & =\text { FEDR }+ \text { PE model } \\
1 & =\text { full hybrid model }(\mathrm{PE}+\mathrm{FE}+\mathrm{RO}+\mathrm{XO}) \\
2 & =\text { partial hybrid model }(\mathrm{PE}+\mathrm{XO})\end{aligned}\end{array}$ & N/A & APMINIT CSC \\
\hline$i_{\text {start } 1}$ & Refractivity level index within $h t d u m$ at $a n t_{r e f}$ & N/A & REFINIT SU \\
\hline$r_{\max }$ & Maximum output range & meters & Calling CSCI \\
\hline$\vartheta_{m x g}$ & $\begin{array}{l}\text { Maximum PE calculation angle for spectral estimation of } \\
\text { grazing angles }\end{array}$ & radians & APMINIT CSC \\
\hline slp & Slope of each segment of terrain & N/A & TERINIT SU \\
\hline$t x$ & Range points of terrain profile & meters & TERINIT SU \\
\hline
\end{tabular}

Table 57. RGTRACE SU output data elements requirements.

\begin{tabular}{|c|c|c|}
\hline Name & Description & Units \\
\hline$i_{g r z}$ & Number of grazing angles computed from ray trace & N/A \\
\hline$\psi_{\text {ray }}$ & $\begin{array}{l}\text { Two-dimensional array containing grazing angles and corresponding ranges } \\
\text { computed from ray trace }\end{array}$ & $\begin{array}{l}\text { radians, } \\
\text { meters }\end{array}$ \\
\hline
\end{tabular}




\subsubsection{Terrain Initialization (TERINIT) SU}

The TERINIT SU examines and initializes terrain arrays for subsequent use in PE calculations. It tests for and determines a range increment if it is found that range/height points are provided in fixed range increments. The minimum terrain height is determined, and the entire terrain profile is adjusted in height so that all internal calculations are referenced to this height. This is done to maximize the PE transform calculation volume.

First, several variables are initialized. The integer flag, $i_{\text {error }}$, which is returned if any errors exist in input data, is set equal to zero. The maximum tangent ray angle, $\alpha_{u}$, from source to terrain peak along the profile path, is set equal to zero. The minimum height of the terrain profile, $h_{\text {minter }}$, is set equal to zero. The transmitting antenna height, ant $t_{\text {ref }}$, relative to the reference height $h_{\text {minter }}$, is set equal to $a n t_{h t}$. The maximum terrain height, $h_{\text {termax }}$, along the profile path, is set equal to zero.

If performing a terrain case ( $f_{\text {ter }}=$ '.true.'), perform steps 1 through 8; otherwise, the SU proceeds to step 9.

1. First, all terrain range points are checked in array terx to ensure they are steadily increasing. If they are not, the error flag $i_{\text {error }}$ is set equal to -17 and the $\mathrm{SU}$ is exited. Otherwise, the SU proceeds to step 2.

2. Next, a test is made to determine whether the first range value is zero. If it is not, the error flag $i_{\text {error }}$ is set equal to -18 and the $\mathrm{SU}$ is exited. Otherwise, the $\mathrm{SU}$ proceeds to step 3.

3. Next, a test is made to determine if the last range point within the terrain profile meets or exceeds $r_{\max }$. If the logical flag lerr6 is '.true.' and if the condition $t e r x_{i_{t p}}<$ $r_{\max }$ is met, then $i_{\text {error }}$ is set equal to -6 and the SU is exited; otherwise, the SU proceeds to step 4.

4. A check is now made to determine if the specified terrain range points are spaced at fixed increments. In this procedure, three variables, $r d i f_{1}, r_{\text {frac }}$, and $r_{\text {difsum }}$ are initialized to ter $x_{2}$-terx $x_{1}$, zero, and $r d i f_{1}$, respectively. The variable $r d i f_{1}$ is the difference between adjacent terrain point ranges. The variable $r_{\text {frac }}$ is the ratio between adjacent terrain point differences. The variable $r_{\text {difsum }}$ is the running sum of adjacent terrain point differences. The final value for $r_{\text {difsum }}$ and maximum $r_{f r a c}$ are determined as

$$
\begin{aligned}
r d i f_{2} & =\operatorname{MAX}\left(10^{-3}, \text { terx }_{i+1}-\operatorname{ter}_{i}\right) ; i=2,3,4, \ldots ., i_{t p}-1 \\
r_{\text {frac }} & =\frac{r d i f_{2}}{r d i f_{1}} \\
r_{\text {dissum }} & =r_{\text {difsum }}+r d i f_{2},
\end{aligned}
$$


where $r d i f_{1}$ is set equal to the previous value of $r d i f_{2}$ before each subsequent calculation of a new $r d i f_{2}$, and $r_{\text {frac }}$ is the maximum of all ratios computed.

5. If it is determined that the terrain points are spaced at fixed range increments, then the range spacing $r_{f i x}$ is set to this increment. Assuming that the range points are not equally spaced, $r_{f i x}$ is initially set equal to zero. If the value of $r_{\text {frac }}$ is less than 1.05, then $r_{f i x}$ is determined from

$$
r_{f i x}=\mathbf{N I N T}\left(\frac{r_{d i f s u m}}{i_{t p}-1}\right)
$$

6. The minimum height $h_{\text {minter }}$ of the terrain profile is now found and the entire terrain profile is adjusted by $h_{\text {minter }}$ such that this is the new zero reference. The adjusted terrain profile is stored in arrays $t x$ and ty (i.e., ty $=$ tery $-h_{\text {minter }}$ for all elements in tery) . Next, the maximum height $h_{\text {termax }}$ of the terrain is also obtained from tery. If $h_{\text {termax }}$ exceeds $h_{\max }$, then $i_{\text {error }}$ is set equal to -8 and the SU is exited. Otherwise, the SU proceeds with step 7.

7. An extra point is added to the arrays $t x$ and $t y$. If $t x_{i_{t p}}$ is less than $r_{\max }$, then $t x_{i_{\text {tpa }}}$ is set equal to $r_{\max }$ times 1.1. The input index $i_{t p a}$ is the number of terrain points used internally in arrays $t x$ and $t y$. If $t x_{i_{t p}}$ is greater or equal to $r_{\max }$, then $t x_{i_{\text {tpa }}}$ is set equal to $t x_{i_{t p}}$ times 1.1. Finally, the array element $t y_{i_{t p a}}$ is set equal to $t y_{i_{t p}}$.

8. The variable $a n t_{r e f}$ is set equal to $a t_{h t}$ plus $t y_{1}$. Next, the array of terrain slopes, $s l p$, and the maximum tangent ray angle, $\alpha_{u}$, from the source to the terrain peak along the profile path are found as follows. The slope, $s l p_{i}$, for each $i^{\text {th }}$ terrain segment is given by

$$
\operatorname{slp} p_{i}=\frac{t y_{i+1}-t y_{i}}{\operatorname{MAX}\left(t x_{i+1}-t x_{i}, 10^{-5}\right)} ; \quad i=1,2,3, \ldots . ., i_{t p a}-1
$$

If the current slope is greater than the slope tolerance of $10^{-5}$, then it is assumed that the terrain profile is no longer flat at the current range and the variable $r_{f l a t}$ is set equal to $t x_{i}$. Next, if the value of $t y_{i}$ is greater than antref, then the maximum tangent angle $\alpha_{u}$ from the source to each terrain point is calculated as

$$
\alpha_{u}=\mathbf{M A X}\left[\mathbf{T A N}^{-1}\left(\frac{t y_{i}-a n t_{r e f}}{t x_{i}}\right)\right] ; \quad i=1,2,3, \ldots, i_{t p a}-1
$$

After $\alpha_{u}$ is determined, $0.5^{\circ}$ is added to its value. 
9. Before exiting, the minimum height $h m_{\text {ref }}$ relative to $h_{\text {minter }}$ is found from the difference between the minimum specified output height $h_{\min }$ and $h_{\text {minter }}$. The maximum height limit $h t_{l i m}$ relative to $h_{\text {minter }}$ is given by the difference between $h_{\max }$ and $h_{\text {minter }}$. If the antenna height $a n t_{\text {ref }}$ is greater than $h t_{\text {lim }}$, the error code $i_{\text {error }}$ is set to -9 .

Table 58 and Table 59 provide identification, description, units of measure, and the computational source for each TERINIT SU input and output data element.

Table 58. TERINIT SU input data element requirements.

\begin{tabular}{c|l|c|c}
\hline Name & \multicolumn{1}{|c|}{ Description } & Units & Source \\
\hline$a n t_{h t}$ & Transmitting antenna height above local ground & meters & Calling CSCI \\
$f_{t e r}$ & $\begin{array}{c}\text { Logical flag indicating if terrain profile has been specified: } \\
\text { '.true.' = terrain profile specified }\end{array}$ & N/A & APMINIT CSC \\
& \multicolumn{1}{|c}{ '.false.' = terrain profile not specified } & & \\
$h_{\max }$ & Maximum output height with respect to mean sea level & meters & Calling CSCI \\
$h_{\min }$ & Minimum output height with respect to mean sea level & meters & Calling CSCI \\
$i_{t p}$ & Number of height/range points in profile & N/A & Calling CSCI \\
$i_{t p a}$ & Number of height/range points pairs in profile $t x, t y$ & N/A & APMINIT CSC \\
lerr6 & User-provided error flag that will trap on certain errors if set & N/A & Calling CSCI \\
& to '.true.' & meters & Calling CSCI \\
$r_{\max }$ & Maximum output range & meters & Calling CSCI \\
$t e r x$ & Range points of terrain profile & meters & Calling CSCI \\
tery & Height points of terrain profile & &
\end{tabular}

Table 59. TERINIT SU output data element requirements.

\begin{tabular}{c|l|c}
\hline Name & \multicolumn{1}{|c|}{ Description } & Units \\
\hline$\alpha_{u}$ & Maximum tangent ray angle from the source to the terrain peak along profile & radians \\
height & Transmitting antenna height relative to the reference height $h_{\text {minter }}$ & meters \\
$h_{\text {minter }}$ & Minimum height of terrain profile & meters \\
$h m_{\text {ref }}$ & Height relative to $h_{\text {minter }}$ & meters \\
$h t_{l i m}$ & User-supplied maximum height relative to $h_{\text {minter }}$, i.e., $h t_{\text {lim }}=h_{\text {max }}-h_{\text {minter }}$ & meters \\
$h t e r m a$ & Maximum terrain height along profile path & meters \\
$x$ & & \\
$i e r r o r$ & Integer value that is returned if errors exist in input data & N/A \\
$r f i x$ & Fixed range increment of terrain profile & meters \\
$r f l a t$ & Maximum range at which the terrain profile remains flat from the source & meters \\
slp & Slope of each segment of terrain & N/A \\
$t x$ & Range points of terrain profile & meters \\
$t y$ & Adjusted height points of terrain profile & meters \\
\hline
\end{tabular}


Table 59. TERINIT SU output data element requirements. (continued)

\begin{tabular}{c|l|c}
\hline Name & \multicolumn{1}{|c|}{ Description } & Units \\
\hline$h_{\text {termax }}$ & Maximum terrain height along profile path & meters \\
$i_{\text {error }}$ & Integer value that is returned if errors exist in input data & N/A \\
$r_{f i x}$ & Fixed range increment of terrain profile & meters \\
$r_{f l a t}$ & Maximum range at which the terrain profile remains flat from the source & meters \\
$s l p$ & Slope of each segment of terrain & N/A \\
$t x$ & Range points of terrain profile & meters \\
$t y$ & Adjusted height points of terrain profile & meters \\
\hline
\end{tabular}

\subsubsection{Trace to Output Range (TRACE_ROUT) SU}

The TRACE_ROUT SU traces a single ray whose launch angle is specified by the calling routine to each output range. The height of this ray is stored at each output range for subsequent proper indexing and accessing of the appropriate sub-models.

Upon entering the SU, four in-line ray trace functions are defined for general parameters $a, b, c$, and $g_{r d}$ : RADA1, AP, RP, and HP. These function definitions are identical to those given in Section 5.1.15.

Next, the propagation angle, range, and height at the beginning of the range step, $a_{0}, r_{0}, h_{0}$, respectively, are initialized to $a_{s}, r_{s}$, and $h_{s}$-the angle, range and height specified by the calling SU. The profile index $j$ is also initialized to $j_{s}$ from the calling SU. The index $j_{r}$ is determined such that rngout $_{j_{r}}$ is the first range point greater than $r_{0}$. The array harray, containing the heights of the traced ray at each output range, is then set equal to 0 for elements 1 through $j_{r}$. Perform steps 1 through 2 for each output range step $i$ from $j_{r}$ to $n_{\text {rout }}$.

1. First, harray $j_{j_{r}}$ is set equal to zero and the variable ro is set equal to $r n g o u t_{j_{r}}$. Next, perform steps 1.a through 1.d until $r_{0}$ has reached ro or $h_{0}$ has reached $h t_{l i m}$.

a. First, the range, $r_{1}$, at the end of the ray trace segment is set equal to ro. Then the current gradient $g_{r d}$ is set equal to $\operatorname{grdum}_{j}$. The angle, $a_{1}$, at the end of the ray trace segment is found from $\operatorname{AP}\left(a_{0}, r_{1}-r_{0}\right)$.

b. If $a_{1}$ is of the opposite sign as $a_{0}$, then $a_{1}$ is set equal to zero and $r_{1}$ is given by $\mathrm{RP}\left(r_{0}, a_{1}-a_{0}\right) . h_{1}$ is then given by $\operatorname{HP}\left(h_{0}, a_{1}, a_{0}\right)$. 
c. Now the value of $h_{1}$ is tested. If the value of $h_{1}$ is greater than or equal to $h_{t d u m_{j+1}}$, then $h_{1}$ is set equal to the minimum of $h t_{\text {lim }}$ or $h t d u m_{j+1}$, and $a_{1}, r_{1}$ and the index $j$ are re-computed as

$$
\begin{aligned}
a_{1} & =\sqrt{\mathbf{R A D A} 1\left(a_{0}, h_{1}-h_{0}\right)} \\
r_{1} & =\mathbf{R P}\left(r_{0}, a_{1}-a_{0}\right) \\
j & =\mathbf{M I N}(\text { lvlep }, j+1) .
\end{aligned}
$$

d. If $h_{1}$ is less than $h t d u m_{j+1}$ but greater than $h t_{\text {lim }}$, then $h_{1}$ is set equal to $h t_{\text {lim }}$ and $a_{1}$ and $r_{1}$ are re-computed as in step 1.c above. The angle, range and height variables $a_{0}, r_{0}$, and $h_{0}$ are now set equal to $a_{1}, r_{1}$, and $h_{1}$ in preparation for the next step. Steps 1.a through 1.d are repeated until $r_{0} \geq$ ro.

2. Once $r_{0}$ has reached ro, harray $_{j_{r}}$ is then set equal to $h_{0}$. The index $j_{r}$ is then incremented by 1 and steps 1 through 2 are repeated for all output range steps or until $h_{0}$ has reached $h t_{\text {lim }}$.

Finally, if the traced ray has reached $h t_{\text {lim }}$ at a range before $r_{\max }$, then harray is set equal to $h t_{\text {lim }}$ for elements from $j_{r}$ to $n_{\text {rout }}$, with the index $i_{h m x}$, indicating the element in harray where this occurs, set equal to $j_{r}$.

Table 60 and Table 61 provide identification, description, units of measure, and

\begin{tabular}{|c|c|c|c|}
\hline Name & Description & Units & Source \\
\hline$a_{s}$ & Propagation angle for start of ray trace & radians & Calling SU \\
\hline grdum & $\begin{array}{l}\text { Array of refractivity gradients defined by profile htdum and } \\
\text { refdum }\end{array}$ & $\begin{array}{l}\text { M-units/ } \\
\text { meter }\end{array}$ & $\begin{array}{l}\text { REFINTER SU } \\
\text { REFINIT SU }\end{array}$ \\
\hline$h_{s}$ & Height for start of ray trace & meters & Calling SU \\
\hline htdum & Height array for current interpolated profile & meters & $\begin{array}{l}\text { REFINTER SU } \\
\text { REFINIT SU }\end{array}$ \\
\hline$h t_{l i m}$ & $\begin{array}{l}\text { User-supplied maximum height relative to } h_{\text {minter }} \text {, i.e., } h t_{\text {lim }} \\
=h_{\text {max }}-h_{\text {minter }}\end{array}$ & meters & TERINIT SU \\
\hline$j_{s}$ & Refractive profile index for start of ray trace & N/A & Calling SU \\
\hline lvlep & Number of height/refractivity levels in profile htdum, refdum & N/A & REFINIT SU \\
\hline$n_{\text {rout }}$ & Number of output height points desired & N/A & Calling CSCI \\
\hline rngout & Array containing all desired output ranges & meters & APMINIT CSC \\
\hline & Range for start of ray trace & meters & Calling SU \\
\hline
\end{tabular}
the computational source for each TRACE_ROUT SU input and output data element.

Table 60. TRACE_ROUT SU input data element requirements. 
Table 61. TRACE_ROUT SU output data element requirements.

\begin{tabular}{c|l|c}
\hline Name & \multicolumn{1}{|c|}{ Description } & Units \\
\hline harray & Array containing heights of traced ray at every output range & meters \\
$i_{h m x}$ & Index in harray where traced height has reached $h t_{l i m}$ & N/A \\
\hline
\end{tabular}

\subsubsection{Trace to next Step (TRACE_STEP) SU}

This SU performs one ray trace step, that, given a starting angle $\left(a_{0}\right)$, range $\left(r_{0}\right)$, and height $\left(h_{0}\right)$, will trace to the first boundary that occurs (refractivity level or surface). It then passes back the ending angle, range, and height for this step and a flag indicating if the ray has hit the surface.

Upon entering the SU, two in-line ray trace functions are defined for general parameters $a, b, c$, and $g_{r d}$ : RADA1, AP, HP, and RP. These function definitions are identical to those in Section 5.1.15.

From the RGTRACE SU, the range $r_{1}$ has already been incremented and is passed as part of the argument list to this SU. If the $r_{1}$ is greater than the next range point in the terrain profile, then $r_{1}$ is set equal to the range value of the next terrain point.

The refractive gradient $g_{r d}$ within the current range step is initialized to grdum $_{j}$. If the gradient is less than $10^{-6}$, then the new angle $a_{1}$ is set equal to the starting angle $a_{0}$ and the new height is computed as

$$
h_{1}=h_{0}+\left(r_{1}-r_{0}\right) \mathbf{T A N}\left(a_{0}\right) \text {. }
$$

If the new height $h_{1}$ is greater than the next level in the refractivity profile, then $h_{1}$ is set equal to the next height in the profile and $r_{1}$ is re-computed as

$$
r_{1}=r_{0}+\frac{\left(h_{1}-h_{0}\right)}{\operatorname{TAN}\left(a_{0}\right)}
$$

If the gradient is greater than $10^{-6}$, then the propagation angle $a_{1}$ at the end of the range step is computed by $\mathbf{A P}\left(a_{0}, r_{1}-r_{0}\right)$. Next, if $\left|a_{0}\right|$ is less than $10^{\circ}$, then if $a_{0}$ and $a_{1}$ differ in sign, $a_{1}$ is set equal to 0 and $r_{1}$ is computed from $\mathbf{R P}\left(r_{0}, a_{1}-a_{0}\right)$. The height of the ray at the end of the range step is next computed by $\mathbf{H P}\left(h_{0}, a_{1}, a_{0}\right)$. If $\left|a_{0}\right|$ is greater than $10^{\circ}$, then $a_{1}$ is set equal to $a_{0}$ and $h_{1}$ is computed as

$$
h_{1}=h_{0}+\left(r_{1}-r_{0}\right) \mathbf{T A N}\left(a_{1}\right)-\frac{\left(r_{1}-r_{0}\right)^{2}}{t w o k a}
$$

where twoka is determined in the GET_K SU. 
Next, the HTCHECK SU is referenced to determine if the height of the ray has fallen below the elevation height of the terrain at the current range step, and if so, a new $a_{1}, r_{1}$, and $h_{1}$ are returned.

Next, it must be determined if the ray has passed through a refractive layer, in which case the index must be adjusted and the range, height, and angle must be computed at the refractive layer transition. For an upward ray, if $a_{1}$ is positive and $h_{1}$ has reached or surpassed the next height level, then $a_{1}, r_{1}, j$, and $h_{1}$, are found as follows. First, $h_{1}$ is set equal to htdum ${ }_{j+1}, j$ is increment by 1 , and $a_{1}$ and $r_{1}$ are given by

$$
\begin{aligned}
& a_{1}=\sqrt{\operatorname{RADA} 1\left(a_{0}, h_{1}-h_{0}\right)} \\
& r_{1}=\operatorname{RP}\left(r_{0}, a_{1}-a_{0}\right) .
\end{aligned}
$$

For a downgoing ray, if $a_{1}$ is less than or equal to 0 , and $h_{1}$ is less than $h t d u m_{j}$, then $h_{1}$ is set equal to $h t d u m_{j}$, and $j$ is set equal to the maximum of 0 or $j-1$. The variables $a_{1}$ and $r_{1}$ are then determined from

$$
\begin{aligned}
& a_{1}=-\sqrt{\operatorname{RADA} 1\left(a_{0}, h_{1}-h_{0}\right)} \\
& r_{1}=\operatorname{RP}\left(r_{0}, a_{1}-a_{0}\right) .
\end{aligned}
$$

Finally, the HTCHECK SU is referenced once again before exiting to determine if this new value of $h_{1}$ has fallen below the height of the current terrain elevation.

\begin{tabular}{|c|c|c|c|}
\hline Name & Description & Units & Source \\
\hline$a_{0}$ & Propagation angle at the start of the ray trace step & radians & Calling SU \\
\hline$f_{\text {ter }}$ & $\begin{array}{l}\text { Logical flag indicating if terrain profile has been specified: } \\
\text { ‘.true.' = terrain profile specified } \\
\text { ‘.false.' = terrain profile not specified }\end{array}$ & N/A & APMINIT CSC \\
\hline grdum & $\begin{array}{l}\text { Array of refractivity gradients defined by profile htdum and } \\
\text { refdum }\end{array}$ & $\begin{array}{l}\text { M-units/ } \\
\text { meter }\end{array}$ & $\begin{array}{l}\text { REFINTER SU } \\
\text { REFINIT SU }\end{array}$ \\
\hline$h_{0}$ & Height at the start of the ray trace step & meters & Calling SU \\
\hline htdum & Height array for current interpolated profile & meters & $\begin{array}{l}\text { REFINTER SU } \\
\text { REFINIT SU }\end{array}$ \\
\hline it & Index of the current terrain segment in arrays $t x$ and ty & N/A & Calling SU \\
\hline
\end{tabular}

Table 62 and Table 63 provide identification, description, units of measure, and the computational source for each TRACE_STEP SU input and output data element.

Table 62. TRACE_STEP SU input data element requirements. 
Table 62. TRACE_STEP SU input data element requirements. (continued)

\begin{tabular}{c|l|c|c}
\hline Name & \multicolumn{1}{|c|}{ Description } & Units & Source \\
\hline$j_{l}$ & Refractive profile index at the start of the ray trace step & N/A & Calling SU \\
$r_{0}$ & Range at the start of the ray trace step & meters & Calling SU \\
twoka & Twice the effective earth radius & meters & GET_K SU \\
$t x$ & Range points of terrain profile & meters & TERINIT SU \\
$t y$ & Height points of terrain profile & meters & TERINIT SU \\
\hline
\end{tabular}

Table 63. TRACE_STEP SU output data element requirements.

\begin{tabular}{|c|c|c|}
\hline Name & Description & Units \\
\hline$a_{1}$ & Propagation angle at the end of the ray trace step & radians \\
\hline$g_{r d}$ & Gradient of the ray trace step & M-units/meter \\
\hline$h_{1}$ & Height at the end of the ray trace step & meters \\
\hline$i_{\text {error }}$ & Integer value that is returned if any errors exist in the computation & N/A \\
\hline ihit & $\begin{array}{l}\text { Integer flag indicating if ray has hit surface: } \\
\text { ihit }=0 \text {; ray has not hit the surface } \\
\text { ihit }=1 \text {; ray has hit the surface }\end{array}$ & N/A \\
\hline$r_{1}$ & Range at the end of the ray trace step & meters \\
\hline
\end{tabular}

\subsubsection{Troposcatter Initialization (TROPOINIT) SU}

The TROPOINIT SU initializes all variables and arrays needed for subsequent troposcatter calculations. The tangent range and tangent angle are determined from the source and the tangent range and tangent angles are determined for all receiver heights and stored in arrays.

Upon entering the SU, the array $\vartheta 1 t$ is allocated for size $i_{P E}$ and initialized to 0 . Next, the GET_K SU is referenced to determine the effective earth radius factor $a_{e k}$ based on a ray launched at the critical angle traced to $h t_{\text {lim. }}$. The array $\vartheta 0$, containing angles used in determining the common volume scattering angle is then determined from

$$
\vartheta 0_{i}=\frac{\text { rngout }_{i}}{a_{e k}} ; \quad \text { for } i=1,2,3, \ldots, n_{\text {rout }} \text {. }
$$

A constant needed in the troposcatter calculation, $r_{f}$, is determined from 0.0419 times the frequency $f_{M H z}$. A second constant needed in the troposcatter calculation, $r t_{1}$, is found from $r_{f}$ times the adjusted transmitting antenna height $a n t_{r e f}$. 
Next, the tangent angle from the source, $\vartheta 1_{s}$, for smooth surface is computed from

$$
\vartheta 1_{s}=\frac{\sqrt{\text { twoka } * a n t_{r e f}}}{a_{e k}} .
$$

The variable $\alpha_{l d}$ is determined from

$$
\begin{aligned}
& \alpha_{l d}=20 \operatorname{LOG}_{10}\left(f\left(\alpha_{d}\right)\right) \\
& \alpha_{d}=\vartheta 1_{s}+10^{-6}
\end{aligned}
$$

where $\alpha_{d}$ represents the lowest direct ray angle in the RO region, and $f\left(\alpha_{d}\right)$ is the antenna pattern factor, obtained from referencing the ANTPAT SU, for the direct angle.

The minimum range, $r_{\text {hor1 }}$, at which the diffraction field solutions are applicable and the intermediate region ends, is determined for smooth surface and zero receiver height. The variable $r_{\text {hor } 1}$ is given by

$$
r_{\text {hor } 1}=\sqrt{\text { twoka } * a n t_{\text {ref }}} \text {. }
$$

Next, the tangent ranges and angles for all output receiver heights are computed and stored in arrays $d 2 s$ and $\vartheta 2 s$, respectively. The minimum ranges at which diffraction field solutions are applicable (for smooth surface) for all output receiver heights are determined and stored in array rdt. Height differences between ant $t_{r e f}$ and each output receiver height are also computed and stored in adif. These arrays are given by

$$
\begin{aligned}
& d 2 s_{i}=\sqrt{2 a_{e k} \text { zout }_{i}}, \\
& \vartheta 2 s_{i}=-\frac{d 2 s_{i}}{a_{e k}}, \quad i=0,1,2, \ldots, n_{\text {zout }} \\
& r d t_{i}=r_{\text {hor }_{1}}+d 2 s_{i} \\
& \text { adif }_{i}=\text { ant }_{\text {ref }}-\text { zout }_{i},
\end{aligned}
$$

where the computation is performed for each $i^{\text {th }}$ output receiver height zout $_{i}$, provided zout $_{i}$ is greater than or equal to 0 , and $i$ ranges from 1 to $n_{\text {zout }}$.

If $f_{\text {ter }}$ is '.true.', then the tangent angles $\vartheta 1 t$ from the source at every PE range is determined as

$$
\vartheta 1 t=\frac{a n t_{r e f}-t y h_{j}}{j \Delta r_{P E}}+\frac{j \Delta r_{P E}}{t w o k a}, \quad j=1,2,3, \ldots, i_{P E}
$$


The index counter $j_{t 2}$ (used in the TROPOSCAT SU) is initialized to 1 . Finally, the troposcatter loss term $t_{l s t}{ }_{w r}$, used in the TROPOSCAT SU is given by

$$
t l s t_{w r}=54.9+30 \mathbf{L O G}_{10}\left(f_{M H z}\right)-\alpha_{l d} \text {. }
$$

Table 64 and Table 65 provide identification, description, units of measure, and the computational source for each TROPOINIT SU input and output data element.

Table 64. TROPOINIT SU input data element requirements

\begin{tabular}{c|l|c|c}
\hline Name & \multicolumn{1}{|c|}{ Description } & Units & Source \\
\hline $\begin{array}{c}\text { ant } t_{r e f} \\
f_{M H z}\end{array}$ & Transmitting antenna height relative to $h_{\text {minter }}$ & meters & TERINIT SU \\
$f_{\text {ter }}$ & Frequency & $\mathrm{MHz}$ & Calling CSCI \\
& \multicolumn{1}{|c}{ '.true.' = terrain profile specified } & N/A terrain profile not specified & APMINIT CSC \\
$i_{P E}$ & Number of PE range steps & & \\
$n_{\text {rout }}$ & Integer number of output range points desired & N/A & PEINIT SU \\
$n_{\text {zout }}$ & Integer number of output height points desired & N/A & Calling CSCI \\
$r n g o u t$ & Array containing all desired output ranges & N/A & Calling CSCI \\
tyh & Adjusted height points of terrain profile at every PE range & meters & APMINIT CSC \\
& step & meters & PEINIT SU \\
zout & Array containing all desired output heights referenced to & meters & APMINIT CSC \\
& $h_{\text {minter }}$ & & \\
\hline
\end{tabular}

Table 65. TROPOINIT SU output data element requirements.

\begin{tabular}{c|l|c}
\hline Name & \multicolumn{1}{|c}{ Description } & Units \\
\hline$a_{e k}$ & Effective earth radius & meters \\
$a d i f$ & Height differences between ant $_{r e f}$ and all output receiver heights & meters \\
$d 2 s$ & Array of tangent ranges for all output receiver heights over smooth surface & meters \\
$e_{k}$ & Effective earth radius factor & N/A \\
$i_{e r r o r}$ & Integer value that is returned if any errors exist in the computation & N/A \\
$j t 2$ & Index counter for $t x$ and ty arrays indicating location of receiver range & N/A \\
$r_{h o r 1}$ & Minimum range at which diffraction field solutions are applicable and the & meters \\
$r d t$ & intermediate region ends, for smooth surface and 0 receiver height. & \\
$r f$ & Array of minimum ranges at which diffraction field solutions are applicable (for & meters \\
$r t_{1}$ & smooth surface) for all output receiver heights. & meters ${ }^{-1}$ \\
& Constant used for troposcatter calculations & N/A
\end{tabular}


Table 65. TROPOINIT SU output data element requirements. (continued)

\begin{tabular}{c|l|c}
\hline Name & \multicolumn{1}{|c}{ Description } & Units \\
\hline$\vartheta 0$ & Array of angles used to determine common volume scattering angle & radians \\
$\vartheta 1_{s}$ & Tangent angle from source (for smooth surface) & radians \\
$\vartheta 1 t$ & Array of tangent angles from source height - used with terrain profile & radians \\
$\vartheta 2 s$ & Array of tangent angles from all output receiver heights - used with smooth & radians \\
& surface & $\mathrm{dB}$ \\
$t l s t_{w r}$ & Troposcatter loss term used in the TROPOSCAT SU & meters \\
twoka & Twice the effective earth radius & \\
\hline
\end{tabular}

\subsubsection{Starter Field Initialization (XYINIT) SU}

The XYINIT SU calculates the complex PE solution at range zero.

Upon entering this SU, several constant terms that will be employed over the entire PE mesh are calculated. The PE mesh is defined by the number of points in the mesh, $n_{f f t}$, and by the mesh size, $\Delta p$. The constant terms include (1) the angle difference between mesh points in p-space $\Delta \theta,(2)$ a height-gain value at the source (transmitter) $a_{t} k_{o}$; and (3) the normalization factor $S_{\text {gain }}$ used in the determination of the complex array containing the field $U$. The normalization factor $s_{\text {gain }}$ is given by

$$
s_{\text {gain }}=\frac{\sqrt{\lambda}}{z_{\max }} .
$$

The height-gain value $a n t k_{o}$ at the source (transmitter) is given by

$$
\operatorname{ant}_{o}=k_{o} a n t_{h t},
$$

where $a n t_{h t}$ is the transmitting antenna height above the local ground in meters.

The complex PE solution $U$ is determined from the antenna pattern factors, elevation angle, and normalization factor according to

$$
\begin{aligned}
& U_{j}=c_{a} s_{\text {gain }}\left[f\left(\alpha_{d}\right) e^{-i p_{j} a n t k_{o}}-f\left(-\alpha_{d}\right) e^{i p_{j} a n t k_{o}}\right] ; \mathrm{H} \text { pol } \\
& U_{j}=c_{a} s_{\text {gain }}\left[f\left(\alpha_{d}\right) e^{-i p_{j} a n t k_{o}}+f\left(-\alpha_{d}\right) e^{i p_{j} a n t k_{o}}\right] ; \mathrm{V} \text { pol } \\
& \alpha_{d}=\operatorname{SIN}^{-1}\left(p_{j}\right), \\
& C_{a}=\left(1-p_{j}^{2}\right)^{-3 / 4},
\end{aligned}
$$


where $p_{j}=j \Delta \theta$ and the antenna pattern factors $f\left(\alpha_{d}\right)$ for the direct path and $f\left(-\alpha_{d}\right)$ for the reflected path are determined by referencing the ANTPAT SU. The index $j$ varies from 0 to $n_{f f t}$.

Next, the upper $1 / 4$ of the field is filtered. A cosine-tapered (Tukey) filter array filt is used for this purpose. The filtered PE field $U$ is given by

$$
U_{j}=\text { filt }_{j-n 34} U_{j} ; \quad j=n_{34}, n_{34}+1, n_{34}+2, \ldots, n_{f f t} \cdot
$$

Finally, the DRST SU is referenced for both the real and imaginary components to transform the complex PE field to z-space before exiting the SU.

Table 66 and Table 67 provide identification, description, units of measure, and the computational source for each XYINIT SU input and output data element.

Table 66. XYINIT SU input data element requirements.

\begin{tabular}{|c|c|c|c|}
\hline Name & Description & Units & Source \\
\hline$a^{\prime} t_{h t}$ & $\begin{array}{l}\text { Transmitting antenna height above local } \\
\text { ground }\end{array}$ & meters & Calling CSCI \\
\hline$\Delta \theta$ & $\begin{array}{l}\text { Angle bin width (i.e., incremental } \\
\text { sine(theta)) }\end{array}$ & radians & PEINIT SU \\
\hline filt & Cosine-tapered (Tukey) filter array & N/A & PEINIT SU \\
\hline$i_{p o l}$ & $\begin{array}{l}\text { Polarization flag: } \\
\qquad \begin{array}{l}0=\text { horizontal polarization } \\
1=\text { vertical polarization }\end{array}\end{array}$ & N/A & Calling SU \\
\hline$k_{o}$ & Free-space wave number & meters $^{-1}$ & APMINIT CSC \\
\hline$\lambda$ & Wavelength & meters & APMINIT CSC \\
\hline $\ln _{\text {fft }}$ & Power of 2 transform size, i.e. $n_{f f t}=2^{l n} f f t$ & N/A & FFTPAR SU \\
\hline$n_{\text {fft }}$ & Transform size & N/A & FFTPAR SU \\
\hline$n_{34}$ & $3 / 4 n_{f f t}$ & N/A & APMINIT CSC \\
\hline$Z_{\max }$ & $\begin{array}{l}\text { Total height of the FFT/PE calculation } \\
\text { domain }\end{array}$ & meters & FFTPAR SU \\
\hline
\end{tabular}

Table 67. XYINIT SU Output Data Element Requirements

\begin{tabular}{c|c|c}
\hline Name & Description & Units \\
\hline$U$ & Transform of complex field & $\mu \mathrm{V} / \mathrm{m}$ \\
\hline
\end{tabular}




\subsection{ADVANCED PROPAGATION MODEL STEP (APMSTEP) CSC}

The APMSTEP SU advances the entire APM CSCI algorithm one output range step, referencing various SUs to calculate the propagation loss at the current output range.

Upon entering the APMSTEP SU, the current output range $r_{\text {out }}$ is updated, the gaseous absorption loss, gas loss (in $\mathrm{dB}$ ), and all $m p f l$ array integer indices for the various calculation regions are initialized. The propaf array is also initialized to a value of -999 . The PESTEP SU is then referenced to determine all propagation loss values within the $\mathrm{PE}$ calculation region. If the PE-only option is specified ( $P E_{\text {flag }}=$ '.true.'), then $m p f l$ is returned with integer indices $j_{p s}$ and $j_{p e}$, corresponding to the start and end, respectively, of propagation factor and loss values within mpfl. Otherwise, the SU proceeds with steps 1 through 3.

1. If APM is executing under the airborne mode ( $i_{\text {hybrid }}=0$ ), then the starting index $j_{a s}$ for the lower angular region is initialized to the maximum of 0 and $i_{z g}$ plus $i_{o}$. The ending index within this region $j_{a e}$ is determined by performing an iterative search to find the index at which the first occurance of zout $t_{j}$ is greater than $h t f e_{i_{s t p}}$. $j_{a e}$ is then set equal to the index $j$. The FEDR SU is then referenced to compute the loss for the lower FE region for the direct ray only, provided $j_{a s}$ is less than $j_{a e}$. Upon returning, $j_{\text {start }}$ is then set equal to $j_{a s}, j_{a s}$ is set equal to $j_{p e}+1, j_{a e}$ is set equal to $n_{\text {zout }}$, and the FEDR SU is again referenced to compute the loss for the upper FE region. The SU then proceeds with step 3 .

2. If APM is executing under the full hybrid mode $\left(i_{\text {hybrid }}=1\right)$ and the current output range is less than the range at which the XO region begins $\left(r_{\text {out }}<r_{\text {tst }}\right)$, the steps 2.a and 2.b are performed.

a. The starting and ending $m p f l$ array indices for FE calculations, $j_{f s}$ and $j_{f e}$, respectively, are determined. For ranges less than $2.5 \mathrm{~km}, j_{f s}$ is set equal to the maximum of 0 and $i_{z g}$ plus $i_{o}$, and $j_{f e}$ is set equal to $n_{z o u t}$. For ranges greater than $2.5 \mathrm{~km}, j_{f s}$ is set equal to the maximum of $j_{p e}+1$, or $j+1$, where $j$ is the first occurance of zout $t_{j}$ that is greater than $h t f e_{i_{s t p}}$ (the output height index that corresponds to the height just above the FE $5^{\circ}$ angle limit). The ending index $j_{f e}$ is set equal to $n_{\text {zout }}$. The FEM SU is then referenced and propagation factor and loss values within the FE region are computed and returned in $m p f l$.

b. If the current output range is greater than $2.5 \mathrm{~km}$, then the starting and ending $m p f l$ array indices for RO calculations, $j_{r s}$ and $j_{r e}$, respectively, are determined. These indices are based on the values of $j_{p s}, j_{p e}, j_{f s}$, and $j_{f e}$ such that at every range step, $j_{r s}$ will always be greater than the ending index of the PE region $\left(j_{p e}\right)$ and $j_{r e}$ will be less than the starting index of the FE region $\left(j_{f s}\right)$. The ROLOSS SU is then referenced and propagation factor and loss values within the RO region are computed and returned in $\mathrm{mpfl}$. 
3. Once the various propagation factor and loss within the various regions have been calculated, the ending index $j_{\text {end }}$ of valid values within $m p f l$ is given by the maximum of $j_{p e}, j_{f e}, j_{r e}$, and $j_{a e}$.

Upon exiting, if the final output range step has been reached, the integer counter $j_{t 2}$, associated with troposcatter calculations, is initialized to 1 .

Table 68 and Table 69 provide identification, description, units of measure, and the computational source for each APMSTEP SU input and output data element.

Table 68. APMSTEP CSC input data element requirements.

\begin{tabular}{|c|c|c|c|}
\hline Name & Description & Units & Source \\
\hline gas $_{a t t}$ & Gaseous absorption attenuation rate & $\mathrm{dB} / \mathrm{km}$ & GASABS SU \\
\hline$h t f e$ & $\begin{array}{l}\text { Array containing the height at each output range separating } \\
\text { the FE region from the RO region (full hybrid mode), or the } \\
\text { FE region from the PE region (partial hybrid mode) }\end{array}$ & meters & FILLHT SU \\
\hline$h t_{l i m}$ & Maximum height relative to $h_{\text {minter }}$ & meters & TERINIT SU \\
\hline$i_{\text {hybrid }}$ & $\begin{array}{l}\text { Integer indicating which sub-models will be used: } \\
\begin{aligned} 0 & =\text { FEDR }+ \text { PE model } \\
1 & =\text { full hybrid model }(\mathrm{PE}+\mathrm{FE}+\mathrm{RO}+\mathrm{XO}) \\
2 & =\text { partial hybrid model }(\mathrm{PE}+\mathrm{XO})\end{aligned}\end{array}$ & N/A & APMINIT CSC \\
\hline$i_{o}$ & $\begin{array}{l}\text { Starting index for } m p f l \text { array: } \\
\qquad \begin{array}{l}0=1^{\text {st }} \text { calculated output point is at surface } \\
1=1^{\text {st }} \text { calculated output point is at height } \Delta z_{\text {out }}\end{array}\end{array}$ & N/A & APMINIT CSC \\
\hline$i_{s t p}$ & Current output range step index & N/A & Calling CSCI \\
\hline$i_{z g}$ & $\begin{array}{l}\text { Number of output height points corresponding to local } \\
\text { ground height at current output range } r_{\text {out }}\end{array}$ & N/A & CALCLOS SU \\
\hline$n o_{P E}$ & $\begin{array}{l}\text { Integer flag indicating if PE calculations are needed: } \\
\begin{aligned} 0 & =\mathrm{PE} \text { calculations needed } \\
1 & =\text { no PE calculations needed }\end{aligned}\end{array}$ & N/A & GETTHMAX SU \\
\hline nrout & Integer number of output range points desired & N/A & Calling CSCI \\
\hline nzout & Integer number of output height points desired & N/A & Calling CSCI \\
\hline$P E_{\text {flag }}$ & $\begin{array}{l}\text { Flag to indicate use of PE algorithm only: } \\
\text { '.true.' = only use PE sub-model } \\
\text { '.false.' = use automatic hybrid model }\end{array}$ & N/A & Calling CSCI \\
\hline$r_{a t z}$ & Range at which $Z_{\text {lim }}$ is reached (used for hybrid model) & meters & APMINIT CSC \\
\hline rngout & Array containing all desired output ranges & meters & APMINIT CSC \\
\hline$r_{\text {pest }}$ & $\begin{array}{l}\text { Range at which loss values from the PE model will start } \\
\text { being calculated }\end{array}$ & meters & GETTHMAX SU \\
\hline$r_{\text {tst }}$ & Range at which to begin RO calculations (equal to $2.5 \mathrm{~km}$ ) & meters & APM_MOD \\
\hline zout & $\begin{array}{l}\text { Array containing all desired output heights referenced to } \\
\text { hminter }\end{array}$ & meters & APMINIT CSC \\
\hline
\end{tabular}


Table 69. APMSTEP CSC output data element requirements.

\begin{tabular}{|c|c|c|}
\hline Name & Description & Units \\
\hline$j_{\text {end }}$ & Index at which valid loss values in $m p f l$ end & N/A \\
\hline$j_{\text {start }}$ & Index at which valid loss values in $m p f l$ start & N/A \\
\hline gas $_{\text {loss }}$ & Gaseous absorption loss at range $r_{\text {out }}$ & $\mathrm{dB}$ \\
\hline jt2 & Index counter for $t x$ and ty arrays indicating location of receiver range & N/A \\
\hline$m p f l$ & Propagation factor and loss array & $\mathrm{cB}$ \\
\hline mpfl_rtg & $\begin{array}{l}\text { Propagation loss and factor at receiver heights specified in the zout_rtg } \\
\text { array }\end{array}$ & $\mathrm{cB}$ \\
\hline$r_{\text {out }}$ & Current desired output range & meters \\
\hline propaf & $\begin{array}{l}\text { Two-dimensional array, containing the propagation angles and factors for } \\
\text { the direct and reflected rays (where applicable) for all output height/range } \\
\text { points }\end{array}$ & $\begin{array}{l}\text { radians, } \\
\text { dB }\end{array}$ \\
\hline
\end{tabular}

\subsubsection{Calculate Propagation Loss (CALCLOS SU)}

The CALCLOS SU determines the propagation factor and loss from the PE region at each output height point at the current output range.

Upon entering the SU, several variables are initialized. The output range, $r_{\text {out }}$, is updated based on the current range step $i_{s t p}$. The height of the terrain at the current and last ranges, $y_{c h}$ and $y_{l h}$, respectively, are determined relative to the reference height, $h m_{r e f}$.

Next, the interpolated ground height, $z_{i n t}$, at the current output range and the number of vertical output points, $i_{z g}$, that correspond to this ground height are determined. First, the interpolated ground height is given by

$$
z_{\text {int }}=y_{\text {last }}+\left(y_{\text {cur }}-y_{\text {last }}\right) x x,
$$

where the parameter $x x$ is given in terms of the PE range step $\Delta r_{P E}$ by

$$
x x=\frac{r_{\text {out }}-r_{\text {last }}}{\Delta r_{P E}} .
$$

Having determined $z_{i n t}, i_{z g}$ is then computed from

$$
i_{\text {zg }}=\mathbf{I N T}\left(\frac{z_{\text {int }}-h m_{\text {ref }}}{\Delta z_{\text {out }}}\right) \text {, }
$$


where $\Delta z_{\text {out }}$ is the output height increment. Next, all elements in array mpfl from 1 to $i_{z g}$ are set to zero, and the index $j_{\text {start }}$, representing beginning valid loss values in the $m p f l$ array, is set to the maximum of 0 or $i_{z g}$, plus $i_{o}$.

If $i_{\text {hybrid }}=0$, then the maximum tangent angle $\alpha_{\text {ter }}$ from the antenna height to the terrain height at the current range is determined from

$$
\alpha_{\text {ter }}=\mathbf{M A X}\left[\mathbf{T A N}^{-1}\left(\frac{z_{\text {int }}-a t_{\text {ref }}-\frac{r_{\text {out }}^{2}}{t w o k a_{\text {down }}}}{r_{\text {out }}}\right), \alpha_{\text {ter }}\right] \text {, }
$$

where $\alpha_{\text {ter }}$ in the argument above is the maximum angle computed from previous references to the CALCLOS SU.

If the current output range is greater than the range $r_{\text {pest }}$ at which PE solutions are valid, then the calculation of loss values is begun. If this condition is not satisfied, then the $m p f l$ array is set to -32767 for values of the array index from $j_{\text {start }}$ up to and including the number of output height points desired $\left(n_{\text {zout }}\right), j_{\text {end }}$ is set equal to $j_{\text {start }}$ and the SU is exited.

Once it is determined that loss calculations will be performed, several parameters are computed. Both parameters $i_{p 1}$ and $i_{p 2}$ are first set to 0 . If the logical variable $f_{\text {ter }}$ is '.true.', then a terrain case is being performed. The two indices $i_{p 1}$ and $i_{p 2}$ are given by

$$
\begin{aligned}
& i_{p 1}=\mathbf{M A X}\left(0, \mathbf{I N T}\left\{\frac{y_{\text {lh }}}{\Delta z_{\text {out }}}\right\}\right) \\
& \left.i_{p 2}=\operatorname{MAX}\left(0, \mathbf{I N T}\left\{\frac{y_{\text {ch }}}{\Delta z_{\text {out }}}\right\}\right)\right) .
\end{aligned}
$$

These indices indicate the first output height point in array zout, where propagation loss will be computed at the last and current PE ranges. Next, the output heights zout $i_{p 1}$ and zout $_{i p 2}$, relative to $y_{\text {last }}$ and $y_{\text {cur }}$, respectively, are checked to make sure they are positive. If not, the two indices $i_{p 1}$ and $i_{p 2}$ are incremented by a value of 1 . For values of the array index from 0 up to $i_{p 1}$, the array of propagation factors $r f a c 1$ at valid height points for range $r_{\text {last }}$ are set to 0 . For values of the array index from 0 up to $i_{p 2}$, the array of propagation factors $r f a c 2$ at valid height points for range $r_{\text {out }}$ are also set 0 . 
If $j_{\text {start }}$ is less than $i_{p 1}$ and $i_{p 2}$, then the variable $i_{z g}$ is recomputed as

$$
i_{z g}=\operatorname{MAX}\left(i_{z g}, \mathbf{M I N}\left(i_{p 1}, i_{p 2}\right)\right)
$$

and the mpfl array at index $i_{z g}$ is set equal to -32766. $j_{\text {start }}$ is then recomputed as the maximum of 0 and $i_{z g}$, and the value of $i_{o}$ is then added to $j_{\text {start }}$.

Next, the height/integer value, $j_{\text {end }}$, indicating the end of valid loss values, is determined as

$$
\begin{aligned}
& j_{\text {end }}=\mathbf{M A X}\left[0, \mathbf{I N T}\left(\frac{z_{\text {lim }}-h m_{\text {ref }}}{\Delta z_{\text {out }}}\right)\right] \quad \text { if } P E_{\text {flag }} \text { is '.true.', otherwise } \\
& j_{\text {end }}=\mathbf{M A X}\left(0, \mathbf{I N T}\left\{\frac{\mathbf{M I N}\left[z_{\text {lim }}, \mathbf{M A X}\left(z_{\text {int }}, h l i m_{i_{\text {stp }}}\right)\right]-h m_{\text {ref }}}{\Delta z_{\text {out }}}\right\}\right) .
\end{aligned}
$$

where $i_{s t p}$ is the current output range step, and hlim $_{i_{s t p}}$ is the height at the current output range step separating the $\mathrm{PE}$ region from the $\mathrm{FE}, \mathrm{RO}$, or $\mathrm{XO}$ regions. Finally, $j_{\text {end }}$ is given by the minimum of $j_{\text {end }}$ (as computed above) and $n_{\text {zout }}$.

If clutter calculations are to be performed ( $C_{l u t}=$ '.true.), the propagation factor at $1 \mathrm{~m}$ above the surface is computed by referencing the FN_GETPFAC function. The propagation factor at 1-m height is computed from the complex PE field at the range step immediately before and after the current output range step by

$$
\begin{gathered}
\left.r f 1=\text { FN_GETPFAC(Ulast, } r_{\text {loglst }}, \Delta z_{P E}, 1.0\right), \\
r f 2=\text { FN_GETPFAC }\left(U, r_{l o g}, \Delta z_{P E}, 1.0\right) .
\end{gathered}
$$

The propagation factor is then interpolated in range to the current output range step by referencing the FN_PLINT function:

$$
\left.\operatorname{Fat}_{1 m_{i_{s p}}}=\text { FN_PLINT( } r f 1, r f 2, x x\right) .
$$

Next, if the value of $j_{\text {end }}$ is less than $j_{\text {start }}$ the $m p f l$ array for indices from $j_{\text {end }}+1$ to $n_{\text {zout }}$ are set equal to -32767 and the SU is exited. Otherwise, the propagation loss values are determined from the propagation factors $r f a c 1_{i}$ and $r f a c 2_{i}$ and from the parameter $x x$ defined earlier in this section. 
If $r_{\text {loglst }}\left(10 \operatorname{LOG}\left(r_{\text {last }}\right)\right)$ is greater than zero (it is initialized to 0 for $\left.i_{s t p}=1\right)$, then the FN_GETPFAC function is referenced to determine the propagation factor $r f a c 1_{i}$, which is given by

$$
\left.r f a c 1_{i}=\text { FN_GETPFAC(Ulast, } r_{\text {loglst }}, \Delta z_{P E}, \text { zout }_{i}-y_{\text {last }}\right), \quad i=i_{p 1}, i_{p 1}+1, \ldots, j_{\text {end }},
$$

where Ulast is the complex field array at the previous PE range. Next, the propagation factor $r f a c 2_{i}$ is given by

$$
r f a c 2_{i}=\text { FN_GETPFAC }\left(U, r_{l o g}, \Delta z_{P E}, \text { zout }_{i}-y_{c u r}\right), \quad i=i_{p 2}, i_{p 2}+1, \ldots, j_{\text {end }},
$$

where $U$ is the complex field array at the current PE range, and $r_{\text {log }}$ is $10 \mathrm{LOG}(r)$.

Next, if a frequency is specified greater than $50 \mathrm{MHz}\left(H F_{\text {flag }}=\right.$ '.false.' $)$ and if using the partial hybrid mode (PE \& XO models), heights corresponding to areas outside the valid PE calculation region are determined and propagation loss is set equal to -32767 within $m p f l$ for those heights. If using the full or partial hybrid modes, the propagation factor at the last PE height point is determined at the previous and current PE ranges. Linear interpolation is then performed to compute the propagation loss at range $r_{\text {out }}$ and height $z_{\text {lim }}$. The loss and height are then stored in array ffrout for subsequent interpolation in the EXTO SU.

Next, the propagation factor and loss at range $r_{\text {out }}$ is found by interpolating between the current and previous PE ranges. The propagation factor in $\mathrm{dB}$, and the propagation loss at range $r_{\text {out }}$ is given by

$$
\begin{aligned}
F_{d B} & \left.=\text { FN_PLINT(rfac1 } 1_{i}, r f a c 2_{i}, x x\right)
\end{aligned} ; i=j_{\text {start }}, j_{\text {start }}+1, \ldots, j_{\text {end }}
$$

where $f s l_{i s t p}$ is the free-space loss in $\mathrm{dB}$ at range $r_{\text {out }}$.

Next, if the propagation factor and propagation angle and are to be computed (lang = '.true.'), the angle information is stored in array propaf by interpolating in height using the values stored in ptr at the current range step. The propagation angle at the current range step is determined by

$$
\begin{gathered}
z f=\frac{\text { zout }_{k}-z_{\text {int }}}{\Delta z_{\text {spec }}}, \\
\operatorname{izsp}=\mathbf{I N T}(z f), \\
f r=z f-i z s p, \\
\text { propaf }_{1, k}=\mathbf{F N \_ P L I N T}\left(p t r_{i z s p, i_{\text {sp }}}, p t r_{i z s p+1, i_{\text {stp }}}, f r\right) ; \quad k=j_{\text {start }}, j_{\text {start }}+1, \ldots, j_{\text {end }},
\end{gathered}
$$

where $\Delta z_{\text {spec }}$ is the height increment at which the propagation angles were spectral estimated. 
If the loss at a receiver height relative to the local ground height is to be computed (lrtg = '.true.'), then the propagation loss at each height specified in array zout_rtg is computed by subsequent references to the FN_GETPFAC function using the field strength arrays at the previous and next PE range steps. The final propagation loss is then interpolated by referencing the FN_PLINT function and stored in array rloss_rtg.

If the troposcatter calculation flag $T_{\text {ropo }}$ is '.true.' and the transmitter height is less than $100 \mathrm{~m}$, then the TROPOSCAT SU is referenced to compute troposcatter loss from height zout $j_{\text {start }}$ to zout $_{j_{\text {end }}}$, and this is added, if necessary, to rloss.

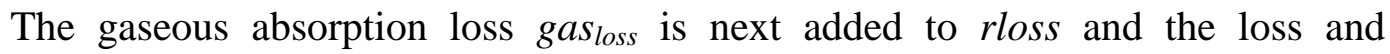
propagation factor in centibels is given by

$$
\begin{aligned}
& m p l_{1, i}=\mathbf{N I N T}\left(10 * r \operatorname{rloss}_{i}\right) \\
& m p l_{2, i}=\mathbf{N I N T}\left(10 *\left(f s l_{i_{s t p}}-\text { rloss }_{i}\right)\right) ; i=j_{\text {start }}, j_{\text {start }}+1, \ldots, j_{\text {end }}
\end{aligned}
$$

with the remaining elements in $m p f l$ set equal to -32767 (i.e., $m p f l_{k}=-32767$ for $k=j_{\text {end }}+1$ to $\left.n_{\text {zout }}\right)$.

Finally, if lang and lrtg are '.true.', then the complete propagation factor is computed from $m p f l$ and rloss_rtg and stored in arrays propaf and $m p f l \_r t g$, respectively, before exiting.

Table 70 and Table 71 provide identification, description, units of measure, and the computational source for each CALCLOS SU input and output data element.

\begin{tabular}{|c|c|c|c|}
\hline Name & Description & Units & Source \\
\hline$a_{\text {nef }}$ & $\begin{array}{l}\text { Transmitting antenna height relative to the } \\
\text { reference height } h_{\text {minter }}\end{array}$ & meters & TERINIT SU \\
\hline$C_{\text {lut }}$ & $\begin{array}{l}\text { Logical flag used to indicate if surface clutter } \\
\text { calculations are desired. }\end{array}$ & logical & Calling CSCI \\
\hline$\Delta r_{P E}$ & PE range step & meters & PEINIT SU \\
\hline$\Delta z_{\text {out }}$ & Output height increment & meters & APMINIT CSC \\
\hline$\Delta z_{P E}$ & PE mesh height increment (bin width in z-space) & meters & FFTPAR SU \\
\hline$\Delta z_{\text {spec }}$ & $\begin{array}{l}\text { Height increment at which the propagation angles } \\
\text { are computed from spectral estimation }\end{array}$ & meters & GETANGLES SU \\
\hline fsl & Free space loss array for output ranges & $\mathrm{dB}$ & APMINIT CSC \\
\hline
\end{tabular}

Table 70. CALCLOS SU input data element requirements. 
Table 70. CALCLOS SU input data element requirements. (continued)

\begin{tabular}{|c|c|c|c|}
\hline Name & Description & Units & Source \\
\hline$f_{\text {ter }}$ & $\begin{array}{l}\text { Logical flag indicating if terrain profile has been } \\
\text { specified: } \\
\text {.true. = terrain profile specified } \\
\text {.false. = terrain profile not specified }\end{array}$ & N/A & APMINIT CSC \\
\hline gas $_{\text {loss }}$ & Gaseous absorption loss at range $r_{\text {out }}$ & $\mathrm{dB}$ & APMSTEP CSC \\
\hline$H F_{\text {flag }}$ & $\begin{array}{l}\text { HF computation flag indicating the frequency } \\
\text { specified is less than } 50 \mathrm{MHz}\end{array}$ & N/A & APMINIT CSC \\
\hline hlim & $\begin{array}{l}\text { Array containing height at each output range } \\
\text { separating the RO region from the PE (at close } \\
\text { ranges) and XO (at far ranges) regions }\end{array}$ & meters & GETTHMAX SU \\
\hline$h_{\text {minter }}$ & Minimum height of terrain profile & meters & TERINIT SU \\
\hline$h m_{\text {ref }}$ & Height relative to $h_{\text {minter }}$ & meters & TERINIT SU \\
\hline$h t f e$ & $\begin{array}{l}\text { Array containing the height at each output range } \\
\text { separating the FE region from the RO region (full } \\
\text { hybrid mode), or the FE region from the PE } \\
\text { region (partial hybrid mode) }\end{array}$ & meters & FILLHT SU \\
\hline$i_{h m x}$ & $\begin{array}{l}\text { Index in harray where traced height has reached } \\
h t_{\text {lim }}\end{array}$ & N/A & TRACE_ROUT SU \\
\hline$i_{\text {hybrid }}$ & $\begin{array}{l}\text { Integer indicating which sub-models will be used: } \\
\begin{aligned} 0 & =\text { pure } \mathrm{PE} \text { model } \\
1 & =\text { full hybrid model }(\mathrm{PE}+\mathrm{FE}+\mathrm{RO}+\mathrm{XO}) \\
2 & =\text { partial hybrid model }(\mathrm{PE}+\mathrm{XO})\end{aligned}\end{array}$ & N/A & APMINIT CSC \\
\hline$i_{o}$ & $\begin{array}{l}\text { Starting index for } m p f l \text { array: } \\
\begin{array}{l}0=1^{\text {st }} \text { calculated output point is at surface } \\
1=1^{\text {st }} \text { calculated output point is at height } \\
\Delta z_{\text {out }}\end{array}\end{array}$ & N/A & APMINIT CSC \\
\hline$i_{s t p}$ & Current output range step index & N/A & Calling SU \\
\hline$i_{x o}$ & Number of range steps in $\mathrm{XO}$ calculation region & N/A & APMINIT CSC \\
\hline lang & $\begin{array}{l}\text { Logical flag indicating if propagation angle and } \\
\text { propagation factor output is desired. }\end{array}$ & N/A & Calling CSCI \\
\hline lrtg & $\begin{array}{l}\text { Logical flag indicating if loss relative to the local } \\
\text { ground height needs to be computed. } \\
\text { '.true.' = Compute loss relative to ground at } \\
\text { heights specified by array zout_rtg. } \\
\text { '.false.' = Do not compute loss. }\end{array}$ & N/A & APMINIT CSC \\
\hline$n_{\text {ang }}$ & $\begin{array}{l}\text { Number of points in the vertical at which to } \\
\text { spectrally estimate propagation angles. }\end{array}$ & N/A & GETANGLES SU \\
\hline$n_{\text {zout }}$ & Integer number of output height points desired & N/A & Calling CSCI \\
\hline$n_{\text {zout_rtg }}$ & $\begin{array}{l}\text { Number of height output points for receiver } \\
\text { heights relative to the local ground elevation. }\end{array}$ & N/A & Calling CSCI \\
\hline$P E_{\text {flag }}$ & $\begin{array}{l}\text { Flag to indicate use of PE algorithm only: } \\
\text { '.true.' = only use PE sub-model } \\
\text { '.false.' = use automatic hybrid model }\end{array}$ & N/A & Calling CSCI \\
\hline$r_{a t z}$ & $\begin{array}{l}\text { Range at which } z_{\text {lim }} \text { is reached (used for hybrid } \\
\text { model) }\end{array}$ & meters & APMINIT CSC \\
\hline
\end{tabular}


Table 70. CALCLOS SU input data element requirements. (continued)

\begin{tabular}{|c|c|c|c|}
\hline Name & Description & Units & Source \\
\hline$r_{\text {last }}$ & Previous PE range & meters & Calling SU \\
\hline$r_{\log }$ & $10 \log (\mathrm{PE}$ range $r$ ) & N/A & PESTEP SU \\
\hline$r_{\text {loglst }}$ & $10 \log \left(\right.$ previous PE range $\left.r_{\text {last }}\right)$ & N/A & PESTEP SU \\
\hline rngout & Array containing all desired output ranges & meters & APMINIT CSC \\
\hline$r_{\text {pest }}$ & $\begin{array}{l}\text { Range at which PE loss values will start being } \\
\text { calculated }\end{array}$ & meters & GETTHMAX SU \\
\hline$T_{\text {ropo }}$ & $\begin{array}{l}\text { Troposcatter calculation flag: } \\
\text { '.false.' = no troposcatter calcs } \\
\text { '.true.' = troposcatter calcs }\end{array}$ & N/A & Calling CSCI \\
\hline twoka $a_{\text {down }}$ & Twice the effective earth radius for downward path & meters & GET_K SU \\
\hline$U$ & Complex field at current PE range $r$ & $\mu \mathrm{V} / \mathrm{m}$ & PESTEP SU \\
\hline Ulast & Complex field at previous PE range $r_{\text {last }}$ & $\mu \mathrm{V} / \mathrm{m}$ & PESTEP SU \\
\hline$y_{\text {cur }}$ & Height of ground at current range $r$ & meters & PESTEP SU \\
\hline$y_{\text {last }}$ & Height of ground at previous range $r_{\text {last }}$ & meters & PESTEP SU \\
\hline zlim & Height limit for PE calculation region & meters & GETTHMAX SU \\
\hline zout & $\begin{array}{l}\text { Array containing all desired output heights } \\
\text { referenced to } h_{\text {minter }}\end{array}$ & meters & APMINIT CSC \\
\hline zout_rtg & $\begin{array}{l}\text { Dynamically allocated array of receiver heights } \\
\text { specified relative to the local ground height. }\end{array}$ & meters & Callng CSCI \\
\hline
\end{tabular}

Table 71. CALCLOS SU output data element requirements.

\begin{tabular}{|c|c|c|}
\hline Name & Description & Units \\
\hline$\alpha_{\text {ter }}$ & Tangent angle from antenna height to terrain height at current range & radians \\
\hline Fat $1 m$ & Propagation factor computed at $1 \mathrm{~m}$ above the surface. & $\mathrm{dB}$ \\
\hline ffrout & $\begin{array}{l}\text { Array of propagation factors at each output range beyond } r_{a t z} \text { and at height } \\
z_{\text {lim }}\end{array}$ & $\mathrm{dB}$ \\
\hline$i_{z g}$ & $\begin{array}{l}\text { Number of output height points corresponding to local ground height at } \\
\text { current output range } r_{\text {out }}\end{array}$ & N/A \\
\hline$j_{\text {end }}$ & Index at which valid loss values in $m p f l$ end & N/A \\
\hline$j_{\text {start }}$ & Index at which valid loss values in $m p f l$ begin & N/A \\
\hline$m p f l$ & Two-dimensional propagation factor and loss array & cB \\
\hline mpfl_rtg & $\begin{array}{l}\text { Propagation loss and factor at receiver heights specified in the zout_rtg } \\
\text { array }\end{array}$ & cB \\
\hline propaf & $\begin{array}{l}\text { Two-dimensional array, containing the propagation angles and factors for } \\
\text { the direct and reflected rays (where applicable) for all output height/range } \\
\text { points }\end{array}$ & $\underset{\mathrm{B}}{\text { radians, } \mathrm{d}}$ \\
\hline rfac1 & Propagation factor at valid output height points from PE field at range $r_{\text {last }}$. & $\mathrm{dB}$ \\
\hline rfac2 & Propagation factor at valid output height points from PE field at range $r$ & $\mathrm{~dB}$ \\
\hline
\end{tabular}


Table 71. CALCLOS SU output data element requirements. (continued)

\begin{tabular}{c|l|c}
\hline Name & \multicolumn{1}{|c|}{ Description } & Units \\
\hline rloss & Propagation loss & $\mathrm{dB}$ \\
rloss_rtg & Propagation loss determined at receiver heights specified in array zout_rtg & $\mathrm{dB}$ \\
zxo & Height of the ground at the current output range step & meters \\
\hline
\end{tabular}

\subsubsection{Current Wind (FN_CURWIND) Function}

The FN_CURWIND function performs a linear interpolation in range to get the current wind speed at the specified range.

Upon entry, the current wind speed, $\omega_{s}$, is initialized to the the final wind speed specified in array wind. If the number of wind speeds specified is greater than 1 then the index, $k$, within the array rngwind is determined such that the current range, $r$, satisfies rngwind $_{k}<r<$ rngwind $_{k+1}$. The wind speed at range $r$ is then determined from linear interpolation:

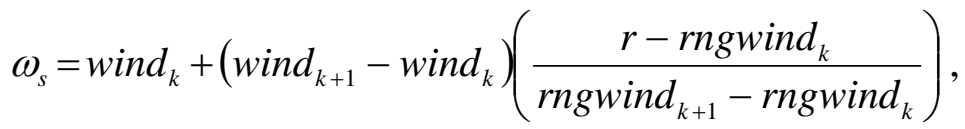

and returned to the calling SU.

Table 72 and Table 73 provide identification, description, units of measure, and the computational source for each FN_CURWIND function input and output data element.

Table 72. FN_CURWIND function input data element requirements.

\begin{tabular}{c|l|c|c}
\hline Name & \multicolumn{1}{|c|}{ Description } & Units & Source \\
\hline$n w$ & Number of wind speeds & N/A & Calling CSCI \\
$r$ & Current range & meters & Calling SU \\
rngwind & Ranges of wind speeds entered: rngwind $_{i}=$ range of & meters & Calling CSCI \\
& $i^{\text {th } \text { wind speed }}$ & \\
wind & Dynamically allocated array of wind speeds. & $\begin{array}{c}\text { meters/ } \\
\text { second }\end{array}$ & Calling CSCI \\
& & \\
\hline
\end{tabular}

Table 73. FN_CURWIND function output data element requirements.

\begin{tabular}{c|c|c}
\hline Name & Description & Units \\
\hline$\omega_{s}$ & Interpolated wind speed & meters/second \\
\hline
\end{tabular}




\subsubsection{Dielectric Constant (FN_DIECON) Function}

The FN_DIECON function extracts the complex dielectric constant at the current range.

Upon entering the function, the index, $k$, within the array rgrnd is determined such that the current range, $r$, satisfies $r g r n d_{k}<r<r g r n d_{k+1}$. The complex dielectric constant is then returned according to diec $=n c^{2}{ }_{k}$, and the function is exited.

Table 74 and Table 75 provide identification, description, units of measure, and the computational source for each FN_DIECON function input and output data element.

Table 74. FN_DIECON function input data element requirements.

\begin{tabular}{c|l|c|c}
\hline Name & \multicolumn{1}{|c|}{ Description } & Units & Source \\
\hline$i_{g r}$ & Number of different ground types specified & N/A & Calling CSCI \\
$n c^{2}$ & Array of complex dielectric constants & N/A & DIEINIT SU \\
$r$ & Current range & meters & Calling SU \\
$r g r n d$ & $\begin{array}{l}\text { Array containing ranges at which varying ground } \\
\text { types apply. }\end{array}$ & meters & Calling CSCI \\
\hline
\end{tabular}

Table 75. FN_DIECON function output data element requirements.

\begin{tabular}{c|l|c}
\hline Name & \multicolumn{1}{|c|}{ Description } & Units \\
\hline diec & Complex dielectric constant at range $r$ & N/A \\
\hline
\end{tabular}

\subsubsection{DOSHIFT SU}

The DOSHIFT SU shifts the field by the number of bins, or PE mesh heights corresponding to the local ground height.

Upon entry, the number of bins to be shifted is determined. First, the difference $y_{\text {diff }}$ between the height of the ground $y_{\text {last }}$ at the previous range and that at the current PE range $y_{\text {cur }}$ is determined from

$$
y_{\text {diff }}=y_{\text {cur }}-y_{\text {last }} \text {. }
$$


The number of bins to be shifted, $k_{b i n}$, is found from

$$
k_{b i n}=\mathbf{N I N T}\left(\frac{\left|y_{d i f f}\right|}{\Delta z_{P E}}\right) .
$$

The PE solution $U$ is then shifted downward if the local ground is currently at a positive slope $\left(y_{\text {diff }}>0\right)$, upward if the local ground is at a negative slope $\left(y_{\text {diff }}<0\right)$, and otherwise not shifted. When the PE solution has been shifted down, the value of the PE solution $U$ for the upper $k_{b i n}$ elements are set to zero. Likewise, when the PE solution has been shifted upwards, the lower $k_{b i n}$ elements are set to zero.

Table 76 and Table 77 provide identification, description, units of measure, and the computational source for each DOSHIFT SU input and output data element.

Table 76. DOSHIFT SU input data element requirements.

\begin{tabular}{c|l|c|c}
\hline Name & \multicolumn{1}{|c|}{ Description } & Units & Source \\
\hline$\Delta z_{P E}$ & PE mesh height increment (bin width in z-space) & meters & FFTPAR SU \\
$n_{f f t}$ & Transform size & N/A & FFTPAR SU \\
$n_{m 1}$ & $n_{f f t}-1$ & N/A & PEINIT SU \\
$U$ & Complex field at range $r$ & $\mu \mathrm{V} / \mathrm{m}$ & PESTEP SU \\
$y_{\text {cur }}$ & Height of ground at current range $r$ & meters & PESTEP SU \\
$y_{\text {last }}$ & Height of ground at previous range $r_{\text {last }}$ & meters & PESTEP SU \\
\hline
\end{tabular}

Table 77. DOSHIFT SU output data element requirements.

\begin{tabular}{c|c|c}
\hline Name & Description & Units \\
\hline$U$ & Complex field at range $r$ & $\mu \mathrm{V} / \mathrm{m}$ \\
\hline
\end{tabular}

\subsubsection{Discrete Sine/Cosine Fast-Fourier Transform (DRST) SU}

A function with a common period, such as a solution to the wave equation, may be represented by a series consisting of sines and cosines. This representation is known as a Fourier series. An analytical transformation of the function, known as a Fourier transform, may be used to obtain a solution for the function.

The solution to the PE approximation to Maxwell's wave equation is obtained by using such a Fourier transformation function. The APM CSCI uses only the real-valued sine or cosine transformation in which the real and imaginary parts of the PE equation are transformed separately. Which transform is performed is dependent on the value of an 
integer flag provided by the calling SU. The Fourier transformation provided with the APM CSCI is described by Bergland (1969) and Cooley (1970).

Other sine/cosine fast Fourier transform (FFT) routines are available in the commercial market, and such a sine/cosine FFT may already be available within another calling CSCI. The selection of which FFT ultimately used by the APM CSCI is left to the application designer as every sine/cosine FFT will have hardware and/or software performance impacts. For this reason, it is beyond the scope of this document to describe the numerical implementation of the FFT algorithm.

Table 78 and Table 79 provide identification, description, units of measure, and the computational source for each DRST SU input and output data element.

Table 78. DRST input data element requirements.

\begin{tabular}{|c|c|c|c|}
\hline Name & Description & Units & Source \\
\hline$i_{\text {flag }}$ & $\begin{array}{l}\text { Flag to indicate which transform to perform } \\
\qquad \begin{array}{l}0=\text { cosine transform } \\
1=\text { sine transform } \\
-1=\text { deallocates all allocated arrays }\end{array}\end{array}$ & N/A & Calling SU \\
\hline $\ln _{f f t}$ & Power of 2 transform size, i.e. $n_{f f t}=2^{l n} f f t$ & N/A & FFTPAR SU \\
\hline$x$ & Field array to be transformed - dimensioned $2^{n} f f t$ in calling SU & $\mu \mathrm{V} / \mathrm{m}$ & Calling SU \\
\hline
\end{tabular}

Table 79. DRST output data element requirements.

\begin{tabular}{c|c|c}
\hline Name & Description & Units \\
\hline$x$ & Transform of field & $\mu \mathrm{V} / \mathrm{m}$ \\
\hline
\end{tabular}

\subsubsection{Flat-Earth Direct Ray (FEDR) SU}

The FEDR SU determines the propagation factor and loss based on FE calculations, for the direct ray path only, for regions above and below the PE maximum propagation angle.

Upon entering the SU the square of the current range, $r_{s q}$, is initialized to $\operatorname{rsqrd}_{i_{s t p}}$. Next, the earth curvature height correction factor $r_{s q k}$ is initialized according to the calculation region:

$$
\begin{aligned}
& r_{s q k}=\frac{r_{s q}}{t w o k a} ; \quad \text { above PE region } \\
& r_{s q k}=\frac{r_{s q}}{t w o k a_{\text {down }}} ; \text { below PE region. }
\end{aligned}
$$


Steps 1 through 2 are performed for all heights within array zoutma with index $j$ varying from $j_{a s}$ to $j_{a e}$.

1. First, the direct ray angle is computed as

$$
\alpha=\mathbf{T A N}^{-1}\left(\frac{\text { zoutma }_{j}-r_{\text {sqk }}}{r_{\text {out }}}\right)
$$

2. Next, if the direct ray $\alpha$ is greater than the tangent angle $\alpha_{\text {ter }}$ produced from the antenna height to the current terrain height, or the calculations are for the upper PE region, then steps 2.a through 2.e are performed.

a. The ANTPAT SU is referenced to obtain the antenna pattern factor $f(\alpha)$ for the direct ray.

b. The path length of the direct ray is then computed:

$$
r_{1}=\sqrt{\left(\text { zoutma }_{j}-r_{\text {sqk }}\right)^{2}+r_{s q}}
$$

c. The propagation factor $\left(F_{d B}\right)$ and loss $(L)$ are then computed from

$$
\begin{aligned}
L & =20 \mathbf{L O G}_{\mathbf{1 0}}\left(r_{1}\right)+p l_{c n s t}-20 \mathbf{L O G}_{\mathbf{1 0}}\left(\mathbf{M A X}\left(f(\alpha), 10^{-13}\right)\right)+r_{1} g a s_{a t t} \\
F_{d B} & =20 \mathbf{L O G}_{\mathbf{1 0}}\left(r_{1}\right)+p l_{c n s t}-L .
\end{aligned}
$$

Note that $F_{d B}$ above is actually 20 times the logarithm of the propagation factor $F$ as defined in most text books.

d. Next, $L$ and $F_{d B}$ are multiplied by 10 and rounded to the nearest integer, then stored in array $m p f l$.

e. If the propagation factor and angle are to be output (lang=`.true.'), then $F_{d B}$ and $\alpha$ are stored in array propaf.

Once the loss has been computed for all heights, the final step is to compute the propagation factor at $1 \mathrm{~m}$ above the surface if clutter computations are to be performed $\left(C_{\text {lut }}=\right.$ '.true.'). The SU is then exited. Table 80 and Table 81 provide identification, description, units of measure, and the computational source for each FEDR SU input and output data element. 
Table 80. FEDR SU input data element requirements.

\begin{tabular}{|c|c|c|c|}
\hline Name & Description & Units & Source \\
\hline$\alpha_{t e r}$ & $\begin{array}{l}\text { Tangent angle from antenna height to terrain height at } \\
\text { current range }\end{array}$ & radians & CALCLOS SU \\
\hline$a n t_{r e f}$ & $\begin{array}{l}\text { Transmitting antenna height relative to the reference } \\
\text { height } h_{\text {minter }}\end{array}$ & meters & TERINIT SU \\
\hline$C_{l u t}$ & $\begin{array}{l}\text { Logical flag used to indicate if surface clutter } \\
\text { calculations are desired. }\end{array}$ & N/A & Calling CSCI \\
\hline $\operatorname{gas}_{a t t}$ & Gaseous absorption attenuation rate & $\mathrm{dB} / \mathrm{km}$ & GASABS SU \\
\hline$i_{\text {flag }}$ & $\begin{array}{l}\text { Flag indicating which portion of the FE region is being } \\
\text { computed. } \\
\begin{aligned} 0=\text { loss is computed for heights above PE region } \\
1=\text { loss is computed for heights below PE region }\end{aligned}\end{array}$ & N/A & Calling SU \\
\hline$i_{s t p}$ & Current output range step index & N/A & Calling CSCI \\
\hline$j_{a e}$ & Ending index within $m p f l$ of FE loss values & N/A & Calling SU \\
\hline$j_{a s}$ & Starting index within $m p f l$ of FE loss values & N/A & Calling SU \\
\hline lang & $\begin{array}{l}\text { Logical flag indicating if propagation angle and } \\
\text { propagation factor output is desired. }\end{array}$ & N/A & Calling CSCI \\
\hline$r_{\text {out }}$ & Current output range & meters & Calling SU \\
\hline$p l_{\text {cnst }}$ & $\begin{array}{l}\text { Constant used in determining propagation loss }\left(p l_{c n s t}=\right. \\
\left.20 \log _{10}\left(2 k_{o}\right)\right)\end{array}$ & $\mathrm{dB} / \mathrm{m}$ & APMINIT CSC \\
\hline rsqrd & Array containing the square of all desired output ranges & meters $^{2}$ & APMINIT CSC \\
\hline twoka & Twice the effective earth radius & meters & GET_K SU \\
\hline twoka $a_{\text {down }}$ & Twice the effective earth radius for downward path & meters & GET_K SU \\
\hline$Z_{C}$ & $\begin{array}{l}\text { Height at which to compute the propagation factor for } \\
\text { clutter computations. The height is specified with respect } \\
\text { to } h m_{\text {ref }}\end{array}$ & meters & APMINIT CSC \\
\hline zoutma & Array output heights relative to "real" ant $t_{r e f}$ & meters & APMINIT CSC \\
\hline
\end{tabular}

Table 81. FEDR SU output data element requirements.

\begin{tabular}{c|l|c}
\hline Name & \multicolumn{1}{|c}{ Description } & Units \\
\hline Fat1m & Propagation factor computed at 1 m above the surface. & $\mathrm{dB}$ \\
$m p f l$ & Array of propagation factor and loss & $\mu \mathrm{V} / \mathrm{m}$ \\
propaf & $\begin{array}{l}\text { Two-dimensional array, containing the propagation angles and factors for } \\
\text { the direct and reflected rays (where applicable) for all output height/range } \\
\text { points }\end{array}$ & $\begin{array}{c}\text { radians,d } \\
\text { B }\end{array}$ \\
\hline
\end{tabular}

\subsubsection{Flat-Earth Model (FEM) SU}

The FEM SU computes propagation loss at a specified range based upon FE approximations. The following steps 1 through 11, are performed for each APM height output point $j$ from $j_{f s}$ to $j_{f e}$. 
1. The receiver height at the $j^{\text {th }}$ output point, zout $j_{\text {, is }}$ first adjusted relative to the antenna height for both the direct and reflected ray paths and is also corrected for earth curvature and average refraction. The receiver heights, $z_{m}$ and $z_{p}$, relative to both the real (direct) and image (reflected) antenna height, respectively, are defined as follows:

$$
\begin{gathered}
z_{m}=\text { zoutma }_{j}-\frac{r_{\text {out }}^{2}}{t w o k a} \\
z_{p}=\text { zoutpa }_{j}-\frac{r_{\text {out }}^{2}}{\text { twoka }}
\end{gathered}
$$

where zoutma $a_{j}$ and zoutpa $a_{j}$ represent the output height zout $t_{j}$ relative to the "real" and "image" antenna heights, respectively, with respect to mean sea level. twoka is twice the effective earth radius as calculated in the FILLHT SU. If the range $r_{\text {out }}$ is less than $2.5 \mathrm{~km}$, then $z_{m}$ and $z_{p}$ are set equal to zoutma and zoutpa $a_{j}$, respectively.

2. Next, the point or range, of reflection, $x_{\text {reflect }}$, is given by

$$
X_{\text {reflect }}=r_{\text {out }} \frac{a n t_{\text {ref }}}{z_{p}}
$$

This quantity is used when referencing the GETREFCOEF SU.

3. The elevation angles for the direct- and reflected-path rays, $\alpha_{d}$ and $\alpha_{r}$, respectively, are given as

$$
\begin{aligned}
& \alpha_{d}=\mathbf{T A N}^{-1}\left(\frac{z_{m}}{r_{\text {out }}}\right) \\
& \alpha_{r}=\mathbf{T A N}^{-1}\left(\frac{z_{p}}{r_{\text {out }}}\right)
\end{aligned}
$$

4. The ANTPAT SU is referenced with the direct-path elevation angle to obtain the antenna pattern factor for the direct-path ray, $f\left(\alpha_{d}\right)$; and with the grazing angle (opposite of the reflected-path ray angle) to obtain the antenna pattern factor for the surface-reflected ray, $f\left(-\alpha_{r}\right)$. 
5. The path lengths for both the direct-path, $r_{1}$, and surface-reflected path, $r_{2}$, are computed from simple right triangle calculations, as

$$
\begin{aligned}
& r_{1}=\sqrt{z_{m}^{2}+r_{\text {out }}^{2}}, \\
& r_{2}=\sqrt{z_{p}{ }^{2}+r_{\text {out }}{ }^{2}}
\end{aligned}
$$

6. The GETREFCOEF SU is referenced with the reflected-path ray angle to obtain the amplitude, $R_{\text {mag }}$, and phase angle, $\varphi$, of the surface-reflection coefficient.

7. From the two path lengths, the surface-reflection phase lag angle, and the free-space wave number, $k_{o}$, the total phase angle is determined as

$$
\Omega=\left(r_{2}-r_{1}\right) k_{o}+\varphi
$$

8. The square of the coherent sum of both the direct-path ray and surface-reflected path ray is computed as

$$
f_{\text {sum }}^{2}=\left|f\left(\alpha_{d}\right)^{2}+R_{\text {mag }}^{2} f\left(-\alpha_{r}\right)^{2}+2 f\left(\alpha_{d}\right) R_{\text {mag }} f\left(-\alpha_{r}\right) \cos (\Omega)\right|
$$

9. The propagation factor in decibels, $F_{d B}$, is computed as

$$
F_{d B}=10 \mathbf{L O G}_{\mathbf{1 0}}\left[\mathbf{M A X}\left(f_{\text {sum }}^{2}, 10^{-25}\right)\right]
$$

A limit of $-250 \mathrm{~dB}$ was put on $F_{d B}$ to avoid underflow problems.

10. The propagation factor and loss for the output point zout $t_{j}$ is calculated and rounded to the nearest centibel as

$$
\begin{aligned}
& \operatorname{mpfl}_{1, j}=\mathbf{N I N T}\left(10\left(L_{f s}-F_{d B}+r_{1} g a s_{a t t}\right)\right) \\
& m p l_{2, j}=\operatorname{NINT}\left(10\left(L_{f s}-m p l_{1, j}\right)\right)
\end{aligned}
$$

where $L_{f s}$ is the free-space loss term in decibels and is given by

$$
L_{f s}=20 \mathbf{L O G}_{10}\left(r_{1}\right)+p l_{c n s t}
$$

11. If the propagation factor and angle are to be output (lang='.true.'), then $\alpha_{d}, \alpha_{r}, f\left(\alpha_{d}\right)$, and $R_{\text {mag }} f\left(\alpha_{r}\right)$, in $\mathrm{dB}$, are stored in array propaf. 
Once the loss has been computed for all heights, the final step is to compute the propagation factor at $1 \mathrm{~m}$ above the surface if clutter computations are to be performed $\left(C_{\text {lut }}=\right.$ '.true.'). The propagation factor is computed at height $z_{c}$ according to steps 1 through 11 above. The SU is then exited.

Table 82 and Table 83 provide identification, description, units of measure, and the computational source for each FEM SU input and output data element.

Table 82. FEM SU input data element requirements.

\begin{tabular}{|c|c|c|c|}
\hline Name & Description & Units & Source \\
\hline$a_{n} t_{r e f}$ & Transmitting antenna height relative to $h_{\text {minter }}$ & meters & TERINIT SU \\
\hline$C_{\text {lut }}$ & $\begin{array}{l}\text { Logical flag used to indicate if surface clutter calculations } \\
\text { are desired. }\end{array}$ & N/A & Calling CSCI \\
\hline gas $_{a t t}$ & Gaseous absorption & $\mathrm{dB} / \mathrm{m}$ & GASABS SU \\
\hline$h t_{l i m}$ & Maximum height relative to $h_{\text {minter }}$ & meters & TERINIT SU \\
\hline$i_{s t p}$ & Current output range step index & N/A & Calling SU \\
\hline$j_{f e}$ & Ending index within $m p f l$ of FE loss values & N/A & Calling SU \\
\hline$j_{f s}$ & Starting index within $m p f l$ of FE loss values & N/A & Calling SU \\
\hline$k_{o}$ & Free-space wavenumber & meters $^{-1}$ & APMINIT CSC \\
\hline lang & $\begin{array}{l}\text { Logical flag indicating if propagation angle and } \\
\text { propagation factor output is desired. }\end{array}$ & N/A & Calling CSCI \\
\hline$p l_{\text {cnst }}$ & $\begin{array}{l}\text { Constant used in determining propagation loss }\left(p l_{\text {cnst }}=20\right. \\
\left.\log _{10}\left(2 k_{o}\right)\right)\end{array}$ & $\mathrm{dB} / \mathrm{m}^{2}$ & APMINIT CSC \\
\hline$r_{\text {out }}$ & Current output range & meters & Calling SU \\
\hline rsqrd & Array containing the square of all desired output ranges & meters $^{2}$ & APMINIT CSC \\
\hline twoka & Twice the effective earth’s radius & meters & GET_K SU \\
\hline$y_{\text {fref }}$ & Ground elevation height at source & meters & APMINIT CSC \\
\hline$z_{c}$ & $\begin{array}{l}\text { Height at which to compute the propagation factor for } \\
\text { clutter computations. The height is specified with respect to } \\
h_{\text {ref }}\end{array}$ & meters & APMINIT CSC \\
\hline zoutma & Array output heights relative to "real" ant $t_{r e f}$ & meters & APMINIT CSC \\
\hline zoutpa & Array output heights relative to "image" ant $t_{r e f}$ & meters & APMINIT CSC \\
\hline
\end{tabular}

Table 83. FEM SU Output Data Element Requirements.

\begin{tabular}{c|l|c}
\hline Name & \multicolumn{1}{|c}{ Description } & Units \\
\hline$\alpha_{d}$ & Direct path ray angle & radians \\
Fat1m & Propagation factor computed at 1 m above the surface. & $\mathrm{dB}$ \\
$m p f l$ & Propagation factor and loss array & $\mathrm{cB}$ \\
propaf & Two-dimensional array, containing the propagation angles and factors for the & radians,d \\
& direct and reflected rays (where applicable) for all output height/range points & $\mathrm{B}$ \\
\hline
\end{tabular}




\subsubsection{Fast-Fourier Transform (FFT) SU}

The FFT SU separates the real and imaginary components of the complex PE field into two real arrays and then references the DRST SU to transform each portion of the PE solution.

For a transform size, $n_{f f t}$, the real and imaginary parts of the complex PE field array $U$, respectively, are found for the index $I$ from 0 to $n_{f f t}$ :

$$
\begin{aligned}
& \operatorname{xdum}_{i}=\operatorname{REAL}\left(U_{i}\right) \\
& \operatorname{ydum}_{i}=\mathbf{I M A G}\left(U_{i}\right)
\end{aligned}
$$

The DRST SU is referenced in turn for $x d u m$ and $y d u m$ along with $I n_{f f t}$, the power of the transform size to the base 2 . The real and imaginary parts of the resulting transform arrays are then converted to the complex array $U$ for $i$ equal 0 to $n_{f f t}$ by

$$
U_{i}=\mathbf{C M P L X}\left(x_{\text {dum }_{i}}, y_{\mathrm{dum}}\right) .
$$

Table 84 and Table 85 provide identification, description, units of measure, and the computational source for each FFT SU input and output data element.

Table 84. FFT SU input data element requirements.

\begin{tabular}{c|l|c|c}
\hline Name & \multicolumn{1}{|c|}{ Description } & Units & Source \\
\hline$n_{f f t}$ & Power of 2 transform size, i.e. $n_{f f t}=2^{l n} f f t$ & N/A & FFTPAR SU \\
$n_{f f t}$ & Transform size & N/A & FFTPAR SU \\
$U$ & Complex field to be transformed & $\mu \mathrm{V} / \mathrm{m}$ & Calling SU \\
\hline
\end{tabular}

Table 85. FFT SU output data element requirements.

\begin{tabular}{c|c|c}
\hline Name & Description & Units \\
\hline$U$ & Transform of complex field & $\mu \mathrm{V} / \mathrm{m}$ \\
\hline
\end{tabular}

\subsubsection{Free Space Range Step (FRSTP) SU}

The FRSTP SU is to propagate the complex PE solution in free space by one range step.

Upon entry the PE field, farray, is transformed to p-space (Fourier space) and its array elements are multiplied by corresponding elements in the free space propagator array, frsp. Before exiting, the PE field is transformed back to z-space. Both transforms are performed by referencing the FFT SU. 
Table 86 and Table 87 provide identification, description, units of measure, and the computational source for each FRSTP SU input and output data element.

Table 86. FRSTP SU input data element requirements.

\begin{tabular}{c|l|c|c}
\hline Name & \multicolumn{1}{|c|}{ Description } & Units & Source \\
\hline farray & Field array to be propagated one range step in free space & $\mu \mathrm{V} / \mathrm{m}$ & Calling SU \\
frsp & Complex free space propagator term array & N/A & PEINIT SU \\
$n_{m 1}$ & $n_{f f t}-1$ & N/A & PEINIT SU \\
\hline
\end{tabular}

Table 87. FRSTP SU output data element requirements.

\begin{tabular}{c|l|c}
\hline Name & Description & Units \\
\hline farray & Propagated field array & $\mu \mathrm{V} / \mathrm{m}$ \\
\hline
\end{tabular}

\subsubsection{FZLIM SU}

The FZLIM SU calculates and stores the outward propagation angle and propagation factor at the top of the PE region for the current PE range. The following steps 1 through 5 are performed for each reference to the FZLIM SU.

1. The FN_GETPFAC function is referenced to determine the propagation factor $F_{d B}$ at height $z_{\text {lim }}-y_{\text {cur }}$.

2. If this is the first reference to the FZLIM SU (iz = 1), then the FN_GETPFAC function is referenced to determine the propagation factor, $F_{d B l s t}$, at the previous $\mathrm{PE}$ range. A linear interpolation is performed on $F_{d B}$ and $F_{d B l s t}$ to compute the propagation factor at range $r_{a t z}$ where the $\mathrm{XO}$ region begins. The interpolated propagation factor and the outward propagation angle, $F r_{a t z}$ and $a_{a t z}$, respectively, are stored in array ffacz. Next, a reference to the SAVEPRO SU is made to store the refractivity profile at the current range from height $z_{\text {lim }}$ to the maximum desired output height.

3. A reference is made to the SPECEST SU to determine the outward propagation angle, $\vartheta_{\text {out }}$. The counter iz is incremented, but is limited to $i z_{\max }$. The propagation factor $F_{d B}$, current PE range $r$, and $\vartheta_{\text {out }}$ (with maximum limit of $a_{a t z}$ ) are stored in $\mathrm{ffacz}_{1, i z}$, $f f a c z_{2, i z}$, and $f f a c z_{3, i z}$, respectively.

4. If iz is greater than 2 , then the propagation angle is checked and slightly altered to avoid extreme spiking when using these angles in the XO region. If $f_{t e r}$ is '.false.' then the angle stored in ffacz is the smaller of $\vartheta_{\text {out }}$ or the previously stored angle. Now, if 
$f_{\text {ter }}$ is '.false.', or conversely, if $f_{\text {ter }}$ is '.true.' AND iz is less than or equal to 10 , then the $i z^{\text {th }}$ angle stored is adjusted and given by

$$
\begin{aligned}
& \alpha_{d i f}=f f a c z_{3, i z}-f f a c z_{3, i z-1} \\
& f f a c z_{3, i z}=f f a c z_{3, i z-1} \pm \operatorname{MIN}\left(\alpha_{d i f}, 10^{-4}\right)
\end{aligned}
$$

where '+' or '-' is used depending on the sign of $\alpha_{\text {dif }}$.

5. Before exiting, a final reference to the SAVEPRO SU is made to store the refractivity profile from height $z_{\text {lim }}$ to the maximum desired output height at the current range.

Table 88 and Table 89 provide identification, description, units of measure, and the computational source for each FZLIM SU input and output data element.

\begin{tabular}{|c|c|c|c|}
\hline Name & Description & Units & Source \\
\hline$a_{a t z}$ & Local ray or propagation angle at height $z_{\text {lim }}$ and range $r_{a t z}$ & radians & APMINIT CSC \\
\hline$\Delta r_{P E}$ & PE range step & meters & PEINIT SU \\
\hline$\Delta z_{P E}$ & PE mesh height increment (bin width in z-space) & meters & FFTPAR SU \\
\hline$f_{\text {ter }}$ & $\begin{array}{l}\text { Logical flag indicating if terrain profile has been specified: } \\
\text {.true. = terrain profile specified } \\
\text {.false. = terrain profile not specified }\end{array}$ & N/A & APMINIT CSC \\
\hline$i z$ & $\begin{array}{l}\text { Number of propagation factor, range, angle triplets stored in } \\
\text { ffacz }\end{array}$ & N/A & $\begin{array}{l}\text { APMINIT CSC } \\
\text { FZLIM SU }\end{array}$ \\
\hline$i z_{\max }$ & $\begin{array}{l}\text { Maximum number of points allocated for arrays associated } \\
\text { with XO calculations }\end{array}$ & N/A & APMINIT CSC \\
\hline$r$ & Current PE range & meters & Calling SU \\
\hline$r_{a t z}$ & Range at which $z_{\text {lim }}$ is reached (used for hybrid model) & meters & APMINIT CSC \\
\hline$r_{\text {last }}$ & Previous PE range & meters & Calling SU \\
\hline$r_{\log }$ & $10 \log _{10}($ PE range $r)$ & N/A & PESTEP SU \\
\hline$r_{\text {loglst }}$ & $10 \log _{10}\left(\right.$ previous PE range $\left.r_{\text {last }}\right)$ & N/A & PESTEP SU \\
\hline$U$ & Complex PE field at range $r$ & $\mu \mathrm{V} / \mathrm{m}$ & PESTEP SU \\
\hline Ulast & Complex PE field at range $r_{\text {last }}$ & $\mu \mathrm{V} / \mathrm{m}$ & PESTEP SU \\
\hline$y_{\text {cur }}$ & Height of ground at current range $r$ & meters & PESTEP SU \\
\hline$y_{\text {last }}$ & Height of ground at previous range $r_{\text {last }}$ & meters & PESTEP SU \\
\hline$z_{\text {lim }}$ & Height limit for PE calculation region & meters & GETTHMAX SU \\
\hline
\end{tabular}

Table 88. FZLIM SU input data element requirements. 
Table 89. FZLIM SU output data element requirements.

\begin{tabular}{|c|c|c|}
\hline Name & Description & Units \\
\hline ffacz & Array containing propagation factor, range, and propagation angle at $z_{\text {lim }}$ & $\begin{array}{l}\mathrm{dB} \text {, meters, } \\
\text { radians }\end{array}$ \\
\hline$i z$ & Number of propagation factor, range, angle triplets stored in ffacz & N/A \\
\hline
\end{tabular}

\subsubsection{Get Propagation Factor (FN_GETPFAC) Function}

The FN_GETPFAC function determines the propagation factor at a specified height.

First, linear interpolation is performed on the magnitudes of the PE field at bins $k$ and $k+1$ to determine the magnitude $p_{\text {mag }}$ of the field at the receiver height, $z_{r}$ :

$$
p_{\text {mag }}=\left|U_{k}\right|+f_{r}\left(\left|U_{k+1}\right|-\left|U_{k}\right|\right)
$$

where the interpolation fraction $f_{r}$ is determined from

$$
\begin{gathered}
f_{r}=\frac{z_{r}}{\Delta z_{P E}}-k ; \quad k \Delta z_{P E} \leq z_{r}<(k+1) \Delta z_{P E} \\
k=\mathbf{I N T}\left(\frac{z_{r}}{\Delta z_{P E}}\right) .
\end{gathered}
$$

$p_{\text {mag }}$ is constrained to be not less than $10^{-20} \mu \mathrm{V} / \mathrm{m}$. Finally, the propagation factor in $\mathrm{dB}$, $F_{d B}$ is given by

$$
F_{d B}=20 \mathbf{L O G}_{\mathbf{1 0}}\left(\operatorname{MAX}\left(p_{\text {mag }}, 10^{-20}\right)\right)+r_{l o g} .
$$

Table 90 and Table 91 provide identification, description, units of measure, and the computational source for each FN_GETPFAC function input and output data element.

Table 90. FN_GETPFAC SU input data element requirements.

\begin{tabular}{c|l|c|l}
\hline Name & \multicolumn{1}{|c|}{ Description } & Units & \multicolumn{1}{|c}{ Source } \\
\hline$\Delta z_{P E}$ & PE mesh height increment (bin width in z-space) & meters & Calling SU \\
$r_{l o g}$ & $10 \log _{10}($ PE range $r)$ & N/A & Calling SU \\
$U$ & Complex PE field at range $r$ & $\mu \mathrm{V} / \mathrm{m}$ & Calling SU \\
$z_{r}$ & Receiver height & meters & Calling SU \\
\hline
\end{tabular}


Table 91. FN_GETPFAC SU output data element requirements.

\begin{tabular}{c|c|c}
\hline Name & Description & Units \\
\hline$F_{d B}$ & Propagation factor at specified height $z_{r}$ & $\mathrm{~dB}$ \\
\hline
\end{tabular}

\subsubsection{Get Reflection Coefficient (GETREFCOEF) SU}

The GETREFCOEF SU computes the Fresnel complex reflection coefficient for a given grazing angle, $\psi$.

Upon entering, the proper dielectric constant $n c_{i}^{2}$ to be applied to the reflected ray must be determined. If the current range is within the $\mathrm{FE}$ and $\mathrm{RO}$ regions, the FN_DIECON function is referenced to determine the correct $n c_{i}^{2}$ for that range. Otherwise, the value for $n c_{i}^{2}$ is set equal to the current value, $n c_{i g}^{2}$ at the PE range. The corresponding dielectric constant $n c_{i}^{2}$ is used in the following equations to compute the reflection coefficient:

$$
\begin{gathered}
\Gamma_{0 V}=\frac{n c_{i}^{2} \operatorname{SIN}(\psi)-\sqrt{n c_{i}^{2}-\operatorname{COS}^{2}(\psi)}}{n c_{i}^{2} \operatorname{SIN}(\psi)+\sqrt{n c_{i}^{2}-\operatorname{COS}^{2}(\psi)}}, \\
\Gamma_{0 H}=\frac{\operatorname{SIN}(\psi)-\sqrt{n c_{i}^{2}-\operatorname{COS}^{2}(\psi)}}{\operatorname{SIN}(\psi)+\sqrt{n c_{i}^{2}-\operatorname{COS}^{2}(\psi)}}
\end{gathered}
$$

where $\Gamma_{0 V}$ and $\Gamma_{0 H}$ represent the reflection coefficients for vertical and horizontal polarization, respectively, and $n c_{i}^{2}$ is computed in the DIEINIT SU and is given by

$$
n c_{i}^{2}=\varepsilon_{i}+j 60 \sigma_{i} \lambda
$$

$\varepsilon_{i}$ and $\sigma_{i}$ are the relative permittivity and conductivity, respectively, to be applied at range rgrnd $_{i}$, and $\lambda$ is the wavelength.

If rough surface calculations are required (ruf = '.true.') the Miller-Brown roughness reduction factor is computed. Wind speeds specified by the calling CSCI are allowed to vary with range, therefore the FN_CURWIND function is referenced to obtain the wind speed $\omega_{s}$ at the current range. The sea surface rms wave height is then computed from

$$
r u f_{h t}=r u f_{f a c} \omega_{s}^{2}
$$


and the roughness reduction factor $\rho$ is determined by

$$
\rho=\left(3.2 x_{g}-2+\sqrt{\left(3.2 x_{g}\right)^{2}-7 x_{g}+9}\right)^{-1 / 2},
$$

where

$$
x_{g}=\frac{1}{2}\left[r u f_{h t} \operatorname{SIN}(\psi)\right]^{2} .
$$

The final reflection coefficient is then computed as $\Gamma_{V, H}=\rho \Gamma_{0 V, 0 H}$.

Lastly, the magnitude and phase of the complex reflection coefficient are determined from

$$
\begin{aligned}
& R_{\text {mag }}=\left|\Gamma_{V, H}\right| \\
& \varphi=\mathbf{T A N}^{-1}\left(\frac{\mathfrak{I}\left(\Gamma_{V, H}\right)}{\mathfrak{R}\left(\Gamma_{V, H}\right)}\right) .
\end{aligned}
$$

\begin{tabular}{|c|c|c|c|}
\hline Name & Description & Units & Source \\
\hline$H F_{\text {flag }}$ & $\begin{array}{l}\text { HF computation flag indicating the frequency specified } \\
\text { is less than } 50 \mathrm{MHz}\end{array}$ & N/A & APMINIT CSC \\
\hline$i_{\text {flag }}$ & $\begin{array}{l}\text { Integer flag indicating what region reflection } \\
\text { coefficient is being computed } \\
0=\mathrm{FE} \text { and RO regions } \\
1=\mathrm{PE} \text { region }\end{array}$ & N/A & Calling SU \\
\hline$i_{g r}$ & Number of different ground types specified & N/A & Calling CSCI \\
\hline$i_{p o l}$ & $\begin{array}{l}\text { Polarization flag: } \\
\qquad \begin{array}{l}0=\text { horizontal polarization } \\
1=\text { vertical polarization }\end{array}\end{array}$ & N/A & Calling CSCI \\
\hline$n c^{2}$ & Array of complex dielectric constants & N/A & DIEINIT SU \\
\hline$n_{w}$ & Number of wind speeds & N/A & Calling CSCI \\
\hline$\psi$ & Grazing angle & radians & Calling SU \\
\hline$r$ & Current calculation range & meters & Calling SU \\
\hline rgrnd & $\begin{array}{l}\text { Array containing ranges at which varying ground types } \\
\text { apply. }\end{array}$ & meters & Calling CSCI \\
\hline rngwind & $\begin{array}{l}\text { Ranges of wind speeds entered: } r n g \text { wind } \\
\text { wind speed }\end{array}$ & meters & Calling CSCI \\
\hline
\end{tabular}

Table 92 and Table 93 provide identification, description, units of measure, and the computational source for each GETREFCOEF SU input and output data element.

Table 92. GETREFCOEF SU input data element requirements. 
Table 92. GETREFCOEF SU input data element requirements. (continued)

\begin{tabular}{|c|c|c|c|}
\hline Name & Description & Units & Source \\
\hline ruf & $\begin{array}{l}\text { Logical flag indicating if rough sea surface calculations } \\
\text { are required } \\
\text { '.true.' = perform rough sea surface calculations } \\
\text { '.false.' = do not perform rough sea surface } \\
\text { calculations }\end{array}$ & N/A & APMINIT CSC \\
\hline$r u f_{f a c}$ & Factor used for wave height calculation & meters $^{-1}$ & APMINIT CSC \\
\hline wind & Array of wind speeds & meters/sec & Calling CSCI \\
\hline
\end{tabular}

Table 93. GETREFCOEF SU output data element requirements.

\begin{tabular}{c|l|c}
\hline Name & \multicolumn{1}{|c|}{ Description } & Units \\
\hline$\Gamma_{V, H}$ & Complex reflection coefficient for vertical $(\mathrm{V})$ and horizontal $(\mathrm{H})$ polarization & N/A \\
$R_{m a g}$ & Magnitude of the reflection coefficient & N/A \\
$\varphi$ & Phase of the reflection coefficient & radians \\
\hline
\end{tabular}

\subsubsection{Get Troposcatter Loss (FN_GET_TLOSS) Function}

The FN_GET_TLOSS function computes loss due to troposcatter and determine the appropriate loss to add to the already calculated propagation loss at a specific transmitter and receiver point over land and water.

Upon entering the function, ftloss is initialized to the propagation loss at the particular receiver height and range, xloss. The range from the receiver to the tangent point $d 2$ is also initialized to that value for smooth surface, $d 2 s e$.

The tangent angle from the receiver, $\vartheta_{2}$, is initialized to the tangent angle from the particular receiver height over smooth surface, $\vartheta 2 s e$. However, if $f_{\text {ter }}=$ '.true.', then the largest tangent angle $a_{2}$ and range $d_{2}$ from the receiver to the tangent point are determined using an iterative loop performed for index $i$ from $j_{t 2}-1$ to $j_{t 1}$ in decrements of -1 as follows:

$$
\begin{gathered}
r_{2}=r_{\text {out }}-i \Delta r_{P E} \\
a_{2}=\frac{h-t y h_{i}}{r_{2}}+\frac{r_{2}}{t w o k a},
\end{gathered}
$$

where $h$ is the reciever height.

If the current $\vartheta_{2}$ value is less than $a_{2}$, then $\vartheta_{2}$ is set equal to $a_{2}$ and $d_{2}$ is set equal to $r_{2}$. The index $i$ is decremented by one and the above calculations are repeated. 
Once the above loop is completed, the final value of $\vartheta_{2}$ is checked. If it is greater than the tangent angle for smooth surface $\vartheta 2$ se (at the same receiver range), then both $\vartheta_{2}$ and $d_{2}$ are set equal to $\vartheta 2 s e$ and $d 2 s e$, respectively.

Next, if $r_{\text {out }}$ is less than the sum of the tangent ranges, $d_{1}$ and $d_{2}$, then the SU is exited. Otherwise, function program flow continues with the next step.

To account for antenna pattern effects over terrain, the ANTPAT SU is referenced using the tangent angle from the source to determine the antenna pattern factor, $f\left(\vartheta_{1}\right)$. The troposcatter loss term is then adjusted from its smooth surface value as

$$
t l s t=t l s t_{s}-20 \mathbf{L O G}_{10}\left[f\left(\vartheta_{1}\right)\right] .
$$

Next, the common volume scattering angle is given by

$$
\theta=\vartheta 0_{i_{s t p}}-\vartheta_{1}-\vartheta_{2} .
$$

The following calculations are made to determine the effective scattering height $h_{o}$ :

$$
\begin{aligned}
a & =\frac{1}{2} \vartheta 0_{i_{\text {stp }}}-\vartheta_{1}+\frac{a n t_{\text {dif }}}{r_{\text {out }}}, \\
b & =\frac{1}{2} \vartheta 0_{i_{\text {stp }}}-\vartheta_{2}-\frac{a n t_{\text {dif }}}{r_{\text {out }}}, \\
s & =\operatorname{MIN}\left(\operatorname{MAX}\left(0.1, \frac{a}{b}\right), 10\right), \\
h_{o} & =\frac{s r_{\text {out }} \theta}{10^{3}\left(1+s^{2}\right)} .
\end{aligned}
$$

The parameter $\eta_{s}$ is then calculated as a function of $h_{o}$ :

$$
\begin{aligned}
& \eta_{s x}=.5696 h_{o}\left(1+s n_{1} e^{-3.8 \times 10^{-6} h_{o}^{6}}\right), \\
& \eta_{s}=\operatorname{MIN}\left(\operatorname{MAX}\left(.01, \eta_{s x}\right), 5\right) .
\end{aligned}
$$

Next, the parameters $c t_{1}$ and $c t_{2}$ are defined as

$$
\begin{aligned}
& c t_{1}=16.3+13.3 \eta_{s}, \\
& c t_{2}=0.4+0.16 \eta_{s}
\end{aligned},
$$


where these are in turn used to calculate the quantities $r_{1}$ and $r_{2}$ :

$$
\begin{aligned}
& r_{1}=\operatorname{MAX}\left(0.1, r t_{1} \theta\right), \\
& r_{2}=\operatorname{MAX}\left(0.1, r_{f} h \theta\right) .
\end{aligned}
$$

The quantity $r_{f}$ was previously determined by referencing the TROPOINIT SU.

$c t_{1}, c t_{2}, r_{1}$, and $r_{2}$ are next used to determine $H_{1}$ and $H_{2}$;

$$
\begin{aligned}
& H_{1}=\mathbf{M A X}\left[0, c t_{1}\left(r_{1}+c t_{2}\right)^{-4 / 3}\right], \\
& H_{2}=\operatorname{MAX}\left[0, c t_{1}\left(r_{2}+c t_{2}\right)^{-4 / 3}\right] .
\end{aligned}
$$

The frequency gain function $H_{o}$ is then determined by

$$
H_{o}=\frac{H_{1}+H_{2}}{2}+\Delta H_{o}
$$

where

$$
\begin{aligned}
& \Delta H_{o}=6\left[.6-\mathbf{L O G}_{10}\left(\eta_{s}\right)\right] \mathbf{L O G}_{10}(s) \mathbf{L O G}_{10}\left(q_{t}\right), \\
& q_{t}=\operatorname{MIN}\left[10, \mathbf{M A X}\left(0.1, \frac{r_{2}}{s r_{1}}\right)\right] .
\end{aligned}
$$

$\Delta H_{o}$ is not allowed to be larger than $1 / 2\left(H_{1}+H_{2}\right)$ and $H_{o}$ is set equal to 0 if it becomes negative.

Next, the troposcatter loss is computed from

$$
t_{\text {loss }}=t l s t+573 \theta+r \log o_{i_{s p p}}+H_{o} .
$$

Finally, through a method of "bold interpolation" the final propagation loss ftloss, is adjusted for loss due to troposcatter according to

$$
\begin{aligned}
L_{\text {dif }} & =\text { ftloss }-t_{\text {loss }}, \\
\text { ftloss } & =t_{\text {loss }} \quad \text { for } L_{\text {dif }} \geq 18 d B, \\
\text { ftloss } & =\text { ftloss }-10 \mathbf{L O G}_{10}\left(1+10^{0.1 L_{\text {dif }}}\right) \text { for } L_{\text {dif }} \geq-18 d B .
\end{aligned}
$$


Table 94 and Table 95 provide identification, description, units of measure, and the computational source for each FN_GET_TLOSS function input and output data element.

Table 94. FN_GET_TLOSS function input data element requirements.

\begin{tabular}{|c|c|c|c|}
\hline Name & Description & Units & Source \\
\hline$a_{\text {dif }}$ & Difference between the transmitter and receiver heights & meters & Calling SU \\
\hline$d 1$ & Range from the transmitter source to the tangent point & meters & Calling SU \\
\hline$d 2 s e$ & $\begin{array}{l}\text { Range from the receiver height to the tangent point over } \\
\text { smooth surface }\end{array}$ & meters & Calling SU \\
\hline$f_{\text {ter }}$ & $\begin{array}{l}\text { Logical flag indicating if terrain profile has been specified: } \\
\text { '.true.' = terrain profile specified } \\
\text { '.false.' = terrain profile not specified }\end{array}$ & N/A & APMINIT CSC \\
\hline$h$ & Receiver height at particular range rout & meters & Calling SU \\
\hline$i_{s t p}$ & Current output range step index & N/A & Calling SU \\
\hline jt1 & Ending index counter for tyh array & N/A & Calling SU \\
\hline jt2 & Starting index counter for tyh array & N/A & Calling SU \\
\hline$r_{f}$ & Constant used for troposcatter calculations & meters $^{-1}$ & TROPOINIT SU \\
\hline rlogo & Array containing 20 times the logarithm of all output ranges & N/A & APMINIT CSC \\
\hline$r_{\text {out }}$ & Current output range & meters & Calling SU \\
\hline$r t_{1}$ & $r_{f} * a n t_{r e f}$ & N/A & TROPOINIT SU \\
\hline$s n_{1}$ & $\begin{array}{l}\text { Surface refractivity term used in troposcatter loss } \\
\text { calculation }\end{array}$ & N/A & Calling SU \\
\hline$\vartheta 0$ & $\begin{array}{l}\text { Array of angles used to determine common volume } \\
\text { scattering angle }\end{array}$ & radians & TROPOINIT SU \\
\hline$\vartheta_{1}$ & Tangent angle from source (for smooth surface) & radians & Calling SU \\
\hline $92 \mathrm{se}$ & Tangent angle from the receiver height over smooth surface & radians & Calling SU \\
\hline$t^{l s t_{s}}$ & Troposcatter loss term for smooth surface & N/A & Calling SU \\
\hline twoka & Twice the effective earth radius & meters & GET_K SU \\
\hline tyh & $\begin{array}{l}\text { Adjusted height points of terrain profile at every PE range } \\
\text { step. }\end{array}$ & meters & PEINIT SU \\
\hline xloss & Propagation loss for receiver height $h$ and range $r_{\text {out }}$ & $\mathrm{dB}$ & Calling SU \\
\hline
\end{tabular}

Table 95. FN_GET_TLOSS function output data element requirements.

\begin{tabular}{c|c|c}
\hline Name & Description & Units \\
\hline ftloss & Propagation loss with troposcatter & $\mathrm{dB}$ \\
\hline
\end{tabular}




\subsubsection{Linear Interpolation (FN_PLINT) Function}

This function performs a linear interpolation on parameters $p l_{1}$ and $p l_{2}$ over a fractional term $f_{\text {rac }}$.

The final interpolated value $p l_{\text {int }}$ is determined according to

$$
p l_{\text {int }}=p l_{1}+f_{\text {rac }}\left(p l_{2}-p l_{1}\right)
$$

and is returned to the calling SU.

Table 96 and Table 97 provide identification, description, units of measure, and the computational source for each FN_PLINT function input and output data element.

Table 96. FN_PLINT function input data element requirements.

\begin{tabular}{c|l|c|l}
\hline Name & \multicolumn{1}{|c|}{ Description } & Units & Source \\
\hline$f_{\text {rac }}$ & Fractional quantity over which to interpolate & N/A & Calling SU \\
$p l_{1}$ & First parameter value & N/A & Calling SU \\
$p l_{2}$ & Second parameter value & N/A & Calling SU \\
\hline
\end{tabular}

Table 97. FN_PLINT function output data element requirements.

\begin{tabular}{c|c|c}
\hline Name & Description & Units \\
\hline$p l_{i n t}$ & Interpolated parameter & N/A \\
\hline
\end{tabular}

\subsubsection{Mixed Fourier Transform (MIXEDFT) SU}

The MIXEDFT SU propagates the PE field in free space one PE range step, applying the Leontovich boundary condition, using the mixed Fourier transform as outlined by Kuttler and Dockery (1991). For finite conducting boundaries (i.e., if vertical polarization is specified or rough surface calculations are required) and the frequency is less than $400 \mathrm{MHz}$, the central difference form of the DMFT is used. If the frequency is greater than $400 \mathrm{MHz}$, the backward difference form of the DMFT is used.

Upon entering the SU, the first and last elements of the $w$ array $w_{0}$ and $w_{n_{f f t}}$ are initialized to 0 . If using the central difference form of the DMFT $\left(i_{a l g}=1\right)$ the following steps 1 through 6 are performed. 
1. The difference field for the vertical PE calculation grid is computed from the PE field as

$$
w_{i}=\frac{U_{i+1}-U_{i-1}}{2 \Delta z_{P E}}+\alpha_{h, v} U_{i} ; \quad i=1,2,3, \ldots . ., n_{m 1}
$$

2. Next, the FRSTP SU is referenced to transform $w$ to p-space, propagate the field forward one PE range step, and is transformed back to z-space upon return.

3. The coefficients used in the central difference form of the mixed transform, $c_{k 1}$ and $c_{k 2}$, are propagated to the new range as follows:

$$
\begin{aligned}
& c_{k 1}=c_{k 1} C_{1 x} \\
& c_{k 2}=c_{k 2} C_{2 x}
\end{aligned} .
$$

4. The particular solution ym of Kuttler's difference equation is then computed as follows:

$$
\begin{aligned}
& y m_{i}=2 \Delta z_{P E} w_{i}+R_{T} w_{i-1} ; \quad i=1,2,3, \ldots, n_{m 1} \\
& y m_{0}=0,
\end{aligned}
$$

where $R_{T}$ is a quadratic root as computed in the GETALN SU.

5. The complex PE field $U$ is then determined from

$$
\begin{aligned}
U_{n-i} & =R_{T}\left(y m_{n-i}-U_{n-i+1}\right) ; \quad i=1,2,3, \ldots, n_{f f t} . \\
U_{0} & =0
\end{aligned}
$$

6. The final step in computing the PE field $U$ is

$$
U_{i}=U_{i}+a_{r} r n_{i}+b_{r} r n_{n-i}(-1)^{n-i} ; i=0,1,2, \ldots, n_{f f t},
$$

where

$$
\begin{aligned}
& a_{r}=c k_{1}-R_{k}\left(1 / 2 U_{0}+1 / 2 U_{n_{f f t}} r n_{n_{f f t}}+\sum_{i=1}^{n_{f f t}-1} U_{i} r n_{i}\right), \\
& b_{r}=c k_{2}-R_{k}\left(1 / 2 U_{0} r n_{n_{f f t}}+1 / 2 U_{n_{f f t}}+\sum_{i=1}^{n_{f f t}-1} U_{n-i} r n_{i}(-1)^{i}\right) .
\end{aligned}
$$


If using the backward difference form of the DMFT $\left(i_{a l g}=2\right)$, then the following steps 1 through 6 are performed.

1. The difference field for the vertical PE calculation grid is computed from the PE field as

$$
w_{i}=U_{i}-R_{T} U_{i-1} ; \quad i=1,2,3, \ldots ., n_{m 1}
$$

2. Next, the FRSTP SU is referenced to transform $w$ to p-space, propagate the field forward one PE range step, and is transformed back to z-space upon return.

3. The coefficient $c m f t$ is then propagated forward one range step via

$$
c m f t=c m f t * c m f t_{x} .
$$

4. The particular solution of the field $U$ is now computed from

$$
\begin{aligned}
U_{i} & =w_{i}+R_{T} U_{i-1} ; \quad i=1,2,3, \ldots, n_{f f t} \\
U_{0} & =0
\end{aligned}
$$

5. The coefficient $a_{r}$ for the homogeneous solution is determined as

$$
a_{r}=\frac{c m f t-\sum_{i=0}^{n_{f f t}-1} U_{i} r n_{i}}{\left(\sum_{i=0}^{n_{f f t}-1} r n_{i}^{2}\right)} .
$$

6. Finally, the PE field $U$ for the backward difference DMFT is computed as the sum of the homogeneous and particular solutions:

$$
U_{i}=U_{i}+a_{r} r n_{i} ; i=0,1,2, \ldots, n_{f f t} .
$$


Table 98 and Table 99 provide identification, description, units of measure, and the computational source for each MIXEDFT SU input and output data element.

Table 98. MIXEDFT SU input data element requirements.

\begin{tabular}{|c|c|c|c|}
\hline Name & Description & Units & Source \\
\hline$\alpha_{h, v}$ & Surface impedance term for horizontal and vertical polarization & N/A & GETALN SU \\
\hline$C_{1 x}$ & $\begin{array}{l}\text { Constant used to propagate } c_{k 1} \text { by one range step in central } \\
\text { difference algorithm }\end{array}$ & N/A & GETALN SU \\
\hline$C_{2 x}$ & $\begin{array}{l}\text { Constant used to propagate } c_{k 2} \text { by one range step in central } \\
\text { difference algorithm }\end{array}$ & N/A & GETALN SU \\
\hline$c k_{1}$ & Coefficient used in central difference form of DMFT & N/A & ALN_INIT SU \\
\hline$c k_{2}$ & Coefficient used in central difference form of DMFT & N/A & ALN_INIT SU \\
\hline $\mathrm{cmft}$ & Coefficient used in backward difference form of DMFT & N/A & ALN_INIT SU \\
\hline $\mathrm{cmft}_{x}$ & $\begin{array}{l}\text { Constant used to propagate } \mathrm{cmft} \text { by one range step in backward } \\
\text { difference algorithm }\end{array}$ & N/A & GETALN SU \\
\hline$\triangle \mathrm{z} 2_{P E}$ & Twice the PE mesh height increment (bin width in z-space) & meters & PEINIT SU \\
\hline$i_{\text {alg }}$ & $\begin{array}{l}\text { Integer flag indicating which DMFT algorithm is being used: } \\
\begin{aligned} 0 & =\text { no DMFT algorithm will be used } \\
1 & =\text { use central difference algorithm } \\
2 & =\text { use backward difference algorithm }\end{aligned}\end{array}$ & N/A & APMINIT CSC \\
\hline$n_{\text {fft }}$ & Transform size & N/A & FFTPAR SU \\
\hline$n_{m 1}$ & $n_{f f t}-1$ & N/A & APMINIT CSC \\
\hline$R_{k}$ & Coefficient used in $C_{1}$ and $C_{2}$ calculations. & N/A & GETALN SU \\
\hline$r n$ & Array of $R_{T}$ to the $i^{\text {th }}$ power (e.g., $r n_{i}=R_{T}^{i}$ ) & N/A & GETALN SU \\
\hline$R_{T}$ & $\begin{array}{l}\text { Complex root of quadratic equation for mixed transform } \\
\text { method based on Kuttler's formulation }\end{array}$ & N/A & GETALN SU \\
\hline$U$ & Complex field at range $r$ & $\mu \mathrm{V} / \mathrm{m}$ & PESTEP SU \\
\hline
\end{tabular}

Table 99. MIXEDFT SU output data element requirements.

\begin{tabular}{c|l|c}
\hline Name & \multicolumn{1}{|c}{ Description } & Units \\
\hline$c k_{1}$ & Coefficient used in central difference form of DMFT & N/A \\
$c k_{2}$ & Coefficient used in central difference form of DMFT & N/A \\
$c m f t$ & Coefficient used in backward difference form of DMFT & N/A \\
$U$ & Complex field at range $r$ & $\mu \mathrm{V} / \mathrm{m}$ \\
$w$ & Difference equation PE field array & $\mu \mathrm{V} / \mathrm{m}$ \\
$y m$ & Field from recursion equation for central difference DMFT & $\mu \mathrm{V} / \mathrm{m}$ \\
\hline
\end{tabular}




\subsubsection{Parabolic Equation Step (PESTEP) SU}

The PESTEP SU computes propagation loss at a specified range based upon the split-step Fourier PE algorithm.

Upon entering the PESTEP SU, if the current output range step, $i_{s t p}$, is equal to 1 , the current PE range $r$ and $r_{\log }$ (10 times the logarithm of $r$ ) are set equal to zero. The current PE range step $i_{P E s t p}$ is also set equal to 0 . An iterative DO WHILE loop is then begun to advance the PE solution such that for the current PE range, a PE solution is calculated from the solution at the previous PE range. This iterative procedure is repeated in the DO WHILE loop until $r$ is greater than the output range $r_{\text {out }}$. The following steps (1 through 8) are performed for each PE range step within the DO WHILE loop.

1. First, if the current PE range, $r$, is greater than zero, then the height of the ground at the previous PE range $y_{\text {last }}$ is set to the height of the ground at the current PE range $y_{\text {cur }}$. Next, the previous PE range $r_{\text {last }}$ is set equal to the current PE range $r$. The complex PE field, $U$, of the previous range is stored in array Ulast for subsequent horizontal interpolation at range $r_{\text {out }}$. In addition, $r$ is incremented by one PE range step, $\Delta r_{P E}$. A new $r_{\text {log }}$ is computed and the PE range step $i_{P E s t p}$ is incremented by one. Finally, the range at which interpolation for range-dependent refractivity profiles is performed, $r_{\text {mid }}$, is also incremented by one-half the PE range step.

2. If performing a terrain case ( $f_{\text {ter }}$ is '.true.'), the ground heights $y_{\text {cur }}$ and $y_{\text {curm }}$, at range $r$ and $r_{\text {mid }}$, respectively, must be determined. $y_{\text {cur }}$ is set equal to the terrain height at the current PE range step, $t y h_{i_{P E s t p}} . y_{\text {curm }}$ is determined as

$$
y_{\text {curm }}=1 / 2\left(t y h_{i_{\text {PEstp }}-1}+y_{\text {cur }}\right) \text {. }
$$

If $y_{\text {cur }}$ is less than $y_{\text {last }}$ and the frequency is greater than $50 \mathrm{MHz}\left(\mathrm{HF}_{\text {flag }}=\right.$ '.false.'), then the DOSHIFT SU is referenced to shift the field accordingly.

3. If the APM CSCI is being used in a range-dependent mode (i.e., the number of profiles $n_{\text {prof }}$ is greater than 1), or if a terrain profile is specified, the REFINTER SU is referenced to compute a new modified refractive index profile, profint, adjusted by the local ground height $y_{\text {curm }}$ at range $r_{\text {mid }}$. If an HF case is being performed and a terrain profile has been specified, then the difference in terrain slopes, $t m_{d i f}$, is computed and a new environmental phase array, envpr, is re-computed as

$$
\text { envpr }_{k}=e^{i\left(\Delta r_{P E} \text { profint }_{k}+k_{0} z_{k} t_{\text {dif }}\right)} ; k=0,1,2, \ldots n_{f f t} \text {, }
$$

Otherwise, envpr is computed with the second term in the exponent set equal to 0 . 
4. The current PE range is then checked against the range of the current ground type given by array rgrnd, and if necessary, the ground type counter $i_{g}$ is incremented. The GETALN SU is then referenced to compute a new surface impedance $\alpha_{h},{ }_{v}$ if vertical polarization is required or if performing rough surface calculations. If performing an HF case, then a new surface impedance is computed at each PE range step.

5. In order to propagate the field in free space one PE range step the MIXEDFT SU is referenced if the DMFT algorithm is required; otherwise, the FRSTP SU is referenced. The field is then multiplied by the environmental array envpr.

6. Next, if the current terrain slope is positive, the DOSHIFT SU is referenced (if not performing an HF case) to shift the field by the appropriate number of bins.

7. If $\mathrm{XO}$ calculations are to be performed $\left(i_{x o} \geq 1\right)$ and the current $\mathrm{PE}$ range is greater than $r_{a t z}$, then the FZLIM SU is referenced to determine and store the outward propagation angle at the top of the PE region for subsequent use in the EXTO SU.

8. Finally, after the output range $r_{\text {out }}$ is reached and the DO WHILE loop exited, the CALCLOS SU is referenced to obtain the propagation loss values at the desired output heights at the current output range $r_{\text {out }}$.

Table 100 and Table 101 provide identification, description, units of measure, and the computational source for each PESTEP SU input and output data element.

Table 100. PESTEP SU input data element requirements.

\begin{tabular}{|c|c|c|c|}
\hline Name & Description & Units & Source \\
\hline$\Delta r_{P E}$ & PE range step & meters & PEINIT SU \\
\hline$\Delta r_{P E 2}$ & $1 / 2 \mathrm{PE}$ range step & meters & PEINIT SU \\
\hline$\Psi$ & Array of interpolated grazing angles at each PE range step & radians & GRAZE_INT SU \\
\hline filt & Cosine-tapered (Tukey) filter array & N/A & PEINIT SU \\
\hline$f_{\text {ter }}$ & $\begin{array}{l}\text { Logical flag indicating if terrain profile has been specified: } \\
\text {.true. = terrain profile specified } \\
\text {.false. = terrain profile not specified }\end{array}$ & N/A & APMINIT CSC \\
\hline$H F_{\text {flag }}$ & $\begin{array}{l}\text { HF computation flag indicating the frequency specified is } \\
\text { less than } 50 \mathrm{MHz}\end{array}$ & N/A & APMINIT CSC \\
\hline$h t$ & PE mesh height array of size $n_{f f t}$ & meters & PEINIT SU \\
\hline$i_{a l g}$ & $\begin{array}{l}\text { Integer flag indicating which DMFT algorithm is being } \\
\text { used: } \\
\qquad \begin{array}{l}0=\text { no DMFT algorithm will be used } \\
1=\text { use central difference algorithm } \\
2=\text { use backward difference algorithm }\end{array}\end{array}$ & N/A & APMINIT CSC \\
\hline
\end{tabular}


Table 100. PESTEP SU input data element requirements. (continued)

\begin{tabular}{|c|c|c|c|}
\hline Name & Description & Units & Source \\
\hline$i_{g}$ & Counter indicating current ground type being modeled & N/A & APMINIT CSC \\
\hline$i_{g r}$ & Number of different ground types specified & N/A & Calling CSCI \\
\hline$i_{P E}$ & Number of PE range steps & N/A & PEINIT SU \\
\hline$i_{P E s t p}$ & Counter indicating current PE range step & N/A & PESTEP SU \\
\hline$i_{p o l}$ & $\begin{array}{l}\text { Polarization flag: } \\
\qquad \begin{array}{l}0=\text { horizontal polarization } \\
1=\text { vertical polarization }\end{array}\end{array}$ & N/A & Calling CSCI \\
\hline$i_{s t p}$ & Current output range step index & N/A & Calling SU \\
\hline$i_{x o}$ & Number of range steps in $\mathrm{XO}$ calculation region & N/A & APMINIT CSC \\
\hline$i z$ & Counter for points stored in $f f a c z$ & N/A & FZLIM SU \\
\hline$i z_{i n c}$ & $\begin{array}{l}\text { Integer increment for storing points at top of PE region (i.e., } \\
\text { points are stored at every } i z_{\text {inc }} \text { range step) }\end{array}$ & N/A & PEINIT SU \\
\hline$n_{\text {fft }}$ & PE Transform size & N/A & FFTPAR SU \\
\hline$n_{34}$ & $3 / 4 n_{f f t}$ & N/A & PEINIT SU \\
\hline$n_{4}$ & $1 / 4 n_{f f t}$ & N/A & PEINIT SU \\
\hline$n_{\text {prof }}$ & Number of refractivity profiles & N/A & Calling CSCI \\
\hline$P E_{\text {flag }}$ & $\begin{array}{l}\text { Flag to indicate use of PE algorithm only: } \\
\text { '.true.' = only use PE sub-model } \\
\text { '.false.' = use automatic hybrid model }\end{array}$ & N/A & Calling CSCI \\
\hline profint & Profile interpolated to every $\Delta z_{P E}$ in height & M-units & REFINTER SU \\
\hline$r$ & Current PE range & meters & PESTEP SU \\
\hline$r_{a t z}$ & Range at which $Z_{\text {lim }}$ is reached (used for hybrid model) & meters & APMINIT CSC \\
\hline rgrnd & $\begin{array}{l}\text { Array containing ranges at which varying ground types } \\
\text { apply. }\end{array}$ & meters & Calling CSCI \\
\hline$r_{\text {last }}$ & Previous PE range & meters & PESTEP SU \\
\hline rlog & $10 \log ($ PE range $r$ ) & N/A & PESTEP SU \\
\hline$r_{\max }$ & Maximum specified range & meters & Calling CSCI \\
\hline$r_{\text {out }}$ & Current output range & meters & Calling SU \\
\hline ruf & $\begin{array}{l}\text { Logical flag indicating if rough sea surface calculations are } \\
\text { required } \\
\text { ‘.true.' = perform rough sea surface calculations } \\
\text { '.false.' = do not perform rough sea surface } \\
\text { calculations }\end{array}$ & N/A & APMINIT CSC \\
\hline tang & Tangent of angle array from terrain slopes. & radians & PEINIT SU \\
\hline tyh & $\begin{array}{l}\text { Adjusted height points of terrain profile at every PE range } \\
\text { step. }\end{array}$ & meters & PEINIT SU \\
\hline$U$ & Complex PE field & $\mu \mathrm{V} / \mathrm{m}$ & PESTEP SU \\
\hline$y_{\text {cur }}$ & Height of ground at current range $r$ & meters & PESTEP SU \\
\hline
\end{tabular}


Table 101. PESTEP SU output data element requirements.

\begin{tabular}{|c|c|c|}
\hline Name & Description & Units \\
\hline envpr & Complex [refractivity] phase term array interpolated every $\Delta z_{P E}$ in height & N/A \\
\hline$i_{g}$ & Counter indicating current ground type being modeled & N/A \\
\hline$i_{\text {PEstp }}$ & Counter indicating current PE range step & N/A \\
\hline$j_{\text {end }}$ & Index at which valid propagation factor and loss values in $m p f l$ end & N/A \\
\hline$j_{\text {start }}$ & Index at which valid propagation factor and loss values in $m p f l$ begin & N/A \\
\hline$m p f l$ & Two-dimensional propagation factor and loss array & $\mathrm{cB}$ \\
\hline mpfl_rtg & Propagation loss and factor at receiver heights specified in the zout_rtg array & cB \\
\hline propaf & $\begin{array}{l}\text { Two-dimensional array, containing the propagation angles and factors for the } \\
\text { direct and reflected rays (where applicable) for all output height/range points }\end{array}$ & $\begin{array}{l}\text { radians, } \\
\mathrm{dB}\end{array}$ \\
\hline$r$ & Current PE range & meters \\
\hline$r_{\text {last }}$ & Previous PE range & meters \\
\hline$r_{l o g}$ & $10 \log (\mathrm{PE}$ range $r)$ & N/A \\
\hline$r_{\text {loglst }}$ & $10 \log$ (previous PE range $r_{\text {last }}$ ) & N/A \\
\hline$r_{\text {mid }}$ & Range at which interpolation for range-dependent profiles is performed & meters \\
\hline$U$ & Complex PE field at range $r$ & $\mu \mathrm{V} / \mathrm{m}$ \\
\hline Ulast & Complex PE field at range $r_{\text {last }}$ & $\mu \mathrm{V} / \mathrm{m}$ \\
\hline$y_{\text {cur }}$ & Height of ground at current range $r$ & meters \\
\hline$y_{\text {curm }}$ & Height of ground at range $r+\Delta r_{P E 2}$ & meters \\
\hline$y_{\text {last }}$ & Height of ground at previous range $r_{\text {last }}$ & meters \\
\hline
\end{tabular}

\subsubsection{Ray Trace (RAYTRACE) SU}

Using standard ray trace techniques, a ray is traced from a starting height ant $t_{r e f}$ and range 0 , with a specified starting elevation angle, $\alpha$, to a termination range, $x_{r}$. As the ray is being traced, an optical path length difference $p l_{d}$ (the difference between the actual path length and $x_{r}$ ) and a derivative of range with respect to elevation angle, $d x d \alpha$, are being continuously computed. If the ray should reflect from the surface, a grazing angle, $\psi$, is determined. Upon reaching the termination range, a terminal elevation angle, $\beta$, is determined along with a termination height, $z_{r}$.

The raytrace is conducted by stepping in profile levels and computing ending values. A number of stepping scenarios, based upon starting and ending elevation angles, determine the program flow of the RAYTRACE SU. These scenarios are a ray that is upgoing, a ray that is downgoing, and a ray which turns around within a layer.

Upon entering the $\mathrm{SU}$, a running range, $x_{\text {sum }}$, the range at which a ray is reflected, $x_{\text {reflect }}, d x d \alpha, p l_{d}, \psi$, and a ray type (direct or reflected) flag, $i_{\text {type }}$, are initialized to zero. A temporary beginning elevation angle, $a_{\text {start }}$, is set equal to $\alpha$, and an environmental profile 
level counter, $i$, is set equal to the array index for the height in the RO region corresponding to the transmitter height, $i_{\text {start }}$.

The sub-steps within the following steps (1 and 2) are now repeated while $x_{\text {sum }}$ remains less than the termination range $x_{r}$. Upon failure to meet this repetition criterion, the $\mathrm{SU}$ program flow continues with step 3 below.

1. The beginning angle $a_{\text {start }}$ is examined to determine if the ray is initially upgoing (i.e., $a_{\text {start }} \geq 0$ ) or downgoing. If it is upgoing, the SU program flow continues with steps 1.a through 1.e, otherwise, the program flow continues with step 2 below.

a. The level counter is examined and if the ray is in the highest layer, (i.e., $i=l_{\text {evels }}$ ), the ending angle, height, range/angle derivative and path length difference are given as

$$
\begin{gathered}
\beta=a_{\text {start }}+\left(x_{r}-x_{\text {sum }}\right) g r_{i}, \\
z_{r}=z r t_{i}+\frac{\beta^{2}-a_{\text {start }}^{2}}{2 g r_{i}}, \\
d x d \alpha=d x d \alpha+\frac{1}{g r_{i}}\left(\frac{\alpha}{\beta}-\frac{\alpha}{a_{\text {start }}}\right), \\
p l_{d}=p l_{d}+\frac{1}{g r_{i}}\left[\left(r m_{i}-\frac{a_{\text {start }}^{2}}{2}\right)\left(\beta-a_{\text {start }}\right)+\frac{1}{3}\left(\beta^{3}-a_{\text {start }}^{3}\right)\right],
\end{gathered}
$$

respectively, where $g r$ is an intermediate M-unit gradient, $r m$ is an intermediate M-unit, and zrt is an intermediate height. Satisfaction of this condition causes the failure of the repetition criterion and the SU program flow continues with step 3 below.

b. If the ray is not in the highest layer, the ray must be examined to determine if it will turn around and become a downgoing ray within the current layer. This is done by looking at the radical term, rad, which will be used in the ending angle calculation. The radical term is given as

$$
r a d=a_{\text {start }}^{2}+q_{i}
$$


where $q$ is an intermediate M-unit difference. If rad is greater than or equal to zero, a solution for the ending angle is possible. The ray will not turn around and the program flow continues with step 1.c; otherwise, the program flow continues with step 1.d.

c. Before calculations can continue, the possible ending range must be compared to the termination range. This possible ending range is determined as

$$
\begin{gathered}
x_{\text {temp }}=x_{\text {sum }}+\frac{\beta-a_{\text {start }}}{g r_{i}}, \\
\beta=\sqrt{r a d} .
\end{gathered}
$$

This possible ending range is compared to the termination range and if it is larger, the ending angle, height, range/angle derivative, and path length difference are computed from equations given in step 1.a. Satisfaction of this condition causes the failure of the repetition criterion and the SU program flow continues with step 3 below.

If the ray has not reached the termination range, $x_{\text {sum }}$ is updated to $x_{\text {temp }}$; the range/angle derivative and path length difference are computed from equations given in step 1.a, where $\beta=\sqrt{\mathrm{rad}}$; $a_{\text {start }}$ is updated to $\beta$; the level counter is incremented by one; and the program flow returns to step 1 above.

d. If the ray has, in fact, turned around within the current layer, a determination must be made for the ray reaching a full range step within the still upgoing segment, for the ray reaching a full range step within the downgoing segment, or the ray exceeding the termination range. The full range step is given by

$$
x_{\text {temp }}=x_{\text {sum }}-\frac{a_{\text {start }}}{g r_{i}},
$$

which is compared to the termination range. If it exceeds the termination range, the ending angle, the ending height, the range/angle derivative, and the path length difference are determined from equations given in step 1.a. Satisfaction of this condition causes the failure of the repetition criterion and the SU program flow continues with step 3 below. If the termination range has not been exceeded, further examination of the ray's segments must be made.

e. At this point, $x_{\text {sum }}$ is updated to $x_{\text {temp }} ; x_{\text {temp }}$ is recalculated as shown in step 1.d; and $x_{\text {temp }}$ is again compared to the termination range. 
If the termination range has been exceeded, the ending angle is given as

$$
\beta=\left(x_{r}-x_{\text {sum }}\right) g r_{i},
$$

and the ending height, the range/angle derivative, and the path length difference are now determined from equations shown in step 1.a. Satisfaction of this condition causes the failure of the repetition criterion and the SU program flow continues with step 3 below.

If the termination range has not been exceeded, $x_{\text {sum }}$ is updated to $x_{\text {temp }} ; \beta$ is updated to $-a_{\text {start }}$; the range/angle derivative and path length difference are determined from equations shown in step 1.a; $a_{\text {start }}$ is updated to $\beta$; and the program flow returns to step 1 above.

2. Note! The equations for the upgoing ray within step 1 above apply equally to the downgoing ray except where specified, otherwise. However, in applying these equations to step 2, the level counter, $i$, within the intermediate M-unit gradient subterm, $g r$, must be reduced by one.

The beginning angle $a_{\text {start }}$ has been examined in step 1 above and the ray has been determined to be initially downgoing. Similar to step 1 above, the ray must be examined to determine if it has turned around and has become an upgoing ray within the current layer. This is done by looking at the radical term, rad, which will be used in the ending angle calculation. This radical term is given as

$$
r a d=a_{\text {start }}^{2}-q_{i-1} .
$$

If rad is greater than or equal to zero, a solution for the ending angle is possible. The ray has not turned around and the program flow continues with steps 2.a through 2.c below; otherwise, the program flow continues with step 2.d.

a. Before calculations can continue, the possible ending range must be compared to the termination range. This possible ending range is determined from the equation given for $x_{\text {temp }}$ in step 1.c, where $\beta$ is now $-\sqrt{\text { rad }}$. This possible ending range is compared to the termination range and if it is larger, the ending angle, the ending height, the range/angle derivative, and the path length difference are computed from equations shown in step 1.a. Satisfaction of this condition causes the failure of the repetition criterion and the SU program flow continues with step 3 below. 
b. If the termination range has not been exceeded, $x_{\text {sum }}$ is updated to $x_{\text {temp }}$; the range/angle derivative and path length difference are computed as shown in step 1.a, where $\beta=-\sqrt{r a d} ; a_{\text {start }}$ is updated to $\beta$; and the level counter is decremented by one.

c. The level counter is examined and if it is zero, the ray has reflected from the surface. In this case, the ray type flag is set to 1 to indicate a reflection, the grazing angle is set as $\psi=\left|a_{\text {start }}\right|$, and $x_{\text {reffect }}$ is set equal to $x_{\text {temp }}$. At this point a symmetry check is made. The idea of symmetry says that the ray will return to its starting height, at twice the reflection range, with an ending elevation angle opposite the starting elevation angle. Symmetry is used for APM speed efficiency so as to preclude redundant ray trace calculations on the upward path back to the starting height. Prior to applying symmetry however, the possible ending range (twice $x_{\text {sum }}$ ) must be compared to the termination range. If the termination range is exceeded by making the symmetry assumption, $a_{\text {start }}$ is updated to $-a_{\text {start }}$ and the assumption is vacated. If not however, the assumption is invoked and $a_{\text {start }}$ is updated to $-\alpha ; x_{\text {sum }}, d x d \alpha$, and $p l_{d}$, are doubled; and the level counter is restored to $i_{\text {start }}$. Control is now returned to the top of step 1 above.

d. From step 2, the ray has turned around within the current layer and is now an upgoing ray. Similar to the upgoing case of step 1 , a determination must be made for the ray reaching a full range step within the still downgoing segment, for the ray reaching a full range step within the upgoing segment, or the ray exceeding the termination range. The full range step is given by $x_{\text {temp }}$ as computed step 1.d.

If the full range step exceeds the termination range, the ending angle, the ending height, the range/angle derivative, and the path length difference are computed from equations shown in step 1.a. Satisfaction of this condition causes the failure of the repetition criterion and the SU program flow continues with section 3 below. If the termination range has not been exceeded, further examination of the ray's segments must be made.

e. At this point, $x_{\text {sum }}$ is updated to $x_{\text {temp }} ; x_{\text {temp }}$ is recalculated as in step 1.d, and $x_{\text {temp }}$ is again compared to the termination range. If the termination range has been exceeded, the ending angle is determined as in step 1.e; the ending height, range/angle derivative, and path length difference are determined as in step 1.a. Satisfaction of this condition causes the failure of the repetition criterion and the SU program flow continues with step 3 below.

If the termination range has not been exceeded, $x_{\text {sum }}$ is updated to $x_{\text {temp }} ; \beta$ is updated to $-a_{\text {start }}$; the range/angle derivative and path length difference are determined as in step 1.a; $a_{\text {start }}$ is updated to $\beta$; and the program flow returns to step 1 above. 
3. Within APM, the terminal elevation angle is not allowed to be equal to zero. Therefore, if its absolute value is less than $10^{-10}$, it is reset to $10^{-10}$ while retaining its present sign.

Table 102 and Table 103 provide identification, description, units of measure, and the computational source for each RAYTRACE SU input and output data element.

Table 102. RAYTRACE SU input data element requirements.

\begin{tabular}{c|l|c|c}
\hline Name & \multicolumn{1}{|c|}{ Description } & Units & Source \\
\hline$\alpha$ & Source elevation angle & radians & Calling SU \\
$g r$ & Intermediate M-unit gradient array, RO region & (M-unit/m)10 $0^{-6}$ & REFINIT SU \\
$i_{\text {start }}$ & Array index for height in RO region corresponding to & N/A & REFINIT SU \\
ant $t_{r e f}$ & & N/A & REFINIT SU \\
levels & Number of levels in $g r, q$ and $z r t$ arrays & 2(M-unit)10 ${ }^{-6}$ & REFINIT SU \\
$r m$ & Intermediate M-unit difference array, RO region & M-unit $10^{-6}$ & REFINIT SU \\
$x_{r}$ & Intermediate M-unit array, RO region & meters & Calling SU \\
& Terminal range - called $x_{R O n}$ in ROCALC SU, & & \\
$z r t$ & equivalent to $r_{\text {out }}$ in calling SU & meters & REFINIT SU \\
\hline
\end{tabular}

Table 103. RAYTRACE SU output data element requirements.

\begin{tabular}{c|l|c}
\hline Name & \multicolumn{1}{|c}{ Description } & Units \\
\hline$\beta$ & Terminal elevation angle & radians \\
$d x d \alpha$ & Derivative of range with respect to elevation angle & meters/radians \\
$i_{t y p e}$ & Ray type (direct or reflected) flag & N/A \\
$p l_{d}$ & Path length from range $x_{r}$ & meters \\
$\psi$ & Grazing angle & radians \\
$x_{\text {reflect }}$ & Range at which ray is reflected & meters \\
$z_{r}$ & Terminal height & meters \\
\hline
\end{tabular}

\subsubsection{Refractivity Interpolation (REFINTER) SU}

The REFINTER SU interpolates horizontally and vertically on the modified refractivity profiles. Profiles are then adjusted so they are relative to the local ground height .

Upon entry, the number of height/refractivity levels, lvlep, for the current profile is set equal to the user-specified number of levels for all profiles specified, Ivlp. For the range-dependent case, all profiles have the same number of levels. 
If there is a range-dependent environment (i.e., $n_{\text {prof }}>1$ ), horizontal interpolation to range $r_{\text {ange }}$ is performed between the two neighboring profiles that are specified relative to mean sea level. In this case the following calculations are made. If $r_{\text {ange }}$ is greater than the range for the next refractivity profile $r v_{2}$, then the index $j$ (indicating the range of the previous refractivity profile) is set equal to the counter for the range of the current profile $i_{s} ; i_{s}$ is then incremented by one. Next, the range of the previous refractivity profile $r v_{1}$ is set equal to $r v_{2}$, and $r v_{2}$ is set equal to the range of the $i_{s}^{\text {th }}$ profile, rngprof $_{i s}$. The fractional range $f v$ for the interpolation is given by

$$
f v=\frac{r_{\text {ange }}-r v_{1}}{r v_{2}-r v_{1}} .
$$

The array refdum, containing M-unit values for the current (interpolated) profile and the array htdum containing height values for the current (interpolated) profile are determined from referencing the FN_PLINT function:

$$
\begin{aligned}
& \text { refdum }_{i}=\mathbf{F N} \_\mathbf{P L I N T}\left(\text { refmsl }_{i, j}, \text { refmsl }_{i, i_{s}}, f v\right) ; i=1,2,3, \ldots \text {...vlep }
\end{aligned}
$$

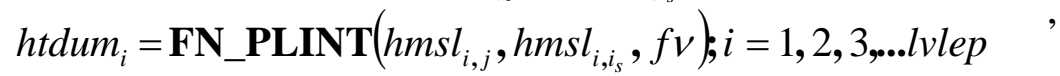

where refmsl and hmsl are Two-dimensional arrays containing refractivity and height, respectively, with respect to mean sea level of each user-specified profile.

The REMDUP SU is referenced to remove duplicate refractivity levels, with lvlep being the number of points in the profile at range $r_{\text {ange }}$. The PROFREF SU is then referenced to adjust the new profile (i.e., refdum and htdum) relative to the internal reference height $h_{\text {minter }}$, corresponding to the minimum height of the terrain profile. The PROFREF SU is then referenced once more to adjust the profile relative to the local ground height $y_{\text {curm }}$, and upon exit from the PROFREF SU, the INTPROF SU is referenced to interpolate vertically on the refractivity profile at each PE mesh height point. This results in the $n_{f f t}$-point profile array profint containing the interpolated M-unit values for the refractivity at $r_{\text {ange }}$, where $n_{f f t}$ is the transform size.

Upon exiting the REFINTER SU, $r v_{1}$ and index $j$ are saved for use upon the next reference of the SU.

Table 104 and Table 105 provide identification, description, units of measure, and the computational source for each REFINTER SU input and output data element. 
Table 104. REFINTER SU input data element requirements.

\begin{tabular}{|c|c|c|c|}
\hline Name & Description & Units & Source \\
\hline$f_{\text {ter }}$ & $\begin{array}{l}\text { Logical flag indicating if terrain profile has been specified: } \\
\text {.true. = terrain profile specified } \\
\text {.false. = terrain profile not specified }\end{array}$ & N/A & APMINIT CSC \\
\hline$h_{\text {minter }}$ & Minimum height of terrain profile & meters & TERINIT SU \\
\hline hmsl & $\begin{array}{l}\text { Two-dimensional array containing heights with respect to } \\
\text { mean sea level of each profile. Array format must be } h m s l_{i, j} \\
=\text { height of } i^{\text {th }} \text { level of } j^{\text {th }} \text { profile. } j=1 \text { for range-independent } \\
\text { cases }\end{array}$ & meters & Calling CSCI \\
\hline$i_{s}$ & Counter for current profile & N/A & $\begin{array}{l}\text { REFINIT SU } \\
\text { REFINTER SU }\end{array}$ \\
\hline$i_{s t p}$ & Current output range step index & N/A & Calling SU \\
\hline lvlp & Number of height/refractivity levels in profiles & N/A & Calling CSCI \\
\hline$n_{\text {prof }}$ & Number of refractivity profiles & N/A & Calling CSCI \\
\hline$r_{\text {ange }}$ & Range for profile interpolation & meters & Calling SU \\
\hline refmsl & $\begin{array}{l}\text { Two-dimensional array containing refractivity with respect } \\
\text { to mean sea level of each profile. Array format must be } \\
\text { refmsl } l_{i, j}=\text { M-unit at } i^{\text {th }} \text { level of } j^{\text {th }} \text { profile. } j=1 \text { for range- } \\
\text { independent cases }\end{array}$ & M-unit & Calling CSCI \\
\hline rngprof & Ranges of each profile. $r n g p r o f_{i}=$ range of $i^{\text {th }}$ profile & meters & Calling CSCI \\
\hline$r v_{2}$ & Range of the next refractivity profile & meters & $\begin{array}{l}\text { REFINIT SU } \\
\text { REFINTER SU }\end{array}$ \\
\hline$y_{\text {curm }}$ & Height of ground midway between last and current PE range & meters & PESTEP SU \\
\hline
\end{tabular}

Table 105. REFINTER SU output data element requirements.

\begin{tabular}{c|l|c}
\hline Name & \multicolumn{1}{|c}{ Description } & Units \\
\hline htdum & Height array for current interpolated profile & meters \\
$i_{s}$ & Counter for current profile & N/A \\
lvlep & Number of height/refractivity levels in profile refdum and $h t d u m$ & N/A \\
profint & Profile interpolated to every $\Delta z_{P E}$ in height & M-units \\
refdum & M-unit array for current interpolated profile & M-units \\
$r v_{2}$ & Range of the next refractivity profile & meters \\
\hline
\end{tabular}

\subsubsection{Ray Optics Calculation (ROCALC) SU}

The ROCALC SU computes the RO components which will be needed (by the ROLOSS SU) in the calculation of propagation loss at a specified range and height within the RO region. These components are the magnitudes for a direct-path and surfacereflected ray, $F d^{2}$ and $F r^{2}$, respectively, and the total phase lag angle, $\Omega$, between the direct-path and surface-reflected rays. 
The RO region may be visualized as having a grid of points superimposed upon it. The grid points are defined at the intersection of a series of lines sloping upward from the origin and a series of vertical lines at varying ranges. The grid point counter $k$ and the vertical lines are defined at varying ranges, two of which are represented by the terms $x_{R O p}$, a range for which the RO calculations were previously performed, and $x_{R O n}$, the next calculation range.

The sloping line with the greatest angle (indicated by $k=k_{\text {max }}$ ) is a function of the maximum APM output height, $h t_{y d i}$, adjusted for terrain and reference heights, and the next calculation range. The sloping line with the least angle (indicated by $k=k_{\text {minp }}$ ) is a function of the height at the top of the PE region and the range of the previous $\mathrm{RO}$ calculations.

The following steps 1 through 4 are performed while the current range, $x$, is greater than $x_{R O n}$.

1. The terms of Table 106 (defined in Table 107) are initialized or updated based upon the RO calculation range counter $i_{R O p}$. If $i_{R O p}$ equals -1 , the terms are initialized; otherwise, the terms are updated. Note that the terms must be computed in the order they appear in the table to insure proper values are assigned to component terms.

Table 106. RO region indices, angles, and ranges.

\begin{tabular}{c|c}
\hline For $i R O p=-1$ (initialize terms) & For $i R O p \neq-1$ (update terms) \\
\hline$i_{R O p}=1$ & $i_{R O p}=1-i_{R O p}$ \\
$i_{R O n}=0$ & $i_{R O n}=1-i_{R O n}$ \\
$\mathbf{N} / \mathbf{A}$ & $x_{R O p}=x_{R O n}$ \\
$k_{\text {minp }}=0$ & $k_{\text {minp }}=k_{\text {minn }}$ \\
$k_{\text {minn }}=0$ & $k_{\text {minn }}=0$ \\
$h t_{y d i f}=h t_{l i m}-y_{f r e f}$ & N/A \\
$d \alpha=\mathbf{M I N}\left(\frac{\mu_{b w r}}{2}, .01745\right)$ & N/A \\
$k_{\max }=88$ & $k_{\text {max }}=\mathbf{M I N}\left(88, \mathbf{I N T}\left(\frac{1000 h t_{y d i f}}{x_{R O p}}\right)+2\right)$
\end{tabular}


Table 106. RO region indices, angles, and ranges. (continued)

\begin{tabular}{c|c}
\hline For $i R O p=-1$ (initialize terms) & For $i R O p \neq-1$ (update terms) \\
\hline $\operatorname{frac}_{R O}=0$ & $\operatorname{frac}_{R O}=\left(\operatorname{MAX}\left(\frac{.001 k_{\max }}{d \alpha}, 5\right)-1\right)^{-1} ;$ for $\operatorname{frac}_{R O}<.25$ \\
N/A & $\Delta x_{R O}=\operatorname{frac}_{R O} x_{R O p}$ \\
$x_{R O n}=x$ & $x_{R O n}=x_{R O p}+\Delta x_{R O}$ \\
\hline
\end{tabular}

2. To calculate the RO components at each vertical point for the next range, $x_{R O n}$, a ray trace within a Newton iteration method is used to find a direct-path ray and a surfacereflected ray which will both originate at the transmitter height, ant ref $_{\text {, and terminate }}$ at the same grid point, $z_{k}$. The results of the iteration are examined and if either of the rays has not been found, an adjustment in the lower boundary of the RO region is made. Following the conclusion of the iterations, the antenna pattern factors for each ray are obtained, a surface reflection coefficient for the surface-reflected ray is computed, and the RO components are calculated.

Prior to all calculations for each vertical point, the ray trace must be initialized with beginning direct-path and surface-reflected ray elevation angles, $\alpha_{d}$ and $\alpha_{r}$, respectively; and derivatives of height with respect to these elevation angles, $d z d \alpha_{d}$ and $d z d \alpha_{r}$. A starting assumption is made that the direct-path ray and the surfacereflected rays are parallel to each other. Thus, $\alpha_{d}$ is initialized as $0.001 k_{\text {max }}$ and $\alpha_{r}$ is initialized as $-\alpha_{d}$. The RAYTRACE SU is referenced separately with $\alpha_{d}$ and $\alpha_{r}$ to obtain termination elevation angles, $\beta_{d}$ and $\beta_{r}$, and the two derivatives of range with respect to elevation angle, $d x d \alpha_{d}$ and $d x d \alpha_{r}$, which are used in turn to compute the needed derivatives of height with respect to elevation angle given as $-\beta_{d} d x d \alpha_{d}$ and $-\beta$ ${ }_{r} d x d \alpha_{r}$.

3. Once the raytrace has been initialized, the following steps 3.a through 3.g are performed for each vertical grid point, $z_{k}$, beginning with $k=k_{\max }$ and subsequently decrementing $k$ downward while $k$ remains $\geq k_{\text {minn }}$. Once $k$ has reached zero, processing continues with step 4 below.

a. The termination height is computed as

$$
z_{k}=x_{R O n} 0.001 k
$$

where $k$ is the grid point counter. 
b. The Newton iteration method to find the direct path ray from antref to $z_{k}$ is started. This iteration is continued until the difference between the ray trace ending height $z_{d}$ and $z_{k}$ is less than a height difference tolerance $z_{\text {tol }}$; but in any case, no more than 10 times. The direct-path elevation angle is given as

$$
\alpha_{d}=\alpha_{d}-\frac{z_{d}-z_{k}}{d z d \alpha_{d}}
$$

where $z_{d}$ and $d z d \alpha_{d}$ are obtained from the ray trace initialization of step 2 above for the first iteration and from the previous iteration for subsequent iterations.

The RAYTRACE SU is referenced and a new $d z d \alpha_{d}$ is calculated as $-\beta_{d} d x d \alpha_{d}$. This new $d z d \alpha_{d}$ is examined and if it is less than $10^{-6}$, or if the ray type flag $i_{\text {type }}$, returned from the RAYTRACE SU, indicates the ray has reflected, the lower boundary of the RO region is adjusted by setting $k_{\text {minn }}$ equal to one more than $k$ and the iteration for the direct ray is stopped.

c. The Newton iteration method to find the surface-reflected ray from $a n t_{r e f}$ to $z_{k}$ is now started. This iteration should be continued until the difference between the ray trace ending height, $z_{r}$ and $z_{k}$ is less than a height difference tolerance $z_{t o l}$; but in any case, no more than 10 times. The reflected-path elevation angle is given as

$$
\alpha_{r}=\alpha_{r}-\frac{z_{r}-z_{k}}{d z d \alpha_{r}}
$$

where $z_{r}$ and $d z d \alpha_{r}$ are obtained from the ray trace initialization of step 2 above for the first iteration and from the previous iteration for subsequent iterations.

The RAYTRACE SU is referenced and a new $d z d \alpha_{r}$ is calculated as $-\beta_{r} d x d \alpha_{r}$. This new $d z d \alpha_{r}$ is examined and if it is less than $10^{-6}$, or if $i_{\text {type }}$ indicates the ray is a direct ray, the lower boundary of the RO region is adjusted by setting $k_{\text {minn }}$ equal to one more than $k$ and the iteration for the surface-reflected ray is stopped.

d. A test is made to determine if the grazing angle, $\psi$, (returned from the RAYTRACE SU) is less than the limiting value, $\psi_{\text {lim }}$, and if so, the lower boundary of the RO region is adjusted by setting $k_{\operatorname{minn}}$ equal to $k$. 
e. The magnitudes for the direct-path and surface-reflected ray, $F d^{2}$ and $F r^{2}$ respectively, are now given as

$$
\begin{gathered}
F d^{2}=\left|\frac{x_{R O n}}{d z d \alpha_{d}}\right| f^{2}\left(\alpha_{d}\right), \\
F r^{2}=\left|\frac{x_{R O n}}{d z d \alpha_{r}}\right|\left[f\left(\alpha_{r}\right) R_{\text {mag }}\right]^{2},
\end{gathered}
$$

where the amplitude of the surface reflection coefficient, $R_{\text {mag }}$, is obtained from a reference to the GETREFCOEF SU; the antenna pattern factors $f\left(\alpha_{d}\right)$ and $f\left(\alpha_{r}\right)$ are obtained from references to the ANTPAT SU; and the derivatives of height with respect to elevation angle are obtained from the RAYTRACE SU within the Newton iteration of steps 3.b and 3.c above.

f. The total phase lag between the direct-path and surface-reflected rays is computed as

$$
\Omega=\left(p l_{r}-p l_{d}\right) k_{o}+\varphi
$$

where the ray path lengths $p l_{d}$ and $p l_{r}$ are obtained from the RAYTRACE SU within the Newton iteration of steps 3.b and 3.c above; the reflection coefficient phase lag angle, $\varphi$, is obtained from a reference to the GETREFCOEF SU; and $k_{o}$ is the free-space wave number.

g. If the propagation angles and factors are to be computed for each separate ray (lang = '.true.'), then the current propagation angles for the direct and reflected rays, $\beta_{d}$ and $\beta_{r}$ respectively, are stored in arrays $R O \alpha_{d i r}$ and $R O \alpha_{r e f}$.

4. If the point counter $k$ has been reduced to zero by the procedures of steps 3.a through 3.g above, the surface values of magnitudes for the direct-path and surface-reflected rays are both set equal to the last value of $F d^{2}$ and the total phase lag between the direct-path and surface-reflected rays is set equal to $-\pi$. If vertical polarization has been specified, then a further calculation is performed for the special case when the RO output height is less than 0 due to the height adjustment of $y_{\text {fref. }}$ A FE calculation is made at this lowest height and replaces the previous values of magnitudes for the direct and surface-reflected paths, along with a new phase lag.

Table 107 and Table 108 provide identification, description, units of measure, and the computational source for each ROCALC SU input and output data element. 
Table 107. ROCALC SU input data element requirements.

\begin{tabular}{|c|c|c|c|}
\hline Name & Description & Units & Source \\
\hline$a^{a n t_{\text {ref }}}$ & $\begin{array}{l}\text { Transmitting antenna height relative to the reference height } \\
h_{\text {minter }}\end{array}$ & meters & TERINIT SU \\
\hline$\mu_{b w r}$ & Antenna vertical beamwidth & radians & Calling CSCI \\
\hline$d \alpha$ & $1 / 2 \mu_{b w r}$ & radians & ROCALC SU \\
\hline $\operatorname{frac}_{R O}$ & RO range interval fraction ( 0.0 to 0.25 ) & N/A & ROCALC SU \\
\hline$h t_{l i m}$ & Maximum height relative to $h_{\text {minter }}$ & meters & TERINIT SU \\
\hline$h t_{y d i f}$ & $h t_{\text {lim }}-y_{\text {fref }}$ & meters & ROCALC SU \\
\hline$i_{p o l}$ & $\begin{array}{l}\text { Polarization flag: } \\
\qquad \begin{array}{l}0=\text { horizontal polarization } \\
1=\text { vertical polarization }\end{array}\end{array}$ & N/A & Calling CSCI \\
\hline$i_{R O n}$ & Array index for next range in $\mathrm{RO}$ region & N/A & ROCALC SU \\
\hline$i_{R O p}$ & Array index for previous range in $\mathrm{RO}$ region & N/A & $\begin{array}{l}\text { APMINIT CSC } \\
\text { ROCALC SU }\end{array}$ \\
\hline$k_{o}$ & Free-space wave number & meters $^{-1}$ & APMINIT CSC \\
\hline$k_{\text {minn }}$ & Array index for minimum angle in $\mathrm{RO}$ region at range $x_{R O n}$ & N/A & ROCALC SU \\
\hline lang & $\begin{array}{l}\text { Propagation angle and factor output flag } \\
\text { '.true.'= Output propagation angle and propagation } \\
\text { factor for direct and reflected ray (where applicable). } \\
\text { '.false.' = Do not output propagation angles and } \\
\text { factors }\end{array}$ & N/A & Calling CSCI \\
\hline$\psi_{\text {lim }}$ & Grazing angle of limiting ray & radians & APMINIT CSC \\
\hline twoka & Twice the effective earth radius & meters & GET_K SU \\
\hline$x$ & Current range & meters & Calling SU \\
\hline$x_{R O n}$ & Next range in RO region & meters & APMINIT CSC \\
\hline$x_{R O p}$ & Previous range in RO region & meters & ROCALC SU \\
\hline$y_{\text {fref }}$ & Ground elevation height at source & meters & APMINIT CSC \\
\hline zoutma & Array output heights relative to "real” $a n t_{r e f}$ & meters & APMINIT CSC \\
\hline zoutpa & Array output heights relative to "image" ant $t_{r e f}$ & meters & APMINIT CSC \\
\hline zro & Array of output heights & meters & APMINIT CSC \\
\hline$z_{\text {tol }}$ & Height tolerance for Newton's method & meters & APMINIT CSC \\
\hline
\end{tabular}

Table 108. ROCALC SU output data element requirements.

\begin{tabular}{c|l|c}
\hline Name & \multicolumn{1}{|c|}{ Description } & Units \\
\hline$d \alpha$ & $1 / 2 \mu_{b w r}$ & radians \\
$\Delta x_{R O}$ & RO range interval & meters \\
$F d^{2}$ & Magnitude array, direct ray & N/A \\
$F r^{2}$ & Magnitude array, reflected ray & N/A
\end{tabular}


Table 108. ROCALC SU output data element requirements. (continued)

\begin{tabular}{c|l|c}
\hline \multicolumn{1}{c|}{ Name } & \multicolumn{1}{|c}{ Description } & Units \\
\hline rrac $_{R O}$ & RO range interval fraction (0.0 to 0.25) & N/A \\
$h t_{y d i f}$ & $h t_{\text {lim }}-y_{f r e f}$ & meters \\
$i_{R O n}$ & Array index for next range in RO region & N/A \\
$i_{R O p}$ & Array index for previous range in RO region & N/A \\
$k_{\max }$ & Array index for maximum angle in RO region at range $x_{R O n}$ & N/A \\
$k_{\operatorname{minn}}$ & Array index for minimum angle in RO region at range $x_{R O n}$ & N/A \\
$k_{\min p}$ & Array index for minimum angle in RO region at range $x_{R O p}$ & N/A \\
$\Omega$ & Total phase angle array & radian \\
$R O \alpha_{\text {dir }}$ & Array of propagation angles for direct rays & radians \\
$R O \alpha_{r e f}$ & Array of propagation angles for reflected rays & radians \\
$x_{R O n}$ & Next range in RO region & meters \\
$x_{R O p}$ & Previous range in RO region & meters \\
\hline
\end{tabular}

\subsubsection{Ray Optics Loss (ROLOSS) SU}

The ROLOSS SU calculates propagation factor and loss values at all valid RO heights at a specified range based upon the components of magnitude for a direct-path and surface-reflected ray, $\mathrm{Fd}^{2}$ and $\mathrm{Fr}^{2}$ respectively, and the total phase lag angle, $\Omega$, between the two rays as determined by the ROCALC SU.

Upon entering the SU, the ROCALC SU is referenced to obtain the current values of the direct and reflected ray magnitudes, along with the phase lag for all heights at the specified range $r_{\text {out }}$.

For computational efficiency, an interpolation from the magnitude and total phase lag angle arrays established by the ROCALC SU is made to obtain these three quantities at each APM vertical output mesh point within the RO region.

From the interpolated phase lag angle and ray magnitudes, a propagation factor is calculated that is used in turn with the free-space propagation loss to obtain a propagation loss at each vertical APM output point.

A range ratio term to be used within the interpolation scheme is defined as

$$
\text { ratio }=\frac{r_{\text {out }}-x_{R O p}}{\Delta x_{R O}}
$$

The phase lag angle and ray magnitude arrays have been filled at grid points defined by a series of sloping lines and the next and previous RO calculation range, $x_{R O n}$ 
and $x_{R O p}$, respectively. Which values to interpolate from are determined by the sloping line immediately above and the sloping line immediately below the current APM output point of interest. To begin the calculations $k_{l o}$ is initialized to $k_{\max }$, the line with the greatest angle.

Perform steps 1 through 6, decrementing downward in APM output points from the maximum output height index in the RO region, $j_{\max }$, to the minimum output height index, in the RO region, $j_{\min }-1$, where the index $j$ varies from $j_{\max }$ to $j_{\min }-1$.

1. Interpolation of $F d^{2}, F r^{2}$, and $\Omega$ values occurs in two stages. The first stage is horizontally, above and below the APM output point (i.e., along the lines $k_{l o}$ and $k_{h i}$ ). These values will be used in turn, in a vertical interpolation stage to obtain values at the APM output point itself. It may be however, that more than one APM output point will fall between two adjacent $k$ lines. In this case, it would be redundant to perform the horizontal interpolation more than once. For this reason, a temporary $k$ line counter is established that will be used in comparison with $k_{l o}$ to determine if interpolation is necessary or if the previously interpolated horizontal values may again be used in the vertical interpolation. This temporary $k$ counter is given by

$$
k_{\text {temp }}=\mathbf{I N T}\left(\frac{1000 \mathrm{zro}_{j}}{r_{\text {out }}}\right),
$$

where $j$ is the APM output point counter, and $z z_{j}$ is the $j^{\text {th }}$ output height point . If the index $j$ has already reached the lowest value of $j_{\min }-1$ and clutter calculations are to be performed, then the height at which to compute the propagation factor is replaced with $z_{c}$, a height of $1 \mathrm{~m}$ above the surface, which is done by replacing $z \mathrm{ro}_{j}$ above with the adjusted height $z_{c}-y_{\text {fref }}$. If $k_{\text {temp }}$ is less than the current $k_{l o}$, the APM output point occurs below the current lower $k$ line and horizontal interpolations must be performed using steps 1.a through 1.c; otherwise, the horizontal interpolations are unnecessary and the SU may proceed with step 2.

a. The lower $k$ line, $k_{l o}$, is reset to $k_{\text {temp }}$ and the upper $k$ line, $k_{h i}$, is set to one more than $k_{l o}$.

b. In preparation for the interpolation, component terms (horizontal differences of direct and surface-reflected magnitudes and phase lag angles) along the $k_{l o}$ and $k_{h i}$ lines are given as

$$
\begin{gathered}
\Delta F d_{l o}^{2}=F d_{i_{R O n}, k_{l o}}^{2}-F d_{i_{R O p}, k_{l o}}^{2}, \\
\Delta F r_{l o}^{2}=F r_{i_{R O O}, k_{l o}}^{2}-F r_{i_{R O p}, k_{l o}}^{2}, \\
\Delta \Omega_{l o}=\Omega_{i_{R O n}, k_{l o}}-\Omega_{i_{R O p}, k_{l o}},
\end{gathered}
$$


and similarly, the propagation angles for the direct and reflected rays are given as

$$
\begin{aligned}
\Delta \alpha d_{l o} & =R O \alpha_{d i r\left(i_{R O O}, k l o\right)}-R O \alpha_{d i r\left(i_{R O O}, k l o\right)}, \\
\Delta \alpha r_{l o} & =R O \alpha_{r e f\left(i_{R O n}, k l o\right)}-R O \alpha_{r e f}\left(i_{R O O}, k l o\right)
\end{aligned}
$$

if lang = '.true.', substituting the index $k_{h i}$ for $k_{l o}$ as appropriate. Note that these horizontal differences need only be calculated while $k_{h i}$ and $k_{l o}$ remain greater than or equal to $k_{\min p}$ and $k_{\operatorname{minn}}$. If these conditions are not met, any continued difference calculations would take place within the PE region, which would yield undesirable results. For failure of these conditions, the previously calculated difference values are used for the lower RO region boundary calculations.

c. If $k_{l o}$ is greater than or equal to $k_{\min }$, the horizontally interpolated direct and surface-reflected magnitudes and phase lag angles along the $k_{l o}$ line can proceed in a forward manor (from $x_{R O p}$ to $r_{\text {out }}$ ). These values are given as

$$
\begin{gathered}
F d_{l o}^{2}=F d_{i_{R O p}, k_{l o}}^{2}+\text { ratio } \Delta F d_{l o}^{2}, \\
F r_{l o}^{2}=F r_{i_{R O p}^{2}, k_{l o}}^{2}+\text { ratio } \Delta F r_{l o}^{2}, \\
\Omega_{l o}=\Omega_{i_{R O p}, k_{l o}}+\text { ratio } \Delta \Omega_{l o}, \\
\alpha d_{l o}=R O \alpha_{d i r\left(i_{R O p}, k_{l o}\right)}+\text { ratio } \Delta \alpha d_{l o}, \\
\alpha r_{l o}=R O \alpha_{\text {ref }\left(i_{R O p}, k_{l o}\right)}+\text { ratio } \Delta \alpha r_{l o} .
\end{gathered}
$$$$
\text { if lang = .'true.'. }
$$

In a like manor, the same equations above are used to get the values along the $k_{h i}$ line by substituting the index $k_{h i}$, assuming, however, $k_{h i}$ is also greater than or equal to $k_{\text {minp }}$. Should $k_{l o}$ or $k_{h i}$ be less than $k_{\text {minp }}$, the interpolation must proceed in a backward manner (from $x_{R O n}$ to $r_{\text {out }}$ ). The above equations may again be used by substituting the index $i_{R O n}$ for $i_{R O p}$ and the value $(1-$ ratio) for ratio.

2. Once the horizontal interpolation of magnitudes and phase lag angles has been accomplished, the vertical interpolation of magnitudes and phase lag angles at the APM output point may proceed as

$$
\begin{aligned}
F d^{2} & =F d_{l o}^{2}+\operatorname{ratio}_{k}\left(F d_{h i}^{2}-F d_{l o}^{2}\right), \\
F r^{2} & =F r_{l o}^{2}+\operatorname{ratio}_{k}\left(F r_{h i}^{2}-F r_{l o}^{2}\right), \\
\Omega & =\Omega_{l o}+\operatorname{ratio}_{k}\left(\Omega_{h i}-\Omega_{l o}\right),
\end{aligned}
$$


and again, if separate values for the direct and reflected rays are desired (lang = '.true.'), these are given by

$$
\begin{aligned}
& \alpha_{d i r}=\alpha d_{l o}+\operatorname{ratio}_{k}\left(\alpha d_{h i}-\alpha d_{l o}\right), \\
& \alpha_{\text {ref }}=\alpha r_{l o}+\operatorname{ratio}_{k}\left(\alpha r_{h i}-\alpha r_{l o}\right) .
\end{aligned}
$$

Where ratio from $_{k_{l o}}$ to $k_{h i}$ is

$$
\text { ratio }_{k}=\frac{1000 \mathrm{zro}_{j}}{r_{\text {out }}}-k_{\text {lo }} .
$$

3. From the magnitudes of the direct and surface-reflected components and the phase lag angle, the square of the propagation factor at the APM output point is given as

$$
F^{2}=\left|F d^{2}+F r^{2}+2 \sqrt{\left|F d^{2} F r^{2}\right| \operatorname{Cos} \Omega}\right|,
$$

which in turn is converted to a propagation factor expressed in decibels by

$$
F_{d B}=10 \mathbf{L O G}_{10}\left[\mathbf{M A X}\left(F^{2}, 10^{-25}\right)\right] .
$$

4. Next, if clutter calculations are to be performed and the $j$ index has reached its lowest value, then the propagation factor is stored in the array ffat $1 \mathrm{~m}$.

5. The total propagation loss and propagation factor is then computed at the $j^{\text {th }}$ APM output point and converted to centibels according to

$$
\begin{aligned}
L_{d B} & =f s l_{i_{s p}}-F_{d B}+g a s_{\text {loss }}, \\
F_{d B} & =f s l_{i_{s p}}-L_{d B}, \\
m p f l_{1, j} & =\operatorname{NINT}\left(10 L_{d B}\right), \\
m p f l_{2, j} & =\operatorname{NINT}\left(10 F_{d B}\right),
\end{aligned}
$$

where $f s l_{i s p}$ is the free space loss at the $i_{s t p}{ }^{\text {th }}$ output range. 
6. Lastly, if the propagation angles and factors for the direct and reflected rays are desired, these are stored separately according to

$$
\begin{aligned}
& \operatorname{propaf}_{1, j}=\alpha_{\text {dir }}, \\
& \operatorname{propaf}_{2, j}=10 \mathrm{LOG}_{10}\left(\mathrm{Fd}^{2}\right), \\
& \operatorname{propaf}_{3, j}=\alpha_{\text {ref }}, \\
& \operatorname{propaf}_{4, j}=10 \mathrm{LOG}_{10}\left(\mathrm{Fr}^{2}\right),
\end{aligned}
$$

Table 109 and Table 110 provide identification, description, units of measure, and the computational source for each ROLOSS SU input and output data element. Table 111

\begin{tabular}{|c|c|c|c|}
\hline Name & Description & Units & Source \\
\hline$C_{\text {lut }}$ & $\begin{array}{l}\text { Logical flag used to indicate if surface clutter calculations } \\
\text { are desired. }\end{array}$ & N/A & Calling CSCI \\
\hline$\Delta x_{R O}$ & RO range interval & meters & ROCALC SU \\
\hline$F d^{2}$ & Magnitude array, direct ray & N/A & ROCALC SU \\
\hline $\mathrm{Fr}^{2}$ & Magnitude array, reflected ray & N/A & ROCALC SU \\
\hline fsl & Free space loss array for output ranges & $\mathrm{dB}$ & APMINIT CSC \\
\hline gas $_{\text {loss }}$ & Gaseous absorption loss at range $r_{\text {out }}$ & $\mathrm{dB}$ & APMSTEP CSC \\
\hline hlim & $\begin{array}{l}\text { Array containing the height at each output range separating } \\
\text { the RO region from the PE (at close ranges) and XO (at far } \\
\text { ranges) regions }\end{array}$ & meters & FILLHT SU \\
\hline$i_{R O n}$ & Array index for next range in $\mathrm{RO}$ region & N/A & ROCALC SU \\
\hline$i_{R O p}$ & Array index for previous range in $\mathrm{RO}$ region & N/A & ROCALC SU \\
\hline$i_{s t p}$ & Current output range step index & N/A & Calling SU \\
\hline$j_{\max }$ & Array index for maximum output height in $\mathrm{RO}$ region & N/A & Calling SU \\
\hline$j_{\min }$ & Array index for minimum output height in $\mathrm{RO}$ region & N/A & Calling SU \\
\hline$k_{\max }$ & Array index for maximum angle in RO region at range $x_{R O n}$ & N/A & ROCALC SU \\
\hline$k_{\min n}$ & Array index for minimum angle in RO region at range $x_{R O n}$ & N/A & ROCALC SU \\
\hline$k_{\min p}$ & Array index for minimum angle in RO region at range $x_{R O p}$ & N/A & ROCALC SU \\
\hline lang & $\begin{array}{l}\text { Propagation angle and factor output flag } \\
\text { '.true.'= Output propagation angle and propagation factor } \\
\text { for direct and reflected ray (where applicable). } \\
\text { ' false' = Do not output propagation angles and factors }\end{array}$ & N/A & CALLING CSCI \\
\hline$\underline{\Omega}$ & Total phase angle array & radians & ROCALC SU \\
\hline
\end{tabular}
identifies terms that are used internal to the ROLOSS SU and whose value must be retained from SU call to SU call for reasons of computational efficiency.

Table 109. ROLOSS SU input data element requirements. 
Table 109. ROLOSS SU input data element requirements. (continued)

\begin{tabular}{c|l|c|c}
\hline Name & \multicolumn{1}{|c|}{ Description } & Units & Source \\
\hline lang & $\begin{array}{c}\text { Propagation angle and factor output flag } \\
\text { '.true.'= Output propagation angle and propagation } \\
\text { factor for direct and reflected ray (where applicable). } \\
\text { '.false.' = Do not output propagation angles and } \\
\text { factors }\end{array}$ & N/A & Calling CSCI \\
& $\begin{array}{c}\text { Total phase angle array } \\
\Omega\end{array}$ & radians & ROCALC SU \\
$R O \alpha_{\text {dir }}$ & Array of propagation angles for direct rays & radians & ROCALC SU \\
$r_{\text {out }}$ & Array of propagation angles for reflected rays & radians & ROCALC SU \\
$x_{R O p}$ & Current output range & meters & Calling SU \\
$y_{\text {fref }}$ & Previous range in RO region & meters & ROCALC SU \\
$z_{C}$ & Height at which to compute propagation factor for clutter & meters & APMINIT CSC \\
& calculations relative to $h m_{\text {ref }}$ & APMINIT CSC \\
zro & Array of output heights in RO region & meters & APMINIT CSC \\
\hline
\end{tabular}

Table 110. ROLOSS SU output data element requirements.

\begin{tabular}{c|l|c}
\hline Name & \multicolumn{1}{|c}{ Description } & Units \\
\hline$m p f l$ & Propagation factor and loss array & $\mathrm{cB}$ \\
\hline propaf & $\begin{array}{l}\text { Two-dimensional array, containing the propagation angles and factors for the direct } \\
\text { and reflected rays (where applicable) for all output height/range points }\end{array}$ & $\begin{array}{c}\mathrm{dB}, \\
\text { radians }\end{array}$ \\
\hline
\end{tabular}

Table 111. ROLOSS SU save data element requirements.

\begin{tabular}{|c|c|c|c|}
\hline Name & Description & Units & Source \\
\hline$\Delta \alpha d_{h i}$ & $\begin{array}{l}\text { Difference in the direct ray angle along the RO step above the } \\
\text { desired point }\end{array}$ & radians & ROLOSS SU \\
\hline$\Delta \alpha d_{l o}$ & $\begin{array}{l}\text { Difference in the direct ray angle along the RO step below the } \\
\text { desired point }\end{array}$ & radians & ROLOSS SU \\
\hline$\Delta \alpha r_{h i}$ & $\begin{array}{l}\text { Difference in the reflected ray angle along the RO step above } \\
\text { the desired point }\end{array}$ & radians & ROLOSS SU \\
\hline$\Delta \alpha r_{l o}$ & $\begin{array}{l}\text { Difference in the reflected ray angle along the RO step below } \\
\text { the desired point }\end{array}$ & radians & ROLOSS SU \\
\hline$\Delta \Omega_{h i}$ & $\begin{array}{l}\text { Difference in total phase lag angle along } \Delta x_{R O} \text { above desired } \\
\text { APM output point }\end{array}$ & radians & ROLOSS SU \\
\hline$\Delta \Omega_{l o}$ & $\begin{array}{l}\text { Difference in total phase lag angle along } \Delta x_{R O} \text { below desired } \\
\text { APM output point }\end{array}$ & radians & ROLOSS SU \\
\hline$\Delta F d_{l o}^{2}$ & $\begin{array}{l}\text { Difference in direct ray magnitude along } \Delta x_{R O} \text { below desired } \\
\text { APM output point }\end{array}$ & N/A & ROLOSS SU \\
\hline
\end{tabular}


Table 111. ROLOSS SU save data element requirements. (continued)

\begin{tabular}{|c|c|c|c|}
\hline Name & Description & Units & Source \\
\hline$\Delta F d_{h i}^{2}$ & $\begin{array}{l}\text { Difference in direct ray magnitude along } \Delta x_{R O} \text { above desired } \\
\text { APM output point }\end{array}$ & N/A & ROLOSS SU \\
\hline$\Delta F r_{l o}^{2}$ & $\begin{array}{l}\text { Difference in reflected ray magnitude along } \Delta x_{R O} \text { below } \\
\text { desired APM output point }\end{array}$ & N/A & ROLOSS SU \\
\hline$\Delta F r_{h i}^{2}$ & $\begin{array}{l}\text { Difference in reflected ray magnitude along } \Delta x_{R O} \text { above } \\
\text { desired APM output point }\end{array}$ & N/A & ROLOSS SU \\
\hline
\end{tabular}

\subsubsection{Save Profile (SAVEPRO) SU}

The SAVEPRO SU stores the gradients and heights of the current refractivity profile, upon each reference to the FZLIM SU, from the top of the PE calculation region to the maximum user-specified height.

Upon entering, the current profile height array htdum is searched to find the index $i$ such that tdum $_{i}$ is the first height in the profile that is greater than the maximum PE calculation height, $z_{\text {lim. }}$. The counter $l_{\text {new }}$ is then initialized to -1 .

Next, the gradients are calculated and stored, along with corresponding heights, as follows

$$
\begin{gathered}
\operatorname{grad}_{l_{\text {new }, \text { iz }}}=\frac{\text { refdum }_{j+1}-\text { refdum }_{j}}{\text { htdum }_{j+1}-\text { htdum }_{j}}, \\
h \operatorname{lr}_{\text {new }, \text { iz }_{1}}=\text { htdum }_{j},
\end{gathered}
$$

where $j$ is incremented by one from $i$ to lvlep- $1, l_{\text {new }}$ is incremented by one with each increment in $j$, and $i z$ represents the range step index for XO calculations.

Before exiting, the last height level in htdum is stored and the final number of levels, $l_{\text {new }}$, in the $i z^{\text {th }}$ profile (represented by grad and $h t r$ ) is stored in array $l v l$.

Table 112 and Table 113 provide identification, description, units of measure, and the computational source for each SAVEPRO SU input and output data element. 
Table 112. SAVEPRO SU input data element requirements.

\begin{tabular}{c|l|c|c}
\hline Name & \multicolumn{1}{|c|}{ Description } & Units & Source \\
\hline htdum & Height array for current profile & meters & REFINTER SU \\
iz & Number of calculation range steps for XO region & N/A & FZLIM SU \\
lvlep & Number of height/refractivity levels in profile refdum and & N/A & REFINTER SU \\
& htdum & & \\
refdum & M-unit array for current profile & M-units & REFINTER SU \\
$z_{\text {lim }}$ & Maximum height in PE calculation region & meters & FFTPAR SU \\
\hline
\end{tabular}

Table 113. SAVEPRO SU output data element requirements.

\begin{tabular}{c|l|c}
\hline Name & \multicolumn{1}{|c|}{ Description } & Units \\
\hline grad & Two-dimensional array containing gradients of each profile used in XO & $\begin{array}{c}\text { M-units } \\
\text { /meter }\end{array}$ \\
& calculations & meters \\
$h t r$ & Two-dimensional array containing heights of each profile used in XO calculations & N/A \\
\hline$v l$ & Number of height levels in each profile used in XO calculations & \multicolumn{1}{|c}{} \\
\hline
\end{tabular}

\subsubsection{Spectral Estimation (SPECEST) SU}

The SPECEST SU determines the outward propagation angle at the top of the PE calculation region, or the grazing angle at the lower part of the PE region, and the propagation angle for all desired output receiver points within the PE region based on spectral estimation. The outward propagation angle is used for XO calculations and the grazing angle is used for rough surface calculations. All other propagation angles are stored in propaf and returned to the calling CSCI.

Upon entering the SPECEST SU, if the outward propagation angle is to be determined ( $i_{\text {flag }}=0$ ), the topmost $n_{p}$ points (within the unfiltered portion) of the complex PE field are separated into their real and imaginary components, $x p$ and $y p$, respectively. If the grazing angle is to be determined ( $\left.i_{\text {flag }}=1\right)$, then the lowest $n_{p}$ points of the complex PE field are used. A window filter is then applied to both real and imaginary component arrays by multiplying each element in $x p$ and $y p$ by each corresponding element in the filter array filtp for indices between $3 / 4 n_{p}$ and $n_{p}$.

Next, the array elements in $x p$ and $y p$ are set to 0 for indices from $n_{p}+1$ to $n_{s}-1$. [Note that both $x p$ and $y p$ are arrays of size $n_{s}$.] The DRST SU is then referenced to obtain the spectral field components.

The spectral amplitudes in $\mathrm{dB}$ are then given by

$$
\operatorname{spectr}_{i}=10 \mathbf{L O G}_{10}\left[\mathbf{M A X}\left(10^{-10}, \sqrt{x p_{i}^{2}+y p_{i}^{2}}\right)\right] ; \quad \mathrm{i}=0,1,2, \ldots, n_{s}-1
$$


Next, a 3-point average is performed on spectr to determine the bin, or index $i_{\text {peak }}$, at which the peak spectral amplitude occurs. Once $i_{\text {peak }}$ has been determined, the outward propagation angle is calculated as

$$
\vartheta_{\text {out }}=\mathbf{S I N}^{-1}\left(\frac{\lambda i_{\text {peak }}}{2 n_{s} \Delta z_{P E}}\right) .
$$

Table 114 and Table 115 provide identification, description, units of measure, and the computational source for each SPECEST SU input and output data element.

Table 114. SPECEST SU input data element requirements.

\begin{tabular}{|c|c|c|c|}
\hline Name & Description & Units & Source \\
\hline$\Delta z_{P E}$ & PE mesh height increment (bin width in z-space) & meters & FFTPAR SU \\
\hline filtp & Array filter for spectral estimation calculations & N/A & APMINIT CSC \\
\hline$i_{\text {flag }}$ & $\begin{array}{l}\text { Flag indicating if spectral estimation is to be performed } \\
\text { on lower PE field or upper PE field } \\
\begin{array}{l}0=\text { upper PE field } \\
1=\text { lower PE field }\end{array}\end{array}$ & N/A & Calling SU \\
\hline$j z_{l i m}$ & PE bin \# corresponding to $z_{l i m}$, i.e., $z_{l i m}=j z_{l i m} \Delta z_{P E}$ & N/A & APMINIT CSC \\
\hline $\ln _{p}$ & $\begin{array}{l}\text { Power of } 2 \text { transform size used in spectral estimation } \\
\text { calculations; i.e., } n_{p}=2^{\ln p}\end{array}$ & N/A & APMINIT CSC \\
\hline$n p_{34}$ & $3 / 4 n_{p}$ & N/A & APMINIT CSC \\
\hline$n_{p}$ & $\begin{array}{l}\text { Number of bins in upper PE region to consider for } \\
\text { spectral estimation. }\end{array}$ & N/A & APMINIT CSC \\
\hline$n_{s}$ & Transform size for spectral estimation calculations & N/A & APMINIT CSC \\
\hline$U$ & Complex field at current PE range $r$ & $\mu \mathrm{V} / \mathrm{m}$ & PESTEP SU \\
\hline$x O_{\text {con }}$ & Constant used in determining $\vartheta_{\text {out }}$ & N/A & APMINIT CSC \\
\hline$y_{\text {cur }}$ & Height of ground at current range $r$ & meters & PESTEP SU \\
\hline
\end{tabular}


Table 115. SPECEST output data element requirements.

\begin{tabular}{c|l|c}
\hline Name & \multicolumn{1}{|c|}{ Description } & Units \\
\hline spectr & Spectral amplitude of field & $\mathrm{dB}$ \\
$\vartheta_{\text {out }}$ & Outward propagation angle at top of PE region & radians \\
$x p$ & Real part of spectral portion of PE field & $\mu \mathrm{V} / \mathrm{m}$ \\
$y p$ & Imaginary part of spectral portion field & $\mu \mathrm{V} / \mathrm{m}$ \\
\hline
\end{tabular}

\subsubsection{Surface Impedance (SURFIMP) SU}

The SURFIMP SU computes the complex normalized surface impedance for rough sea surface conditions, given the wind speed and the frequency. The SURFIMP SU implements a modified version Sailors (1997) of the original Barrick (1971a, 1971b) model for the normalized rough sea surface impedance for HF frequencies.

The SU begins by converting the internal wind speed input variable, ws, from meters/second to knots using the conversion:

$$
w s(\text { knots })=1.94 w s(\mathrm{~m} / \mathrm{s}) \text {. }
$$

The SU then populates, from internal DATA statements, real polynomial coefficient arrays appropriate to the real (cons1r, cons $2 r$, cons $3 r$, cons $4 r$, cons $5 r$ ) and imaginary (cons1i, cons2i, cons3i, cons4i, cons5i) parts of the complex normalized surface impedance. The arrays appropriate for the real part of the normalized impedance are then passed, in five sequential function calls, along with the wind speed, to the FN_POLY5 SU for the determination of the five polynomial coefficients required for input to the FN_POLY4 SU. The polynomial coefficients returned from the sequential FN_POLY5 SU calls are stored in the constr array variable which is then passed to the FN_POLY4 SU, along with the frequency $f_{M H z}$, for determination of the real part of the complex normalized rough sea surface impedance, $\xi_{r}$.

This process is then repeated, passing the appropriate coefficient arrays (cons1i, cons $2 i$, cons $3 i$, cons $4 i$, cons5i) along with the wind speed variable to the FN_POLY5 SU for determination of the polynomial coefficients for FN_POLY4. These coefficients are then passed to the FN_POLY4 SU, along with the frequency $f_{M H z}$, for determination of the imaginary part of the normalized rough sea surface impedance, $\xi_{i}$.

The output of the SURFIMP SU is the double precision complex normalized rough sea surface impedance given by

$$
\xi=\xi_{r}+j \xi_{i}
$$


Table 116 and Table 117 provide identification, description, units of measure, and the computational source for each SURFIMP SU input and output data element.

Table 116. SURFIMP input data element requirements.

\begin{tabular}{c|l|c|c}
\hline Name & Description & Units & Source \\
\hline$w S$ & Wind speed & meters/second & Calling SU \\
$f_{\mathrm{MHz}}$ & Frequency & $\mathrm{MHz}$ & Calling SU \\
\hline
\end{tabular}

Table 117. SURFIMP output data element requirements.

\begin{tabular}{c|c|c}
\hline Name & Description & Units \\
\hline$\xi$ & Normalized rough sea surface impedance & N/A \\
\hline
\end{tabular}

\subsubsection{Troposcatter (TROPOSCAT) SU}

The TROPOSCAT SU calculates the loss due to troposcatter at and beyond the radio horizon for an array of receiver heights.

Upon entering the TROPOSCAT SU, the current output range $r_{\text {out }}$ is updated, and the surface refractivity and associated variables are also initialized. The surface refractivity $s n_{r e f}$ is initialized according to

$$
s n_{r e f}=\frac{1}{2}\left(\text { snref }_{t x}+\text { snref }_{0}\right) .
$$

A term used in the troposcatter transmission loss calculation, $s n_{1}$, is determined from

$$
s n_{1}=0.031-0.00232 s n_{\text {ref }}+5.67 \times 10^{-6} s n_{\text {ref }}^{2},
$$

along with a loss term tlst $_{s}$ for smooth surface as

$$
t l s t_{s}=t l s t_{w r}-.2 s n_{r e f} \text {. }
$$

Next, the tangent angle from the source to the surface, $\vartheta_{1}$, is initialized to its value for smooth surface, $\vartheta_{1 s}$. If performing a terrain case $\left(f_{t e r}=\right.$ '.true.'), the index $j_{t 2}$ is initialized where the first occurance of the condition $j_{t 2} \Delta r_{P E}>r_{\text {out }}$ is met. The index $j_{t 1}$ is set equal to the index location within $\vartheta 1 t$, up to $j_{t 2}-1$, where the minimum value occurs. The tangent angle from the source height $\vartheta_{1}$ is then initialized to $\vartheta 1 t_{j_{t 1}}$ and the corresponding range $d_{1}$ is initialized $j_{t 1} \Delta r_{P E}$. 
For each output height index $j$ from $j_{s}$ to $j_{e}$, the following steps are performed:

1. If running a smooth surface case ( $f_{\text {ter }}=$ '.false.') and $r_{\text {out }}$ is less than the minimum range $r d t_{j}$ at which diffraction field solutions are applicable for the current height, then the index is iterated until the loop is exited. Otherwise, the SU program flow continues with step 2 .

2. The function FN_GET_TLOSS is referenced to determine the troposcatter loss and returns the total loss for each height zout $j_{j}$. The total loss is then stored in array rloss.

If receiver heights relative to the local ground are specified, then steps 1 to 2 above are also performed for all heights in zout_rtg from 1 to $n_{\text {zout_rtg }}$ with appropriate initialization of angle and height variables before referencing FN_GET_TLOSS. The final loss values are then stored in array rloss_rtg.

Table 118 and Table 119 provide identification, description, units of measure, and the computational source for each TROPOSCAT SU input and output data element.

Table 118. TROPOSCAT SU input data element requirements.

\begin{tabular}{c|l|c|c}
\hline Name & \multicolumn{1}{|c|}{ Description } & Units & Source \\
\hline$a d i f$ & $\begin{array}{l}\text { Height differences between } \text { ant }_{\text {ref }} \text { and all output receiver } \\
\text { heights }\end{array}$ & meters & TROPOINIT SU \\
$d 2 s$ & $\begin{array}{l}\text { Array of tangent ranges for all output receiver heights over } \\
\text { smooth surface }\end{array}$ & meters & TROPOINIT SU \\
$\Delta r_{P E}$ & PE range step & meters & PEINIT SU \\
$e_{k}$ & Effective earth's radius factor & N/A & GET_K SU \\
$f_{t e r}$ & Logical flag indicating if terrain profile has been specified: & N/A & APMINIT CSC \\
& \multicolumn{1}{|c}{ '.false.' = terrain profile specified } \\
$h_{m i n t e r}$ & Minimum height of terrain profile & & \\
$i_{P E}$ & Number of PEecified & meters & TERINIT SU \\
$i_{s t p}$ & Current output range step index & N/A & PEINIT SU \\
$j_{e}$ & Ending receiver height index at which to compute & N/A & Calling SU \\
$j_{s}$ & troposcatter loss & N/A & Calling SU \\
$j_{t 2}$ & Starting receiver height index at which to compute & N/A & Calling SU \\
& troposcatter loss & & \\
& Index counter for tyh array indicating location of receiver & N/A & TROPOINIT SU \\
& range & APMSTEP CSC
\end{tabular}


Table 118. TROPOSCAT SU input data element requirements. (continued)

\begin{tabular}{|c|c|c|c|}
\hline Name & Description & Units & Source \\
\hline$r d t$ & $\begin{array}{l}\text { Array of minimum ranges at which diffraction field } \\
\text { solutions are applicable (for smooth surface) for all output } \\
\text { receiver heights. }\end{array}$ & meters & TROPOINIT SU \\
\hline rloss & Propagation loss array & $\mathrm{dB}$ & Calling SU \\
\hline rloss_rtg & $\begin{array}{l}\text { Propagation loss computed relative to the local ground } \\
\text { height at heights specified by zout_rtg }\end{array}$ & $\mathrm{dB}$ & Calling SU \\
\hline rngout & Array containing all desired output ranges & meters & APMINIT CSC \\
\hline snrefo & $\begin{array}{l}\text { Surface refractivity taken from the refractivity profile with } \\
\text { respect to mean sea level }\end{array}$ & M-unit & REFINIT SU \\
\hline snref $_{t x}$ & Surface refractivity at transmitter & M-unit & REFINIT SU \\
\hline$\vartheta 0$ & $\begin{array}{l}\text { Array of angles used to determine common volume } \\
\text { scattering angle }\end{array}$ & radians & TROPOINIT SU \\
\hline$\vartheta_{1 s}$ & Tangent angle from source (for smooth surface) & radians & TROPOINIT SU \\
\hline$\vartheta 2 \mathrm{~s}$ & $\begin{array}{l}\text { Array of tangent angles from all output receiver heights - } \\
\text { used with smooth surface }\end{array}$ & radians & TROPOINIT SU \\
\hline$\vartheta 1 t$ & $\begin{array}{l}\text { Array of tangent angles from source height - used with } \\
\text { terrain profile }\end{array}$ & radians & TROPOINIT SU \\
\hline$t l s t_{w r}$ & Troposcatter loss term & $\mathrm{dB}$ & TROPOINIT SU \\
\hline twoka & Twice the effective earth radius & meters & GET_K SU \\
\hline$y_{\text {ground }}$ & Height of the surface at the current output range step & meters & Calling SU \\
\hline zout & $\begin{array}{l}\text { Array containing all desired output heights referenced to } \\
h_{\text {minter }}\end{array}$ & meters & APMINIT CSC \\
\hline zout_rtg & $\begin{array}{l}\text { Receiver heights specified relative to the local ground } \\
\text { height. }\end{array}$ & meters & Calling CSCI \\
\hline
\end{tabular}

Table 119. TROPOSCAT SU output data element requirements.

\begin{tabular}{c|l|c}
\hline Name & \multicolumn{1}{|c|}{ Description } & Units \\
\hline rloss & Propagation loss array & $\mathrm{dB}$ \\
\hline rloss_rtg & $\begin{array}{l}\text { Propagation loss computed relative to the local ground height at heights specified by } \\
\text { zout_rtg }\end{array}$ & $\mathrm{dB}$ \\
\hline
\end{tabular}

\subsection{EXTENDED OPTICS INITIALIZATION (XOINIT) CSC}

The XOINIT CSC initializes the range, height, and angle arrays in preparation for XO calculations and to reference the CLUTTER SU if clutter values are desired. 
Upon entering the XOINIT CSC, if $C_{\text {lut }}$ is '.true.' the CLUTTER SU is referenced to compute the clutter-to-noise ratio $(C N R)$. Next, the value of $i_{x o s t p}$ is tested. If $i_{x o s t p}$ is equal to 0 , then the APMCLEAN SU is referenced to deallocate all arrays used in the APM application and the CSC is exited. If $i_{\text {xostp }}$ is greater than 0 , then the following procedure is performed.

The arrays curang and curng, used for storage of traced local angles and ranges, respectively, are allocated and initialized to the range and angle values stored in ffacz. The array curht is allocated and initialized to the height of the top of the PE calculation region, $z_{\text {lim. }}$. The array igrd, used for storage of starting refractivity gradient level [at which to begin ray tracing], is allocated and initialized to 0 . The two-dimensional array prfhxo, containing final output heights and propagation factors, along with the dummy array dum, used for temporary storage, are also allocated and initialized to 0 .

If $f_{\text {ter }}$ is '.true.', then the MEANFILT SU is referenced twice to perform a 9-point smoothing operation on the angle values, using dum for temporary storage of angles after the first pass smoothing operation. Next, the starting height index at which to begin XO calculations, $j_{x s t a r t}$ is initialized to the ending height index for PE calculations, $j_{\text {end }}$, plus one. Finally, dum is deallocated before exiting.

Table 120 and Table 121 provide identification, description, units of measure, and the computational source for each XOINIT CSC input and output data element.

Table 120. XOINIT CSC input data element requirements.

\begin{tabular}{|c|c|c|c|}
\hline Name & Description & Units & Source \\
\hline$C_{\text {lut }}$ & $\begin{array}{l}\text { Logical flag used to indicate if surface clutter calculations } \\
\text { are desired. }\end{array}$ & N/A & Calling CSCI \\
\hline$f f a c z$ & $\begin{array}{l}\text { Array containing propagation factor, range, and } \\
\text { propagation angle at } Z_{\text {lim }}\end{array}$ & $\begin{array}{l}\mathrm{dB}, \\
\text { meters, } \\
\text { radians }\end{array}$ & FZLIM SU \\
\hline$f_{\text {ter }}$ & $\begin{array}{l}\text { Logical flag indicating if terrain profile has been specified: } \\
\text {.true. = terrain profile specified } \\
\text {.false. = terrain profile not specified }\end{array}$ & N/A & APMINIT CSC \\
\hline$i_{\text {xostp }}$ & Current output range step index for XO calculations & N/A & Calling SU \\
\hline$i z$ & $\begin{array}{l}\text { Number of propagation factor, range, angle triplets stored } \\
\text { in ffacz }\end{array}$ & N/A & $\begin{array}{l}\text { FZLIM SU } \\
\text { APMINIT CSC }\end{array}$ \\
\hline$i z_{\max }$ & $\begin{array}{l}\text { Maximum number of points allocated for arrays associated } \\
\text { with XO calculations }\end{array}$ & N/A & APMINIT CSC \\
\hline$j_{\text {end }}$ & Ending index within $m p f l$ of PE loss values & N/A & Calling SU \\
\hline$z_{\text {lim }}$ & Height limit for PE calculation region & meters & GETTHMAX SU \\
\hline
\end{tabular}


Table 121. XOINIT CSC output data element requirements.

\begin{tabular}{c|l|c}
\hline Name & \multicolumn{1}{|c}{ Description } & Units \\
\hline CNR & Array of clutter-to-noise ratio values & $\mathrm{dB}$ \\
curang & Array of current local angles for each ray being traced in XO region & radians \\
curht & Array of current local heights for each ray being traced in XO region & meters \\
curng & Array of current local ranges for each ray being traced in XO region & meters \\
$i_{\text {error }}$ & Error flag & N/A \\
igrd & Integer indexes indicating at what refractive gradient level to begin ray tracing & N/A \\
& for next XO range step for each ray in XO region. & N/A \\
$j_{x s t a r t}$ & Starting index within mpfl of XO loss values & dB,meters \\
prfhxo & Two-dimensional array of propagation factor and heights for each ray traced & \\
& in XO region to range $r_{\text {out }}$ &
\end{tabular}

\subsubsection{APM Clean (APMCLEAN) SU}

The APMCLEAN SU deallocates all dynamically dimensioned arrays used in one complete run of APM calculations.

Upon entry, all arrays that were dynamically allocated at the beginning of the current application are now deallocated.

Table 122 and Table 123 provide identification, description, units of measure, and the computational source for each APMCLEAN SU input and output data element.

Table 122. APMCLEAN SU input data element requirements.

\begin{tabular}{|c|c|c|c|}
\hline Name & Description & Units & Source \\
\hline adif & Height array used for troposcatter calculations & meters & TROPOINIT SU \\
\hline curang & $\begin{array}{l}\text { Array of current local angles for each ray being } \\
\text { traced in XO region }\end{array}$ & radians & $\begin{array}{l}\text { EXTO SU } \\
\text { XOINIT CSC }\end{array}$ \\
\hline curht & $\begin{array}{l}\text { Array of current local heights for each ray being } \\
\text { traced in XO region }\end{array}$ & meters & $\begin{array}{l}\text { EXTO SU } \\
\text { XOINIT CSC }\end{array}$ \\
\hline curng & $\begin{array}{l}\text { Array of current local ranges for each ray being } \\
\text { traced in XO region }\end{array}$ & meters & $\begin{array}{l}\text { EXTO SU } \\
\text { XOINIT CSC }\end{array}$ \\
\hline$d 2 s$ & $\begin{array}{l}\text { Array of tangent ranges for all output receiver } \\
\text { heights over smooth surface }\end{array}$ & meters & TROPOINIT SU \\
\hline dielec & $\begin{array}{l}\text { Two-dimensional array containing the relative } \\
\text { permittivity and conductivity; } \text { dielec }_{1, i} \text { and } \\
\text { dielec }_{2, i} \text {, respectively. }\end{array}$ & $\mathrm{N} / \mathrm{A}, \mathrm{S} / \mathrm{m}$ & $\begin{array}{l}\text { Calling CSCI, } \\
\text { DIEINIT SU }\end{array}$ \\
\hline envpr & $\begin{array}{l}\text { Complex [refractivity] phase term array } \\
\text { interpolated every } \Delta z_{P E} \text { in height }\end{array}$ & N/A & $\begin{array}{l}\text { PEINIT SU } \\
\text { PESTEP SU }\end{array}$ \\
\hline
\end{tabular}


Table 122. APMCLEAN SU input data element requirements. (continued)

\begin{tabular}{|c|c|c|c|}
\hline Name & Description & Units & Source \\
\hline ffacz & $\begin{array}{l}\text { Array containing propagation factor, range, and } \\
\text { propagation angle at } Z_{\text {lim }}\end{array}$ & $\begin{array}{l}\mathrm{dB}, \\
\text { meters, } \\
\text { radians }\end{array}$ & FZLIM SU \\
\hline ffat1m & $\begin{array}{l}\text { Propagation factor array computed at } 1 \mathrm{~m} \text { above } \\
\text { the surface. }\end{array}$ & $\mathrm{dB}$ & $\begin{array}{l}\text { CALCLOS SU, } \\
\text { FEDR SU, } \\
\text { FEM SU, } \\
\text { ROLOSS SU }\end{array}$ \\
\hline ffrout & $\begin{array}{l}\text { Array of propagation factors at each output } \\
\text { range beyond } r_{a t z} \text { and at height } z_{\text {lim }}\end{array}$ & $\mathrm{dB}$ & CALCLOS SU \\
\hline filt & Cosine-tapered (Tukey) filter array & N/A & PEINIT SU \\
\hline filtp & Array filter for spectral estimation calculations & N/A & APMINIT CSC \\
\hline frsp & Complex free space propagator term array & N/A & PEINIT SU \\
\hline$f s l$ & Free space loss array for output ranges & $\mathrm{dB}$ & APMINIT CSC \\
\hline$\gamma c$ & $\begin{array}{l}\text { Dynamically allocated array of constants } \\
\text { describing the backscattering effectiveness of } \\
\text { the surface }\end{array}$ & $\mathrm{dB}$ & Calling CSCI \\
\hline prng & $\begin{array}{l}\text { Dynamically allocated array of ranges } \\
\text { corresponding to the values in } \gamma c\end{array}$ & meters & Calling CSCI \\
\hline$g r$ & Intermediate M-unit gradient array, RO region & (M-unit/m) $10^{-6}$ & REFINIT SU \\
\hline grad & $\begin{array}{l}\text { Two-dimensional array containing gradients of } \\
\text { each profile used in XO calculations }\end{array}$ & $\begin{array}{l}\text { M-units } \\
\text { /meter }\end{array}$ & SAVEPRO SU \\
\hline grdum & $\begin{array}{l}\text { Array of refractivity gradients defined by profile } \\
\text { htdum and refdum }\end{array}$ & $\begin{array}{l}\text { M-units } \\
\text { /meter }\end{array}$ & $\begin{array}{l}\text { REFINIT SU } \\
\text { REFINTER SU }\end{array}$ \\
\hline hfangr & $\begin{array}{l}\text { Array of user-defined cut-back angles. This is } \\
\text { used only for user-defined height-finder antenna } \\
\text { type. }\end{array}$ & radians & APMINIT CSC \\
\hline hlim & $\begin{array}{l}\text { Array containing height at each output range } \\
\text { separating the RO region from the PE (at close } \\
\text { ranges) and XO (at far ranges) regions }\end{array}$ & meters & GETTHMAX SU \\
\hline href & Heights of refractivity profile with respect to $y_{\text {ref }}$ & meters & PROFINT SU \\
\hline ht & PE mesh height array of size $n_{f f t}$ & meters & PEINIT SU \\
\hline htdum & Height array for current interpolated profile & meters & $\begin{array}{l}\text { REFINIT SU } \\
\text { REFINTER SU }\end{array}$ \\
\hline htfe & $\begin{array}{l}\text { Array containing the height at each output range } \\
\text { separating the FE region from the RO region } \\
\text { (full hybrid mode), or the FE region from the PE } \\
\text { region (partial hybrid mode) }\end{array}$ & meters & FILLHT SU \\
\hline$h t r$ & $\begin{array}{l}\text { Two-dimensional array containing heights of } \\
\text { each profile used in XO calculations }\end{array}$ & meters & SAVEPRO SU \\
\hline igrd & $\begin{array}{l}\text { Integer indexes indicating at what refractive } \\
\text { gradient level to begin ray tracing for next XO } \\
\text { range step for each ray in XO region. }\end{array}$ & N/A & XOINIT CSC \\
\hline
\end{tabular}


Table 122. APMCLEAN SU input data element requirements. (continued)

\begin{tabular}{|c|c|c|c|}
\hline Name & Description & Units & Source \\
\hline igrnd & 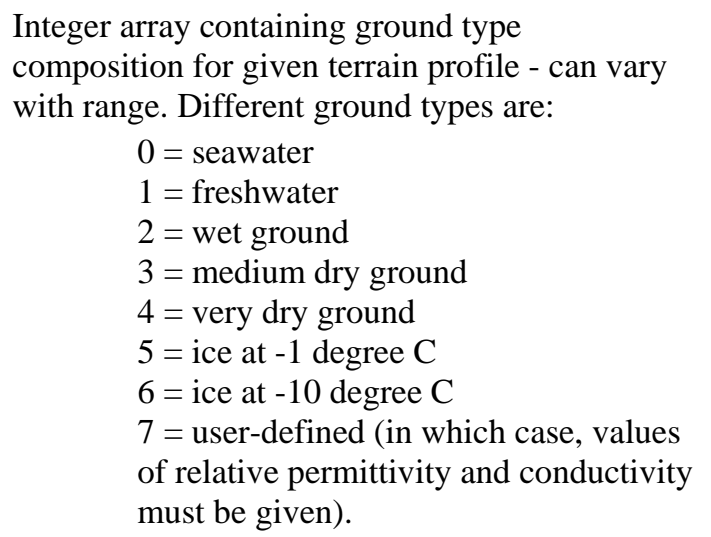 & N/A & Calling CSCI \\
\hline$l v l$ & $\begin{array}{l}\text { Number of height levels in each profile used in } \\
\text { XO calculations }\end{array}$ & N/A & SAVEPRO SU \\
\hline$n c^{2}$ & Array of complex dielectric constants & N/A & DIEINIT SU \\
\hline prfhxo & $\begin{array}{l}\text { Array of propagation factor and heights for each } \\
\text { ray traced in XO region to range } r_{\text {out }}\end{array}$ & $\mathrm{dB}$,meters & XOINIT CSC \\
\hline profint & Profile interpolated to every $\Delta z_{P E}$ in height & M-units & REFINTER SU \\
\hline$\Psi$ & $\begin{array}{l}\text { Array of interpolated grazing angles at each PE } \\
\text { range step }\end{array}$ & radians & GRAZE_INT SU \\
\hline$q$ & Intermediate M-unit difference array, RO region & 2M-unit $10^{-6}$ & REFINIT SU \\
\hline$r d t$ & $\begin{array}{l}\text { Array of minimum ranges at which diffraction } \\
\text { field solutions are applicable (for smooth } \\
\text { surface) for all output receiver heights. }\end{array}$ & meters & TROPOINIT SU \\
\hline refdum & M-unit array for current interpolated profile & M-units & $\begin{array}{l}\text { REFINIT SU } \\
\text { REFINTER SU }\end{array}$ \\
\hline refref & Refractivity profile with respect to $y_{\text {ref }}$ & M-units & PROFINT SU \\
\hline rfac1 & $\begin{array}{l}\text { Propagation factor at valid output height points } \\
\text { from PE field at range } r_{\text {last }}\end{array}$ & $\mathrm{dB}$ & CALCLOS SU \\
\hline rfac2 & $\begin{array}{l}\text { Propagation factor at valid output height points } \\
\text { from PE field at range } r\end{array}$ & $\mathrm{~dB}$ & CALCLOS SU \\
\hline rgrnd & $\begin{array}{l}\text { Array containing ranges at which varying } \\
\text { ground types apply. }\end{array}$ & meters & Calling CSCI \\
\hline rlogo & $\begin{array}{l}\text { Array containing } 20 \text { times the logarithm of all } \\
\text { output ranges }\end{array}$ & N/A & APMINIT CSC \\
\hline rloss & Propagation loss & $\mathrm{dB}$ & $\begin{array}{l}\text { ALLARRAY_APM } \\
\text { CALCLOS SU } \\
\text { EXTO SU } \\
\text { TROPOSCAT SU }\end{array}$ \\
\hline $\begin{array}{l}\text { rloss_rt } \\
g\end{array}$ & $\begin{array}{l}\text { Propagation loss computed relative to the local } \\
\text { ground height at heights specified by zout_rtg }\end{array}$ & $\mathrm{dB}$ & $\begin{array}{l}\text { ALLARRAY_APM } \\
\text { CALCLOS SU } \\
\text { TROPOSCAT SU }\end{array}$ \\
\hline$r m$ & Intermediate M-unit array, RO region & M-unit $10^{-6}$ & REFINIT SU \\
\hline rngout & Array containing all desired output ranges & meters & APMINIT CSC \\
\hline
\end{tabular}


Table 122. APMCLEAN SU input data element requirements. (continued)

\begin{tabular}{|c|c|c|c|}
\hline Name & Description & Units & Source \\
\hline$r n$ & Array of $R_{T}$ to the $i^{\text {th }}$ power (e.g., $r n_{i}=R_{T}^{i}$ ) & N/A & GETALN SU \\
\hline rsqrd & $\begin{array}{l}\text { Array containing the square of all desired output } \\
\text { ranges }\end{array}$ & meters $^{2}$ & APMINIT CSC \\
\hline spectr & Spectral amplitude of field & $\mathrm{dB}$ & SPECEST SU \\
\hline 90 & $\begin{array}{l}\text { Array of angles used to determine common } \\
\text { volume scattering angle }\end{array}$ & radians & TROPOINIT SU \\
\hline $92 \mathrm{~s}$ & $\begin{array}{l}\text { Array of tangent angles from all output receiver } \\
\text { heights - used with smooth surface }\end{array}$ & radians & TROPOINIT SU \\
\hline$\vartheta 1 t$ & $\begin{array}{l}\text { Array of tangent angles from source height - } \\
\text { used with terrain profile }\end{array}$ & radians & TROPOINIT SU \\
\hline$\Theta_{\text {rout }}$ & $\begin{array}{l}\text { Two-dimensional array containing the } \\
\text { propagation angle spectrally estimated from PE } \\
\text { at } n_{\text {ang }} \text { height points and at every output range } \\
\text { step } r_{\text {out }}\end{array}$ & radians & RET_GRAZE SU \\
\hline tang & Tangent of angle array from terrain slopes. & radians & PEINIT SU \\
\hline tyh & Adjusted height points of terrain profile & meters & PEINIT SU \\
\hline$U$ & Complex PE field & $\mu \mathrm{V} / \mathrm{m}$ & PESTEP SU \\
\hline udum & Real or imaginary part of complex field array & $\mu \mathrm{V} / \mathrm{m}$ & FFT SU \\
\hline Ulast & Complex PE field at range $r_{\text {last }}$ & $\mu \mathrm{V} / \mathrm{m}$ & PESTEP SU \\
\hline$w$ & Difference equation of complex PE field & $\mu \mathrm{V} / \mathrm{m}^{2}$ & PESTEP SU \\
\hline$x p$ & Real part of spectral portion of PE field & $\mu \mathrm{V} / \mathrm{m}$ & SPECEST SU \\
\hline$y m$ & Particular solution of difference equation & $\mu \mathrm{V} / \mathrm{m}$ & PESTEP SU \\
\hline yp & Imaginary part of spectral portion field & $\mu \mathrm{V} / \mathrm{m}$ & SPECEST SU \\
\hline zout & $\begin{array}{l}\text { Array containing all desired output heights } \\
\text { referenced to } h_{\text {minter }}\end{array}$ & meters & APMINIT CSC \\
\hline zoutma & Output height point relative to "real” ant $t_{r e f}$ & meters & APMINIT CSC \\
\hline zoutpa & Output height point relative to "image" $a n t_{\text {ref }}$ & meters & APMINIT CSC \\
\hline$z R O$ & Array of output heights in RO region & meters & APMINIT CSC \\
\hline$z r t$ & Intermediate height array, RO region & meters & REFINIT SU \\
\hline$z X O$ & $\begin{array}{l}\text { Height of the ground at the current output range } \\
\text { step }\end{array}$ & meters & CALCLOS SU \\
\hline
\end{tabular}

Table 123. APMCLEAN CSC output data element requirements.

\begin{tabular}{c|c|c}
\hline Name & Description & Units \\
\hline$i_{\text {error }}$ & Error flag indicator: non-zero if error has occurred in deallocation procedure & N/A \\
\hline
\end{tabular}




\subsubsection{Clutter-to-Noise (CLUTTER) SU}

The CLUTTER SU calculates the returned CNR at each range $r_{\text {out }}$ based on the radar range equation.

The reflectivity computed for that portion of the path over water is based on a modification to the Georgia Institute of Technology (GIT) model. However, the GIT model is valid for frequencies above $1 \mathrm{GHz}$. Therefore, for paths over water and for frequencies less than $1 \mathrm{GHz}$, the reflectivity is determined using the same model as that over land, detailed below.

Upon entering the SU the noise power, $P_{N}$, and a constant, Con, based on system parameters and used in the radar equation, are determined according to

$$
\begin{aligned}
& \text { Con }=10 \operatorname{LOG}_{10}\left(\frac{\lambda^{2} P_{t} 10^{3}}{(4 \pi)^{3}}\right)+2 G-L_{\text {sys }}, \\
& P_{N}=10 \operatorname{LOG}_{10}\left(\frac{4 \times 10^{-15}}{\tau}\right)+N_{f},
\end{aligned}
$$

where $\tau$ is the system pulse length in microseconds, $P_{t}$ is the transmitter peak power in $\mathrm{kW}, G$ is the transmitting antenna gain (transmitting and receiving antenna are assumed to be equal), $L_{s y s}$, is the assumed system loss, and $N_{f}$ is the noise figure in $\mathrm{dB}$.

Other constants used in determining the area of the clutter cell are computed for subsequent use in the GIT model and for clutter cross section model used over land:

$$
\begin{aligned}
& A c_{G I T}=\frac{\theta_{\text {hbw }} c_{o} \tau}{2 \sqrt{2}}, \\
& A C x_{\text {low }}=c_{o} \tau \operatorname{TAN}\left(\theta_{\text {hbw }} / 2\right), \\
& \operatorname{ACX}_{\text {high }}=\pi \operatorname{TAN}\left(\mu_{\text {bwr }} / 2\right) \operatorname{TAN}\left(\theta_{\text {hbw }} / 2\right) .
\end{aligned}
$$

Next, if any of the path is over water and the frequency is greater than $1 \mathrm{GHz}$, then the GIT flag, $l_{G I T}$, is set to '.true.' and the GIT_INIT SU (if only one wind speed has been specified) and the SPMINIT SU are referenced to initialize all necessary variables used in computing the clutter over water.

Next, steps 1 through 9 are performed for each index $i$ ranging from 1 to $n_{\text {rout }}$. 
1. The grazing angle, $\psi_{\text {rout }}$, and range, $\mathrm{r}_{\text {out }}$ are initialized:

$$
\begin{aligned}
& \psi_{\text {rout }}=\text { grz }_{-} \text {rout }_{i} \\
& r_{\text {out }}=\text { rngout }_{i}
\end{aligned}
$$

If the current range corresponds to that part of the path over land or the frequency is less than $1 \mathrm{GHz}$ ( $l_{G I T}=$ '.false.'), then the SU continues with steps 2 through 3; otherwise, the SU proceeds with steps 4 through 8.

2. The area of the clutter cell, $A_{c}$, is determined based on high and low grazing angle formulas:

$$
\begin{aligned}
& A c_{\text {low }}=\frac{A c x_{\text {low }} r_{\text {out }}}{\operatorname{COS}\left(\psi_{\text {rout }}\right)}, \\
& A c_{\text {high }}=\frac{A c x_{\text {high }} r_{\text {out }}^{2}}{\operatorname{SIN}\left(\psi_{\text {rout }}\right)}, \\
& A_{c}=10 L O G_{10}\left(\operatorname{MIN}\left(A c_{\text {low }}, A c_{\text {high }}\right)\right) .
\end{aligned}
$$

3. The reflectivity, or clutter cross section per unit area, $\sigma^{\circ}$, over land is then determined from

$$
\sigma^{o}=\gamma C_{\text {igrc }}+10 \operatorname{LOG}_{10}\left[\operatorname{SIN}\left(\psi_{\text {rout }}\right)\right] \text {, }
$$

where igrc is the index counter for the $\gamma c$ array at the current range. The SU then proceeds to step 9.

4. If the current range corresponds to that portion of the path over water, then the GIT_INIT SU is referenced if more than one wind speed has been specified. The range at which the current grazing angle occurs for a standard atmosphere is then determined from

$$
r_{\text {spm }}=\sqrt{a_{\text {ekst }}^{2} \psi_{\text {rout }}^{2}+2 a_{\text {ekst }} a n t_{\text {ref }}}-a_{\text {ekst }} \psi_{\text {rout }} .
$$

5. The propagation factor (in $\mathrm{dB}$ ) for a standard atmosphere at this range, $F_{\text {spm }}$, is then obtained from referencing the SPM SU. 
6. Next, several variables are computed for use in computing the reflectivity:

$$
\begin{aligned}
& \sigma_{\varphi}=\sigma_{\text {term }} \psi_{\text {rout }}, \\
& a_{i}=\frac{\sigma_{\varphi}^{4}}{\left(1+\sigma_{\varphi}^{4}\right)}, \\
& a_{u}=e^{a u_{\text {term }}\left(1-2.8 \psi_{\text {rout }}\right)},
\end{aligned}
$$

where $\sigma_{\text {term }}$ and $a u_{\text {term }}$ are determined in the GIT_INIT SU.

7. The GIT reflectivity, $\sigma_{G I T}^{o}$, is then computed as

$$
\begin{aligned}
& \sigma_{H}^{o}=10 L O G_{10}\left(3.9 \times 10^{-6} \lambda \psi_{\text {rout }}^{0.4} a_{i} a_{u} a_{w}\right), \text { for } 3000 \leq f_{M H z}<10,000 \mathrm{MHz} ; \\
& \sigma_{V}^{o}=\sigma_{H}^{o}+\sigma_{V H 2}+1.27 L N\left(\psi_{\text {rout }}+10^{-4}\right) \\
& \sigma_{H}^{o}=\text { as above, } \\
& \sigma_{V}^{o}=\sigma_{H}^{o}+\sigma_{V H 1}+2.46 L N\left(\psi_{\text {rout }}+10^{-4}\right) \text { for } f_{M H z}<3000 \mathrm{MHz} ; \\
& \sigma_{H}^{o}=10 L O G_{10}\left(5.78 \times 10^{-6} \psi_{\text {rout }}^{0.547} a_{i} a_{u} a_{w}\right), \text { for } f_{M H z} \geq 10,000 \mathrm{MHz}, \\
& \sigma_{V}^{o}=\sigma_{H}^{o}+\sigma_{V H 3}+1.31 L N\left(\psi_{\text {rout }}\right) \\
& \sigma_{G I T}^{o}=\sigma_{H}^{o}, \text { for } H \text { pol, } \\
& \sigma_{G I T}^{o}=\sigma_{V}^{o}, \text { for } V \text { pol, }
\end{aligned}
$$

where the terms $a_{w}$ and $\sigma_{V H 1-3}$ are determined in the GIT_INIT SU.

8. The reflectivity and the area of the clutter cell for over water paths are then computed according to

$$
\begin{aligned}
& \sigma^{o}=\sigma_{G I T}^{o}-2 F_{\text {spm }}, \\
& A_{c}=10 L O G_{10}\left(r_{\text {out }} A c_{G I T}\right) .
\end{aligned}
$$

9. The return clutter power at the current range $r_{\text {out }}$ is then computed:

$$
C=C o n+\sigma^{o}+2 \text { ffat } 1 m_{i}+A_{c}-40 L O G_{10}\left(r_{\text {out }}\right) .
$$


Once the clutter power has been computed for all ranges and stored in an array, the clutter-to-noise ratio is determined for all ranges by subtracting the noise power:

$$
C N R_{i}=C_{i}-P_{N} \text {, for } i=1,2,3, \ldots n_{\text {rout }} .
$$

Table 124 and Table 125 provide identification, description, units of measure, and the computational source for each CLUTTER SU input and output data element.

Table 124. CLUTTER SU input data element requirements.

\begin{tabular}{|c|c|c|c|}
\hline Name & Description & Units & Source \\
\hline$a_{e k s t}$ & 4/3 effective earth's radius & meters & APM_MOD \\
\hline$a_{n} t_{r e f}$ & Transmitting antenna height relative to $h_{\text {minter }}$ & meters & APMINIT CSC \\
\hline$f_{M H z}$ & Frequency in $\mathrm{MHz}$ & $\mathrm{MHz}$ & Calling CSCI \\
\hline ffat1m & $\begin{array}{l}\text { Propagation factor array computed at } 1 \mathrm{~m} \text { above } \\
\text { the surface. }\end{array}$ & $\mathrm{dB}$ & $\begin{array}{l}\text { CALCLOS SU, } \\
\text { FEDR SU, } \\
\text { FEM SU, } \\
\text { ROLOSS SU }\end{array}$ \\
\hline$f_{\text {ter }}$ & $\begin{array}{l}\text { Logical flag indicating if terrain profile has been } \\
\text { specified: } \\
\text {.true. = terrain profile specified } \\
\text {.false. = terrain profile not specified }\end{array}$ & N/A & APMINIT CSC \\
\hline$G$ & Gain of transmit/receive antennas & $\mathrm{dBi}$ & Calling CSCI \\
\hline$\gamma c$ & $\begin{array}{l}\text { Dynamically allocated array of constants } \\
\text { describing the backscattering effectiveness of } \\
\text { the surface }\end{array}$ & $\mathrm{dB}$ & Calling CSCI \\
\hline yrng & $\begin{array}{l}\text { Dynamically allocated array of ranges } \\
\text { corresponding to the values in } \gamma c\end{array}$ & meters & Calling CSCI \\
\hline$\Psi_{\text {rout }}$ & Array of grazing angles at each output range $r_{\text {out }}$ & radians & RET_GRAZE SU \\
\hline$i_{g c}$ & $\begin{array}{l}\text { Number of } \gamma c \text { values for a particular application } \\
\text { of APM }\end{array}$ & N/A & Calling CSCI \\
\hline$i_{o}$ & $\begin{array}{l}\text { Starting index for mpfl array: } \\
\begin{array}{l}0=1^{\text {st }} \text { calculated output point is at surface } \\
1=1^{\text {st }} \text { calculated output point is at height } \\
\Delta z_{\text {out }}\end{array}\end{array}$ & N/A & APMINIT CSC \\
\hline$i_{P E}$ & Number of PE range steps & N/A & PEINIT SU \\
\hline$i_{p o l}$ & $\begin{array}{l}\text { Polarization flag: } \\
\qquad \begin{array}{l}0=\text { horizontal polarization } \\
1=\text { vertical polarization }\end{array}\end{array}$ & N/A & Calling CSCI \\
\hline$\lambda$ & Wavelength & meters & APMINIT CSC \\
\hline$L_{\text {sys }}$ & Miscellaneous system losses & $\mathrm{dB}$ & Calling CSCI \\
\hline$\mu_{b w r}$ & Antenna vertical beamwidth & radians & APMINIT CSC \\
\hline$N_{f}$ & Noise figure & dB & Calling CSCI \\
\hline$n_{\text {rout }}$ & Integer number of output range points desired & N/A & Calling CSCI \\
\hline$n_{w}$ & Number of wind speeds & N/A & Calling CSCI \\
\hline$P_{t}$ & Transmitter peak power & $\mathrm{kW}$ & Calling CSCI \\
\hline
\end{tabular}


Table 124. CLUTTER SU input data element requirements. (continued)

\begin{tabular}{|c|c|c|c|}
\hline Name & Description & Units & Source \\
\hline rlogo & $\begin{array}{l}\text { Array containing } 20 \text { times the logarithm of all } \\
\text { output ranges }\end{array}$ & $\mathrm{dB}$ & APMINIT CSC \\
\hline rngout & Array containing all desired output ranges & meters & APMINIT CSC \\
\hline ruf & $\begin{array}{l}\text { Logical flag indicating if rough sea surface } \\
\text { calculations are required } \\
\text { ‘.true.' = perform rough sea surface } \\
\text { calculations } \\
\text { '.false.' = do not perform rough sea surface } \\
\text { calculations }\end{array}$ & N/A & APMINIT CSC \\
\hline$\theta_{h b w}$ & Antenna horizontal beam width & radians & APMINIT CSC \\
\hline$\tau$ & Pulse length/width & $\mu$ sec & Calling CSCI \\
\hline$y_{\text {fref }}$ & Ground elevation height at source & meters & APMINIT CSC \\
\hline$z_{c}$ & $\begin{array}{l}\text { Height at which to compute propagation factor } \\
\text { for clutter calculations relative to } h m_{r e f}\end{array}$ & meters & APMINIT CSC \\
\hline
\end{tabular}

Table 125. CLUTTER SU output data element requirements.

\begin{tabular}{c|c|c}
\hline Name & Description & Units \\
\hline$C N R$ & Clutter-to-noise ratio array & $\mathrm{dB}$ \\
\hline
\end{tabular}

\subsubsection{Diffraction Loss (FN_DLOSS) Function}

The FN_DLOSS function computes the diffraction region loss based on the CCIR model. Please refer to Hitney et al. (1984) for a complete description.

\subsubsection{Get Theta (GETTHETA) SU}

The GETTHETA SU computes the optical phase-lag difference angle based on the reflection range. Please refer to Hitney et al (1984). for a complete description.

\subsubsection{GIT Initialization (GIT_INIT) SU}

The GIT_INIT SU initializes all variables used in the calculation of the reflectivity based on the GIT model.

Upon entering the SU, the wind speed, $\omega_{s}$, at the current range is determined by referencing the FN_CURWIND function. Next, the average wave height, $h_{\text {avg }}$, and wind direction are initialized according to

$$
\begin{aligned}
& h_{\text {avg }}=\left(\frac{\omega_{s}}{8.67}\right)^{2.5}, \\
& \omega_{d}=\text { wind }_{\text {dir }}(\pi / 180) .
\end{aligned}
$$


The following terms used in determining the upwind/downwind factor, $a_{u}$, and wind speed factor, $a_{w}$, are then computed:

$$
\begin{aligned}
& q_{w}=1.1(\lambda+0.015)^{-0.4}, \\
& a u_{\text {term }}=0.2 \operatorname{COS}\left(\omega_{d}\right)(\lambda+0.015)^{-0.4} \text { for } f_{M H z}<10000 ; \\
& q_{w}=1.93 \lambda^{-0.04}, \\
& a u_{\text {term }}=0.25 \operatorname{COS}\left(\omega_{d}\right) \lambda^{-0.33} \text { for } f_{M H z} \geq 10000
\end{aligned}
$$

Finally, the wind speed factor and several variables used in computing the reflectivity are determined:

$$
\begin{aligned}
a_{w} & =\left(\frac{1.94 \omega_{s}}{\left[1+\omega_{s} / 15.4\right]}\right)^{q_{w}}, \\
\sigma_{\text {term }} & =\frac{(14.4 \lambda+5.5) h_{\text {avg }}}{\lambda}, \\
\sigma_{V H 1} & =22.2+3.76 \mathrm{LN}(\lambda)-1.73 \mathrm{LN}\left(h_{\text {avg }}+0.015\right), \\
\sigma_{V H 2} & =9.7+1.09 \mathrm{LN}(\lambda)-1.05 \mathrm{LN}\left(h_{\text {avg }}+0.015\right), \\
\sigma_{V H 3} & =18.55+3.43 \mathrm{LN}(\lambda)-1.38 \mathrm{LN}\left(h_{\text {avg }}\right) .
\end{aligned}
$$

Table 126 and Table 127 provide identification, description, units of measure, and the computational source for each GIT_INIT SU input and output data element.

Table 126. GIT_INIT SU input data element requirements.

\begin{tabular}{c|l|c|c}
\hline Name & \multicolumn{1}{|c|}{ Description } & Units & Source \\
\hline$f_{\mathrm{MHz}}$ & Frequency in $\mathrm{MHz}$ & $\mathrm{MHz}$ & Calling CSCI \\
$\lambda$ & Wavelength & meters & APMINIT CSC \\
$n_{w}$ & Number of wind speeds & $\mathrm{N} / \mathrm{A}$ & Calling CSCI \\
rngwind & Ranges of wind speeds entered: rngwind $_{i}$ & meters & Calling CSCI \\
= range of $i^{\text {th }}$ wind speed & & \\
rout & Current output range & Calling SU \\
wind & Array of wind speeds & Calling CSCI \\
winddir & Angle between antenna boresight and & degrees & Calling CSCI \\
& upwind direction & & \\
\hline
\end{tabular}


Table 127. GIT_INIT SU output data element requirements.

\begin{tabular}{c|l|c}
\hline Name & \multicolumn{1}{|c}{ Description } & Units \\
\hline$a u_{t e r m}$ & Term in computing the upwind/downwind factor & N/A \\
$a_{w}$ & Wind speed factor & N/A \\
$\sigma_{t e r m}$ & Term used in computing the GIT reflectivity & N/A \\
$\sigma_{V H 1-3}$ & Terms used in computing the GIT reflectivity & N/A \\
\hline
\end{tabular}

\subsubsection{GofZ (GOFZ) Function}

The GOFZ function computes the diffraction region height-gain in decibels, based on the CCIR diffraction model. Please refer to Hitney et al.(1984) for a complete description.

\subsubsection{Mean Filter (MEANFILT) SU}

The MEANFILT SU performs a $i_{s z}$-point average smoothing operation on the array passed to it.

The array arbef is passed to the SU, along with the number of points over which to perform the smoothing operation, $i_{s z}$. Once the smoothing operation has been performed, the resulting "smoothed" points are stored in araft and passed back to the calling routine. The operation is performed as follows:

$$
\operatorname{araft}_{k}=\frac{1}{i_{S Z}} \sum_{i=k-m^{\prime}}^{k+m^{\prime}} \operatorname{arbef}_{i} \quad \text { for } k=m^{\prime}+1, m^{\prime}+2, \ldots ., m-m^{\prime},
$$

where $m^{\prime}$ is $1 / 2\left(i_{s z}-1\right)$ and $m$ is the size of the array arbef.

Table 128 and Table 129 provide identification, description, units of measure, and the computational source for each MEANFILT SU input and output data element.

Table 128. MEANFILT SU input data element requirements.

\begin{tabular}{c|l|c|c}
\hline Name & \multicolumn{1}{|c|}{ Description } & Units & Source \\
\hline arbef & Array of angles before smoothing operation & radians & Calling SU \\
$i_{s z}$ & Number of points over which to perform average smoothing & N/A & Calling SU \\
$m$ & Size of array arbef & N/A & Calling SU \\
\hline
\end{tabular}

Table 129. MEANFILT SU output data element requirements.

\begin{tabular}{c|c|c}
\hline Name & Description & Units \\
\hline araft & Array of angles after smoothing operation & radians \\
\hline
\end{tabular}




\subsubsection{Optical Region Limit (OPLIMIT) SU}

The OPLIMIT SU calculates the maximum range in the optical region and the corresponding loss at that range. Please refer to Hitney et al. (1984) for a complete description.

\subsubsection{Optical Difference (OPTICF) SU}

The OPTICF SU calculate the optical path-length difference angle by solving a cubic equation for the reflection point range. Please refer to Hitney et al. (1984) for a complete description.

\subsubsection{R1 Iteration (R1ITER) SU}

The R1ITER SU finds the range of the reflection point corresponding to a particular launch angle. Please refer to Hitney et al. (1984) for a complete description.

\subsubsection{Standard Propagation Model Initialization (SPM_INIT) SU}

The SPM_INIT SU initializes many of the variables used throughout the SPM SU. Please refer to Hitney et al. (1984) for a complete description.

\subsubsection{Standard Propagation Model (SPM) SU}

The SPM SU computes the propagation factor for a standard atmosphere only, with the assumption of omnidirectional antenna patterns. Please refer to Hitney et al. (1984) for a complete description.

\subsection{EXTENDED OPTICS STEP (XOSTEP) CSC}

The XOSTEP CSC calculates the propagation loss in the XO region for one output range step.

Upon entering the XOSTEP CSC, the current execution mode is checked to determine if XO calculations will be necessary ( $\left.i_{h y b r i d} \neq 0\right)$ ). If $i_{\text {hybrid }}$ is 0 , then the CSC is exited.

If $i_{\text {hybrid }}$ is not equal to 0 , the output range $r_{\text {out }}$, and the gaseous absorption loss

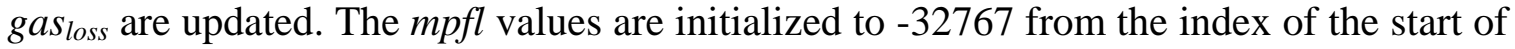
XO calculations, $j_{\text {xstart }}$, to the maximum number of height output points, $n_{\text {zout }}$ The EXTO $\mathrm{SU}$ is then referenced to calculate propagation factor and loss values in the XO region. Propagation factor and loss values are returned in $m p f l$ from index $j_{x s t a r t}$ to $j_{x e}$.

If $\mathrm{FE}$ and RO calculations need to be performed ( $\left.i_{\text {hybrid }}=1\right)$, then the indices $j_{f s}$ and $j_{f e}$, indicating the height index at which to start and end FE calculations, respectively, are determined. The FEM SU is then referenced to compute propagation factor and loss values for heights zout $j_{f s}$ to zout $_{j e}$. Similarly for RO calculations, the indices $j_{r s}$ and $j_{r e}$ are 
determined, and the ROLOSS SU is referenced to compute propagation factor and loss values for heights zout $_{j_{r s}}$ to zout $_{j_{r e}}$.

Finally, the index $j_{x e n d}$ is set equal to the maximum of $j_{x e}, j_{f e}$, and $j_{r e}$. If the last range value has been reached $\left(i_{s t p}=n_{\text {rout }}\right)$, then the APMCLEAN SU is referenced to deallocate all arrays allocated for the APM application.

Table 130 and Table 131 provide identification, description, units of measure, and the computational source for each XOSTEP CSC input and output data element.

Table 130. XOSTEP CSC input data element requirements.

\begin{tabular}{|c|c|c|c|}
\hline Name & Description & Units & Source \\
\hline$g a s_{a t t}$ & Gaseous absorption attenuation rate & $\mathrm{dB} / \mathrm{km}$ & GASABS SU \\
\hline$h t f e$ & $\begin{array}{l}\text { Array containing the height at each output range separating } \\
\text { the FE region from the RO region (full hybrid mode), or the } \\
\text { FE region from the PE region (partial hybrid mode) }\end{array}$ & meters & FILLHT SU \\
\hline$h t_{\text {lim }}$ & Maximum height relative to $h_{\text {minter }}$ & meters & TERINIT SU \\
\hline$i_{\text {hybrid }}$ & $\begin{array}{l}\text { Integer indicating which sub-models will be used: } \\
\begin{aligned} 0 & =\text { pure PE model } \\
1 & =\text { full hybrid model }(\mathrm{PE}+\mathrm{FE}+\mathrm{RO}+\mathrm{XO}) \\
2 & =\text { partial hybrid model }(\mathrm{PE}+\mathrm{XO})\end{aligned}\end{array}$ & N/A & APMINIT CSC \\
\hline$i_{s t p}$ & Current output range step index & N/A & Calling CSCI \\
\hline$j_{x s t a r t}$ & $\begin{array}{l}\text { Index at which valid propagation factor and loss values in } \\
m p f l \text { start }\end{array}$ & N/A & Calling CSCI \\
\hline$n_{\text {zout }}$ & Integer number of output height points desired & N/A & Calling CSCI \\
\hline rngout & Array containing all desired output ranges & meters & APMINIT CSC \\
\hline zout & $\begin{array}{l}\text { Array containing all desired output heights referenced to } \\
h_{\text {minter }}\end{array}$ & meters & APMINIT CSC \\
\hline
\end{tabular}

Table 131. XOSTEP CSC output data element requirements.

\begin{tabular}{c|l|c}
\hline Name & \multicolumn{1}{|c|}{ Description } & Units \\
\hline gas $_{\text {loss }}$ & Loss due to gaseous absorption & $\mathrm{dB}$ \\
$j_{\text {xend }}$ & Index at which valid propagation factor and loss values in $m p f l$ end & N/A \\
$m p f l$ & Propagation factor and loss array & $\mathrm{cB}$ \\
$r_{\text {out }}$ & Current desired output range & meters \\
\hline
\end{tabular}




\subsubsection{Extended Optics (EXTO) SU}

The EXTO SU calculates propagation factor and loss based on XO techniques. The SU performs a ray trace on all rays within one output range step and returns the propagation factor and loss up to the necessary height; storing all angle, height, and range information for subsequent ray tracing upon the next reference to the SU.

Upon entering the SU, internal one-line ray trace functions are defined as

$$
\begin{aligned}
\operatorname{RADA} 1(a, b) & =a^{2}+2 g_{r d} b, \\
\operatorname{RP}(a, b) & =a+\frac{b}{g_{r d}}, \\
\operatorname{AP}(a, b) & =a+b g_{r d}, \\
\mathbf{H P}(a, b, c) & =a+\frac{b^{2}-c^{2}}{2 g_{r d}} .
\end{aligned}
$$

Next, the starting and ending index counters $i z_{s}, i z_{e}$, respectively, for the local angle, range, and height arrays; and the refractivity profile starting index $i_{r p s}$ are initialized to 1 for the first reference to the EXTO SU. The index $i z_{e}$ is then determined such that curng $_{\mathrm{iz}_{e}} \leq r_{\text {out }}<$ curng $_{\mathrm{iz}} e^{+1}$. The integer counter $\mathrm{k}$, indicating the number of propagation factor and heights in array prfhxo, is initialized to 0 .

Ray trace steps 1 through 3 are performed for each ray; i.e., for each $j^{\text {th }}$ angle, range, and height triplet, for $j$ ranging from $i z_{s}$ to $i z_{e}$.

1. At the start of the ray trace, the current local angle $\left(a_{0}\right)$, range $\left(r_{0}\right)$, height $\left(h_{0}\right)$, and refractive gradient index $\left(i_{\text {grad }}\right)$ are initialized to curang $_{j}, c_{\text {curng }}$, curht $t_{j}$, and igrd $_{j}$, respectively. Next, refractive profile index, $i_{r p}$, is initialized to the maximum of $j$ or $i_{r p s}$. Finally, the refractivity gradient, $g_{r d}$ is set equal to the gradient at the $i_{\text {grad }}$ th level of the $i_{r p}{ }^{\text {th }}$ profile, $\operatorname{grad}_{i_{\text {grad }}, i_{r p}}$. Ray trace steps 1.a through 1.d are then performed until the current local range $r_{0}$ becomes greater than or equal to $r_{\text {out }}$.

a. The ending range, $r_{1}$, in the ray trace segment is set equal to the minimum of $f f a c i_{i p p}+1,2$ or $r_{\text {out }}$. If $i_{r p}$ is equal to the number of stored triplets, $i z$, then $r_{1}$ is set equal to $r_{\text {out }}$.

b. The $j^{\text {th }}$ ray is then traced to $r_{1}$ and the resulting angle and height at the end of the segment is determined via the in-line functions as

$$
\begin{aligned}
& a_{1}=\operatorname{AP}\left(a_{0}, r_{1}-r_{0}\right), \\
& h_{1}=\operatorname{HP}\left(h_{0}, a_{1}, a_{0}\right) .
\end{aligned}
$$


c. The ending height $h_{1}$ is then compared to the next height level in the current refractivity profile, $h t r_{\text {grad }^{+1, i} i_{r p}}$, and if $h_{1}$ is greater than this height level, it is set equal to $h t r_{i_{\text {grad }}+1, i_{r p}}$ and a new $a_{1}$ and $r_{1}$ are computed from

$$
\begin{aligned}
& a_{1}=\sqrt{\operatorname{RADA} 1\left(a_{0}, h_{1}-h_{0}\right)} . \\
& r_{1}=\operatorname{RP}\left(r_{0}, a_{1}-a_{0}\right)
\end{aligned} .
$$

$i_{\text {grad }}$ is then set to the minimum of $i_{\text {grad }}+1$ or $l v l_{i r p}-1$.

d. The starting angle, range, and height for the next ray trace segment is updated, and if necessary, the refractivity profile index $i_{r p}$ is updated to the minimum of $i_{r p}+1$ or $i z_{e}$. Steps 1.a through 1.d are then repeated for the next ray segment.

2. Once the ray has been traced to a range of $r_{\text {out }}$ or greater, the current angle, range, and height arrays, curang, curng, and curht, respectively, are updated to the values for $a_{0}$, $r_{0}$, and $h_{0}$ for subsequent references to the EXTO SU.

3. The counter $k$ for the propagation factor and height array is incremented by one and the array is updated according to

$$
\begin{aligned}
& \operatorname{prfhxo}_{k, 1}=f f a c z_{j, 1}, \\
& \operatorname{prfhxo}_{k, 2}=h_{0} .
\end{aligned}
$$

Once all rays have been traced, the starting profile index $i_{r p s}$ is updated to $i z_{e}$ for the next reference to the EXTO SU, and the counter $k$ is again incremented by one and the last values of prfhxo updated as follows,

$$
\begin{aligned}
\text { prfhxo }_{k, 1} & =\text { ffrout }_{i_{s p}, 1}, \\
\text { prfhxo }_{k, 2} & =\text { ffrout }_{i_{s p}, 2} .
\end{aligned}
$$

The number of traced XO height points, $n_{x o}$, at the current output range is then set to $k$. Note that at this point, all output heights in $\operatorname{prfhxo}_{0: n_{x 0^{2}}}$ are decreasing from $p r f h x o_{1,2}$ to prfhxo $_{n_{X 0^{2}}}$ and all traced heights in curht are decreasing from curht $t_{i_{S}}$ to curht $_{i_{z_{e}}}$.

The starting index $i z_{s}$ is then adjusted [for the next reference to the EXTO SU] if the topmost traced height ${ } u r h t_{i_{S}}$ is greater than $h t_{l i m}$. If performing a terrain case, the

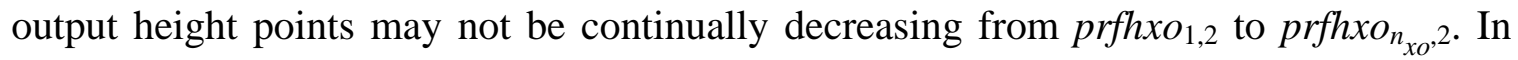
this case, prfhxo is sorted such that all height values are steadily decreasing. The ending index, $j_{x e}$, at which XO propagation factor and loss values will be calculated and stored 
in $m p f l$, is set equal to $n_{\text {zout }}$ and adjusted, if necessary, such that zout $_{j x}$ is less than $\operatorname{prfhxo}_{1,2}$. Now, the counter index $i x$ is initialized to $n_{x o}$. Next, the propagation factor is determined via linear interpolation on the values in prfhxo. Steps 1 through 2 are performed for each output height point zout $t_{j}$ for $j$ varying from $j_{x s}$ to $j_{x e}$.

1. The counter $i x$ is adjusted (if necessary) such that $\operatorname{prfhxo}_{i x, 2} \leq$ zout $_{j}<$ prfhxo $_{i x-1,2}$.

2. The propagation factor $\left(F_{d B}\right)$, propagation loss (rloss), and propagation angle (propaf $f_{1, j}$ ) at height zout $t_{j}$ are then calculated according to

$$
\begin{aligned}
& \text { frac }=\frac{\text { zout }_{j}-\text { prfhxo }}{{\text { prfh } x o_{1 x-1,2}}_{1}-\text { prfhxo }_{1 x, 2}}, \\
& F_{d B}=\text { FN_PLIN }\left(p r f h x o_{x, 1}, p r f h x o_{1 x-1,1}, \text { frac }\right) \text {, } \\
& \text { rloss }_{j}=f s l_{i_{s p}}-F_{d B} \text {, } \\
& \text { propa } f_{1, j}=\text { FN_PLIN }\left\{p r f h x a_{1,3}, p r f h x a_{1 x-1,3} \text {, frac }\right) \text {. }
\end{aligned}
$$

Once all propagation loss values have been computed, the TROPOSCAT SU is referenced to compute troposcatter loss, if necessary. Finally, the loss due to gasesous absorption is added to rloss and then converted to centibels and stored in mpfl before exiting.

Table 132 and Table 133 provide identification, description, units of measure, and the computational source for each EXTO SU input and output data element. Table 134 identifies terms that are used internal to the EXTO SU and whose value must be retained from SU call to SU call for reasons of computational efficiency. 
Table 132. EXTO SU input data element requirements.

\begin{tabular}{|c|c|c|c|}
\hline Name & Description & Units & Source \\
\hline curang & $\begin{array}{l}\text { Array of current local angles for each ray being traced in XO } \\
\text { region }\end{array}$ & radians & $\begin{array}{l}\text { EXTO SU } \\
\text { XOINIT CSC }\end{array}$ \\
\hline curht & $\begin{array}{l}\text { Array of current local heights for each ray being traced in } \mathrm{XO} \\
\text { region }\end{array}$ & meters & $\begin{array}{l}\text { EXTO SU } \\
\text { XOINIT CSC }\end{array}$ \\
\hline curng & $\begin{array}{l}\text { Array of current local ranges for each ray being traced in XO } \\
\text { region }\end{array}$ & meters & $\begin{array}{l}\text { EXTO SU } \\
\text { XOINIT CSC }\end{array}$ \\
\hline ffacz & $\begin{array}{l}\text { Array containing propagation factor, range, and propagation } \\
\text { angle at } Z_{\text {lim }}\end{array}$ & $\begin{array}{l}\mathrm{dB}, \\
\text { meters, } \\
\text { radians }\end{array}$ & FZLIM SU \\
\hline ffrout & $\begin{array}{l}\text { Array of propagation factors at each output range beyond } r_{a t z} \\
\text { and at height } z_{\text {lim }}\end{array}$ & $\mathrm{dB}$ & CALCLOS SU \\
\hline fsl & Free space loss array for output ranges & $\mathrm{dB}$ & APMINIT CSC \\
\hline$f_{\text {ter }}$ & $\begin{array}{l}\text { Logical flag indicating if terrain profile has been specified: } \\
\text {.true. = terrain profile specified } \\
\text {.false. = terrain profile not specified }\end{array}$ & N/A & APMINIT CSC \\
\hline gas $_{\text {loss }}$ & Gaseous absorption loss at range $r_{\text {out }}$ & $\mathrm{dB}$ & APMSTEP CSC \\
\hline grad & $\begin{array}{l}\text { Two-dimensional array containing gradients of each profile } \\
\text { used in XO calculations }\end{array}$ & $\begin{array}{l}\text { M-units } \\
\text { /meter }\end{array}$ & SAVEPRO SU \\
\hline hlim & $\begin{array}{l}\text { Array containing height at each output range separating the } \\
\text { RO region from the PE (at close ranges) and XO (at far } \\
\text { ranges) regions }\end{array}$ & meters & GETTHMAX SU \\
\hline$h t_{\text {lim }}$ & Maximum height relative to $h_{\text {minter }}$ & meters & TERINIT SU \\
\hline htr & $\begin{array}{l}\text { Two-dimensional array containing heights of each profile } \\
\text { used in XO calculations }\end{array}$ & meters & SAVEPRO SU \\
\hline igrd & $\begin{array}{l}\text { Integer indexes indicating at what refractive gradient level to } \\
\text { begin ray tracing for next XO range step for each ray in XO } \\
\text { region. }\end{array}$ & N/A & XOINIT CSC \\
\hline$i_{\text {ratz }}$ & $\begin{array}{l}\text { Index of output range step in which to begin storing } \\
\text { propagation factor and outgoing angle for XO region }\end{array}$ & N/A & APMINIT CSC \\
\hline$i_{s t p}$ & Current output range step index & N/A & Calling SU \\
\hline$T_{\text {ropo }}$ & $\begin{array}{l}\text { Troposcatter calculation flag: } \\
\text { '.false.'= no troposcatter calcs } \\
\text { '.true.' = troposcatter calcs }\end{array}$ & N/A & Calling CSCI \\
\hline$i z$ & $\begin{array}{l}\text { Number of propagation factor, range, angle triplets stored in } \\
\text { ffacz }\end{array}$ & N/A & FZLIM SU \\
\hline$j_{x s}$ & Index at which valid loss values in $m p f l$ start & N/A & Calling SU \\
\hline$l v l$ & $\begin{array}{l}\text { Number of height levels in each profile used in } \mathrm{XO} \\
\text { calculations }\end{array}$ & N/A & SAVEPRO SU \\
\hline$n_{\text {zout }}$ & Integer number of output height points desired & N/A & Calling CSCI \\
\hline$r_{\text {out }}$ & Current output range & meters & Calling SU \\
\hline zout & $\begin{array}{l}\text { Array containing all desired output heights referenced to } \\
h_{\text {minter }}\end{array}$ & meters & APMINIT CSC \\
\hline ZXO & Height of the ground at the current output range step & meters & CALCLOS SU \\
\hline
\end{tabular}


Table 133. EXTO SU output data element requirements.

\begin{tabular}{|c|c|c|}
\hline Name & Description & Units \\
\hline curang & Array of current local angles for each ray being traced in XO region & radians \\
\hline curht & Array of current local heights for each ray being traced in $\mathrm{XO}$ region & meters \\
\hline curng & Array of current local ranges for each ray being traced in XO region & meters \\
\hline hlim & $\begin{array}{l}\text { Array containing height at each output range separating the RO } \\
\text { region from the PE (at close ranges) and XO (at far ranges) regions }\end{array}$ & meters \\
\hline igrd & $\begin{array}{l}\text { Integer indexes indicating at what refractive gradient level to begin } \\
\text { ray tracing for next XO range step for each ray in XO region }\end{array}$ & N/A \\
\hline$j_{x e}$ & Index at which valid loss values in $m p f l$ end & N/A \\
\hline$m p f l$ & Two-dimensional propagation factor and loss array & cB \\
\hline prfhxo & $\begin{array}{l}\text { Two-dimensional array of propagation factor and heights for each } \\
\text { ray traced in XO region to range } r_{\text {out }}\end{array}$ & dB,meters \\
\hline propaf & $\begin{array}{l}\text { Two-dimensional array, containing the propagation angles and } \\
\text { factors for the direct and reflected rays (where applicable) for all } \\
\text { output height/range points }\end{array}$ & radians, $\mathrm{N} / \mathrm{A}$ \\
\hline rloss & Propagation loss & $\mathrm{dB}$ \\
\hline
\end{tabular}

Table 134. EXTO SU save data element requirements.

\begin{tabular}{c|l|c}
\hline Name & \multicolumn{1}{|c|}{ Description } & Units \\
\hline$i_{r p s}$ & Starting index counter for refractivity profiles & N/A \\
$i z_{e}$ & Ending index in curang, curng, and curht to trace to $r_{\text {out }}$ & N/A \\
$i z_{s}$ & Starting index in curang, curng, and curht to trace to $r_{\text {out }}$ & N/A \\
\hline
\end{tabular}

\subsection{Return Grazing Angle (RET_GRAZE) CSC}

The RET_GRAZE CSC interpolates grazing angles to every output range step $r_{\text {out }}$, and if propagation angles are desired everywhere (lang = '.true.'), the SU will also interpolate the propagation angles in height at every output range.

Upon entering the SU, if lang = '.true.', the two-dimensional array $\Theta_{\text {rout, }}$ is allocated and initialized. $\Theta_{\text {rout }}$ will contain all the interpolated propagation angles at each output height and every output range.

For every output range rngout $_{i}$ where $i$ varies from 1 to $n_{\text {rout }}$, steps 1 through 2 are performed: 
1. The grazing angle at the current output range is determined by referencing FN_PLINT:

$$
\begin{gathered}
\Psi_{\text {rout }_{i}}=\text { FN_PLINT }\left(\Psi_{i s}, \Psi_{\text {isp } 1}, x\right), \\
\text { is }=\operatorname{INT}\left(\frac{r n g o u t_{i}}{\Delta r_{P E}}\right), \\
\text { isp } 1=\operatorname{MIN}\left(i s+1, i_{P E}\right), \\
x=\frac{\text { rngout }_{i}-i s \Delta r_{\text {grz }}}{\Delta r_{\text {grz }}} .
\end{gathered}
$$

where

If lang = '.true.', then the SU continues with step 2; otherwise, step 1 is repeated for all values of $i$ to $n_{\text {rout }}$.

2. Parameters in calculating all propagation angles at the current output range are determined according to

$$
\begin{aligned}
& i p=\operatorname{INT}\left(\frac{r n g o u t_{i}}{\Delta r_{\text {grz }}}\right), \\
& i p p 1=\operatorname{MIN}\left(i p+1, i_{g P E}\right), \\
& x=\frac{\text { rngout }_{i}-i p \Delta r_{\text {grz }}}{\Delta r_{\text {grz }}} .
\end{aligned}
$$

The propagation angles are then determined for all values of in from 1 to $n_{\text {ang }}$ by referencing FN_PLINT:

$$
\Theta_{\text {rout }_{i n, i}}=\text { FN_PLINT }\left(\Theta_{p_{i n, i p}}, \Theta_{p_{i n, i p p 1}}, x\right) .
$$

If the current value of $\Theta_{p}$ lies outside the PE calculation region (value = -999), $i$ is incremented and step 2 is repeated for the next output range.

Finally, array $\Theta_{p}$ is deallocated before exiting.

Table 135 and Table 136 provide identification, description, units of measure, and the computational source for each RET_GRAZE CSC input and output data element. 
Table 135. RET_GRAZE CSC input data element requirements.

\begin{tabular}{|c|c|c|c|}
\hline Name & Description & Units & Source \\
\hline$\Delta r_{g r z}$ & PE range step used for calculation of grazing angles & meters & APMINIT CSC \\
\hline$\Delta r_{P E}$ & PE range step & meters & PEINIT SU \\
\hline$i_{g P E}$ & Number of grazing angles computed from spectral estimation & N/A & GETANGLES SU \\
\hline$i_{P E}$ & Number of PE range steps & N/A & PEINIT SU \\
\hline lang & $\begin{array}{l}\text { Propagation angle and factor output flag } \\
\text { '.true.'= Output propagation angle and propagation } \\
\text { factor for direct and reflected ray (where applicable). } \\
\text { '.false.' = Do not output propagation angles and factors }\end{array}$ & N/A & Calling CSCI \\
\hline$n_{\text {ang }}$ & $\begin{array}{l}\text { Number of points in the vertical at which to spectrally } \\
\text { estimate propagation angles. }\end{array}$ & N/A & GETANGLES SU \\
\hline$n_{\text {rout }}$ & Integer number of output range points desired & N/A & Calling CSCI \\
\hline rngout & Array containing all desired output ranges & meters & APMINIT CSC \\
\hline$\Psi$ & Array of interpolated grazing angles at each PE range step & radians & GRAZE_INT SU \\
\hline$\Theta_{p}$ & $\begin{array}{l}\text { Two-dimensional array containing the propagation angle } \\
\text { estimated from PE at } n_{\text {ang }} \text { height points and at every PE } \\
\text { calculation range step during the initialization routine. } \\
\text { Format is } \Theta_{p}(i, j)=\text { propagation angle at the } i^{\text {th }} \text { height point } \\
\left(i=1 \text { to } n_{a n g}\right) \text { and } j^{\text {th }} \text { PE range step }\left(j=0 \text { to } i_{g P E}\right) \text {. }\end{array}$ & radians & GETANGLES SU \\
\hline
\end{tabular}

Table 136. RET_GRAZE SU output data element requirements.

\begin{tabular}{c|l|c}
\hline Name & \multicolumn{1}{|c}{ Description } & Units \\
\hline$\Psi_{\text {rout }}$ & Array of grazing angles at each output range $r_{\text {out }}$ & radians \\
$\Theta_{\text {rout }}$ & $\begin{array}{l}\text { Two-dimensional array containing the propagation angle spectrally estimated } \\
\text { from PE at } n_{\text {ang }} \text { height points and at every output range step } r_{\text {out }}\end{array}$ & radians \\
$i_{\text {error }}$ & Integer value that is returned if an error occurs in called routine & N/A \\
\hline
\end{tabular}

\section{REQUIREMENTS TRACEABILITY}

This section provides the traceability of the design of the APM CSCI Table 137 presents this traceability between the corresponding sections of the Software Requirements Specification (SRS) and the Software Design Description (SDD) and between the various components of the APM CSCI. 
Table 137. Traceability Matrix between the SRS and the SDD.

\begin{tabular}{|c|c|c|c|}
\hline \multicolumn{2}{|c|}{ Software Requirements Specification } & \multicolumn{2}{|c|}{ Software Design Description } \\
\hline SRS Requirement Name & $\begin{array}{c}\text { SRS } \\
\text { Paragraph } \\
\text { Number }\end{array}$ & Software Design Description Name & $\begin{array}{c}\text { SDD } \\
\text { Paragraph } \\
\text { Number }\end{array}$ \\
\hline CSCI Capability Requirements & 3.1 & CSCI-WIDE DESIGN DECISIONS & 3. \\
\hline CSCI Capability Requirements & 3.1 & CSCI Components & 4.1 \\
\hline CSCI Capability Requirements & 3.1 & Concept of Execution & 4.2 \\
\hline $\begin{array}{l}\text { Advance Propagation Initialization } \\
\text { (APMINIT) CSC }\end{array}$ & 3.1 .1 & $\begin{array}{l}\text { Advance Propagation Initialization } \\
\text { (APMINIT) CSC }\end{array}$ & 5.1 \\
\hline $\begin{array}{l}\text { Allocate Arrays APM } \\
\text { (ALLARRAY_APM) SU }\end{array}$ & 3.1.1.1 & $\begin{array}{l}\text { Allocate Arrays APM } \\
\text { (ALLARRAY_APM) SU }\end{array}$ & 5.1 .1 \\
\hline $\begin{array}{l}\text { Allocate Array PE } \\
\text { (ALLARRAY_PE) SU }\end{array}$ & 3.1.1.2 & $\begin{array}{l}\text { Allocate Array PE } \\
\text { (ALLARRAY_PE) SU }\end{array}$ & 5.1 .2 \\
\hline $\begin{array}{l}\text { Allocate Array RO } \\
\text { (ALLARRAY_RO) SU }\end{array}$ & 3.1.1.3 & $\begin{array}{l}\text { Allocate Array RO } \\
\text { (ALLARRAY_RO) SU }\end{array}$ & 5.1 .3 \\
\hline $\begin{array}{l}\text { Allocate Array } \\
\text { (ALLARRAY_XORUF) SU }\end{array}$ & 3.1.1.4 & $\begin{array}{l}\text { Allocate Array } \\
\text { (ALLARRAY_XORUF) SU }\end{array}$ & 5.1 .4 \\
\hline $\begin{array}{l}\text { Alpha Impedance Initialization } \\
\text { (ALN_INIT) SU }\end{array}$ & 3.1.1.5 & $\begin{array}{l}\text { Alpha Impedance Initialization } \\
\text { (ALN_INIT) SU }\end{array}$ & 5.1 .5 \\
\hline Antenna Pattern (ANTPAT) SU & 3.1.1.6 & Antenna Pattern (ANTPAT) SU & 5.1 .6 \\
\hline APM Status (APMSTATUS) SU & 3.1.1.7 & APM Status (APMSTATUS) SU & 5.1 .7 \\
\hline $\begin{array}{l}\text { Dielectric Initialization (DIEINIT) } \\
\text { SU }\end{array}$ & 3.1.1.8 & $\begin{array}{l}\text { Dielectric Initialization (DIEINIT) } \\
\text { SU }\end{array}$ & 5.1 .8 \\
\hline FFT Parameters (FFTPAR) SU & 3.1.1.9 & FFT Parameters (FFTPAR) SU & 5.1 .9 \\
\hline Fill Height Arrays (FILLHT) SU & 3.1.1.10 & Fill Height Arrays (FILLHT) SU & 5.1 .10 \\
\hline $\begin{array}{l}\text { Gaseous Absorption (GASABS) } \\
\text { SU }\end{array}$ & 3.1.1.11 & $\begin{array}{l}\text { Gaseous Absorption (GASABS) } \\
\text { SU }\end{array}$ & 5.1 .11 \\
\hline $\begin{array}{l}\text { Get Effective Earth Radius Factor } \\
\text { (GET_K) SU }\end{array}$ & 3.1.1.12 & $\begin{array}{l}\text { Get Effective Earth Radius Factor } \\
(\text { GET_K) SU }\end{array}$ & 5.1 .12 \\
\hline $\begin{array}{l}\text { Get Alpha Impedance (GETALN) } \\
\text { SU }\end{array}$ & 3.1.1.13 & $\begin{array}{l}\text { Get Alpha Impedance (GETALN) } \\
\text { SU }\end{array}$ & 5.1 .13 \\
\hline Get Angles (GETANGLES) SU & 3.1.1.14 & Get Angles (GETANGLES) SU & 5.1 .14 \\
\hline $\begin{array}{l}\text { Get Maximum Angle (GETTHMAX) } \\
\text { SU }\end{array}$ & 3.1.1.15 & $\begin{array}{l}\text { Get Maximum Angle (GETTHMAX) } \\
\text { SU }\end{array}$ & 5.1 .15 \\
\hline $\begin{array}{l}\text { Grazing Angle Interpolation } \\
\text { (GRAZE_INT) SU }\end{array}$ & 3.1.1.16 & $\begin{array}{l}\text { Grazing Angle Interpolation } \\
\text { (GRAZE_INT) SU }\end{array}$ & 5.1 .16 \\
\hline Height Check (HTCHECK) SU & 3.1.1.17 & Height Check (HTCHECK) SU & 5.1 .17 \\
\hline
\end{tabular}


Table 137. Traceability Matrix between the SRS and the SDD. (continued)

\begin{tabular}{|c|c|c|c|}
\hline \multicolumn{2}{|c|}{ Software Requirements Specification } & \multicolumn{2}{|c|}{ Software Design Description } \\
\hline SRS Requirement Name & $\begin{array}{c}\text { SRS } \\
\text { Paragraph } \\
\text { Number }\end{array}$ & $\begin{array}{c}\text { Software Design Description } \\
\text { Name }\end{array}$ & $\begin{array}{c}\text { SDD } \\
\text { Paragraph } \\
\text { Number }\end{array}$ \\
\hline $\begin{array}{l}\text { Interpolate Profile (INTPROF) } \\
\text { SU }\end{array}$ & 3.1.1.18 & $\begin{array}{l}\text { Interpolate Profile (INTPROF) } \\
\text { SU }\end{array}$ & 5.1 .18 \\
\hline PE Initialization (PEINIT) SU & 3.1.1.19 & PE Initialization (PEINIT) SU & 5.1 .19 \\
\hline Poly 4 (FN_POLY4) Function & 3.1.1.20 & Poly 4 (FN_POLY4) Function & 5.1 .20 \\
\hline Poly 5 (FN_POLY5) Function & 3.1.1.21 & Poly 5 (FN_POLY5) Function & 5.1 .21 \\
\hline $\begin{array}{l}\text { Profile Reference (PROFREF) } \\
\text { SU }\end{array}$ & 3.1.1.22 & $\begin{array}{l}\text { Profile Reference (PROFREF) } \\
\text { SU }\end{array}$ & 5.1 .22 \\
\hline $\begin{array}{l}\text { Refractivity Initialization (RefInit) } \\
\text { SU }\end{array}$ & 3.1.1.23 & $\begin{array}{l}\text { Refractivity Initialization } \\
\text { (REFINIT) SU }\end{array}$ & 5.1 .23 \\
\hline $\begin{array}{l}\text { Remove Duplicate Refractivity } \\
\text { Levels (REMDUP) SU }\end{array}$ & 3.1.1.24 & $\begin{array}{l}\text { Remove Duplicate Refractivity } \\
\text { Levels (REMDUP) SU }\end{array}$ & 5.1 .24 \\
\hline RG Trace (RGTRACE) SU & 3.1.1.25 & RG Trace (RGTRACE) SU & 5.1 .25 \\
\hline $\begin{array}{l}\text { Terrain Initialization (TERINIT) } \\
\text { SU }\end{array}$ & 3.1.1.26 & $\begin{array}{l}\text { Terrain Initialization (TERINIT) } \\
\text { SU }\end{array}$ & 5.1 .26 \\
\hline $\begin{array}{l}\text { Trace to Output Range } \\
\text { (TRACE_ROUT) SU }\end{array}$ & 3.1.1.27 & $\begin{array}{l}\text { Trace to Output Range } \\
\text { (TRACE_ROUT) SU }\end{array}$ & 5.1 .27 \\
\hline $\begin{array}{l}\text { Trace to Next Step } \\
\text { (TRACE_STEP) SU }\end{array}$ & 3.1.1.28 & $\begin{array}{l}\text { Trace to Next Step } \\
\text { (TRACE_STEP) SU }\end{array}$ & 5.1 .28 \\
\hline $\begin{array}{l}\text { Troposcatter Initialization } \\
\text { (TROPOINIT) SU }\end{array}$ & 3.1.1.29 & $\begin{array}{l}\text { Troposcatter Initialization } \\
\text { (TROPOINIT) SU }\end{array}$ & 5.1 .29 \\
\hline $\begin{array}{l}\text { Starter Field Initialization } \\
(\text { XYINIT) SU }\end{array}$ & 3.1.1.30 & $\begin{array}{l}\text { Starter Field Initialization } \\
\text { (XYINIT) SU }\end{array}$ & 5.1 .30 \\
\hline $\begin{array}{l}\text { Advance Propagation Model } \\
\text { Step (APMSTEP) CSC }\end{array}$ & 3.1 .2 & $\begin{array}{l}\text { Advance Propagation Model } \\
\text { Step (APMSTEP) CSC }\end{array}$ & 5.2 \\
\hline $\begin{array}{l}\text { Calculate Propagation Loss } \\
\text { (CALCLOS) SU }\end{array}$ & 3.1.2.1 & $\begin{array}{l}\text { Calculate Propagation Loss } \\
\text { (CALCLOS) SU }\end{array}$ & 5.2 .1 \\
\hline $\begin{array}{l}\text { Current Wind (FN_CURWIND) } \\
\text { Function }\end{array}$ & 3.1.2.2 & $\begin{array}{l}\text { Current Wind (FN_CURWIND) } \\
\text { Function }\end{array}$ & 5.2 .2 \\
\hline $\begin{array}{l}\text { Dielectric Constant } \\
\text { (FN_DIECON) Function }\end{array}$ & 3.1.2.3 & $\begin{array}{l}\text { Dielectric Constant } \\
\text { (FN_DIECON) Function }\end{array}$ & 5.2 .3 \\
\hline DOSHIFT SU & 3.1.2.4 & DOSHIFT SU & 5.2 .4 \\
\hline $\begin{array}{l}\text { Discrete Sine/Cosine Fast } \\
\text { Fourier Transform (DRST) SU }\end{array}$ & 3.1.2.5 & $\begin{array}{l}\text { Discrete Sine/Cosine Fast } \\
\text { Fourier Transform (DRST) SU }\end{array}$ & 5.2 .5 \\
\hline
\end{tabular}


Table 137. Traceability Matrix between the SRS and the SDD. (continued)

\begin{tabular}{|c|c|c|c|}
\hline \multicolumn{2}{|c|}{ Software Requirements Specification } & \multicolumn{2}{|c|}{ Software Design Description } \\
\hline SRS Requirement Name & $\begin{array}{c}\text { SRS } \\
\text { Paragraph } \\
\text { Number }\end{array}$ & $\begin{array}{c}\text { Software Design Description } \\
\text { Name }\end{array}$ & $\begin{array}{c}\text { SDD } \\
\text { Paragraph } \\
\text { Number }\end{array}$ \\
\hline Flat Earth Direct Ray (FEDR) SU & 3.1.2.6 & $\begin{array}{l}\text { Flat Earth Direct Ray (FEDR) } \\
\text { SU }\end{array}$ & 5.2 .6 \\
\hline Flat Earth Model (FEM) SU & 3.1.2.7 & Flat Earth Model (FEM) SU & 5.2 .7 \\
\hline Fast Fourier Transform (FFT) SU & 3.1.2.8 & $\begin{array}{l}\text { Fast-Fourier Transform (FFT) } \\
\text { SU }\end{array}$ & 5.2 .8 \\
\hline $\begin{array}{l}\text { Free Space Range Step } \\
\text { (FRSTP) SU }\end{array}$ & 3.1.2.9 & $\begin{array}{l}\text { Free Space Range Step } \\
\text { (FRSTP) SU }\end{array}$ & 5.2 .9 \\
\hline FZLIM SU & 3.1.2.10 & FZLIM SU & 5.2 .10 \\
\hline $\begin{array}{l}\text { Get Propagation Factor } \\
\text { (FN_GETPFAC) Function }\end{array}$ & 3.1.2.11 & $\begin{array}{l}\text { Get Propagation Factor } \\
\text { (FN_GETPFAC) Function }\end{array}$ & 5.2 .11 \\
\hline $\begin{array}{l}\text { Get Reflection Coefficient } \\
\text { (GETREFCOEF) SU }\end{array}$ & 3.1.2.12 & $\begin{array}{l}\text { Get Reflection Coefficient } \\
\text { (GETREFCOEF) SU }\end{array}$ & 5.2 .12 \\
\hline $\begin{array}{l}\text { Get Troposcatter Loss } \\
\text { (FN_GET_TLOSS) Function }\end{array}$ & 3.1.2.13 & $\begin{array}{l}\text { Get Troposcatter Loss } \\
\text { (FN_GET_TLOSS) Function }\end{array}$ & 5.2 .13 \\
\hline $\begin{array}{l}\text { Linear Interpolation (FN_PLINT) } \\
\text { Function }\end{array}$ & 3.1.2.14 & $\begin{array}{l}\text { Linear Interpolation (FN_PLINT) } \\
\text { Function }\end{array}$ & 5.2 .14 \\
\hline $\begin{array}{l}\text { Mixed Fourier Transform } \\
\text { (MIXEDFT) SU }\end{array}$ & 3.1.2.15 & $\begin{array}{l}\text { Mixed Fourier Transform } \\
\text { (MIXEDFT) SU }\end{array}$ & 5.2 .15 \\
\hline $\begin{array}{l}\text { Parabolic Equation Step } \\
\text { (PESTEP) SU }\end{array}$ & 3.1.2.16 & $\begin{array}{l}\text { Parabolic Equation Step } \\
\text { (PESTEP) SU }\end{array}$ & 5.2 .16 \\
\hline Ray Trace (RAYTRACE) SU & 3.1.2.17 & Ray Trace (RAYTRACE) SU & 5.2 .17 \\
\hline $\begin{array}{l}\text { Refractivity Interpolation } \\
\text { (REFINTER) SU }\end{array}$ & 3.1.2.18 & $\begin{array}{l}\text { Refractivity Interpolation } \\
\text { (REFINTER) SU }\end{array}$ & 5.2 .18 \\
\hline $\begin{array}{l}\text { Ray Optics Calculation } \\
\text { (ROCALC) SU }\end{array}$ & 3.1.2.19 & $\begin{array}{l}\text { Ray Optics Calculation } \\
\text { (ROCALC) SU }\end{array}$ & 5.2 .19 \\
\hline Ray Optics Loss (ROLOSS) SU & 3.1.2.20 & Ray Optics Loss (ROLOSS) SU & 5.2 .20 \\
\hline Save Profile (SAVEPRO) SU & 3.1.2.21 & Save Profile (SAVEPRO) SU & 5.2 .21 \\
\hline $\begin{array}{l}\text { Spectral Estimation (SPECEST) } \\
\text { SU }\end{array}$ & 3.1.2.22 & $\begin{array}{l}\text { Spectral Estimation (SPECEST) } \\
\text { SU }\end{array}$ & 5.2 .22 \\
\hline $\begin{array}{l}\text { Surface Impedance (SURFIMP) } \\
\text { SU }\end{array}$ & 3.1.2.23 & $\begin{array}{l}\text { Surface Impedance (SURFIMP) } \\
\text { SU }\end{array}$ & 5.2 .23 \\
\hline Troposcatter (TROPOSCAT) SU & 3.1.2.24 & Troposcatter (TROPOSCAT) SU & 5.2 .24 \\
\hline $\begin{array}{l}\text { Extended Optics Initialization } \\
\text { (XOINIT) CSC }\end{array}$ & 3.1 .3 & $\begin{array}{l}\text { Extended Optics Initialization } \\
\text { (XOINIT) CSC }\end{array}$ & 5.3 \\
\hline
\end{tabular}


Table 137. Traceability Matrix between the SRS and the SDD. (continued)

\begin{tabular}{|c|c|c|c|}
\hline \multicolumn{2}{|c|}{ Software Requirements Specification } & \multicolumn{2}{|c|}{ Software Design Description } \\
\hline SRS Requirement Name & $\begin{array}{c}\text { SRS } \\
\text { Paragraph } \\
\text { Number }\end{array}$ & $\begin{array}{c}\text { Software Design Description } \\
\text { Name }\end{array}$ & $\begin{array}{c}\text { SDD } \\
\text { Paragraph } \\
\text { Number }\end{array}$ \\
\hline $\begin{array}{l}\text { Advanced Propagation Model } \\
\text { Clean (APMCLEAN) CSC }\end{array}$ & 3.1.3.1 & $\begin{array}{l}\text { Advanced Propagation Model } \\
\text { Clean (APMCLEAN) CSC }\end{array}$ & 5.3 .1 \\
\hline Clutter-to-Noise (CLUTTER) SU & 3.1.3.2 & Clutter-to-Noise (CLUTTER) SU & 5.3 .2 \\
\hline $\begin{array}{l}\text { Diffraction LoSS (FN_DLOSS) } \\
\text { Function) }\end{array}$ & 3.1.3.3 & $\begin{array}{l}\text { Diffraction Loss (FN_DLOSS) } \\
\text { Function) }\end{array}$ & 5.3 .3 \\
\hline Get Theta (GETTHETA) SU & 3.1.3.4 & Get Theta (GETTHETA) SU & 5.3 .4 \\
\hline GIT Initialization (GIT_INIT) SU & 3.1.3.5 & GIT Initialization (GIT_INIT) SU & 5.3 .5 \\
\hline GofZ (GOFZ) Function & 3.1.3.6 & GofZ (GOFZ) Function & 5.3 .6 \\
\hline Mean Filter (MEANFILT) SU & 3.1.3.7 & Mean Filter (MEANFILT) SU & 5.3 .7 \\
\hline $\begin{array}{l}\text { Optical Region Limit (OPLIMIT) } \\
\text { SU }\end{array}$ & 3.1.3.8 & $\begin{array}{l}\text { Optical Region Limit (OPLIMIT) } \\
\text { SU }\end{array}$ & 5.3 .8 \\
\hline Optical Difference (OPTICF) SU & 3.1.3.9 & Optical Difference (OPTICF) SU & 5.3 .9 \\
\hline R1 Iteration (R1ITER) SU & 3.1.3.10 & R1 Iteration (R1ITER) SU & 5.3 .10 \\
\hline $\begin{array}{l}\text { Standard Propagation Model } \\
\text { Initialization (SPM_INIT) SU }\end{array}$ & 3.1.3.11 & $\begin{array}{l}\text { Standard Propagation Model } \\
\text { Initialization (SPM_INIT) SU }\end{array}$ & 5.3 .11 \\
\hline $\begin{array}{l}\text { Standard Propagation Model } \\
\text { (SPM) SU }\end{array}$ & 3.1.3.12 & $\begin{array}{l}\text { Standard Propagation Model } \\
\text { (SPM) SU }\end{array}$ & 5.3 .12 \\
\hline $\begin{array}{l}\text { Extended Optics Step (XOSTEP) } \\
\text { CSC }\end{array}$ & 3.1 .4 & $\begin{array}{l}\text { Extended Optics Step } \\
\text { (XOSTEP) CSC }\end{array}$ & 5.4 \\
\hline Extended Optics (EXTO) SU & 3.1.4.1 & Extended Optics (EXTO) SU & 5.4 .1 \\
\hline $\begin{array}{l}\text { Return Grazing Angle } \\
\text { (RET_GRAZE) CSC }\end{array}$ & 3.1 .5 & $\begin{array}{l}\text { Return Grazing Angle } \\
\text { (RET_GRAZE) CSC }\end{array}$ & 5.5 \\
\hline $\begin{array}{l}\text { CSCI External Interface } \\
\text { Requirements }\end{array}$ & 3.2 & External Interface & 4.3 .2 \\
\hline $\begin{array}{l}\text { CSCI Internal Interface } \\
\text { Requirements }\end{array}$ & 3.3 & Internal Interface & 4.3 .3 \\
\hline CSCI Internal Data Requirements & 3.4 & Internal Data & 4.3 .4 \\
\hline $\begin{array}{l}\text { Environmental Radio Refractivity } \\
\text { field Data Element }\end{array}$ & 3.5 .1 & $\begin{array}{l}\text { Environmental Radio Refractivity } \\
\text { field Data Element }\end{array}$ & 7.2 \\
\hline Terrain Profile Data Element & 3.5 .2 & Terrain Profile Data Element & 7.3 \\
\hline $\begin{array}{l}\text { Implementation and Application } \\
\text { Considerations }\end{array}$ & 3.10 .1 & $\begin{array}{l}\text { Implementation and Application } \\
\text { Considerations }\end{array}$ & 7.1 \\
\hline
\end{tabular}




\section{NOTES}

\subsection{APM CSCI IMPLEMENTATION AND APPLICATION CONSIDERATIONS}

The calling external CSCI application will determine the employment of the APM CSCI. However, the intensive computational nature of the APM CSCI must be considered when designing an efficient calling application. For this reason, the APM CSCI is designed with flexibility for various hardware suites and computer resource management considerations. This APM CSCI applies only to a coverage and loss diagram application. The following highly recommended guidelines are provided to aid in the design of a coverage or loss diagram application that will most efficiently employ the APM CSCI.

The APM CSCI propagation loss calculations are independent of any target or receiver considerations, therefore, for any EM emitter, one execution of the APM CSCI may be used to create both a coverage diagram and a loss diagram. Since execution time and computer memory allocation should be a consideration when employing this model, it is most efficient and appropriate to execute the APM CSCI for a particular EM system/environmental/terrain combination before executing any application. The output of the APM CSCI would be stored in a file that would be accessed by multiple applications.

For example, the external application operator may desire a coverage diagram for one particular radar system. At the beginning of the coverage diagram application, a check would be made for the existence of a previously created APM CSCI output file appropriate for the EM system, environmental, and terrain conditions. If such a file exists, the propagation loss values would be read from the file and used to create the coverage diagram. If the file does not exist, the APM CSCI would be executed to create one. As the APM CSCI is executing, its output could be routed simultaneously to a graphics display device and a file. This file could then be used in the loss diagram application should the operator also choose it. Two distinct applications, therefore, are achieved with only one execution of the APM CSCI. Additionally, should the operator desire an individual coverage diagram for each of multiple targets, or a single coverage diagram illustrating radar detection of a low-flying missile superimposed on a coverage diagram illustrating his own radar's vulnerability as defined by the missile's ESM receiver, only a single execution of the APM CSCI would be required, thereby saving valuable computer resources.

\subsection{ENVIRONMENTAL RADIO REFRACTIVITY FIELD DATA ELEMENTS}

The radio-refractivity field, i.e. the profiles of M-units versus height, should consist of vertical piece-wise linear profiles specified by couplets of height in meters with respect to mean sea level and modified refractivity (M-units) at multiple arbitrary ranges. All vertical profiles must contain the same number of vertical data points and be specified 
such that each numbered data point corresponds to like-numbered points (i.e., features) in the other profiles. The first numbered data point of each profile must correspond to a height of zero mean sea level and the last numbered data point must correspond to a height such that the modified refractivity for all greater heights is well represented by extrapolation using the two highest profile points specified.

With the inclusion of terrain and allowing the terrain profile to fall below mean sea level, refractivity profiles can also be provided in which the first level is less than 0 (or below mean sea level). For a terrain profile that falls below mean sea level at some point, the assumption is that the minimum height may be less than the first height in any refractivity profile specified. Therefore, an extrapolation flag, $i_{\text {extra }}$, must be specified to indicate how the APM CSCI should extrapolate from the first refractivity level to the minimum height along the terrain profile. Setting $i_{\text {extra }}$ to 0 will cause the APM CSCI to extrapolate to the minimum height using a standard atmosphere gradient; setting $i_{\text {extra }}$ to 1 will cause the APM CSCI to extrapolate to the minimum height using the gradient determined from the first two levels of the refractivity profile.

Within each profile, each numbered data point must correspond to a height greater than or equal to the height of the previous data point. Note that this requirement allows for a profile which contains redundant data points. Note also that all significant features of the refractivity profiles must be specified, even if they are above the maximum output height specified for a particular application of APM.
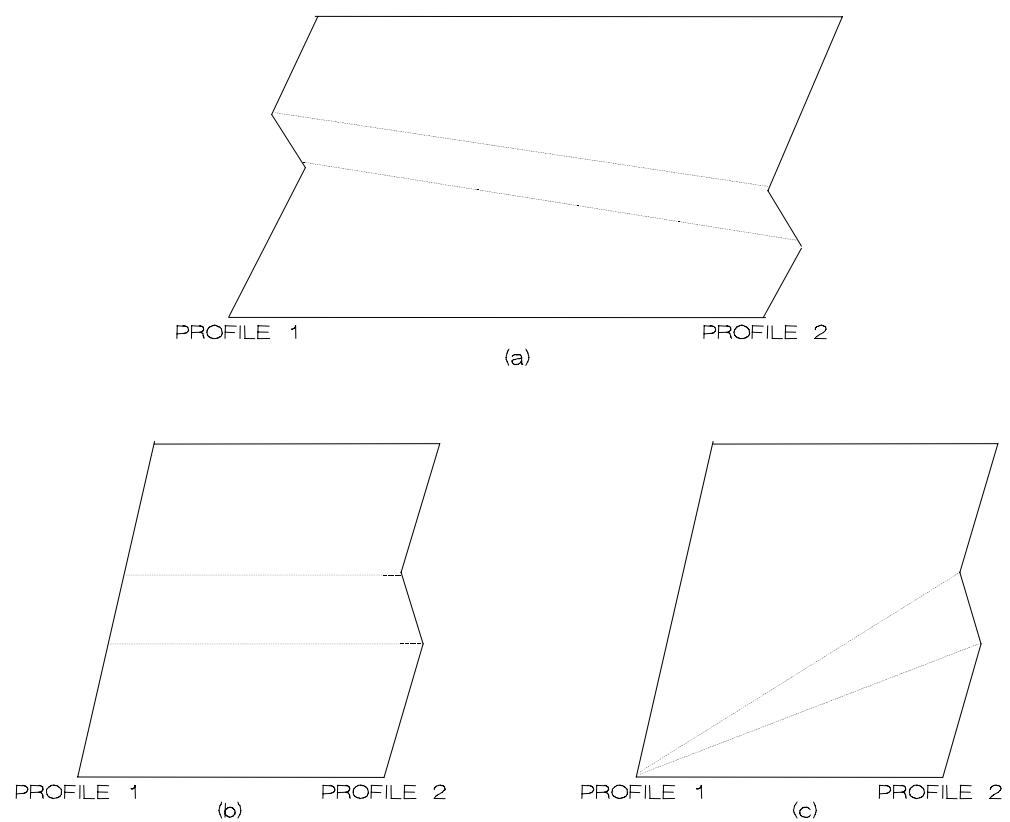

Figure 3. Idealized M-unit profiles (solid) and lines of interpolation (dashed). 
The external CSCI application designer and the external application operator share responsibility for determining appropriate environmental inputs. For example, a loss diagram may be used to consider a surface-to-surface radar detection problem. Since the operator is interested in surface-to-surface, he/she may truncate the profile while assuming that effects from elevated ducting conditions are negligible. It may be, however, that the elevated duct does indeed produce a significant effect. The operator should ensure therefore that the maximum height of the profile allows for the inclusion of all significant refractive features.

This specification allows a complicated refractivity field to be described with a minimum of data points. For example, a field in which a single trapping layer linearly descends with increasing range can be described with just two profiles containing only four data points each, frame (a) of Figure 3. In the same manner, other evolutions of refractive layers may be described. Frames (b) and (c) of Figure 3 show two possible scenarios for the development of a trapping layer. The scenario of choice is the one that is consistent with the true thermodynamical and hydrological layering of the atmosphere.

Two external implementation data variables applicable to both the external application operator and to the calling application designer are $r_{\max }$, the maximum APM CSCI output range, and $h_{\max }$, the maximum APM CSCI output height. These two parameters are required by the APM CSCI to determine the horizontal and vertical resolution, respectively, for internal range and height calculations based on the current values of $n_{\text {rout }}$ and $n_{\text {zout }}$. Any value of $r_{\max }$ and $h_{\max }$ is allowed for the convenience of the external application operator and the calling application designer, provided $r_{\max } \geq 5 \mathrm{~km}$, and $h_{\max } \geq 100 \mathrm{~m}$. For example, the external application operator may desire a coverage diagram that extends to a range of 500 kilometers $(\mathrm{km})$. In addition to accommodating the desires of the operator, specification of such a convenient maximum range eases the burden for the application designer in determining incremental tick marks for the horizontal axis of the display.

Provided the value of the parameter lerr12 is set to '.false.', if the furthest environment profile range is less than $r_{\max }$, the APM CSCI will automatically create an environment profile at $r_{\max }$ equal to the last profile specified, making the environment homogeneous from the range of the last profile specified to $r_{\max }$. For example, a profile is input with an accompanying range of $450 \mathrm{~km}$. If the external application operator chooses an $r_{\max }$ of $500 \mathrm{~km}$, the APM CSCI will continue loss calculations to $500 \mathrm{~km}$, keeping the refractivity environment homogeneous from $450 \mathrm{~km}$ to $500 \mathrm{~km}$.

If lerr12 is set to '.true.' and the furthest environment profile range is less than $r_{\max }$, then an error will be returned in $i_{\text {error }}$ from the APMINIT CSC, which allows the external CSCI application designer greater flexibility in how environment data are handled. 


\subsection{TERRAIN PROFILE DATA ELEMENT}

The terrain profile should consist of linear piecewise segments specified as range/height pairs. All range values must be increasing, and the first terrain height value must be at range zero. General ground composition types can be specified (Table 4), along with corresponding ranges over which the ground type is to be applied. If ground type "User Defined" is specified $\left(\right.$ igrnd $_{i}=7$ ), then numeric values of relative permittivity and conductivity must be given.

The maximum height, $h_{\max }$, must always be greater than the minimum height, $h_{\min }$. Also, a value of $h_{\max }$ must be given such that it is larger than the maximum elevation height along a specified terrain profile.

Provided lerr6 is set to '.false.', if the furthest range point in the terrain profile is less than $r_{\max }$, the APM CSCI will automatically create a height/range pair as part of the terrain profile at $r_{\max }$ with elevation height equal to the last height specified in the profile, making the terrain profile flat from the range of the last profile point specified to $r_{\max }$. For example, a terrain profile is input where the last height/range pair is $50 \mathrm{~m}$ in height with an accompanying range of $95 \mathrm{~km}$. If the external application operator chooses an $r_{\text {max }}$ of $100 \mathrm{~km}$, the APM CSCI will continue loss calculations to $100 \mathrm{~km}$, keeping the terrain profile flat from 95 to $100 \mathrm{~km}$ with an elevation height of $50 \mathrm{~m}$.

If lerr6 is set to '.true.' and the furthest range point is less than $r_{\max }$, then an error will be returned in $i_{\text {error }}$ from the APMINIT SU, which allow the external CSCI application designer greater flexibility in how terrain data are handled.

\subsection{ACRONYM AND ABBREVIATIONS}

Table 138 is a glossary of acronyms and abbreviations used within this document.

Table 138. Acronyms and Abbreviations

\begin{tabular}{c|l}
\hline Term & \\
MIN & Minimum of variables within parenthesis \\
MAX & Maximum of variables within parenthesis \\
APM & Advanced Propagation Model \\
Centibel & One-hundredth of the logarithm of a quantity \\
COMMON BLOCK & $\begin{array}{l}\text { Allows two or more FORTRAN SUs to share variables without having to } \\
\text { pass them as arguments } \\
\text { COS }\end{array}$ \\
CMPLX & Cosine function \\
& Data conversion to complex number
\end{tabular}


Table 138. Acronyms and Abbreviations. (continued)

\begin{tabular}{|c|c|}
\hline Term & Definition \\
\hline $\mathrm{CSCl}$ & Computer software configuration item \\
\hline $\mathrm{dB}$ & Decibel \\
\hline decibel & 10 times the logarithm of a quantity \\
\hline EM & electromagnetic \\
\hline FE & Flat earth \\
\hline FFT & Fast-Fourier Transform \\
\hline FORTRAN & Formula Translation \\
\hline IMAG & Imaginary part of complex number \\
\hline INT & Integer value of \\
\hline $\mathrm{km}$ & Kilometers \\
\hline $\mathrm{LOG}_{10}$ & Logarithm to base 10 \\
\hline LN & Natural logarithm \\
\hline $\mathrm{m}$ & Meters \\
\hline M & Modified refractivity units \\
\hline $\mathrm{MHz}$ & MegaHertz \\
\hline M-unit & Refractivity measurement unit \\
\hline$\mu \mathrm{V} / \mathrm{m}$ & Microvolts per meter \\
\hline N/A & Not applicable \\
\hline NINT & Round real number \\
\hline PE & Parabolic Equation \\
\hline p space & Phase space \\
\hline RADA1 & Angle trace function \\
\hline radian & Unit of angular measurement \\
\hline REAL & Real part of complex number \\
\hline RO & Ray Optics \\
\hline SIGN & Sign transfer function \\
\hline $\operatorname{SIN}$ & Sine function \\
\hline $\mathrm{SIN}^{-1}$ & Inverse sine function \\
\hline $\mathrm{S} / \mathrm{m}$ & Conductivity unit Siemens per meter \\
\hline $\operatorname{Sin}(X) / x$ & Sine $(X) / X$ \\
\hline SRS & Software Requirements Specification \\
\hline SU & Software unit \\
\hline $\mathrm{TAN}^{-1}$ & Inverse tangent function \\
\hline z-space & Height space \\
\hline
\end{tabular}




\subsection{SDD VARIABLE NAME, FORTRAN VARIABLE NAME CROSS REFERENCE}

Table 139 is a cross reference of variable names used within the body of this document and the FORTRAN variable names as used in the APM CSCI source code. Included are the SDD variable name, its description, the FORTRAN source code name, and the designation of the FORTRAN COMMON BLOCK name, if applicable. Note that all dynamically allocated arrays are declared PUBLIC and are common to all SUs containing the APM_MOD module.

Table 139. Variable name cross reference.

\begin{tabular}{|c|c|c|c|}
\hline $\begin{array}{l}\text { SDD } \\
\text { variable } \\
\text { name }\end{array}$ & Description & $\begin{array}{l}\text { FORTRAN } \\
\text { source } \\
\text { code name }\end{array}$ & $\begin{array}{l}\text { FORTRAN } \\
\text { common } \\
\text { block name }\end{array}$ \\
\hline$a_{0}$ & Angle at start of ray trace step & $\mathrm{a} 0$ & N/A \\
\hline$a_{1}$ & Angle at end of ray trace step & a1 & N/A \\
\hline$a_{2}$ & Tangent angle for receiver height $z o u t_{j}$ & ang2 & N/A \\
\hline$a_{a t z}$ & $\begin{array}{l}\text { Local ray or propagation angle at height } z_{\text {lim }} \text { and range } \\
r_{a t z}\end{array}$ & aatz & APM_VAR \\
\hline$a b s_{\text {hum }}$ & Absolute humidity near the surface & abshum & REFRACTIVITY \\
\hline$a_{\text {crit }}$ & Critical angle (angle above which no rays are trapped) & acrit & APM_VAR \\
\hline$a_{\text {cut }}$ & Tangent angle from antenna height to radio horizon & acut & APM_VAR \\
\hline adif & $\begin{array}{l}\text { Height differences between } a n t_{r e f} \text { and all output receiver } \\
\text { heights }\end{array}$ & $\operatorname{adif}()$ & N/A \\
\hline$a_{e k}$ & Effective earth radius & aek & APM_VAR \\
\hline$a_{e k s t}$ & $4 / 3$ effective earth’s radius & aekst & N/A \\
\hline ainc $_{1}$ & $\begin{array}{l}\text { Angular increment for ray tracing to determine grazing } \\
\text { angles }\end{array}$ & ainc1 & N/A \\
\hline $\operatorname{ainc}_{2}$ & $\begin{array}{l}\text { Angular increment for ray tracing to determine grazing } \\
\text { angles }\end{array}$ & ainc2 & N/A \\
\hline $\operatorname{ainc}_{3}$ & $\begin{array}{l}\text { Angular increment for ray tracing to determine grazing } \\
\text { angles }\end{array}$ & ainc3 & N/A \\
\hline$a_{\text {launch }}$ & $\begin{array}{l}\text { Launch angle used which, when traced, separates PE and } \\
\text { XO regions from the RO region }\end{array}$ & alaunch & APM_VAR \\
\hline$\alpha$ & Source elevation angle & ang & N/A \\
\hline$\alpha_{d}$ & Direct-path ray angle & alphad & APM_VAR \\
\hline$\alpha_{\text {dif }}$ & $\begin{array}{l}\text { The difference between current and previous outgoing } \\
\text { propagation angles }\end{array}$ & angdif & N/A \\
\hline$\alpha_{l d}$ & $\begin{array}{l}\text { LOG of antenna pattern factor for } \alpha_{d} \text { where } \alpha_{d} \text { represents } \\
\text { lowest direct ray angle in optical region }\end{array}$ & ald & N/A \\
\hline$\alpha_{l i m}$ & Elevation angle of the RO limiting ray & alflim & N/A \\
\hline
\end{tabular}


Table 139. Variable name cross reference. (continued)

\begin{tabular}{|c|c|c|c|}
\hline $\begin{array}{c}\text { SDD } \\
\text { variable } \\
\text { name }\end{array}$ & Description & $\begin{array}{c}\text { FORTRAN } \\
\text { source } \\
\text { code name }\end{array}$ & $\begin{array}{c}\text { FORTRAN } \\
\text { common } \\
\text { block name }\end{array}$ \\
\hline$\alpha_{p a t}$ & Elevation angle relative to the antenna elevation angle & udif & N/A \\
\hline$\alpha_{r}$ & Reflected-path ray angle & alphar & N/A \\
\hline$\alpha_{t e r}$ & Tangent angle from antenna height to current terrain height & $\begin{array}{l}\text { alphax, } \\
\text { terang }\end{array}$ & N/A \\
\hline$\alpha_{u}$ & $\begin{array}{l}\text { Maximum tangent ray angle from the source to the terrain } \\
\text { peak along profile height }\end{array}$ & angu & N/A \\
\hline$\alpha_{h, v}$ & $\begin{array}{l}\text { Surface impedance term for horizontal or vertical } \\
\text { polarization }\end{array}$ & alphaq & APM_VAR \\
\hline$a_{m \lim }$ & $\begin{array}{l}\text { Elevation angle of RO limiting ray in radians. Used to } \\
\text { initialize launch angle in the GETTHMAX SU }\end{array}$ & amlim & N/A \\
\hline$a_{m x c u r}$ & $\begin{array}{l}\text { Maximum local angle along the traced ray up to } z_{\text {lim }} \text { (with } \\
\text { minimum limit } a_{\text {mlim }} \text { ) }\end{array}$ & amxcur & N/A \\
\hline ant $_{f a c}$ & Antenna pattern parameter (depends on $i_{p a t}$ and $\mu_{b w}$ ) & afac & APM_VAR \\
\hline$a n t_{h t}$ & Transmitting antenna height above local ground & antht & SYSTEMVAR \\
\hline ant $_{\mathrm{ko}}$ & Height-gain value at source & antko & N/A \\
\hline ant $_{\text {ref }}$ & Transmitting antenna height relative to $h_{\text {minter }}$ & antref & APM_VAR \\
\hline$a_{s}$ & Propagation angle for start of ray trace & as & N/A \\
\hline araft & Array of angles after smoothing operation & $\operatorname{araft}()$ & N/A \\
\hline arbef & Array of angles before smoothing operation & arbef() & N/A \\
\hline$a_{\text {start }}$ & Elevation angle at start of ray step & astart & N/A \\
\hline$a_{\text {test }}$ & $\begin{array}{l}\text { Tangent angle used for automatic calculation of maximum } \\
\text { propagation angle. Only used for modes } i_{\text {hybrid }}=0,2 \text {. }\end{array}$ & atest & N/A \\
\hline$\beta$ & Terminal elevation angle & $\mathrm{ab}$ & N/A \\
\hline$\beta_{d}$ & Direct ray terminal elevation angle & betad & N/A \\
\hline$\beta_{r}$ & Reflected ray terminal elevation angle & betar & N/A \\
\hline$c_{a}$ & Wide-angle propagator correction term & cak & N/A \\
\hline$C_{1 x}$ & $\begin{array}{l}\text { Constant used to propagate } c_{k 1} \text { by one range step in central } \\
\text { difference algorithm }\end{array}$ & $\mathrm{c} 1 \mathrm{x}$ & APM_VAR \\
\hline$C_{2 x}$ & $\begin{array}{l}\text { Constant used to propagate } c_{k 2} \text { by one range step in central } \\
\text { difference algorithm }\end{array}$ & c2x & APM_VAR \\
\hline$c k_{1}$ & Coefficient used in central difference form of DMFT & ck1 & APM_VAR \\
\hline$c k_{2}$ & Coefficient used in central difference form of DMFT & ck2 & APM_VAR \\
\hline$C_{\text {lut }}$ & $\begin{array}{l}\text { Logical flag used to indicate if surface clutter calculations } \\
\text { are desired. }\end{array}$ & clut & SYSTEMVAR \\
\hline cmft & Coefficient used in backward difference form of DMFT & $\mathrm{cmft}$ & APM_VAR \\
\hline$c m f t_{x}$ & $\begin{array}{l}\text { Constant used to propagate } \mathrm{cmft} \text { by one range step in } \\
\text { backward difference algorithm }\end{array}$ & cmft_x & APM_VAR \\
\hline CNR & Clutter-to-Noise ratio array & cnr_dB() & N/A \\
\hline
\end{tabular}


Table 139. Variable name cross reference. (continued)

\begin{tabular}{|c|c|c|c|}
\hline $\begin{array}{c}\text { SDD } \\
\text { variable } \\
\text { name }\end{array}$ & Description & $\begin{array}{c}\text { FORTRAN } \\
\text { source } \\
\text { code name }\end{array}$ & $\begin{array}{c}\text { FORTRAN } \\
\text { common } \\
\text { block name }\end{array}$ \\
\hline$c_{o}$ & Speed of light $\left(299.79 \times 10^{-6} \mathrm{~m} / \mathrm{s}\right)$ & $\mathrm{c} 0$ & N/A \\
\hline con & $10^{-6} k_{o}$ & con & APM_VAR \\
\hline$c n_{p 75}$ & Factor used in calculating filtp array & cnp75 & N/A \\
\hline$c t_{1}$ & $\begin{array}{l}\text { Quantity defined in equ. } 124 \text { in EREPS } 3.0 \text { User's Manual } \\
\text { NRaD TD 2648, pp. } 106\end{array}$ & ct1 & N/A \\
\hline$c t_{2}$ & $\begin{array}{l}\text { Quantity defined in equ. } 125 \text { in EREPS } 3.0 \text { User’s Manual } \\
\text { NRaD TD 2648, pp. } 106\end{array}$ & ct2 & N/A \\
\hline curang & $\begin{array}{l}\text { Array of current local angles for each ray being traced in } \mathrm{XO} \\
\text { region }\end{array}$ & curang() & N/A \\
\hline curht & $\begin{array}{l}\text { Array of current local heights for each ray being traced in } \mathrm{XO} \\
\text { region }\end{array}$ & curht() & N/A \\
\hline curng & $\begin{array}{l}\text { Array of current local ranges for each ray being traced in } \mathrm{XO} \\
\text { region }\end{array}$ & curng() & N/A \\
\hline$\Delta F d_{l o}^{2}$ & $\begin{array}{l}\text { Difference in direct ray magnitude along } \Delta x_{R O} \text { below desired } \\
\text { APM output point }\end{array}$ & dfsdlo & N/A \\
\hline$\Delta F d_{h i}^{2}$ & $\begin{array}{l}\text { Difference in direct ray magnitude along } \Delta x_{R O} \text { above desired } \\
\text { APM output point }\end{array}$ & dfsdhi & N/A \\
\hline$\Delta F r_{l o}^{2}$ & $\begin{array}{l}\text { Difference in reflected ray magnitude along } \Delta x_{R O} \text { below } \\
\text { desired APM output point }\end{array}$ & dfsrlo & N/A \\
\hline$\Delta F r_{h i}^{2}$ & $\begin{array}{l}\text { Difference in reflected ray magnitude along } \Delta x_{R O} \text { above } \\
\text { desired APM output point }\end{array}$ & dfsrhi & N/A \\
\hline$\Delta H_{o}$ & $\begin{array}{l}\text { Frequency gain function correction term defined in equ. } 127 \text { in } \\
\text { EREPS 3.0 User's Manual NRaD TD 2648, pp. } 106\end{array}$ & delho & N/A \\
\hline$\Delta \Omega_{h i}$ & $\begin{array}{l}\text { Difference in total phase lag angle along } \Delta x_{R O} \text { above desired } \\
\text { APM output point }\end{array}$ & danghi & N/A \\
\hline$\Delta \Omega_{10}$ & $\begin{array}{l}\text { Difference in total phase lag angle along } \Delta x_{R O} \text { below desired } \\
\text { APM output point }\end{array}$ & danglo & N/A \\
\hline$\Delta p$ & Mesh size in angle- (or p-) space & delp & APM_VAR \\
\hline$\Delta r_{g r z}$ & PE range step used for calculation of grazing angles & drgrz & APM_VAR \\
\hline$\Delta r_{\text {out }}$ & Output range step & drout & APM_VAR \\
\hline$\Delta r_{P E}$ & PE range step & $\mathrm{dr}$ & APM_VAR \\
\hline$\Delta r_{P E 2}$ & $1 / 2 \mathrm{PE}$ range step & $\mathrm{dr} 2$ & APM_VAR \\
\hline$\Delta r_{\text {temp }}$ & Range step for ray tracing & drtemp & N/A \\
\hline$\Delta \theta$ & Angle difference between mesh points in p-space & dtheta & APM_VAR \\
\hline$\Delta x_{R O}$ & $\mathrm{RO}$ range interval & delxRO & APM_VAR \\
\hline$\Delta z_{\text {out }}$ & Output height increment & dzout & APM_VAR \\
\hline$\Delta z_{P E}$ & PE mesh height increment (bin width in z-space) & delz & APM_VAR \\
\hline$d_{1}$ & Range from source to tangent point & $\mathrm{d} 1$ & N/A \\
\hline
\end{tabular}


Table 139. Variable name cross reference. (continued)

\begin{tabular}{|c|c|c|c|}
\hline $\begin{array}{c}\text { SDD } \\
\text { variable } \\
\text { name }\end{array}$ & Description & $\begin{array}{c}\text { FORTRAN } \\
\text { source } \\
\text { code name }\end{array}$ & $\begin{array}{c}\text { FORTRAN } \\
\text { common } \\
\text { block name }\end{array}$ \\
\hline$d_{2}$ & Range from receiver to tangent point & $\mathrm{d} 2$ & N/A \\
\hline$d 2 s$ & $\begin{array}{l}\text { Array of tangent ranges for all output receiver heights over } \\
\text { smooth surface }\end{array}$ & $\mathrm{d} 2 \mathrm{~s}()$ & N/A \\
\hline$d \alpha$ & $1 / 2 \mu_{b w r}$ & dalpha & N/A \\
\hline dielec & $\begin{array}{l}\text { Two-dimensional array containing the relative permittivity } \\
\text { and conductivity; } \text { dielec }_{1, i} \text { and } \text { dielec }_{2, i} \text {, respectively. }\end{array}$ & dielec(,) & N/A \\
\hline$d x d \alpha$ & Derivative of range with respect to elevation angle & dxda & N/A \\
\hline$d x d \alpha_{d}$ & Derivative of range with respect to $\alpha_{d}$ & dxdad & N/A \\
\hline$d x d \alpha_{r}$ & Derivative of range with respect to $\alpha_{r}$ & dxdar & N/A \\
\hline$d z d \alpha_{d}$ & Derivative of height with respect to $\alpha_{d}$ & dzdad & N/A \\
\hline$d z d \alpha_{r}$ & Derivative of height with respect to $\alpha_{r}$ & dzdar & N/A \\
\hline$e_{k}$ & Effective earth’s radius factor & ek & APM_VAR \\
\hline envpr & Complex [refractivity] phase term array & envpr() & N/A \\
\hline$\varepsilon_{r}$ & Relative permittivity & epsilon & N/A \\
\hline$\eta_{s}$ & $\begin{array}{l}\text { Quantity defined in equ. } 126 \text { in EREPS } 3.0 \text { User’s Manual } \\
\text { NRaD TD 2648, pp. } 106\end{array}$ & etas & N/A \\
\hline$f(\alpha)$ & Antenna pattern factor for angle $\alpha$ & patfac & N/A \\
\hline$f\left(\vartheta_{1}\right)$ & Antenna pattern factor for angle $\vartheta_{1}$ & factr & N/A \\
\hline$f\left(\alpha_{d}\right)$ & Antenna pattern factor for direct ray & facd & N/A \\
\hline$f\left(-\alpha_{r}\right)$ & Antenna pattern factor for reflected ray & facr & N/A \\
\hline farray & Field array to be propagated one range step in free space & farray() & N/A \\
\hline$F d^{2}$ & Magnitude array, direct ray & dmagsq(,) & APM_VAR \\
\hline$F_{d B}$ & Propagation factor in $\mathrm{dB}$ & ff, facdb & N/A \\
\hline$F_{d B l s t}$ & Propagation factor in $\mathrm{dB}$ at previous range & pfdblst & N/A \\
\hline ffat1m & Propagation factor array computed at $1 \mathrm{~m}$ above the surface. & ffat1m_dB() & N/A \\
\hline$f f a c z$ & $\begin{array}{l}\text { Two-dimensional array containing propagation factor, } \\
\text { range, and propagation angle at } z_{\text {lim }}\end{array}$ & ffacz(,) & N/A \\
\hline ffrout & $\begin{array}{l}\text { Array of propagation factors at each output range beyond } \\
r_{a t z} \text { and at height } z_{l i m}\end{array}$ & ffrout() & N/A \\
\hline filt & Cosine-tapered (Tukey) filter array & filt() & N/A \\
\hline filtp & Array filter for spectral estimation calculations & filtp() & N/A \\
\hline$f_{M H z}$ & Frequency in $\mathrm{MHz}$ & freq & SYSTEMVAR \\
\hline$f_{\text {rqg }}$ & $\begin{array}{l}\text { Frequency in } \mathrm{MHz} \text { at which to perform grazing angle } \\
\text { calculations }\end{array}$ & frqg & N/A \\
\hline$f_{\text {norm }}$ & Normalization factor & fnorm & APM_VAR \\
\hline$f_{r}$ & Fractional bin used for interpolation & $\mathrm{fr}$ & N/A \\
\hline
\end{tabular}


Table 139. Variable name cross reference. (continued)

\begin{tabular}{|c|c|c|c|}
\hline $\begin{array}{c}\text { SDD } \\
\text { variable } \\
\text { name }\end{array}$ & Description & $\begin{array}{l}\text { FORTRAN } \\
\text { source } \\
\text { code name }\end{array}$ & $\begin{array}{c}\text { FORTRAN } \\
\text { common } \\
\text { block name }\end{array}$ \\
\hline$F r^{2}$ & Magnitude array, reflected ray & rmagsq(,) & APM_VAR \\
\hline $\operatorname{frac}_{R O}$ & RO range interval fraction ( 0.0 to 0.25 ) & fracRO & N/A \\
\hline$F r_{a t z}$ & Propagation factor in $\mathrm{dB}$ at range $r_{a t z}$ and height $z_{\text {lim }}$ & pfratz & N/A \\
\hline frsp & Complex free space propagator term array & $\operatorname{frsp}()$ & N/A \\
\hline$f_{\text {sum }}^{2}$ & Square of coherent sum of directand reflected rays & ffac2 & N/A \\
\hline$f_{\text {ter }}$ & $\begin{array}{l}\text { Logical flag indicating if terrain profile has been } \\
\text { specified: } \\
\text {.true. = terrain profile specified } \\
\text {.false. = terrain profile not specified }\end{array}$ & fter & APM_VAR \\
\hline fv & Fraction range for profile interpolation & $\mathrm{fv}$ & N/A \\
\hline$\gamma_{a}$ & Surface specific attenuation & gammaa & REFRACTIVITY \\
\hline$\gamma c$ & $\begin{array}{l}\text { Dynamically allocated array of constants describing the } \\
\text { backscattering effectiveness of the surface }\end{array}$ & gammac() & TERRAIN \\
\hline$\gamma_{0}$ & Oxygen absorption & gammao & N/A \\
\hline yrng & $\begin{array}{l}\text { Dynamically allocated array of ranges corresponding to } \\
\text { the values in } \gamma c\end{array}$ & gamrng() & TERRAIN \\
\hline$\gamma_{w}$ & Water absorption & gammaw & N/A \\
\hline$\Gamma_{h, v}$ & $\begin{array}{l}\text { Complex reflection coefficient for horizontal or vertical } \\
\text { polarization }\end{array}$ & refcoef & N/A \\
\hline$G$ & Gain of transmit/receive antennas & antgain & SYSTEMVAR \\
\hline gas $_{a t t}$ & Gaseous absorption attenuation rate & gasatt & APM_VAR \\
\hline gas $_{\text {loss }}$ & Gaseous absorption loss at range $r_{\text {out }}$ & gasloss & APM_VAR \\
\hline$g r$ & Intermediate M-unit gradient array, RO region & $\operatorname{gr}()$ & N/A \\
\hline grad & $\begin{array}{l}\text { Two-dimensional array containing gradients of each } \\
\text { profile used in XO calculations }\end{array}$ & $\operatorname{grad}()$, & N/A \\
\hline$g_{r d}$ & Refractivity gradient & grd & N/A \\
\hline grdum & $\begin{array}{l}\text { Array of refractivity gradients defined by profile htdum } \\
\text { and refdum }\end{array}$ & grdum() & N/A \\
\hline$h_{0}$ & Height at start of ray trace step & h0 & N/A \\
\hline$h_{1}$ & Height at end of ray trace step & h1 & N/A \\
\hline$H_{1}$ & $\begin{array}{l}\text { Quantity defined in equ. } 120 \text { in EREPS } 3.0 \text { User's } \\
\text { Manual NRaD TD 2648, pp. } 106\end{array}$ & hor1 & N/A \\
\hline$H_{2}$ & $\begin{array}{l}\text { Quantity defined in equ. } 121 \text { in EREPS } 3.0 \text { User's } \\
\text { Manual NRaD TD 2648, pp. } 106\end{array}$ & hor2 & N/A \\
\hline hfang & Cut-back angles in degrees & hfang() & N/A \\
\hline hfangr & Array of height-finder cut-back angles in radians & hfangr() & N/A \\
\hline hffac & Cut-back antenna pattern factors & hffac() & N/A \\
\hline
\end{tabular}


Table 139. Variable name cross reference. (continued)

\begin{tabular}{|c|c|c|c|}
\hline $\begin{array}{l}\text { SDD } \\
\text { variable } \\
\text { name }\end{array}$ & Description & $\begin{array}{c}\text { FORTRAN } \\
\text { source } \\
\text { code name }\end{array}$ & $\begin{array}{c}\text { FORTRAN } \\
\text { common } \\
\text { block name }\end{array}$ \\
\hline$H F_{\text {flag }}$ & $\begin{array}{l}\text { HF computation flag indicating the frequency specified is less } \\
\text { than } 50 \mathrm{MHz}\end{array}$ & hf_flag & APM_VAR \\
\hline$h_{\text {large }}$ & $\begin{array}{l}\text { Maximum height limit for last level in height/refractivity } \\
\text { profiles }\end{array}$ & hlarge & N/A \\
\hline hlim & $\begin{array}{l}\text { Array containing the height at each output range separating the } \\
\text { RO region from the PE (at close ranges) and XO (at far ranges) } \\
\text { regions }\end{array}$ & $\operatorname{hlim}()$ & N/A \\
\hline$h_{\max }$ & Maximum output height with respect to mean sea level & hmax & INPUTVAR \\
\hline$h_{\min }$ & Minimum output height with respect to mean sea level & hmin & INPUTVAR \\
\hline$h_{\text {minter }}$ & Minimum height of terrain profile & hminter & APM_VAR \\
\hline$h m_{\text {ref }}$ & Height relative to $h_{\text {minter }}$ & hmref & APM_VAR \\
\hline hmsl & $\begin{array}{l}\text { Two-dimensional array containing heights with respect to } \\
\text { mean sea level of each profile. Array format must be } h m s l_{i, j}= \\
\text { height of } i^{\text {th }} \text { level of } j^{\text {th }} \text { profile; } j=1 \text { for range-independent cases }\end{array}$ & hmsl(,) & N/A \\
\hline$h_{o}$ & $\begin{array}{l}\text { Effective scattering height - defined in equ. } 109 \text { in EREPS } 3.0 \\
\text { User’s Manual NRaD TD 2648, pp. } 105\end{array}$ & h0 & N/A \\
\hline$H_{o}$ & $\begin{array}{l}\text { Frequency gain function defined in equ. } 119 \text { in EREPS } 3.0 \\
\text { User’s Manual NRaD TD 2648, pp. } 106\end{array}$ & bigh & N/A \\
\hline href & Heights of refractivity profile with respect to $y_{\text {ref }}$ & href() & N/A \\
\hline$h_{s}$ & Height for start of ray trace & hs & N/A \\
\hline$h_{\text {start }}$ & Starting height for ray trace to fill array hlim & hstart & N/A \\
\hline$h t$ & PE mesh height array of size $n_{f f t}$ & ht( $)$ & N/A \\
\hline htdum & Height array for current interpolated profile & htdum() & N/A \\
\hline htemp & Heights at which ray is traced to every range in rtemp & htemp() & APM_VAR \\
\hline$h_{\text {ter }}$ & Height of terrain at end of ray trace step & hter & N/A \\
\hline$h_{\text {termax }}$ & Maximum terrain height along profile path & htermax & N/A \\
\hline$h_{\text {test }}$ & $\begin{array}{l}\text { Minimum height at which all trapping refractivity features are } \\
\text { below }\end{array}$ & htest & N/A \\
\hline htfe & $\begin{array}{l}\text { Array containing the height at each output range separating the } \\
\text { FE region from the RO region (full hybrid mode), or the FE } \\
\text { region from the PE region (partial hybrid mode) }\end{array}$ & htfe() & N/A \\
\hline$h_{\text {thick }}$ & $\begin{array}{l}\text { Thickness of highest trapping layer from all refractivity } \\
\text { profiles }\end{array}$ & hthick & N/A \\
\hline$h t_{l i m}$ & Maximum height relative to $h_{\text {minter }}$ & htlim & APM_VAR \\
\hline$h t r$ & $\begin{array}{l}\text { Two-dimensional array containing heights of each profile used } \\
\text { in XO calculations }\end{array}$ & htr() & N/A \\
\hline$h_{\text {trap }}$ & Height of highest trapping layer from all refractivity profiles & htrap & N/A \\
\hline$h t_{y d i f}$ & $h t_{\text {lim }}-y_{f r e f}$ & htydif & APM_VAR \\
\hline
\end{tabular}


Table 139. Variable name cross reference. (continued)

\begin{tabular}{|c|c|c|c|}
\hline $\begin{array}{c}\text { SDD } \\
\text { variable } \\
\text { name }\end{array}$ & Description & $\begin{array}{l}\text { FORTRAN } \\
\text { source } \\
\text { code name }\end{array}$ & $\begin{array}{c}\text { FORTRAN } \\
\text { common } \\
\text { block name }\end{array}$ \\
\hline$i_{a l g}$ & $\begin{array}{l}\text { Integer flag indicating which DMFT algorithm is being } \\
\text { used: } \\
\qquad \begin{array}{l}0=\text { no DMFT algorithm will be used } \\
1=\text { use central difference algorithm } \\
2=\text { use backward difference algorithm }\end{array}\end{array}$ & ialg & APM_VAR \\
\hline$i_{a p}$ & $\begin{array}{l}\text { Index indicating when the local ray angle becomes } \\
\text { positive in array raya }\end{array}$ & iap & APM_VAR \\
\hline$i_{e r r}$ & Return error code & ierr & N/A \\
\hline$i_{\text {error }}$ & $\begin{array}{l}\text { Error flag - traps for various errors dependent on the } \\
\text { calling SU }\end{array}$ & ierror & N/A \\
\hline$i_{\text {extra }}$ & $\begin{array}{l}\text { Extrapolation flag for refractivity profiles entered in } \\
\text { combination with terrain below below mean sea level } \\
0=\text { extrapolate to minimum terrain height standard } \\
\text { atmosphere gradient } \\
1=\text { extrapolate to minimum terrain height using first } \\
\text { gradient in profile }\end{array}$ & iextra & REFRACTIVITY \\
\hline$i_{\text {flag }}$ & $\begin{array}{l}\text { Flag indicating whether to determine maximum FFT size } \\
n_{\text {fft }} \text { based on given } \Theta_{\max } \text { and } z_{\text {lim }} \text { or determine } z_{\text {lim }} \text { based } \\
\text { on given } \Theta_{\max } \text { and FFT size } n_{\text {fft }} \text {. }\end{array}$ & iflag & N/A \\
\hline$i_{\text {flag }}$ & $\begin{array}{l}\text { Flag to indicate which transform to perform } \\
\begin{array}{l}0=\text { cosine transform } \\
1=\text { sine transform } \\
-1=\text { deallocates all allocated arrays }\end{array}\end{array}$ & iflag & N/A \\
\hline$i_{\text {flag }}$ & $\begin{array}{l}\text { Integer flag indicating what region reflection coefficient } \\
\text { is being computed } \\
\quad 0=\mathrm{FE} \text { and RO regions } \\
1=\mathrm{PE} \text { region }\end{array}$ & iflag & N/A \\
\hline$i_{g}$ & Counter indicating current ground type being modeled & ig & APM_VAR \\
\hline$i_{g c}$ & Number of $\gamma c$ values for a particular application of APM & igc & TERRAIN \\
\hline$i_{g P E}$ & $\begin{array}{l}\text { Number of grazing angles computed from spectral } \\
\text { estimation }\end{array}$ & igpe & APM_VAR \\
\hline$i_{g r}$ & Number of different ground types specified & igr & TERRAIN \\
\hline$i_{\text {grad }}$ & Index of current gradient level in grad & igrad & N/A \\
\hline igrd & $\begin{array}{l}\text { Integer indexes indicating at what refractive gradient } \\
\text { level to begin ray tracing for next XO range step for each } \\
\text { ray in XO region }\end{array}$ & $\operatorname{igrd}()$ & N/A \\
\hline
\end{tabular}


Table 139. Variable name cross reference. (continued)

\begin{tabular}{|c|c|c|c|}
\hline $\begin{array}{c}\text { SDD } \\
\text { variable } \\
\text { name }\end{array}$ & Description & $\begin{array}{l}\text { FORTRAN } \\
\text { source } \\
\text { code name }\end{array}$ & $\begin{array}{c}\text { FORTRAN } \\
\text { common } \\
\text { block name }\end{array}$ \\
\hline igrnd & $\begin{array}{l}\text { Integer array containing ground type composition for given } \\
\text { terrain profile - can vary with range. Different ground types } \\
\text { are: } \\
\qquad \begin{array}{l}0=\text { seawater } \\
1 \text { = freshwater } \\
2 \text { = wet ground } \\
3=\text { medium dry ground } \\
4=\text { very dry ground } \\
5=\text { ice at }-1 \text { degree } C \\
6=\text { ice at }-10 \text { degree } C \\
7=\text { user-defined (in which case, values of relative } \\
\text { permittivity and conductivity must be given). }\end{array}\end{array}$ & igrnd() & N/A \\
\hline$i_{g r z}$ & Number of grazing angles computed from ray trace & igrz & APM_VAR \\
\hline$i_{h m x}$ & $\begin{array}{l}\text { Output range step index where height } h t_{l i m} \text { is reached in } \\
\text { array hlim }\end{array}$ & ihmx & APM_VAR \\
\hline$i_{\text {hybrid }}$ & $\begin{aligned} \text { Integer indicating which sub-models will be used: } & \\
0 & =\text { pure PE model } \\
1 & =\text { full hybrid model }(\mathrm{PE}+\mathrm{FE}+\mathrm{RO}+\mathrm{XO}) \\
2 & =\text { partial hybrid model }(\mathrm{PE}+\mathrm{XO})\end{aligned}$ & ihybrid & APM_VAR \\
\hline$i_{o}$ & $\begin{array}{l}\text { Starting index for } m p f l \text { array: } \\
\qquad \begin{array}{l}0=1^{\text {st }} \text { calculated output point is at surface } \\
1=1^{\text {st }} \text { calculated output point is at height } \Delta z_{\text {out }}\end{array}\end{array}$ & io & APM_VAR \\
\hline$i_{\text {org }}$ & $\begin{array}{c}\text { Integer flag indicating origin of calling SU } \\
0=\text { called from APMINIT CSC } \\
1=\text { called from TROPOINIT SU }\end{array}$ & iorg & N/A \\
\hline$i_{p 1}$ & $\begin{array}{l}\text { First output height point index in zout where propagation } \\
\text { loss will be computed at previous PE range }\end{array}$ & ip1 & N/A \\
\hline$i_{p 2}$ & $\begin{array}{l}\text { First output height point index in zout where propagation } \\
\text { loss will be computed at current PE range }\end{array}$ & ip2 & N/A \\
\hline$i_{\text {pat }}$ & $\begin{array}{l}\text { Antenna pattern type } \\
\qquad \begin{aligned} & 1=\text { Omni-directional } \\
& 2=\text { Gaussian } \\
& 3=\text { Sine }(\mathrm{x}) / \mathrm{x} \\
& 4=\text { Cosecant-squared } \\
& 5=\text { Generic height-finder } \\
& 6=\text { User-defined height-finder } \\
& 7=\text { User-defined antenna pattern } \\
& 8=\text { Quarter-wave vertical dipole (should be used only } \\
& \text { for HF applications) }\end{aligned}\end{array}$ & ipat & SYSTEMVAR \\
\hline$i_{P E}$ & Number of PE range steps & ipe & APM_VAR \\
\hline$i_{\text {peak }}$ & Bin \# in spectr corresponding to the peak magnitude & ipeak & N/A \\
\hline$i_{\text {PEstp }}$ & Counter indicating current PE range step & ipestp & N/A \\
\hline$i_{p l}$ & $\begin{array}{c}\text { Polarization flag } \\
0=\text { horizontal } \\
1=\text { vertical }\end{array}$ & ipl & N/A \\
\hline
\end{tabular}


Table 139. Variable name cross reference. (continued)

\begin{tabular}{|c|c|c|c|}
\hline $\begin{array}{l}\text { SDD } \\
\text { variable } \\
\text { name }\end{array}$ & Description & $\begin{array}{l}\text { FORTRAN } \\
\text { source } \\
\text { code name }\end{array}$ & $\begin{array}{c}\text { FORTRAN } \\
\text { common } \\
\text { block name }\end{array}$ \\
\hline$i_{p o l}$ & $\begin{array}{l}\text { Polarization flag: } \\
\qquad \begin{array}{l}0=\text { horizontal polarization } \\
1=\text { vertical polarization }\end{array}\end{array}$ & ipol & SYSTEMVAR \\
\hline$i_{\text {quit }}$ & $\begin{array}{l}\text { Integer flag indicating to quit tracing current ray and begin } \\
\text { again with a new launch angle }\end{array}$ & iquit & N/A \\
\hline$i_{\text {ratz }}$ & $\begin{array}{l}\text { Index of output range step in which to begin storing } \\
\text { propagation factor and outgoing angle for XO region }\end{array}$ & iratz & APM_VAR \\
\hline$i_{R O n}$ & Array index for next range in $\mathrm{RO}$ region & iROn & APM_VAR \\
\hline$i_{R O p}$ & Array index for previous range in $\mathrm{RO}$ region & iROp & APM_VAR \\
\hline$i_{r p}$ & $\begin{array}{l}\text { Counter for current refractivity/gradient profile being used } \\
\text { from grad }\end{array}$ & irp & N/A \\
\hline$i_{r p s}$ & Starting index counter for refractivity profiles & irps & N/A \\
\hline$i_{\text {rtemp }}$ & Temporary number of range steps (used for ray tracing) & irtemp & N/A \\
\hline$i_{s}$ & Counter for current profile & is & APM_VAR \\
\hline$i_{\text {start }}$ & Array index for height in RO region corresponding to $a n t_{r e f}$ & istart & APM_VAR \\
\hline$i_{\text {start } 1}$ & Refractivity level index within $h t d u m$ at $a n t_{r e f}$ & istart1 & APM_VAR \\
\hline$i_{s t p}$ & Current output range step index & istp & N/A \\
\hline$i_{s z}$ & Number of points over which to perform average smoothing & isz & N/A \\
\hline$i_{t p}$ & Number of height/range points in profile & itp & TERRAIN \\
\hline$i_{\text {tpa }}$ & Number of height/range points pairs in profile $t x$, ty & itpa & APM_VAR \\
\hline$i_{\text {type }}$ & $\begin{aligned} \text { Ray type (direct or reflected) flag } \\
\begin{aligned} 0 & =\text { direct } \\
1 & =\text { reflected }\end{aligned}\end{aligned}$ & itype & N/A \\
\hline$i_{x o}$ & Number of range steps in $\mathrm{XO}$ calculation region & ixo & APM_VAR \\
\hline$i_{\text {xostp }}$ & Current output range step index for XO calculations & ixostp & N/A \\
\hline iz & $\begin{array}{l}\text { Number of propagation factor, range, angle triplets stored in } \\
\text { ffacz }\end{array}$ & iz & APM_VAR \\
\hline$i_{z g}$ & $\begin{array}{l}\text { Number of output height points corresponding to local } \\
\text { ground height at current output range rout }\end{array}$ & izg & APM_VAR \\
\hline$i z_{\text {inc }}$ & $\begin{array}{l}\text { Integer increment for storing points at top of PE region (i.e., } \\
\text { points are stored at every } i z_{i n c} \text { range step) }\end{array}$ & izinc & APM_VAR \\
\hline$i z_{\max }$ & $\begin{array}{l}\text { Maximum number of points allocated for arrays associated } \\
\text { with XO calculations }\end{array}$ & izmax & APM_VAR \\
\hline$j_{a e}$ & Ending index within $m p f l$ of airborne loss values & jae & N/A \\
\hline$j_{a s}$ & Starting index within $m p f l$ of airborne loss values & jas & N/A \\
\hline$j_{e}$ & $\begin{array}{l}\text { Ending receiver height index at which to compute } \\
\text { troposcatter loss }\end{array}$ & je & N/A \\
\hline$j_{\text {end }}$ & Index at which valid loss values in $m p f l$ end & jend & N/A \\
\hline$j_{f e}$ & Ending index within $m p f l$ of FE loss values & jfe & N/A \\
\hline
\end{tabular}


Table 139. Variable name cross reference. (continued)

\begin{tabular}{|c|c|c|c|}
\hline $\begin{array}{c}\text { SDD } \\
\text { variable } \\
\text { name }\end{array}$ & Description & $\begin{array}{l}\text { FORTRAN } \\
\text { source } \\
\text { code name }\end{array}$ & $\begin{array}{c}\text { FORTRAN } \\
\text { common } \\
\text { block name }\end{array}$ \\
\hline$j_{f s}$ & Starting index within $m p f l$ of FE loss values & jfs & N/A \\
\hline$j_{\max }$ & Array index for maximum output height in RO region & jmax & N/A \\
\hline$j_{\min }$ & Array index for minimum output height in RO region & jmin & N/A \\
\hline$j_{p e}$ & Ending index within $m p f l$ of PE loss values & jpe & N/A \\
\hline$j_{p s}$ & Starting index within $m p f l$ of PE loss values & jps & N/A \\
\hline$j_{r e}$ & Ending index within $m p f l$ of RO loss values & jre & N/A \\
\hline$j_{r s}$ & Starting index within $m p f l$ of RO loss values & jrs & N/A \\
\hline$j_{s}$ & Refractive profile index for start of ray trace & js & N/A \\
\hline$j_{s}$ & $\begin{array}{l}\text { Starting receiver height index at which to compute } \\
\text { troposcatter loss }\end{array}$ & js & N/A \\
\hline$j_{\text {start }}$ & Index at which valid loss values in $m p f l$ start & jstart & N/A \\
\hline$j t 2$ & $\begin{array}{l}\text { Index counter for } t x \text { and ty arrays indicating location of } \\
\text { receiver range }\end{array}$ & jt2 & APM_VAR \\
\hline$j_{x e}$ & Index at which valid loss values in $m p f l$ end & jxe & N/A \\
\hline$j_{x s}$ & Index at which valid loss values in $m p f l$ start & jxs & N/A \\
\hline$j_{x s t a r t}$ & Starting index within $m p f l$ of XO loss values & jxstart & N/A \\
\hline$j z_{\text {lim }}$ & PE bin \# corresponding to $z_{\text {lim }}$, i.e., $z_{\text {lim }}=j z_{l i m} \Delta z_{P E}$ & jzlim & APM_VAR \\
\hline$k_{a b s}$ & $\begin{array}{l}\text { Gaseous absorption calculation flag: } \\
0=\text { no absorption loss } \\
1=\text { compute absorption loss based on air temperature } t_{\text {air }} \\
\text { and absolute humidity } a b s_{\text {hum }} \\
2=\text { compute absorption loss based on specified } \\
\text { absorption attenuation rate } \gamma_{a}\end{array}$ & kabs & N/A \\
\hline$k_{b i n}$ & Number of bins complex PE field is to be shifted & kbin & N/A \\
\hline$k_{h i}$ & $k$ index above desired point & khi & N/A \\
\hline$k_{l o}$ & $k$ index below desired point & klo & N/A \\
\hline$k_{\max }$ & Array index for maximum angle in RO region at range $x_{R O n}$ & kmax & APM_VAR \\
\hline$k_{\min n}$ & Array index for minimum angle in RO region at range $x_{R O n}$ & kminn & APM_VAR \\
\hline$k_{\min }$ & Array index for minimum angle in RO region at range $x_{R O p}$ & kminp & APM_VAR \\
\hline$k_{o}$ & Free-space wavenumber & fko & APM_VAR \\
\hline$k_{\text {temp }}$ & Temporary $k_{l o}$ value & klotmp & N/A \\
\hline$\lambda$ & Wavelength & wl & APM_VAR \\
\hline$L$ & Propagation loss & dloss & N/A \\
\hline lang & $\begin{array}{l}\text { Propagation angle and factor output flag } \\
\text { '.true.'= Output propagation angle and propagation } \\
\text { factor for direct and reflected ray (where applicable). } \\
\text { '.false.' = Do not output propagation angles and factors }\end{array}$ & lang & INPUT_VAR \\
\hline$L_{d i f}$ & Difference between propagation loss and troposcatter loss & dif & N/A \\
\hline
\end{tabular}


Table 139. Variable name cross reference. (continued)

\begin{tabular}{|c|c|c|c|}
\hline $\begin{array}{c}\text { SDD } \\
\text { variable } \\
\text { name }\end{array}$ & Description & $\begin{array}{c}\text { FORTRAN } \\
\text { source } \\
\text { code name }\end{array}$ & $\begin{array}{c}\text { FORTRAN } \\
\text { common } \\
\text { block name }\end{array}$ \\
\hline$l_{\text {duct }}$ & $\begin{array}{l}\text { Logical flag indicating if surface-based duct profile has } \\
\text { been specified } \\
\text { ‘.true'. = surface-based duct exists } \\
\text { '.false.' = no surface-based duct exists }\end{array}$ & lduct & APM_VAR \\
\hline lerr6 & $\begin{array}{l}\text { User-provided error flag that will trap on certain errors if } \\
\text { set to '.true.’ }\end{array}$ & lerr6 & ERRORFLAG \\
\hline lerr12 & $\begin{array}{l}\text { User-provided error flag that will trap on certain errors if } \\
\text { set to 'true.' }\end{array}$ & lerr12 & ERRORFLAG \\
\hline$l_{\text {evap }}$ & $\begin{array}{l}\text { Logical flag indicating if evaporation duct profile has } \\
\text { been specified } \\
\text { ‘.true'. = evaporation duct exists } \\
\text { ‘.false.' = no evaporation duct exists }\end{array}$ & levap & APM_VAR \\
\hline levels & Number of levels in $g r, q$ and $z r t$ arrays & levels & APM_VAR \\
\hline$L_{f s}$ & Free space loss & fsloss & N/A \\
\hline$l_{\text {graze }}$ & $\begin{array}{l}\text { Logical flag indicating if grazing angles were computed } \\
\text { for a particular application of APM }\end{array}$ & lgraze & N/A \\
\hline$l_{\text {new }}$ & Temporary refractivity level counter & newl & N/A \\
\hline $\ln _{f f t}$ & Power of 2 transform size, i.e. $n_{f f t}=2^{l n} f f t$ & $\ln$ & APM_VAR \\
\hline $\ln _{\min }$ & Minimum power of 2 transform size & $\operatorname{lnmin}$ & APM_VAR \\
\hline $\ln _{p}$ & $\begin{array}{l}\text { Power of } 2 \text { transform size used in spectral estimation } \\
\text { calculations; i.e., } n_{p}=2^{\ln p}\end{array}$ & $\operatorname{lnp}$ & APM_VAR \\
\hline$L_{\text {sys }}$ & Miscellaneous system losses & sysloss & SYSTEMVAR \\
\hline$l v l$ & $\begin{array}{l}\text { Number of height levels in each profile used in } \mathrm{XO} \\
\text { calculations }\end{array}$ & $\operatorname{lvl}()$ & N/A \\
\hline lvlep & $\begin{array}{l}\text { Number of height/refractivity levels in profile refdum } \\
\text { and htdum }\end{array}$ & lvlep & APM_VAR \\
\hline lvlp & Number of height/refractivity levels in profiles & lvlp & REFRACTIVITY \\
\hline$m$ & Size of array arbef & $\mathrm{m}$ & N/A \\
\hline$m p f l$ & Two-dimensional propagation factor and loss array & mpfl & N/A \\
\hline mpfl_rtg & $\begin{array}{l}\text { Propagation loss and factor at receiver heights specified } \\
\text { in the zout_rtg array }\end{array}$ & mpfl_rtg & N/A \\
\hline$\mu_{o}$ & Antenna elevation angle in degrees & elev & SYSTEMVAR \\
\hline$\mu_{o r}$ & Antenna pattern elevation angle in radians & elv & APM_VAR \\
\hline$\mu_{b w}$ & Antenna vertical beamwidth in degrees & bwidth & SYSTEMVAR \\
\hline$\mu_{b w r}$ & Antenna vertical beamwidth in radians & bw & APM_VAR \\
\hline$\mu_{\text {lim }}$ & Limiting elevation angle - no more than $10^{\circ}$ & elv_lim & N/A \\
\hline$\mu_{\max }$ & $\begin{array}{l}\text { Limiting angle for } \operatorname{Sin}(\mathrm{X}) / \mathrm{X} \text { and generic height finder } \\
\text { antenna pattern factors }\end{array}$ & umax & APM_VAR \\
\hline$n_{34}$ & $3 / 4 n_{f f t}$ & n34 & APM_VAR \\
\hline
\end{tabular}


Table 139. Variable name cross reference. (continued)

\begin{tabular}{|c|c|c|c|}
\hline $\begin{array}{l}\text { SDD } \\
\text { variable } \\
\text { name }\end{array}$ & Description & $\begin{array}{c}\text { FORTRAN } \\
\text { source } \\
\text { code name }\end{array}$ & $\begin{array}{c}\text { FORTRAN } \\
\text { common } \\
\text { block name }\end{array}$ \\
\hline$n_{4}$ & $1 / 4 n_{f f t}$ & nf4 & APM_VAR \\
\hline$n_{\text {ang }}$ & $\begin{array}{l}\text { Number of points in the vertical at which to spectrally } \\
\text { estimate propagation angles }\end{array}$ & nang & APM_VAR \\
\hline$n c^{2}$ & Array of complex dielectric constants & $\operatorname{cn} 2()$ & N/A \\
\hline$N_{f}$ & Noise figure & qnoisef & SYSTEMVAR \\
\hline$n_{f f t}$ & Transform size & $\mathrm{n}$ & APM_VAR \\
\hline$n_{\text {facs }}$ & $\begin{array}{l}\text { Number of user-defined cut-back angles and cut-back } \\
\text { pattern factors }\end{array}$ & nfacs & SYSTEMVAR \\
\hline nlvl & Number of levels in new profile & nlvl & APM_VAR \\
\hline$n_{m 1}$ & $n_{f f t}-1$ & $\mathrm{~nm} 1$ & APM_VAR \\
\hline$n o_{P E}$ & $\begin{array}{l}\text { Integer flag indicating if PE calculations are needed: } \\
\begin{array}{l}0=\mathrm{PE} \text { calculations needed } \\
1=\text { no PE calculations needed }\end{array}\end{array}$ & nope & APM_VAR \\
\hline$n_{p 34}$ & $3 / 4 n_{p}$ & np34 & APM_VAR \\
\hline$n_{p 4}$ & $1 / 4 n_{p}$ & np4 & APM_VAR \\
\hline$n_{p}$ & $\begin{array}{l}\text { Number of bins in upper PE region to consider for } \\
\text { spectral estimation }\end{array}$ & npnts & APM_VAR \\
\hline$n_{\text {prof }}$ & Number of refractivity profiles & nprof & REFRACTIVITY \\
\hline$n_{\text {ray }}$ & $\begin{array}{l}\text { Number of rays used for ray trace to determine grazing } \\
\text { angles }\end{array}$ & nray & $\mathrm{N} / \mathrm{A}$ \\
\hline$n_{\text {rout }}$ & Integer number of output range points desired & nrout & INPUTVAR \\
\hline$n_{s}$ & Transform size for spectral estimation calculations & ns & APM_VAR \\
\hline$n_{x o}$ & Number of rays traced, i.e., height points, in XO region & nxo & N/A \\
\hline$n_{\text {zout }}$ & Integer number of output height points desired & nzout & INPUTVAR \\
\hline$n_{\text {zout_rtg }}$ & $\begin{array}{l}\text { Number of height output points for receiver heights } \\
\text { relative to the local ground elevation. }\end{array}$ & nzout_rtg & INPUTVAR \\
\hline$n_{w}$ & Number of wind speeds & nw & REFRACTIVITY \\
\hline$\Omega$ & Total phase angle & phdif & N/A \\
\hline$\Omega$ & Total phase angle array & omega(,) & N/A \\
\hline$\omega_{s}$ & Interpolated wind speed & $\begin{array}{l}\text { ws, } \\
\text { windsp }\end{array}$ & N/A \\
\hline$P E_{\text {flag }}$ & $\begin{array}{c}\text { Flag to indicate use of PE algorithm only: } \\
\text { '.true.' = only use PE sub-model } \\
\text { '.false.' = use automatic hybrid model }\end{array}$ & peflag & INPUTVAR \\
\hline$p_{\text {elev }}$ & Sine of antenna elevation angle & pelev & APM_VAR \\
\hline$\varphi$ & Phase lag angle of reflected ray & rphase & N/A \\
\hline$p l_{\text {cnst }}$ & $\begin{array}{l}\text { Constant used in determining propagation loss }\left(p l_{c n s t}=\right. \\
\left.20 \log _{10}\left(2 k_{o}\right)\right)\end{array}$ & plcnst & APM_VAR \\
\hline$p l_{d}$ & Path length difference from range $x$ for direct ray & pld & N/A \\
\hline
\end{tabular}


Table 139. Variable name cross reference. (continued)

\begin{tabular}{|c|c|c|c|}
\hline $\begin{array}{c}\text { SDD } \\
\text { variable } \\
\text { name }\end{array}$ & Description & $\begin{array}{c}\text { FORTRAN } \\
\text { source } \\
\text { code name }\end{array}$ & $\begin{array}{c}\text { FORTRAN } \\
\text { common } \\
\text { block name }\end{array}$ \\
\hline$p l_{r}$ & Path length difference from range $x$ for reflected ray & plr & N/A \\
\hline$p_{\text {mag }}$ & Interpolated magnitude of complex PE field & pmag & N/A \\
\hline prfhxo & $\begin{array}{l}\text { Two-dimensional array of propagation factor and heights } \\
\text { for each ray traced in XO region to range } r_{\text {out }}\end{array}$ & prfh_xo & N/A \\
\hline profint & Profile interpolated to every $\Delta z_{P E}$ in height & profint() & N/A \\
\hline propaf & $\begin{array}{l}\text { Two-dimensional array, containing the propagation } \\
\text { angles and factors for the direct and reflected rays (where } \\
\text { applicable) for all output height/range points }\end{array}$ & propaf() & N/A \\
\hline$P_{t}$ & Transmitter peak power & tx_pow & SYSTEMVAR \\
\hline$\psi$ & Grazing angle & $\begin{array}{l}\text { psi, } \\
\text { angle }\end{array}$ & N/A \\
\hline$\Psi$ & $\begin{array}{l}\text { Array of interpolated grazing angles at each PE range } \\
\text { step }\end{array}$ & graze() & N/A \\
\hline$\Psi_{\text {rout }}$ & Array of grazing angles at each output range $r_{\text {out }}$ & graze_at_rout() & N/A \\
\hline$\psi_{\lim }$ & Grazing angle of limiting ray & psilim & APM_VAR \\
\hline$\psi_{P E}$ & $\begin{array}{l}\text { Array containing grazing angles computed from spectral } \\
\text { estimation of PE field }\end{array}$ & grz_pe() & N/A \\
\hline$\psi_{\text {ray }}$ & $\begin{array}{l}\text { Two-dimensional array containing grazing angles and } \\
\text { corresponding ranges computed from ray trace }\end{array}$ & grz_ray(,) & N/A \\
\hline$q$ & Intermediate M-unit difference array, RO region & $\mathrm{q}()$ & N/A \\
\hline$q_{t}$ & $\begin{array}{l}\text { Quantity defined in equ. } 128 \text { in EREPS } 3.0 \text { User’s } \\
\text { Manual NRaD TD 2648, pp. } 107\end{array}$ & qt & N/A \\
\hline$r$ & Current PE range & $\mathrm{r}$ & N/A \\
\hline$r_{0}$ & Range at start of ray trace step & r0 & N/A \\
\hline$r_{1}$ & Range at end of ray trace step & r1 & N/A \\
\hline$r_{1}$ & Path length for direct-ray path & r1 & N/A \\
\hline$r_{1}$ & $\begin{array}{l}\text { Quantity defined in equ. } 122 \text { in EREPS } 3.0 \text { User’s } \\
\text { Manual NRaD TD 2648, pp. } 106\end{array}$ & r1 & N/A \\
\hline$r_{2}$ & Path length for reflected-ray path & r2 & N/A \\
\hline$r_{2}$ & $\begin{array}{l}\text { Quantity defined in equ. } 123 \text { in EREPS } 3.0 \text { User's } \\
\text { Manual NRaD TD 2648, pp. } 106\end{array}$ & r2 & N/A \\
\hline$r_{\text {ange }}$ & Range for profile interpolation & range & N/A \\
\hline ratio & Fractional range term used for interpolation & ratiox & N/A \\
\hline ratio $_{k}$ & Fraction of one $k$ index ( 0 to 1 ) & ratiok & N/A \\
\hline$r_{a t z}$ & Range at which $z_{\text {lim }}$ is reached (used for hybrid model) & ratz & APM_VAR \\
\hline raya & $\begin{array}{l}\text { Array containing all local angles of traced ray } a_{\text {launch }} \text { at } \\
\text { each } i_{\text {rtemp }} \text { range }\end{array}$ & raya() & APM_VAR \\
\hline$r_{\text {crit }}$ & Minimum M-unit value above height $a n t_{r e f}$ & rcrit & N/A \\
\hline rdif & Range difference between adjacent terrain points & rdif1 & N/A \\
\hline
\end{tabular}


Table 139. Variable name cross reference. (continued)

\begin{tabular}{|c|c|c|c|}
\hline $\begin{array}{l}\text { SDD } \\
\text { variable } \\
\text { name }\end{array}$ & Description & $\begin{array}{c}\text { FORTRAN } \\
\text { source } \\
\text { code name }\end{array}$ & $\begin{array}{c}\text { FORTRAN } \\
\text { common } \\
\text { block name }\end{array}$ \\
\hline$r d i f_{2}$ & Range difference between adjacent terrain points & rdif2 & N/A \\
\hline$r_{\text {difsum }}$ & Sum of adjacent terrain point differences & rdifsum & N/A \\
\hline$r d t$ & $\begin{array}{l}\text { Array of minimum ranges at which diffraction field solutions } \\
\text { are applicable (for smooth surface) for all output receiver } \\
\text { heights }\end{array}$ & $\operatorname{rdt}()$ & N/A \\
\hline refdum & M-unit array for current interpolated profile & refdum() & N/A \\
\hline refmsl & $\begin{array}{l}\text { Two-dimensional array containing refractivity with respect to } \\
\text { mean sea level of each profile. Array format must be refms } l_{i, j} \\
=\text { M-unit at } i^{\text {th }} \text { level of } j^{\text {th }} \text { profile; } j=1 \text { for range-independent } \\
\text { cases }\end{array}$ & refmsl(,) & N/A \\
\hline refref & Refractivity profile with respect to $y_{\text {ref }}$ & refref() & N/A \\
\hline$r_{f}$ & Constant used for troposcatter calculations & $\mathrm{rf}$ & APM_VAR \\
\hline rfac1 & $\begin{array}{l}\text { Propagation factor at valid output height points from PE field } \\
\text { at range } r_{\text {last }}\end{array}$ & $\operatorname{rfac1}()$ & N/A \\
\hline rfac2 & $\begin{array}{l}\text { Propagation factor at valid output height points from PE field } \\
\text { at range } r\end{array}$ & $\operatorname{rfac} 2()$ & N/A \\
\hline$r_{f i x}$ & Fixed range increment of terrain profile & rfix & N/A \\
\hline$r_{\text {flat }}$ & $\begin{array}{l}\text { Maximum range at which the terrain profile remains flat from } \\
\text { the source }\end{array}$ & rflat & N/A \\
\hline$r_{\text {frac }}$ & Ratio between adjacent terrain point differences & rfrac & N/A \\
\hline rgrnd & Array containing ranges at which varying ground types apply & $\operatorname{rgrnd}()$ & N/A \\
\hline$r_{h o r}$ & Radio horizon range & rhor & APM_VAR \\
\hline$r_{\text {hor } 1}$ & $\begin{array}{l}\text { Minimum range at which diffraction field solutions are } \\
\text { applicable - determined for } 0 \text { receiver height }\end{array}$ & rdhor1 & APM_VAR \\
\hline$R_{k}$ & $\begin{array}{l}\text { Constant used to compute coefficients in central difference } \\
\text { form of the DMFT }\end{array}$ & rk & APM_VAR \\
\hline$r_{\text {last }}$ & Previous PE range & rlast & N/A \\
\hline$r_{l o g}$ & $10 \log _{10}(\mathrm{PE}$ range $r)$ & rlog & APM_VAR \\
\hline rlogo & Array containing 20 times the logarithm of all output ranges & rlogo() & N/A \\
\hline$r_{\text {loglst }}$ & $10 \log _{10}$ (previous PE range $r_{\text {last }}$ ) & rloglst & APM_VAR \\
\hline rloss & Propagation loss & rloss() & N/A \\
\hline rloss_rtg & $\begin{array}{l}\text { Propagation loss computed relative to the local ground height } \\
\text { at heights specified by zout_rtg }\end{array}$ & rloss_rtg() & N/A \\
\hline$r m$ & Intermediate M-unit array, RO region & $\operatorname{rm}()$ & N/A \\
\hline$R_{\text {mag }}$ & Magnitude of reflection coefficient & rmag & N/A \\
\hline$r_{\max }$ & Maximum specified range & $\operatorname{rmax}$ & INPUTVAR \\
\hline$r_{\text {mid }}$ & $\begin{array}{l}\text { Range at which interpolation for range-dependent profiles is } \\
\text { performed }\end{array}$ & rmid & N/A \\
\hline$r m_{\max }$ & Maximum M-unit value of refractivity profile at range 0 & rmmax & N/A \\
\hline
\end{tabular}


Table 139. Variable name cross reference. (continued)

\begin{tabular}{|c|c|c|c|}
\hline $\begin{array}{l}\text { SDD } \\
\text { variable } \\
\text { name }\end{array}$ & Description & $\begin{array}{l}\text { FORTRAN } \\
\text { source } \\
\text { code name }\end{array}$ & $\begin{array}{c}\text { FORTRAN } \\
\text { common } \\
\text { block name }\end{array}$ \\
\hline$r m_{\min }$ & Minimum M-unit value of refractivity profile at range 0 & rmmin & N/A \\
\hline$r m_{t x}$ & M-unit value at height $a n t_{r e f}$ & rmtx & APM_VAR \\
\hline$r_{\text {mult }}$ & PE range step multiplication factor & rmult & INPUTVAR \\
\hline$r n$ & Array of $R_{T}$ to the $i^{\text {th }}$ power (e.g., $r n_{i}=R_{T}^{i}$ ) & $\mathrm{rn}()$ & N/A \\
\hline rngout & Array containing all desired output ranges & rngout() & N/A \\
\hline rngprof & Ranges of each profile: $r n g p r o f_{i}=$ range of $i^{\text {th }}$ profile & rngprof() & N/A \\
\hline rngwind & $\begin{array}{l}\text { Ranges of wind speeds entered: } r n g w i n d_{i}=\text { range of } i^{\text {th }} \text { wind } \\
\text { speed }\end{array}$ & rngwind() & N/A \\
\hline$r_{o}$ & Current ending range for ray trace step & ro & N/A \\
\hline$R O \alpha_{d i r}$ & $\begin{array}{l}\text { Array of propagation angles of direct rays determined in the } \\
\text { RO region }\end{array}$ & ROdir_ang() & APM_VAR \\
\hline$R O \alpha_{r e f}$ & $\begin{array}{l}\text { Array of propagation angles of reflected rays determined in } \\
\text { the RO region }\end{array}$ & ROref_ang() & APM_VAR \\
\hline$r_{\text {out }}$ & Current output range & rout & N/A \\
\hline$r_{\text {pest }}$ & Range at which PE loss values will start being calculated & rpest & APM_VAR \\
\hline$r_{s}$ & Range for start of ray trace & rs & N/A \\
\hline$r_{\text {skip }}$ & Approximate range interval of skip zone if duct is present & rskip & N/A \\
\hline$r_{\text {slope }}$ & Ray slope used in determining reflection point over terrain & rslope & N/A \\
\hline$r_{s q}$ & Square of current output range & rsq & N/A \\
\hline$r_{s q k}$ & Earth curvature correction factor & rsqk & N/A \\
\hline rsqrd & Array containing the square of all desired output ranges & $\operatorname{rsqrd}()$ & N/A \\
\hline$R_{T}$ & $\begin{array}{l}\text { Complex root of quadratic equation for mixed transform } \\
\text { method based on Kuttler's formulation }\end{array}$ & $\mathrm{rt}$ & APM_VAR \\
\hline$r t_{1}$ & $r_{f}$ multiplied by $a n t_{r e f}$ & r1t & APM_VAR \\
\hline rtemp & Range steps for tracing to determine maximum PE angle & rtemp() & APM_VAR \\
\hline$r_{t s t}$ & Range at which to begin RO calculations (equal to $2.5 \mathrm{~km}$ ) & rtst & N/A \\
\hline ruf & $\begin{array}{l}\text { Logical flag indicating if rough sea surface calculations are } \\
\text { required } \\
\text { '.true.' = perform rough sea surface calculations } \\
\text { '.false.' = do not perform rough sea surface calculations }\end{array}$ & ruf & APM_VAR \\
\hline$r u f_{f a c}$ & Factor used for wave height calculation & ruf_fac & APM_VAR \\
\hline$r u f_{h t}$ & Sea surface rms wave height & ruf_ht & APM_VAR \\
\hline$r v_{1}$ & Range of the previous refractivity profile & rv1 & N/A \\
\hline$r v_{2}$ & Range of the next refractivity profile & rv2 & APM_VAR \\
\hline$\sigma$ & Conductivity & sigma & N/A \\
\hline$s$ & $\begin{array}{l}\text { Quantity defined equ. } 110 \text { in EREPS } 3.0 \text { User's Manual } \\
\text { NRaD TD 2648, pp. } 105\end{array}$ & s & N/A \\
\hline
\end{tabular}


Table 139. Variable name cross reference. (continued)

\begin{tabular}{|c|c|c|c|}
\hline $\begin{array}{c}\text { SDD } \\
\text { variable } \\
\text { name }\end{array}$ & Description & $\begin{array}{c}\text { FORTRAN } \\
\text { source } \\
\text { code name }\end{array}$ & $\begin{array}{c}\text { FORTRAN } \\
\text { common } \\
\text { block name }\end{array}$ \\
\hline$s_{b w}$ & Sine of antenna vertical beam width & sbw & APM_VAR \\
\hline$S_{\text {gain }}$ & Normalization factor used in starter field calculation & sgain & N/A \\
\hline $\operatorname{slp}$ & Slope of each segment of terrain & $\operatorname{slp}()$ & N/A \\
\hline$s n_{1}$ & Term used in troposcatter loss calculation & sn1 & N/A \\
\hline snref & Surface refractivity & snref & N/A \\
\hline snrefo & $\begin{array}{l}\text { Surface refractivity taken from the refractivity profile } \\
\text { with respect to mean sea level }\end{array}$ & snref_0 & APM_VAR \\
\hline snref $f_{t x}$ & Surface refractivity at transmitter & snref_tx & APM_VAR \\
\hline spectr & Spectral amplitude of field & spectr() & N/A \\
\hline$\vartheta 0$ & $\begin{array}{l}\text { Array of angles used to determine common volume } \\
\text { scattering angle }\end{array}$ & theta0() & N/A \\
\hline$\vartheta_{1}$ & Tangent angle from source height & theta1 & N/A \\
\hline$\vartheta_{2}$ & Tangent angle from receiver height & theta2 & N/A \\
\hline$\vartheta 1_{s}$ & Tangent angle from source (for smooth surface) & theta1s & APM_VAR \\
\hline$\vartheta 2 \mathrm{~s}$ & $\begin{array}{l}\text { Array of tangent angles from all output receiver } \\
\text { heights - used with smooth surface }\end{array}$ & theta2s() & N/A \\
\hline$\vartheta 1 t$ & $\begin{array}{l}\text { Array of tangent angles from source height - used with } \\
\text { terrain profile }\end{array}$ & th1() & N/A \\
\hline$\theta_{\text {hbw }}$ & Antenna horizontal beam width & horbw & SYSTEMVAR \\
\hline$\Theta_{\max }$ & Maximum propagation angle in PE calculations & thetamax & N/A \\
\hline$\vartheta_{m \times g}$ & $\begin{array}{l}\text { Maximum PE calculation angle for spectral estimation } \\
\text { of grazing angles }\end{array}$ & thmxg & N/A \\
\hline$\Theta_{75}$ & $\begin{array}{l}\text { 75\% of maximum propagation angle in PE } \\
\text { calculations }\end{array}$ & theta75 & APM_VAR \\
\hline$\vartheta_{\text {out }}$ & $\begin{array}{l}\text { Outgoing propagation angle determined at top of } \mathrm{PE} \\
\text { region }\end{array}$ & thout & N/A \\
\hline$\theta$ & Common volume scattering angle & theta & N/A \\
\hline$\Theta_{\text {rout }}$ & $\begin{array}{l}\text { Two-dimensional array containing the propagation } \\
\text { angle spectrally estimated from PE at } n_{\text {ang }} \text { height } \\
\text { points and at every output range step } r_{\text {out }}\end{array}$ & ptheta_rout(,) & N/A \\
\hline$\Theta_{p}$ & $\begin{array}{l}\text { Two-dimensional array containing the propagation } \\
\text { angle estimated from PE at } n_{\text {ang }} \text { height points and at } \\
\text { every PE calculation range step during the } \\
\text { initialization routine. }\end{array}$ & ptheta(,) & N/A \\
\hline$\theta_{t}$ & $\begin{array}{l}\text { Angular interval limit for ray trace in determining } \\
\text { grazing angles }\end{array}$ & degt & N/A \\
\hline$t_{\text {air }}$ & Air temperature near the surface & tair & REFRACTIVITY \\
\hline$\tau$ & Pulse length/width & pulse_len & SYSTEMVAR \\
\hline tang & Tangent of angle array from terrain slopes. & $\operatorname{tang}()$ & N/A \\
\hline
\end{tabular}


Table 139. Variable name cross reference. (continued)

\begin{tabular}{|c|c|c|c|}
\hline $\begin{array}{l}\text { SDD } \\
\text { variable } \\
\text { name }\end{array}$ & Description & $\begin{array}{l}\text { FORTRAN } \\
\text { source } \\
\text { code name }\end{array}$ & $\begin{array}{l}\text { FORTRAN } \\
\text { common } \\
\text { block name }\end{array}$ \\
\hline terx & Range points of terrain profile & $\operatorname{terx}()$ & N/A \\
\hline tery & Height points of terrain profile & tery () & N/A \\
\hline$t h_{\max }$ & Visible portion of maximum PE calculation angle & thmax & INPUTVAR \\
\hline$t_{\text {loss }}$ & Troposcatter loss in $\mathrm{dB}$ & tloss & N/A \\
\hline tlst & Troposcatter loss term & tlst & N/A \\
\hline tlst $_{\mathrm{s}}$ & Troposcatter loss term for smooth surface case & tlsts & N/A \\
\hline$t^{t} s t_{w r}$ & Troposcatter loss term used in TROPOSCAT SU & tsltwr & APM_VAR \\
\hline$T_{\text {ropo }}$ & $\begin{array}{l}\text { Troposcatter calculation flag: } \\
\text { '.false.'= no troposcatter calcs } \\
\text { '.true.' = troposcatter calcs }\end{array}$ & tropo & INPUTVAR \\
\hline twoka & Twice the effective earth’s radius & twoka & APM_VAR \\
\hline twoka $a_{\text {down }}$ & Twice the effective earth radius for downward path & twoka_down & APM_VAR \\
\hline$t x$ & Range points of terrain profile & $\operatorname{tx}()$ & N/A \\
\hline ty & Adjusted height points of terrain profile & $\operatorname{ty}()$ & N/A \\
\hline tyh & $\begin{array}{l}\text { Adjusted height points of sampled terrain profile at every } \\
\text { PE range step }\end{array}$ & $\operatorname{tyh}()$ & N/A \\
\hline$U$ & Complex field at current PE range $r$ & $\mathrm{u}()$ & N/A \\
\hline Udum & $\begin{array}{l}\text { Dummy array used for temporary storage of real or } \\
\text { imaginary part of complex PE field array } U\end{array}$ & udum() & N/A \\
\hline Ulast & Complex field at previous PE range $r_{\text {last }}$ & ulst() & N/A \\
\hline$w$ & Difference equation of complex PE field & $\mathrm{w}()$ & N/A \\
\hline wind & Array of wind speeds & wind() & N/A \\
\hline wind $_{\text {dir }}$ & Angle between antenna boresight and upwind direction & wind_dir & REFRACTIVITY \\
\hline$x$ & Current output range & $\mathrm{x}$ & N/A \\
\hline$x$ & $\begin{array}{l}\text { Field array to be transformed - dimensioned } 2^{n_{f f t}} \text { in calling } \\
\text { SU }\end{array}$ & $\mathrm{x}()$ & N/A \\
\hline xdum & Real part of complex field array & xdum() & N/A \\
\hline$x O_{\text {con }}$ & Constant used in determining $\vartheta_{\text {out }}$ & xocon & APM_VAR \\
\hline$x p$ & Real part of spectral field & $\mathrm{xp}()$ & N/A \\
\hline$x_{r}$ & Terminal range - called $x_{R O n}$ in ROCALC SU & rout & N/A \\
\hline$x_{\text {reflect }}$ & Range at which ray is reflected & xreflect & N/A \\
\hline$x_{R O n}$ & Next range in RO region & xROn & APM_VAR \\
\hline$X_{R O p}$ & Previous range in RO region & xROp & APM_VAR \\
\hline$x_{\text {temp }}$ & Temporary range in ray trace step & xtemp & N/A \\
\hline$x_{\text {sum }}$ & Running sum of range during ray trace & xsum & N/A \\
\hline$x x$ & Fractional range for interpolation & $\mathrm{xx}$ & N/A \\
\hline
\end{tabular}


Table 139. Variable name cross reference. (continued)

\begin{tabular}{|c|c|c|c|}
\hline $\begin{array}{c}\text { SDD } \\
\text { variable } \\
\text { name } \\
\end{array}$ & Description & $\begin{array}{c}\text { FORTRAN } \\
\text { source } \\
\text { code name }\end{array}$ & $\begin{array}{c}\text { FORTRAN } \\
\text { common } \\
\text { block name }\end{array}$ \\
\hline$y_{c h}$ & Height of terrain at the current PE range relative to $h m_{\text {ref }}$ & ych & N/A \\
\hline$y_{\text {cur }}$ & Height of ground at current range $r$ & ycur & APM_VAR \\
\hline$y_{\text {curm }}$ & Height of ground midway between last and current PE range & ycurm & APM_VAR \\
\hline$y_{\text {diff }}$ & $y_{\text {cur }}-y_{\text {last }}$ & ydiff & N/A \\
\hline ydum & Imaginary part of complex field array & ydum() & N/A \\
\hline$y_{\text {fref }}$ & Ground elevation height at source & yfref & APM_VAR \\
\hline$y_{\text {last }}$ & Height of ground at previous range $r_{\text {last }}$ & ylast & APM_VAR \\
\hline$y_{l h}$ & Height of terrain at the previous PE range relative to $h m_{r e f}$ & ylh & N/A \\
\hline$y m$ & Particular solution of difference equation & $\mathrm{ym}()$ & N/A \\
\hline yp & Imaginary part of spectral field & ур() & N/A \\
\hline$y_{\text {ref }}$ & Ground elevation height at current range & yref & N/A \\
\hline$z_{c}$ & $\begin{array}{l}\text { Height at which to compute propagation factor for clutter } \\
\text { calculations relative to } h m_{\text {ref }}\end{array}$ & $\mathrm{zc}$ & APM_VAR \\
\hline$z_{d}$ & Terminal height of direct ray & $\mathrm{zd}$ & N/A \\
\hline$z_{\text {int }}$ & Interpolated terrain elevation at current output range & zint & N/A \\
\hline$z_{k}$ & Height of $k^{\text {th }}$ RO index & $\mathrm{zk}$ & N/A \\
\hline$Z_{\text {lim }}$ & Height limit for PE calculation region & zlim & APM_VAR \\
\hline$Z_{\text {limt }}$ & $h t_{l i m}-10^{-5}$ & zlimt & N/A \\
\hline$Z_{\max }$ & Total height of the FFT/PE calculation domain & zmax & APM_VAR \\
\hline zout & Array containing all desired output heights referenced to $h_{\text {minter }}$ & zout() & N/A \\
\hline zoutma & Array output heights relative to "real" ant $t_{r e f}$ & zoutma() & N/A \\
\hline zoutpa & Array output heights relative to "image" ant $t_{r e f}$ & zoutpa() & N/A \\
\hline zout_rtg & $\begin{array}{l}\text { Dynamically allocated array of receiver heights specified relative } \\
\text { to the local ground height. }\end{array}$ & zout_rtg() & INPUTVAR \\
\hline$z_{r}$ & Receiver height & height, zr & N/A \\
\hline zro & Array of output heights in $\mathrm{RO}$ region & zro() & $\mathrm{N} / \mathrm{A}$ \\
\hline$z r t$ & Intermediate height array, RO region & $\operatorname{zrt}()$ & N/A \\
\hline$z_{\text {test }}$ & Height in PE region that must be reached for hybrid model & ztest & N/A \\
\hline$z_{t o l}$ & Height tolerance for Newton's method & ztol & APM_VAR \\
\hline zXO & Height of the ground at the current output range step & $\mathrm{zxo}()$ & N/A \\
\hline
\end{tabular}




\section{SOFTWARE TEST DESCRIPTION}

FOR THE

ADVANCED PROPAGATION MODEL CSCI

(Version 2.1.04)

20 December 2006 


\section{CONTENTS}

1. SCOPE

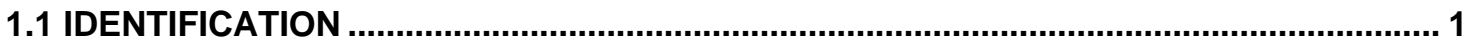

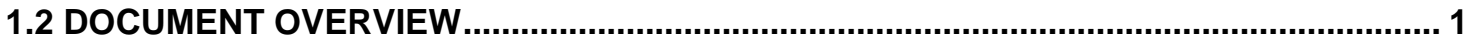

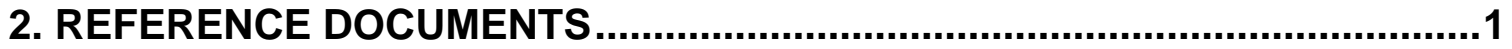

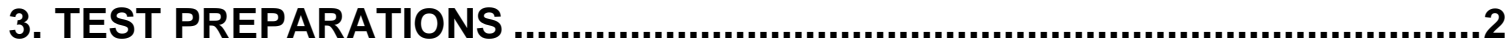

3.1 HARDWARE PREPARATION

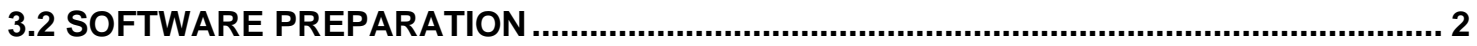

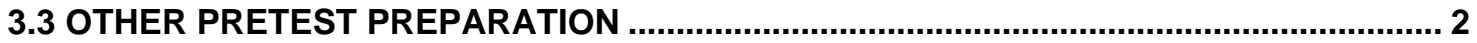

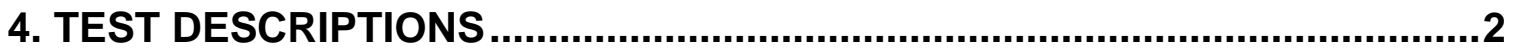

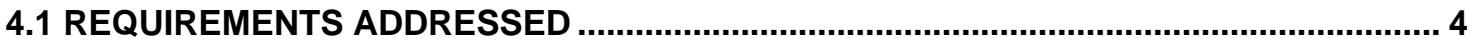

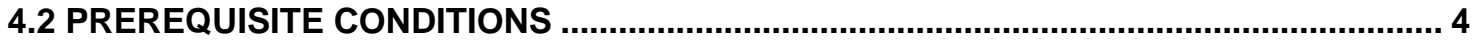

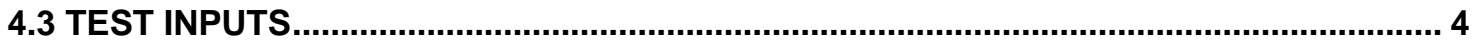

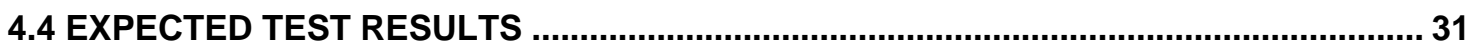

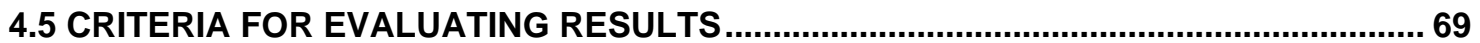

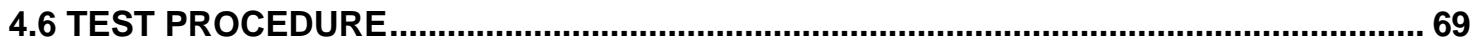

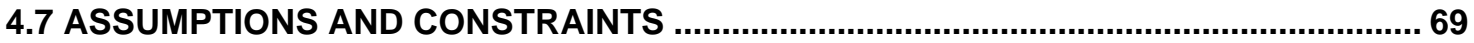

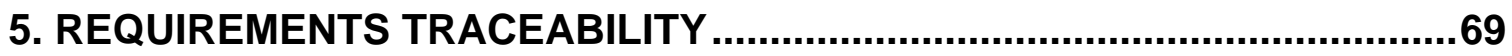

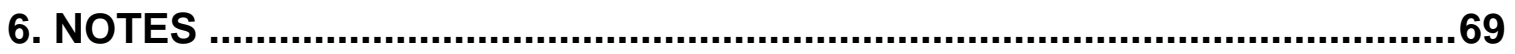

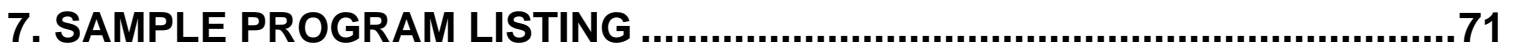

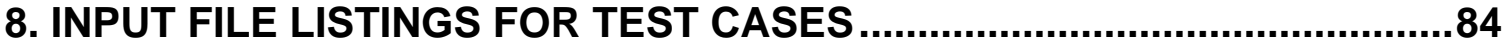

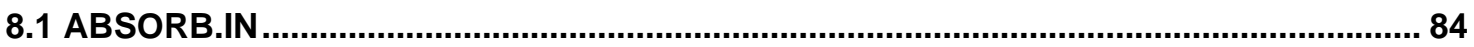

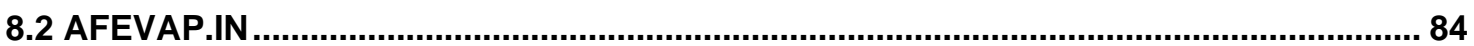

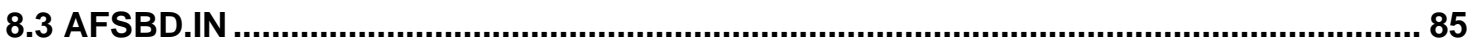

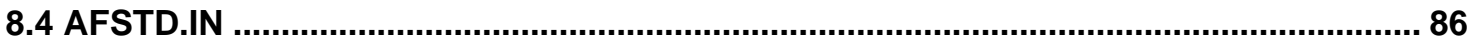

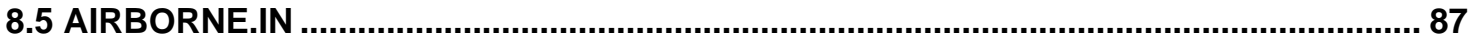

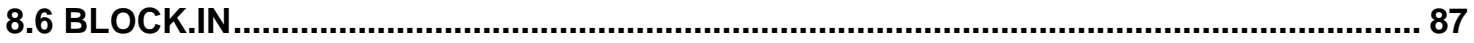

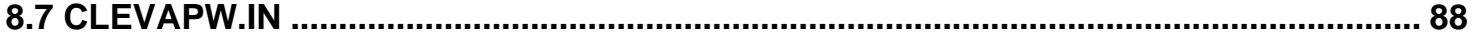

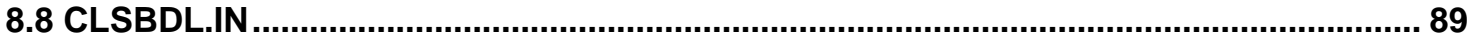

8.9 CLSBDW.IN.................................................................................................................. 91

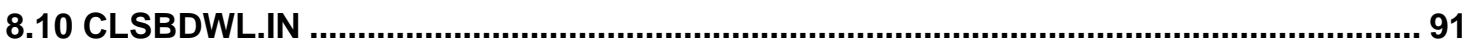

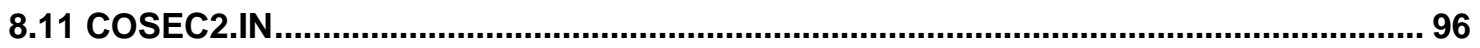

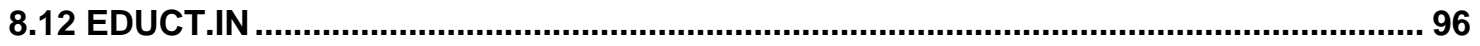

8.13 EDUCTRF.IN ................................................................................................................ 97

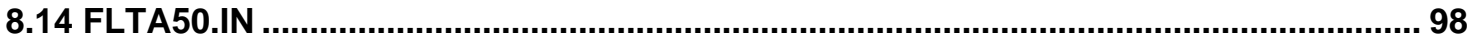

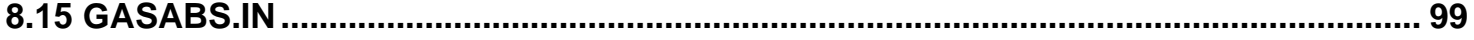

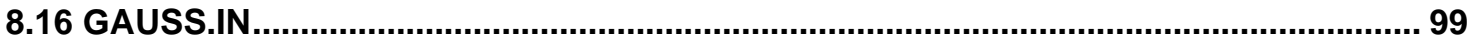

8.17 HEIGHT_RTG............................................................................................................ 100

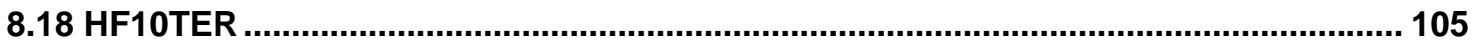

8.19 HF20QWVD ................................................................................................................. 109

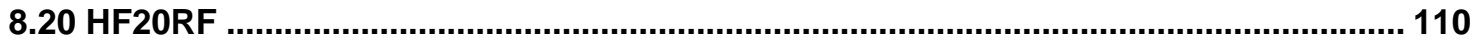

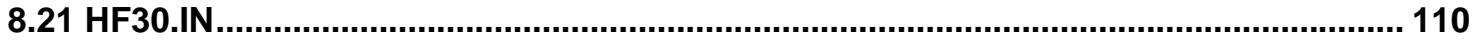

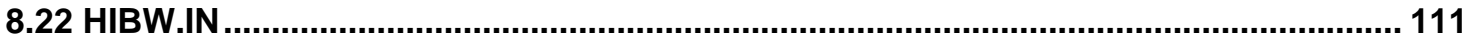

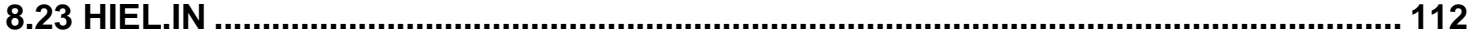

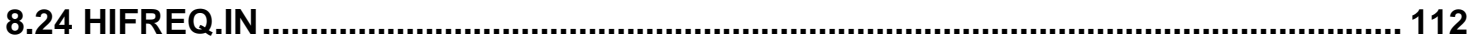

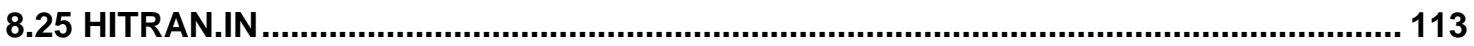

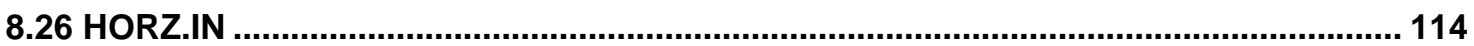

8.27 HTFIND.IN ................................................................................................................ 114

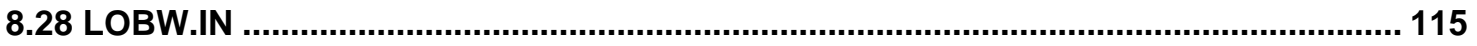




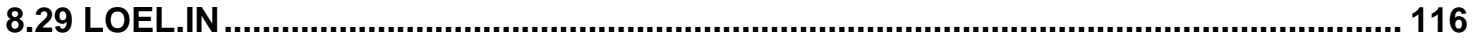

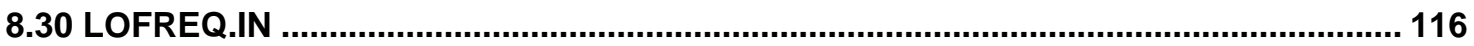

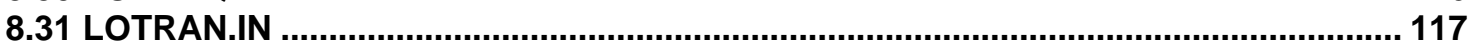

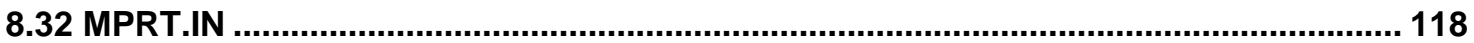

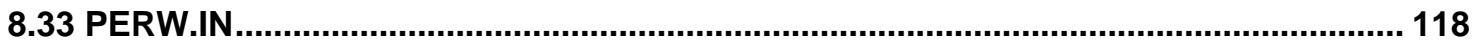

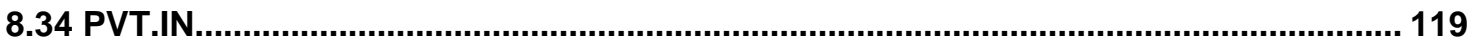

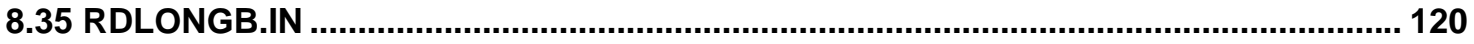

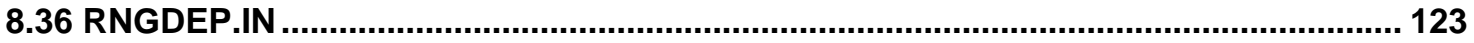

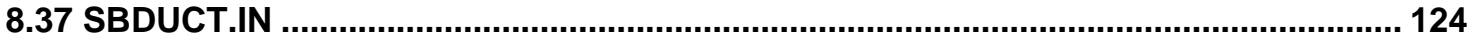

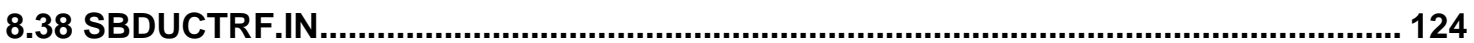

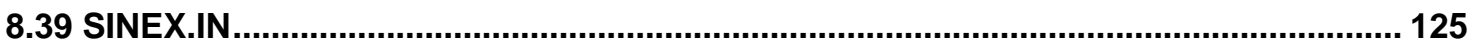

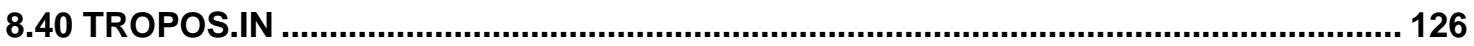

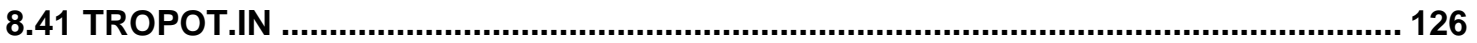

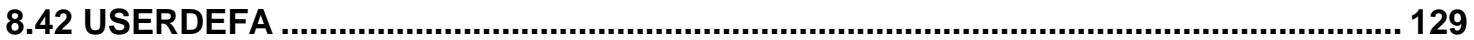

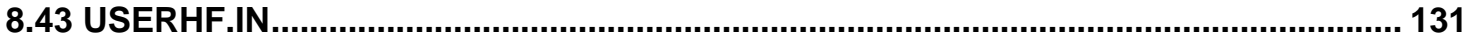

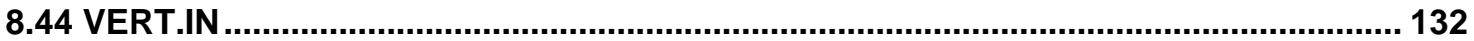

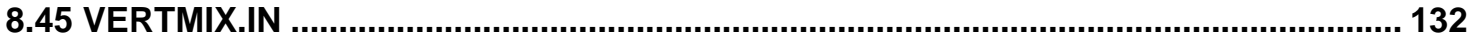

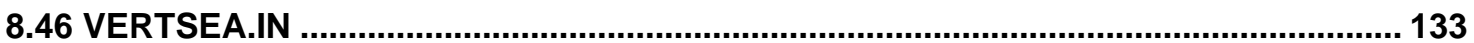

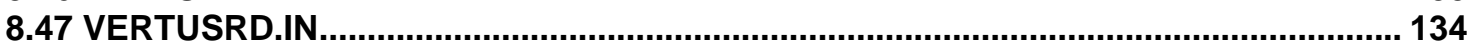

8.48 WEDGE.IN .................................................................................................................. 134

\section{TABLES}

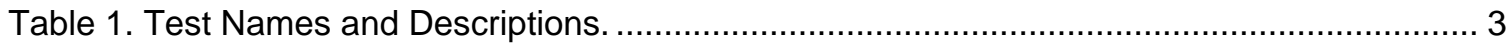

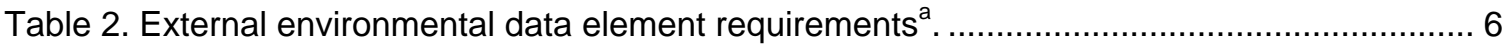

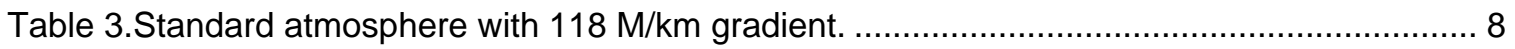

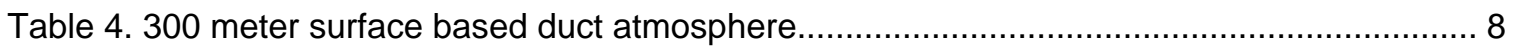

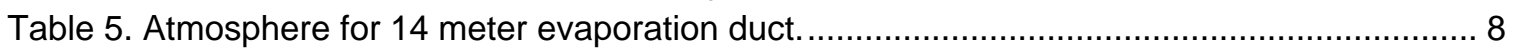

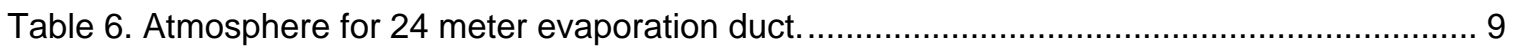

Table 7. Range-dependent atmosphere, standard atmosphere to surface-based duct. ............... 9

Table 8. Range-dependent atmosphere, surface-based duct to high elevated duct. .................... 9

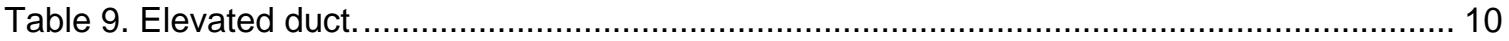

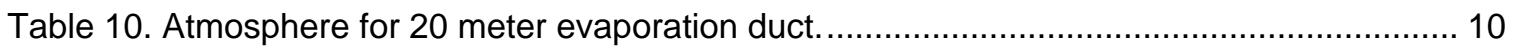

Table 11. External EM system data element requirements. ..................................................... 12

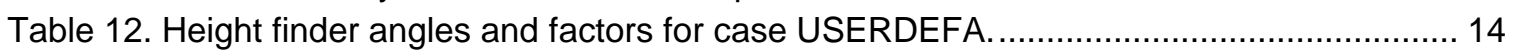

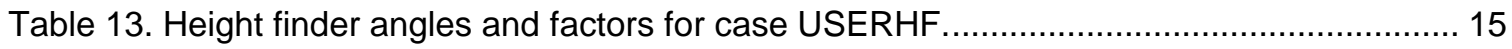

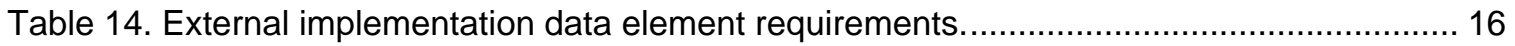

Table 15. Heights relative to ground for test case HEIGHT_RTG. ......................................... 17

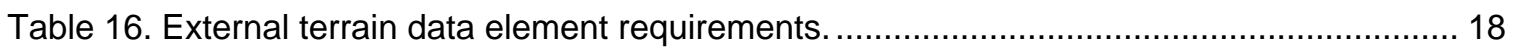

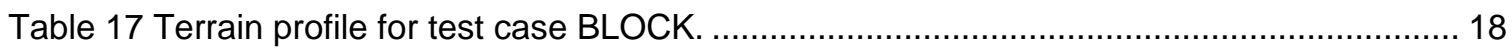

Table 18. Terrain profile for test case CLSBDL ................................................................. 19

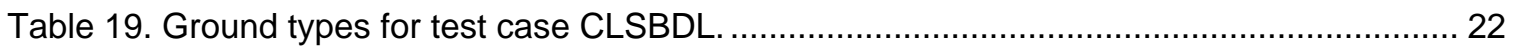

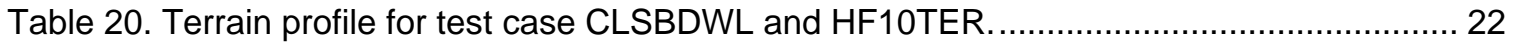

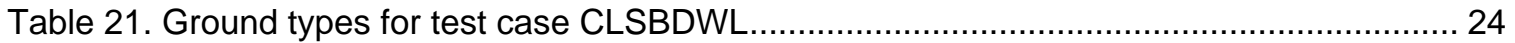

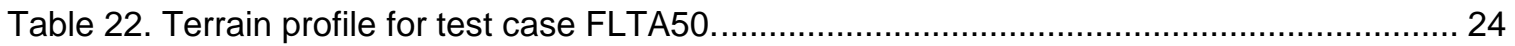

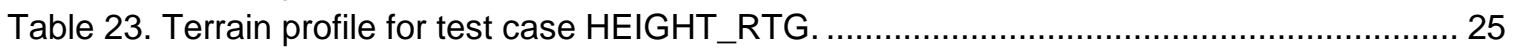

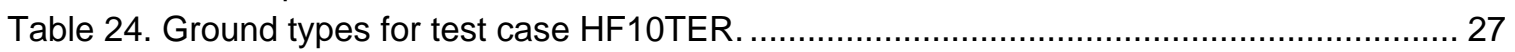

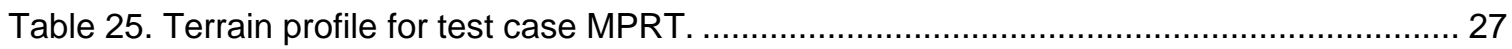

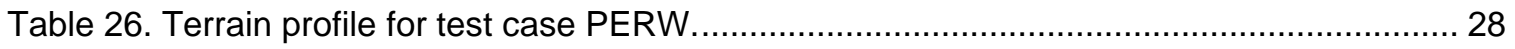

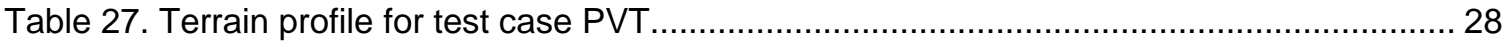




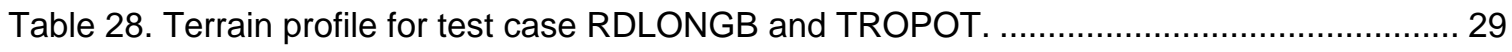

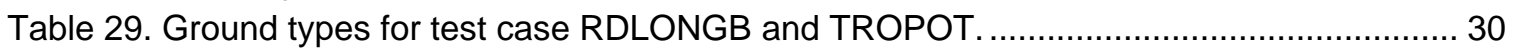

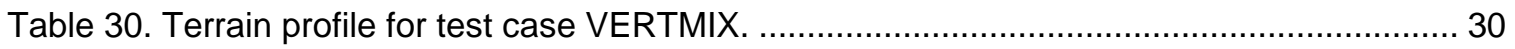

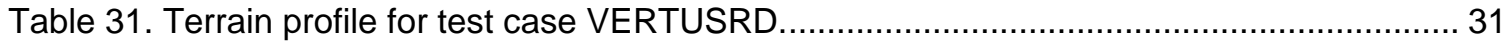

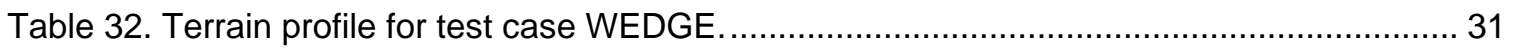

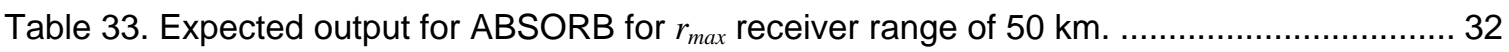

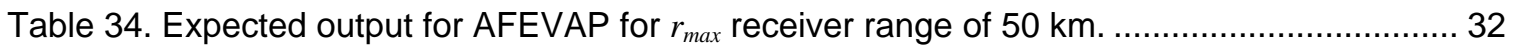

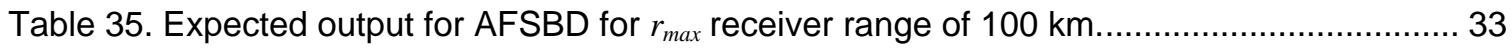

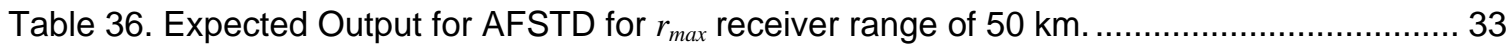

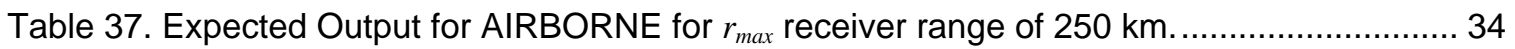

Table 38. Expected output for BLOCK for $r_{\max }$ receiver range of $60 \mathrm{~km}$. ................................... 34

Table 39. Expected output for CLEVAPW for receiver height of 0 and $500 \mathrm{~m}$, plus clutter-to-noise

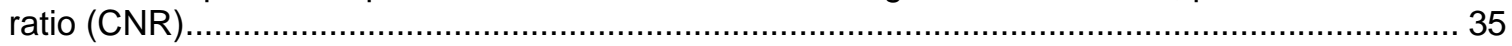

Table 40. Expected output for CLSBDL for receiver height of 500 and $1000 \mathrm{~m}$, plus clutter-to-

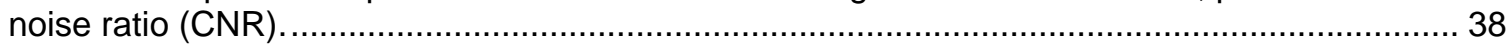

Table 41. Expected output for CLSBDW for receiver height of 500 and $1000 \mathrm{~m}$, plus clutter-to-

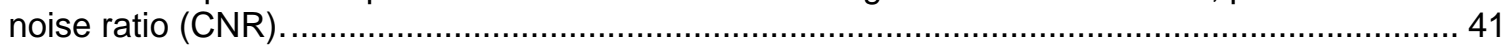

Table 42. Expected output for CLSBDWL for receiver height of 1500 and $3000 \mathrm{~m}$, plus clutter-to-

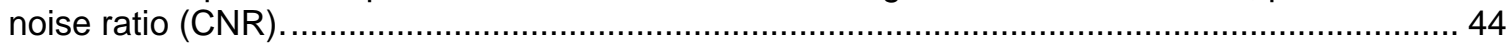

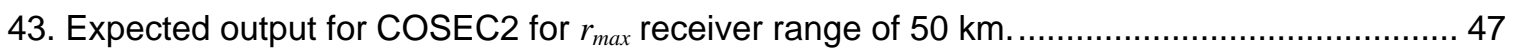

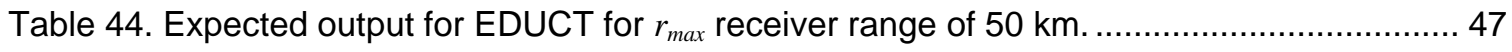

Table 45. Expected output for EDUCTRF for $r_{\max }$ receiver range of $100 \mathrm{~km}$. .......................... 48

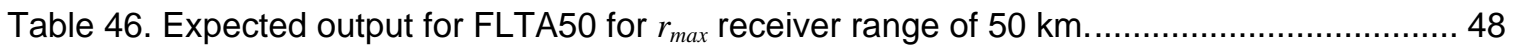

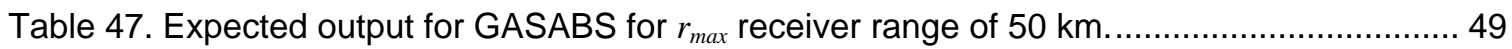

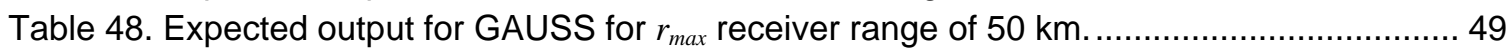

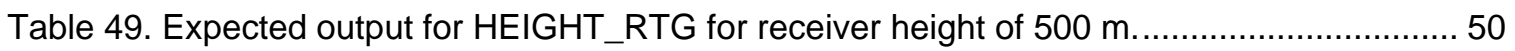

Table 50. Expected output for HF10TER for receiver heights of 0, 1000, and $2000 \mathrm{~m} \ldots \ldots \ldots \ldots . . . . . .51$

Table 51. Expected output for HF20QWVD for $r_{\max }$ receiver range of $100 \mathrm{~km}$. .........................5 52

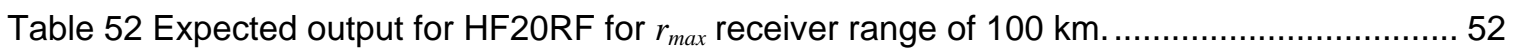

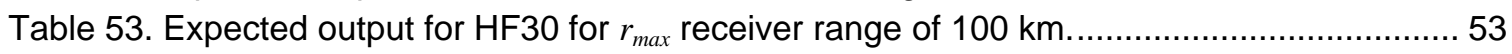

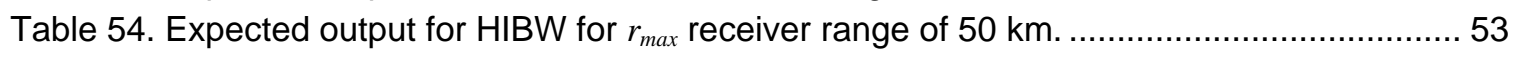

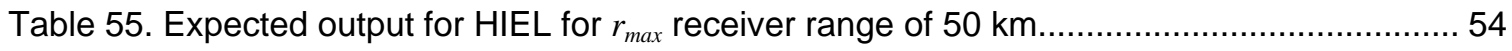

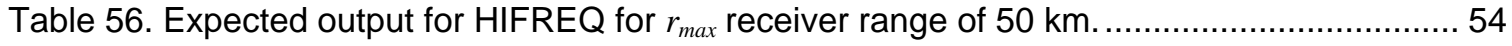

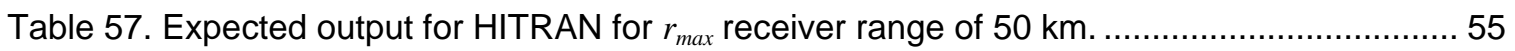

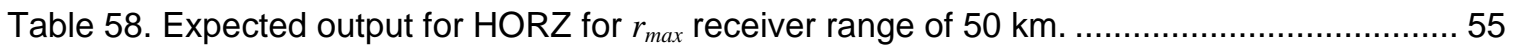

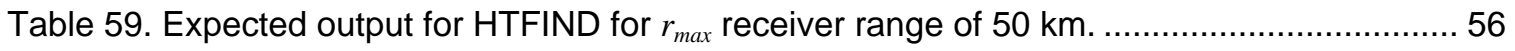

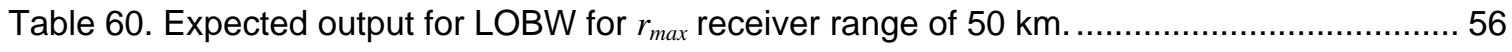

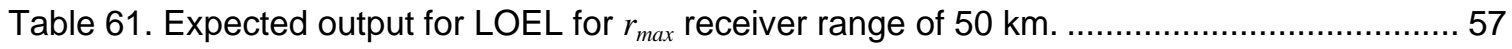

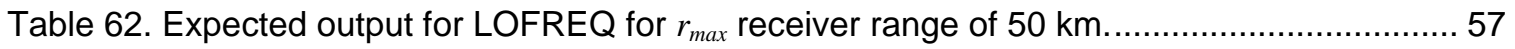

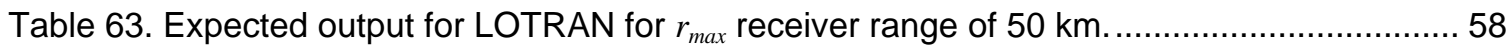

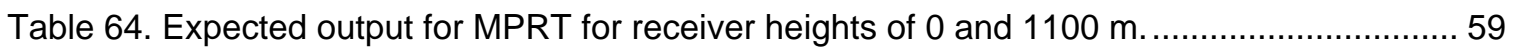

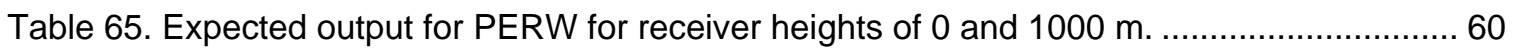

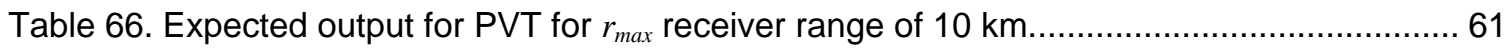

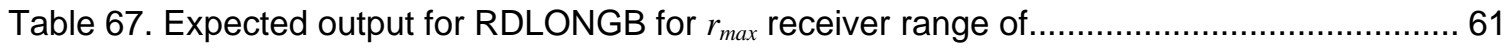

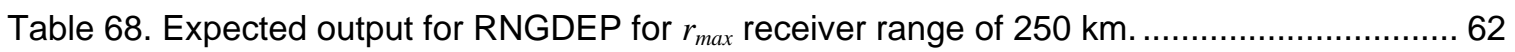

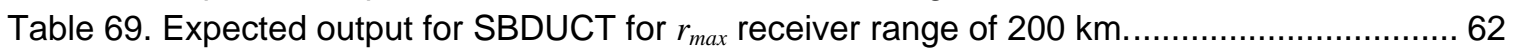

Table 70. Expected output for SBDUCTRF for $r_{\text {max }}$ receiver range of $200 \mathrm{~km}$............................ 63

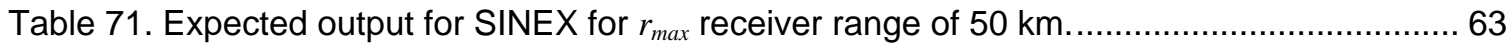




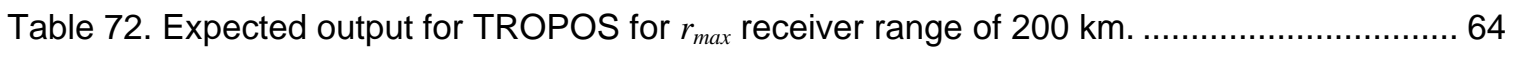

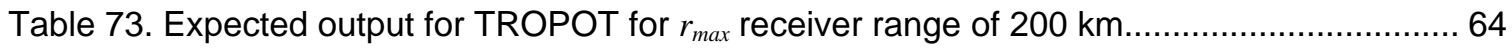

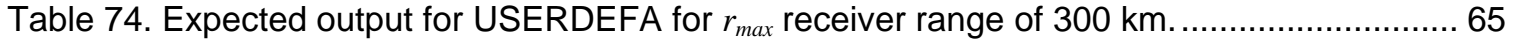

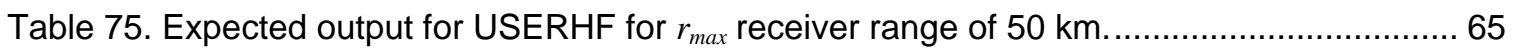

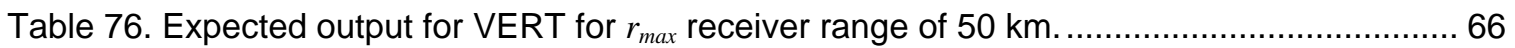

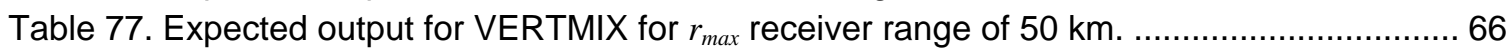

Table 78. Expected output for VERTSEA for $r_{\max }$ receiver range of ...................................... 67

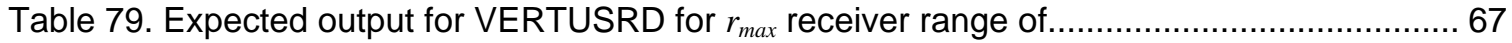

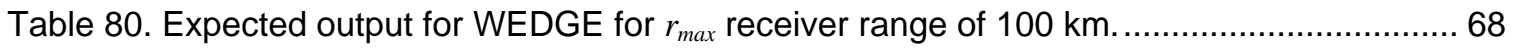

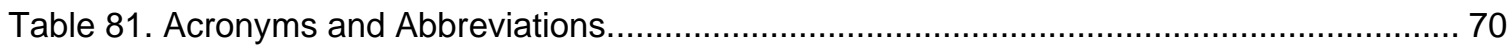




\section{SCOPE}

\subsection{IDENTIFICATION}

Advanced Propagation Model (APM) Version 2.1.04 computer software configuration item (CSCI). The purpose of the APM CSCI is to calculate rangedependent electromagnetic (EM) system propagation loss and propagation factor within a heterogeneous atmospheric medium over variable terrain, where the radio-frequency index of refraction is allowed to vary both vertically and horizontally. Numerous external applications require EM-system propagation loss values. The APM model described by this document may be applied to two such external applications, one which displays propagation loss on a range versus height scale (commonly referred to as a coverage diagram) and one which displays propagation loss on a propagation loss versus range/height scale (commonly referred to as a loss diagram).

\subsection{DOCUMENT OVERVIEW}

This document specifies the test cases and test procedures necessary to perform qualification testing of the APM CSCI. A discussion of precise input values of each input variable required to perform the test together with final expected test results is presented.

\section{REFERENCE DOCUMENTS}

1. Commander-In-Chief, Pacific Fleet Meteorological Requirement (PAC MET) 87-04, "Range Dependent Electromagnetic Propagation Models."

2. Naval Oceanographic Office, "Software Documentation Standards and Coding Requirements for Environmental System Product Development,” April 1990.

3. Space and Naval Warfare Systems Center San Diego (SSC SD), "Software Requirements Specification for the Advanced Propagation Model (APM) CSCI (Version 1.3.1),” Aug. 1998.

4. Space and Naval Warfare Systems Center San Diego (SSC SD), "Software Design Document for the Advanced Propagation Model (APM) CSCI (Version 1.3.1)," Aug. 1998.

5. Space and Naval Warfare Systems Center San Diego (SSC SD), "Software Design Document for the Advanced Propagation Model (APM) CSCI,” TD 3033, Aug. 1998. 


\section{TEST PREPARATIONS}

\subsection{HARDWARE PREPARATION}

Not applicable

\subsection{SOFTWARE PREPARATION}

A short driver program, APMMAIN.F90, is provided in Section 7. This program exercises the main software components, APMINIT CSC, APMSTEP CSC, XOINIT CSC, and XOSTEP CSC that comprise the APM CSCI. The driver program demonstrates how to access the APM CSCI and to exercise the test cases listed in the following sections. It is written to read all necessary input data for the test cases from files in a specific format. All necessary input information is presented in tables in Section 4.3 and the input files for each test case are listed in Section 8.

One of the main features of APM is the use of dynamic allocation in most of the arrays used for numeric calculations and as inputs to the model. The external CSCI application designer must be careful to properly allocate memory and initialize all variable and array inputs to APM. Ultimately, it is the responsibility of the external CSCI application designer to provide the necessary input in the form required by the APM CSCI.

\subsection{OTHER PRETEST PREPARATION}

None.

\section{TEST DESCRIPTIONS}

The test specification for the APM CSCI consists of 48 separate tests that exercise all subroutines and functions of the CSCI. For ease of testing, each of these 48 tests is given a name describing which portion of the APM CSCI is being exercised. All 48 tests and their descriptions are listed in Table 1. 
Table 1. Test Names and Descriptions.

\begin{tabular}{|c|c|}
\hline Test Name & Description \\
\hline ABSORB & Gaseous absorption attenuation rate is specified. \\
\hline AFEVAP & $\begin{array}{l}\text { Enables the computation of propagation angles and factors for an } \\
\text { evaporation duct environment. }\end{array}$ \\
\hline AFSBD & $\begin{array}{l}\text { Enables the computation of propagation angles and factors for a surface- } \\
\text { based duct environment. }\end{array}$ \\
\hline AFSTD & $\begin{array}{l}\text { Enables the computation of propagation angles and factors for a standard } \\
\text { atmosphere. }\end{array}$ \\
\hline AIRBORNE & Airborne platform for antenna height. \\
\hline BLOCK & $\begin{array}{l}\text { The terrain profile consists of a vertical flat-topped block or obstacle in } \\
\text { which the terrain slope is undefined. }\end{array}$ \\
\hline CLEVAPW & $\begin{array}{l}\text { Computes clutter-to-noise ratio for an evaporation duct environment where } \\
\text { the propagation path is entirely over water. }\end{array}$ \\
\hline CLSBDL & $\begin{array}{l}\text { Computes clutter-to-noise ratio for a surface-based duct environment where } \\
\text { the propagation path is entirely over land. }\end{array}$ \\
\hline CLSBDW & $\begin{array}{l}\text { Computes clutter-to-noise ratio for a surface-based duct environment where } \\
\text { the propagation path is entirely over water. }\end{array}$ \\
\hline CLSBDWL & $\begin{array}{l}\text { Computes clutter-to-noise ratio for a surface-based duct environment where } \\
\text { the propagation path is a mixed land-sea path. }\end{array}$ \\
\hline COSEC2 & Antenna pattern is of cosecant-squared type. \\
\hline EDUCT & The refractivity consists of a 14 meter evaporation duct profile. \\
\hline EDUCTRF & $\begin{array}{l}\text { The refractivity consists of a } 14 \text { meter evaporation duct in the presence of } \\
\text { rough seas with wind speed of } 10 \mathrm{~m} / \mathrm{s} \text {. }\end{array}$ \\
\hline FLTA50 & Raised flat land with antenna height of $50 \mathrm{~m}$. \\
\hline GASABS & $\begin{array}{l}\text { The surface absolute humidity and surface air temperature are specified in } \\
\text { order to compute a gaseous absorption attenuation rate. }\end{array}$ \\
\hline GAUSS & Antenna pattern is of Gaussian type. \\
\hline HEIGHT_RTG & $\begin{array}{l}\text { Computes the propagation loss/factor for specific heights relative to the } \\
\text { local ground height. }\end{array}$ \\
\hline HF10TER & HF (10 MHz) emitter where the propagation path is entirely over land. \\
\hline HF20QWVD & $\begin{array}{l}\mathrm{HF}(20 \mathrm{MHz}) \text { emitter with quarter-wave dipole antenna; propagation path is } \\
\text { entirely over water. }\end{array}$ \\
\hline HF20RF & $\begin{array}{l}\mathrm{HF}(20 \mathrm{MHz}) \text { emitter with quarter-wave dipole antenna; propagation path is } \\
\text { over a rough sea surface with a wind speed of } 10 \mathrm{~m} / \mathrm{s} \text {. }\end{array}$ \\
\hline HF30 & HF (30 MHz) emitter over a smooth sea surface. \\
\hline HIBW & Large vertical beamwidth is specified. \\
\hline HIEL & High elevation angle is specified. \\
\hline HIFREQ & High frequency. \\
\hline HITRAN & High transmitter antenna height. \\
\hline HORZ & Horizontal polarization antenna and standard atmosphere. \\
\hline HTFIND & Antenna pattern is of generic height-finder type. \\
\hline LOBW & Small vertical beamwidth is specified. \\
\hline LOEL & Low elevation angle is specified. \\
\hline
\end{tabular}


Table 1. Test Names and Descriptions. (Continued)

\begin{tabular}{|c|c|}
\hline Test Name & Description \\
\hline LOFREQ & Low frequency. \\
\hline LOTRAN & Low transmitter antenna height. \\
\hline MPRT & Mid-path reflection over wedge. \\
\hline PERW & Propagation over rounded wedge using PE model only. \\
\hline PVT & Parabolic valley with short range. \\
\hline RDLONGB & $\begin{array}{l}\text { Range-dependent refractivity over a DTED-extracted terrain profile from } \\
\text { Long Beach to Point Mugu, using vertical polarization and generic ground } \\
\text { composition types. }\end{array}$ \\
\hline RNGDEP & Range-dependent refractivity over smooth earth (over-water case). \\
\hline SBDUCT & 300 meter surface-based duct, over-water case. \\
\hline SBDUCTRF & $\begin{array}{l}\text { Exercises rough surface model for surface-based duct case, with wind } \\
\text { speed of } 10 \mathrm{~m} / \mathrm{s} \text {. }\end{array}$ \\
\hline SINEX & Antenna pattern is of Sine $(X) / X$ type. \\
\hline TROPOS & Exercises troposcatter model for smooth surface (over-water case). \\
\hline TROPOT & Exercises troposcatter model for terrain case. \\
\hline USERDEFA & User-defined antenna pattern with explicit power and angle information. \\
\hline USERHF & $\begin{array}{l}\text { Antenna pattern is of specific height finder type, with user-specified cut- } \\
\text { back angles and power factors. }\end{array}$ \\
\hline VERT & $\begin{array}{l}\text { Vertical polarization antenna is specified (short range over-water case, } \\
\text { standard atmosphere). }\end{array}$ \\
\hline VERTMIX & Vertical polarization antenna over mixed land-sea terrain path. \\
\hline VERTSEA & $\begin{array}{l}\text { Vertical polarization antenna is specified (long range over-water case, } \\
\text { ducting atmosphere). }\end{array}$ \\
\hline VERTUSRD & Vertical polarization antenna and user-specified dielectric ground constants. \\
\hline WEDGE & The terrain profile consists of a triangular wedge. \\
\hline
\end{tabular}

\subsection{REQUIREMENTS ADDRESSED}

Not applicable.

\subsection{PREREQUISITE CONDITIONS}

None.

\subsection{TEST INPUTS}

Although there are actual values for all input parameters listed in the input files in Section 8, some are ignored depending on the values of certain input parameters. Those input parameters that are inapplicable depending on the test case, are listed as "N/A" in the tables. Note that for all test cases, the error flags lerr6 and lerr12 are set to 
“.TRUE.”. These flags allow for extra error control regarding terrain and refractivity inputs. We recommend that these error flags always be set to ".TRUE.". However, we allowed the capability of the external applications designer to bypass these error controls according to the application.

The external environmental data element requirements are listed in Table 2 for each test name, with Table 3 through Table 9 providing specific height and M-unit values. The external EM system data element requirements are listed Table 11. 
Table 2. External environmental data element requirements ${ }^{\mathrm{a}}$.

\begin{tabular}{|c|c|c|c|c|c|c|c|c|c|c|c|}
\hline Test Name & $\begin{array}{c}\text { hmsl; refmsl } \\
\text { Table }\end{array}$ & $n_{\text {prof }}$ & lvlp & $\begin{array}{c}\text { rngprof }^{b} \\
\text { Table }\end{array}$ & $\begin{array}{l}a b s_{\text {hum }} \\
\left(\mathrm{g} / \mathrm{m}^{3}\right)\end{array}$ & $\begin{array}{c}t_{\text {air }} \\
\left({ }^{\circ} \mathrm{C}\right)\end{array}$ & $\begin{array}{c}\gamma_{a} \\
(\mathrm{~dB} / \mathrm{km})\end{array}$ & $n_{w}$ & $\begin{array}{c}\text { rngwind } \\
(\mathrm{km})\end{array}$ & $\begin{array}{l}\text { wind } \\
(\mathrm{m} / \mathrm{s})\end{array}$ & $\begin{array}{l}\text { wind }_{\text {dir }} \\
\text { (deg) }\end{array}$ \\
\hline ABSORB & 3 & 1 & 2 & 0. & 0. & 0. & .146 & 0 & $\mathrm{~N} / \mathrm{A}$ & $\mathrm{N} / \mathrm{A}$ & 0. \\
\hline AFEVAP & 6 & 1 & 17 & 0. & 0. & 0. & 0. & 0 & N/A & $\mathrm{N} / \mathrm{A}$ & 0. \\
\hline AFSBD & 4 & 1 & 4 & 0 . & 0. & 0. & 0. & 0 & $\mathrm{~N} / \mathrm{A}$ & $\mathrm{N} / \mathrm{A}$ & 0. \\
\hline AFSTD & 3 & 1 & 2 & 0. & 0. & 0. & 0. & 0 & $N / A$ & $\mathrm{~N} / \mathrm{A}$ & 0. \\
\hline AIRBORNE & 9 & 1 & 5 & 0. & 0. & 0. & 0. & 0 & N/A & $\mathrm{N} / \mathrm{A}$ & 0. \\
\hline BLOCK & 3 & 1 & 2 & 0. & 7.5 & 0. & 0. & 0 & $\mathrm{~N} / \mathrm{A}$ & $\mathrm{N} / \mathrm{A}$ & 0. \\
\hline CLEVAPW & 10 & 1 & 50 & 0. & 0. & 0. & 0. & 1 & 0. & 10. & 0. \\
\hline CLSBDL & 4 & 1 & 4 & 0. & 0. & 0. & 0. & 0 & $N / A$ & $\mathrm{~N} / \mathrm{A}$ & 0. \\
\hline CLSBDW & 4 & 1 & 4 & 0. & 0. & 0. & 0. & 1 & 0. & 10. & 0. \\
\hline CLSBDWL & 4 & 1 & 4 & 0. & 0. & 0. & 0. & 1 & 0. & 10. & 45. \\
\hline COSEC2 & 3 & 1 & 2 & 0. & 0. & 0. & 0. & 0 & $N / A$ & $N / A$ & 0. \\
\hline EDUCT & 5 & 1 & 21 & 0. & 0. & 0. & 0. & 0 & $\mathrm{~N} / \mathrm{A}$ & $\mathrm{N} / \mathrm{A}$ & 0. \\
\hline EDUCTRF & 5 & 1 & 21 & 0. & 0. & 0. & 0. & 1 & 0. & 10. & 0. \\
\hline FLTA50 & 3 & 1 & 2 & 0. & 0. & 0. & 0. & 0 & N/A & $\mathrm{N} / \mathrm{A}$ & 0. \\
\hline GASABS & 3 & 1 & 2 & 0. & 10. & 25. & 0. & 0 & $N / A$ & $N / A$ & 0. \\
\hline GAUSS & 3 & 1 & 2 & 0. & 0. & 0. & 0. & 0 & $\mathrm{~N} / \mathrm{A}$ & N/A & 0. \\
\hline HEIGHT_RTG & 3 & 1 & 2 & 0. & 0. & 0. & 0. & 0 & N/A & $\mathrm{N} / \mathrm{A}$ & 0. \\
\hline HF10TER & 3 & 1 & 2 & 0. & 0. & 0. & 0. & 0 & N/A & $\mathrm{N} / \mathrm{A}$ & 0. \\
\hline HF20QWVD & 3 & 1 & 2 & 0. & 0. & 0. & 0. & 0 & $N / A$ & $\mathrm{~N} / \mathrm{A}$ & 0. \\
\hline HF20RF & 3 & 1 & 2 & 0. & 0. & 0. & 0. & 1 & 0. & 10. & 0. \\
\hline HF30 & 3 & 1 & 2 & 0. & 0. & 0. & 0. & 0 & N/A & $\mathrm{N} / \mathrm{A}$ & 0. \\
\hline HIBW & 3 & 1 & 2 & 0. & 0. & 0. & 0. & 0 & N/A & $\mathrm{N} / \mathrm{A}$ & 0. \\
\hline HIEL & 3 & 1 & 2 & 0. & 0. & 0. & 0. & 0 & N/A & N/A & 0. \\
\hline HIFREQ & 3 & 1 & 2 & 0. & 0. & 0. & 0. & 0 & N/A & N/A & 0. \\
\hline HITRAN & 3 & 1 & 2 & 0. & 0. & 0. & 0. & 0 & N/A & N/A & 0. \\
\hline HORZ & 3 & 1 & 2 & 0. & 0. & 0. & 0. & 0 & $N / A$ & $N / A$ & 0. \\
\hline
\end{tabular}


Table 2. External environmental data element requirements ${ }^{\mathrm{a}}$. (Continued)

\begin{tabular}{|c|c|c|c|c|c|c|c|c|c|c|c|}
\hline Test Name & $\begin{array}{c}\text { hmsl; refmsl } \\
\text { Table }\end{array}$ & $n_{\text {prof }}$ & lvlp & $\begin{array}{c}\text { rngprof }^{\text {b }} \\
\text { Table }\end{array}$ & $\begin{array}{l}a b s_{\text {hum }} \\
\left(\mathrm{g} / \mathrm{m}^{3}\right)\end{array}$ & $\begin{array}{c}t_{\text {air }} \\
\left({ }^{\circ} \mathrm{C}\right) \\
\end{array}$ & $\begin{array}{c}\gamma_{a} \\
(\mathrm{~dB} / \mathrm{km})\end{array}$ & $n_{w}$ & $\begin{array}{c}\text { rngwind } \\
(\mathrm{km})\end{array}$ & $\begin{array}{l}\text { wind } \\
(\mathrm{m} / \mathrm{s})\end{array}$ & $\begin{array}{l}\text { wind }_{\text {dir }} \\
\text { (deg) }\end{array}$ \\
\hline HTFIND & 3 & 1 & 2 & 0. & 0. & 0 & 0. & 0 & N/A & $\mathrm{N} / \mathrm{A}$ & 0. \\
\hline LOBW & 3 & 1 & 2 & 0. & 0. & 0. & 0. & 0 & N/A & N/A & 0. \\
\hline LOEL & 3 & 1 & 2 & 0. & 0. & 0. & 0. & 0 & $\mathrm{~N} / \mathrm{A}$ & N/A & 0. \\
\hline LOFREQ & 3 & 1 & 2 & 0. & 0. & 0. & 0. & 0 & N/A & $\mathrm{N} / \mathrm{A}$ & 0. \\
\hline LOTRAN & 3 & 1 & 2 & 0. & 0. & 0. & 0. & 0 & N/A & $\mathrm{N} / \mathrm{A}$ & 0. \\
\hline MPRT & 3 & 1 & 2 & 0. & 7.5 & 0. & 0. & 0 & N/A & N/A & 0. \\
\hline PERW & 3 & 1 & 2 & 0. & 7.5 & 0. & 0. & 0 & N/A & $\mathrm{N} / \mathrm{A}$ & 0. \\
\hline PVT & 3 & 1 & 2 & 0. & 7.5 & 0. & 0. & 0 & N/A & $\mathrm{N} / \mathrm{A}$ & 0. \\
\hline RDLONGB & 7 & 2 & 4 & 7 & 0. & 0. & 0. & 0. & $\mathrm{~N} / \mathrm{A}$ & $\mathrm{N} / \mathrm{A}$ & 0. \\
\hline RNGDEP & 8 & 2 & 4 & 8 & 0. & 0. & 0. & 0. & N/A & N/A & 0. \\
\hline SBDUCT & 4 & 1 & 4 & 0. & 0. & 0. & 0. & 0. & N/A & N/A & 0. \\
\hline SBDUCTRF & 4 & 1 & 4 & 0. & 0. & 0. & 0. & 1. & 0. & 10. & 0. \\
\hline SINEX & 3 & 1 & 2 & 0. & 0. & 0. & 0. & 0. & N/A & $\mathrm{N} / \mathrm{A}$ & 0. \\
\hline TROPOS & 3 & 1 & 2 & 0. & 0. & 0. & 0. & 0. & N/A & $\mathrm{N} / \mathrm{A}$ & 0. \\
\hline TROPOT & 3 & 1 & 2 & 0. & 0. & 0. & 0. & 0. & $\mathrm{~N} / \mathrm{A}$ & $\mathrm{N} / \mathrm{A}$ & 0. \\
\hline USERDEFA & 4 & 1 & 4 & 0. & 0. & 0. & 0. & 0. & N/A & $\mathrm{N} / \mathrm{A}$ & 0. \\
\hline USERHF & 3 & 1 & 2 & 0. & 0. & 0. & 0. & 0. & N/A & $\mathrm{N} / \mathrm{A}$ & 0. \\
\hline VERT & 3 & 1 & 2 & 0. & 0. & 0. & 0. & 0. & N/A & $\mathrm{N} / \mathrm{A}$ & 0. \\
\hline VERTMIX & 3 & 1 & 2 & 0. & 0. & 0. & 0. & 0. & N/A & N/A & 0. \\
\hline VERTSEA & 4 & 1 & 4 & 0. & 0. & 0. & 0. & 0. & N/A & $\mathrm{N} / \mathrm{A}$ & 0. \\
\hline VERTUSRD & 3 & 1 & 2 & 0. & 0. & 0. & 0. & 0. & N/A & N/A & 0. \\
\hline WEDGE & 3 & 1 & 2 & 0. & 0. & 0. & 0. & 0. & N/A & $\mathrm{N} / \mathrm{A}$ & \\
\hline
\end{tabular}

${ }^{\mathrm{a}}$ The interpolation flag, iextra, is set to 0 for all test cases.

${ }^{\mathrm{b}}$ The refractivity profile range is in meters except for cases RDLONGB and RNGDEP, which refer to the specific Table number. 
Table 3.Standard atmosphere with $118 \mathrm{M} / \mathrm{km}$ gradient.

\begin{tabular}{c|c|c}
\hline$i$ & $\begin{array}{c}\text { hmsl }_{i, 1} \\
\text { (meters) }\end{array}$ & $\begin{array}{c}\text { refmsl } \\
\text { (M-unit) }\end{array}$ \\
\hline 1 & 0. & 350. \\
2 & 1000. & 468. \\
\hline
\end{tabular}

Table 4. 300 meter surface based duct atmosphere.

\begin{tabular}{c|c|c}
\hline$i$ & $\begin{array}{c}h m s l_{i, 1} \\
\text { (meters) }\end{array}$ & $\begin{array}{c}\text { refmsl }_{i, 1} \\
\text { (M-unit) }\end{array}$ \\
\hline 1 & 0. & 339.0 \\
2 & 250. & 368.5 \\
3 & 300. & 319.0 \\
4 & 1500. & 460.6 \\
\hline
\end{tabular}

Table 5. Atmosphere for 14 meter evaporation duct.

\begin{tabular}{c|c|c}
\hline$i$ & $\begin{array}{c}h_{m s l_{i, 1}} \\
\text { (meters) }\end{array}$ & $\begin{array}{c}\text { refmsl } \\
\text { (M-unit) }\end{array}$ \\
\hline 1 & 0.000 & 339.00 \\
2 & 0.040 & 335.10 \\
3 & 0.100 & 333.66 \\
4 & 0.200 & 332.60 \\
5 & 0.398 & 331.54 \\
6 & 0.794 & 330.51 \\
7 & 1.585 & 329.53 \\
8 & 4.362 & 328.65 \\
9 & 6.310 & 327.96 \\
10 & 12.589 & 327.68 \\
11 & 14.000 & 327.67 \\
12 & 25.119 & 328.13 \\
13 & 39.811 & 329.25 \\
14 & 50.119 & 330.18 \\
15 & 63.096 & 331.44 \\
16 & 79.433 & 334.32 \\
17 & 100.000 & 335.33 \\
18 & 125.893 & 338.20 \\
19 & 158.489 & 341.92 \\
20 & 199.526 & 346.69 \\
21 & 209.526 & 347.87 \\
\hline & &
\end{tabular}


Table 6. Atmosphere for 24 meter evaporation duct.

\begin{tabular}{c|c|c}
\hline$i$ & $\begin{array}{c}\text { hmsl }_{i, 1} \\
\text { (meters) }\end{array}$ & $\begin{array}{c}\text { refmsl } \\
\text { (M-unit) }\end{array}$ \\
\hline 1 & 0.0 & 0.0 \\
2 & 0.135 & -20.40 \\
3 & 0.223 & -21.89 \\
4 & 0.368 & -23.37 \\
5 & 0.607 & -24.84 \\
6 & 1.000 & -26.29 \\
7 & 1.649 & -27.71 \\
8 & 2.718 & -29.08 \\
9 & 4.482 & -30.35 \\
10 & 7.389 & -31.49 \\
11 & 12.182 & -32.39 \\
12 & 20.086 & -32.90 \\
13 & 24.000 & -32.95 \\
14 & 33.115 & -32.78 \\
15 & 54.598 & -31.59 \\
16 & 90.017 & -28.66 \\
17 & 148.413 & -22.86 \\
\hline
\end{tabular}

Table 7. Range-dependent atmosphere, standard atmosphere to surface-based duct.

\begin{tabular}{|c|c|c|c|c|}
\hline \multirow[b]{2}{*}{$i$} & \multicolumn{2}{|c|}{$\begin{array}{c}\text { Standard Atmosphere } \\
\text { rngprof } f_{1}=0 \mathrm{~km}\end{array}$} & \multicolumn{2}{|c|}{$\begin{array}{l}\text { Surface-based Duct } \\
\text { rngprof } f_{2}=100 \mathrm{~km}\end{array}$} \\
\hline & $\begin{array}{c}h^{\prime} m s l_{i, 1} \\
\text { (meters) }\end{array}$ & $\begin{array}{l}\text { refmsl }_{, 1} \\
\text { (M-unit) }\end{array}$ & $\begin{array}{c}h^{h} l_{i, 2} \\
\text { (meters) }\end{array}$ & $\begin{array}{l}\text { refmsl }_{i, 2} \\
\text { (M-unit) }\end{array}$ \\
\hline 1 & 0. & 350. & 0. & 339.0 \\
\hline 2 & 0. & 350. & 250. & 368.5 \\
\hline 3 & 0. & 350. & 300. & 319.0 \\
\hline 4 & 1000. & 468. & 1000. & 401.6 \\
\hline
\end{tabular}

Table 8. Range-dependent atmosphere, surface-based duct to high elevated duct.

\begin{tabular}{|c|c|c|c|c|}
\hline \multirow[b]{2}{*}{$i$} & \multicolumn{2}{|c|}{$\begin{array}{l}\text { Surface-based Duct } \\
\text { rngprof }_{1}=0 . \mathrm{km}\end{array}$} & \multicolumn{2}{|c|}{$\begin{array}{l}\text { High Elevated Duct } \\
\text { rngprof } f_{2}=250 . \mathrm{km}\end{array}$} \\
\hline & $\begin{array}{l}h_{m s l} l_{i, 1} \\
\text { meters }\end{array}$ & $\begin{array}{c}\text { refmsl }_{i, 1} \\
\text { M-unit }\end{array}$ & $\begin{array}{l}\text { hmsl } \\
\text { meters }\end{array}$ & $\begin{array}{l}\text { refmsl }_{i, 2} \\
\text { M-unit }\end{array}$ \\
\hline 1 & 0. & 330. & 0. & 330. \\
\hline 2 & 100. & 342.5 & 600. & 405. \\
\hline 3 & 230. & 312.5 & 730. & 375. \\
\hline 4 & 2000. & 517.8 & 2000. & 522.3 \\
\hline
\end{tabular}


Table 9. Elevated duct.

\begin{tabular}{c|c|c}
\hline$i$ & $\begin{array}{c}h_{m s l_{i, 1}} \\
\text { (meters) }\end{array}$ & $\begin{array}{c}\text { refmsl } \\
\text { (M-unit) }\end{array}$ \\
\hline 1 & 0. & 209.2 \\
2 & 1100. & 339.0 \\
3 & 1500. & 386.2 \\
4 & 1625. & 361.5 \\
5 & 5625. & 833.5 \\
\hline
\end{tabular}

Table 10. Atmosphere for 20 meter evaporation duct.

\begin{tabular}{c|c|c}
\hline$i$ & $\begin{array}{c}h_{m s l}, 1 \\
\text { (meters) }\end{array}$ & $\begin{array}{c}\text { refmsl } \\
\text { (M-unit) }\end{array}$ \\
\hline 1 & 0.000000 & 339.000000 \\
2 & 0.833333 & 318.405284 \\
3 & 1.666667 & 316.841934 \\
4 & 2.500000 & 315.968883 \\
5 & 3.333333 & 315.378476 \\
6 & 4.166667 & 314.942950 \\
7 & 5.000000 & 314.605389 \\
8 & 5.833333 & 314.335435 \\
9 & 6.666667 & 314.114965 \\
10 & 7.500000 & 313.932289 \\
11 & 8.333333 & 313.779427 \\
12 & 9.166667 & 313.650685 \\
13 & 10.000000 & 313.541859 \\
14 & 10.833333 & 313.449758 \\
15 & 11.666667 & 313.371900 \\
16 & 12.500000 & 313.306318 \\
17 & 13.333333 & 313.251426 \\
18 & 14.166667 & 313.205927 \\
19 & 15.000000 & 313.168748 \\
20 & 15.833333 & 313.138987 \\
21 & 16.666667 & 313.115883 \\
22 & 17.500000 & 313.098787 \\
23 & 18.333333 & 313.087139 \\
24 & 19.166667 & 313.080455 \\
25 & 20.000000 & 313.078311 \\
26 & 20.833333 & 313.080339 \\
27 & 21.666667 & 313.086209 \\
28 & 22.500000 & 313.095632 \\
29 & 23.333333 & 313.108350 \\
\hline & & \\
\hline
\end{tabular}


Table 10. Atmosphere for 20 meter evaporation duct (continued).

\begin{tabular}{c|c|c}
\hline$i$ & $\begin{array}{c}\text { hmsl }_{i, 1} \\
\text { (meters) }\end{array}$ & $\begin{array}{c}\text { refmsl } \\
\text { (M-unit) }\end{array}$ \\
\hline 30 & 24.166667 & 313.124130 \\
31 & 25.000000 & 313.142767 \\
32 & 25.833333 & 313.164071 \\
33 & 26.666667 & 313.187874 \\
34 & 27.500000 & 313.214022 \\
35 & 28.333333 & 313.242374 \\
36 & 29.166667 & 313.272804 \\
37 & 30.000000 & 313.305194 \\
38 & 30.833333 & 313.339436 \\
39 & 31.666667 & 313.375432 \\
40 & 32.500000 & 313.413091 \\
41 & 33.333333 & 313.452328 \\
42 & 34.166667 & 313.493066 \\
43 & 35.000000 & 313.535231 \\
44 & 35.833333 & 313.578758 \\
45 & 36.666667 & 313.623583 \\
46 & 37.500000 & 313.669648 \\
47 & 38.333333 & 313.716898 \\
48 & 39.166667 & 313.765283 \\
49 & 40.000000 & 313.814755 \\
50 & 1200.000000 & 444.851829 \\
\hline & &
\end{tabular}


Table 11. External EM system data element requirements.

\begin{tabular}{|c|c|c|c|c|c|c|c|c|c|c|c|c|c|}
\hline Test Name & $\begin{array}{c}f_{M H z} \\
(\mathrm{MHz})\end{array}$ & $\begin{array}{c}a n t_{h t} \\
\text { (meters) }\end{array}$ & $i_{p a t}^{a}$ & $i_{p o l}^{\mathrm{b}}$ & $\begin{array}{c}\mu_{b w} \\
(\text { deg) }\end{array}$ & $\begin{array}{c}\mu_{0} \\
\text { (deg) }\end{array}$ & $C_{\text {lut }}$ & $\begin{array}{l}\text { ant }_{\text {gain }} \\
\text { (dBi) }\end{array}$ & $\begin{array}{c}\theta_{\text {hbw }} \\
\text { (deg) }\end{array}$ & $\begin{array}{c}\tau \\
(\mu \mathrm{sec})\end{array}$ & $\begin{array}{c}N_{f} \\
(\mathrm{~dB})\end{array}$ & $\begin{array}{l}L_{\text {sys }} \\
(\mathrm{dB})\end{array}$ & $\begin{array}{c}P_{t} \\
(\mathrm{~kW})\end{array}$ \\
\hline ABSORB & 20000. & 25. & 1 & 0 & N/A & $\mathrm{N} / \mathrm{A}$ & false. & $N / A$ & $\mathrm{~N} / \mathrm{A}$ & N/A & $\mathrm{N} / \mathrm{A}$ & $\mathrm{N} / \mathrm{A}$ & $\mathrm{N} / \mathrm{A}$ \\
\hline AFEVAP & 3000 & 25 & 1 & 0 & $N / A$ & N/A & false. & $\mathrm{N} / \mathrm{A}$ & $N / A$ & $N / A$ & $\mathrm{~N} / \mathrm{A}$ & $N / A$ & $\mathrm{~N} / \mathrm{A}$ \\
\hline AFSBD & 3000 & 25 & 1 & 0 & N/A & N/A & false. & N/A & N/A & N/A & N/A & $\mathrm{N} / \mathrm{A}$ & N/A \\
\hline AFSTD & 1000 & 25 & 1 & 0 & $N / A$ & $N / A$ & false. & $N / A$ & $N / A$ & $N / A$ & $N / A$ & $N / A$ & $N / A$ \\
\hline AIRBORNE & 900. & 2500. & 1 & 0 & $N / A$ & $N / A$ & false. & $N / A$ & N/A & $N / A$ & N/A & $N / A$ & $N / A$ \\
\hline BLOCK & 1000. & 101. & 1 & 1 & $N / A$ & N/A & false. & N/A & $N / A$ & $N / A$ & $\mathrm{~N} / \mathrm{A}$ & $N / A$ & $N / A$ \\
\hline CLEVAPW & 10,000 . & 25. & 3 & 1 & 2 & 0. & true. & 32. & 1.5 & 1.3 & 10.0 & 8.4 & 285. \\
\hline CLSBDL & 3000. & 15. & 5 & 0 & 1.5 & 0.5 & true. & 39. & 2.0 & 9.0 & 5.5 & 3.0 & 2000. \\
\hline CLSBDW & 3000. & 15. & 5 & 0 & 1.5 & 0.5 & true. & 39. & 2.0 & 9.0 & 5.5 & 3.0 & 2000. \\
\hline CLSBDWL & 5600. & 15. & 3 & 0 & 16. & 0. & true. & 30. & 1.5 & 1.3 & 5.0 & 3.0 & 230. \\
\hline COSEC2 & 1000. & 25. & 4 & 0 & 1. & 0. & false. & $N / A$ & N/A & N/A & N/A & $N / A$ & N/A \\
\hline EDUCT & 10,000 . & 15. & 2 & 0 & 5. & 0. & false. & $N / A$ & N/A & $N / A$ & $N / A$ & $N / A$ & $\mathrm{~N} / \mathrm{A}$ \\
\hline EDUCTRF & 10,000 . & 15. & 2 & 0 & 5. & 0. & false. & N/A & N/A & N/A & $N / A$ & $N / A$ & $N / A$ \\
\hline FLTA50 & 1000. & 50. & 1 & 1 & $N / A$ & N/A & false. & N/A & $N / A$ & N/A & $\mathrm{N} / \mathrm{A}$ & $N / A$ & N/A \\
\hline GASABS & 20,000 . & 25. & 1 & 0 & $N / A$ & N/A & false. & $N / A$ & $N / A$ & $N / A$ & $N / A$ & $N / A$ & $\mathrm{~N} / \mathrm{A}$ \\
\hline GAUSS & 1000. & 25. & 2 & 0 & 1. & 0. & false. & $N / A$ & $N / A$ & N/A & $N / A$ & $N / A$ & $N / A$ \\
\hline HEIGHT_RTG & 162.4 & 54.864 & 1 & 1 & N/A & N/A & false. & N/A & $N / A$ & $N / A$ & $\mathrm{~N} / \mathrm{A}$ & $N / A$ & N/A \\
\hline HF10TER & 10. & 20. & 1 & 1 & $N / A$ & N/A & false. & $N / A$ & N/A & N/A & $N / A$ & $N / A$ & N/A \\
\hline HF20QWVD & 20. & 20. & 8 & 1 & N/A & N/A & false. & N/A & N/A & N/A & N/A & N/A & N/A \\
\hline HF2ORF & 20. & 20. & 8 & 1 & N/A & N/A & false. & N/A & N/A & N/A & N/A & N/A & $\mathrm{N} / \mathrm{A}$ \\
\hline HF30 & 30. & 10. & 1 & 1 & $N / A$ & N/A & false. & $N / A$ & $N / A$ & $N / A$ & $N / A$ & N/A & $N / A$ \\
\hline HIBW & 1000. & 25. & 3 & 0 & 45. & 0. & false. & $N / A$ & N/A & N/A & N/A & $N / A$ & $\mathrm{~N} / \mathrm{A}$ \\
\hline HIEL & 1000. & 25. & 2 & 0 & 1. & 10. & false. & $\mathrm{N} / \mathrm{A}$ & $N / A$ & $N / A$ & $\mathrm{~N} / \mathrm{A}$ & N/A & $N / A$ \\
\hline HIFREQ & 20,000 . & 25. & 1 & 0 & N/A & N/A & false. & N/A & N/A & N/A & $\mathrm{N} / \mathrm{A}$ & N/A & N/A \\
\hline HITRAN & 1000. & 100. & 1 & 0 & N/A & $N / A$ & false. & N/A & N/A & $N / A$ & N/A & N/A & N/A \\
\hline HORZ & 1000. & 25. & 1 & 0 & N/A & N/A & false. & N/A & N/A & N/A & N/A & $N / A$ & N/A \\
\hline HTFIND & 1000. & 25. & 5 & 0 & 2. & 0. & false. & N/A & N/A & N/A & $\mathrm{N} / \mathrm{A}$ & N/A & N/A \\
\hline LOBW & 1000. & 25. & 2 & 0 & 0.5 & 0. & false. & N/A & $N / A$ & N/A & $\mathrm{N} / \mathrm{A}$ & N/A & $\mathrm{N} / \mathrm{A}$ \\
\hline LOEL & 1000. & 25. & 2 & 0 & 1. & -10. & false. & $N / A$ & $N / A$ & $N / A$ & $N / A$ & $N / A$ & N/A \\
\hline LOFREQ & 100. & 25. & 1 & 0 & N/A & N/A & false. & N/A & N/A & N/A & N/A & N/A & N/A \\
\hline LOTRAN & 1000. & 1.5 & 1 & 0 & N/A & $N / A$ & false. & N/A & N/A & N/A & $N / A$ & N/A & N/A \\
\hline
\end{tabular}


Table 11. External EM system data element requirements (continued).

\begin{tabular}{|c|c|c|c|c|c|c|c|c|c|c|c|c|c|}
\hline Test Name & $\begin{array}{c}f_{M H z} \\
(\mathrm{MHz})\end{array}$ & $\begin{array}{c}a n t_{h t} \\
\text { (meters) }\end{array}$ & $i_{\text {pat }}{ }^{a}$ & $i_{p o l}^{\mathrm{b}}$ & $\begin{array}{c}\mu_{b w} \\
\text { (deg) }\end{array}$ & $\begin{array}{c}\mu_{0} \\
\text { (deg) }\end{array}$ & $C_{\text {lut }}$ & $\begin{array}{l}\text { ant }_{\text {gain }} \\
\text { (dBi) }\end{array}$ & $\begin{array}{c}\theta_{\text {hbw }} \\
\text { (deg) }\end{array}$ & $\begin{array}{c}\tau \\
(\mu \mathrm{sec})\end{array}$ & $\begin{array}{c}N_{f} \\
(\mathrm{~dB})\end{array}$ & $\begin{array}{l}L_{\text {sys }} \\
(\mathrm{dB})\end{array}$ & $\begin{array}{c}P_{t} \\
(\mathrm{~kW})\end{array}$ \\
\hline MPRT & 300. & 800. & 2 & 1 & 0.5 & -2.5 & false. & $\mathrm{N} / \mathrm{A}$ & $\mathrm{N} / \mathrm{A}$ & $N / A$ & $\mathrm{~N} / \mathrm{A}$ & $\mathrm{N} / \mathrm{A}$ & $\mathrm{N} / \mathrm{A}$ \\
\hline PERW & 300 & 10. & 1 & 1 & $N / A$ & N/A & false. & $\mathrm{N} / \mathrm{A}$ & N/A & N/A & $\mathrm{N} / \mathrm{A}$ & $\mathrm{N} / \mathrm{A}$ & $\mathrm{N} / \mathrm{A}$ \\
\hline PVT & 500. & 10. & 1 & 1 & N/A & N/A & false. & $\mathrm{N} / \mathrm{A}$ & $N / A$ & $N / A$ & N/A & N/A & $\mathrm{N} / \mathrm{A}$ \\
\hline RDLONGB & 150. & 100. & 1 & 0 & $\mathrm{~N} / \mathrm{A}$ & $N / A$ & false. & $\mathrm{N} / \mathrm{A}$ & N/A & N/A & $\mathrm{N} / \mathrm{A}$ & $\mathrm{N} / \mathrm{A}$ & $\mathrm{N} / \mathrm{A}$ \\
\hline RNGDEP & 3000. & 25. & 1 & 0 & N/A & $\mathrm{N} / \mathrm{A}$ & false. & $\mathrm{N} / \mathrm{A}$ & $N / A$ & N/A & N/A & $\mathrm{N} / \mathrm{A}$ & N/A \\
\hline SBDUCT & 3000. & 25. & 2 & 0 & 5. & 0. & false. & $N / A$ & $N / A$ & $N / A$ & $N / A$ & $N / A$ & $N / A$ \\
\hline SBDUCTRF & 3000. & 25. & 2 & 1 & 5. & 0. & false. & $\mathrm{N} / \mathrm{A}$ & $N / A$ & N/A & $\mathrm{N} / \mathrm{A}$ & $\mathrm{N} / \mathrm{A}$ & $\mathrm{N} / \mathrm{A}$ \\
\hline SINEX & 1000. & 25. & 3 & 0 & 1. & 0. & false. & $\mathrm{N} / \mathrm{A}$ & N/A & N/A & N/A & $\mathrm{N} / \mathrm{A}$ & $\mathrm{N} / \mathrm{A}$ \\
\hline TROPOS & 100. & 25. & 1 & 0 & $\mathrm{~N} / \mathrm{A}$ & N/A & false. & $\mathrm{N} / \mathrm{A}$ & N/A & N/A & $\mathrm{N} / \mathrm{A}$ & N/A & N/A \\
\hline TROPOT & 100. & 25. & 1 & 0 & $\mathrm{~N} / \mathrm{A}$ & $N / A$ & false. & $N / A$ & N/A & N/A & $N / A$ & N/A & $N / A$ \\
\hline USERDEFA ${ }^{c}$ & 900. & 6. & 7 & 0 & $N / A$ & $N / A$ & false. & $\mathrm{N} / \mathrm{A}$ & N/A & $\mathrm{N} / \mathrm{A}$ & $\mathrm{N} / \mathrm{A}$ & $\mathrm{N} / \mathrm{A}$ & $\mathrm{N} / \mathrm{A}$ \\
\hline USERHF $^{d}$ & 1000. & 25. & 6 & 0 & 1. & 0. & false. & $N / A$ & $N / A$ & $N / A$ & $N / A$ & $\mathrm{~N} / \mathrm{A}$ & $N / A$ \\
\hline VERT & 1000. & 25. & 1 & 1 & N/A & $N / A$ & false. & $\mathrm{N} / \mathrm{A}$ & N/A & N/A & $\mathrm{N} / \mathrm{A}$ & $\mathrm{N} / \mathrm{A}$ & $\mathrm{N} / \mathrm{A}$ \\
\hline VERTMIX & 100. & 10. & 1 & 1 & $N / A$ & $N / A$ & false. & $N / A$ & $N / A$ & $N / A$ & $N / A$ & $\mathrm{~N} / \mathrm{A}$ & $N / A$ \\
\hline VERTSEA & 100. & 25. & 1 & 1 & N/A & N/A & false. & $\mathrm{N} / \mathrm{A}$ & N/A & N/A & $\mathrm{N} / \mathrm{A}$ & $\mathrm{N} / \mathrm{A}$ & $\mathrm{N} / \mathrm{A}$ \\
\hline VERTUSRD & 100. & 10. & 1 & 1 & $\mathrm{~N} / \mathrm{A}$ & N/A & false. & $\mathrm{N} / \mathrm{A}$ & N/A & N/A & $\mathrm{N} / \mathrm{A}$ & N/A & $\mathrm{N} / \mathrm{A}$ \\
\hline WEDGE & 1000. & 25. & 1 & 0 & N/A & $N / A$ & false. & N/A & $N / A$ & $N / A$ & $N / A$ & $N / A$ & $\mathrm{~N} / \mathrm{A}$ \\
\hline
\end{tabular}

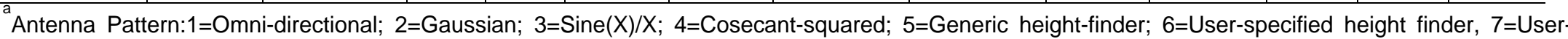
defined; 8=Quarter-wave dipole antenna pattern.

${ }^{b}$ Polarization: 0=Horizontal; $1=$ Vertical

${ }^{\mathrm{C}}$ See Table 12 for $h f f a n g$ and $h f f a c$ parameters $\left(n_{\text {facs }}=54\right)$..

${ }^{\mathrm{d}}$ See Table 13 for $h$ ffang and $h$ ffac parameters $\left(n_{\text {facs }}=10\right)$. 
Table 12. Height finder angles and factors for case USERDEFA.

\begin{tabular}{|c|c|c|}
\hline$i$ & $\begin{array}{l}\text { hfang }_{i} \\
\text { (deg) }\end{array}$ & hffac \\
\hline 1 & -17 & .017 \\
\hline 2 & -16 & .044 \\
\hline 3 & -15 & .080 \\
\hline 4 & -14 & .126 \\
\hline 5 & -13 & .182 \\
\hline 6 & -12 & .245 \\
\hline 7 & -11 & .316 \\
\hline 8 & -10 & .389 \\
\hline 9 & -9 & .479 \\
\hline 10 & -8 & .556 \\
\hline 11 & -7 & .631 \\
\hline 12 & -6 & .716 \\
\hline 13 & -5 & .785 \\
\hline 14 & -4 & .861 \\
\hline 15 & -3 & .912 \\
\hline 16 & -2 & .966 \\
\hline 17 & -1 & .998 \\
\hline 18 & 0 & 1.00 \\
\hline 19 & 1 & 1.00 \\
\hline 20 & 2 & .966 \\
\hline 21 & 3 & .902 \\
\hline 22 & 4 & .822 \\
\hline 23 & 5 & .742 \\
\hline 24 & 6 & .646 \\
\hline 25 & 7 & .569 \\
\hline 26 & 8 & .501 \\
\hline 27 & 9 & .452 \\
\hline 28 & 10 & .422 \\
\hline 29 & 11 & .402 \\
\hline 30 & 12 & .389 \\
\hline 31 & 13 & .375 \\
\hline 32 & 14 & .359 \\
\hline 33 & 15 & .339 \\
\hline 34 & 16 & .305 \\
\hline 35 & 17 & .276 \\
\hline 36 & 18 & .245 \\
\hline 37 & 19 & .221 \\
\hline 38 & 20 & .210 \\
\hline 39 & 21 & .199 \\
\hline 40 & 22 & .190 \\
\hline 41 & 23 & .180 \\
\hline
\end{tabular}


Table 12. Antenna pattern angles and factors for case USERDEFA (continued).

\begin{tabular}{c|c|c}
\hline$i$ & $\begin{array}{c}h f_{\text {fang }} \\
\text { (deg) }\end{array}$ & hffac $_{i}$ \\
\hline 42 & 24 & .164 \\
43 & 25 & .148 \\
44 & 26 & .130 \\
45 & 27 & .110 \\
46 & 28 & .095 \\
47 & 29 & .077 \\
48 & 30 & .070 \\
49 & 31 & .065 \\
50 & 32 & .058 \\
51 & 33 & .050 \\
52 & 34 & .039 \\
53 & 35 & .031 \\
54 & 36 & .025 \\
\hline
\end{tabular}

Table 13. Height finder angles and factors for case USERHF.

\begin{tabular}{c|c|c}
\hline$i$ & $\begin{array}{c}\text { hfang } \\
\text { (deg) }\end{array}$ & hffac $_{i}$ \\
\hline 1 & 1.0 & 0.9 \\
2 & 1.5 & 0.8 \\
3 & 2.0 & 0.7 \\
4 & 2.5 & 0.6 \\
5 & 3.0 & 0.5 \\
6 & 3.5 & 0.4 \\
7 & 4.0 & 0.3 \\
8 & 4.5 & 0.2 \\
9 & 5.0 & 0.1 \\
10 & 5.5 & 0.0 \\
\hline
\end{tabular}

The external implementation data element requirements that must be specified for each test are listed in Table 14. For all cases except those noted, $T_{\text {ropo }}$ is '.true.', and $h_{\text {min }}$ is 0.0 meters. Note: the lang flag should only be used for over-water propagation paths and should not be enabled for cases where any portion of the path is over land. 
Table 14. External implementation data element requirements.

\begin{tabular}{|c|c|c|c|c|c|c|c|c|c|c|}
\hline Test Name & $\begin{array}{c}h_{\max } \\
\text { (meters) }\end{array}$ & $n_{\text {rout }}$ & $n_{\text {zout }}$ & $P E_{\text {flag }}$ & $\begin{array}{r}r_{\max } \\
(\mathrm{km})\end{array}$ & $r_{\text {mult }}$ & $\begin{array}{r}t h_{\max } \\
\text { (deg) }\end{array}$ & lerr6 & lerr12 & lang \\
\hline ABSORB & 200. & 1 & 20 & false. & 50. & $N / A$ & $N / A$ & true & true. & false. \\
\hline AFEVAP & 1000. & 1 & 20 & false. & 50. & N/A & N/A & true & true. & true. \\
\hline AFSBD & 3000. & 1 & 20 & false. & 100. & N/A & N/A & .true & true. & true. \\
\hline AFSTD & 1000. & 1 & 20 & false. & 50. & N/A & N/A & true & true. & true. \\
\hline AIRBORNE & 5000. & 1 & 20 & false. & 250. & N/A & N/A & true & true. & false. \\
\hline BLOCK & 400. & 1 & 20 & false. & 60. & N/A & $N / A$ & true & true. & false. \\
\hline CLEVAPW & 1000. & 100 & 2 & false. & 100. & N/A & $N / A$ & false. & true. & false. \\
\hline CLSBDL & 1000. & 100 & 2 & false. & 100. & N/A & N/A & false. & true. & false. \\
\hline CLSBDW & 1000. & 100 & 2 & false. & 100. & N/A & N/A & false. & true. & false. \\
\hline CLSBDWL & 3000. & 100 & 2 & false. & 200. & $\mathrm{~N} / \mathrm{A}$ & $N / A$ & false. & true. & false. \\
\hline COSEC2 & 2000. & 1 & 20 & false. & 50. & N/A & N/A & true. & .true. & false. \\
\hline EDUCT & 200. & 1 & 20 & false. & 50. & N/A & N/A & true. & true. & false. \\
\hline EDUCTRF & 200. & 1 & 20 & false. & 100. & N/A & N/A & true. & true. & false. \\
\hline FLTA50 & 100. & 1 & 20 & false. & 50. & N/A & $N / A$ & true. & true. & false. \\
\hline GASABS & 200. & 1 & 20 & false. & 50. & N/A & N/A & true. & true. & false. \\
\hline GAUSS & 2000. & 1 & 20 & false. & 50. & N/A & N/A & true. & true. & false. \\
\hline HEIGHT_RTG ${ }^{a}$ & 500. & 20 & 1 & false. & 18.41 & $\mathrm{~N} / \mathrm{A}$ & $N / A$ & false. & true. & false. \\
\hline HF10TER & 2000. & 30 & 2 & false. & 249. & N/A & N/A & true. & true. & false. \\
\hline HF20QWVD & 1000. & 1 & 20 & false. & 100. & N/A & N/A & true. & true. & false. \\
\hline HF20RF & 1000. & 1 & 20 & false. & 100. & N/A & N/A & true. & true. & false. \\
\hline HF30 & 1000. & 1 & 20 & false. & 100. & N/A & N/A & true. & true. & false. \\
\hline HIBW & 2000. & 1 & 20 & false. & 50. & N/A & N/A & .true. & .true. & false. \\
\hline HIEL & 20,000 & 1 & 20 & false. & 50. & N/A & N/A & true. & true. & false. \\
\hline HIFREQ & 200. & 1 & 20 & false. & 50. & N/A & N/A & true. & .true. & false. \\
\hline HITRAN & 1000. & 1 & 20 & false. & 50. & N/A & N/A & true. & true. & false. \\
\hline HORZ & 2000. & 1 & 20 & false. & 50. & N/A & N/A & true. & true. & false. \\
\hline HTFIND & 2000. & 1 & 20 & false. & 50. & N/A & N/A & true. & true. & false. \\
\hline LOBW & 2000. & 1 & 20 & false. & 50. & N/A & N/A & true. & true. & false. \\
\hline LOEL & 20,000 & 1 & 20 & false. & 50. & $N / A$ & $N / A$ & true. & true. & false. \\
\hline LOFREQ & 5000. & 1 & 20 & false. & 50. & N/A & N/A & true. & true. & false. \\
\hline LOTRAN & 10,000 & 1 & 20 & false. & 50. & N/A & N/A & true. & true. & false. \\
\hline MPRT & 1100. & 30 & 1 & false. & 60. & $\mathrm{~N} / \mathrm{A}$ & N/A & true. & true. & false. \\
\hline PERW & 1000. & 20 & 1 & true. & 50. & 1. & 10. & .true. & .true. & false. \\
\hline PVT & 2000. & 1 & 20 & false. & 10. & N/A & N/A & true. & true. & false. \\
\hline RDLONGB & 1000. & 1 & 20 & false. & 100. & N/A & N/A & true. & true. & false. \\
\hline RNGDEP & 2000. & 1 & 20 & false. & 250. & N/A & N/A & true. & true. & false. \\
\hline SBDUCT & 5000. & 1 & 20 & false. & 200. & N/A & N/A & true. & true. & false. \\
\hline SBDUCTRF & 1000. & 1 & 20 & false. & 200. & $N / A$ & $N / A$ & .true. & .true. & false. \\
\hline
\end{tabular}


Table 14. External implementation data element requirements (continued).

\begin{tabular}{|c|c|c|c|c|c|c|c|c|c|c|}
\hline Test Name & $\begin{array}{c}h_{\max } \\
\text { (meters) }\end{array}$ & $n_{\text {rout }}$ & $n_{\text {zout }}$ & $P E_{\text {flag }}$ & $\begin{array}{c}r_{\max } \\
(\mathrm{km})\end{array}$ & $r_{\text {mult }}$ & $\begin{array}{c}t h_{\max } \\
\text { (deg) }\end{array}$ & lerr6 & lerr12 & lang \\
\hline SINEX & 2000. & 1 & 20 & false. & 50. & $N / A$ & N/A & true. & .true. & false. \\
\hline TROPOS $^{b}$ & 2000. & 1 & 20 & false. & 200. & N/A & $N / A$ & true. & true. & false. \\
\hline TROPOT $^{b}$ & 2000. & 1 & 20 & false. & 200. & $\mathrm{~N} / \mathrm{A}$ & N/A & true. & true. & false. \\
\hline USERDEFA & 3000. & 1 & 20 & false. & 300. & $N / A$ & N/A & true. & true. & false. \\
\hline USERHF & 2000. & 1 & 20 & false. & 50. & $N / A$ & $\mathrm{~N} / \mathrm{A}$ & true. & true. & false. \\
\hline VERT & 2000. & 1 & 20 & false. & 50. & N/A & N/A & true. & true. & false. \\
\hline VERTMIX & 1000. & 1 & 20 & false. & 50. & N/A & N/A & true. & true. & false. \\
\hline VERTSEA & 1000. & 1 & 20 & false. & 300. & N/A & $N / A$ & true. & true. & false. \\
\hline VERTUSRD & 1000. & 1 & 20 & false. & 50. & $N / A$ & N/A & .true. & true. & false. \\
\hline WEDGE & 1000. & 1 & 20 & false. & 100. & $N / A$ & N/A & true. & true. & false. \\
\hline
\end{tabular}

Table 15. Heights relative to ground for test case HEIGHT_RTG.

\begin{tabular}{c|c}
\hline$i$ & $\begin{array}{c}\text { zout_rtg } \\
\text { (meters) }\end{array}$ \\
\hline 1 & 1.2 \\
2 & 2.5 \\
3 & 5.1 \\
\hline
\end{tabular}

The external terrain data element requirements are only applicable to select test cases and are listed below in Table 16. All test cases where no terrain profile is specified implies the propagation path is entirely over sea water. Terrain profiles used for these specific test cases are listed in Table 17 through Table 32. 
Table 16. External terrain data element requirements.

\begin{tabular}{|c|c|c|c|c|c|c|c|c|c|}
\hline Test Name & $\begin{array}{c}\text { terx, tery } \\
\text { Table }\end{array}$ & $i_{t p}$ & $i_{g r}$ & igrnd & $\begin{array}{c}\text { rgrnd } \\
(\mathrm{km})\end{array}$ & $\begin{array}{l}\text { dielec } \\
\left(\varepsilon_{r} \sigma\right)^{\mathrm{a}}\end{array}$ & $i_{g c}$ & $\begin{array}{c}\gamma c \\
(\mathrm{~dB})\end{array}$ & $\begin{array}{l}\gamma r n g \\
(\mathrm{~km})\end{array}$ \\
\hline BLOCK & Table 17 & 6 & 1 & 7 & 0. & $(7.5, .01)$ & $N / A$ & $N / A$ & $\mathrm{~N} / \mathrm{A}$ \\
\hline CLSBDL & Table 18 & 374 & 2 & Table 19 & Table 19 & $N / A$ & 2 & Table 19 & Table 19 \\
\hline CLSBDWL & Table 20 & 265 & 2 & Table 21 & Table 21 & $N / A$ & 2 & Table 21 & Table 21 \\
\hline FLTA50 & Table 22 & 2 & 1 & 7 & 0. & $(7.0, .01)$ & $\mathrm{N} / \mathrm{A}$ & $N / A$ & $N / A$ \\
\hline HEIGHT_RTG & Table 23 & 304 & 1 & 3 & 0. & N/A & N/A & N/A & N/A \\
\hline HF10TER & Table 20 & 265 & 2 & Table 24 & Table 24 & Table 24 & $\mathrm{~N} / \mathrm{A}$ & $N / A$ & $N / A$ \\
\hline MPRT & Table 25 & 5 & 1 & 7 & 0. & $(7.5,0.01)$ & $N / A$ & $N / A$ & $N / A$ \\
\hline PERW & Table 26 & 11 & 1 & 7 & 0. & $(7.5,0.01)$ & $N / A$ & $N / A$ & $N / A$ \\
\hline PVT & Table 27 & 17 & 1 & 7 & 0. & $(7.5,0.01)$ & N/A & $\mathrm{N} / \mathrm{A}$ & $\mathrm{N} / \mathrm{A}$ \\
\hline RDLONGB & Table 28 & 167 & 6 & Table 29 & Table 29 & $N / A$ & $\mathrm{~N} / \mathrm{A}$ & N/A & N/A \\
\hline TROPOT & Table 28 & 167 & 6 & Table 29 & Table 29 & $N / A$ & $N / A$ & N/A & N/A \\
\hline VERTMIX & Table 30 & 2 & 2 & Table 30 & Table 30 & N/A & $N / A$ & $\mathrm{~N} / \mathrm{A}$ & $\mathrm{N} / \mathrm{A}$ \\
\hline VERTUSRD & Table 31 & 2 & 1 & 7 & 0. & $(3 ., 6 e-4)$ & $N / A$ & $\mathrm{~N} / \mathrm{A}$ & $\mathrm{N} / \mathrm{A}$ \\
\hline WEDGE & Table 32 & 5 & 1 & 0 & 0. & $\mathrm{~N} / \mathrm{A}$ & $\mathrm{N} / \mathrm{A}$ & $\mathrm{N} / \mathrm{A}$ & $\mathrm{N} / \mathrm{A}$ \\
\hline
\end{tabular}

Table 17 Terrain profile for test case BLOCK.

\begin{tabular}{c|c|c}
\hline$i$ & $\begin{array}{c}\text { ter } x_{i} \\
(\mathrm{~km})\end{array}$ & $\begin{array}{c}\text { tery } \\
\text { (meters) }\end{array}$ \\
\hline 1 & 0. & 1. \\
2 & 10. & 1. \\
3 & 10. & 200. \\
4 & 40. & 200. \\
5 & 40. & 1 \\
6 & 60. & 1 \\
\hline
\end{tabular}


Table 18. Terrain profile for test case CLSBDL

\begin{tabular}{|c|c|c|c|c|c|c|c|c|}
\hline$i$ & $\begin{array}{l}\text { terx } \\
(\mathrm{km})\end{array}$ & $\begin{array}{c}\text { tery }_{i} \\
\text { (meters) }\end{array}$ & $i$ & $\begin{array}{l}\text { ter } x_{i} \\
(\mathrm{~km})\end{array}$ & $\begin{array}{c}\text { tery }_{i} \\
\text { (meters) }\end{array}$ & $i$ & $\begin{array}{l}\text { terx }_{i} \\
(\mathrm{~km})\end{array}$ & $\begin{array}{c}\text { tery }_{i} \\
\text { (meters) }\end{array}$ \\
\hline 1 & 0.00 & 91.0 & 43 & 10.50 & 37.9 & 85 & 21.00 & 10.8 \\
\hline 2 & 0.25 & 81.9 & 44 & 10.75 & 36.7 & 86 & 21.25 & 11.4 \\
\hline 3 & 0.50 & 66.5 & 45 & 11.00 & 36.8 & 87 & 21.50 & 11.7 \\
\hline 4 & 0.75 & 53.6 & 46 & 11.25 & 34.9 & 88 & 21.75 & 10.6 \\
\hline 5 & 1.00 & 42.3 & 47 & 11.50 & 31.7 & 89 & 22.00 & 8.5 \\
\hline 6 & 1.25 & 40.6 & 48 & 11.75 & 32.5 & 90 & 22.25 & 8.0 \\
\hline 7 & 1.50 & 44.8 & 49 & 12.00 & 35.8 & 91 & 22.50 & 8.7 \\
\hline 8 & 1.75 & 53.4 & 50 & 12.25 & 32.7 & 92 & 22.75 & 9.0 \\
\hline 9 & 2.00 & 64.4 & 51 & 12.50 & 25.6 & 93 & 23.00 & 9.1 \\
\hline 10 & 2.25 & 62.8 & 52 & 12.75 & 22.9 & 94 & 23.25 & 8.7 \\
\hline 11 & 2.50 & 53.6 & 53 & 13.00 & 22.9 & 95 & 23.50 & 7.9 \\
\hline 12 & 2.75 & 54.9 & 54 & 13.25 & 22.9 & 96 & 23.75 & 6.7 \\
\hline 13 & 3.00 & 62.6 & 55 & 13.50 & 22.9 & 97 & 24.00 & 5.1 \\
\hline 14 & 3.25 & 63.2 & 56 & 13.75 & 22.7 & 98 & 24.25 & 5.2 \\
\hline 15 & 3.50 & 59.4 & 57 & 14.00 & 22.4 & 99 & 24.50 & 6.3 \\
\hline 16 & 3.75 & 57.1 & 58 & 14.25 & 22.3 & 100 & 24.75 & 7.0 \\
\hline 17 & 4.00 & 55.7 & 59 & 14.50 & 22.4 & 101 & 25.00 & 7.4 \\
\hline 18 & 4.25 & 53.2 & 60 & 14.75 & 22.4 & 102 & 25.25 & 7.6 \\
\hline 19 & 4.50 & 49.8 & 61 & 15.00 & 22.5 & 103 & 25.50 & 7.6 \\
\hline 20 & 4.75 & 43.7 & 62 & 15.25 & 20.3 & 104 & 25.75 & 9.9 \\
\hline 21 & 5.00 & 36.0 & 63 & 15.50 & 16.7 & 105 & 26.00 & 13.8 \\
\hline 22 & 5.25 & 31.5 & 64 & 15.75 & 15.2 & 106 & 26.25 & 15.2 \\
\hline 23 & 5.50 & 28.9 & 65 & 16.00 & 15.2 & 107 & 26.50 & 15.2 \\
\hline 24 & 5.75 & 24.0 & 66 & 16.25 & 15.2 & 108 & 26.75 & 15.2 \\
\hline 25 & 6.00 & 17.7 & 67 & 16.50 & 15.2 & 109 & 27.00 & 15.2 \\
\hline 26 & 6.25 & 15.2 & 68 & 16.75 & 15.3 & 110 & 27.25 & 15.2 \\
\hline 27 & 6.50 & 15.2 & 69 & 17.00 & 15.3 & 111 & 27.50 & 15.2 \\
\hline 28 & 6.75 & 15.2 & 70 & 17.25 & 15.3 & 112 & 27.75 & 17.6 \\
\hline 29 & 7.00 & 15.2 & 71 & 17.50 & 15.3 & 113 & 28.00 & 21.4 \\
\hline 30 & 7.25 & 17.6 & 72 & 17.75 & 15.2 & 114 & 28.25 & 22.9 \\
\hline 31 & 7.50 & 21.4 & 73 & 18.00 & 15.2 & 115 & 28.50 & 22.9 \\
\hline 32 & 7.75 & 24.6 & 74 & 18.25 & 15.2 & 116 & 28.75 & 23.7 \\
\hline 33 & 8.00 & 27.4 & 75 & 18.50 & 15.2 & 117 & 29.00 & 25.1 \\
\hline 34 & 8.25 & 30.3 & 76 & 18.75 & 15.2 & 118 & 29.25 & 25.7 \\
\hline 35 & 8.50 & 33.3 & 77 & 19.00 & 15.2 & 119 & 29.50 & 25.8 \\
\hline 36 & 8.75 & 33.2 & 78 & 19.25 & 15.2 & 120 & 29.75 & 24.0 \\
\hline 37 & 9.00 & 31.3 & 79 & 19.50 & 15.2 & 121 & 30.00 & 21.0 \\
\hline 38 & 9.25 & 30.0 & 80 & 19.75 & 13.1 & 122 & 30.25 & 19.8 \\
\hline 39 & 9.50 & 29.2 & 81 & 20.00 & 9.7 & 123 & 30.50 & 19.7 \\
\hline 40 & 9.75 & 33.1 & 82 & 20.25 & 8.4 & 124 & 30.75 & 18.3 \\
\hline 41 & 10.00 & 40.1 & 83 & 20.50 & 8.5 & 125 & 31.00 & 16.1 \\
\hline
\end{tabular}


Table 18. Terrain profile for test case CLSBDL (continued).

\begin{tabular}{|c|c|c|c|c|c|c|c|c|}
\hline$i$ & $\begin{array}{l}\text { terx }_{i} \\
(\mathrm{~km}) \\
\end{array}$ & $\begin{array}{c}\text { tery }_{i} \\
\text { (meters) }\end{array}$ & $i$ & $\begin{array}{l}\operatorname{ter}_{i} \\
(\mathrm{~km}) \\
\end{array}$ & $\begin{array}{c}\text { tery }_{i} \\
\text { (meters) }\end{array}$ & $i$ & $\begin{array}{l}\operatorname{ter}_{i} \\
(\mathrm{~km}) \\
\end{array}$ & $\begin{array}{c}\text { tery }_{i} \\
\text { (meters) }\end{array}$ \\
\hline 127 & 31.50 & 12.5 & 169 & 42.00 & 50.3 & 211 & 52.50 & 24.5 \\
\hline 128 & 31.75 & 14.1 & 170 & 42.25 & 51.6 & 212 & 52.75 & 24.7 \\
\hline 129 & 32.00 & 17.9 & 171 & 42.50 & 51.6 & 213 & 53.00 & 24.8 \\
\hline 130 & 32.25 & 19.3 & 172 & 42.75 & 51.7 & 214 & 53.25 & 27.5 \\
\hline 131 & 32.50 & 19.3 & 173 & 43.00 & 51.7 & 215 & 53.50 & 31.9 \\
\hline 132 & 32.75 & 15.7 & 174 & 43.25 & 50.0 & 216 & 53.75 & 33.8 \\
\hline 133 & 33.00 & 9.9 & 175 & 43.50 & 46.9 & 217 & 54.00 & 34.0 \\
\hline 134 & 33.25 & 6.4 & 176 & 43.75 & 48.0 & 218 & 54.25 & 34.0 \\
\hline 135 & 33.50 & 4.4 & 177 & 44.00 & 51.8 & 219 & 54.50 & 33.7 \\
\hline 136 & 33.75 & 4.2 & 178 & 44.25 & 53.3 & 220 & 54.75 & 35.6 \\
\hline 137 & 34.00 & 5.2 & 179 & 44.50 & 53.3 & 221 & 55.00 & 39.0 \\
\hline 138 & 34.25 & 7.3 & 180 & 44.75 & 53.0 & 222 & 55.25 & 43.6 \\
\hline 139 & 34.50 & 10.1 & 181 & 45.00 & 52.4 & 223 & 55.50 & 48.8 \\
\hline 140 & 34.75 & 11.1 & 182 & 45.25 & 50.3 & 224 & 55.75 & 57.6 \\
\hline 141 & 35.00 & 11.1 & 183 & 45.50 & 47.0 & 225 & 56.00 & 68.9 \\
\hline 142 & 35.25 & 11.0 & 184 & 45.75 & 43.7 & 226 & 56.25 & 76.5 \\
\hline 143 & 35.50 & 11.0 & 185 & 46.00 & 40.4 & 227 & 56.50 & 81.7 \\
\hline 144 & 35.75 & 9.9 & 186 & 46.25 & 38.6 & 228 & 56.75 & 82.0 \\
\hline 145 & 36.00 & 8.3 & 187 & 46.50 & 37.9 & 229 & 57.00 & 78.8 \\
\hline 146 & 36.25 & 7.1 & 188 & 46.75 & 33.2 & 230 & 57.25 & 77.9 \\
\hline 147 & 36.50 & 6.3 & 189 & 47.00 & 25.8 & 231 & 57.50 & 78.5 \\
\hline 148 & 36.75 & 7.9 & 190 & 47.25 & 22.7 & 232 & 57.75 & 77.4 \\
\hline 149 & 37.00 & 11.0 & 191 & 47.50 & 22.4 & 233 & 58.00 & 75.2 \\
\hline 150 & 37.25 & 14.6 & 192 & 47.75 & 20.2 & 234 & 58.25 & 77.8 \\
\hline 151 & 37.50 & 18.5 & 193 & 48.00 & 16.7 & 235 & 58.50 & 83.4 \\
\hline 152 & 37.75 & 22.3 & 194 & 48.25 & 15.2 & 236 & 58.75 & 87.8 \\
\hline 153 & 38.00 & 26.2 & 195 & 48.50 & 15.2 & 237 & 59.00 & 91.4 \\
\hline 154 & 38.25 & 30.1 & 196 & 48.75 & 15.2 & 238 & 59.25 & 94.0 \\
\hline 155 & 38.50 & 33.9 & 197 & 49.00 & 15.2 & 239 & 59.50 & 96.1 \\
\hline 156 & 38.75 & 34.7 & 198 & 49.25 & 15.2 & 240 & 59.75 & 92.3 \\
\hline 157 & 39.00 & 33.5 & 199 & 49.50 & 15.2 & 241 & 60.00 & 84.7 \\
\hline 158 & 39.25 & 29.3 & 200 & 49.75 & 17.5 & 242 & 60.25 & 83.6 \\
\hline 159 & 39.50 & 23.0 & 201 & 50.00 & 21.3 & 243 & 60.50 & 87.1 \\
\hline 160 & 39.75 & 19.6 & 202 & 50.25 & 25.1 & 244 & 60.75 & 91.0 \\
\hline 161 & 40.00 & 18.1 & 203 & 50.50 & 29.0 & 245 & 61.00 & 95.0 \\
\hline 162 & 40.25 & 19.1 & 204 & 50.75 & 32.8 & 246 & 61.25 & 98.8 \\
\hline 163 & 40.50 & 21.8 & 205 & 51.00 & 36.6 & 247 & 61.50 & 102.5 \\
\hline 164 & 40.75 & 25.8 & 206 & 51.25 & 38.1 & 248 & 61.75 & 104.8 \\
\hline 165 & 41.00 & 30.6 & 207 & 51.50 & 38.1 & 249 & 62.00 & 106.1 \\
\hline 166 & 41.25 & 36.5 & 208 & 51.75 & 33.9 & 250 & 62.25 & 109.6 \\
\hline 167 & 41.50 & 43.1 & 209 & 52.00 & 27.0 & 251 & 62.50 & 114.3 \\
\hline 168 & 41.75 & 47.4 & 210 & 52.25 & 24.3 & 252 & 62.75 & 117.0 \\
\hline
\end{tabular}


Table 18. Terrain profile for test case CLSBDL (continued).

\begin{tabular}{|c|c|c|c|c|c|c|c|c|}
\hline$i$ & $\begin{array}{l}\operatorname{ter}_{i} \\
(\mathrm{~km})\end{array}$ & $\begin{array}{c}\text { tery }_{i} \\
\text { (meters) }\end{array}$ & $i$ & $\begin{array}{l}\text { ter } x_{i} \\
(\mathrm{~km})\end{array}$ & $\begin{array}{c}\text { tery }_{i} \\
\text { (meters) }\end{array}$ & $i$ & $\begin{array}{l}\operatorname{ter}_{i} \\
(\mathrm{~km})\end{array}$ & $\begin{array}{c}\text { tery }_{i} \\
\text { (meters) }\end{array}$ \\
\hline 253 & 63.00 & 118.4 & 295 & 73.50 & 79.9 & 337 & 84.00 & 56.7 \\
\hline 254 & 63.25 & 119.8 & 296 & 73.75 & 78.0 & 338 & 84.25 & 51.5 \\
\hline 255 & 63.50 & 121.3 & 297 & 74.00 & 76.0 & 339 & 84.50 & 48.1 \\
\hline 256 & 63.75 & 119.2 & 298 & 74.25 & 71.5 & 340 & 84.75 & 46.5 \\
\hline 257 & 64.00 & 114.8 & 299 & 74.50 & 65.1 & 341 & 85.00 & 46.0 \\
\hline 258 & 64.25 & 111.2 & 300 & 74.75 & 60.9 & 342 & 85.25 & 45.7 \\
\hline 259 & 64.50 & 108.0 & 301 & 75.00 & 58.2 & 343 & 85.50 & 45.7 \\
\hline 260 & 64.75 & 107.7 & 302 & 75.25 & 53.8 & 344 & 85.75 & 47.8 \\
\hline 261 & 65.00 & 109.4 & 303 & 75.50 & 48.1 & 345 & 86.00 & 51.3 \\
\hline 262 & 65.25 & 106.9 & 304 & 75.75 & 45.7 & 346 & 86.25 & 50.7 \\
\hline 263 & 65.50 & 101.3 & 305 & 76.00 & 45.7 & 347 & 86.50 & 47.2 \\
\hline 264 & 65.75 & 96.7 & 306 & 76.25 & 50.7 & 348 & 86.75 & 49.7 \\
\hline 265 & 66.00 & 92.7 & 307 & 76.50 & 59.1 & 349 & 87.00 & 56.5 \\
\hline 266 & 66.25 & 90.1 & 308 & 76.75 & 68.3 & 350 & 87.25 & 59.8 \\
\hline 267 & 66.50 & 88.3 & 309 & 77.00 & 78.2 & 351 & 87.50 & 60.6 \\
\hline 268 & 66.75 & 87.6 & 310 & 77.25 & 82.8 & 352 & 87.75 & 60.7 \\
\hline 269 & 67.00 & 87.7 & 311 & 77.50 & 83.5 & 353 & 88.00 & 60.2 \\
\hline 270 & 67.25 & 86.5 & 312 & 77.75 & 81.6 & 354 & 88.25 & 62.1 \\
\hline 271 & 67.50 & 84.4 & 313 & 78.00 & 77.8 & 355 & 88.50 & 65.9 \\
\hline 272 & 67.75 & 82.3 & 314 & 78.25 & 74.0 & 356 & 88.75 & 71.6 \\
\hline 273 & 68.00 & 80.5 & 315 & 78.50 & 70.2 & 357 & 89.00 & 78.9 \\
\hline 274 & 68.25 & 74.2 & 316 & 78.75 & 68.9 & 358 & 89.25 & 82.4 \\
\hline 275 & 68.50 & 64.8 & 317 & 79.00 & 69.4 & 359 & 89.50 & 83.4 \\
\hline 276 & 68.75 & 66.5 & 318 & 79.25 & 71.8 & 360 & 89.75 & 83.8 \\
\hline 277 & 69.00 & 75.7 & 319 & 79.50 & 75.5 & 361 & 90.00 & 83.8 \\
\hline 278 & 69.25 & 83.0 & 320 & 79.75 & 77.2 & 362 & 90.25 & 83.4 \\
\hline 279 & 69.50 & 89.0 & 321 & 80.00 & 77.3 & 363 & 90.50 & 82.6 \\
\hline 280 & 69.75 & 94.6 & 322 & 80.25 & 77.2 & 364 & 90.75 & 80.2 \\
\hline 281 & 70.00 & 100.0 & 323 & 80.50 & 77.0 & 365 & 91.00 & 76.7 \\
\hline 282 & 70.25 & 105.7 & 324 & 80.75 & 74.6 & 366 & 91.25 & 71.1 \\
\hline 283 & 70.50 & 111.8 & 325 & 81.00 & 70.7 & 367 & 91.50 & 64.0 \\
\hline 284 & 70.75 & 114.3 & 326 & 81.25 & 69.1 & 368 & 91.75 & 64.0 \\
\hline 285 & 71.00 & 114.3 & 327 & 81.50 & 69.0 & 369 & 92.00 & 69.1 \\
\hline 286 & 71.25 & 112.1 & 328 & 81.75 & 69.0 & 370 & 92.25 & 72.7 \\
\hline 287 & 71.50 & 108.2 & 329 & 82.00 & 68.9 & 371 & 92.50 & 75.2 \\
\hline 288 & 71.75 & 104.3 & 330 & 82.25 & 68.8 & 372 & 92.75 & 76.2 \\
\hline 289 & 72.00 & 100.4 & 331 & 82.50 & 68.6 & 373 & 93.00 & 76.2 \\
\hline 290 & 72.25 & 96.0 & 332 & 82.75 & 68.6 & 374 & 93.25 & 76.2 \\
\hline 291 & 72.50 & 91.1 & 333 & 83.00 & 68.6 & & & \\
\hline 292 & 72.75 & 87.0 & 334 & 83.25 & 68.6 & & & \\
\hline 293 & 73.00 & 83.4 & 335 & 83.50 & 68.6 & & & \\
\hline 294 & 73.25 & 81.2 & 336 & 83.75 & 64.2 & & & \\
\hline
\end{tabular}


Table 19. Ground types for test case CLSBDL.

\begin{tabular}{c|c|c|c|c}
\hline$i_{g r}, i_{g c}$ & grnd $_{i}^{\mathrm{a}}$ & $\begin{array}{c}\operatorname{rgrnd}_{i} \\
(\mathrm{~km})\end{array}$ & $\begin{array}{c}\gamma c \\
(\mathrm{~dB})\end{array}$ & $\begin{array}{c}\gamma r n g \\
(\mathrm{~km})\end{array}$ \\
\hline 1 & 4 & 0. & -7. & 0. \\
2 & 3 & 45. & -10. & 45. \\
\hline
\end{tabular}

${ }^{a}$ Ground composition type: $0=$ sea water; $1=$ fresh water; $2=$ =wet ground; 3=medium dry ground; $4=$ very dry ground; $5=$ ice at $-1^{\circ} \mathrm{C} ; 6=$ ice at $-10^{\circ} \mathrm{C} ; 7=$ user-defined permittivity and conductivity.

Table 20. Terrain profile for test case CLSBDWL and HF10TER.

\begin{tabular}{|c|c|c|c|c|c|c|c|c|}
\hline$i$ & $\begin{array}{l}\text { ter }_{i} \\
(\mathrm{~km})\end{array}$ & $\begin{array}{c}\text { tery }_{i} \\
\text { (meters) }\end{array}$ & $i$ & $\begin{array}{l}\text { ter }_{i} \\
(\mathrm{~km}) \\
\end{array}$ & $\begin{array}{c}\text { tery }_{i} \\
\text { (meters) }\end{array}$ & $i$ & $\begin{array}{l}\text { ter }_{i} \\
(\mathrm{~km}) \\
\end{array}$ & $\begin{array}{c}\text { tery }_{i} \\
\text { (meters) }\end{array}$ \\
\hline 1 & 0 & 0 & 34 & 118.1818 & 100 & 67 & 136.9318 & 9 \\
\hline 2 & 100 & 0 & 35 & 118.75 & 106 & 68 & 137.5 & 6 \\
\hline 3 & 100.5682 & 2 & 36 & 119.3182 & 100 & 69 & 138.0682 & 5 \\
\hline 4 & 101.1364 & 2 & 37 & 119.8864 & 108 & 70 & 138.6364 & 7 \\
\hline 5 & 101.7045 & 2 & 38 & 120.4545 & 89 & 71 & 139.2045 & 5 \\
\hline 6 & 102.2727 & 3 & 39 & 121.0227 & 90 & 72 & 139.7727 & 8 \\
\hline 7 & 102.8409 & 4 & 40 & 121.5909 & 95 & 73 & 140.3409 & 14 \\
\hline 8 & 103.4091 & 5 & 41 & 122.1591 & 89 & 74 & 140.9091 & 7 \\
\hline 9 & 103.9773 & 4 & 42 & 122.7273 & 107 & 75 & 141.4773 & 12 \\
\hline 10 & 104.5455 & 7 & 43 & 123.2955 & 97 & 76 & 142.0455 & 10 \\
\hline 11 & 105.1136 & 6 & 44 & 123.8636 & 108 & 77 & 142.6136 & 8 \\
\hline 12 & 105.6818 & 9 & 45 & 124.4318 & 87 & 78 & 143.1818 & 14 \\
\hline 13 & 106.25 & 12 & 46 & 125 & 76 & 79 & 143.75 & 15 \\
\hline 14 & 106.8182 & 9 & 47 & 125.5682 & 73 & 80 & 144.3182 & 18 \\
\hline 15 & 107.3864 & 9 & 48 & 126.1364 & 88 & 81 & 144.8864 & 29 \\
\hline 16 & 107.9545 & 8 & 49 & 126.7045 & 86 & 82 & 145.4545 & 78 \\
\hline 17 & 108.5227 & 10 & 50 & 127.2727 & 101 & 83 & 146.0227 & 76 \\
\hline 18 & 109.0909 & 19 & 51 & 127.8409 & 101 & 84 & 146.5909 & 89 \\
\hline 19 & 109.6591 & 21 & 52 & 128.4091 & 92 & 85 & 147.1591 & 139 \\
\hline 20 & 110.2273 & 27 & 53 & 128.9773 & 65 & 86 & 147.7273 & 168 \\
\hline 21 & 110.7955 & 32 & 54 & 129.5455 & 62 & 87 & 148.2955 & 173 \\
\hline 22 & 111.3636 & 32 & 55 & 130.1136 & 47 & 88 & 148.8636 & 184 \\
\hline 23 & 111.9318 & 47 & 56 & 130.6818 & 59 & 89 & 149.4318 & 193 \\
\hline 24 & 112.5 & 43 & 57 & 131.25 & 44 & 90 & 150 & 232 \\
\hline 25 & 113.0682 & 58 & 58 & 131.8182 & 33 & 91 & 150.5682 & 227 \\
\hline 26 & 113.6364 & 82 & 59 & 132.3864 & 21 & 92 & 151.1364 & 264 \\
\hline 27 & 114.2045 & 75 & 60 & 132.9545 & 20 & 93 & 151.7045 & 222 \\
\hline 28 & 114.7727 & 96 & 61 & 133.5227 & 21 & 94 & 152.2727 & 267 \\
\hline 29 & 115.3409 & 63 & 62 & 134.0909 & 11 & 95 & 152.8409 & 247 \\
\hline 30 & 115.9091 & 100 & 63 & 134.6591 & 7 & 96 & 153.4091 & 287 \\
\hline 31 & 116.4773 & 123 & 64 & 135.2273 & 7 & 97 & 153.9773 & 363 \\
\hline 32 & 117.0455 & 98 & 65 & 135.7955 & 4 & 98 & 154.5455 & 427 \\
\hline 33 & 117.6136 & 95 & 66 & 136.3636 & 12 & 99 & 155.1136 & 399 \\
\hline
\end{tabular}


Table 20. Terrain profile for test case CLSBDWL and HF10TER. (Continued)

\begin{tabular}{|c|c|c|c|c|c|c|c|c|}
\hline$i$ & $\begin{array}{l}\operatorname{terx}_{i} \\
(\mathrm{~km}) \\
\end{array}$ & $\begin{array}{c}\text { tery }_{i} \\
\text { (meters) }\end{array}$ & $i$ & $\begin{array}{l}\operatorname{ter}_{i} \\
(\mathrm{~km}) \\
\end{array}$ & $\begin{array}{c}\text { tery }_{i} \\
\text { (meters) }\end{array}$ & $i$ & $\begin{array}{l}\operatorname{terx}_{i} \\
(\mathrm{~km}) \\
\end{array}$ & $\begin{array}{c}\text { tery }_{i} \\
\text { (meters) }\end{array}$ \\
\hline 100 & 155.6818 & 344 & 142 & 179.5455 & 682 & 184 & 203.4091 & 548 \\
\hline 101 & 156.25 & 258 & 143 & 180.1136 & 544 & 185 & 203.9773 & 551 \\
\hline 102 & 156.8182 & 188 & 144 & 180.6818 & 477 & 186 & 204.5455 & 546 \\
\hline 103 & 157.3864 & 182 & 145 & 181.25 & 509 & 187 & 205.1136 & 545 \\
\hline 104 & 157.9545 & 94 & 146 & 181.8182 & 510 & 188 & 205.6818 & 547 \\
\hline 105 & 158.5227 & 85 & 147 & 182.3864 & 546 & 189 & 206.25 & 556 \\
\hline 106 & 159.0909 & 63 & 148 & 182.9545 & 582 & 190 & 206.8182 & 569 \\
\hline 107 & 159.6591 & 43 & 149 & 183.5227 & 844 & 191 & 207.3864 & 576 \\
\hline 108 & 160.2273 & 18 & 150 & 184.0909 & 873 & 192 & 207.9545 & 610 \\
\hline 109 & 160.7955 & 16 & 151 & 184.6591 & 776 & 193 & 208.5227 & 636 \\
\hline 110 & 161.3636 & 16 & 152 & 185.2273 & 819 & 194 & 209.0909 & 634 \\
\hline 111 & 161.9318 & 13 & 153 & 185.7955 & 830 & 195 & 209.6591 & 704 \\
\hline 112 & 162.5 & 21 & 154 & 186.3636 & 814 & 196 & 210.2273 & 736 \\
\hline 113 & 163.0682 & 20 & 155 & 186.9318 & 860 & 197 & 210.7955 & 719 \\
\hline 114 & 163.6364 & 22 & 156 & 187.5 & 870 & 198 & 211.3636 & 702 \\
\hline 115 & 164.2045 & 26 & 157 & 188.0682 & 993 & 199 & 211.9318 & 714 \\
\hline 116 & 164.7727 & 27 & 158 & 188.6364 & 901 & 200 & 212.5 & 691 \\
\hline 117 & 165.3409 & 31 & 159 & 189.2045 & 886 & 201 & 213.0682 & 676 \\
\hline 118 & 165.9091 & 45 & 160 & 189.7727 & 946 & 202 & 213.6364 & 671 \\
\hline 119 & 166.4773 & 58 & 161 & 190.3409 & 911 & 203 & 214.2045 & 671 \\
\hline 120 & 167.0455 & 64 & 162 & 190.9091 & 1025 & 204 & 214.7727 & 708 \\
\hline 121 & 167.6136 & 87 & 163 & 191.4773 & 1123 & 205 & 215.3409 & 668 \\
\hline 122 & 168.1818 & 92 & 164 & 192.0455 & 1262 & 206 & 215.9091 & 674 \\
\hline 123 & 168.75 & 112 & 165 & 192.6136 & 1424 & 207 & 216.4773 & 688 \\
\hline 124 & 169.3182 & 124 & 166 & 193.1818 & 1460 & 208 & 217.0455 & 638 \\
\hline 125 & 169.8864 & 144 & 167 & 193.75 & 1442 & 209 & 217.6136 & 661 \\
\hline 126 & 170.4545 & 178 & 168 & 194.3182 & 1348 & 210 & 218.1818 & 652 \\
\hline 127 & 171.0227 & 154 & 169 & 194.8864 & 1152 & 211 & 218.75 & 673 \\
\hline 128 & 171.5909 & 172 & 170 & 195.4545 & 940 & 212 & 219.3182 & 673 \\
\hline 129 & 172.1591 & 192 & 171 & 196.0227 & 1256 & 213 & 219.8864 & 665 \\
\hline 130 & 172.7273 & 192 & 172 & 196.5909 & 1111 & 214 & 220.4545 & 703 \\
\hline 131 & 173.2955 & 196 & 173 & 197.1591 & 943 & 215 & 221.0227 & 671 \\
\hline 132 & 173.8636 & 216 & 174 & 197.7273 & 1037 & 216 & 221.5909 & 685 \\
\hline 133 & 174.4318 & 222 & 175 & 198.2955 & 931 & 217 & 222.1591 & 730 \\
\hline 134 & 175 & 234 & 176 & 198.8636 & 759 & 218 & 222.7273 & 722 \\
\hline 135 & 175.5682 & 236 & 177 & 199.4318 & 673 & 219 & 223.2955 & 737 \\
\hline 136 & 176.1364 & 262 & 178 & 200 & 702 & 220 & 223.8636 & 709 \\
\hline 137 & 176.7045 & 287 & 179 & 200.5682 & 607 & 221 & 224.4318 & 752 \\
\hline 138 & 177.2727 & 372 & 180 & 201.1364 & 649 & 222 & 225 & 767 \\
\hline 139 & 177.8409 & 546 & 181 & 201.7045 & 576 & 223 & 225.5682 & 774 \\
\hline 140 & 178.4091 & 699 & 182 & 202.2727 & 551 & 224 & 226.1364 & 728 \\
\hline 141 & 178.9773 & 821 & 183 & 202.8409 & 548 & 225 & 226.7045 & 749 \\
\hline
\end{tabular}


Table 20. Terrain profile for test case CLSBDWL and HF10TER. (Continued)

\begin{tabular}{c|c|c||c|c|c||c|c|c}
\hline$i$ & $\begin{array}{c}\text { ter } x_{i} \\
(\mathrm{~km})\end{array}$ & $\begin{array}{c}\text { tery } \\
(\text { meters })\end{array}$ & $i$ & $\begin{array}{c}\text { ter } x_{i} \\
(\mathrm{~km})\end{array}$ & $\begin{array}{c}\text { tery } \\
(\text { meters })\end{array}$ & $i$ & $\begin{array}{c}\text { ter } x_{i} \\
(\mathrm{~km})\end{array}$ & $\begin{array}{c}\text { ter }_{i} \\
(\text { meters })\end{array}$ \\
\hline 226 & 227.2727 & 761 & 240 & 235.2273 & 1094 & 254 & 243.1818 & 964 \\
227 & 227.8409 & 759 & 241 & 235.7955 & 1249 & 255 & 243.75 & 897 \\
228 & 228.4091 & 815 & 242 & 236.3636 & 1334 & 256 & 244.3182 & 861 \\
229 & 228.9773 & 836 & 243 & 236.9318 & 1286 & 257 & 244.8864 & 806 \\
230 & 229.5455 & 896 & 244 & 237.5 & 1235 & 258 & 245.4545 & 796 \\
231 & 230.1136 & 924 & 245 & 238.0682 & 1181 & 259 & 246.0227 & 780 \\
232 & 230.6818 & 956 & 246 & 238.6364 & 1165 & 260 & 246.5909 & 773 \\
233 & 231.25 & 1136 & 247 & 239.2045 & 1196 & 261 & 247.1591 & 767 \\
234 & 231.8182 & 1187 & 248 & 239.7727 & 1207 & 262 & 247.7273 & 764 \\
235 & 232.3864 & 1353 & 249 & 240.3409 & 1257 & 263 & 248.2955 & 761 \\
236 & 232.9545 & 1313 & 250 & 240.9091 & 1177 & 264 & 248.8636 & 753 \\
237 & 233.5227 & 1153 & 251 & 241.4773 & 1237 & 265 & 249.4318 & 750 \\
238 & 234.0909 & 1111 & 252 & 242.0455 & 1186 & & & \\
239 & 234.6591 & 1095 & 253 & 242.6136 & 1085 & & & \\
\hline
\end{tabular}

Table 21. Ground types for test case CLSBDWL

\begin{tabular}{c|c|c|c|c}
\hline$i_{g r}, i_{g c}$ & igrnd $_{i}^{\mathrm{a}}$ & $\begin{array}{c}\text { rgrnd }_{i} \\
(\mathrm{~km})\end{array}$ & $\begin{array}{c}\gamma c \\
(\mathrm{~dB})\end{array}$ & $\begin{array}{c}\gamma r n g \\
(\mathrm{~km})\end{array}$ \\
\hline 1 & 0 & 0. & -5. & 0. \\
2 & 4 & 100. & -10. & 100. \\
\hline
\end{tabular}

Table 22. Terrain profile for test case FLTA50.

\begin{tabular}{c|c|c}
\hline$i$ & $\begin{array}{c}\text { ter } x_{i} \\
(\mathrm{~km})\end{array}$ & $\begin{array}{c}\text { tery } \\
\text { (meters) }\end{array}$ \\
\hline 1 & 0. & 10. \\
2 & 50. & 10. \\
\hline
\end{tabular}


Table 23. Terrain profile for test case HEIGHT_RTG.

\begin{tabular}{|c|c|c|c|c|c|c|c|c|}
\hline$i$ & $\begin{array}{l}\text { terx }_{i} \\
(\mathrm{~km})\end{array}$ & $\begin{array}{c}\text { tery }_{i} \\
\text { (meters) }\end{array}$ & $i$ & $\begin{array}{l}\text { terx }_{i} \\
(\mathrm{~km})\end{array}$ & $\begin{array}{c}\text { tery }_{i} \\
\text { (meters) }\end{array}$ & $i$ & $\begin{array}{l}\text { terx }_{i} \\
(\mathrm{~km})\end{array}$ & $\begin{array}{c}\text { tery }_{i} \\
\text { (meters) }\end{array}$ \\
\hline 1 & 0.000 & 299.505 & 43 & 2.552 & 194.509 & 85 & 5.104 & 129.424 \\
\hline 2 & 0.061 & 293.102 & 44 & 2.613 & 191.783 & 86 & 5.165 & 128.345 \\
\hline 3 & 0.122 & 285.291 & 45 & 2.674 & 189.084 & 87 & 5.226 & 126.420 \\
\hline 4 & 0.182 & 275.476 & 46 & 2.734 & 186.385 & 88 & 5.286 & 124.172 \\
\hline 5 & 0.243 & 274.000 & 47 & 2.795 & 184.601 & 89 & 5.347 & 122.607 \\
\hline 6 & 0.304 & 282.185 & 48 & 2.856 & 183.542 & 90 & 5.408 & 122.185 \\
\hline 7 & 0.365 & 295.347 & 49 & 2.917 & 183.000 & 91 & 5.469 & 121.980 \\
\hline 8 & 0.425 & 302.937 & 50 & 2.977 & 182.743 & 92 & 5.529 & 119.549 \\
\hline 9 & 0.486 & 303.296 & 51 & 3.038 & 182.000 & 93 & 5.590 & 115.950 \\
\hline 10 & 0.547 & 303.015 & 52 & 3.099 & 182.000 & 94 & 5.651 & 112.351 \\
\hline 11 & 0.608 & 304.000 & 53 & 3.160 & 182.000 & 95 & 5.712 & 109.501 \\
\hline 12 & 0.668 & 303.744 & 54 & 3.220 & 179.587 & 96 & 5.772 & 106.397 \\
\hline 13 & 0.729 & 300.168 & 55 & 3.281 & 174.642 & 97 & 5.833 & 103.135 \\
\hline 14 & 0.790 & 296.820 & 56 & 3.342 & 169.241 & 98 & 5.894 & 100.155 \\
\hline 15 & 0.851 & 292.997 & 57 & 3.403 & 164.599 & 99 & 5.955 & 97.891 \\
\hline 16 & 0.911 & 287.759 & 58 & 3.463 & 160.716 & 100 & 6.015 & 96.565 \\
\hline 17 & 0.972 & 281.854 & 59 & 3.524 & 156.889 & 101 & 6.076 & 96.000 \\
\hline 18 & 1.033 & 276.646 & 60 & 3.585 & 153.416 & 102 & 6.137 & 96.030 \\
\hline 19 & 1.094 & 272.799 & 61 & 3.646 & 151.279 & 103 & 6.198 & 96.592 \\
\hline 20 & 1.154 & 269.115 & 62 & 3.706 & 147.165 & 104 & 6.258 & 97.472 \\
\hline 21 & 1.215 & 263.611 & 63 & 3.767 & 143.896 & 105 & 6.319 & 98.371 \\
\hline 22 & 1.276 & 257.254 & 64 & 3.828 & 139.232 & 106 & 6.380 & 99.271 \\
\hline 23 & 1.337 & 245.911 & 65 & 3.889 & 134.218 & 107 & 6.441 & 100.171 \\
\hline 24 & 1.398 & 243.898 & 66 & 3.950 & 128.083 & 108 & 6.502 & 101.071 \\
\hline 25 & 1.458 & 243.155 & 67 & 4.010 & 124.264 & 109 & 6.562 & 101.970 \\
\hline 26 & 1.519 & 240.320 & 68 & 4.071 & 123.043 & 110 & 6.623 & 103.117 \\
\hline 27 & 1.580 & 237.236 & 69 & 4.132 & 122.844 & 111 & 6.684 & 104.770 \\
\hline 28 & 1.641 & 235.571 & 70 & 4.193 & 123.099 & 112 & 6.745 & 105.716 \\
\hline 29 & 1.701 & 233.148 & 71 & 4.253 & 123.944 & 113 & 6.805 & 107.516 \\
\hline 30 & 1.762 & 228.323 & 72 & 4.314 & 124.425 & 114 & 6.866 & 108.996 \\
\hline 31 & 1.823 & 224.033 & 73 & 4.375 & 123.526 & 115 & 6.927 & 110.273 \\
\hline 32 & 1.884 & 219.266 & 74 & 4.436 & 123.481 & 116 & 6.988 & 111.914 \\
\hline 33 & 1.944 & 214.502 & 75 & 4.496 & 123.203 & 117 & 7.048 & 113.714 \\
\hline 34 & 2.005 & 213.180 & 76 & 4.557 & 122.460 & 118 & 7.109 & 115.514 \\
\hline 35 & 2.066 & 213.000 & 77 & 4.618 & 121.908 & 119 & 7.170 & 117.313 \\
\hline 36 & 2.127 & 212.574 & 78 & 4.679 & 121.691 & 120 & 7.231 & 119.113 \\
\hline 37 & 2.187 & 211.037 & 79 & 4.739 & 121.538 & 121 & 7.291 & 120.858 \\
\hline 38 & 2.248 & 208.621 & 80 & 4.800 & 121.278 & 122 & 7.352 & 122.435 \\
\hline 39 & 2.309 & 205.922 & 81 & 4.861 & 122.138 & 123 & 7.413 & 126.256 \\
\hline 40 & 2.370 & 203.222 & 82 & 4.922 & 128.631 & 124 & 7.474 & 129.869 \\
\hline 41 & 2.430 & 200.523 & 83 & 4.982 & 131.102 & 125 & 7.534 & 133.931 \\
\hline 42 & 2.491 & 197.785 & 84 & 5.043 & 130.169 & 126 & 7.595 & 138.091 \\
\hline
\end{tabular}


Table 23. Terrain profile for test case HEIGHT_RTG.

\begin{tabular}{|c|c|c|c|c|c|c|c|c|}
\hline$i$ & $\begin{array}{l}\text { terx } \\
(\mathrm{km})\end{array}$ & $\begin{array}{c}\text { tery }_{i} \\
\text { (meters) }\end{array}$ & $i$ & $\begin{array}{l}\text { ter } x_{i} \\
(\mathrm{~km})\end{array}$ & $\begin{array}{c}\text { tery }_{i} \\
\text { (meters) }\end{array}$ & $i$ & $\begin{array}{l}\text { terx } \\
(\mathrm{km})\end{array}$ & $\begin{array}{c}\text { tery }_{i} \\
\text { (meters) }\end{array}$ \\
\hline 127 & 7.656 & 143.134 & 169 & 10.208 & 177.921 & 211 & 12.760 & 155.000 \\
\hline 128 & 7.717 & 146.594 & 170 & 10.269 & 177.924 & 212 & 12.821 & 155.000 \\
\hline 129 & 7.778 & 148.177 & 171 & 10.329 & 177.827 & 213 & 12.881 & 155.000 \\
\hline 130 & 7.838 & 149.890 & 172 & 10.390 & 177.204 & 214 & 12.942 & 155.000 \\
\hline 131 & 7.899 & 150.397 & 173 & 10.451 & 177.540 & 215 & 13.003 & 155.000 \\
\hline 132 & 7.960 & 150.576 & 174 & 10.512 & 177.876 & 216 & 13.064 & 155.000 \\
\hline 133 & 8.021 & 149.898 & 175 & 10.573 & 176.820 & 217 & 13.125 & 155.000 \\
\hline 134 & 8.081 & 146.486 & 176 & 10.633 & 176.887 & 218 & 13.185 & 155.000 \\
\hline 135 & 8.142 & 148.353 & 177 & 10.694 & 177.890 & 219 & 13.246 & 155.000 \\
\hline 136 & 8.203 & 150.580 & 178 & 10.755 & 179.717 & 220 & 13.307 & 155.000 \\
\hline 137 & 8.264 & 151.340 & 179 & 10.816 & 183.114 & 221 & 13.368 & 155.000 \\
\hline 138 & 8.324 & 151.342 & 180 & 10.876 & 187.030 & 222 & 13.428 & 155.000 \\
\hline 139 & 8.385 & 151.344 & 181 & 10.937 & 187.587 & 223 & 13.489 & 155.000 \\
\hline 140 & 8.446 & 151.346 & 182 & 10.998 & 186.186 & 224 & 13.550 & 155.000 \\
\hline 141 & 8.507 & 151.349 & 183 & 11.059 & 184.961 & 225 & 13.611 & 155.000 \\
\hline 142 & 8.567 & 151.351 & 184 & 11.119 & 181.541 & 226 & 13.671 & 155.000 \\
\hline 143 & 8.628 & 151.354 & 185 & 11.180 & 176.874 & 227 & 13.732 & 155.000 \\
\hline 144 & 8.689 & 148.733 & 186 & 11.241 & 170.821 & 228 & 13.793 & 155.000 \\
\hline 145 & 8.750 & 146.851 & 187 & 11.302 & 166.223 & 229 & 13.854 & 155.000 \\
\hline 146 & 8.810 & 145.405 & 188 & 11.362 & 162.408 & 230 & 13.914 & 155.000 \\
\hline 147 & 8.871 & 144.259 & 189 & 11.423 & 159.207 & 231 & 13.975 & 155.000 \\
\hline 148 & 8.932 & 141.215 & 190 & 11.484 & 157.475 & 232 & 14.036 & 155.000 \\
\hline 149 & 8.993 & 137.404 & 191 & 11.545 & 154.210 & 233 & 14.097 & 155.000 \\
\hline 150 & 9.053 & 135.142 & 192 & 11.605 & 152.143 & 234 & 14.157 & 155.000 \\
\hline 151 & 9.114 & 131.871 & 193 & 11.666 & 156.158 & 235 & 14.218 & 155.000 \\
\hline 152 & 9.175 & 133.486 & 194 & 11.727 & 158.057 & 236 & 14.279 & 155.000 \\
\hline 153 & 9.236 & 135.960 & 195 & 11.788 & 158.445 & 237 & 14.340 & 155.000 \\
\hline 154 & 9.297 & 138.960 & 196 & 11.849 & 158.874 & 238 & 14.401 & 155.000 \\
\hline 155 & 9.357 & 141.996 & 197 & 11.909 & 159.644 & 239 & 14.461 & 155.109 \\
\hline 156 & 9.418 & 145.272 & 198 & 11.970 & 159.410 & 240 & 14.522 & 155.337 \\
\hline 157 & 9.479 & 149.519 & 199 & 12.031 & 158.268 & 241 & 14.583 & 155.336 \\
\hline 158 & 9.540 & 152.000 & 200 & 12.092 & 157.187 & 242 & 14.644 & 155.327 \\
\hline 159 & 9.600 & 152.000 & 201 & 12.152 & 155.000 & 243 & 14.704 & 155.026 \\
\hline 160 & 9.661 & 152.149 & 202 & 12.213 & 155.000 & 244 & 14.765 & 155.000 \\
\hline 161 & 9.722 & 152.765 & 203 & 12.274 & 155.000 & 245 & 14.826 & 155.000 \\
\hline 162 & 9.783 & 153.698 & 204 & 12.335 & 155.000 & 246 & 14.887 & 155.000 \\
\hline 163 & 9.843 & 155.393 & 205 & 12.395 & 155.000 & 247 & 14.947 & 155.000 \\
\hline 164 & 9.904 & 158.023 & 206 & 12.456 & 155.000 & 248 & 15.008 & 155.000 \\
\hline 165 & 9.965 & 161.649 & 207 & 12.517 & 155.000 & 249 & 15.069 & 155.000 \\
\hline 166 & 10.026 & 165.081 & 208 & 12.578 & 155.000 & 250 & 15.130 & 155.000 \\
\hline 167 & 10.086 & 169.421 & 209 & 12.638 & 155.000 & 251 & 15.190 & 155.526 \\
\hline 168 & 10.147 & 174.600 & 210 & 12.699 & 155.000 & 252 & 15.251 & 159.420 \\
\hline
\end{tabular}


Table 23. Terrain profile for test case HEIGHT_RTG.

\begin{tabular}{c|c|c||c|c|c||c|c|c}
\hline$i$ & $\begin{array}{c}\text { ter }_{i} \\
(\mathrm{~km})\end{array}$ & $\begin{array}{c}\text { tery }_{i} \\
(\text { meters })\end{array}$ & $i$ & $\begin{array}{c}\text { ter } x_{i} \\
(\mathrm{~km})\end{array}$ & $\begin{array}{c}\text { tery } \\
(\text { meters })\end{array}$ & $\begin{array}{c}\text { ter }_{i} \\
(\mathrm{~km})\end{array}$ & $\begin{array}{c}\text { ter }_{i} \\
(\text { meters })\end{array}$ \\
\hline 253 & 15.312 & 163.313 & 271 & 16.406 & 182.000 & 289 & 17.499 & 194.094 \\
254 & 15.373 & 166.669 & 272 & 16.466 & 182.000 & 290 & 17.560 & 193.410 \\
255 & 15.433 & 169.488 & 273 & 16.527 & 182.000 & 291 & 17.621 & 193.332 \\
256 & 15.494 & 172.388 & 274 & 16.588 & 182.000 & 292 & 17.682 & 192.703 \\
257 & 15.555 & 174.689 & 275 & 16.649 & 182.000 & 293 & 17.742 & 192.442 \\
258 & 15.616 & 176.772 & 276 & 16.709 & 182.000 & 294 & 17.803 & 191.841 \\
259 & 15.677 & 178.643 & 277 & 16.770 & 182.000 & 295 & 17.864 & 191.503 \\
260 & 15.737 & 179.691 & 278 & 16.831 & 182.000 & 296 & 17.925 & 191.423 \\
261 & 15.798 & 181.120 & 279 & 16.892 & 182.000 & 297 & 17.985 & 191.425 \\
262 & 15.859 & 182.000 & 280 & 16.953 & 182.000 & 298 & 18.046 & 191.014 \\
263 & 15.920 & 182.000 & 281 & 17.013 & 182.000 & 299 & 18.107 & 190.116 \\
264 & 15.980 & 182.000 & 282 & 17.074 & 182.000 & 300 & 18.168 & 189.431 \\
265 & 16.041 & 182.000 & 283 & 17.135 & 182.000 & 301 & 18.229 & 189.545 \\
266 & 16.102 & 182.000 & 284 & 17.196 & 182.000 & 302 & 18.289 & 190.451 \\
267 & 16.163 & 182.000 & 285 & 17.256 & 182.000 & 303 & 18.350 & 191.606 \\
268 & 16.223 & 182.000 & 286 & 17.317 & 186.555 & 304 & 18.411 & 193.116 \\
269 & 16.284 & 182.000 & 287 & 17.378 & 191.465 & & & \\
270 & 16.345 & 182.000 & 288 & 17.439 & 193.821 & & & \\
\hline
\end{tabular}

Table 24. Ground types for test case HF10TER.

\begin{tabular}{c|c|c|c}
\hline$i_{g r}, i_{g c}$ & igrnd $_{i}^{\mathrm{a}}$ & $\begin{array}{c}\operatorname{rgrnd}_{i} \\
(\mathrm{~km})\end{array}$ & $\begin{array}{c}\text { dielec } \\
\left(\varepsilon_{r}, \sigma\right)^{\mathrm{a}}\end{array}$ \\
\hline 1 & 7 & 0. & $(80 ., 4.0)$ \\
2 & 7 & 80. & $(5.0,0.0001)$ \\
\hline \multicolumn{2}{r}{${ }^{\mathrm{a}} \varepsilon_{r}=$ relative permittivity; $\sigma=$ conductivity $(\mathrm{S} / \mathrm{m})$}
\end{tabular}

Table 25. Terrain profile for test case MPRT.

\begin{tabular}{c|c|c}
\hline$i$ & $\begin{array}{c}\text { ter } x_{i} \\
(\mathrm{~km})\end{array}$ & $\begin{array}{c}\text { tery } \\
\text { (meters) }\end{array}$ \\
\hline 1 & 0. & 0. \\
2 & 10. & 0. \\
3 & 30. & 600. \\
4 & 50. & 0. \\
5 & 60. & 0. \\
\hline
\end{tabular}


Table 26. Terrain profile for test case PERW.

\begin{tabular}{c|c|c}
\hline$i$ & $\begin{array}{c}\text { ter } x_{i} \\
(\mathrm{~km})\end{array}$ & $\begin{array}{c}\text { tery }_{i} \\
(\text { meters })\end{array}$ \\
\hline 1 & 0. & 0. \\
2 & 18.75 & 0. \\
3 & 20.312 & 210. \\
4 & 21.875 & 320. \\
5 & 23.4375 & 375. \\
6 & 25.00 & 390. \\
7 & 26.565 & 375. \\
8 & 28.125 & 320. \\
9 & 31.250 & 90. \\
10 & 32.8125 & 0. \\
11 & 50.00 & 0. \\
\hline
\end{tabular}

Table 27. Terrain profile for test case PVT.

\begin{tabular}{c|c|c}
\hline$i$ & $\begin{array}{c}\text { ter } x_{i} \\
(\mathrm{~km})\end{array}$ & $\begin{array}{c}\text { tery } \\
\text { (meters) }\end{array}$ \\
\hline 1 & 0.00 & 625. \\
2 & 3.17 & 476. \\
3 & 6.34 & 347. \\
4 & 9.51 & 239. \\
5 & 12.69 & 151. \\
6 & 15.87 & 83. \\
7 & 19.04 & 35. \\
8 & 22.22 & 7. \\
9 & 25.00 & 0. \\
10 & 27.78 & 7. \\
11 & 30.96 & 35. \\
12 & 34.13 & 83. \\
13 & 37.13 & 151. \\
14 & 40.49 & 239. \\
15 & 43.66 & 347. \\
16 & 46.83 & 476. \\
17 & 50. & 625. \\
\hline
\end{tabular}


Table 28. Terrain profile for test case RDLONGB and TROPOT.

\begin{tabular}{|c|c|c|c|c|c|c|c|c|}
\hline$i$ & $\begin{array}{l}\text { terx }_{i} \\
(\mathrm{~km})\end{array}$ & $\begin{array}{c}\text { tery }_{I} \\
\text { (meters) }\end{array}$ & $i$ & $\begin{array}{l}\operatorname{terx}_{i} \\
(\mathrm{~km}) \\
\end{array}$ & $\begin{array}{c}\text { tery }_{I} \\
\text { (meters) }\end{array}$ & $i$ & $\begin{array}{l}\text { terx }_{i} \\
(\mathrm{~km}) \\
\end{array}$ & $\begin{array}{c}\text { tery }_{I} \\
\text { (meters) }\end{array}$ \\
\hline 1 & 0.0 & 8.0 & 57 & 20.10 & 22.0 & 113 & 79.20 & 184.0 \\
\hline 2 & .30 & 8.0 & 58 & 20.40 & 23.0 & 114 & 79.50 & 226.0 \\
\hline 3 & .60 & 9.0 & 59 & 20.70 & 24.0 & 115 & 79.80 & 152.0 \\
\hline 4 & .90 & 9.0 & 60 & 21.00 & 24.0 & 116 & 80.10 & 201.0 \\
\hline 5 & 1.20 & 10.0 & 61 & 21.30 & 25.0 & 117 & 80.40 & 244.0 \\
\hline 6 & 1.50 & 11.0 & 62 & 21.60 & 26.0 & 118 & 80.70 & 152.0 \\
\hline 7 & 1.80 & 12.0 & 63 & 21.90 & 27.0 & 119 & 81.00 & 143.0 \\
\hline 8 & 2.10 & 13.0 & 64 & 22.20 & 27.0 & 120 & 81.30 & 91.0 \\
\hline 9 & 2.40 & 14.0 & 65 & 22.50 & 28.0 & 121 & 81.60 & 107.0 \\
\hline 10 & 2.70 & 15.0 & 66 & 22.80 & 29.0 & 122 & 81.90 & 152.0 \\
\hline 11 & 3.00 & 17.0 & 67 & 23.40 & 29.0 & 123 & 82.20 & 152.0 \\
\hline 12 & 3.30 & 19.0 & 68 & 23.70 & 30.0 & 124 & 82.50 & 170.0 \\
\hline 13 & 3.60 & 21.0 & 69 & 24.60 & 30.0 & 125 & 82.80 & 152.0 \\
\hline 14 & 3.90 & 23.0 & 70 & 24.90 & 32.0 & 126 & 83.10 & 66.0 \\
\hline 15 & 4.20 & 25.0 & 71 & 25.20 & 34.0 & 127 & 83.40 & 70.0 \\
\hline 16 & 4.50 & 27.0 & 72 & 25.50 & 38.0 & 128 & 83.70 & 121.0 \\
\hline 17 & 4.80 & 28.0 & 73 & 26.10 & 38.0 & 129 & 84.00 & 152.0 \\
\hline 18 & 5.10 & 30.0 & 74 & 26.40 & 36.0 & 130 & 84.30 & 170.0 \\
\hline 19 & 5.40 & 31.0 & 75 & 26.70 & 34.0 & 131 & 84.60 & 141.0 \\
\hline 20 & 5.70 & 31.0 & 76 & 27.00 & 32.0 & 132 & 84.90 & 139.0 \\
\hline 21 & 6.00 & 29.0 & 77 & 27.30 & 27.0 & 133 & 85.20 & 147.0 \\
\hline 22 & 6.30 & 23.0 & 78 & 27.60 & 15.0 & 134 & 85.50 & 177.0 \\
\hline 23 & 6.60 & 14.0 & 79 & 27.90 & 6.0 & 135 & 85.80 & 152.0 \\
\hline 24 & 6.90 & 9.0 & 80 & 28.20 & 1.0 & 136 & 86.10 & 61.0 \\
\hline 25 & 7.20 & 7.0 & 81 & 28.50 & 0.0 & 137 & 86.70 & 61.0 \\
\hline 26 & 7.50 & 7.0 & 82 & 64.50 & 0.0 & 138 & 87.00 & 70.0 \\
\hline 27 & 7.80 & 9.0 & 83 & 64.80 & 8.0 & 139 & 87.30 & 44.0 \\
\hline 28 & 8.10 & 11.0 & 84 & 65.10 & 30.0 & 140 & 87.60 & 11.0 \\
\hline 29 & 8.40 & 14.0 & 85 & 65.40 & 39.0 & 141 & 87.90 & 1.0 \\
\hline 30 & 8.70 & 13.0 & 86 & 65.70 & 61.0 & 142 & 89.40 & 1.0 \\
\hline 31 & 9.30 & 13.0 & 87 & 66.60 & 61.0 & 143 & 89.70 & 61.0 \\
\hline 32 & 9.60 & 12.0 & 88 & 66.90 & 24.0 & 144 & 90.00 & 84.0 \\
\hline 33 & 9.90 & 11.0 & 89 & 67.20 & 14.0 & 145 & 90.30 & 152.0 \\
\hline 34 & 10.20 & 8.0 & 90 & 67.50 & 26.0 & 146 & 90.60 & 152.0 \\
\hline 35 & 10.80 & 8.0 & 91 & 67.80 & 16.0 & 147 & 90.90 & 101.0 \\
\hline 36 & 11.10 & 7.0 & 92 & 68.10 & 1.0 & 148 & 91.20 & 40.0 \\
\hline 37 & 12.60 & 7.0 & 93 & 68.40 & 1.0 & 149 & 91.50 & 15.0 \\
\hline 38 & 12.90 & 6.0 & 94 & 68.70 & 0.0 & 150 & 91.80 & 20.0 \\
\hline
\end{tabular}


Table 28. Terrain profile for test case RDLONGB and TROPOT.

\begin{tabular}{c|c|c||c|c|c||c|c|c}
\hline$i$ & $\begin{array}{c}\text { ter } x_{i} \\
(\mathrm{~km})\end{array}$ & $\begin{array}{c}\text { tery }_{i} \\
(\text { meters })\end{array}$ & $i$ & $\begin{array}{c}\text { ter } x_{i} \\
(\mathrm{~km})\end{array}$ & $\begin{array}{c}\text { ter }_{i} \\
(\text { meters })\end{array}$ & $\begin{array}{c}\text { ter } x_{i} \\
(\mathrm{~km})\end{array}$ & $\begin{array}{c}\text { ter }_{i} \\
(\text { meters })\end{array}$ \\
\hline 39 & 14.40 & 6.0 & 95 & 73.80 & 0.0 & 151 & 92.10 & 2.0 \\
40 & 14.70 & 7.0 & 96 & 74.10 & 1.0 & 152 & 92.40 & 10.0 \\
41 & 15.00 & 8.0 & 97 & 74.40 & 1.0 & 153 & 92.70 & 4.0 \\
42 & 15.30 & 8.0 & 98 & 74.70 & 10.0 & 154 & 93.00 & 1.0 \\
43 & 15.60 & 9.0 & 99 & 75.00 & 8.0 & 155 & 93.30 & 1.0 \\
44 & 15.90 & 10.0 & 100 & 75.30 & 39.0 & 156 & 93.60 & 0.0 \\
45 & 16.20 & 11.0 & 101 & 75.60 & 45.0 & 157 & 93.90 & 1.0 \\
46 & 16.50 & 11.0 & 102 & 75.90 & 53.0 & 158 & 96.30 & 1.0 \\
47 & 16.80 & 12.0 & 103 & 76.20 & 61.0 & 159 & 96.60 & 0.0 \\
48 & 17.40 & 12.0 & 104 & 76.50 & 61.0 & 160 & 96.90 & 1.0 \\
49 & 17.70 & 13.0 & 105 & 76.80 & 82.0 & 161 & 97.50 & 1.0 \\
50 & 18.00 & 13.0 & 106 & 77.10 & 61.0 & 162 & 97.80 & 2.0 \\
51 & 18.30 & 14.0 & 107 & 77.40 & 78.0 & 163 & 98.10 & 3.0 \\
52 & 18.60 & 15.0 & 108 & 77.70 & 61.0 & 164 & 99.30 & 3.0 \\
53 & 18.90 & 16.0 & 109 & 78.00 & 129.0 & 165 & 99.60 & 2.0 \\
54 & 19.20 & 18.0 & 110 & 78.30 & 30.0 & 166 & 99.90 & 2.0 \\
55 & 19.50 & 20.0 & 111 & 78.60 & 46.0 & 167 & 100.20 & 1.0 \\
56 & 19.80 & 21.0 & 112 & 78.90 & 159.0 & & & \\
\hline
\end{tabular}

Table 29. Ground types for test case RDLONGB and TROPOT.

\begin{tabular}{c|c|c}
\hline$i_{q r}$ & igrnd $_{i}^{\mathrm{a}}$ & $\begin{array}{c}\text { rgrnd }_{i} \\
(\mathrm{~km})\end{array}$ \\
\hline 1 & 2 & 0. \\
2 & 0 & 28.5 \\
3 & 3 & 64.8 \\
4 & 0 & 68.7 \\
5 & 4 & 74.1 \\
6 & 0 & 100.2 \\
\hline
\end{tabular}

${ }^{\mathrm{a}}$ Ground composition type: $0=$ sea water; $1=$ fresh water; $2=$ wet ground; $3=$ medium dry ground; $4=$ very dry ground; $5=$ ice at $-1^{\circ} \mathrm{C} ; 6=$ ice at $-10^{\circ} \mathrm{C} ; 7=$ user-defined permittivity and conductivity.

Table 30. Terrain profile for test case VERTMIX.

\begin{tabular}{c|c|c|c|c|c}
\hline$i$ & $\begin{array}{c}\operatorname{ter}_{i} \\
(\mathrm{~km})\end{array}$ & $\begin{array}{c}\text { tery } \\
(\text { meters })\end{array}$ & $i_{g r}$ & igrnd $_{i}{ }^{\mathrm{a}}$ & $\begin{array}{c}\text { rgrnd }_{i} \\
(\mathrm{~km})\end{array}$ \\
\hline 1 & 0. & 0. & 1 & 4 & 0. \\
2 & 50. & 0. & 2 & 0 & 25.0 \\
\hline
\end{tabular}

a Ground composition type: 0=sea water; 1=fresh water; 2=wet ground; 3=medium dry ground; $4=$ very dry ground; $5=$ ice at $-1^{\circ} \mathrm{C} ; 6=$ ice at $-10^{\circ} \mathrm{C} ; 7=$ user-defined permittivity and conductivity. 
Table 31. Terrain profile for test case VERTUSRD.

\begin{tabular}{c|c|c}
\hline & $\begin{array}{c}\text { ter } x_{i} \\
(\mathrm{~km})\end{array}$ & $\begin{array}{c}\text { tery }_{i} \\
\text { (meters) }\end{array}$ \\
\hline 1 & 0. & 0. \\
2 & 50. & 0. \\
\hline
\end{tabular}

Table 32. Terrain profile for test case WEDGE.

\begin{tabular}{c|c|c}
\hline$i$ & $\operatorname{ter}_{i}$ & $\begin{array}{c}\text { tery } \\
(\mathrm{km})\end{array}$ \\
\hline 1 & 0. & 0. \\
2 & 45.0 & 0. \\
3 & 50.0 & 200. \\
4 & 55.0 & 0. \\
5 & 100.0 & 0. \\
\hline
\end{tabular}

\subsection{EXPECTED TEST RESULTS}

The expected test results listing propagation loss versus height values for each of the 48 test cases are listed in tabular form inTable 33 through Table 80. 
Table 33. Expected output for ABSORB for $r_{\max }$ receiver range of $50 \mathrm{~km}$.

\begin{tabular}{c|c|c}
\hline $\begin{array}{c}\text { Height } \\
\text { (meters) }\end{array}$ & $\begin{array}{c}\text { Prop. } \\
\text { Loss } \\
(\mathrm{dB})\end{array}$ & $\begin{array}{c}\text { Prop. } \\
\text { Factor } \\
(\mathrm{dB})\end{array}$ \\
\hline 10.0 & 212.7 & -60.2 \\
20.0 & 199.3 & -46.8 \\
30.0 & 188.9 & -36.5 \\
40.0 & 180.1 & -27.6 \\
50.0 & 172.2 & -19.8 \\
60.0 & 165.5 & -13 \\
70.0 & 160.1 & -7.6 \\
80.0 & 156.7 & -4.3 \\
90.0 & 156.5 & -4 \\
100.0 & 163.2 & -10.7 \\
110.0 & 159.3 & -6.9 \\
120.0 & 156 & -3.6 \\
130.0 & 167.8 & -15.3 \\
140.0 & 155.7 & -3.3 \\
150.0 & 163 & -10.5 \\
160.0 & 156.1 & -3.6 \\
170.0 & 161.9 & -9.4 \\
180.0 & 155.7 & -3.3 \\
190.0 & 164.5 & -12 \\
200.0 & 154.9 & -2.5 \\
\hline & &
\end{tabular}

Table 34. Expected output for AFEVAP for $r_{\text {max }}$ receiver range of $50 \mathrm{~km}$.

\begin{tabular}{c|c|c}
\hline $\begin{array}{c}\text { Height } \\
\text { (meters) }\end{array}$ & $\begin{array}{c}\text { Prop. } \\
\text { Loss } \\
\text { (dB) }\end{array}$ & $\begin{array}{c}\text { Prop. } \\
\text { Factor } \\
(\mathrm{dB})\end{array}$ \\
\hline 50 & 133.7 & 2.3 \\
100 & 143.8 & -7.8 \\
150 & 135.8 & 0.2 \\
200 & 131.1 & 4.9 \\
250 & 142 & -6.1 \\
300 & 132.1 & 3.9 \\
350 & 133.1 & 2.8 \\
400 & 135.7 & 0.2 \\
450 & 131.2 & 4.8 \\
500 & 139.7 & -3.7 \\
550 & 130.4 & 5.6 \\
600 & 146.5 & -10.5 \\
650 & 130.1 & 5.8 \\
700 & 163.8 & -27.8 \\
750 & 130.1 & 5.9 \\
800 & 154.5 & -18.6 \\
850 & 130.1 & 5.9 \\
900 & 147.1 & -11.2 \\
950 & 130.2 & 5.8 \\
1000 & 142.8 & -6.8 \\
\hline & &
\end{tabular}


Table 35. Expected output for AFSBD for $r_{\text {max }}$ receiver range of $100 \mathrm{~km}$.

\begin{tabular}{c|c|c}
\hline $\begin{array}{c}\text { Height } \\
\text { (meters) }\end{array}$ & $\begin{array}{c}\text { Prop. } \\
\text { Loss } \\
(\mathrm{dB})\end{array}$ & $\begin{array}{c}\text { Prop. } \\
\text { Factor } \\
(\mathrm{dB})\end{array}$ \\
\hline 150 & 138.8 & 3.2 \\
300 & 166.3 & -24.3 \\
450 & 156.2 & -14.2 \\
600 & 143.1 & -1.1 \\
750 & 140.1 & 1.9 \\
900 & 148.9 & -6.9 \\
1050 & 138 & 4 \\
1200 & 143.9 & -1.9 \\
1350 & 139.7 & 2.3 \\
1500 & 138 & 4 \\
1650 & 155.6 & -13.6 \\
1800 & 137.1 & 4.9 \\
1950 & 139.6 & 2.4 \\
2100 & 147.8 & -5.8 \\
2250 & 136.8 & 5.2 \\
2400 & 139.1 & 2.8 \\
2550 & 151.9 & -9.9 \\
2700 & 137.2 & 4.8 \\
2850 & 137.5 & 4.5 \\
3000 & 155.1 & -13.1 \\
\hline & &
\end{tabular}

Table 36. Expected Output for AFSTD for $r_{\text {max }}$ receiver range of $50 \mathrm{~km}$.

\begin{tabular}{c|c|c}
\hline $\begin{array}{c}\text { Height } \\
\text { (meters) }\end{array}$ & $\begin{array}{c}\text { Prop. } \\
\text { Loss } \\
(\mathrm{dB})\end{array}$ & $\begin{array}{c}\text { Prop. } \\
\text { Factor } \\
(\mathrm{dB})\end{array}$ \\
\hline 50 & 143.8 & -17.4 \\
100 & 133.6 & -7.2 \\
150 & 127.3 & -0.8 \\
200 & 123.3 & 3.1 \\
250 & 121.3 & 5.2 \\
300 & 121.1 & 5.3 \\
350 & 123.2 & 3.3 \\
400 & 129.7 & -3.3 \\
450 & 137.6 & -11.2 \\
500 & 124.9 & 1.5 \\
550 & 121.3 & 5.1 \\
600 & 120.6 & 5.8 \\
650 & 122.3 & 4.1 \\
700 & 128.1 & -1.7 \\
750 & 141.2 & -14.8 \\
800 & 125.3 & 1.1 \\
850 & 121.3 & 5.1 \\
900 & 120.5 & 5.9 \\
950 & 122.1 & 4.3 \\
1000 & 127.6 & -1.1 \\
\hline & &
\end{tabular}


Table 37. Expected Output for AIRBORNE for $r_{\max }$ receiver range of $250 \mathrm{~km}$.

\begin{tabular}{c|c|c}
\hline $\begin{array}{c}\text { Height } \\
\text { (meters) }\end{array}$ & $\begin{array}{c}\text { Prop. } \\
\text { Loss } \\
(\mathrm{dB})\end{array}$ & $\begin{array}{c}\text { Prop. } \\
\text { Factor } \\
(\mathrm{dB})\end{array}$ \\
\hline 250 & 136.9 & 2.6 \\
500 & 136 & 3.5 \\
750 & 138.1 & 1.4 \\
1000 & 140 & -0.5 \\
1250 & 138.2 & 1.3 \\
1500 & 133.1 & 6.4 \\
1750 & 142.8 & -3.3 \\
2000 & 142.4 & -2.9 \\
2250 & 143 & -3.5 \\
2500 & 142.4 & -2.9 \\
2750 & 141.9 & -2.4 \\
3000 & 136.9 & 2.6 \\
3250 & 141 & -1.5 \\
3500 & 141 & -1.5 \\
3750 & 139.5 & 0 \\
4000 & 143.2 & -3.7 \\
4250 & 140 & -0.5 \\
4500 & 144 & -4.5 \\
4750 & 138.4 & 1.1 \\
5000 & 138.9 & 0.6 \\
\hline & &
\end{tabular}

Table 38. Expected output for BLOCK for $r_{\max }$ receiver range of $60 \mathrm{~km}$.

\begin{tabular}{c|c|c}
\hline $\begin{array}{c}\text { Height } \\
\text { (meters) }\end{array}$ & $\begin{array}{c}\text { Prop. } \\
\text { Loss } \\
\text { (dB) }\end{array}$ & $\begin{array}{c}\text { Prop. } \\
\text { Factor } \\
\text { (dB) }\end{array}$ \\
\hline 0 & -3276.6 & -3276.6 \\
20 & 216.1 & -88.1 \\
40 & 209.4 & -81.4 \\
60 & 207.8 & -79.8 \\
80 & 209 & -81 \\
100 & 207.2 & -79.2 \\
120 & 203 & -75 \\
140 & 200.7 & -72.7 \\
160 & 198.6 & -70.6 \\
180 & 195.1 & -67 \\
200 & 191.6 & -63.6 \\
220 & 188.2 & -60.2 \\
240 & 184.4 & -56.4 \\
260 & 180.6 & -52.6 \\
280 & 176.9 & -48.9 \\
300 & 173.3 & -45.3 \\
320 & 170 & -42 \\
340 & 167.1 & -39.1 \\
360 & 164.7 & -36.7 \\
380 & 162.6 & -34.6 \\
400 & 160.7 & -32.7 \\
\hline & &
\end{tabular}


Table 39. Expected output for CLEVAPW for receiver height of 0 and $500 \mathrm{~m}$, plus clutterto-noise ratio (CNR).

\begin{tabular}{|c|c|c|c|c|c|}
\hline & \multicolumn{2}{|c|}{ Rec. height at $0 \mathrm{~m}$} & \multicolumn{2}{|c|}{ Rec. height at $500 \mathrm{~m}$} & \multirow[b]{2}{*}{$\begin{array}{l}\text { CNR } \\
\text { (dB) }\end{array}$} \\
\hline $\begin{array}{l}\text { Range } \\
(\mathrm{km})\end{array}$ & $\begin{array}{l}\text { Prop. } \\
\text { Loss } \\
\text { (dB) }\end{array}$ & $\begin{array}{l}\text { Prop. } \\
\text { Factor } \\
\text { (dB) }\end{array}$ & $\begin{array}{l}\text { Prop. } \\
\text { Loss } \\
\text { (dB) }\end{array}$ & $\begin{array}{l}\text { Prop. } \\
\text { Factor } \\
\text { (dB) }\end{array}$ & \\
\hline 1 & 119.9 & -7.4 & 143.8 & -30.5 & 53.1 \\
\hline 2 & 121.7 & -3.3 & 149.2 & -30.5 & 52.2 \\
\hline 3 & -3276.7 & -3276.7 & 152.5 & -30.4 & 46.5 \\
\hline 4 & -3276.7 & -3276.7 & 155 & -30.5 & 40 \\
\hline 5 & 133.2 & -6.8 & 156.9 & -30.4 & 38.9 \\
\hline 6 & 135.8 & -7.8 & 158.5 & -30.5 & 34.7 \\
\hline 7 & 137.8 & -8.4 & 159.7 & -30.4 & 31.6 \\
\hline 8 & 139.5 & -9 & 160.9 & -30.3 & 28.8 \\
\hline 9 & 141.1 & -9.6 & 162.1 & -30.6 & 26.2 \\
\hline 10 & 142.6 & -10.2 & 162.7 & -30.3 & 23.7 \\
\hline 11 & 144 & -10.7 & 163.9 & -30.7 & 21.5 \\
\hline 12 & 145.2 & -11.2 & 166 & -32 & 19.5 \\
\hline 13 & 146.3 & -11.6 & 154.8 & -20 & 17.7 \\
\hline 14 & 147.3 & -11.9 & 150.1 & -14.7 & 16 \\
\hline 15 & 148.2 & -12.2 & 147.6 & -11.6 & 14.6 \\
\hline 16 & 149 & -12.5 & 146.4 & -9.9 & 13.3 \\
\hline 17 & 149.8 & -12.7 & 145.2 & -8.1 & 12 \\
\hline 18 & 150.5 & -13 & 144.9 & -7.4 & 10.7 \\
\hline 19 & 151.3 & -13.3 & 143.8 & -5.8 & 9.4 \\
\hline 20 & 152 & -13.6 & 144.2 & -5.7 & 8.2 \\
\hline 21 & 152.8 & -13.9 & 144.2 & -5.3 & 6.9 \\
\hline 22 & 153.5 & -14.2 & 143.8 & -4.5 & 5.7 \\
\hline 23 & 154.2 & -14.5 & 142.9 & -3.2 & 4.5 \\
\hline 24 & 154.9 & -14.8 & 143.9 & -3.9 & 3.3 \\
\hline 25 & 155.5 & -15.1 & 142.9 & -2.5 & 2.2 \\
\hline 26 & 156.1 & -15.4 & 144.3 & -3.6 & 1.1 \\
\hline 27 & 156.8 & -15.7 & 143.7 & -2.7 & 0 \\
\hline 28 & 157.4 & -16 & 143 & -1.7 & -1.1 \\
\hline 29 & 158 & -16.3 & 143 & -1.3 & -2.1 \\
\hline 30 & 158.5 & -16.5 & 143.1 & -1.1 & -3 \\
\hline 31 & 159.1 & -16.8 & 143.2 & -0.9 & -3.9 \\
\hline 32 & 159.6 & -17 & 143.3 & -0.8 & -4.9 \\
\hline 33 & 160.1 & -17.3 & 144 & -1.2 & -5.7 \\
\hline 34 & 160.6 & -17.5 & 145.4 & -2.4 & -6.6 \\
\hline
\end{tabular}


Table 39. Expected output for CLEVAPW for receiver height of 0 and $500 \mathrm{~m}$, plus clutterto-noise ratio (CNR).

\begin{tabular}{|c|c|c|c|c|c|}
\hline & \multicolumn{2}{|c|}{ Rec. height at $0 \mathrm{~m}$} & \multicolumn{2}{|c|}{ Rec. height at $500 \mathrm{~m}$} & \multirow[b]{2}{*}{$\begin{array}{l}\text { CNR } \\
\text { (dB) }\end{array}$} \\
\hline $\begin{array}{c}\text { Range } \\
(\mathrm{km})\end{array}$ & $\begin{array}{l}\text { Prop. } \\
\text { Loss } \\
\text { (dB) }\end{array}$ & $\begin{array}{l}\text { Prop. } \\
\text { Factor } \\
\text { (dB) }\end{array}$ & $\begin{array}{l}\text { Prop. } \\
\text { Loss } \\
\text { (dB) }\end{array}$ & $\begin{array}{l}\text { Prop. } \\
\text { Factor } \\
\text { (dB) }\end{array}$ & \\
\hline 35 & 161 & -17.7 & 145.4 & -2 & -6.6 \\
\hline 36 & 161.4 & -17.9 & 143.8 & -0.2 & -7.3 \\
\hline 37 & 161.8 & -18 & 144.6 & -0.8 & -8 \\
\hline 38 & 162.2 & -18.1 & 146.4 & -2.4 & -9.2 \\
\hline 39 & 162.5 & -18.2 & 144.1 & 0.1 & -9.8 \\
\hline 40 & 162.9 & -18.4 & 145.9 & -1.5 & -10.4 \\
\hline 41 & 163.2 & -18.5 & 145.5 & -0.8 & -11 \\
\hline 42 & 163.6 & -18.6 & 145.1 & -0.2 & -11.6 \\
\hline 43 & 163.9 & -18.8 & 146.7 & -1.6 & -12.2 \\
\hline 44 & 164.2 & -18.9 & 145 & 0.3 & -12.7 \\
\hline 45 & 164.6 & -19.1 & 147.1 & -1.6 & -13.3 \\
\hline 46 & 164.9 & -19.2 & 145.5 & 0.2 & -13.8 \\
\hline 47 & 165.2 & -19.3 & 146.6 & -0.7 & -14.4 \\
\hline 48 & 165.5 & -19.5 & 146.9 & -0.8 & -14.9 \\
\hline 49 & 165.8 & -19.6 & 145.6 & 0.6 & -15.5 \\
\hline 50 & 166.2 & -19.8 & 148.9 & -2.4 & -16.1 \\
\hline 51 & 166.5 & -19.9 & 145.6 & 1 & -16.7 \\
\hline 52 & 166.9 & -20.1 & 147.7 & -1 & -17.3 \\
\hline 53 & 167.2 & -20.3 & 148.2 & -1.2 & -17.9 \\
\hline 54 & 167.6 & -20.5 & 145.8 & 1.3 & -18.6 \\
\hline 55 & 168 & -20.7 & 149 & -1.7 & -19.2 \\
\hline 56 & 168.4 & -20.9 & 148.3 & -0.9 & -19.9 \\
\hline 57 & 168.7 & -21.2 & 146.1 & 1.4 & -20.6 \\
\hline 58 & 169.1 & -21.4 & 148.9 & -1.2 & -21.3 \\
\hline 59 & 169.5 & -21.6 & 149.7 & -1.9 & -22 \\
\hline 60 & 169.9 & -21.9 & 146.7 & 1.3 & -22.7 \\
\hline 61 & 170.2 & -22.1 & 147.7 & 0.4 & -23.3 \\
\hline 62 & 170.6 & -22.3 & 151.2 & -2.9 & -23.9 \\
\hline 63 & 170.9 & -22.4 & 146.2 & 2.2 & -24.5 \\
\hline 64 & 171.2 & -22.6 & 148.1 & 0.5 & -25 \\
\hline 65 & 171.5 & -22.8 & 152 & -3.3 & -25.5 \\
\hline 66 & 171.7 & -22.9 & 152.1 & -3.3 & -26 \\
\hline 67 & 172 & -23 & 148.7 & 0.3 & -26.4 \\
\hline 68 & 172.2 & -23.1 & 147 & 2.1 & -26.8 \\
\hline 69 & 172.4 & -23.2 & 147.6 & 1.6 & -27.2 \\
\hline 70 & 172.7 & -23.3 & 150.1 & -0.7 & -27.5 \\
\hline
\end{tabular}


Table 39. Expected output for CLEVAPW for receiver height of 0 and $500 \mathrm{~m}$, plus clutterto-noise ratio (CNR).

\begin{tabular}{|c|c|c|c|c|c|}
\hline & \multicolumn{2}{|c|}{ Rec. height at $0 \mathrm{~m}$} & \multicolumn{2}{|c|}{ Rec. height at $500 \mathrm{~m}$} & \multirow[b]{2}{*}{$\begin{array}{l}\text { CNR } \\
\text { (dB) }\end{array}$} \\
\hline $\begin{array}{c}\text { Range } \\
(\mathrm{km})\end{array}$ & $\begin{array}{l}\text { Prop. } \\
\text { Loss } \\
\text { (dB) }\end{array}$ & $\begin{array}{l}\text { Prop. } \\
\text { Factor } \\
\text { (dB) }\end{array}$ & $\begin{array}{l}\text { Prop. } \\
\text { Loss } \\
\text { (dB) }\end{array}$ & $\begin{array}{l}\text { Prop. } \\
\text { Factor } \\
(\mathrm{dB})\end{array}$ & \\
\hline 71 & 172.9 & -23.4 & 153.6 & -4.2 & -27.9 \\
\hline 72 & 173.1 & -23.5 & 151.7 & -2.1 & -28.2 \\
\hline 73 & 173.3 & -23.5 & 147.8 & 1.9 & -28.6 \\
\hline 74 & 173.5 & -23.6 & 147.7 & 2.1 & -28.9 \\
\hline 75 & 173.7 & -23.7 & 151.2 & -1.3 & -29.3 \\
\hline 76 & 173.9 & -23.8 & 155.2 & -5.1 & -29.6 \\
\hline 77 & 174.1 & -23.9 & 152.6 & -2.4 & -30 \\
\hline 78 & 174.3 & -24 & 148.8 & 1.5 & -30.4 \\
\hline 79 & 174.5 & -24.1 & 148.1 & 2.3 & -30.8 \\
\hline 80 & 174.8 & -24.2 & 149.8 & 0.7 & -31.2 \\
\hline 81 & 175 & -24.4 & 154 & -3.4 & -31.6 \\
\hline 82 & 175.2 & -24.5 & 155.9 & -5.2 & -32 \\
\hline 83 & 175.5 & -24.7 & 152 & -1.2 & -32.5 \\
\hline 84 & 175.7 & -24.8 & 149.6 & 1.3 & -32.9 \\
\hline 85 & 176 & -25 & 149 & 2 & -33.4 \\
\hline 86 & 176.3 & -25.1 & 150 & 1.2 & -33.9 \\
\hline 87 & 176.5 & -25.3 & 152.5 & -1.3 & -34.3 \\
\hline 88 & 176.8 & -25.5 & 156.8 & -5.5 & -34.8 \\
\hline 89 & 177 & -25.6 & 159.4 & -7.9 & -35.3 \\
\hline 90 & 177.3 & -25.8 & 157 & -5.5 & -35.8 \\
\hline 91 & 177.6 & -25.9 & 153.5 & -1.8 & -36.2 \\
\hline 92 & 177.8 & -26.1 & 151.4 & 0.3 & -36.7 \\
\hline 93 & 178 & -26.2 & 150.6 & 1.3 & -37.1 \\
\hline 94 & 178.3 & -26.4 & 150.6 & 1.4 & -37.5 \\
\hline 95 & 178.5 & -26.5 & 151.3 & 0.7 & -37.9 \\
\hline 96 & 178.7 & -26.6 & 152.7 & -0.6 & -38.3 \\
\hline 97 & 178.9 & -26.8 & 154.8 & -2.7 & -38.7 \\
\hline 98 & 179.2 & -26.9 & 157.9 & -5.6 & -39.1 \\
\hline 99 & 179.4 & -27 & 161.5 & -9.1 & -39.4 \\
\hline 100 & 179.6 & -27.1 & 162.8 & -10.3 & -39.8 \\
\hline
\end{tabular}


Table 40. Expected output for CLSBDL for receiver height of 500 and $1000 \mathrm{~m}$, plus clutter-to-noise ratio (CNR).

\begin{tabular}{|c|c|c|c|c|c|}
\hline & \multicolumn{2}{|c|}{ Rec. height at $500 \mathrm{~m}$} & \multicolumn{2}{|c|}{ Rec. height at $1000 \mathrm{~m}$} & \multirow[b]{2}{*}{$\begin{array}{l}\text { CNR } \\
\text { (dB) }\end{array}$} \\
\hline $\begin{array}{c}\text { Range } \\
(\mathrm{km})\end{array}$ & $\begin{array}{l}\text { Prop. } \\
\text { Loss } \\
\text { (dB) }\end{array}$ & $\begin{array}{l}\text { Prop. } \\
\text { Factor } \\
\text { (dB) }\end{array}$ & $\begin{array}{l}\text { Prop. } \\
\text { Loss } \\
\text { (dB) }\end{array}$ & $\begin{array}{l}\text { Prop. } \\
\text { Factor } \\
\text { (dB) }\end{array}$ & \\
\hline 1 & -3276.7 & -3276.7 & -3276.7 & -3276.7 & -33.7 \\
\hline 2 & -3276.7 & -3276.7 & -3276.7 & -3276.7 & 54.8 \\
\hline 3 & -3276.7 & -3276.7 & -3276.7 & -3276.7 & 81.8 \\
\hline 4 & -3276.7 & -3276.7 & -3276.7 & -3276.7 & 80.1 \\
\hline 5 & -3276.7 & -3276.7 & -3276.7 & -3276.7 & 36.8 \\
\hline 6 & -3276.7 & -3276.7 & -3276.7 & -3276.7 & 28.1 \\
\hline 7 & -3276.7 & -3276.7 & -3276.7 & -3276.7 & 65.3 \\
\hline 8 & -3276.7 & -3276.7 & -3276.7 & -3276.7 & 88.7 \\
\hline 9 & -3276.7 & -3276.7 & -3276.7 & -3276.7 & 76.9 \\
\hline 10 & -3276.7 & -3276.7 & -3276.7 & -3276.7 & 90.8 \\
\hline 11 & -3276.7 & -3276.7 & -3276.7 & -3276.7 & 77.6 \\
\hline 12 & -3276.7 & -3276.7 & -3276.7 & -3276.7 & 89.9 \\
\hline 13 & -3276.7 & -3276.7 & -3276.7 & -3276.7 & 59.2 \\
\hline 14 & -3276.7 & -3276.7 & -3276.7 & -3276.7 & 64.5 \\
\hline 15 & -3276.7 & -3276.7 & -3276.7 & -3276.7 & 67.3 \\
\hline 16 & -3276.7 & -3276.7 & -3276.7 & -3276.7 & 56.8 \\
\hline 17 & -3276.7 & -3276.7 & -3276.7 & -3276.7 & 61.7 \\
\hline 18 & -3276.7 & -3276.7 & -3276.7 & -3276.7 & 64 \\
\hline 19 & -3276.7 & -3276.7 & -3276.7 & -3276.7 & 64.5 \\
\hline 20 & -3276.7 & -3276.7 & -3276.7 & -3276.7 & 46.3 \\
\hline 21 & -3276.7 & -3276.7 & -3276.7 & -3276.7 & 71.5 \\
\hline 22 & -3276.7 & -3276.7 & -3276.7 & -3276.7 & 47.9 \\
\hline 23 & -3276.7 & -3276.7 & -3276.7 & -3276.7 & 57.8 \\
\hline 24 & -3276.7 & -3276.7 & -3276.7 & -3276.7 & 46.2 \\
\hline 25 & 129.5 & 0.5 & -3276.7 & -3276.7 & 59.1 \\
\hline 26 & 130.8 & -0.5 & -3276.7 & -3276.7 & 93.3 \\
\hline 27 & 130.3 & 0.3 & -3276.7 & -3276.7 & 61 \\
\hline 28 & 131.9 & -1 & -3276.7 & -3276.7 & 96.5 \\
\hline 29 & 132 & -0.8 & -3276.7 & -3276.7 & 82.1 \\
\hline 30 & 131.6 & -0.1 & -3276.7 & -3276.7 & 42.4 \\
\hline 31 & 133.2 & -1.4 & -3276.7 & -3276.7 & 35.2 \\
\hline 32 & 131.7 & 0.4 & -3276.7 & -3276.7 & 49.5 \\
\hline 33 & 132.7 & -0.4 & -3276.7 & -3276.7 & 14.4 \\
\hline 34 & 133 & -0.4 & -3276.7 & -3276.7 & 30.6 \\
\hline 35 & 134.1 & -1.2 & -3276.7 & -3276.7 & 34.5 \\
\hline 36 & 134.3 & -1.2 & -3276.7 & -3276.7 & 19.7 \\
\hline
\end{tabular}


Table 40. Expected output for CLSBDL for receiver height of 500 and $1000 \mathrm{~m}$, plus clutter-to-noise ratio (CNR).

\begin{tabular}{|c|c|c|c|c|c|}
\hline \multirow[b]{2}{*}{$\begin{array}{c}\text { Range } \\
(\mathrm{km})\end{array}$} & \multicolumn{2}{|c|}{ Rec. height at $500 \mathrm{~m}$} & \multicolumn{2}{|c|}{ Rec. height at $1000 \mathrm{~m}$} & \multirow[b]{2}{*}{$\begin{array}{l}\text { CNR } \\
\text { (dB) }\end{array}$} \\
\hline & $\begin{array}{l}\text { Prop. } \\
\text { Loss } \\
\text { (dB) }\end{array}$ & $\begin{array}{l}\text { Prop. } \\
\text { Factor } \\
\text { (dB) }\end{array}$ & $\begin{array}{l}\text { Prop. } \\
\text { Loss } \\
\text { (dB) }\end{array}$ & $\begin{array}{l}\text { Prop. } \\
\text { Factor } \\
\text { (dB) }\end{array}$ & \\
\hline 37 & 135.2 & -1.8 & -3276.7 & -3276.7 & 46.3 \\
\hline 38 & 135.4 & -1.8 & -3276.7 & -3276.7 & 75.5 \\
\hline 39 & 134.2 & -0.4 & -3276.7 & -3276.7 & 54 \\
\hline 40 & 136 & -2 & -3276.7 & -3276.7 & 28.6 \\
\hline 41 & 134.8 & -0.6 & -3276.7 & -3276.7 & 50.2 \\
\hline 42 & 135.6 & -1.2 & -3276.7 & -3276.7 & 86.6 \\
\hline 43 & 135.1 & -0.5 & -3276.7 & -3276.7 & 46.6 \\
\hline 44 & 135.9 & -1.1 & -3276.7 & -3276.7 & 43.7 \\
\hline 45 & 136.8 & -1.7 & -3276.7 & -3276.7 & 30.6 \\
\hline 46 & 136.9 & -1.7 & -3276.7 & -3276.7 & 5.1 \\
\hline 47 & 136.5 & -1 & -3276.7 & -3276.7 & -20.6 \\
\hline 48 & 137.5 & -1.9 & -3276.7 & -3276.7 & -8.9 \\
\hline 49 & 137.1 & -1.3 & -3276.7 & -3276.7 & 11.4 \\
\hline 50 & 138.3 & -2.4 & -3276.7 & -3276.7 & 3.9 \\
\hline 51 & 136.9 & -0.7 & -3276.7 & -3276.7 & 27.1 \\
\hline 52 & 137.2 & -0.9 & -3276.7 & -3276.7 & -14.6 \\
\hline 53 & 137.7 & -1.2 & 136.4 & 0 & 23.6 \\
\hline 54 & 138.5 & -1.9 & 136.1 & 0.5 & 48.3 \\
\hline 55 & 139.4 & -2.6 & 136.5 & 0.3 & 39.6 \\
\hline 56 & 138.3 & -1.3 & 137.2 & -0.3 & 74.3 \\
\hline 57 & 139.6 & -2.5 & 138.3 & -1.2 & 67.9 \\
\hline 58 & 141.3 & -4 & 138.6 & -1.3 & 73.9 \\
\hline 59 & 138.9 & -1.5 & 138.2 & -0.8 & 94.5 \\
\hline 60 & 139.3 & -1.8 & 138.6 & -1.1 & 53.8 \\
\hline 61 & 141.1 & -3.4 & 138.9 & -1.2 & 86 \\
\hline 62 & 143.1 & -5.3 & 138.6 & -0.7 & 92.2 \\
\hline 63 & 138.7 & -0.8 & 139.6 & -1.6 & 88.1 \\
\hline 64 & 144.7 & -6.6 & 138.7 & -0.5 & 51.8 \\
\hline 65 & 143.9 & -5.7 & 139.8 & -1.6 & 61.8 \\
\hline 66 & 141.8 & -3.5 & 139.2 & -0.9 & 22.4 \\
\hline 67 & 143.8 & -5.3 & 139.6 & -1.1 & 37.2 \\
\hline 68 & 140.2 & -1.6 & 139.6 & -0.9 & 27.4 \\
\hline 69 & 142.1 & -3.3 & 140.6 & -1.8 & 37.1 \\
\hline 70 & 142.6 & -3.7 & 139.8 & -0.9 & 77.5 \\
\hline 71 & 146.4 & -7.4 & 142.2 & -3.2 & 70.3 \\
\hline 72 & 143.3 & -4.2 & 140.3 & -1.2 & 29.1 \\
\hline
\end{tabular}


Table 40. Expected output for CLSBDL for receiver height of 500 and $1000 \mathrm{~m}$, plus clutter-to-noise ratio (CNR).

\begin{tabular}{c|c|c||c|c||c}
\hline & \multicolumn{2}{|c||}{ Rec. height at $500 \mathrm{~m}$} & \multicolumn{2}{|c||}{ Rec. height at $1000 \mathrm{~m}$} & \\
\hline $\begin{array}{c}\text { Range } \\
(\mathrm{km})\end{array}$ & $\begin{array}{c}\text { Prop. } \\
\text { Loss } \\
(\mathrm{dB})\end{array}$ & $\begin{array}{c}\text { Prop. } \\
\text { Factor } \\
(\mathrm{dB})\end{array}$ & $\begin{array}{c}\text { Prop. } \\
\text { Loss } \\
(\mathrm{dB})\end{array}$ & $\begin{array}{c}\text { Prop. } \\
\text { Factor } \\
(\mathrm{dB})\end{array}$ & $\begin{array}{c}\text { CNR } \\
(\mathrm{dB})\end{array}$ \\
\hline 73 & 146.3 & -7.1 & 139.6 & -0.4 & 15.1 \\
74 & 151.9 & -12.6 & 142.2 & -2.9 & 22 \\
75 & 140.3 & -0.8 & 139.7 & -0.2 & -1.6 \\
76 & 143.7 & -4.1 & 142.4 & -2.8 & 5 \\
77 & 147.3 & -7.5 & 141.6 & -1.8 & 48 \\
78 & 158 & -18.2 & 139.7 & 0.1 & 29.5 \\
79 & 150.2 & -10.3 & 142.4 & -2.4 & 29.6 \\
80 & 144.9 & -4.9 & 142.8 & -2.8 & 46.6 \\
81 & 150 & -9.9 & 141 & -0.8 & 21.6 \\
82 & 147.8 & -7.5 & 141.9 & -1.7 & 30.3 \\
83 & 148.8 & -8.4 & 142.1 & -1.7 & 38.5 \\
84 & 156.8 & -16.3 & 142.5 & -2.1 & 1.5 \\
85 & 147.6 & -7 & 144.5 & -4 & 18.6 \\
86 & 144.7 & -4.1 & 143.9 & -3.2 & 32.3 \\
87 & 145.2 & -4.4 & 141.1 & -0.3 & 51.8 \\
88 & 154.3 & -13.5 & 140.6 & 0.3 & 36.3 \\
89 & 146.9 & -6 & 142 & -1.1 & 77.7 \\
90 & 147.5 & -6.4 & 142.7 & -1.6 & 53.4 \\
91 & 163.6 & -22.4 & 142.3 & -1.1 & 13.4 \\
92 & 150.9 & -9.7 & 142.5 & -1.2 & 17.4 \\
93 & 147.9 & -6.5 & 143.1 & -1.8 & 50.1 \\
94 & 153.3 & -11.9 & 143.2 & -1.8 & 48.1 \\
95 & 149.8 & -8.2 & 143.6 & -2 & 39.5 \\
96 & 151.5 & -9.8 & 143.7 & -2.1 & 46.8 \\
97 & 147.9 & -6.2 & 142.7 & -1 & 53.2 \\
98 & 149.8 & -8 & 144.9 & -3.1 & 48 \\
99 & 157.8 & -15.9 & 148 & -6.1 & 39.6 \\
100 & 161.9 & -20 & 149.3 & -7.3 & 47.4 \\
\hline & & & & &
\end{tabular}


Table 41. Expected output for CLSBDW for receiver height of 500 and $1000 \mathrm{~m}$, plus clutter-to-noise ratio (CNR).

\begin{tabular}{|c|c|c|c|c|c|}
\hline & Rec. $\mathrm{h}$ & at $500 \mathrm{~m}$ & Rec. he & $1000 \mathrm{~m}$ & \\
\hline $\begin{array}{c}\text { Range } \\
(\mathrm{km})\end{array}$ & $\begin{array}{l}\text { Prop. } \\
\text { Loss } \\
\text { (dB) }\end{array}$ & $\begin{array}{l}\text { Prop. } \\
\text { Factor } \\
(\mathrm{dB})\end{array}$ & $\begin{array}{l}\text { Prop. } \\
\text { Loss } \\
\text { (dB) }\end{array}$ & $\begin{array}{l}\text { Prop. } \\
\text { Factor } \\
(\mathrm{dB})\end{array}$ & $\begin{array}{l}\text { CNR } \\
(\mathrm{dB})\end{array}$ \\
\hline 1 & 102.9 & 0 & 104.9 & 0 & 91.8 \\
\hline 2 & 108.3 & 0 & 109 & 0 & 90.9 \\
\hline 3 & 111.6 & 0 & 112 & 0 & 81.3 \\
\hline 4 & 114.1 & 0 & 114.3 & 0 & 72.4 \\
\hline 5 & 116 & 0 & 116.1 & 0 & 65.2 \\
\hline 6 & 117.6 & 0 & 117.7 & 0 & 59.3 \\
\hline 7 & 118.9 & 0 & 119 & 0 & 53.6 \\
\hline 8 & 120 & 0 & 120.1 & 0 & 48.1 \\
\hline 9 & 121.1 & 0 & 121.1 & 0 & 42.8 \\
\hline 10 & 122 & 0 & 122 & 0 & 36.9 \\
\hline 11 & 122.8 & 0 & 122.9 & 0 & 33 \\
\hline 12 & 123.6 & 0 & 123.6 & 0 & 29.5 \\
\hline 13 & 124.3 & 0 & 124.3 & 0 & 26.2 \\
\hline 14 & 124.9 & 0 & 124.9 & 0 & 22.9 \\
\hline 15 & 125.5 & 0 & 125.5 & 0 & 19.6 \\
\hline 16 & 126.1 & 0 & 126.1 & 0 & 16.3 \\
\hline 17 & 126.6 & 0 & 126.6 & 0 & 13.5 \\
\hline 18 & 127.1 & 0 & 127.1 & 0 & 10.5 \\
\hline 19 & 127.5 & 0 & 127.6 & 0 & 7.5 \\
\hline 20 & 128 & 0 & 128.1 & 0 & 4.2 \\
\hline 21 & 128.4 & 0 & 128.5 & -0.1 & 1 \\
\hline 22 & 128.8 & 0 & 128.9 & -0.1 & -0.9 \\
\hline 23 & 129.2 & 0 & 129.3 & -0.1 & -4.7 \\
\hline 24 & 129.6 & 0 & 129.7 & -0.1 & -7.1 \\
\hline 25 & 129.9 & 0 & 130 & -0.1 & -10.6 \\
\hline 26 & 130.3 & 0 & 130.4 & -0.1 & -12.5 \\
\hline 27 & 130.6 & 0 & 130.7 & -0.1 & -20.9 \\
\hline 28 & 130.9 & 0 & 131 & -0.1 & -19 \\
\hline 29 & 131.2 & 0 & 131.3 & -0.1 & -18.6 \\
\hline 30 & 131.5 & 0 & 131.6 & -0.1 & -28.3 \\
\hline 31 & 131.8 & 0 & 131.9 & -0.1 & -29 \\
\hline 32 & 132.1 & 0 & 132.2 & -0.1 & -28 \\
\hline 33 & 132.4 & 0 & 132.5 & -0.1 & -37.6 \\
\hline 34 & 132.5 & 0.1 & 132.8 & -0.1 & -37.5 \\
\hline 35 & 132.9 & -0.1 & 133 & -0.1 & -27.7 \\
\hline 36 & 133.5 & -0.4 & 133.3 & -0.2 & -47.2 \\
\hline
\end{tabular}


Table 41. Expected output for CLSBDW for receiver height of 500 and $1000 \mathrm{~m}$, plus clutter-to-noise ratio (CNR).

\begin{tabular}{|c|c|c|c|c|c|}
\hline \multirow[b]{2}{*}{$\begin{array}{c}\text { Range } \\
(\mathrm{km})\end{array}$} & \multicolumn{2}{|c|}{ Rec. height at $500 \mathrm{~m}$} & \multicolumn{2}{|c|}{ Rec. height at $1000 \mathrm{~m}$} & \multirow[b]{2}{*}{$\begin{array}{l}\text { CNR } \\
\text { (dB) }\end{array}$} \\
\hline & $\begin{array}{l}\text { Prop. } \\
\text { Loss } \\
\text { (dB) }\end{array}$ & $\begin{array}{l}\text { Prop. } \\
\text { Factor } \\
\text { (dB) }\end{array}$ & $\begin{array}{l}\text { Prop. } \\
\text { Loss } \\
\text { (dB) }\end{array}$ & $\begin{array}{l}\text { Prop. } \\
\text { Factor } \\
\text { (dB) }\end{array}$ & \\
\hline 37 & 134.1 & -0.8 & 133.5 & -0.2 & -36.2 \\
\hline 38 & 134.3 & -0.7 & 133.8 & -0.2 & -34.1 \\
\hline 39 & 134.2 & -0.4 & 134 & -0.2 & -38.5 \\
\hline 40 & 133.8 & 0.3 & 134.2 & -0.2 & -36.1 \\
\hline 41 & 133.3 & 0.9 & 134.4 & -0.2 & -32.7 \\
\hline 42 & 133.2 & 1.3 & 134.7 & -0.2 & -45.9 \\
\hline 43 & 133.3 & 1.4 & 134.9 & -0.2 & -25.8 \\
\hline 44 & 133.8 & 1.1 & 135.1 & -0.2 & -30.2 \\
\hline 45 & 134.6 & 0.5 & 135.3 & -0.2 & -30 \\
\hline 46 & 135.6 & -0.4 & 135.5 & -0.3 & -36.2 \\
\hline 47 & 136.9 & -1.5 & 135.7 & -0.3 & -25 \\
\hline 48 & 138.2 & -2.6 & 135.9 & -0.3 & -24.2 \\
\hline 49 & 139.3 & -3.5 & 136.1 & -0.3 & -43.7 \\
\hline 50 & 139.8 & -3.8 & 136.3 & -0.3 & -20.8 \\
\hline 51 & 139.5 & -3.3 & 136.5 & -0.3 & -20.3 \\
\hline 52 & 138.6 & -2.3 & 136.6 & -0.3 & -23.6 \\
\hline 53 & 137.9 & -1.4 & 136.8 & -0.3 & -25.6 \\
\hline 54 & 137.1 & -0.4 & 137 & -0.4 & -39.7 \\
\hline 55 & 136.4 & 0.4 & 137.2 & -0.4 & -27 \\
\hline 56 & 135.9 & 1.1 & 137.3 & -0.4 & -15.2 \\
\hline 57 & 135.5 & 1.6 & 137.5 & -0.4 & -12.6 \\
\hline 58 & 135.3 & 1.9 & 137.7 & -0.4 & -16.7 \\
\hline 59 & 135.3 & 2.1 & 137.8 & -0.4 & -17.7 \\
\hline 60 & 135.3 & 2.3 & 138 & -0.5 & -8.8 \\
\hline 61 & 135.4 & 2.3 & 138.2 & -0.5 & -2.8 \\
\hline 62 & 135.7 & 2.1 & 138.3 & -0.5 & -1.7 \\
\hline 63 & 136 & 2 & 138.4 & -0.4 & -4.5 \\
\hline 64 & 136.4 & 1.7 & 138.5 & -0.4 & -2.2 \\
\hline 65 & 137 & 1.3 & 138.7 & -0.5 & 3.3 \\
\hline 66 & 137.5 & 0.9 & 139 & -0.6 & 5.8 \\
\hline 67 & 138.1 & 0.4 & 139.3 & -0.8 & 6.9 \\
\hline 68 & 138.8 & -0.2 & 139.7 & -1.1 & 8.6 \\
\hline 69 & 139.6 & -0.8 & 140.1 & -1.3 & 10 \\
\hline 70 & 140.4 & -1.5 & 140.3 & -1.4 & 10.6 \\
\hline 71 & 141.2 & -2.2 & 140.4 & -1.4 & 11.7 \\
\hline 72 & 142.2 & -3 & 140.3 & -1.2 & 14 \\
\hline
\end{tabular}


Table 41. Expected output for CLSBDW for receiver height of 500 and $1000 \mathrm{~m}$, plus clutter-to-noise ratio (CNR).

\begin{tabular}{|c|c|c|c|c|c|}
\hline & Rec. $\mathrm{h}$ & at $500 \mathrm{~m}$ & Rec. he & $\mathrm{t} 1000 \mathrm{~m}$ & \\
\hline $\begin{array}{c}\text { Range } \\
(\mathrm{km})\end{array}$ & $\begin{array}{l}\text { Prop. } \\
\text { Loss } \\
\text { (dB) }\end{array}$ & $\begin{array}{l}\text { Prop. } \\
\text { Factor } \\
\text { (dB) }\end{array}$ & $\begin{array}{l}\text { Prop. } \\
\text { Loss } \\
\text { (dB) }\end{array}$ & $\begin{array}{l}\text { Prop. } \\
\text { Factor } \\
\text { (dB) }\end{array}$ & $\begin{array}{l}\text { CNR } \\
(\mathrm{dB})\end{array}$ \\
\hline 73 & 143.2 & -3.9 & 140.1 & -0.9 & 16.8 \\
\hline 74 & 144.2 & -4.8 & 139.8 & -0.5 & 19.3 \\
\hline 75 & 145.2 & -5.7 & 139.6 & -0.1 & 21.8 \\
\hline 76 & 146.4 & -6.8 & 139.4 & 0.2 & 24.6 \\
\hline 77 & 147.6 & -7.9 & 139.2 & 0.5 & 27.7 \\
\hline 78 & 148.7 & -8.9 & 139.2 & 0.6 & 30.9 \\
\hline 79 & 149.8 & -9.9 & 139.3 & 0.7 & 39 \\
\hline 80 & 151 & -11 & 139.5 & 0.5 & 43.2 \\
\hline 81 & 152 & -11.8 & 139.9 & 0.3 & 46.1 \\
\hline 82 & 153.2 & -13 & 140.3 & -0.1 & 49 \\
\hline 83 & 153.8 & -13.4 & 141 & -0.6 & 51.8 \\
\hline 84 & 154.7 & -14.2 & 141.7 & -1.2 & 54.4 \\
\hline 85 & 155.4 & -14.8 & 142.5 & -1.9 & 56.6 \\
\hline 86 & 156 & -15.3 & 143.4 & -2.7 & 58.2 \\
\hline 87 & 156.7 & -15.9 & 144.2 & -3.5 & 59.4 \\
\hline 88 & 156.8 & -15.9 & 145 & -4.1 & 60.2 \\
\hline 89 & 156.9 & -15.9 & 145.5 & -4.6 & 60.6 \\
\hline 90 & 157.2 & -16.1 & 145.7 & -4.7 & 60.3 \\
\hline 91 & 157.3 & -16.1 & 145.6 & -4.4 & 59.7 \\
\hline 92 & 158 & -16.7 & 145.1 & -3.9 & 58.5 \\
\hline 93 & 157.7 & -16.4 & 144.4 & -3 & 56.7 \\
\hline 94 & 158.4 & -17 & 143.8 & -2.3 & 55 \\
\hline 95 & 157.8 & -16.2 & 143.2 & -1.7 & 52.6 \\
\hline 96 & 158.8 & -17.1 & 142.7 & -1 & 50.9 \\
\hline 97 & 159.6 & -17.9 & 142.2 & -0.4 & 48.7 \\
\hline 98 & 159.4 & -17.5 & 141.8 & 0 & 46.7 \\
\hline 99 & 160.3 & -18.4 & 141.5 & 0.4 & 44.3 \\
\hline 100 & 160.3 & -18.3 & 141.2 & 0.8 & 40.6 \\
\hline
\end{tabular}


Table 42. Expected output for CLSBDWL for receiver height of 1500 and $3000 \mathrm{~m}$, plus clutter-to-noise ratio (CNR).

\begin{tabular}{|c|c|c|c|c|c|}
\hline \multirow[b]{2}{*}{$\begin{array}{c}\text { Range } \\
(\mathrm{km})\end{array}$} & \multicolumn{2}{|c|}{ Rec. height at $1500 \mathrm{~m}$} & \multicolumn{2}{|c|}{ Rec. height at $3000 \mathrm{~m}$} & \multirow[b]{2}{*}{$\begin{array}{l}\text { CNR } \\
\text { (dB) }\end{array}$} \\
\hline & $\begin{array}{l}\text { Prop. } \\
\text { Loss } \\
\text { (dB) }\end{array}$ & $\begin{array}{l}\text { Prop. } \\
\text { Factor } \\
\text { (dB) }\end{array}$ & $\begin{array}{l}\text { Prop. } \\
\text { Loss } \\
\text { (dB) }\end{array}$ & $\begin{array}{l}\text { Prop. } \\
\text { Factor } \\
\text { (dB) }\end{array}$ & \\
\hline 2 & 145.8 & -30.5 & 149 & -30.5 & 58.3 \\
\hline 4 & 150.4 & -30.4 & 151.9 & -30.5 & 39.7 \\
\hline 6 & 134.2 & -11 & 154.3 & -30.4 & 25.6 \\
\hline 8 & 131.3 & -5.7 & 156.4 & -30.4 & 14.3 \\
\hline 10 & 131 & -3.4 & 147.9 & -20.1 & 3.5 \\
\hline 12 & 131.6 & -2.5 & 140.2 & -11 & -4.5 \\
\hline 14 & 132 & -1.6 & 138.2 & -7.7 & -11.9 \\
\hline 16 & 132.9 & -1.3 & 137.2 & -5.5 & -19.1 \\
\hline 18 & 133.1 & -0.6 & 136.6 & -4 & -26.1 \\
\hline 20 & 134.1 & -0.7 & 136.6 & -3.1 & -33 \\
\hline 22 & 135.3 & -1.1 & 137.4 & -3 & -39.7 \\
\hline 24 & 135.2 & -0.2 & 137.3 & -2.2 & -46.2 \\
\hline 26 & 136.9 & -1.1 & 137.4 & -1.6 & -52.2 \\
\hline 28 & 136.2 & 0.2 & 138.1 & -1.7 & -57.8 \\
\hline 30 & 136.6 & 0.3 & 138.5 & -1.5 & -69 \\
\hline 32 & 137.9 & -0.4 & 138.7 & -1.2 & -73.3 \\
\hline 34 & 138.7 & -0.6 & 139.4 & -1.4 & -93.1 \\
\hline 36 & 139.1 & -0.5 & 139.9 & -1.3 & -80.2 \\
\hline 38 & 138.3 & 0.7 & 139.6 & -0.6 & -84.8 \\
\hline 40 & 138.9 & 0.6 & 140.7 & -1.3 & -87.2 \\
\hline 42 & 139.4 & 0.5 & 141.2 & -1.3 & -83.9 \\
\hline 44 & 139.5 & 0.8 & 141.2 & -0.9 & -92.5 \\
\hline 46 & 139.8 & 0.8 & 140.7 & 0 & -76.5 \\
\hline 48 & 141.5 & -0.4 & 142.2 & -1.2 & -73.5 \\
\hline 50 & 143.2 & -1.8 & 141.3 & 0.1 & -97.2 \\
\hline 52 & 141 & 0.8 & 142.8 & -1 & -71.1 \\
\hline 54 & 142.2 & -0.2 & 143.1 & -1 & -67.6 \\
\hline 56 & 143.4 & -1.1 & 142.7 & -0.3 & -67.1 \\
\hline 58 & 141.8 & 0.9 & 142.6 & 0.1 & -72.4 \\
\hline 60 & 144.7 & -1.7 & 142.8 & 0.2 & -61.4 \\
\hline 62 & 142.4 & 0.9 & 143.3 & 0 & -71.8 \\
\hline 64 & 144.5 & -1 & 144.1 & -0.6 & -44 \\
\hline 66 & 144.1 & -0.3 & 145.1 & -1.3 & -39.8 \\
\hline 68 & 143.1 & 1 & 144.9 & -0.8 & -38.2 \\
\hline 70 & 147.3 & -3 & 143.8 & 0.5 & -40.2 \\
\hline 72 & 143.4 & 1.1 & 144.5 & 0 & -34.4 \\
\hline
\end{tabular}


Table 42. Expected output for CLSBDWL for receiver height of 1500 and $3000 \mathrm{~m}$, plus clutter-to-noise ratio (CNR).

\begin{tabular}{|c|c|c|c|c|c|}
\hline \multirow[b]{2}{*}{$\begin{array}{c}\text { Range } \\
(\mathrm{km})\end{array}$} & \multicolumn{2}{|c|}{ Rec. height at $1500 \mathrm{~m}$} & \multicolumn{2}{|c|}{ Rec. height at $3000 \mathrm{~m}$} & \multirow[b]{2}{*}{$\begin{array}{l}\text { CNR } \\
\text { (dB) }\end{array}$} \\
\hline & $\begin{array}{l}\text { Prop. } \\
\text { Loss } \\
\text { (dB) }\end{array}$ & $\begin{array}{l}\text { Prop. } \\
\text { Factor } \\
\text { (dB) }\end{array}$ & $\begin{array}{l}\text { Prop. } \\
\text { Loss } \\
\text { (dB) }\end{array}$ & $\begin{array}{l}\text { Prop. } \\
\text { Factor } \\
\text { (dB) }\end{array}$ & \\
\hline 74 & 144.5 & 0.3 & 146.3 & -1.5 & -24.4 \\
\hline 76 & 148.3 & -3.3 & 144.5 & 0.5 & -12.7 \\
\hline 78 & 144.3 & 1 & 145.7 & -0.5 & -1.8 \\
\hline 80 & 144.3 & 1.2 & 146.1 & -0.6 & 11.4 \\
\hline 82 & 148.8 & -3.1 & 145.2 & 0.5 & 18.3 \\
\hline 84 & 147.4 & -1.5 & 147.2 & -1.3 & 21.6 \\
\hline 86 & 144.4 & 1.7 & 145.4 & 0.7 & 18.1 \\
\hline 88 & 145.3 & 1 & 147.6 & -1.3 & -10 \\
\hline 90 & 149.9 & -3.4 & 146 & 0.5 & 14 \\
\hline 92 & 149.6 & -2.9 & 147.3 & -0.6 & 20.9 \\
\hline 94 & 145.7 & 1.1 & 147.4 & -0.5 & 19.3 \\
\hline 96 & 145 & 2 & 146.4 & 0.7 & 19.8 \\
\hline 98 & 147.2 & 0.1 & 149.3 & -2.1 & 12.8 \\
\hline 100 & 152.1 & -4.7 & 146.5 & 0.9 & 6.8 \\
\hline 102 & 151.5 & -4 & 148.1 & -0.5 & -19.3 \\
\hline 104 & 147.4 & 0.3 & 149.2 & -1.5 & 2.9 \\
\hline 106 & 145.8 & 2.1 & 146.7 & 1.2 & 30 \\
\hline 108 & 146.2 & 1.8 & 149 & -0.9 & 11.1 \\
\hline 110 & 148.7 & -0.4 & 149.9 & -1.6 & 13 \\
\hline 112 & 153.4 & -5 & 147.2 & 1.2 & 26 \\
\hline 114 & 155.3 & -6.7 & 148.7 & -0.1 & -13.7 \\
\hline 116 & 150.6 & -1.9 & 151.4 & -2.7 & 17.4 \\
\hline 118 & 147.9 & 1 & 148.4 & 0.4 & -30.8 \\
\hline 120 & 146.8 & 2.2 & 147.8 & 1.2 & -32 \\
\hline 122 & 147 & 2.1 & 150.8 & -1.7 & -53.7 \\
\hline 124 & 148.4 & 0.9 & 151.6 & -2.3 & -48.8 \\
\hline 126 & 151.2 & -1.8 & 148.5 & 0.9 & -62.1 \\
\hline 128 & 155.8 & -6.3 & 148.3 & 1.2 & -58.9 \\
\hline 130 & 159.9 & -10.3 & 151.2 & -1.6 & -118.4 \\
\hline 132 & 154.7 & -4.9 & 153 & -3.2 & -101.4 \\
\hline 134 & 151.4 & -1.5 & 149.8 & 0.2 & -108 \\
\hline 136 & 149.4 & 0.7 & 148.5 & 1.6 & -102.2 \\
\hline 138 & 148.5 & 1.7 & 149.9 & 0.3 & -74 \\
\hline 140 & 148.1 & 2.2 & 153.6 & -3.2 & -96.4 \\
\hline 142 & 148.3 & 2.2 & 153.4 & -2.9 & -68.5 \\
\hline 144 & 149 & 1.6 & 150.1 & 0.5 & -66.6 \\
\hline
\end{tabular}


Table 42. Expected output for CLSBDWL for receiver height of 1500 and $3000 \mathrm{~m}$, plus clutter-to-noise ratio (CNR).

\begin{tabular}{c|c|c||c|c||c}
\hline & \multicolumn{2}{|c||}{ Rec. height at 1500 m } & \multicolumn{2}{|c||}{ Rec. height at 3000 m } & \\
\hline $\begin{array}{c}\text { Range } \\
(\mathrm{km})\end{array}$ & $\begin{array}{c}\text { Prop. } \\
\text { Loss } \\
\text { (dB) }\end{array}$ & $\begin{array}{c}\text { Prop. } \\
\text { Factor } \\
\text { (dB) }\end{array}$ & $\begin{array}{c}\text { Prop. } \\
\text { Loss } \\
\text { (dB) }\end{array}$ & $\begin{array}{c}\text { Prop. } \\
\text { Factor }\end{array}$ & $\begin{array}{c}\text { CNR } \\
(\mathrm{dB})\end{array}$ \\
\hline 146 & 151.2 & -0.5 & 148.9 & 1.8 & -44.6 \\
148 & 152.4 & -1.5 & 150 & 0.8 & 8.1 \\
150 & 158.1 & -7.2 & 153.4 & -2.5 & 14.6 \\
152 & 159.8 & -8.7 & 155.8 & -4.8 & -29.4 \\
154 & 161.7 & -10.6 & 152.6 & -1.4 & -31.9 \\
156 & 168.8 & -17.5 & 150 & 1.2 & -267.8 \\
158 & 167.5 & -16.1 & 149.5 & 1.9 & -317.4 \\
160 & 158.4 & -6.9 & 150.7 & 0.8 & -297.7 \\
162 & 160.4 & -8.8 & 153.9 & -2.3 & -246.4 \\
164 & 157.9 & -6.2 & 157.6 & -5.9 & -88.4 \\
166 & 154.9 & -3.1 & 155.2 & -3.4 & -76.9 \\
168 & 155.5 & -3.6 & 151.9 & 0 & -71.1 \\
170 & 156.1 & -4.1 & 150.3 & 1.7 & -79.3 \\
172 & 155.8 & -3.6 & 150.1 & 2 & -82.4 \\
174 & 156.1 & -3.9 & 151.3 & 1 & -82.5 \\
176 & 157 & -4.7 & 153.9 & -1.6 & -87.2 \\
178 & 157 & -4.6 & 158.1 & -5.7 & -30.8 \\
180 & 158.8 & -6.2 & 159.8 & -7.3 & -328.1 \\
182 & 161.7 & -9 & 155.9 & -3.3 & -319.2 \\
184 & 165.1 & -12.4 & 152.9 & -0.2 & -66.9 \\
186 & 162.4 & -9.6 & 151.3 & 1.5 & -114.6 \\
188 & 163.5 & -10.6 & 150.8 & 2.1 & -22 \\
190 & 171.5 & -18.5 & 151.3 & 1.7 & -93.4 \\
192 & 161 & -8 & 152.6 & 0.5 & -21.5 \\
194 & 179.5 & -26.4 & 155 & -1.8 & -225.5 \\
196 & 180 & -26.7 & 158.9 & -5.6 & -389 \\
198 & 193.2 & -39.8 & 170.4 & -17 & -388 \\
200 & 196 & -42.6 & 160.4 & -7 & -391.4 \\
\hline
\end{tabular}


Table 43. Expected output for COSEC2 for $r_{\text {max }}$ receiver range of $50 \mathrm{~km}$.

\begin{tabular}{c|c|c}
\hline $\begin{array}{c}\text { Height } \\
\text { (meters) }\end{array}$ & $\begin{array}{c}\text { Prop. } \\
\text { Loss } \\
(\mathrm{dB})\end{array}$ & $\begin{array}{c}\text { Prop. } \\
\text { Factor } \\
(\mathrm{dB})\end{array}$ \\
\hline 100 & 134.4 & -8 \\
200 & 124.1 & 2.3 \\
300 & 122.3 & 4.1 \\
400 & 129.7 & -3.3 \\
500 & 126.5 & -0.1 \\
600 & 123.5 & 2.9 \\
700 & 128 & -1.6 \\
800 & 126.9 & -0.4 \\
900 & 125.7 & 0.7 \\
1000 & 126.4 & 0 \\
1100 & 127 & -0.5 \\
1200 & 127.8 & -1.4 \\
1300 & 128.7 & -2.2 \\
1400 & 129.4 & -3 \\
1500 & 130.1 & -3.6 \\
1600 & 130.7 & -4.3 \\
1700 & 131.3 & -4.9 \\
1800 & 131.8 & -5.4 \\
1900 & 132.4 & -5.9 \\
2000 & 132.8 & -6.4 \\
\hline & &
\end{tabular}

Table 44. Expected output for EDUCT for $r_{\text {max }}$ receiver range of $50 \mathrm{~km}$.

\begin{tabular}{c|c|c}
\hline $\begin{array}{c}\text { Height } \\
\text { (meters) }\end{array}$ & $\begin{array}{c}\text { Prop. } \\
\text { Loss } \\
\text { (dB) }\end{array}$ & $\begin{array}{c}\text { Prop. } \\
\text { Factor } \\
\text { (dB) }\end{array}$ \\
\hline 10 & 142.8 & 3.6 \\
20 & 147.5 & -1.1 \\
30 & 150.1 & -3.6 \\
40 & 152.5 & -6.1 \\
50 & 156 & -9.6 \\
60 & 158.6 & -12.2 \\
70 & 154.1 & -7.7 \\
80 & 149.5 & -3 \\
90 & 146.3 & 0.1 \\
100 & 144.3 & 2.2 \\
110 & 143.1 & 3.4 \\
120 & 142.7 & 3.7 \\
130 & 143.2 & 3.2 \\
140 & 145.1 & 1.3 \\
150 & 149.4 & -3 \\
160 & 162.2 & -15.8 \\
170 & 151.8 & -5.4 \\
180 & 145.2 & 1.3 \\
190 & 142.4 & 4.1 \\
200 & 141.5 & 4.9 \\
\hline & &
\end{tabular}


Table 45. Expected output for EDUCTRF for $r_{\max }$ receiver range of 100 $\mathrm{km}$.

\begin{tabular}{c|c|c}
\hline $\begin{array}{c}\text { Height } \\
\text { (meters) }\end{array}$ & $\begin{array}{c}\text { Prop. } \\
\text { Loss } \\
(\mathrm{dB})\end{array}$ & $\begin{array}{c}\text { Prop. } \\
\text { Factor } \\
(\mathrm{dB})\end{array}$ \\
\hline 10 & 155 & -2.5 \\
20 & 158.2 & -5.7 \\
30 & 160 & -7.6 \\
40 & 160.7 & -8.2 \\
50 & 161 & -8.6 \\
60 & 161.3 & -8.8 \\
70 & 161.4 & -8.9 \\
80 & 161.5 & -9 \\
90 & 161.5 & -9.1 \\
100 & 161.6 & -9.2 \\
110 & 161.7 & -9.2 \\
120 & 161.7 & -9.3 \\
130 & 161.8 & -9.4 \\
140 & 161.8 & -9.4 \\
150 & 162 & -9.5 \\
160 & 162.1 & -9.6 \\
170 & 162.1 & -9.7 \\
180 & 162.3 & -9.8 \\
190 & 162.4 & -9.9 \\
200 & 162.6 & -10.1 \\
\hline & &
\end{tabular}

Table 46. Expected output for FLTA50 for $r_{\max }$ receiver range of $50 \mathrm{~km}$.

\begin{tabular}{c|c|c}
\hline $\begin{array}{c}\text { Height } \\
\text { (meters) }\end{array}$ & $\begin{array}{c}\text { Prop. } \\
\text { Loss } \\
\text { (dB) }\end{array}$ & $\begin{array}{c}\text { Prop. } \\
\text { Factor } \\
\text { (dB) }\end{array}$ \\
\hline 0 & -3276.6 & -3276.6 \\
5 & -3276.6 & -3276.6 \\
10 & 190.3 & -63.9 \\
15 & 159 & -32.5 \\
20 & 152.7 & -26.3 \\
25 & 148.9 & -22.5 \\
30 & 146.1 & -19.7 \\
35 & 143.8 & -17.3 \\
40 & 141.8 & -15.3 \\
45 & 140 & -13.6 \\
50 & 138.4 & -12 \\
55 & 137 & -10.5 \\
60 & 135.6 & -9.2 \\
65 & 134.4 & -8 \\
70 & 133.3 & -6.8 \\
75 & 132.2 & -5.8 \\
80 & 131.2 & -4.7 \\
85 & 130.2 & -3.8 \\
90 & 129.4 & -2.9 \\
95 & 128.5 & -2.1 \\
100 & 127.8 & -1.3 \\
\hline & &
\end{tabular}


Table 47. Expected output for GASABS for $r_{\text {max }}$ receiver range of $50 \mathrm{~km}$.

\begin{tabular}{c|c|c}
\hline $\begin{array}{c}\text { Height } \\
\text { (meters) }\end{array}$ & $\begin{array}{c}\text { Prop. } \\
\text { Loss } \\
(\mathrm{dB})\end{array}$ & $\begin{array}{c}\text { Prop. } \\
\text { Factor } \\
(\mathrm{dB})\end{array}$ \\
\hline 10 & 212.7 & -60.2 \\
20 & 199.2 & -46.8 \\
30 & 188.9 & -36.5 \\
40 & 180.1 & -27.6 \\
50 & 172.2 & -19.8 \\
60 & 165.5 & -13 \\
70 & 160.1 & -7.6 \\
80 & 156.7 & -4.2 \\
90 & 156.5 & -4 \\
100 & 163.1 & -10.7 \\
110 & 159.3 & -6.9 \\
120 & 156 & -3.6 \\
130 & 167.8 & -15.3 \\
140 & 155.7 & -3.3 \\
150 & 163 & -10.5 \\
160 & 156.1 & -3.6 \\
170 & 161.9 & -9.4 \\
180 & 155.7 & -3.3 \\
190 & 164.4 & -12 \\
200 & 154.9 & -2.5 \\
\hline & &
\end{tabular}

Table 48. Expected output for GAUSS for $r_{\text {max }}$ receiver range of $50 \mathrm{~km}$.

\begin{tabular}{c|c|c}
\hline $\begin{array}{c}\text { Height } \\
\text { (meters) }\end{array}$ & $\begin{array}{c}\text { Prop. } \\
\text { Loss } \\
\text { (dB) }\end{array}$ & $\begin{array}{c}\text { Prop. } \\
\text { Factor } \\
(\mathrm{dB})\end{array}$ \\
\hline 100 & 133.7 & -7.2 \\
200 & 123.5 & 2.9 \\
300 & 121.7 & 4.8 \\
400 & 130.7 & -4.3 \\
500 & 127.1 & -0.6 \\
600 & 124 & 2.4 \\
700 & 133 & -6.6 \\
800 & 132.2 & -5.7 \\
900 & 129.6 & -3.2 \\
1000 & 139 & -12.6 \\
1100 & 140 & -13.5 \\
1200 & 138.1 & -11.7 \\
1300 & 148.3 & -21.9 \\
1400 & 150.4 & -23.9 \\
1500 & 149.5 & -23.1 \\
1600 & 160.5 & -34.1 \\
1700 & 163.6 & -37.1 \\
1800 & 163.7 & -37.3 \\
1900 & 175.5 & -49.1 \\
2000 & 179.6 & -53.1 \\
\hline
\end{tabular}


Table 49. Expected output for HEIGHT_RTG for receiver height of $500 \mathrm{~m}$.

\begin{tabular}{c|c|c}
\hline $\begin{array}{c}\text { Range } \\
(\mathrm{km})\end{array}$ & $\begin{array}{c}\text { Prop. } \\
\text { Loss } \\
(\mathrm{dB})\end{array}$ & $\begin{array}{c}\text { Prop. } \\
\text { Factor } \\
(\mathrm{dB})\end{array}$ \\
\hline 0.92 & -3276.7 & -3276.7 \\
1.84 & -3276.7 & -3276.7 \\
2.76 & -3276.7 & -3276.7 \\
3.68 & -3276.7 & -3276.7 \\
4.6 & 89.6 & 0.3 \\
5.52 & 94.3 & -2.8 \\
6.44 & 94.1 & -1.2 \\
7.36 & 93.4 & 0.6 \\
8.28 & 92.2 & 2.8 \\
9.21 & 94.4 & 1.5 \\
10.13 & 94.5 & 2.3 \\
11.05 & 93.6 & 4 \\
11.97 & 99.8 & -1.5 \\
12.89 & 96.5 & 2.4 \\
13.81 & 103.9 & -4.5 \\
14.73 & 97.7 & 2.4 \\
15.65 & 97.4 & 3.1 \\
16.57 & 99.5 & 1.6 \\
17.49 & 105.1 & -3.6 \\
18.41 & 100 & 1.9 \\
\hline & &
\end{tabular}


Table 50. Expected output for HF10TER for receiver heights of 0, 1000, and $2000 \mathrm{~m}$.

\begin{tabular}{|c|c|c|c|c|c|c|}
\hline \multirow[b]{2}{*}{$\begin{array}{c}\text { Rang } \\
\mathrm{e} \\
(\mathrm{km})\end{array}$} & \multicolumn{2}{|c|}{ Rec. height at $0 \mathrm{~m}$} & \multicolumn{2}{|c|}{ Rec. height at $1000 \mathrm{~m}$} & \multicolumn{2}{|c|}{ Rec. height at $2000 \mathrm{~m}$} \\
\hline & $\begin{array}{l}\text { Prop. } \\
\text { Loss } \\
\text { (dB) } \\
\end{array}$ & $\begin{array}{l}\text { Prop. } \\
\text { Factor } \\
\text { (dB) }\end{array}$ & $\begin{array}{l}\text { Prop. } \\
\text { Loss } \\
(\mathrm{dB})\end{array}$ & $\begin{array}{l}\text { Prop. } \\
\text { Factor } \\
(\mathrm{dB})\end{array}$ & $\begin{array}{l}\text { Prop. } \\
\text { Loss } \\
\text { (dB) }\end{array}$ & $\begin{array}{l}\text { Prop. } \\
\text { Factor } \\
(\mathrm{dB})\end{array}$ \\
\hline 8.3 & 65.5 & 5.4 & 66.9 & 3.9 & 70.8 & 0 \\
\hline 16.6 & 71.6 & 5.3 & 72.6 & 4.3 & 73 & 3.9 \\
\hline 24.9 & 75.2 & 5.2 & 76.2 & 4.1 & 76.1 & 4.3 \\
\hline 33.2 & 77.8 & 5.1 & 79.4 & 3.5 & 78.7 & 4.1 \\
\hline 41.5 & 80 & 4.8 & 82.2 & 2.6 & 80.8 & 4.1 \\
\hline 49.8 & 81.9 & 4.5 & 84.6 & 1.8 & 82.6 & 3.8 \\
\hline 58.1 & 83.6 & 4.1 & 86.8 & 0.9 & 84.3 & 3.4 \\
\hline 66.4 & 85.2 & 3.7 & 88.9 & 0 & 85.8 & 3 \\
\hline 74.7 & 86.7 & 3.2 & 90.8 & -0.9 & 87.2 & 2.7 \\
\hline 83 & 110.9 & -20.1 & 92.7 & -1.9 & 88.4 & 2.4 \\
\hline 91.3 & 118.4 & -26.8 & 93 & -1.3 & 90.1 & 1.6 \\
\hline 99.6 & 122.8 & -30.4 & 94.6 & -2.2 & 89.9 & 2.5 \\
\hline 107.9 & -3276.6 & -3276.6 & 93.1 & 0 & 91.1 & 2 \\
\hline 116.2 & -3276.6 & -3276.6 & 94.6 & -0.9 & 93.4 & 0.4 \\
\hline 124.5 & -3276.6 & -3276.6 & 96.8 & -2.5 & 95.4 & -1.1 \\
\hline 132.8 & -3276.6 & -3276.6 & 98.5 & -3.6 & 93.6 & 1.3 \\
\hline 141.1 & -3276.6 & -3276.6 & 100.8 & -5.3 & 93.8 & 1.7 \\
\hline 149.4 & -3276.6 & -3276.6 & 103.2 & -7.3 & 94.8 & 1.1 \\
\hline 157.7 & -3276.6 & -3276.6 & 106.6 & -10.2 & 96.8 & -0.4 \\
\hline 166 & -3276.6 & -3276.6 & 106.2 & -9.3 & 98.6 & -1.7 \\
\hline 174.3 & -3276.6 & -3276.6 & 109.2 & -12 & 100.8 & -3.5 \\
\hline 182.6 & -3276.6 & -3276.6 & 111.1 & -13.5 & 103.1 & -5.4 \\
\hline 190.9 & -3276.6 & -3276.6 & -3276.6 & -3276.6 & 103 & -5 \\
\hline 199.2 & -3276.6 & -3276.6 & 138.5 & -40 & 104 & -5.6 \\
\hline 207.5 & -3276.6 & -3276.6 & 131.3 & -32.5 & 110.6 & -11.9 \\
\hline 215.8 & -3276.6 & -3276.6 & 129.1 & -30 & 115.9 & -16.7 \\
\hline 224.1 & -3276.6 & -3276.6 & 128.2 & -28.7 & 119.9 & -20.4 \\
\hline 232.4 & -3276.6 & -3276.6 & -3276.6 & -3276.6 & 121.4 & -21.7 \\
\hline 240.7 & -3276.6 & -3276.6 & -3276.6 & -3276.6 & 124 & -23.9 \\
\hline 249 & -3276.6 & -3276.6 & 152.1 & -51.7 & 125.8 & -25.4 \\
\hline
\end{tabular}


Table 51. Expected output for HF20QWVD for $r_{\text {max }}$ receiver range of 100 km.

\begin{tabular}{c|c|c}
\hline $\begin{array}{c}\text { Height } \\
\text { (meters) }\end{array}$ & $\begin{array}{c}\text { Prop. } \\
\text { Loss } \\
(\mathrm{dB})\end{array}$ & $\begin{array}{c}\text { Prop. } \\
\text { Factor } \\
(\mathrm{dB})\end{array}$ \\
\hline 0 & 114.9 & -16.4 \\
50 & 117 & -18.5 \\
100 & 118.6 & -20.1 \\
150 & 118.8 & -20.3 \\
200 & 117.6 & -19.1 \\
250 & 115.8 & -17.3 \\
300 & 114.1 & -15.6 \\
350 & 112.6 & -14.2 \\
400 & 111.4 & -12.9 \\
450 & 110.4 & -11.9 \\
500 & 109.5 & -11 \\
550 & 108.8 & -10.3 \\
600 & 108.2 & -9.8 \\
650 & 107.8 & -9.3 \\
700 & 107.4 & -8.9 \\
750 & 107.1 & -8.6 \\
800 & 106.8 & -8.3 \\
850 & 106.6 & -8.1 \\
900 & 106.4 & -7.9 \\
950 & 106.1 & -7.7 \\
1000 & 105.9 & -7.4 \\
\hline & &
\end{tabular}

Table 52 Expected output for HF20RF for $r_{\max }$ receiver range of $100 \mathrm{~km}$.

\begin{tabular}{c|c|c}
\hline $\begin{array}{c}\text { Height } \\
\text { (meters) }\end{array}$ & $\begin{array}{c}\text { Prop. } \\
\text { Loss } \\
\text { (dB) }\end{array}$ & $\begin{array}{c}\text { Prop. } \\
\text { Factor } \\
\text { (dB) }\end{array}$ \\
\hline 0 & 119.8 & -21.3 \\
50 & 122.6 & -24.1 \\
100 & 123.5 & -25 \\
150 & 121.7 & -23.3 \\
200 & 119.3 & -20.8 \\
250 & 117.1 & -18.7 \\
300 & 115.4 & -16.9 \\
350 & 113.9 & -15.5 \\
400 & 112.7 & -14.3 \\
450 & 111.7 & -13.2 \\
500 & 110.9 & -12.4 \\
550 & 110.2 & -11.7 \\
600 & 109.6 & -11.1 \\
650 & 109.1 & -10.6 \\
700 & 108.7 & -10.2 \\
750 & 108.4 & -9.9 \\
800 & 108.1 & -9.7 \\
850 & 107.9 & -9.5 \\
900 & 107.8 & -9.3 \\
950 & 107.6 & -9.1 \\
1000 & 107.4 & -9 \\
\hline & &
\end{tabular}


Table 53. Expected output for HF30 for $r_{\text {max }}$ receiver range of $100 \mathrm{~km}$.

\begin{tabular}{c|c|c}
\hline $\begin{array}{c}\text { Height } \\
\text { (meters) }\end{array}$ & $\begin{array}{c}\text { Prop. } \\
\text { Loss } \\
\text { (dB) }\end{array}$ & $\begin{array}{c}\text { Prop. } \\
\text { Factor } \\
\text { (dB) }\end{array}$ \\
\hline 0 & 128.9 & -26.9 \\
50 & 131.9 & -29.9 \\
100 & 131.1 & -29.1 \\
150 & 128.2 & -26.2 \\
200 & 125.6 & -23.6 \\
250 & 123.4 & -21.5 \\
300 & 121.7 & -19.7 \\
350 & 120.2 & -18.2 \\
400 & 118.9 & -16.9 \\
450 & 117.7 & -15.7 \\
500 & 116.6 & -14.6 \\
550 & 115.6 & -13.7 \\
600 & 114.8 & -12.8 \\
650 & 113.9 & -11.9 \\
700 & 113.2 & -11.2 \\
750 & 112.5 & -10.5 \\
800 & 111.8 & -9.8 \\
850 & 111.2 & -9.2 \\
900 & 110.6 & -8.6 \\
950 & 110.1 & -8.1 \\
1000 & 109.6 & -7.6 \\
\hline & &
\end{tabular}

Table 54. Expected output for HIBW for $r_{\max }$ receiver range of $50 \mathrm{~km}$.

\begin{tabular}{c|c|c}
\hline $\begin{array}{c}\text { Height } \\
\text { (meters) }\end{array}$ & $\begin{array}{c}\text { Prop. } \\
\text { Loss } \\
\text { (dB) }\end{array}$ & $\begin{array}{c}\text { Prop. } \\
\text { Factor } \\
\text { (dB) }\end{array}$ \\
\hline 100 & 133.6 & -7.2 \\
200 & 123.3 & 3.1 \\
300 & 121.1 & 5.3 \\
400 & 129.7 & -3.3 \\
500 & 124.9 & 1.5 \\
600 & 120.6 & 5.8 \\
700 & 128.1 & -1.7 \\
800 & 125.3 & 1.1 \\
900 & 120.5 & 5.9 \\
1000 & 127.6 & -1.1 \\
1100 & 125.6 & 0.8 \\
1200 & 120.5 & 6 \\
1300 & 127.5 & -1.1 \\
1400 & 125.6 & 0.8 \\
1500 & 120.5 & 6 \\
1600 & 127.5 & -1 \\
1700 & 125.6 & 0.8 \\
1800 & 120.5 & 5.9 \\
1900 & 127.4 & -1 \\
2000 & 125.7 & 0.8 \\
\hline
\end{tabular}


Table 55. Expected output for HIEL for $r_{\text {max }}$ receiver range of $50 \mathrm{~km}$.

\begin{tabular}{c|c|c}
\hline $\begin{array}{c}\text { Height } \\
\text { (meters) }\end{array}$ & $\begin{array}{c}\text { Prop. } \\
\text { Loss } \\
\text { (dB) }\end{array}$ & $\begin{array}{c}\text { Prop. } \\
\text { Factor } \\
(\mathrm{dB})\end{array}$ \\
\hline 1000 & 376.4 & -250 \\
2000 & 376.4 & -250 \\
3000 & 376.4 & -250 \\
4000 & 376.4 & -250 \\
5000 & 364.2 & -237.7 \\
6000 & 258.9 & -132.4 \\
7000 & 184.7 & -58.1 \\
8000 & 140.8 & -14.2 \\
9000 & 126.6 & 0 \\
10000 & 141.2 & -14.6 \\
11000 & 183.7 & -57.1 \\
12000 & 253.1 & -126.4 \\
13000 & 348.2 & -221.5 \\
14000 & 376.7 & -250 \\
15000 & 376.8 & -250 \\
16000 & 376.8 & -250 \\
17000 & 376.9 & -250 \\
18000 & 376.9 & -250 \\
19000 & 377 & -250 \\
20000 & 377.1 & -250 \\
\hline & &
\end{tabular}

Table 56. Expected output for HIFREQ for $r_{\max }$ receiver range of $50 \mathrm{~km}$.

\begin{tabular}{c|c|c}
\hline $\begin{array}{c}\text { Height } \\
(\mathrm{km})\end{array}$ & $\begin{array}{c}\text { Prop. } \\
\text { Loss } \\
(\mathrm{dB})\end{array}$ & $\begin{array}{c}\text { Prop. } \\
\text { Factor } \\
(\mathrm{dB})\end{array}$ \\
\hline 10 & 205.4 & -52.9 \\
20 & 192 & -39.5 \\
30 & 181.6 & -29.2 \\
40 & 172.8 & -20.3 \\
50 & 164.9 & -12.5 \\
60 & 158.2 & -5.7 \\
70 & 152.8 & -0.3 \\
80 & 149.4 & 3 \\
90 & 149.2 & 3.3 \\
100 & 155.9 & -3.4 \\
110 & 152 & 0.4 \\
120 & 148.7 & 3.7 \\
130 & 160.5 & -8 \\
140 & 148.4 & 4 \\
150 & 155.7 & -3.2 \\
160 & 148.8 & 3.7 \\
170 & 154.6 & -2.1 \\
180 & 148.4 & 4 \\
190 & 157.2 & -4.7 \\
200 & 147.6 & 4.8 \\
\hline
\end{tabular}


Table 57. Expected output for HITRAN for $r_{\text {max }}$ receiver range of $50 \mathrm{~km}$.

\begin{tabular}{c|c|c}
\hline $\begin{array}{c}\text { Height } \\
(\mathrm{m})\end{array}$ & $\begin{array}{c}\text { Prop. } \\
\text { Loss } \\
(\mathrm{dB})\end{array}$ & $\begin{array}{c}\text { Prop. } \\
\text { Factor } \\
(\mathrm{dB})\end{array}$ \\
\hline 50 & 126.3 & 0.1 \\
100 & 121.8 & 4.7 \\
150 & 138.1 & -11.7 \\
200 & 121.4 & 5 \\
250 & 134.6 & -8.2 \\
300 & 122 & 4.4 \\
350 & 124.4 & 2 \\
400 & 127.7 & -1.3 \\
450 & 120.9 & 5.5 \\
500 & 131.4 & -5 \\
550 & 123.2 & 3.3 \\
600 & 121.5 & 5 \\
650 & 147.9 & -21.5 \\
700 & 121.7 & 4.7 \\
750 & 122.3 & 4.1 \\
800 & 137.7 & -11.3 \\
850 & 121.1 & 5.3 \\
900 & 123.2 & 3.2 \\
950 & 132.5 & -6.1 \\
1000 & 120.8 & 5.6 \\
\hline & &
\end{tabular}

Table 58. Expected output for HORZ for $r_{\text {max }}$ receiver range of $50 \mathrm{~km}$.

\begin{tabular}{c|c|c}
\hline $\begin{array}{c}\text { Height } \\
\text { (meters) }\end{array}$ & $\begin{array}{c}\text { Prop. } \\
\text { Loss } \\
(\mathrm{dB})\end{array}$ & $\begin{array}{c}\text { Prop. } \\
\text { Factor } \\
(\mathrm{dB})\end{array}$ \\
\hline 100 & 133.6 & -7.2 \\
200 & 123.3 & 3.1 \\
300 & 121.1 & 5.3 \\
400 & 129.7 & -3.3 \\
500 & 124.9 & 1.5 \\
600 & 120.6 & 5.8 \\
700 & 128.1 & -1.7 \\
800 & 125.3 & 1.1 \\
900 & 120.5 & 5.9 \\
1000 & 127.6 & -1.1 \\
1100 & 125.6 & 0.9 \\
1200 & 120.5 & 6 \\
1300 & 127.5 & -1 \\
1400 & 125.6 & 0.8 \\
1500 & 120.5 & 6 \\
1600 & 127.4 & -1 \\
1700 & 125.6 & 0.8 \\
1800 & 120.5 & 6 \\
1900 & 127.4 & -1 \\
2000 & 125.6 & 0.8 \\
\hline & &
\end{tabular}


Table 59. Expected output for HTFIND for $r_{\max }$ receiver range of $50 \mathrm{~km}$.

\begin{tabular}{c|c|c}
\hline $\begin{array}{c}\text { Height } \\
\text { (meters) }\end{array}$ & $\begin{array}{c}\text { Prop. } \\
\text { Loss } \\
(\mathrm{dB})\end{array}$ & $\begin{array}{c}\text { Prop. } \\
\text { Factor } \\
(\mathrm{dB})\end{array}$ \\
\hline 100 & 133.6 & -7.2 \\
200 & 123.4 & 3 \\
300 & 121.5 & 4.9 \\
400 & 130 & -3.6 \\
500 & 125.8 & 0.7 \\
600 & 122.1 & 4.3 \\
700 & 128.8 & -2.3 \\
800 & 126.9 & -0.5 \\
900 & 124.4 & 2 \\
1000 & 127.1 & -0.7 \\
1100 & 126.5 & -0.1 \\
1200 & 126.4 & 0 \\
1300 & 126.4 & 0 \\
1400 & 126.4 & 0 \\
1500 & 126.4 & 0 \\
1600 & 126.4 & 0 \\
1700 & 126.4 & 0 \\
1800 & 126.4 & 0 \\
1900 & 126.4 & 0 \\
2000 & 126.4 & 0 \\
\hline & &
\end{tabular}

Table 60. Expected output for LOBW for $r_{\max }$ receiver range of $50 \mathrm{~km}$.

\begin{tabular}{c|c|c}
\hline $\begin{array}{c}\text { Height } \\
\text { (meters) }\end{array}$ & $\begin{array}{c}\text { Prop. } \\
\text { Loss } \\
(\mathrm{dB})\end{array}$ & $\begin{array}{c}\text { Prop. } \\
\text { Factor } \\
(\mathrm{dB})\end{array}$ \\
\hline 100 & 133.8 & -7.4 \\
200 & 124 & 2.4 \\
300 & 123.2 & 3.2 \\
400 & 132.9 & -6.4 \\
500 & 133 & -6.6 \\
600 & 134.1 & -7.7 \\
700 & 146.4 & -19.9 \\
800 & 151.8 & -25.3 \\
900 & 156.6 & -30.2 \\
1000 & 171.5 & -45.1 \\
1100 & 181.5 & -55.1 \\
1200 & 190.5 & -64 \\
1300 & 208.4 & -81.9 \\
1400 & 222.4 & -96 \\
1500 & 235.6 & -109.2 \\
1600 & 256.6 & -130.1 \\
1700 & 274.6 & -148.2 \\
1800 & 292 & -165.6 \\
1900 & 316.2 & -189.7 \\
2000 & 338.1 & -211.7 \\
\hline & &
\end{tabular}


Table 61. Expected output for LOEL for $r_{\max }$ receiver range of $50 \mathrm{~km}$.

\begin{tabular}{c|c|c}
\hline $\begin{array}{c}\text { Height } \\
\text { (meters) }\end{array}$ & $\begin{array}{c}\text { Prop. } \\
\text { Loss } \\
(\mathrm{dB})\end{array}$ & $\begin{array}{c}\text { Prop. } \\
\text { Factor } \\
(\mathrm{dB})\end{array}$ \\
\hline 1000 & 376.4 & -250 \\
2000 & 376.4 & -250 \\
3000 & 376.4 & -250 \\
4000 & 376.4 & -250 \\
5000 & 358.3 & -231.8 \\
6000 & 254.6 & -128.2 \\
7000 & 181.9 & -55.4 \\
8000 & 139.6 & -13.1 \\
9000 & 126.9 & -0.3 \\
10000 & 142.9 & -16.3 \\
11000 & 186.8 & -60.2 \\
12000 & 257.6 & -130.9 \\
13000 & 354 & -227.3 \\
14000 & 376.7 & -250 \\
15000 & 376.8 & -250 \\
16000 & 376.8 & -250 \\
17000 & 376.9 & -250 \\
18000 & 376.9 & -250 \\
19000 & 377 & -250 \\
20000 & 377.1 & -250 \\
\hline & &
\end{tabular}

Table 62. Expected output for LOFREQ for $r_{\max }$ receiver range of $50 \mathrm{~km}$.

\begin{tabular}{c|c|c}
\hline $\begin{array}{c}\text { Height } \\
\text { (meters) }\end{array}$ & $\begin{array}{c}\text { Prop. } \\
\text { Loss } \\
\text { (dB) }\end{array}$ & $\begin{array}{c}\text { Prop. } \\
\text { Factor } \\
(\mathrm{dB})\end{array}$ \\
\hline 250 & 116.7 & -10.3 \\
500 & 109 & -2.6 \\
750 & 105 & 1.4 \\
1000 & 102.6 & 3.8 \\
1250 & 101.2 & 5.2 \\
1500 & 100.5 & 5.9 \\
1750 & 100.5 & 5.9 \\
2000 & 101 & 5.4 \\
2250 & 102.3 & 4.1 \\
2500 & 104.5 & 1.9 \\
2750 & 108.4 & -1.9 \\
3000 & 116.9 & -10.5 \\
3250 & 119.4 & -13 \\
3500 & 109.2 & -2.7 \\
3750 & 104.9 & 1.5 \\
4000 & 102.6 & 3.9 \\
4250 & 101.2 & 5.2 \\
4500 & 100.6 & 5.9 \\
4750 & 100.5 & 6 \\
5000 & 101 & 5.5 \\
\hline
\end{tabular}


Table 63. Expected output for LOTRAN for $r_{\max }$ receiver range of $50 \mathrm{~km}$.

\begin{tabular}{c|c|c}
\hline $\begin{array}{c}\text { Height } \\
\text { (meters) }\end{array}$ & $\begin{array}{c}\text { Prop. } \\
\text { Loss } \\
(\mathrm{dB})\end{array}$ & $\begin{array}{c}\text { Prop. } \\
\text { Factor } \\
(\mathrm{dB})\end{array}$ \\
\hline 500 & 133.8 & -7.3 \\
1000 & 126.3 & 0.2 \\
1500 & 122.9 & 3.5 \\
2000 & 121.2 & 5.3 \\
2500 & 120.5 & 6 \\
3000 & 120.7 & 5.8 \\
3500 & 121.8 & 4.6 \\
4000 & 124.1 & 2.3 \\
4500 & 128.5 & -2 \\
5000 & 140.4 & -14 \\
5500 & 134.2 & -7.7 \\
6000 & 126.7 & -0.2 \\
6500 & 123.2 & 3.3 \\
7000 & 121.4 & 5.1 \\
7500 & 120.7 & 5.8 \\
8000 & 120.8 & 5.8 \\
8500 & 121.7 & 4.9 \\
9000 & 123.6 & 3 \\
9500 & 127.1 & -0.6 \\
10000 & 134.8 & -8.2 \\
\hline & &
\end{tabular}


Table 64. Expected output for MPRT for receiver heights of 0 and $1100 \mathrm{~m}$.

\begin{tabular}{|c|c|c|c|c|}
\hline \multirow[b]{2}{*}{$\begin{array}{c}\text { Rang } \\
\mathrm{e} \\
(\mathrm{km})\end{array}$} & \multicolumn{2}{|c|}{ Rec. height at $0 \mathrm{~m}$} & \multicolumn{2}{|c|}{ Rec. height at $1100 \mathrm{~m}$} \\
\hline & $\begin{array}{l}\text { Prop. } \\
\text { Loss } \\
\text { (dB) }\end{array}$ & $\begin{array}{l}\text { Prop. } \\
\text { Factor } \\
\text { (dB) }\end{array}$ & $\begin{array}{l}\text { Prop. } \\
\text { Loss } \\
\text { (dB) }\end{array}$ & $\begin{array}{l}\text { Prop. } \\
\text { Factor } \\
\text { (dB) }\end{array}$ \\
\hline 2 & 369.9 & -281.9 & 348.1 & -260 \\
\hline 4 & 377.6 & -283.6 & 361.7 & -267.6 \\
\hline 6 & 389.7 & -292.2 & 376.8 & -279.2 \\
\hline 8 & 374.3 & -274.3 & 357.8 & -257.7 \\
\hline 10 & 274.5 & -172.5 & 365.6 & -263.6 \\
\hline 12 & -3276.6 & -3276.6 & 313.5 & -209.9 \\
\hline 14 & -3276.6 & -3276.6 & 265.1 & -160.2 \\
\hline 16 & -3276.6 & -3276.6 & 261.7 & -155.6 \\
\hline 18 & -3276.6 & -3276.6 & 265.6 & -158.5 \\
\hline 20 & -3276.6 & -3276.6 & 206.2 & -98.2 \\
\hline 22 & -3276.6 & -3276.6 & 149 & -40.1 \\
\hline 24 & -3276.6 & -3276.6 & 123.4 & -13.8 \\
\hline 26 & -3276.6 & -3276.6 & 121.9 & -11.6 \\
\hline 28 & -3276.6 & -3276.6 & 134.8 & -23.9 \\
\hline 30 & -3276.6 & -3276.6 & 156.8 & -45.2 \\
\hline 32 & -3276.6 & -3276.6 & 181 & -68.9 \\
\hline 34 & -3276.6 & -3276.6 & 207.2 & -94.6 \\
\hline 36 & -3276.6 & -3276.6 & 233.8 & -120.6 \\
\hline 38 & -3276.6 & -3276.6 & 244 & -130.4 \\
\hline 40 & -3276.6 & -3276.6 & 245.4 & -131.4 \\
\hline 42 & -3276.6 & -3276.6 & 246.8 & -132.3 \\
\hline 44 & -3276.6 & -3276.6 & 249 & -134.1 \\
\hline 46 & -3276.6 & -3276.6 & 252.2 & -136.9 \\
\hline 48 & -3276.6 & -3276.6 & 256.8 & -141.1 \\
\hline 50 & 316.7 & -200.7 & 261.6 & -145.7 \\
\hline 52 & 327.9 & -211.6 & 267 & -150.7 \\
\hline 54 & 322.1 & -205.5 & 271.8 & -155.2 \\
\hline 56 & 321.3 & -204.3 & 275.8 & -158.9 \\
\hline 58 & 320.1 & -202.8 & 279.4 & -162.1 \\
\hline 60 & 319.7 & -202.2 & 282.1 & -164.5 \\
\hline
\end{tabular}


Table 65. Expected output for PERW for receiver heights of 0 and $1000 \mathrm{~m}$.

\begin{tabular}{c|c|c||c|c}
\hline \multicolumn{1}{|c||}{} & \multicolumn{2}{|c||}{ Rec. height at 0 m } & \multicolumn{2}{c}{ Rec. height at $1100 \mathrm{~m}$} \\
\hline $\begin{array}{c}\text { Rang } \\
\mathrm{e}\end{array}$ & $\begin{array}{c}\text { Prop. } \\
\text { Loss }\end{array}$ & $\begin{array}{c}\text { Prop. } \\
\text { Factor } \\
(\mathrm{dB})\end{array}$ & $\begin{array}{c}\text { Prop. } \\
\text { Loss } \\
(\mathrm{dB})\end{array}$ & $\begin{array}{c}\text { Prop. } \\
\text { Factor } \\
(\mathrm{dB})\end{array}$ \\
\hline 2.5 & 122.7 & -32.8 & 179.8 & -89.9 \\
5 & 135 & -39 & 129.6 & -33.6 \\
7.5 & 142.3 & -42.8 & 95.9 & 3.6 \\
10 & 147.7 & -45.7 & 110.7 & -8.7 \\
12.5 & 152 & -48.1 & 99.7 & 4.2 \\
15 & 155.7 & -50.2 & 102.4 & 3.1 \\
17.5 & 158.9 & -52.1 & 110.2 & -3.3 \\
20 & -3276.6 & -3276.6 & 119.7 & -11.6 \\
22.5 & -3276.6 & -3276.6 & 111.8 & -2.8 \\
25 & -3276.6 & -3276.6 & 108.7 & 1.2 \\
27.5 & -3276.6 & -3276.6 & 108.1 & 2.7 \\
30 & -3276.6 & -3276.6 & 106.9 & 4.6 \\
32.5 & -3276.6 & -3276.6 & 107.6 & 4.6 \\
35 & 196.5 & -83.6 & 109 & 3.8 \\
37.5 & 191 & -77.5 & 109.3 & 4.2 \\
40 & 187.8 & -73.8 & 110.3 & 3.8 \\
42.5 & 186.1 & -71.5 & 109.8 & 4.7 \\
45 & 185.2 & -70.1 & 109.5 & 5.6 \\
47.5 & 184.8 & -69.3 & 109.1 & 6.4 \\
50 & 184.8 & -68.8 & 111.9 & 4.1 \\
\hline & & & &
\end{tabular}


Table 66. Expected output for PVT for $r_{\max }$ receiver range of $10 \mathrm{~km}$.

\begin{tabular}{c|c|c}
\hline $\begin{array}{c}\text { Height } \\
\text { (meters) }\end{array}$ & $\begin{array}{c}\text { Prop. } \\
\text { Loss } \\
(\mathrm{dB})\end{array}$ & $\begin{array}{c}\text { Prop. } \\
\text { Factor } \\
(\mathrm{dB})\end{array}$ \\
\hline 0 & -3276.6 & -3276.6 \\
100 & -3276.6 & -3276.6 \\
200 & -3276.6 & -3276.6 \\
300 & 101.3 & 5.1 \\
400 & 113.6 & -7.1 \\
500 & 103.9 & 2.6 \\
600 & 105.2 & 1.2 \\
700 & 109.5 & -3 \\
800 & 104.1 & 2.3 \\
900 & 107 & -0.5 \\
1000 & 107.7 & -1.3 \\
1100 & 106.2 & 0.3 \\
1200 & 105.8 & 0.7 \\
1300 & 102.9 & 3.6 \\
1400 & 108.5 & -2.1 \\
1500 & 104.1 & 2.3 \\
1600 & 107.5 & -1.1 \\
1700 & 103.3 & 3.1 \\
1800 & 104.7 & 1.7 \\
1900 & 111 & -4.6 \\
2000 & 104 & 2.4 \\
\hline & &
\end{tabular}

Table 67. Expected output for RDLONGB for $r_{\max }$ receiver range of $100 \mathrm{~km}$.

\begin{tabular}{c|c|c}
\hline $\begin{array}{c}\text { Height } \\
\text { (meters) }\end{array}$ & $\begin{array}{c}\text { Prop. } \\
\text { Loss } \\
(\mathrm{dB})\end{array}$ & $\begin{array}{c}\text { Prop. } \\
\text { Factor } \\
(\mathrm{dB})\end{array}$ \\
\hline 50 & 138.7 & -22.8 \\
100 & 133.1 & -17.1 \\
150 & 129.3 & -13.3 \\
200 & 126.4 & -10.5 \\
250 & 126.8 & -10.8 \\
300 & 129.5 & -13.5 \\
350 & 125 & -9.1 \\
400 & 121.7 & -5.7 \\
450 & 119.1 & -3.2 \\
500 & 118.5 & -2.5 \\
550 & 118.5 & -2.5 \\
600 & 117.1 & -1.1 \\
650 & 114.6 & 1.4 \\
700 & 112.6 & 3.4 \\
750 & 113.6 & 2.4 \\
800 & 112.6 & 3.4 \\
850 & 111 & 5 \\
900 & 110.9 & 5.1 \\
950 & 110.9 & 5.1 \\
1000 & 110.1 & 5.8 \\
\hline & &
\end{tabular}


Table 68. Expected output for RNGDEP for $r_{\text {max }}$ receiver range of $250 \mathrm{~km}$.

\begin{tabular}{c|c|c}
\hline $\begin{array}{c}\text { Height } \\
\text { (meters) }\end{array}$ & $\begin{array}{c}\text { Prop. } \\
\text { Loss } \\
(\mathrm{dB})\end{array}$ & $\begin{array}{c}\text { Prop. } \\
\text { Factor } \\
(\mathrm{dB})\end{array}$ \\
\hline 100 & 199.8 & -49.9 \\
200 & 195.8 & -45.9 \\
300 & 202.8 & -52.8 \\
400 & 178.6 & -28.7 \\
500 & 141.9 & 8 \\
600 & 135.4 & 14.5 \\
700 & 150.9 & -0.9 \\
800 & 164.2 & -14.2 \\
900 & 166.8 & -16.9 \\
1000 & 182.9 & -33 \\
1100 & 196.7 & -46.8 \\
1200 & 197.4 & -47.4 \\
1300 & 200.7 & -50.8 \\
1400 & 195.1 & -45.2 \\
1500 & 192.9 & -43 \\
1600 & 191.5 & -41.5 \\
1700 & 192.4 & -42.4 \\
1800 & 195.5 & -45.5 \\
1900 & 194.5 & -44.6 \\
2000 & 193.3 & -43.3 \\
\hline & &
\end{tabular}

Table 69. Expected output for SBDUCT for $r_{\max }$ receiver range of $200 \mathrm{~km}$.

\begin{tabular}{c|c|c}
\hline $\begin{array}{c}\text { Height } \\
\text { (meters) }\end{array}$ & $\begin{array}{c}\text { Prop. } \\
\text { Loss } \\
\text { (dB) }\end{array}$ & $\begin{array}{c}\text { Prop. } \\
\text { Factor } \\
(\mathrm{dB})\end{array}$ \\
\hline 250 & 139.9 & 8.1 \\
500 & 166 & -18 \\
750 & 157 & -9 \\
1000 & 161.3 & -13.3 \\
1250 & 174.6 & -26.6 \\
1500 & 169.5 & -21.4 \\
1750 & 158.6 & -10.6 \\
2000 & 150.8 & -2.8 \\
2250 & 146.9 & 1.1 \\
2500 & 147.7 & 0.3 \\
2750 & 165.7 & -17.7 \\
3000 & 145.1 & 3 \\
3250 & 148 & 0 \\
3500 & 147 & 1 \\
3750 & 145.3 & 2.7 \\
4000 & 149.7 & -1.7 \\
4250 & 144.4 & 3.7 \\
4500 & 149.9 & -1.9 \\
4750 & 144.5 & 3.5 \\
5000 & 148 & 0 \\
\hline & &
\end{tabular}


Table 70. Expected output for SBDUCTRF for $r_{\max }$ receiver range of 200 km.

\begin{tabular}{c|c|c}
\hline $\begin{array}{c}\text { Height } \\
\text { (meters) }\end{array}$ & $\begin{array}{c}\text { Prop. } \\
\text { Loss } \\
(\mathrm{dB})\end{array}$ & $\begin{array}{c}\text { Prop. } \\
\text { Factor } \\
(\mathrm{dB})\end{array}$ \\
\hline 0 & 182.2 & -34.2 \\
50 & 141.7 & 6.3 \\
100 & 136.5 & 11.5 \\
150 & 141.5 & 6.5 \\
200 & 141.5 & 6.5 \\
250 & 141 & 7 \\
300 & 152.5 & -4.4 \\
350 & 171.6 & -23.6 \\
400 & 182.7 & -34.7 \\
450 & 173 & -25 \\
500 & 169.4 & -21.4 \\
550 & 165.7 & -17.7 \\
600 & 163.3 & -15.3 \\
650 & 160.9 & -12.9 \\
700 & 159.9 & -11.9 \\
750 & 159.2 & -11.1 \\
800 & 159.2 & -11.2 \\
850 & 159.4 & -11.4 \\
900 & 160.1 & -12 \\
950 & 161.2 & -13.1 \\
1000 & 162.5 & -14.5 \\
\hline & &
\end{tabular}

Table 71. Expected output for SINEX for $r_{\max }$ receiver range of $50 \mathrm{~km}$.

\begin{tabular}{c|c|c}
\hline $\begin{array}{c}\text { Height } \\
\text { (meters) }\end{array}$ & $\begin{array}{c}\text { Prop. } \\
\text { Loss } \\
\text { (dB) }\end{array}$ & $\begin{array}{c}\text { Prop. } \\
\text { Factor } \\
\text { (dB) }\end{array}$ \\
\hline 100 & 133.7 & -7.2 \\
200 & 123.5 & 3 \\
300 & 121.6 & 4.8 \\
400 & 130.7 & -4.3 \\
500 & 127 & -0.6 \\
600 & 124.1 & 2.4 \\
700 & 133.3 & -6.8 \\
800 & 133 & -6.6 \\
900 & 131.9 & -5.4 \\
1000 & 143.2 & -16.8 \\
1100 & 151.3 & -24.8 \\
1200 & 150.9 & -24.5 \\
1300 & 157.9 & -31.5 \\
1400 & 156.1 & -29.6 \\
1500 & 150.9 & -24.5 \\
1600 & 157.9 & -31.5 \\
1700 & 156.1 & -29.7 \\
1800 & 150.9 & -24.5 \\
1900 & 157.9 & -31.5 \\
2000 & 156.1 & -29.7 \\
\hline & &
\end{tabular}


Table 72. Expected output for TROPOS for $r_{\max }$ receiver range of $200 \mathrm{~km}$.

\begin{tabular}{c|c|c}
\hline $\begin{array}{c}\text { Height } \\
\text { (meters) }\end{array}$ & $\begin{array}{c}\text { Prop. } \\
\text { Loss } \\
(\mathrm{dB})\end{array}$ & $\begin{array}{c}\text { Prop. } \\
\text { Factor } \\
(\mathrm{dB})\end{array}$ \\
\hline 100 & 165.2 & -46.7 \\
200 & 164.6 & -46.1 \\
300 & 164.5 & -46 \\
400 & 164.4 & -46 \\
500 & 164.4 & -46 \\
600 & 164.2 & -45.7 \\
700 & 163.4 & -45 \\
800 & 162.1 & -43.6 \\
900 & 160.2 & -41.8 \\
1000 & 158 & -39.6 \\
1100 & 155.7 & -37.2 \\
1200 & 153.4 & -34.9 \\
1300 & 151.2 & -32.7 \\
1400 & 149 & -30.5 \\
1500 & 146.9 & -28.4 \\
1600 & 144.9 & -26.5 \\
1700 & 143.1 & -24.6 \\
1800 & 141.3 & -22.8 \\
1900 & 139.6 & -21.1 \\
2000 & 138 & -19.6 \\
& &
\end{tabular}

Table 73. Expected output for TROPOT for $r_{\text {max }}$ receiver range of $200 \mathrm{~km}$..

\begin{tabular}{c|c|c}
\hline $\begin{array}{c}\text { Height } \\
\text { (meters) }\end{array}$ & $\begin{array}{c}\text { Prop. } \\
\text { Loss } \\
\text { (dB) }\end{array}$ & $\begin{array}{c}\text { Prop. } \\
\text { Factor } \\
\text { (dB) }\end{array}$ \\
\hline 100 & 164.8 & -46.3 \\
200 & 163.8 & -45.3 \\
300 & 162.8 & -44.3 \\
400 & 161.2 & -42.7 \\
500 & 159.2 & -40.7 \\
600 & 157 & -38.6 \\
700 & 155.1 & -36.6 \\
800 & 153.5 & -35.1 \\
900 & 152.4 & -33.9 \\
1000 & 151.6 & -33.2 \\
1100 & 151.2 & -32.7 \\
1200 & 150.6 & -32.2 \\
1300 & 149.6 & -31.1 \\
1400 & 147.9 & -29.4 \\
1500 & 145.9 & -27.4 \\
1600 & 143.9 & -25.4 \\
1700 & 142.1 & -23.7 \\
1800 & 140.6 & -22.2 \\
1900 & 139.1 & -20.6 \\
2000 & 137.4 & -18.9 \\
\hline & &
\end{tabular}


Table 74. Expected output for USERDEFA for $r_{\max }$ receiver range of 300 km.

\begin{tabular}{c|c|c}
\hline $\begin{array}{c}\text { Height } \\
\text { (meters) }\end{array}$ & $\begin{array}{c}\text { Prop. } \\
\text { Loss } \\
(\mathrm{dB})\end{array}$ & $\begin{array}{c}\text { Prop. } \\
\text { Factor } \\
(\mathrm{dB})\end{array}$ \\
\hline 150 & 137.7 & 3.3 \\
300 & 138.8 & 2.3 \\
450 & 159 & -17.9 \\
600 & 153.2 & -12.2 \\
750 & 151.3 & -10.2 \\
900 & 152.6 & -11.5 \\
1050 & 154.8 & -13.8 \\
1200 & 158.9 & -17.9 \\
1350 & 168.6 & -27.5 \\
1500 & 165 & -24 \\
1650 & 156.5 & -15.4 \\
1800 & 153.6 & -12.5 \\
1950 & 153 & -11.9 \\
2100 & 152.2 & -11.1 \\
2250 & 151 & -10 \\
2400 & 151 & -10 \\
2550 & 151.8 & -10.7 \\
2700 & 152.5 & -11.4 \\
2850 & 153.7 & -12.6 \\
3000 & 155.3 & -14.2 \\
\hline & &
\end{tabular}

Table 75. Expected output for USERHF for $r_{\max }$ receiver range of $50 \mathrm{~km}$.

\begin{tabular}{c|c|c}
\hline $\begin{array}{c}\text { Height } \\
\text { (meters) }\end{array}$ & $\begin{array}{c}\text { Prop. } \\
\text { Loss } \\
\text { (dB) }\end{array}$ & $\begin{array}{c}\text { Prop. } \\
\text { Factor } \\
\text { (dB) }\end{array}$ \\
\hline 100 & 133.6 & -7.2 \\
200 & 123.8 & 2.6 \\
300 & 122.6 & 3.8 \\
400 & 128.9 & -2.5 \\
500 & 126.7 & -0.3 \\
600 & 126 & 0.4 \\
700 & 126.4 & 0 \\
800 & 126.4 & 0 \\
900 & 126.4 & 0 \\
1000 & 126.4 & 0 \\
1100 & 127.3 & -0.9 \\
1200 & 127.3 & -0.9 \\
1300 & 127.3 & -0.9 \\
1400 & 127.3 & -0.9 \\
1500 & 128.4 & -1.9 \\
1600 & 128.4 & -1.9 \\
1700 & 128.4 & -1.9 \\
1800 & 128.4 & -1.9 \\
1900 & 128.4 & -1.9 \\
2000 & 129.5 & -3.1 \\
\hline & &
\end{tabular}


Table 76. Expected output for VERT for $r_{\max }$ receiver range of $50 \mathrm{~km}$.

\begin{tabular}{c|c|c}
\hline $\begin{array}{c}\text { Height } \\
\text { (meters) }\end{array}$ & $\begin{array}{c}\text { Prop. } \\
\text { Loss } \\
(\mathrm{dB})\end{array}$ & $\begin{array}{c}\text { Prop. } \\
\text { Factor } \\
(\mathrm{dB})\end{array}$ \\
\hline 0 & 186.9 & -60.5 \\
100 & 133.8 & -7.3 \\
200 & 123.5 & 2.9 \\
300 & 121.4 & 5.1 \\
400 & 129.3 & -2.9 \\
500 & 125.9 & 0.5 \\
600 & 121.3 & 5.1 \\
700 & 127.8 & -1.4 \\
800 & 127 & -0.6 \\
900 & 121.7 & 4.8 \\
1000 & 127.2 & -0.8 \\
1100 & 127.9 & -1.5 \\
1200 & 122.1 & 4.4 \\
1300 & 126.9 & -0.5 \\
1400 & 128.5 & -2 \\
1500 & 122.5 & 4 \\
1600 & 126.7 & -0.3 \\
1700 & 128.8 & -2.4 \\
1800 & 122.8 & 3.6 \\
1900 & 126.5 & -0.1 \\
2000 & 129.1 & -2.7 \\
\hline & &
\end{tabular}

Table 77. Expected output for VERTMIX for $r_{\max }$ receiver range of $50 \mathrm{~km}$.

\begin{tabular}{c|c|c}
\hline $\begin{array}{c}\text { Height } \\
\text { (meters) }\end{array}$ & $\begin{array}{c}\text { Prop. } \\
\text { Loss } \\
\text { (dB) }\end{array}$ & $\begin{array}{c}\text { Prop. } \\
\text { Factor } \\
(\mathrm{dB})\end{array}$ \\
\hline 0 & 150.7 & -44.3 \\
50 & 141.8 & -35.4 \\
100 & 134.7 & -28.3 \\
150 & 130.4 & -24 \\
200 & 127.2 & -20.8 \\
250 & 124.6 & -18.2 \\
300 & 122.4 & -16 \\
350 & 120.5 & -14.1 \\
400 & 118.9 & -12.5 \\
450 & 117.6 & -11.1 \\
500 & 116.4 & -10 \\
550 & 115.4 & -9 \\
600 & 114.5 & -8.1 \\
650 & 113.7 & -7.3 \\
700 & 113.3 & -6.9 \\
750 & 112.6 & -6.2 \\
800 & 112 & -5.6 \\
850 & 111.4 & -5 \\
900 & 110.8 & -4.4 \\
950 & 110.3 & -3.9 \\
1000 & 109.8 & -3.4 \\
\hline & &
\end{tabular}


Table 78. Expected output for VERTSEA for $r_{\max }$ receiver range of 300 km.

\begin{tabular}{c|c|c}
\hline $\begin{array}{c}\text { Height } \\
\text { (meters) }\end{array}$ & $\begin{array}{c}\text { Prop. } \\
\text { Loss } \\
\text { (dB) }\end{array}$ & $\begin{array}{c}\text { Prop. } \\
\text { Factor } \\
\text { (dB) }\end{array}$ \\
\hline 0 & 145.6 & -23.6 \\
50 & 136.4 & -14.4 \\
100 & 129.9 & -7.9 \\
150 & 127.1 & -5.1 \\
200 & 126.7 & -4.7 \\
250 & 129.2 & -7.2 \\
300 & 135.9 & -13.9 \\
350 & 143.8 & -21.8 \\
400 & 147.6 & -25.7 \\
450 & 146.3 & -24.3 \\
500 & 144.9 & -22.9 \\
550 & 144.2 & -22.3 \\
600 & 144 & -22 \\
650 & 144 & -22 \\
700 & 144.2 & -22.2 \\
750 & 144.6 & -22.6 \\
800 & 145 & -23 \\
850 & 145.5 & -23.5 \\
900 & 145.9 & -23.9 \\
950 & 146.3 & -24.3 \\
1000 & 146.6 & -24.6 \\
\hline & &
\end{tabular}

Table 79. Expected output for VERTUSRD for $r_{\text {max }}$ receiver range of $50 \mathrm{~km}$.

\begin{tabular}{c|c|c}
\hline $\begin{array}{c}\text { Height } \\
\text { (meters) }\end{array}$ & $\begin{array}{c}\text { Prop. } \\
\text { Loss } \\
\text { (dB) }\end{array}$ & $\begin{array}{c}\text { Prop. } \\
\text { Factor } \\
\text { (dB) }\end{array}$ \\
\hline 0 & 174.6 & -68.2 \\
50 & 140.5 & -34.1 \\
100 & 134 & -27.5 \\
150 & 129.8 & -23.4 \\
200 & 126.8 & -20.3 \\
250 & 124.3 & -17.9 \\
300 & 122.2 & -15.8 \\
350 & 120.5 & -14.1 \\
400 & 119 & -12.5 \\
450 & 117.7 & -11.2 \\
500 & 116.5 & -10.1 \\
550 & 115.4 & -9 \\
600 & 114.5 & -8.1 \\
650 & 113.7 & -7.2 \\
700 & 113.3 & -6.9 \\
750 & 112.6 & -6.2 \\
800 & 112 & -5.6 \\
850 & 111.4 & -5 \\
900 & 110.8 & -4.4 \\
950 & 110.3 & -3.9 \\
1000 & 109.8 & -3.4 \\
\hline
\end{tabular}


Table 80. Expected output for WEDGE for $r_{\max }$ receiver range of $100 \mathrm{~km}$.

\begin{tabular}{c|c|c}
\hline $\begin{array}{c}\text { Height } \\
\text { (meters) }\end{array}$ & $\begin{array}{c}\text { Prop. } \\
\text { Loss } \\
(\mathrm{dB})\end{array}$ & $\begin{array}{c}\text { Prop. } \\
\text { Factor } \\
(\mathrm{dB})\end{array}$ \\
\hline 50 & 154.5 & -22 \\
100 & 154.7 & -22.3 \\
150 & 155.7 & -23.3 \\
200 & 155.5 & -23 \\
250 & 154 & -21.6 \\
300 & 152.1 & -19.7 \\
350 & 150.3 & -17.9 \\
400 & 149 & -16.6 \\
450 & 148.1 & -15.6 \\
500 & 146.8 & -14.4 \\
550 & 144 & -11.5 \\
600 & 139.7 & -7.3 \\
650 & 135.4 & -2.9 \\
700 & 131.6 & 0.9 \\
750 & 128.3 & 4.1 \\
800 & 126.3 & 6.2 \\
850 & 126.9 & 5.6 \\
900 & 127.8 & 4.6 \\
950 & 127.4 & 5 \\
1000 & 130.2 & 2.2 \\
50 & 154.5 & -22 \\
\hline & &
\end{tabular}




\subsection{CRITERIA FOR EVALUATING RESULTS}

The calculated propagation loss in $\mathrm{dB}$ should match the numerical values in each table at each of the levels shown to within $0.1 \mathrm{~dB}(1 \mathrm{cB})$. APM rounds its output loss values to the nearest $1 \mathrm{cB}$, and hence it is possible for differences of $1 \mathrm{cB}$ to exist between different implementations of APM. It is expected, however, that in most cases the values will match those inTable 33 through Table 80 exactly.

\subsection{TEST PROCEDURE}

1. Compile for execution, the APM CSCI, the driver program APMMAIN.F90, and the module APM_MOD.F90.

2. An input data file has been provided, as a text file, for each test case.

3. The APM CSCI is executed in a form that reads the input data file, performs the calculations, and writes the output to a text file.

4. The output file is compared to the final expected test results to determine satisfactory performance.

\subsection{ASSUMPTIONS AND CONSTRAINTS}

Input data elements are assumed to be constrained by the limits listed within Tables 1 through 4 of the Software Requirements Specification (Ref. 3).

\section{REQUIREMENTS TRACEABILITY}

The provided driver program that accesses the APM CSCI will create an output file for each test case. The output file will have the same prefix name as the input file. The extension is ".OUT". This output file contains height in meters and corresponding propagation loss in $\mathrm{dB}$ that should correspond to the entries in Table 33 through Table 80 for each test case.

The provided program APMMAIN.FOR, when compiled with the APM CSCI, will read the provided input files containing all necessary information for each test case. Each input file is named for each test case, with a “.IN" extension.

\section{NOTES}

Table 81 is a glossary of acronyms and abbreviations used within this document. 
Table 81. Acronyms and Abbreviations.

\begin{tabular}{|c|c|}
\hline Term & Definition \\
\hline$a b s_{\text {hum }}$ & Surface absolute humidity $\left(\mathrm{g} / \mathrm{m}^{3}\right)$ \\
\hline ant $_{\text {gain }}$ & Antenna gain $(\mathrm{dBi})$ \\
\hline$a_{h t}$ & Antenna height \\
\hline APM & Advanced Propagation Model \\
\hline $\mathrm{cB}$ & centibel \\
\hline$C_{l u t}$ & Logical flag used to indicate if surface clutter calculations are desired \\
\hline CSC & Computer Software Component \\
\hline CSCI & Computer Software Configuration Item \\
\hline $\mathrm{dB}$ & Decibel \\
\hline dielec & 2-dimensional array of relative permittivity and conductivity \\
\hline EM & Electromagnetic \\
\hline FORTRAN & Formula Translation \\
\hline$f_{M H z}$ & EM system frequency (MHz) \\
\hline$\gamma_{a}$ & Surface specific attenuation rate $(\mathrm{dB} / \mathrm{km})$ \\
\hline$\gamma_{c}$ & Array of "backscattering effectiveness" term used in reflectivity computation (dB) \\
\hline rrng & Array of corresponding ranges for $\gamma_{c}(\mathrm{~km})$ \\
\hline hfang & User-defined height-finder power reduction angle array (deg) \\
\hline hffac & User-defined power reduction factor array \\
\hline$h_{\max }$ & Maximum height output for a particularapplication of APM. \\
\hline$h_{\min }$ & Minimum height output for a particular application of APM. \\
\hline hmsl & Refractivity profile height array \\
\hline$i_{\text {extra }}$ & Extrapolation flag for refractivity profiles entered below mean sea level \\
\hline$i_{g c}$ & Number of $\gamma_{c}$ values and corresponding ranges \\
\hline$i_{g r}$ & Number of ground composition types for particular application of APM \\
\hline igrnd & Ground composition type array \\
\hline$i_{\text {pat }}$ & Antenna pattern \\
\hline$i_{\text {pol }}$ & Antenna polarization \\
\hline lang & $\begin{array}{l}\text { Logical flag indicating if propagation angle and propagation factor output for specific ray } \\
\text { paths is desired }\end{array}$ \\
\hline lerr6 & Controlling logical flag for error 6 \\
\hline lerr12 & Controlling logical flag for error 12 \\
\hline
\end{tabular}


Table 81. Acronyms and Abbreviations. (Continued)

\begin{tabular}{|c|c|}
\hline Term & Definition \\
\hline$L_{\text {sys }}$ & System loss (dB) \\
\hline lvlp & Number of levels in refractivity profiles for particular application of APM \\
\hline $\mathrm{km}$ & kilometers \\
\hline $\mathrm{m}$ & meters \\
\hline N/A & Not applicable \\
\hline$N_{f}$ & Noise figure $(\mathrm{dB})$ \\
\hline$n_{\text {facs }}$ & $\begin{array}{l}\text { Number of power reduction factors and cut-back angles for user-defined height finder } \\
\text { radar }\end{array}$ \\
\hline$n_{\text {prof }}$ & Number of refractivity profiles for particular application of APM \\
\hline$n_{\text {rout }}$ & Number of range output points for a particular application of APM. \\
\hline$n_{w}$ & Number of wind speeds \\
\hline$n_{\text {zout }}$ & Number of height output points for a particular application of APM. \\
\hline$n_{\text {zout_rtg }}$ & Number of height output points relative to ground for a particular application of APM. \\
\hline$P E_{\text {flag }}$ & Logical flag indicating PE-only mode \\
\hline$P_{t}$ & Transmitter power $(\mathrm{kW})$ \\
\hline refmsl & Refractivity profile M-unit array \\
\hline rgrnd & Gound composition type range array \\
\hline$r_{\max }$ & Maximum range output for a particular application of APM. \\
\hline$r_{\text {mult }}$ & PE range step multiplier \\
\hline rngprof & Refractivity profile range array \\
\hline rngwind & Range array of wind speeds \\
\hline$t_{\text {air }}$ & Surface air temperature $\left({ }^{\circ} \mathrm{C}\right)$ \\
\hline$\tau$ & Pulse width/length (microseconds) \\
\hline terx & Terrain profile range array \\
\hline tery & Terrain profile height array \\
\hline$\theta_{\text {hbw }}$ & Antenna horizontal beam width (degrees) \\
\hline$t h_{\max }$ & Visible portion of maximum PE propagation angle \\
\hline$T_{\text {ropo }}$ & Logical flag to include troposcatter calculations \\
\hline$\mu_{b w}$ & Antenna vertical beam width (degrees) \\
\hline$\mu_{o}$ & antenna elevation angle (degrees) \\
\hline wind & Wind speed array $(\mathrm{m} / \mathrm{s})$ \\
\hline wind $_{\text {dir }}$ & Wind direction relative to boresight (degrees) \\
\hline zout_rtg & Array of receiver heights relative to local surface elevation (m) \\
\hline
\end{tabular}




\section{SAMPLE PROGRAM LISTING}

The sample driver program APMMAIN.F90, which exercises the APM CSCI, is provided below.

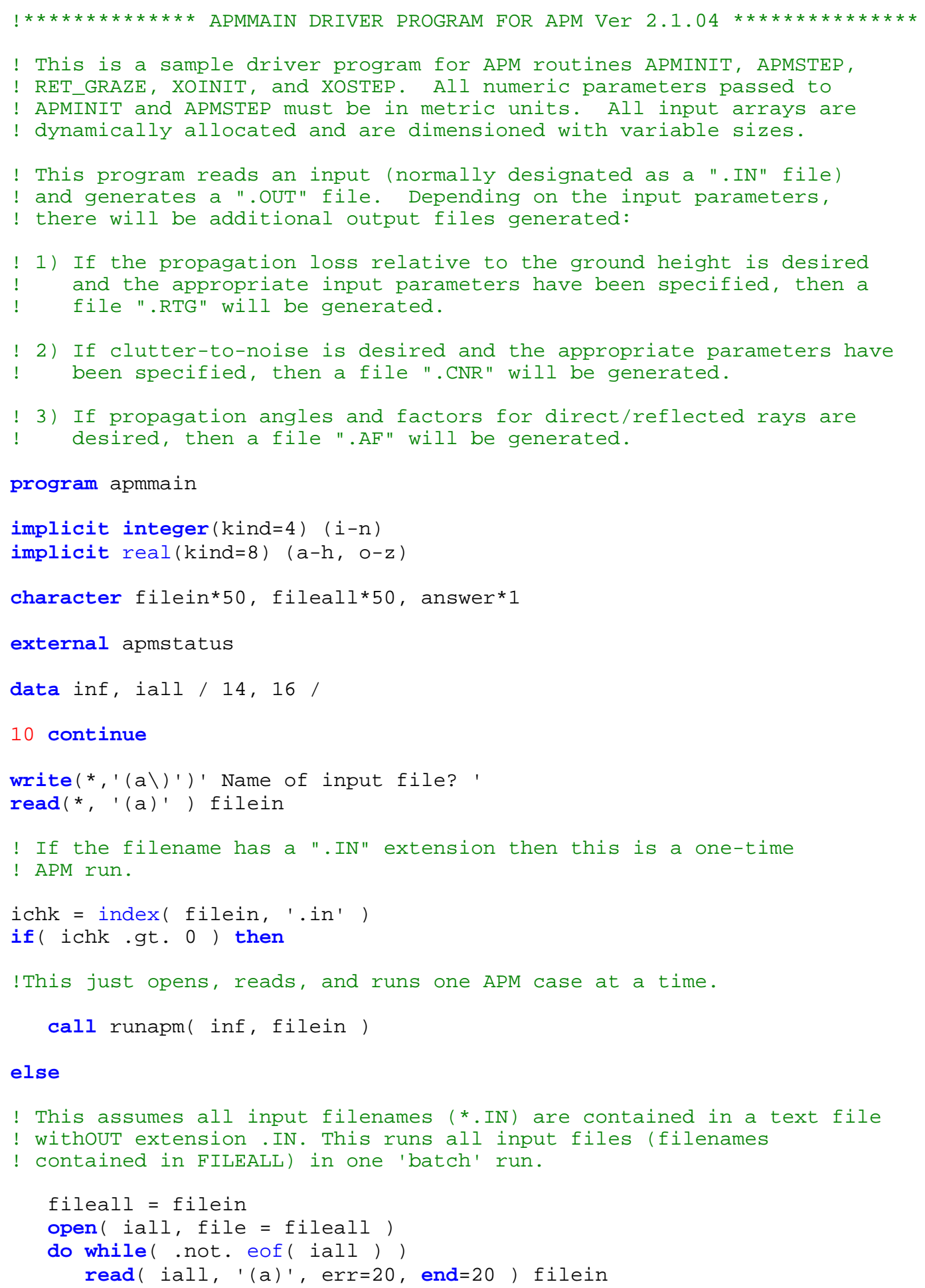




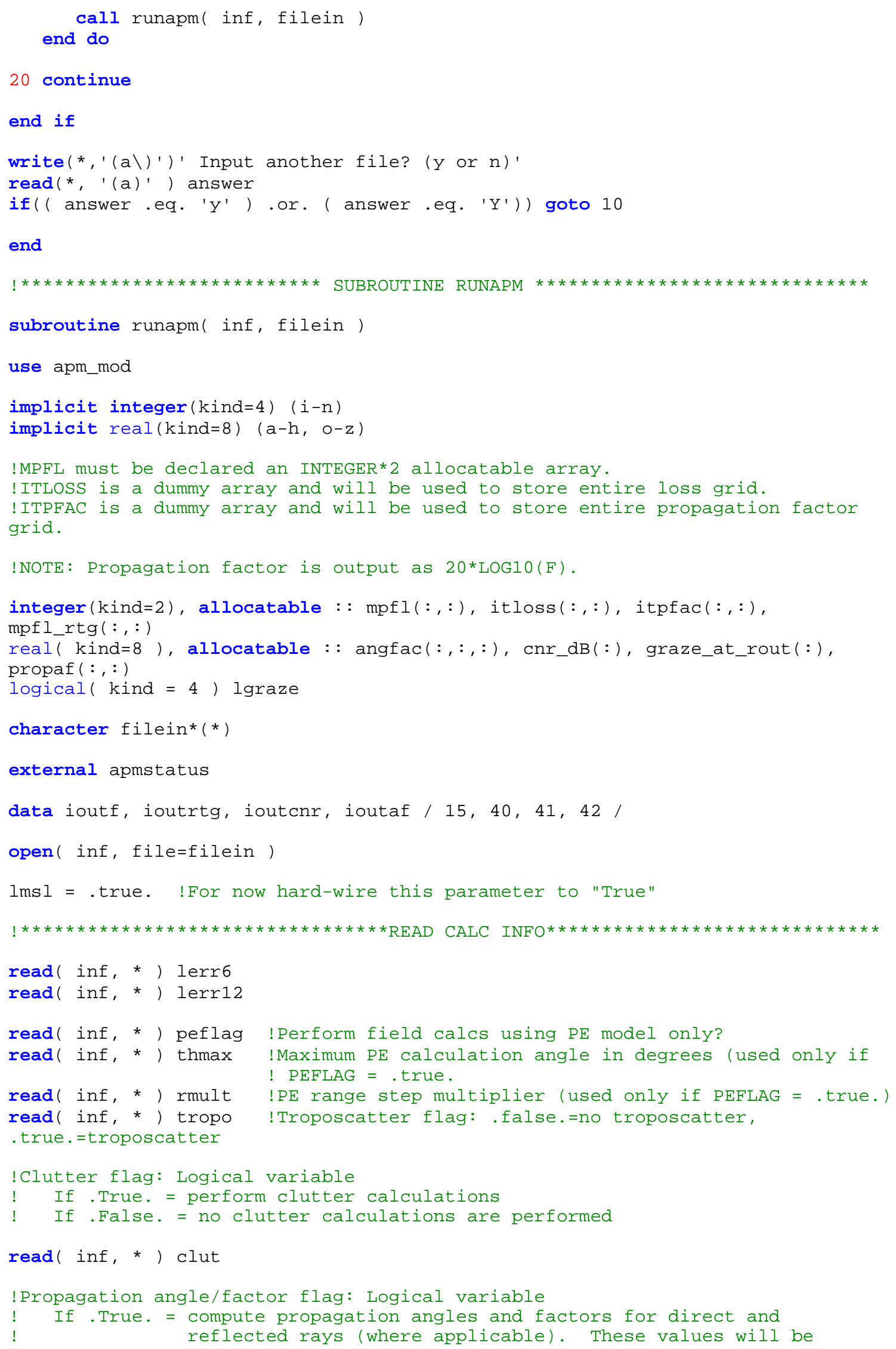




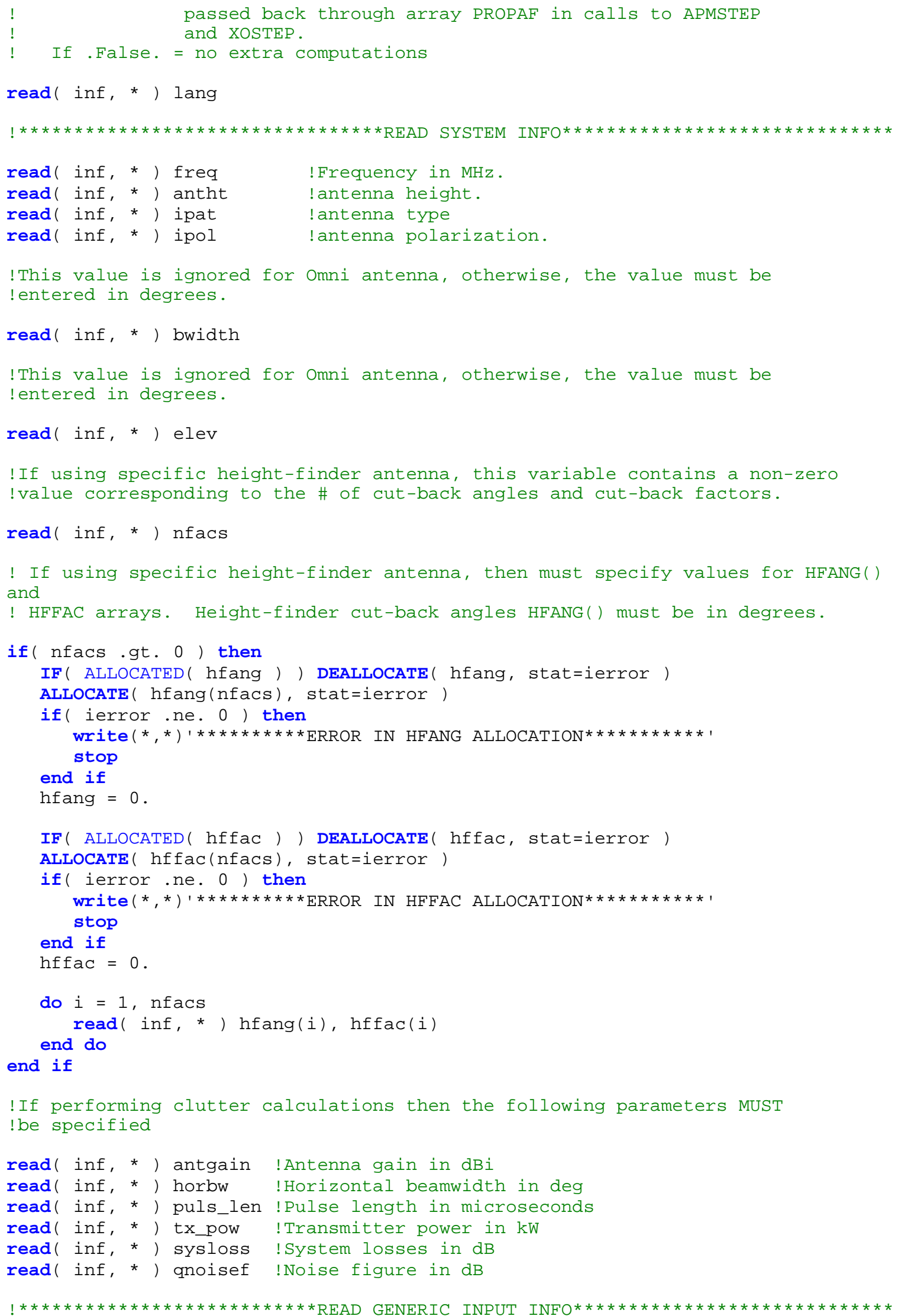




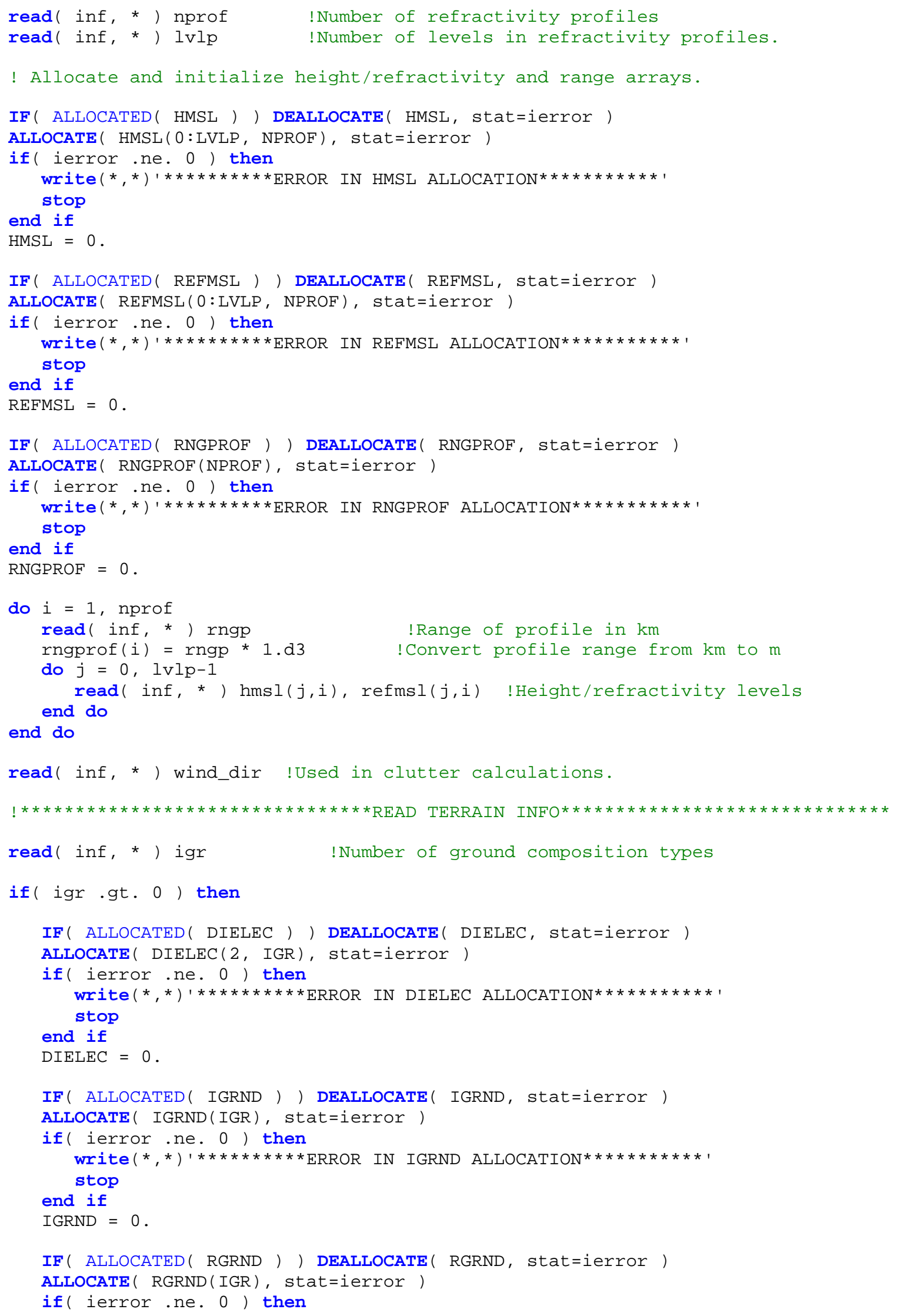




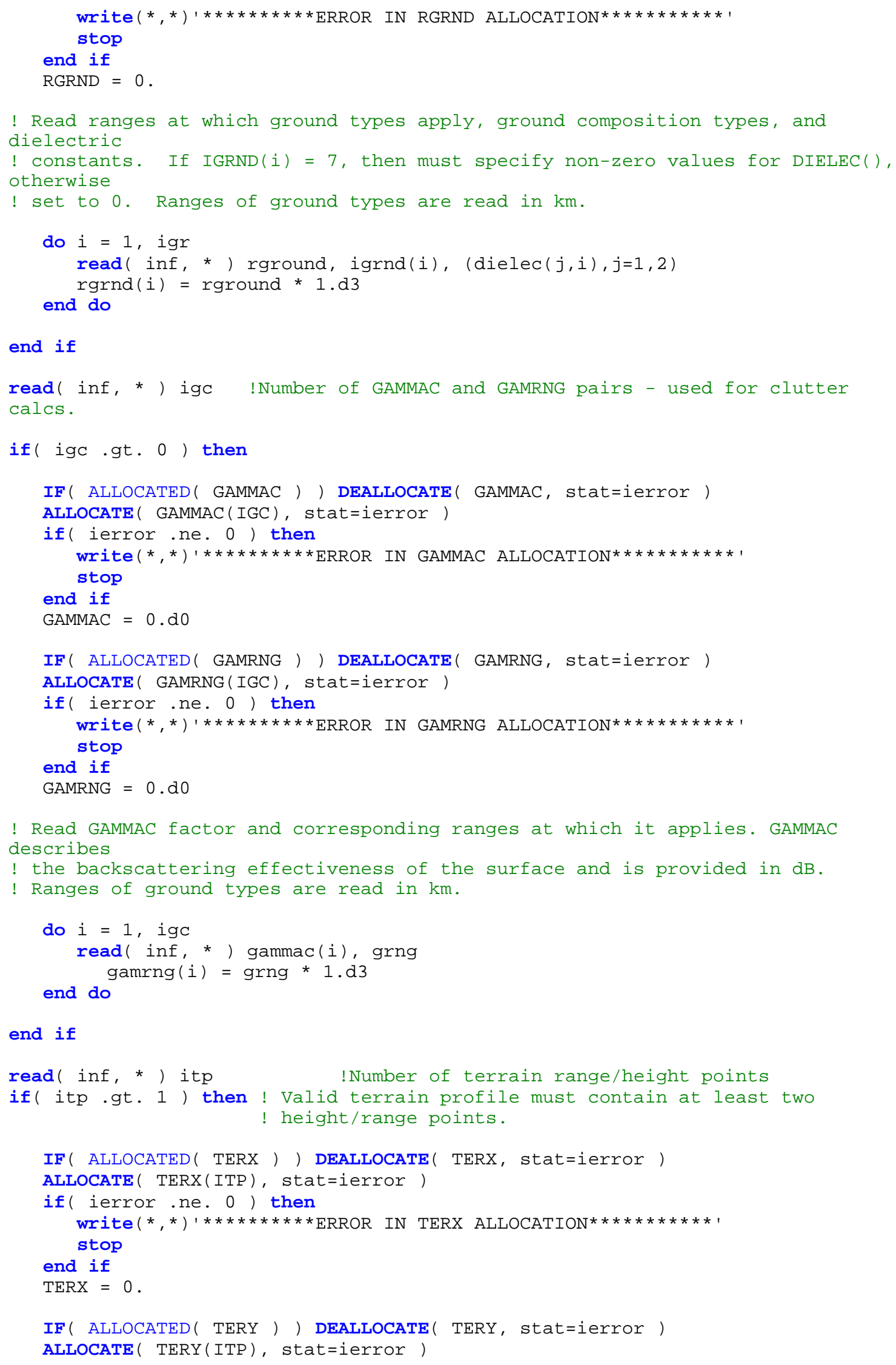




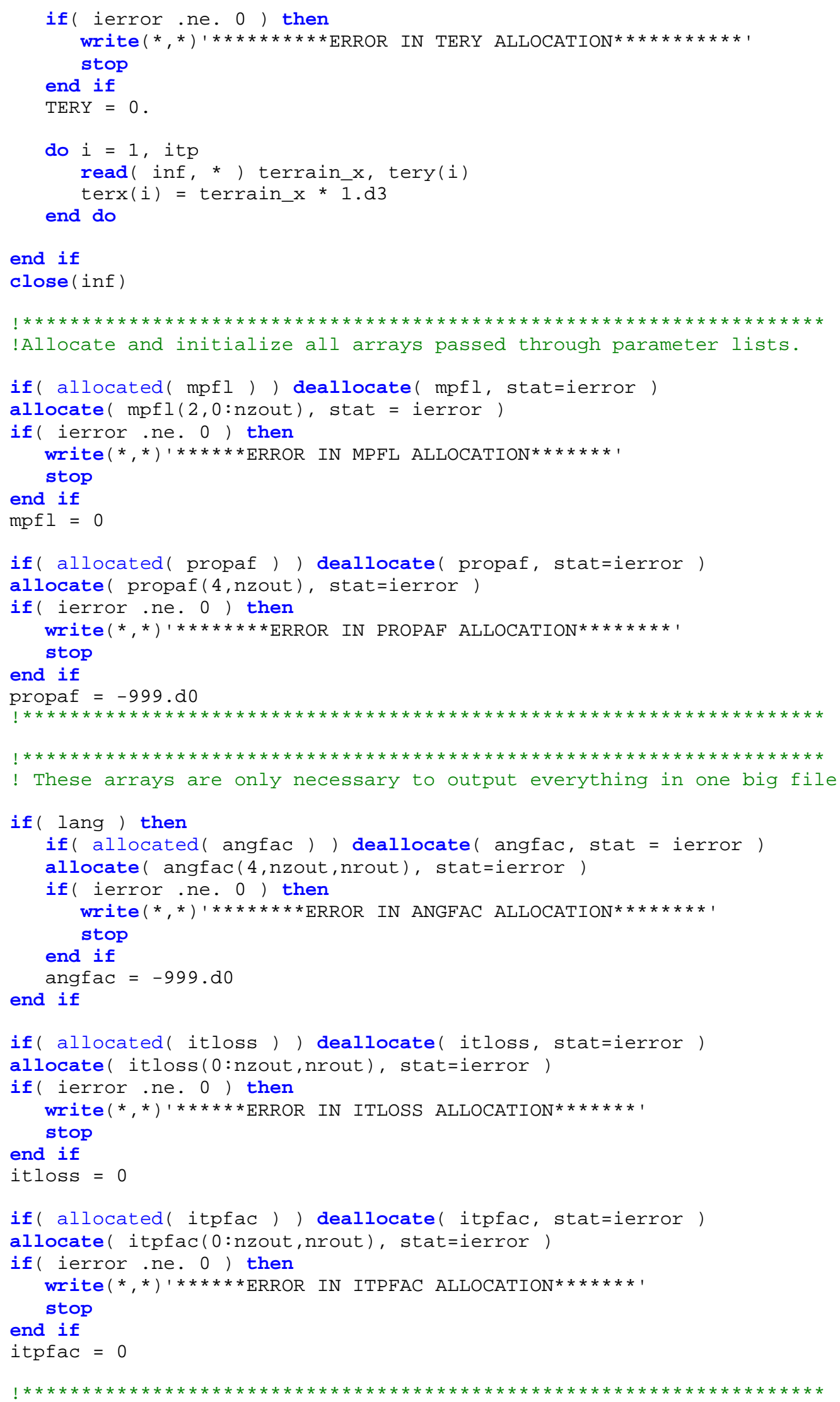


! Allocate and initialize CNR_dB array with proper size

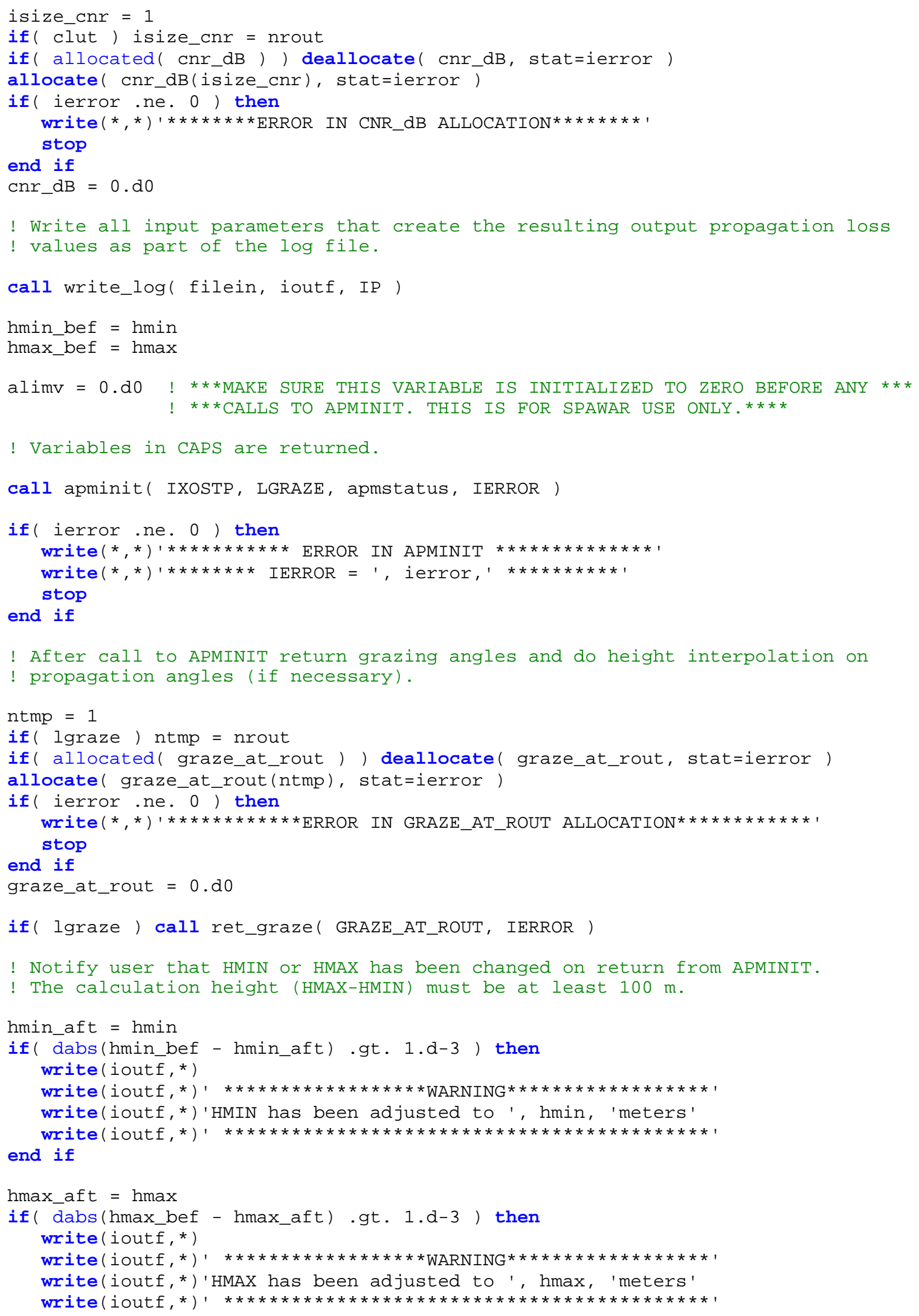


end if

! Create and write header info for extraneous files.

call write_extra( ip, filein, ioutrtg, ioutcnr, ioutaf )

! $* * * * * * * * * * * * * * * * * * * * *$ START OF MAIN IPM LOOP $* * * * * * * * * * * * * * * * * * * * * * * * * * *$

do istp $=1$, nrout

! JSTART $=$ start of valid loss points, JEND $=$ end of valid loss

! points. If at a range where extended optics will be applied, then

! JEND will be the index at top of PE region in MPFL().

call apmstep( istp, ROUT, MPFL, JSTART, JEND, MPFL_RTG, PROPAF )

write $\left(*,{ }^{*}\right)$ 'range in $\mathrm{km}=$ ', rout*1.d-3 !Output to screen

! Store loss and propagation factor points in 2-dim. grid for later output to file.

itloss $(0:$ nzout, istp $)=\operatorname{mpfl}(1,0:$ nzout $)$ !prop loss

itpfac $(\odot:$ nzout, istp $)=\operatorname{mpfl}(2,0:$ nzout $)$ !prop factor

!**** Output to separate file for loss relative to ground.******

!*** NOTE: ALL HEIGHTS SPECIFIED FOR LOSS RELATIVE TO GROUND ARE ASSUMED TO BE AT

!RELATIVELy LOW ALtitude - I.E., ALL heightS ARE CONTAINEd WITHIN THE FE, RO, OR PE

! REGIONS.

if $(\operatorname{lrtg})$ then

write( ioutrtg, ' $((\mathrm{f} 10.1,5 x) \backslash)$ ') rout*1.d-3, $\left(\operatorname{dble}\left(\operatorname{mpfl} \_r t g(1, i){ }^{*} 1\right)\right.$, $i=1$, nzout_rtg )

end if

write( ioutrtg, *)

! If necessary move values in PROPAF to larger array ANGFAC for later

! output after calls to XOINIT \& XOSTEP.

if ( lang $)$ angfac $(1: 4,1:$ jend, istp $)=\operatorname{propaf}(1: 4,1:$ jend $)$

end do

if ( $\operatorname{lrtg})$ close(ioutrtg)

! Initialize variables to be used in $\mathrm{xO}$ model.

call xoinit( graze_at_rout, ixostp, jend, JXSTART, CNR_dB, IERROR )

if ( ierror.gt. $\odot$ ) then

write $(*, *) 1 * * * * * * * \operatorname{ERROR}$ IN XOINIT*******1

stop

end if

! Output height increment DZOUT, as determined in APMINIT, is computed as

! DZOUT $=($ HMAX-HMIN $) /$ float $($ NZOUT $)$

! Output range increment DROUT, as determined in APMINIT, is computed as

! DROUT = RMAX / float $($ NROUT $)$

$! * * * *$ Output to separate file for clutter-to-noise ratio computed in XOINIT

if ( clut ) then

do $i=1$, nrout

write( ioutcnr, '(2(f10.1,2x))') drout*real(i,8)*1.d-3, cnr_dB(i) 


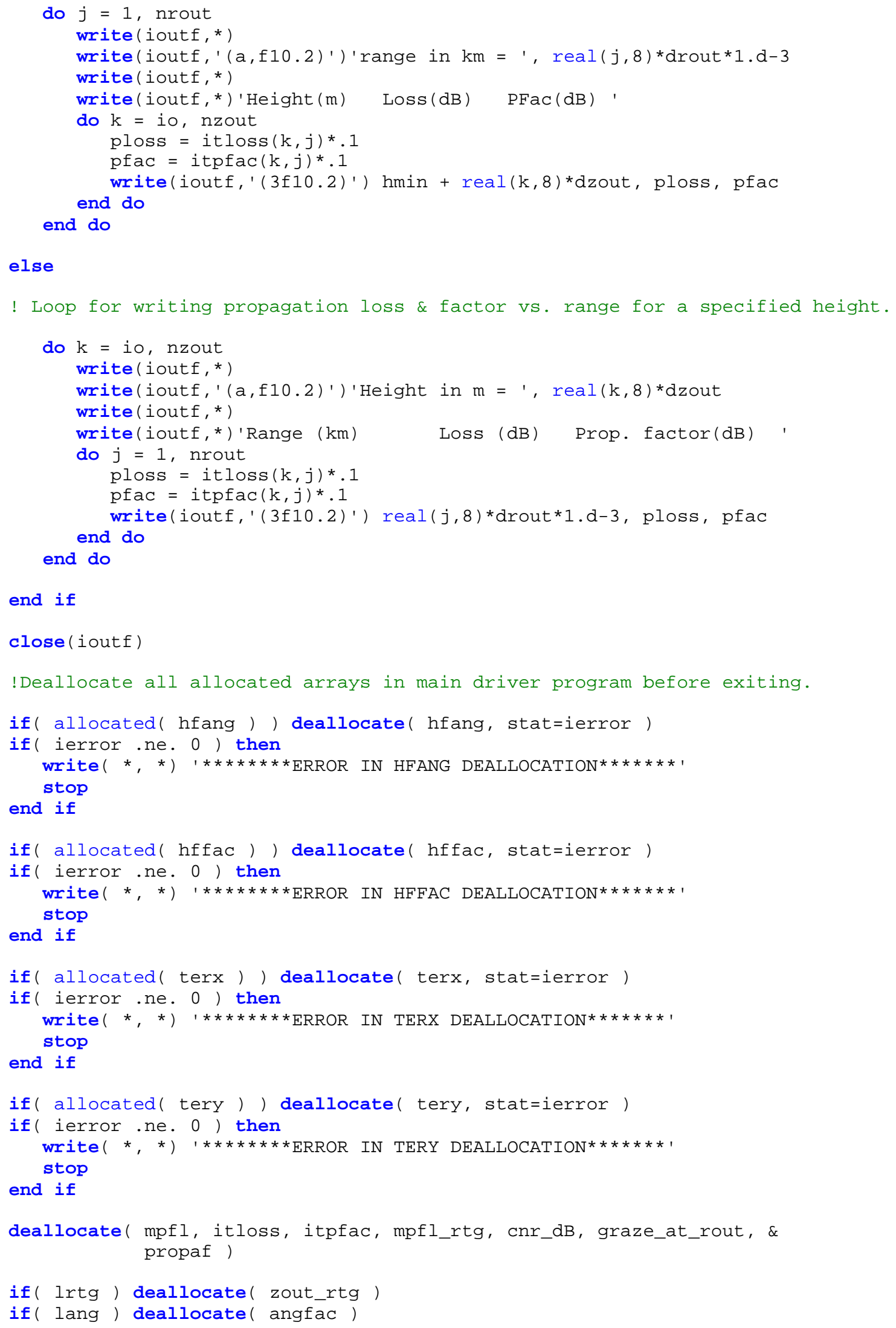




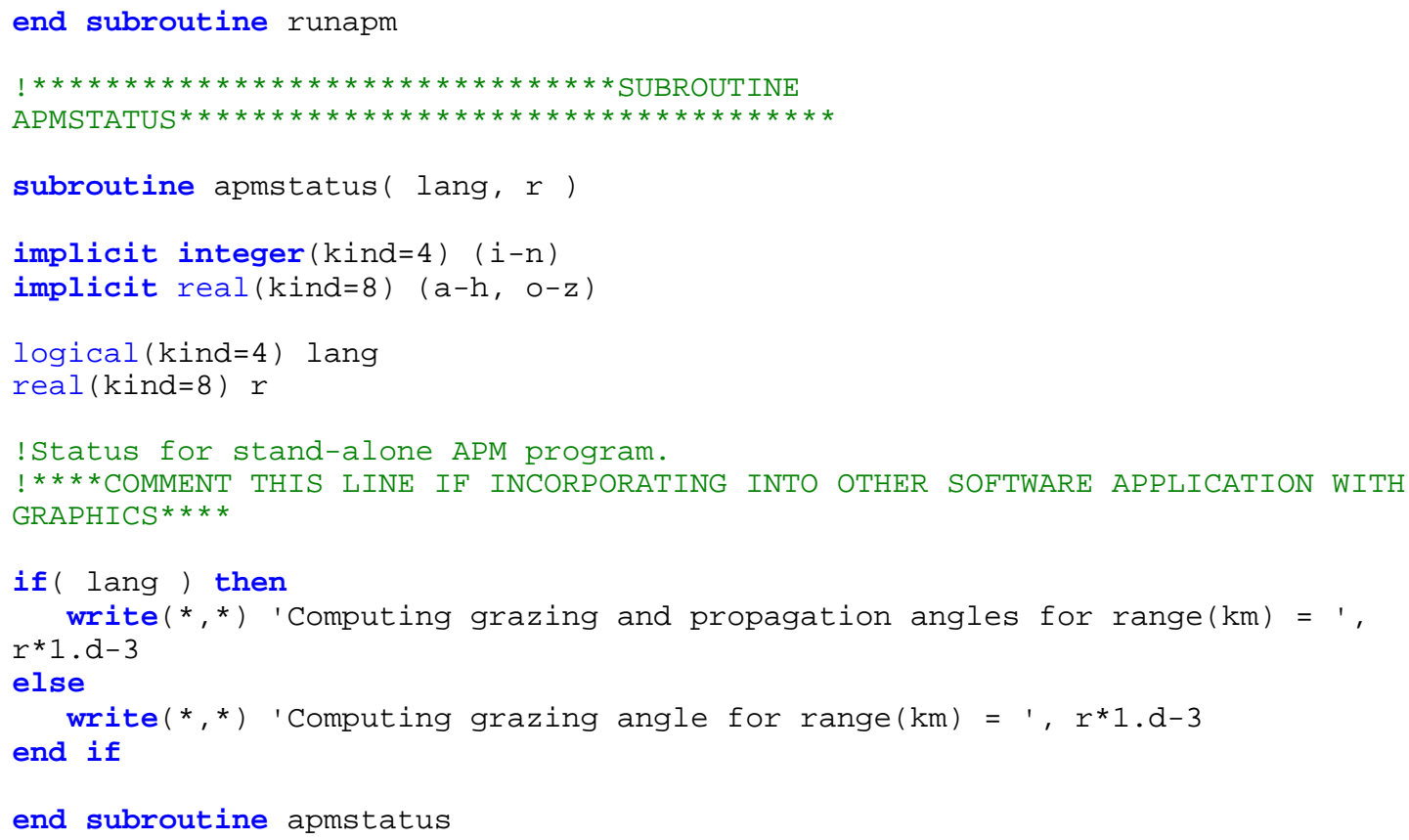




\section{INPUT FILE LISTINGS FOR TEST CASES}

Each test case, when using the sample driver program APMMAIN.F90, shall consist of an input file (TestName.IN) and an output file (TestName.OUT). The input file's contents are listed in sections 8.1 through 8.28. The output file's contents, consisting of couplets of height in meters and propagation loss and propagation factor in $\mathrm{dB}$, are listed in Table 33 through Table 80.

\subsection{ABSORB.IN}

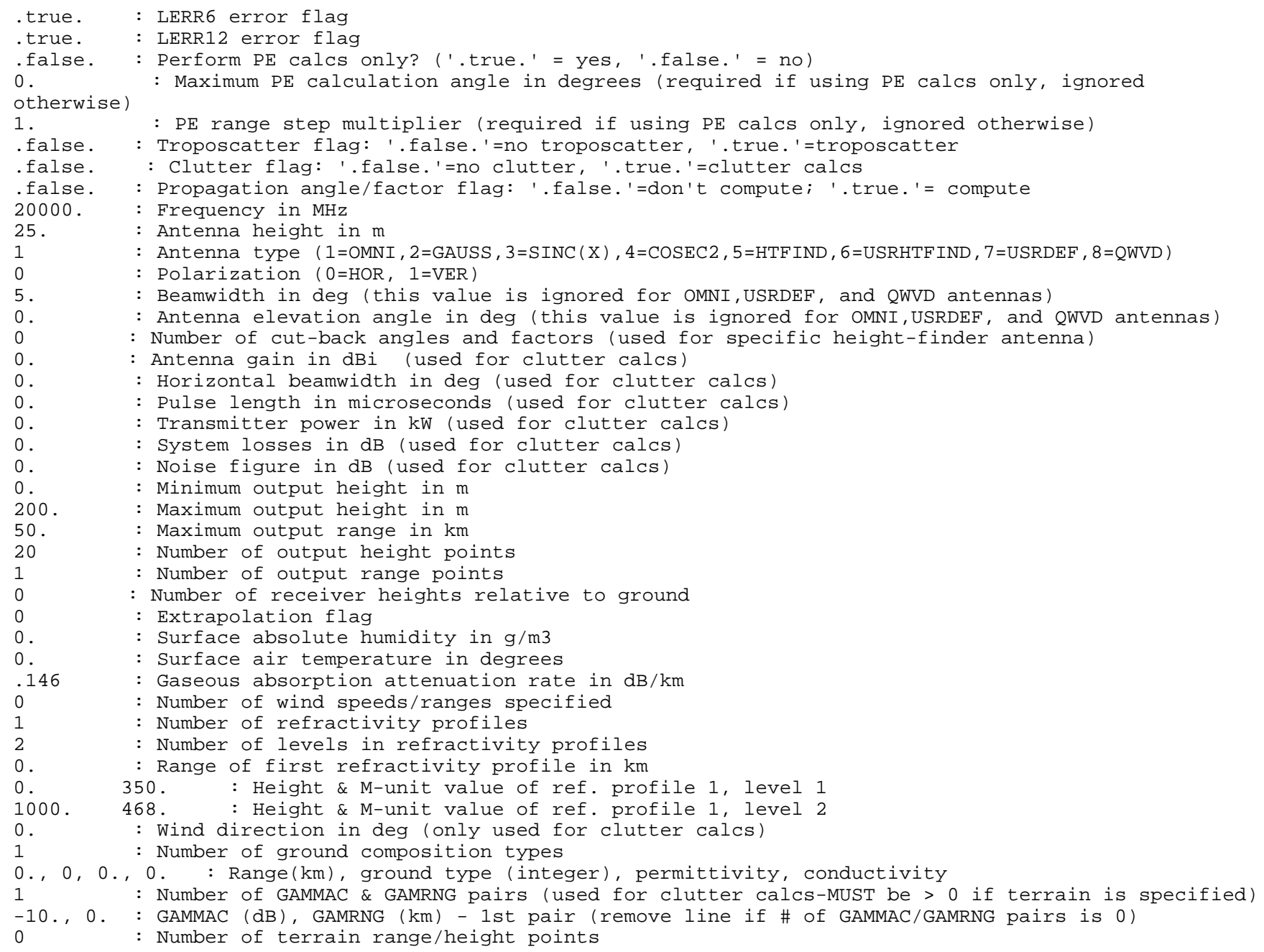

\subsection{AFEVAP.IN}

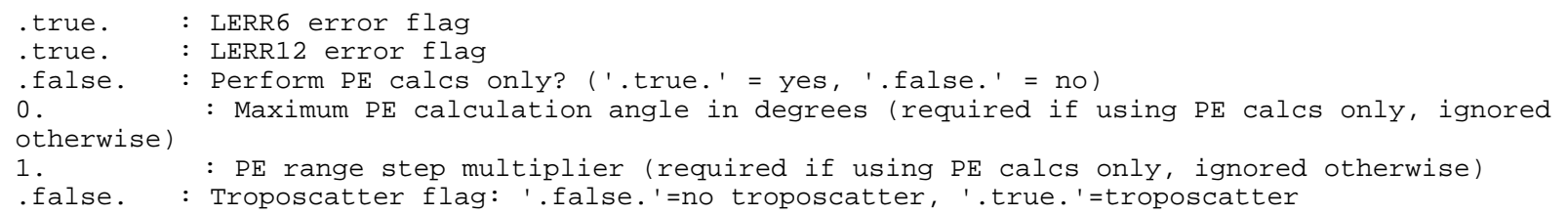




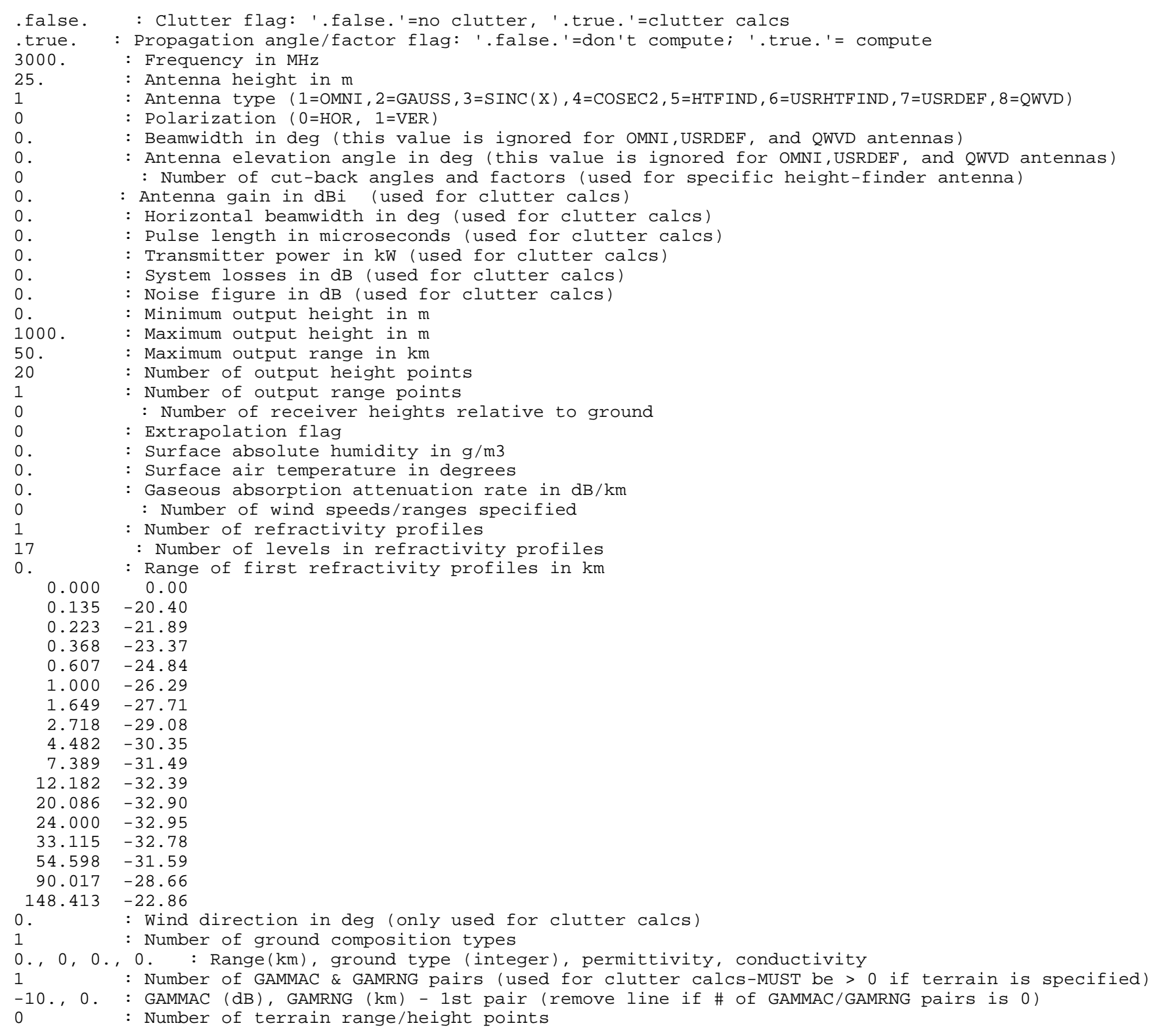

\subsection{AFSBD.IN}

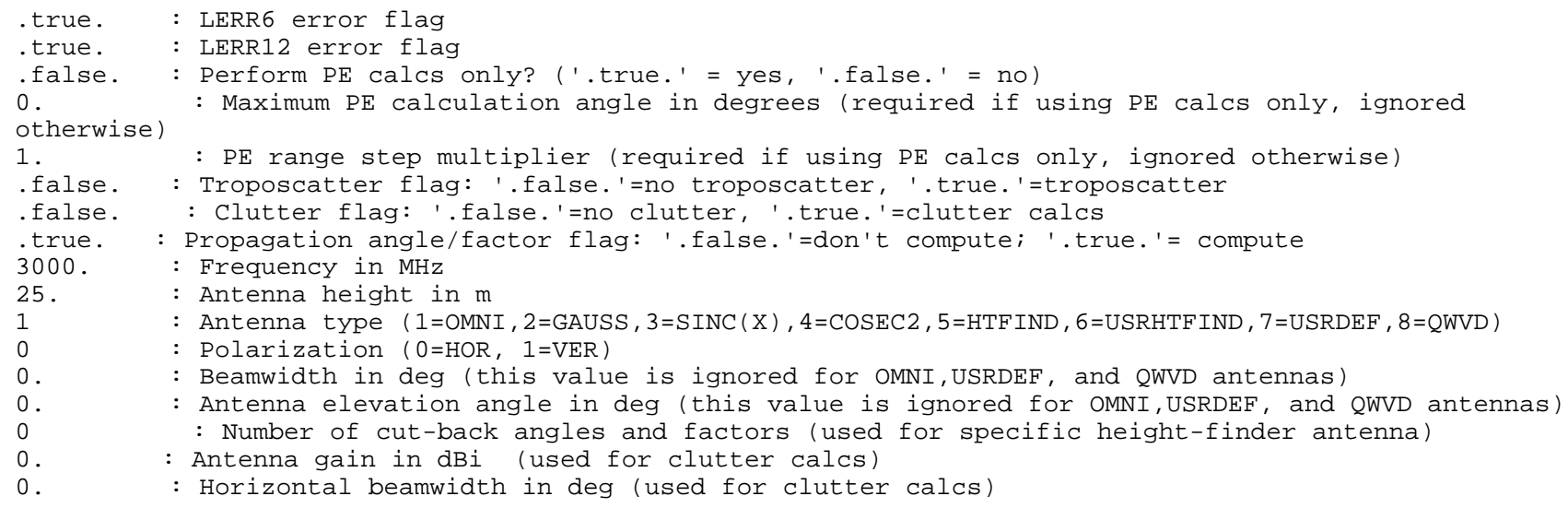




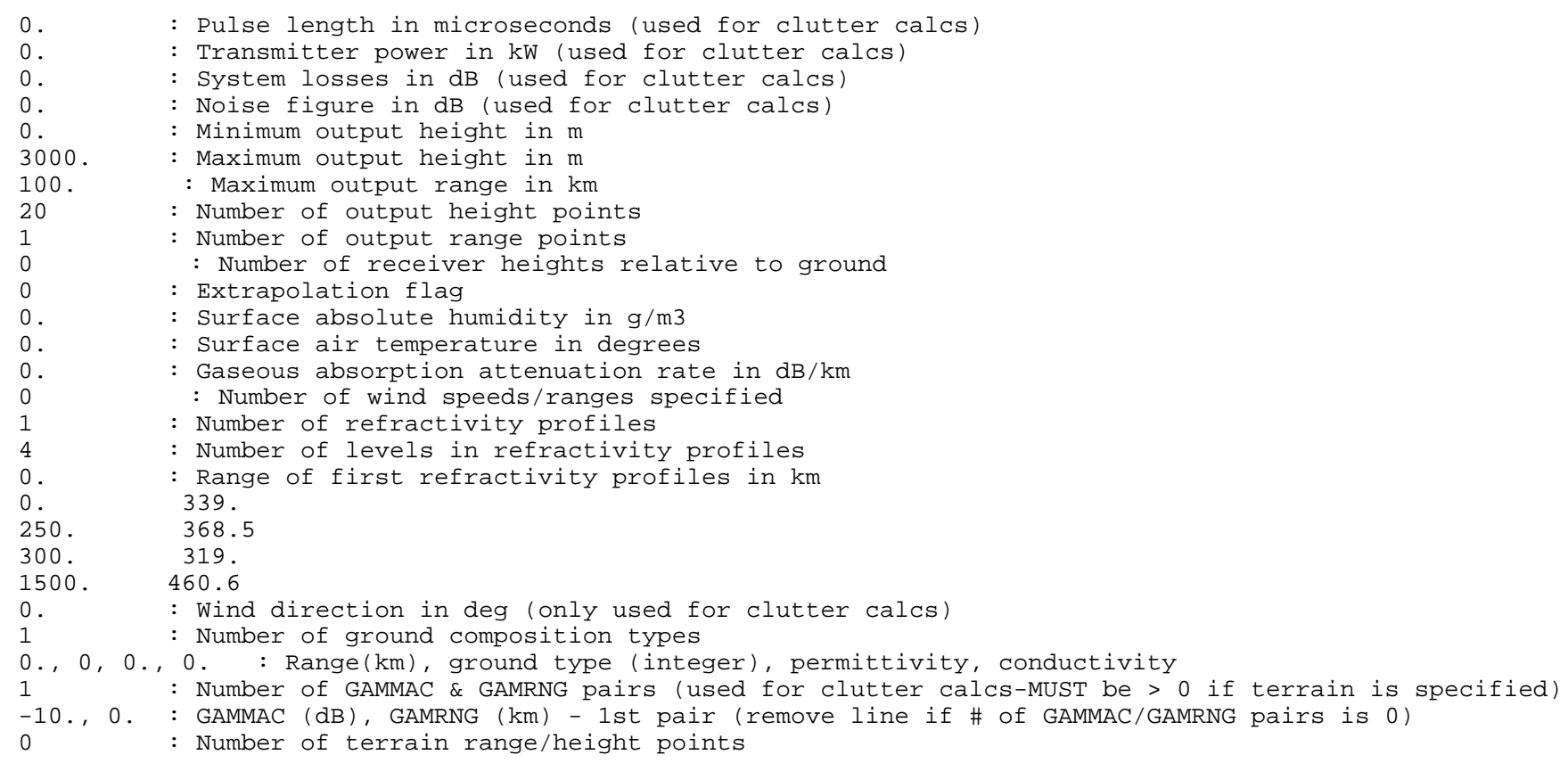

\subsection{AFSTD.IN}

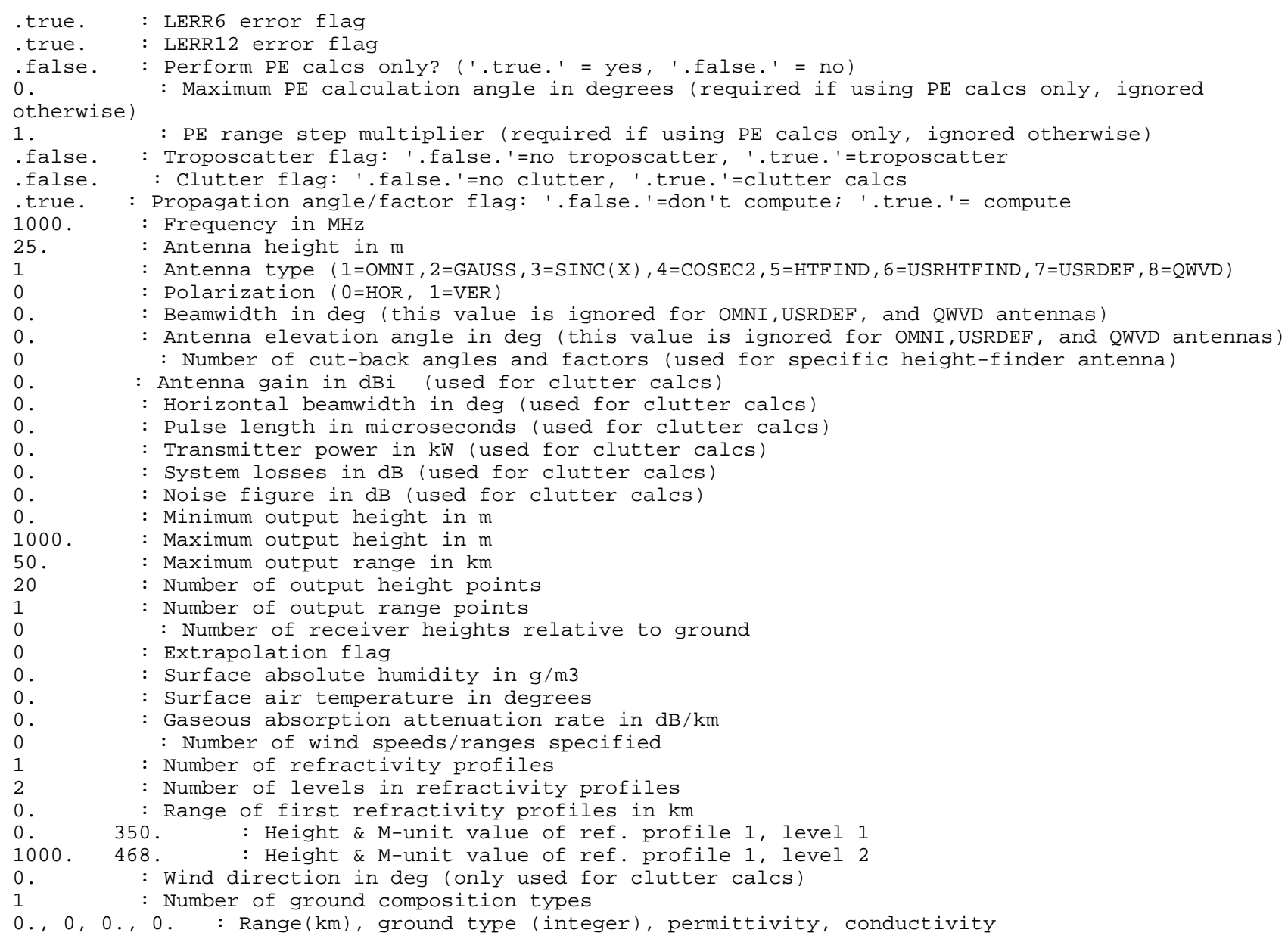


1 : Number of GAMMAC \& GAMRNG pairs (used for clutter calcs-MUST be > $\odot$ if terrain is specified) $-10 ., 0 . \quad$ : GAMMAC $(\mathrm{dB})$, GAMRNG $(\mathrm{km})$ - 1st pair (remove line if \# of GAMMAC/GAMRNG pairs is 0 )

\subsection{AIRBORNE.IN}

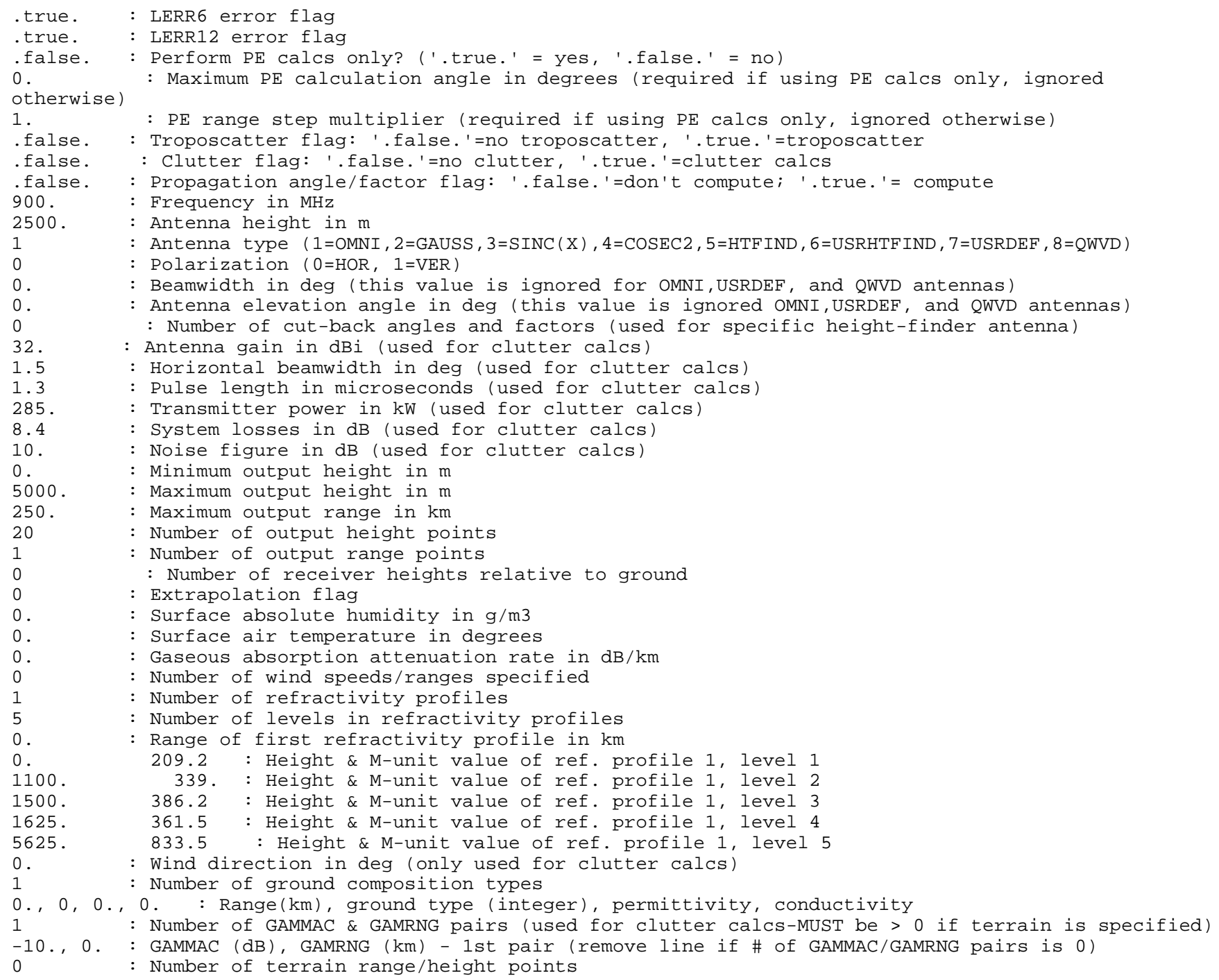

\subsection{BLOCK.IN}

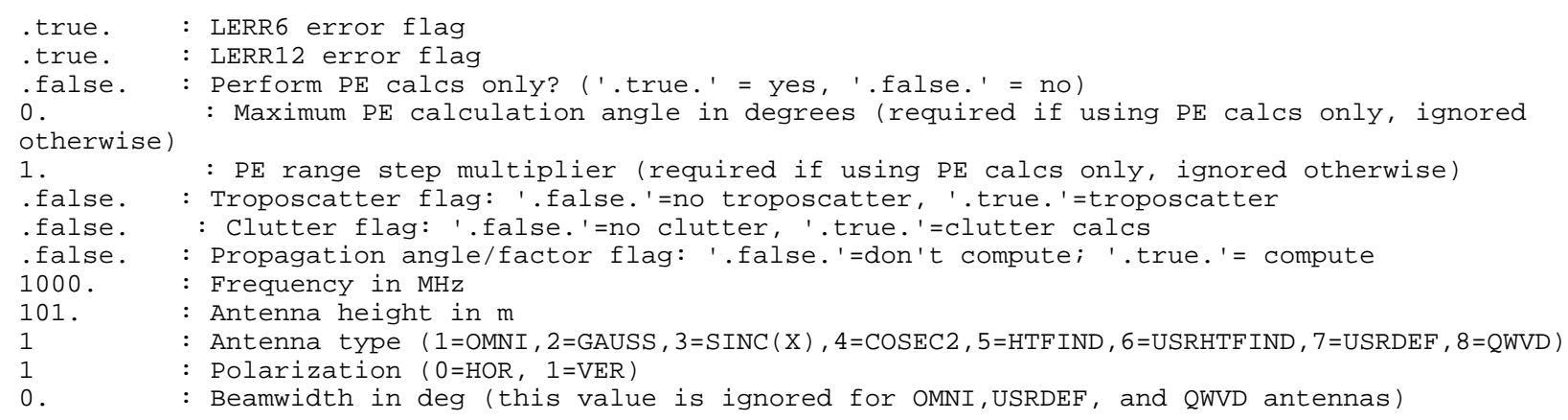




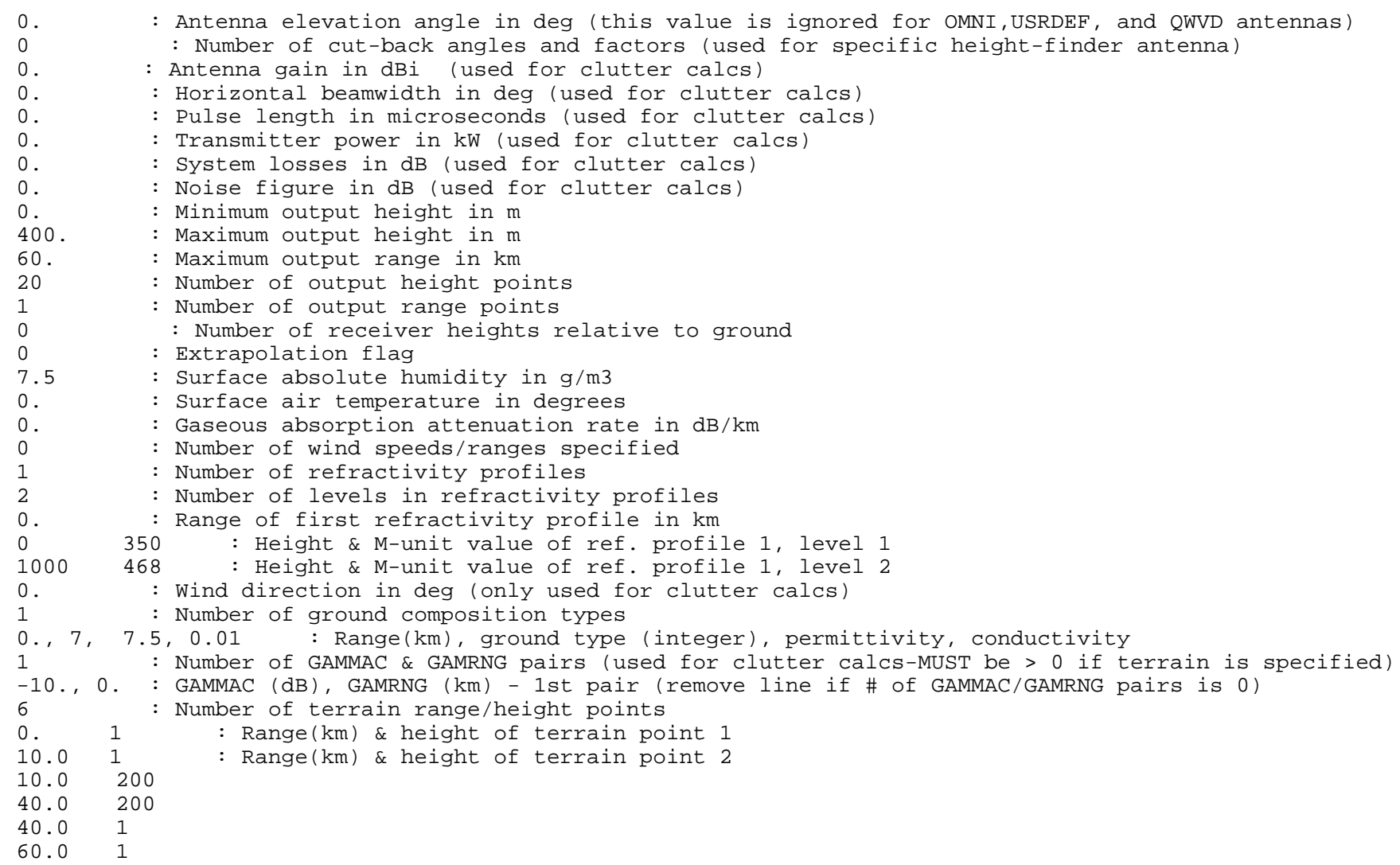

\subsection{CLEVAPW.IN}

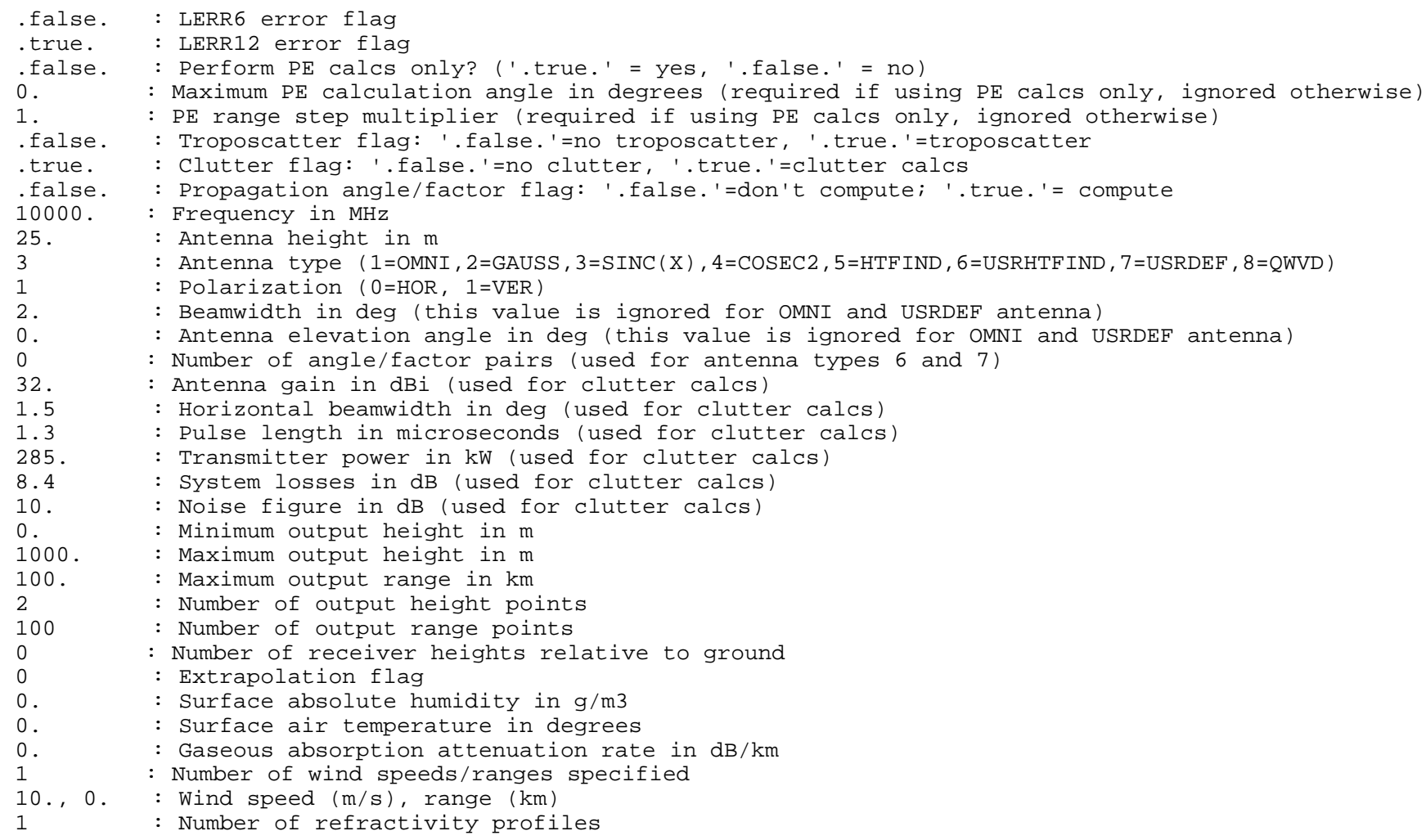




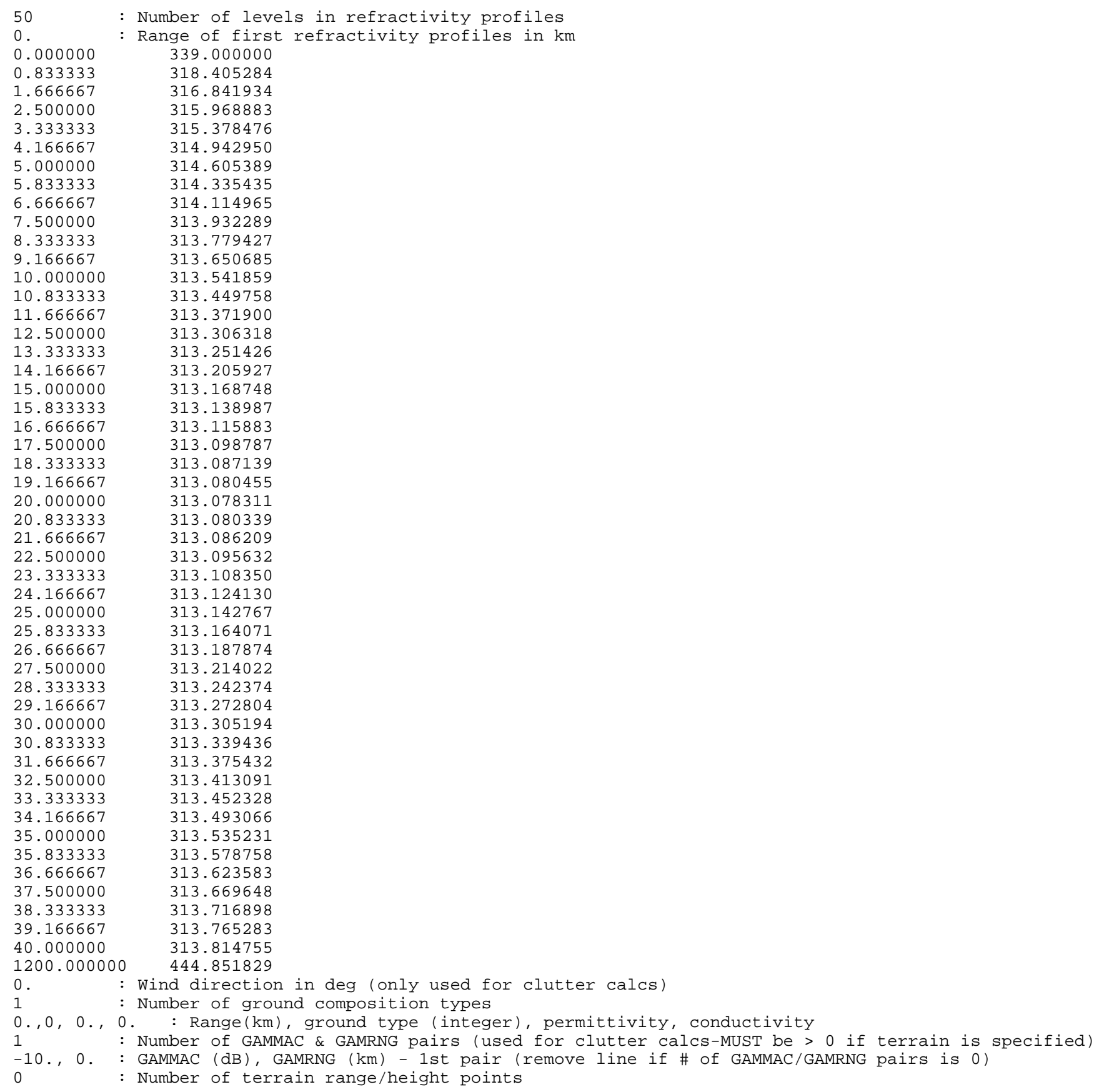

\subsection{CLSBDL.IN}

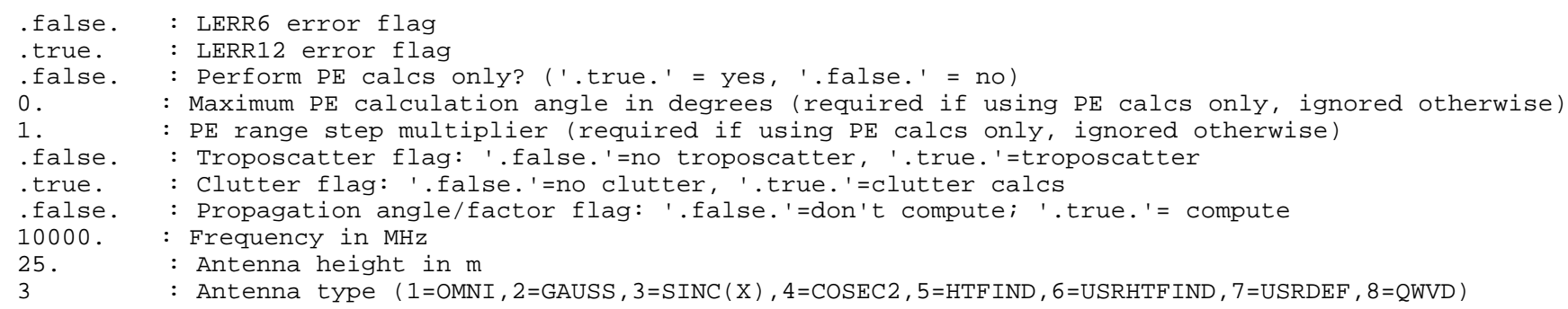




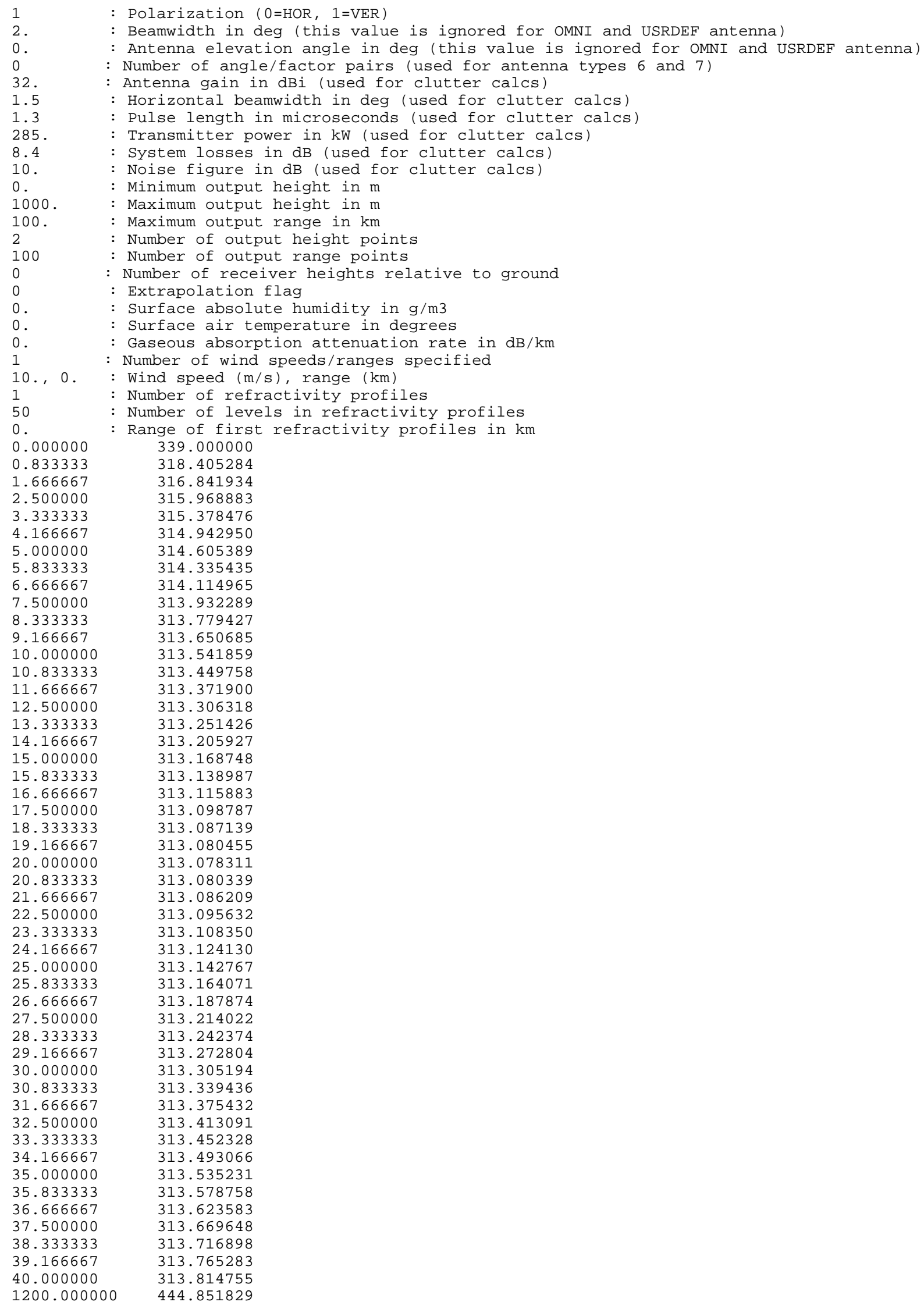




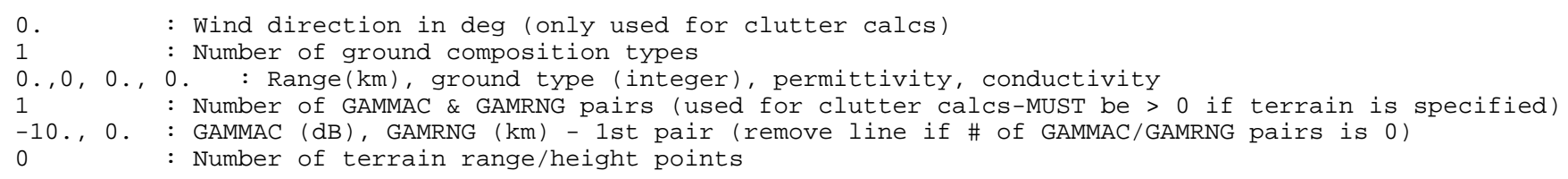

\subsection{CLSBDW.IN}

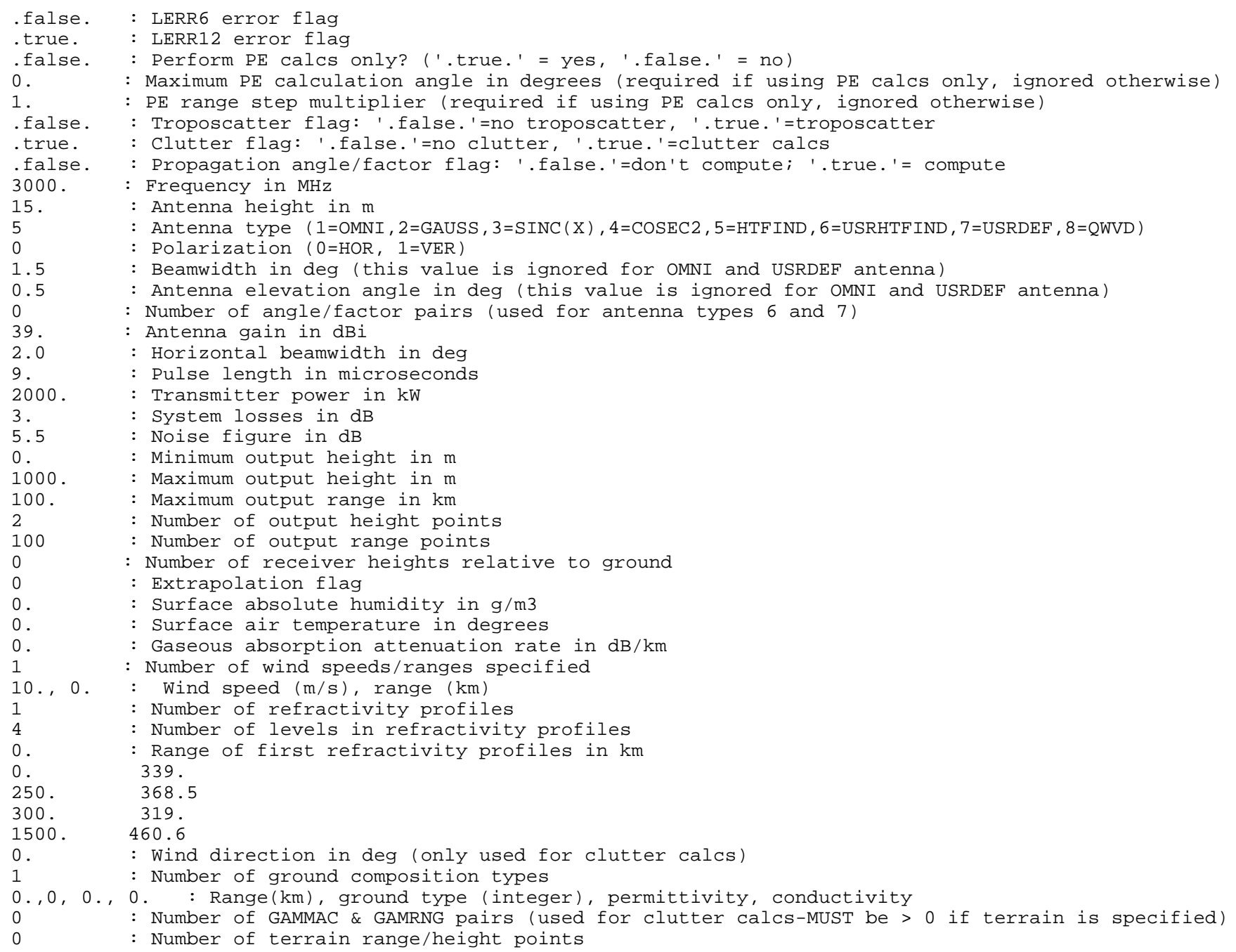

\subsection{CLSBDWL.IN}

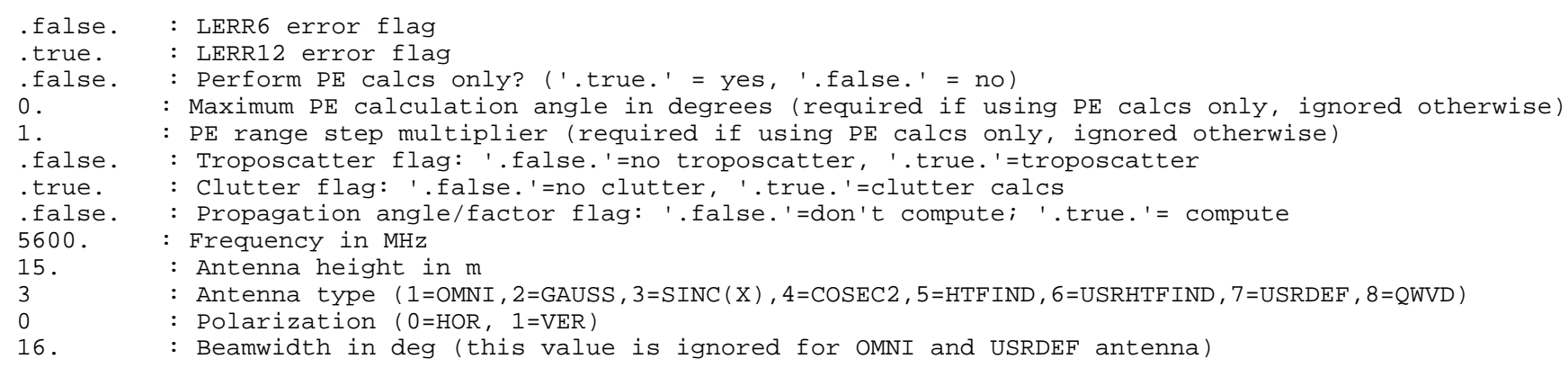




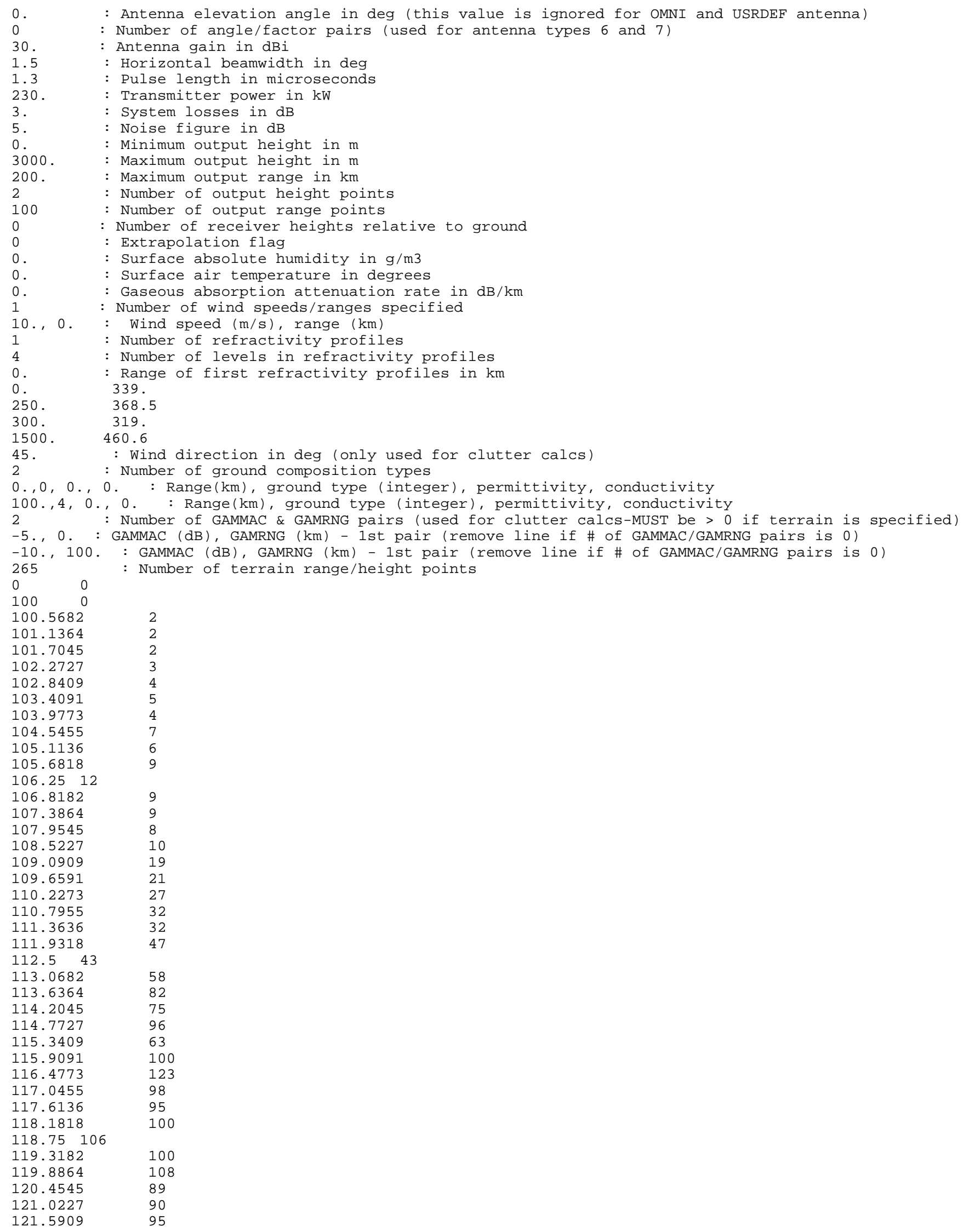




\begin{tabular}{|c|c|}
\hline & \\
\hline 122.1591 & 89 \\
\hline 122.7273 & 107 \\
\hline 123.2955 & 97 \\
\hline 123.8636 & 108 \\
\hline 124.4318 & 87 \\
\hline $125 \quad 76$ & \\
\hline 125.5682 & 73 \\
\hline 126.1364 & 88 \\
\hline 126.7045 & 86 \\
\hline 127.2727 & 101 \\
\hline 127.8409 & 101 \\
\hline 128.4091 & 92 \\
\hline 128.9773 & 65 \\
\hline 129.5455 & 62 \\
\hline 130.1136 & 47 \\
\hline 130.6818 & 59 \\
\hline $131.25 \quad 44$ & \\
\hline 131.8182 & 33 \\
\hline 132.3864 & 21 \\
\hline 132.9545 & 20 \\
\hline 133.5227 & 21 \\
\hline 134.0909 & 11 \\
\hline 134.6591 & 7 \\
\hline 135.2273 & 7 \\
\hline 135.7955 & 4 \\
\hline 136.3636 & 12 \\
\hline 136.9318 & 9 \\
\hline 137.56 & \\
\hline 138.0682 & 5 \\
\hline 138.6364 & 7 \\
\hline 139.2045 & 5 \\
\hline 139.7727 & 8 \\
\hline 140.3409 & 14 \\
\hline 140.9091 & 7 \\
\hline 141.4773 & 12 \\
\hline 142.0455 & 10 \\
\hline 142.6136 & 8 \\
\hline 143.1818 & 14 \\
\hline $143.75 \quad 15$ & \\
\hline 144.3182 & 18 \\
\hline 144.8864 & 29 \\
\hline 145.4545 & 78 \\
\hline 146.0227 & 76 \\
\hline 146.5909 & 89 \\
\hline 147.1591 & 139 \\
\hline 147.7273 & 168 \\
\hline 148.2955 & 173 \\
\hline 148.8636 & 184 \\
\hline 149.4318 & 193 \\
\hline $150 \quad 232$ & \\
\hline 150.5682 & 227 \\
\hline 151.1364 & 264 \\
\hline 151.7045 & 222 \\
\hline 152.2727 & 267 \\
\hline 152.8409 & 247 \\
\hline $153.4 \odot 91$ & 287 \\
\hline 153.9773 & 363 \\
\hline 154.5455 & 427 \\
\hline 155.1136 & 399 \\
\hline 155.6818 & 344 \\
\hline $156.25 \quad 258$ & \\
\hline 156.8182 & 188 \\
\hline 157.3864 & 182 \\
\hline 157.9545 & 94 \\
\hline 158.5227 & 85 \\
\hline 159.0909 & 63 \\
\hline 159.6591 & 43 \\
\hline 160.2273 & 18 \\
\hline 160.7955 & 16 \\
\hline 161.3636 & 16 \\
\hline 161.9318 & 13 \\
\hline $162.5 \quad 21$ & \\
\hline 163.0682 & 20 \\
\hline 163.6364 & 22 \\
\hline 164.2045 & 26 \\
\hline
\end{tabular}




\begin{tabular}{|c|c|}
\hline & \\
\hline 164.7727 & 27 \\
\hline 165.3409 & 31 \\
\hline 165.9091 & 45 \\
\hline 166.4773 & 58 \\
\hline 167.0455 & 64 \\
\hline 167.6136 & 87 \\
\hline 168.1818 & 92 \\
\hline $168.75 \quad 112$ & \\
\hline 169.3182 & 124 \\
\hline 169.8864 & 144 \\
\hline 170.4545 & 178 \\
\hline 171.0227 & 154 \\
\hline 171.5909 & 172 \\
\hline 172.1591 & 192 \\
\hline 172.7273 & 192 \\
\hline 173.2955 & 196 \\
\hline 173.8636 & 216 \\
\hline 174.4318 & 222 \\
\hline $175 \quad 234$ & \\
\hline 175.5682 & 236 \\
\hline 176.1364 & 262 \\
\hline 176.7045 & 287 \\
\hline 177.2727 & 372 \\
\hline 177.8409 & 546 \\
\hline 178.4091 & 699 \\
\hline 178.9773 & 821 \\
\hline 179.5455 & 682 \\
\hline 180.1136 & 544 \\
\hline 180.6818 & 477 \\
\hline $181.25 \quad 509$ & \\
\hline 181.8182 & 510 \\
\hline 182.3864 & 546 \\
\hline 182.9545 & 582 \\
\hline 183.5227 & 844 \\
\hline 184.0909 & 873 \\
\hline 184.6591 & 776 \\
\hline 185.2273 & 819 \\
\hline 185.7955 & 830 \\
\hline 186.3636 & 814 \\
\hline 186.9318 & 860 \\
\hline 187.5870 & \\
\hline 188.0682 & 993 \\
\hline 188.6364 & 901 \\
\hline 189.2045 & 886 \\
\hline 189.7727 & 946 \\
\hline 190.3409 & 911 \\
\hline 190.9091 & 1025 \\
\hline 191.4773 & 1123 \\
\hline 192.0455 & 1262 \\
\hline 192.6136 & 1424 \\
\hline 193.1818 & 1460 \\
\hline $193.75 \quad 1442$ & \\
\hline 194.3182 & 1348 \\
\hline 194.8864 & 1152 \\
\hline 195.4545 & 940 \\
\hline 196.0227 & 1256 \\
\hline 196.5909 & 1111 \\
\hline 197.1591 & 943 \\
\hline 197.7273 & 1037 \\
\hline 198.2955 & 931 \\
\hline 198.8636 & 759 \\
\hline 199.4318 & 673 \\
\hline $200 \quad 702$ & \\
\hline 200.5682 & 607 \\
\hline 201.1364 & 649 \\
\hline 201.7045 & 576 \\
\hline 202.2727 & 551 \\
\hline 202.8409 & 548 \\
\hline 203.4091 & 548 \\
\hline 203.9773 & 551 \\
\hline 204.5455 & 546 \\
\hline 205.1136 & 545 \\
\hline 205.6818 & 547 \\
\hline $206.25 \quad 556$ & \\
\hline 206.8182 & \\
\hline
\end{tabular}




\begin{tabular}{|c|c|}
\hline 207.3864 & 576 \\
\hline 207.9545 & 610 \\
\hline 208.5227 & 636 \\
\hline 209.0909 & 634 \\
\hline 209.6591 & 704 \\
\hline 210.2273 & 736 \\
\hline 210.7955 & 719 \\
\hline 211.3636 & 702 \\
\hline 211.9318 & 714 \\
\hline $212.5 \quad 691$ & \\
\hline 213.0682 & 676 \\
\hline 213.6364 & 671 \\
\hline 214.2045 & 671 \\
\hline 214.7727 & 708 \\
\hline 215.3409 & 668 \\
\hline 215.9091 & 674 \\
\hline 216.4773 & 688 \\
\hline 217.0455 & 638 \\
\hline 217.6136 & 661 \\
\hline 218.1818 & 652 \\
\hline $218.75 \quad 673$ & \\
\hline 219.3182 & 673 \\
\hline 219.8864 & 665 \\
\hline 220.4545 & 703 \\
\hline 221.0227 & 671 \\
\hline 221.5909 & 685 \\
\hline 222.1591 & 730 \\
\hline 222.7273 & 722 \\
\hline 223.2955 & 737 \\
\hline 223.8636 & 709 \\
\hline 224.4318 & 752 \\
\hline $225 \quad 767$ & \\
\hline 225.5682 & 774 \\
\hline 226.1364 & 728 \\
\hline 226.7045 & 749 \\
\hline 227.2727 & 761 \\
\hline 227.8409 & 759 \\
\hline 228.4091 & 815 \\
\hline 228.9773 & 836 \\
\hline 229.5455 & 896 \\
\hline 230.1136 & 924 \\
\hline 230.6818 & 956 \\
\hline 231.251136 & \\
\hline 231.8182 & 1187 \\
\hline 232.3864 & 1353 \\
\hline 232.9545 & 1313 \\
\hline 233.5227 & 1153 \\
\hline 234.0909 & 1111 \\
\hline 234.6591 & 1095 \\
\hline 235.2273 & 1094 \\
\hline 235.7955 & 1249 \\
\hline 236.3636 & 1334 \\
\hline 236.9318 & 1286 \\
\hline $237.5 \quad 1235$ & \\
\hline 238.0682 & 1181 \\
\hline 238.6364 & 1165 \\
\hline 239.2045 & 1196 \\
\hline 239.7727 & 1207 \\
\hline 240.3409 & 1257 \\
\hline 240.9091 & 1177 \\
\hline 241.4773 & 1237 \\
\hline 242.0455 & 1186 \\
\hline 242.6136 & 1085 \\
\hline 243.1818 & 964 \\
\hline $243.75 \quad 897$ & \\
\hline 244.3182 & 861 \\
\hline 244.8864 & 806 \\
\hline 245.4545 & 796 \\
\hline 246.0227 & 780 \\
\hline 246.5909 & 773 \\
\hline 247.1591 & 767 \\
\hline 247.7273 & 764 \\
\hline 248.2955 & 761 \\
\hline 248.8636 & 753 \\
\hline 249.4318 & 750 \\
\hline
\end{tabular}




\subsection{COSEC2.IN}

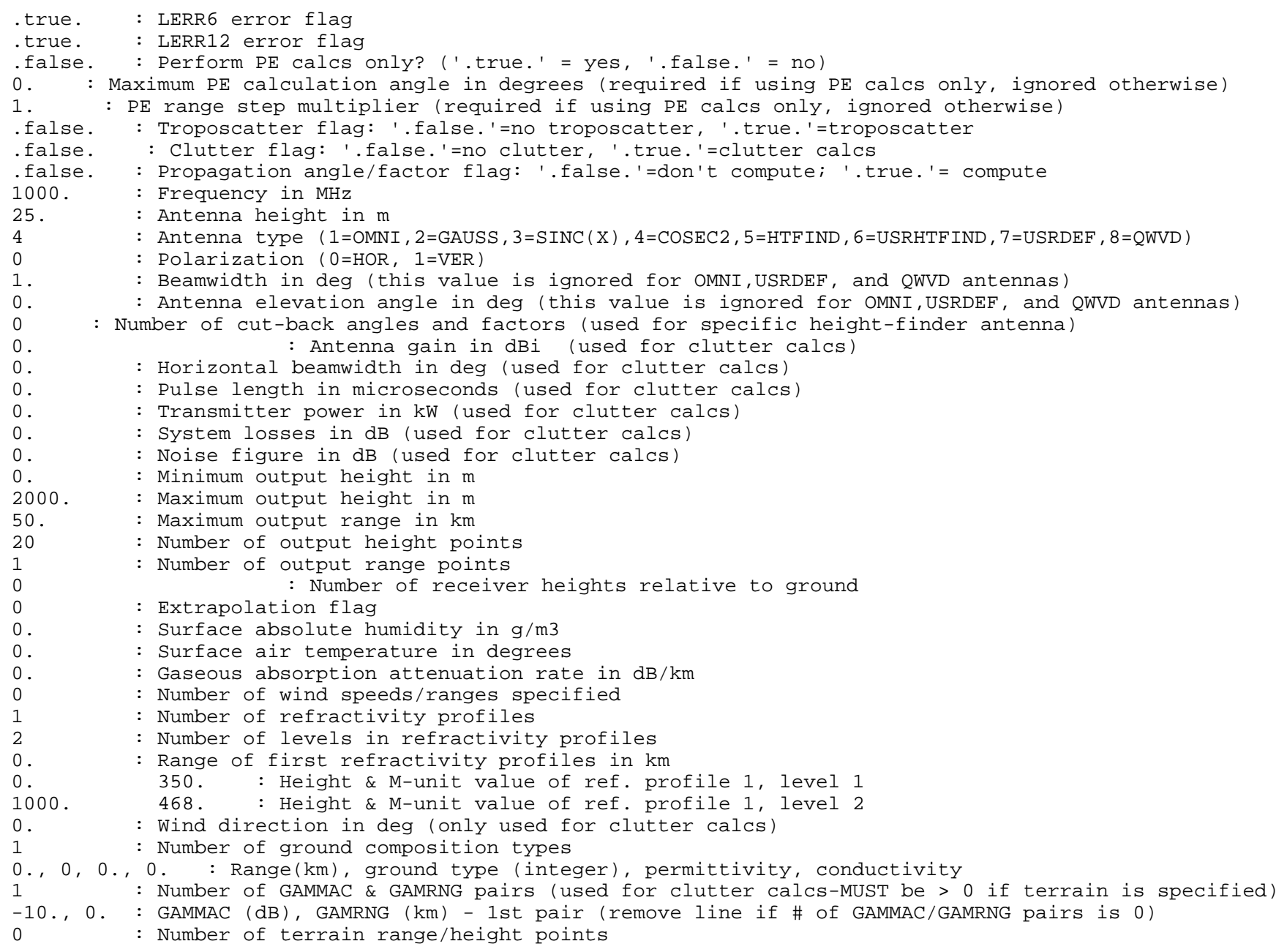

\subsection{EDUCT.IN}

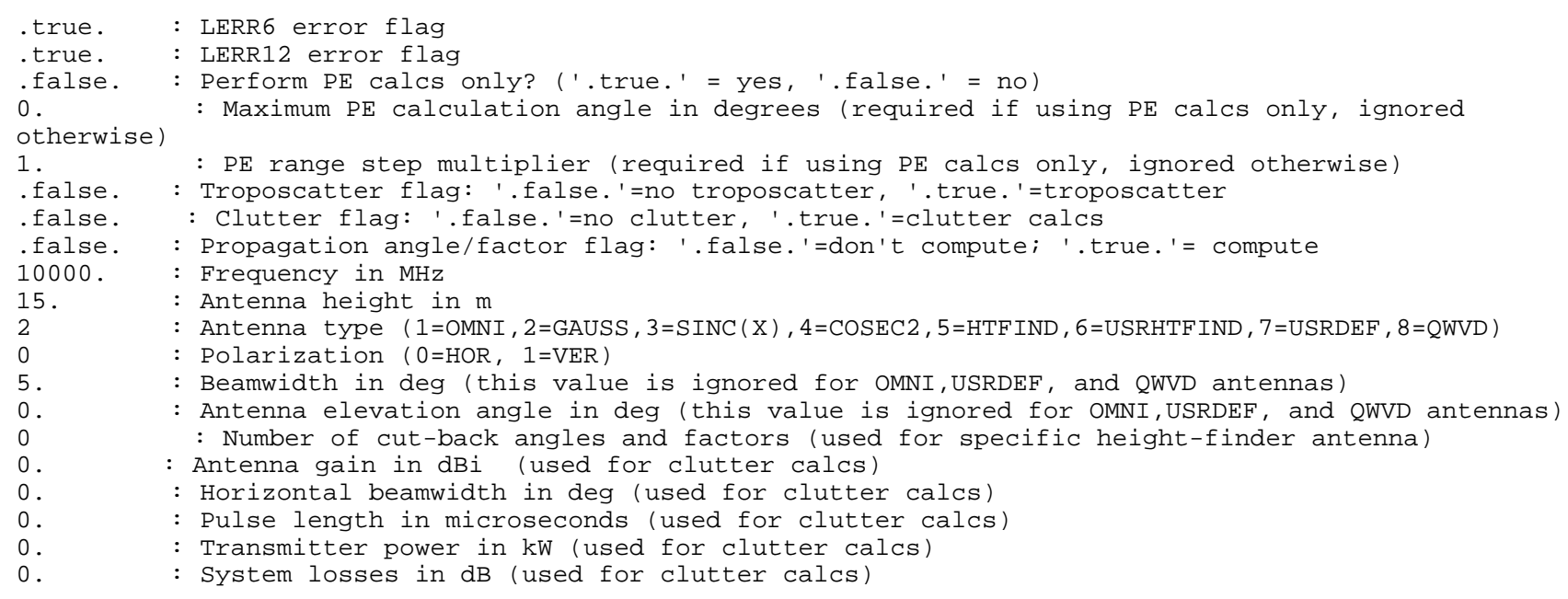




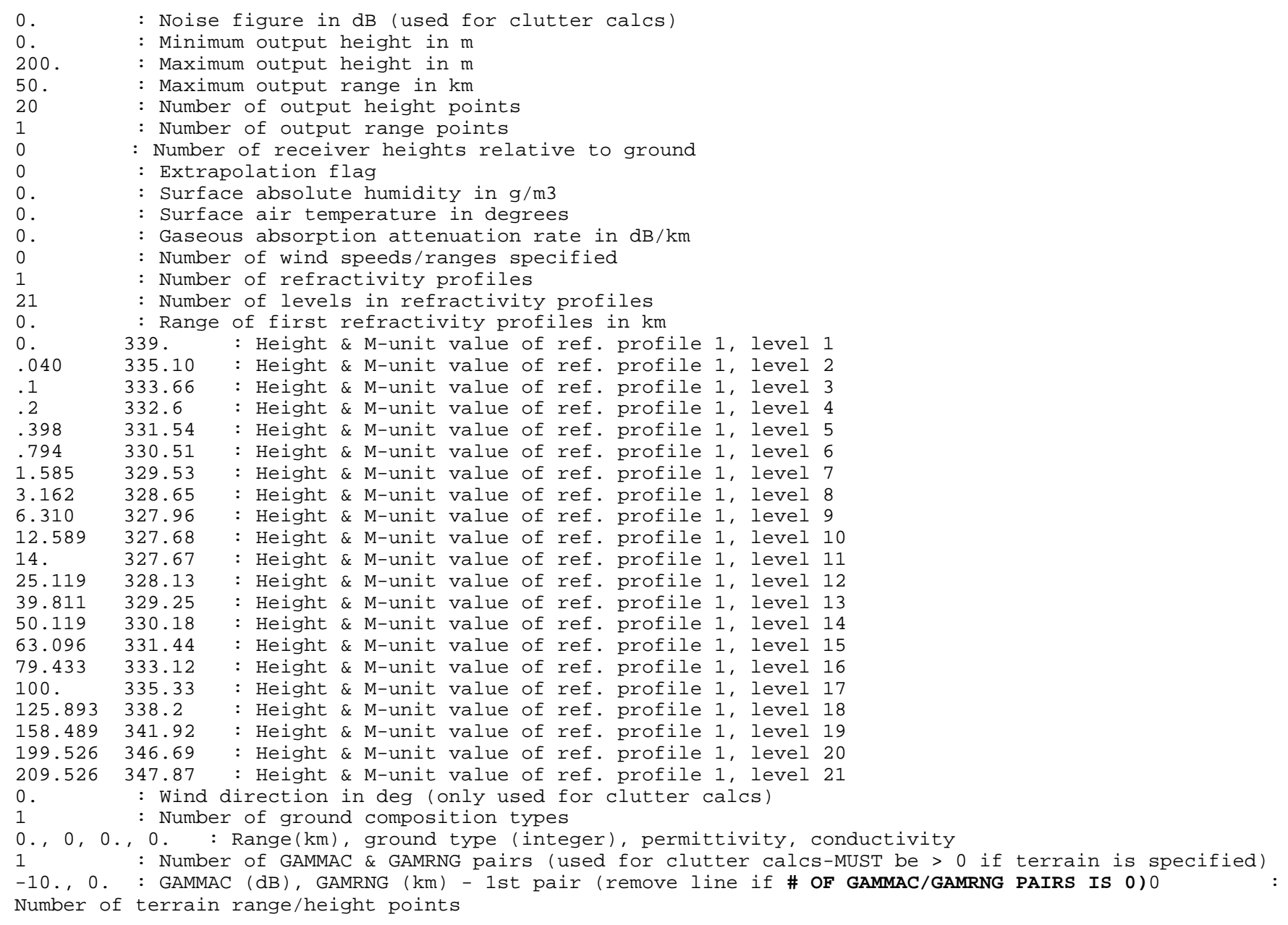

\subsection{EDUCTRF.IN}

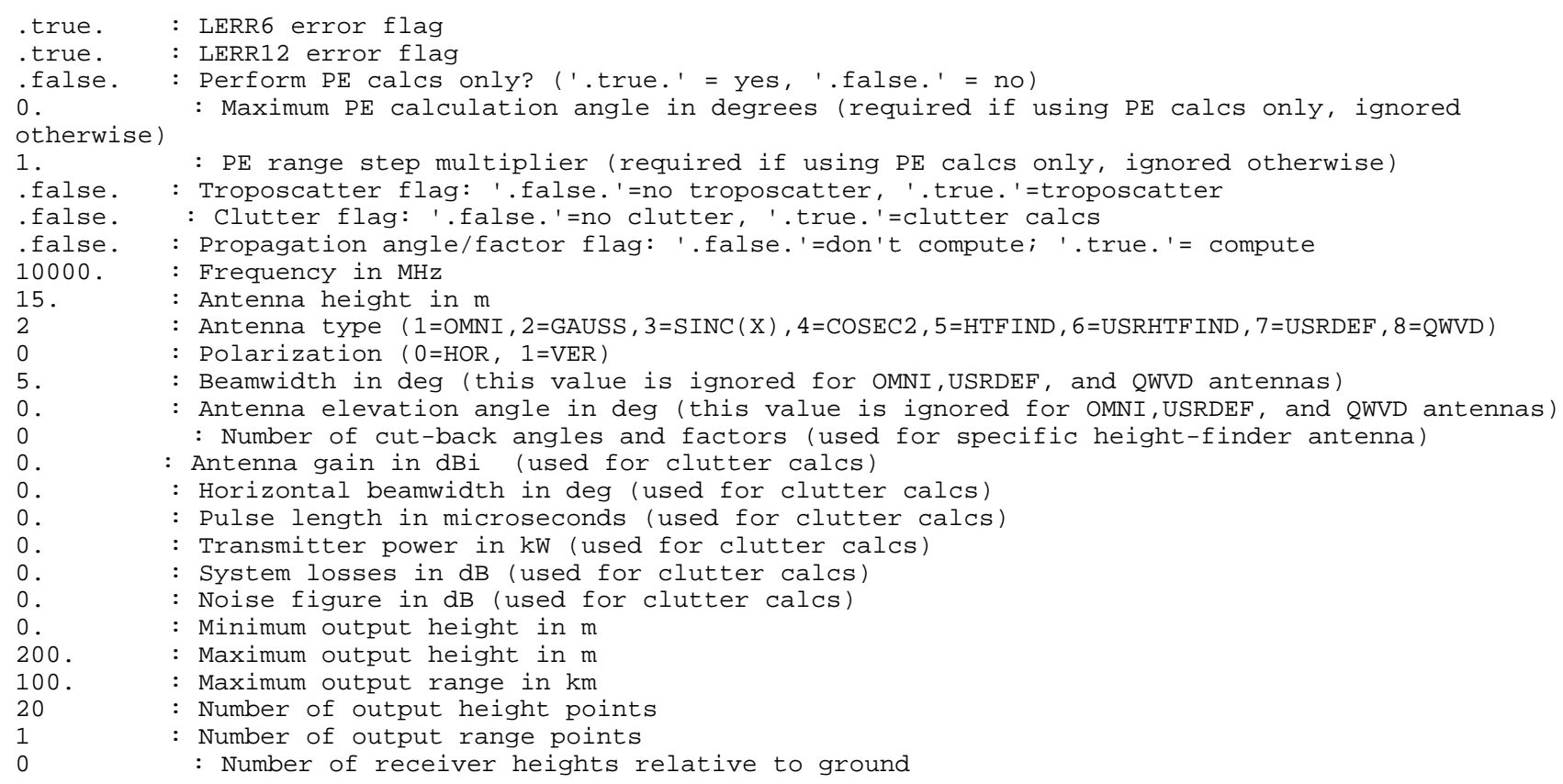




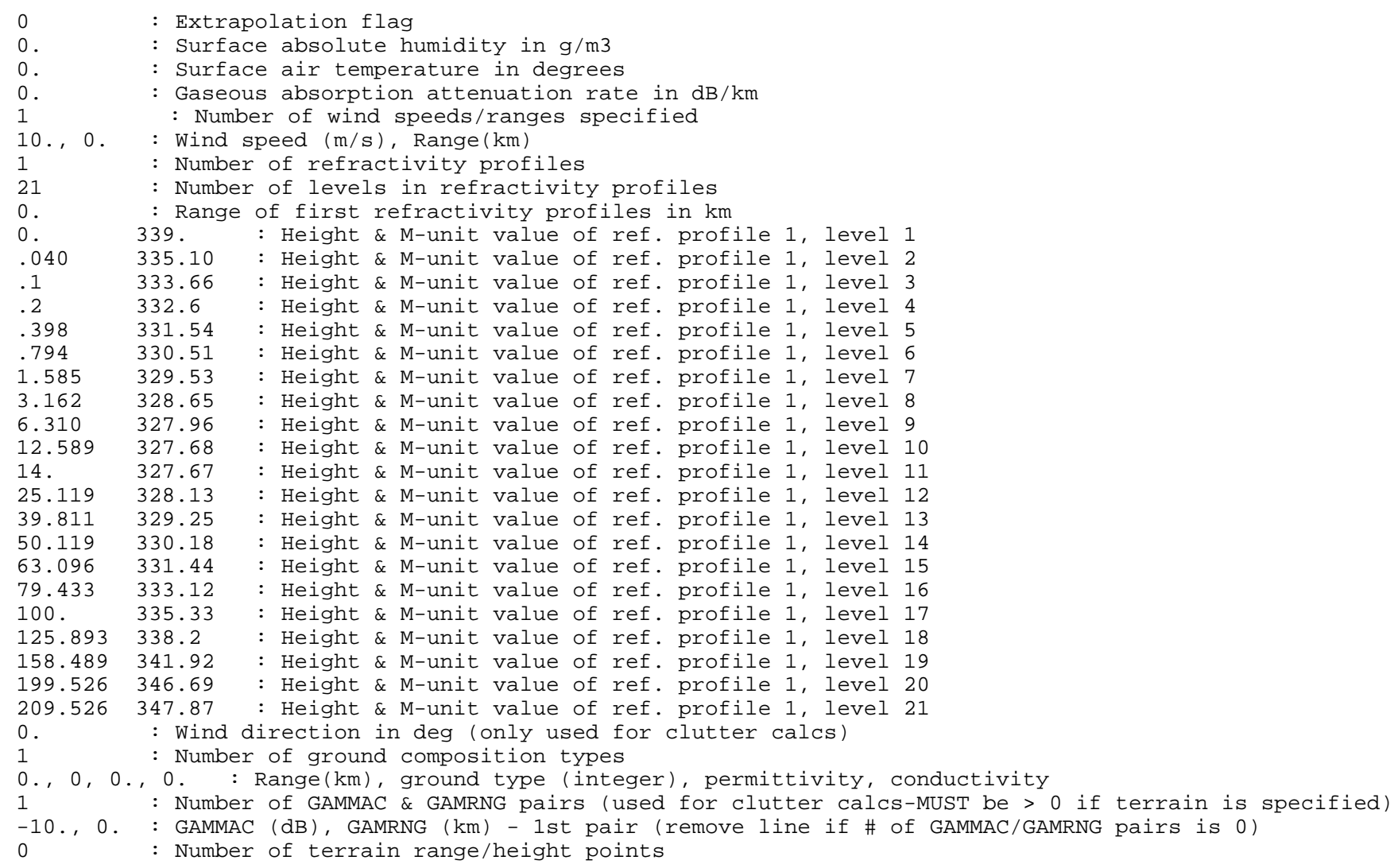

\subsection{FLTA50.IN}

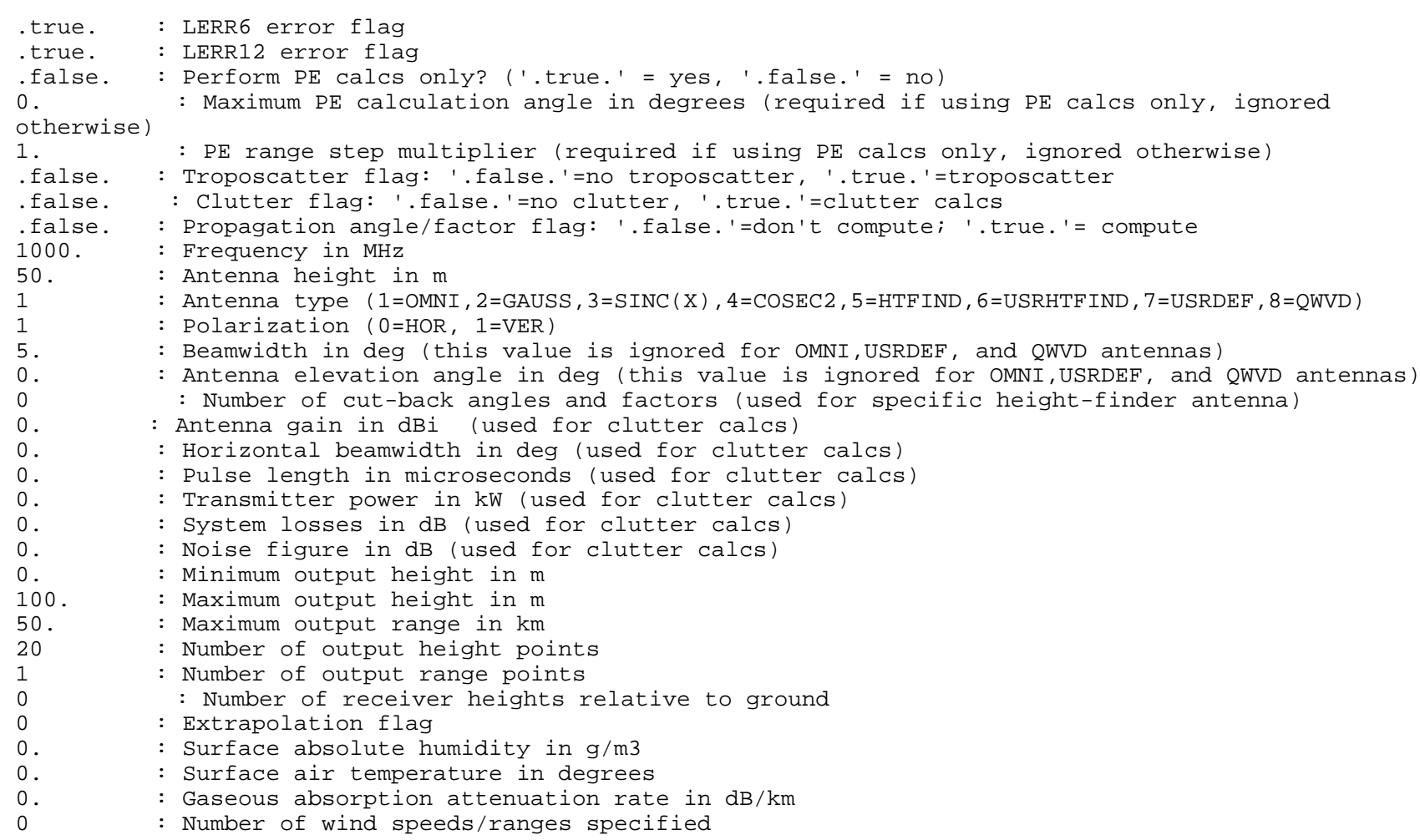




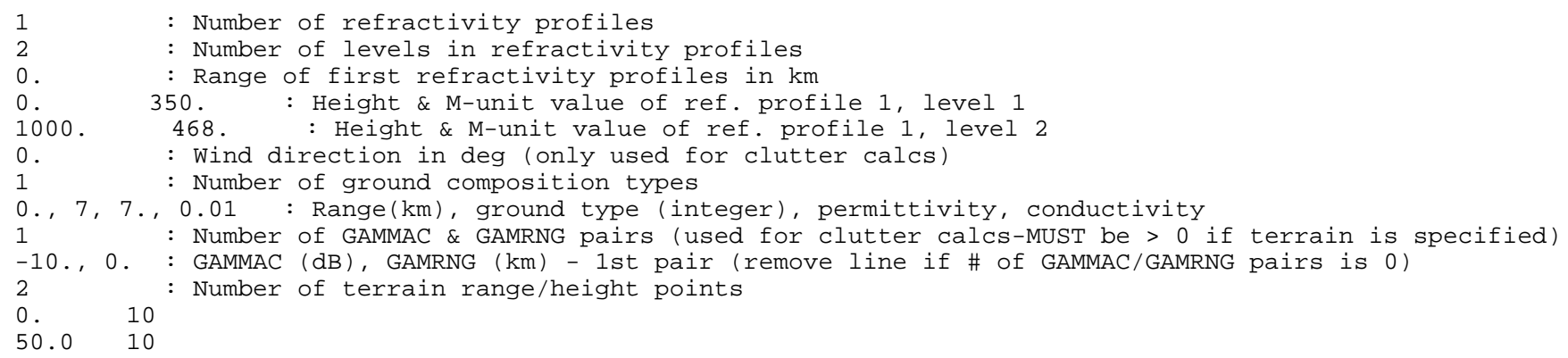

\subsection{GASABS.IN}

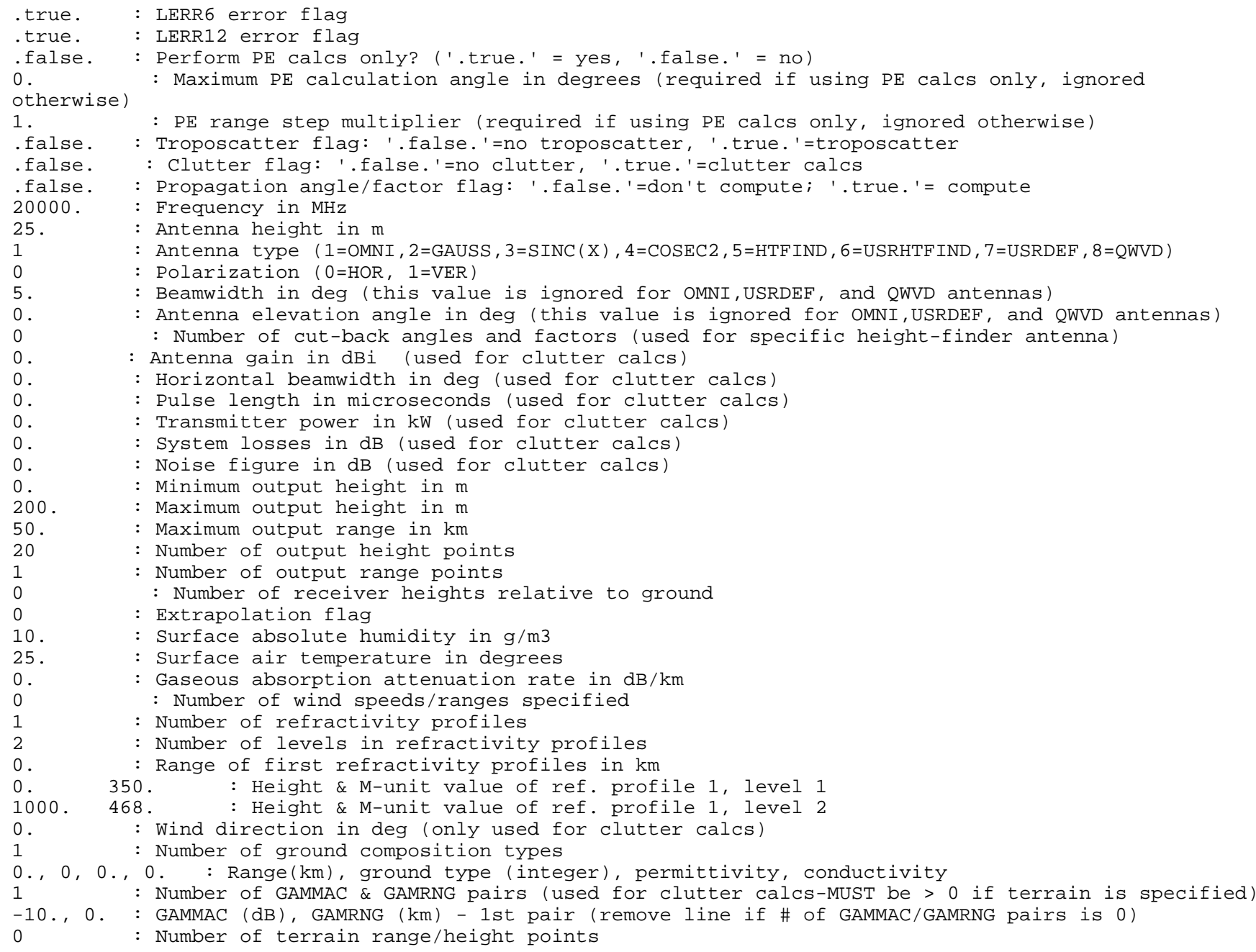

\subsection{GAUSS.IN}

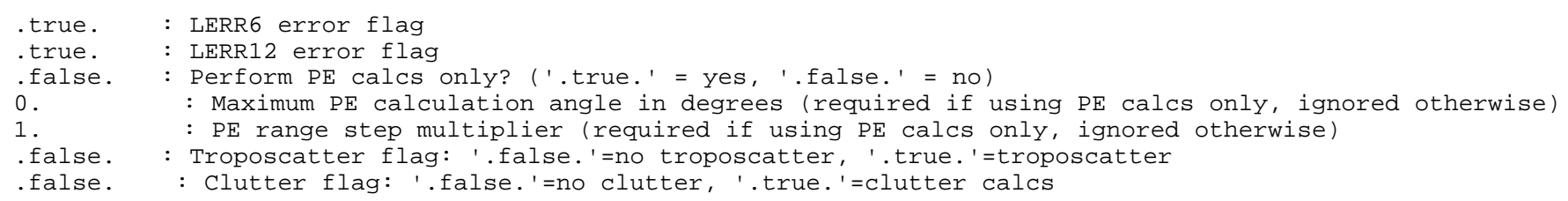




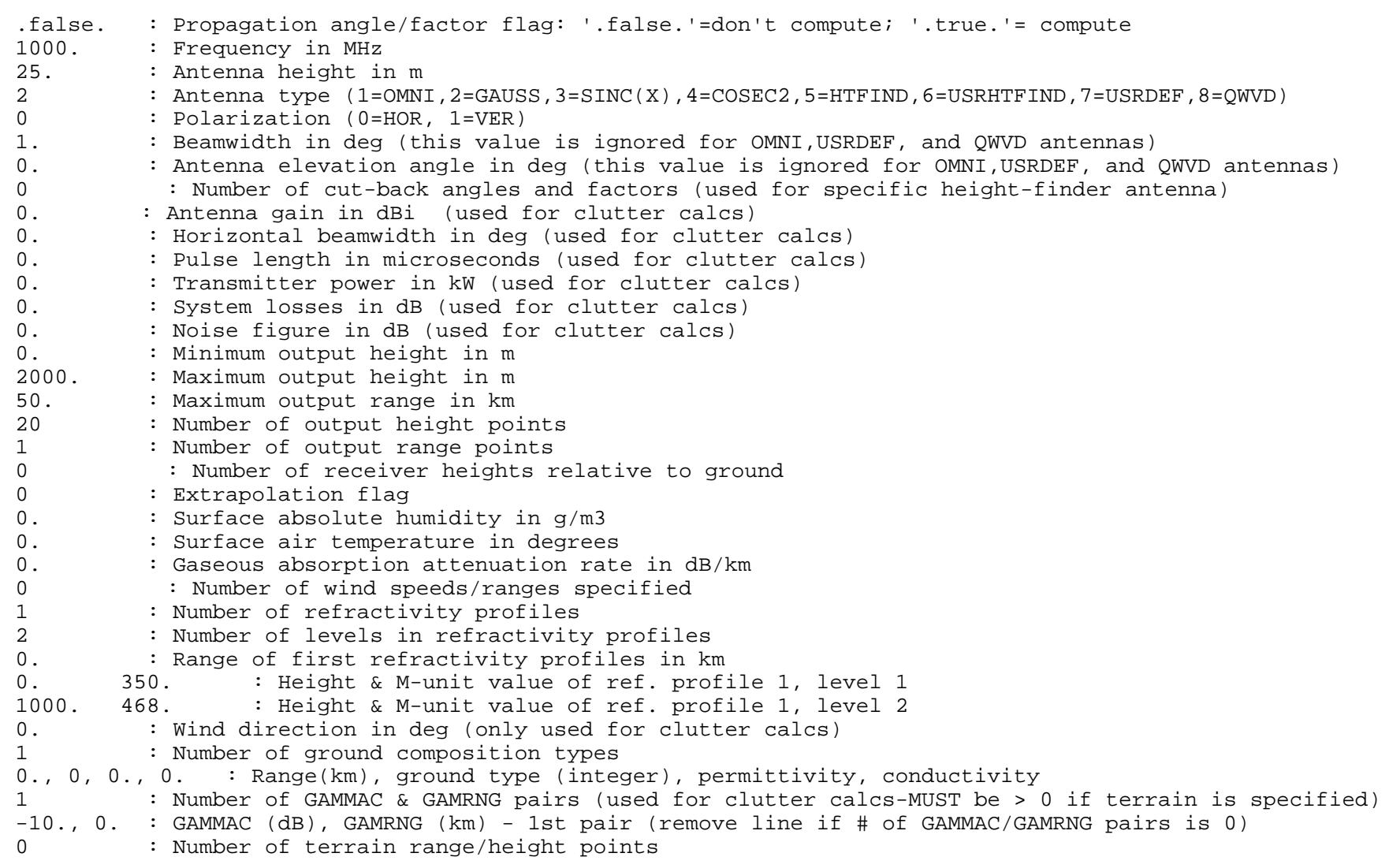

\subsection{HEIGHT_RTG}

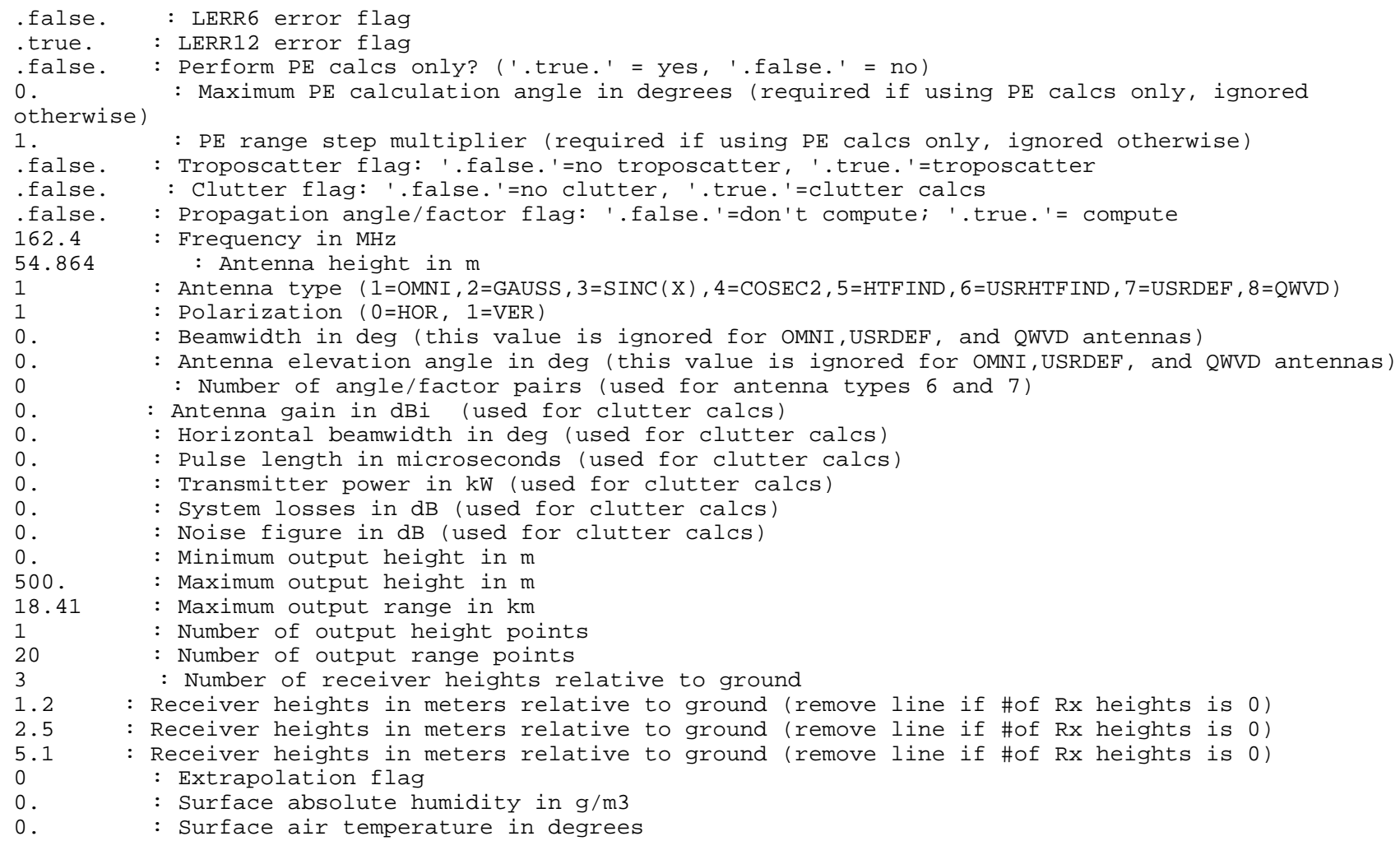




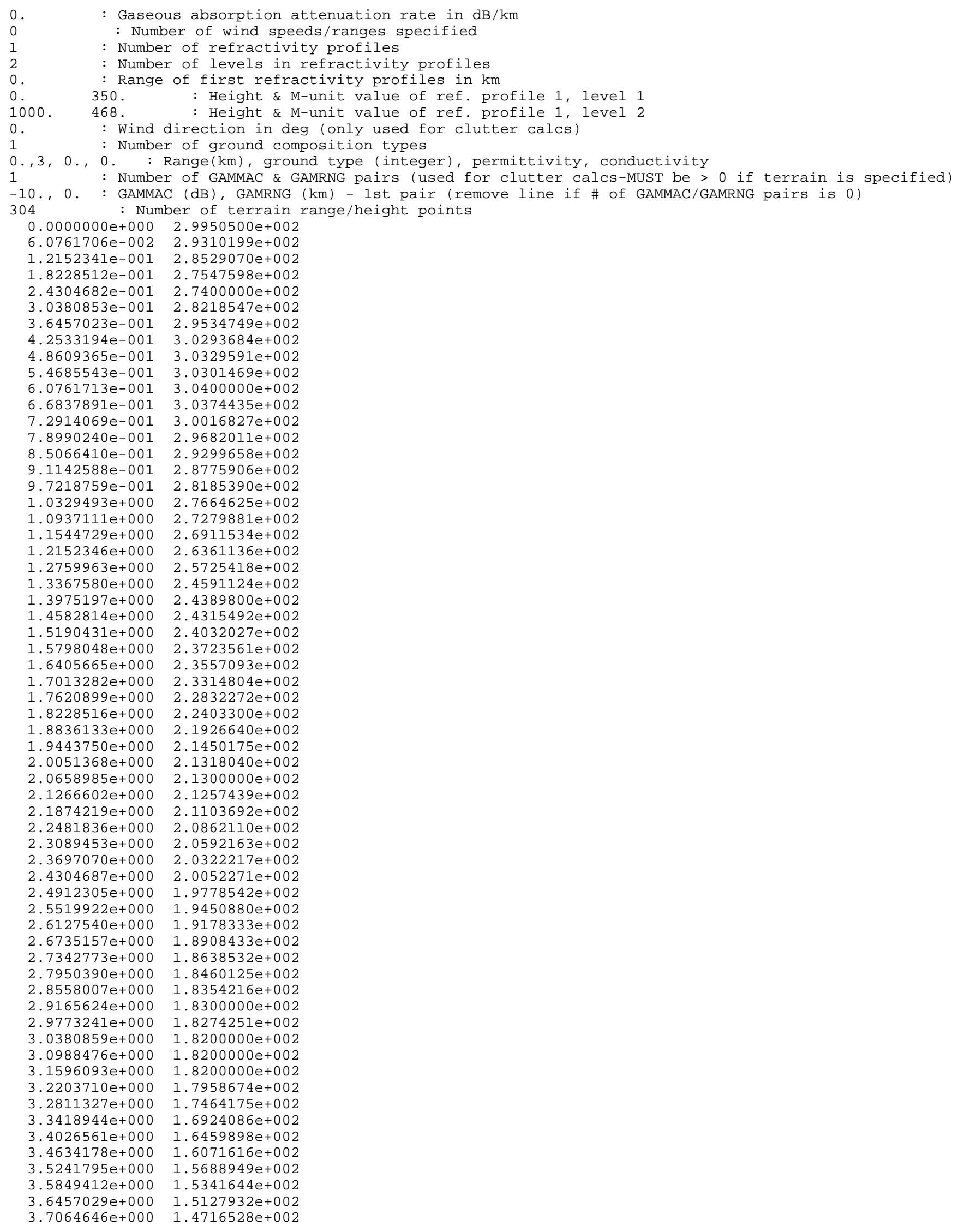


3. $7672263 \mathrm{e}+\odot \odot \odot$

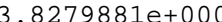

3. $8887498 \mathrm{e}+000$

3. $9495115 \mathrm{e}+000$

4. $0102732 \mathrm{e}+000$

4. $0710348 \mathrm{e}+000$

4. $1317965 \mathrm{e}+00 \%$

$4.1925582 \mathrm{e}+0 \odot \odot$

$4.2533201 \mathrm{e}+00 \odot$

$4.3140818 \mathrm{e}+0 \odot \odot$

$4.3748436 \mathrm{e}+\odot \odot \odot$

$4.4356053 \mathrm{e}+000$

4. $4963670 \mathrm{e}+000$

$4.5571287 \mathrm{e}+00 \odot$

4. $6178904 \mathrm{e}+\odot \odot \odot$

$4.6786521 \mathrm{e}+0 \odot 0$

$4.7394138 \mathrm{e}+000$

$4.8001755 \mathrm{e}+000$

$4.8609372 \mathrm{e}+000$

$4.9216989 \mathrm{e}+00 \odot$

4. $9824606 \mathrm{e}+00 \odot$

$5.0432223 \mathrm{e}+000$

5. $1039840 \mathrm{e}+000$

$5.1647457 \mathrm{e}+000$

$5.2255074 \mathrm{e}+\odot \odot \odot$

$5.2862691 \mathrm{e}+000$

$5.3470308 \mathrm{e}+000$

$5.4077925 \mathrm{e}+000$

$5.4685543 \mathrm{e}+000$

$5.5293160 \mathrm{e}+000$

$5.5900777 \mathrm{e}+000$

5. $6508394 \mathrm{e}+000$

$5.7116012 \mathrm{e}+000$

$5.7723629 \mathrm{e}+000$

$5.8331246 \mathrm{e}+000$

$5.8938865 \mathrm{e}+000$

$5.9546483 \mathrm{e}+0 \odot \odot$

$6.0154100 \mathrm{e}+000$

6. $0761717 \mathrm{e}+000$

6. $1369335 \mathrm{e}+000$

$6.1976953 \mathrm{e}+000$

$6.2584570 \mathrm{e}+000$

$6.3192187 \mathrm{e}+00 \odot$

$6.3799804 \mathrm{e}+000$

$6.4407422 \mathrm{e}+00 \mathrm{0}$

$6.5015040 \mathrm{e}+000$

$6.5622658 \mathrm{e}+00 \mathrm{0}$

6. $6230275 \mathrm{e}+000$

$6.6837892 \mathrm{e}+000$

$6.7445509 \mathrm{e}+000$

$6.8053127 \mathrm{e}+000$

$6.8660744 \mathrm{e}+000$

$6.9268362 \mathrm{e}+0 \odot \odot$

$6.9875980 \mathrm{e}+00 \odot$

$7.0483599 \mathrm{e}+000$

7. $1091217 \mathrm{e}+0 \odot \odot$

$7.1698835 \mathrm{e}+0 \odot \odot$

$7.2306452 \mathrm{e}+000$

$7.2914070 \mathrm{e}+000$

$7.3521688 \mathrm{e}+0 \odot \odot$

$7.4129305 \mathrm{e}+000$

$7.4736924 \mathrm{e}+00 \odot$

$7.5344542 \mathrm{e}+000$

$7.5952161 \mathrm{e}+0 \odot \odot$

$7.6559780 \mathrm{e}+0 \odot \odot$

$7.7167398 \mathrm{e}+0 \circ \odot$

$7.7775017 \mathrm{e}+000$

$7.8382635 \mathrm{e}+0 \odot \odot$

$7.8990254 \mathrm{e}+0 \odot \odot$

$7.9597872 \mathrm{e}+0 \odot \odot$

8. $0205491 \mathrm{e}+000$

8. $.8813109 \mathrm{e}+00 \odot$

$8.1420727 \mathrm{e}+00 \mathrm{0}$

8. $2028345 \mathrm{e}+00 \mathrm{0}$

$8.2635962 \mathrm{e}+0 \odot \odot$
$1.4389636 \mathrm{e}+0 \odot 2$

. 3923192e+002

. $3421815 \mathrm{e}+002$

1. $2808321 \mathrm{e}+002$

$2426357 \mathrm{e}+\odot \odot 2$

1. $2304306 \mathrm{e}+002$

$1.2284360 \mathrm{e}+002$

1. $2309907 \mathrm{e}+0 \odot 2$

1. $2394364 \mathrm{e}+002$

1. $2442499 \mathrm{e}+002$

1. $2352635 \mathrm{e}+002$

$1.2348088 \mathrm{e}+002$

$2320301 \mathrm{e}+002$

1. $2245950 \mathrm{e}+002$

1. $2190808 \mathrm{e}+002$

1. $2169107 \mathrm{e}+002$

1. $2153794 \mathrm{e}+002$

1. $2127808 \mathrm{e}+002$

1. $2213782 \mathrm{e}+002$

1. $2863134 \mathrm{e}+002$

$3110240 \mathrm{e}+002$

$1.3016863 \mathrm{e}+002$

. 2942360e+002

1. $2834528 \mathrm{e}+002$

1. $2642025 \mathrm{e}+002$

1. $2417204 \mathrm{e}+002$

1. $2260712 \mathrm{e}+0 \odot 2$

1. $2218517 \mathrm{e}+002$

1. $2197993 \mathrm{e}+\odot \odot 2$

$1.1954888 \mathrm{e}+0 \odot 2$

$1.1594994 \mathrm{e}+002$

1. $1235100 \mathrm{e}+002$

1. $0950149 \mathrm{e}+002$

1. $0639660 \mathrm{e}+002$

1. $0313536 \mathrm{e}+002$

1. $0015476 \mathrm{e}+002$

9. 7891421e+001

$9.6564563 \mathrm{e}+001$

9. $6000000 \mathrm{e}+001$

. $6030080 \mathrm{e}+001$

$9.6591542 \mathrm{e}+001$

. $7471706 \mathrm{e}+001$

9. $8371421 \mathrm{e}+001$

$9.9271143 e+001$

1. $0017087 \mathrm{e}+002$

1. $0107060 \mathrm{e}+002$

1. $0197034 \mathrm{e}+002$

1. $0311686 \mathrm{e}+0 \odot 2$

1. $0476984 \mathrm{e}+0 \odot 2$

1. $0571601 \mathrm{e}+002$

1. $0751559 e+002$

1. $0899628 \mathrm{e}+002$

$1.1027310 \mathrm{e}+002$

1. $1191438 \mathrm{e}+002$

$1.1371398 \mathrm{e}+002$

1. $1551360 \mathrm{e}+\odot \odot 2$

1. $1731322 \mathrm{e}+002$

$1.1911284 \mathrm{e}+002$

1. $2085786 \mathrm{e}+002$

1. $2243519 \mathrm{e}+002$

1. $2625557 \mathrm{e}+002$

1. $2986853 \mathrm{e}+002$

1. $3393133 \mathrm{e}+002$

1. $3809119 \mathrm{e}+0 \odot 2$

1. $4313355 \mathrm{e}+002$

$1.4659434 \mathrm{e}+002$

1. $4817706 \mathrm{e}+002$

1. $4988962 \mathrm{e}+002$

1. $5039665 \mathrm{e}+002$

1. $5057576 \mathrm{e}+0 \odot 2$

1. $4989814 \mathrm{e}+002$

1. $4648570 \mathrm{e}+002$

1. $4835292 \mathrm{e}+002$

$1.5058030 \mathrm{e}+002$

1. $5133977 \mathrm{e}+\odot \odot 2$ 
$8.3243579 \mathrm{e}+000$ $8.3851197 \mathrm{e}+\Theta \odot \Theta$

$8.4458815 \mathrm{e}+000$

$8.5066434 \mathrm{e}+000$

$8.5674052 \mathrm{e}+000$

$8.6281670 \mathrm{e}+000$

$8.6889288 \mathrm{e}+000$

$8.7496905 \mathrm{e}+00 \mathrm{0}$

$8.8104523 \mathrm{e}+000$

$8.8712141 \mathrm{e}+00 \mathrm{0}$

$8.9319758 \mathrm{e}+000$

$8.9927375 \mathrm{e}+000$

9. $0534993 e+000$

$9.1142610 \mathrm{e}+00 \odot$

$9.1750227 \mathrm{e}+\Theta \odot \odot$

$9.2357844 \mathrm{e}+000$

$9.2965462 \mathrm{e}+00 \mathrm{O}$

$9.3573079 \mathrm{e}+000$

$9.4180696 \mathrm{e}+000$

$9.4788314 \mathrm{e}+000$

$9.5395931 \mathrm{e}+000$

$9.6003548 \mathrm{e}+000$

$9.6611165 \mathrm{e}+000$

$9.7218782 \mathrm{e}+00 \Theta$

$9.7826400 \mathrm{e}+\odot \odot \odot$

$9.8434017 \mathrm{e}+000$

$9.9041634 \mathrm{e}+000$

$9.9649251 \mathrm{e}+000$

1. $0025687 \mathrm{e}+001$

$1.0086448 \mathrm{e}+001$

1. $0147210 \mathrm{e}+001$

1. $0207972 \mathrm{e}+001$

$1.0268734 \mathrm{e}+001$

1. $0329495 \mathrm{e}+001$

$1.0390257 \mathrm{e}+001$

$1.0451019 \mathrm{e}+001$

1. $0511780 \mathrm{e}+001$

$1.0572542 \mathrm{e}+001$

1. $0633304 \mathrm{e}+001$

1. $0694065 \mathrm{e}+001$

$1.0754827 \mathrm{e}+001$

1. $0815589 \mathrm{e}+001$

1. $0876351 \mathrm{e}+001$

1. $0937112 \mathrm{e}+001$

1. $0997874 \mathrm{e}+001$

$1.1058636 \mathrm{e}+001$

1. $1119397 \mathrm{e}+001$

1. $1180159 \mathrm{e}+001$

1. $1240921 \mathrm{e}+001$

$1.1301683 \mathrm{e}+001$

1. $1362444 \mathrm{e}+001$

1. $1423206 \mathrm{e}+001$

$1.1483968 \mathrm{e}+001$

$1.1544729 \mathrm{e}+001$

1. $1605491 \mathrm{e}+001$

1. $1666253 \mathrm{e}+001$

$1.1727015 \mathrm{e}+001$

$1.1787776 \mathrm{e}+001$

1. $1848538 \mathrm{e}+001$

1. $1909300 \mathrm{e}+001$

$1.1970061 \mathrm{e}+001$

1. $2030823 \mathrm{e}+001$

1. $2091585 \mathrm{e}+001$

1. 2152347e+001

1. $2213108 \mathrm{e}+001$

1. $2273870 \mathrm{e}+001$

1. $2334632 \mathrm{e}+001$

1. $2395393 \mathrm{e}+001$

1. $2456155 \mathrm{e}+001$

1. $2516917 \mathrm{e}+0 \odot 1$

1. $2577678 \mathrm{e}+001$

1. $2638440 \mathrm{e}+001$

1. $2699202 \mathrm{e}+001$

1. $2759964 \mathrm{e}+001$

1. $2820725 \mathrm{e}+0 \odot 1$
$1.5134179 \mathrm{e}+002$

1. 5134395e+002

. $5134624 \mathrm{e}+002$

$1.5134867 \mathrm{e}+002$

$5135123 \mathrm{e}+\odot \odot 2$

1. $5135393 \mathrm{e}+002$

$1.4873290 \mathrm{e}+002$

4685104e+0०2

1. $4540519 \mathrm{e}+002$

. $4425930 \mathrm{e}+002$

$1.4121540 \mathrm{e}+002$

$1.3740409 \mathrm{e}+002$

$3514239 \mathrm{e}+002$

1. $3187107 \mathrm{e}+002$

$1.3348579 \mathrm{e}+002$

1. 3596048e+0०2

1. $3895951 \mathrm{e}+002$

$1.4199633 \mathrm{e}+002$

1. $4527156 \mathrm{e}+002$

1. $4951941 \mathrm{e}+002$

$1.5200000 \mathrm{e}+002$

1. $5200000 \mathrm{e}+002$

1. $5214912 \mathrm{e}+002$

1. $5276486 \mathrm{e}+002$

$1.5369799 \mathrm{e}+002$

$1.5539260 \mathrm{e}+002$

1. $5802300 \mathrm{e}+002$

1. $6164928 \mathrm{e}+002$

$1.6508088 \mathrm{e}+002$

1. $6942093 \mathrm{e}+002$

$1.7460048 \mathrm{e}+002$

$1.7792114 \mathrm{e}+0 \circ 2$

1. $7792418 \mathrm{e}+002$

$1.7782666 \mathrm{e}+002$

1. $7720373 \mathrm{e}+002$

1. $7754017 \mathrm{e}+\odot \odot 2$

1. $7787615 \mathrm{e}+0 \odot 2$

1. $7682012 \mathrm{e}+002$

$1.7688705 \mathrm{e}+002$

$1.7788968 \mathrm{e}+002$

1. $7971680 \mathrm{e}+\odot \odot 2$

$1.8311357 \mathrm{e}+002$

$1.8702959 \mathrm{e}+002$

$1.8758687 \mathrm{e}+\odot \odot 2$

$1.8618577 \mathrm{e}+002$

1. $8496084 \mathrm{e}+002$

$1.8154109 \mathrm{e}+002$

1. $7687416 \mathrm{e}+\odot \odot 2$

1. $7082137 \mathrm{e}+0 \odot 2$

$1.6622341 \mathrm{e}+002$

1. $6240798 \mathrm{e}+002$

1. $5920693 \mathrm{e}+0 \odot 2$

1. $5747477 \mathrm{e}+002$

1. $5420958 \mathrm{e}+0 \odot 2$

1. $5214325 \mathrm{e}+002$

1. $5615842 \mathrm{e}+\odot \odot 2$

1. $5805664 \mathrm{e}+0 \odot 2$

$1.5844509 \mathrm{e}+002$

$1.5887424 \mathrm{e}+002$

1. $5964437 \mathrm{e}+002$

$1.5940992 \mathrm{e}+002$

$1.5826843 \mathrm{e}+002$

1. $5718705 \mathrm{e}+002$

1. $5500000 \mathrm{e}+002$

1. $5500000 \mathrm{e}+002$

1. $5500000 \mathrm{e}+002$

1. $5500000 \mathrm{e}+002$

1. $5500000 \mathrm{e}+002$

$1.5500000 \mathrm{e}+002$

1. $5500000 \mathrm{e}+002$

1. $5500000 \mathrm{e}+002$

1. $5500000 \mathrm{e}+002$

1. $5500000 \mathrm{e}+002$

1. $5500000 \mathrm{e}+0 \odot 2$

1. $5500000 \mathrm{e}+002$ 
$1.2881487 \mathrm{e}+0 \odot 1 \quad 1.550000 \odot \mathrm{e}+\odot \odot 2$

$1.2942249 \mathrm{e}+001 \quad 1.5500000 \mathrm{e}+002$

$1.3003010 \mathrm{e}+\odot \odot 1 \quad 1.550000 \odot \mathrm{e}+\odot \odot 2$

$1.3063772 \mathrm{e}+001 \quad 1.5500000 \mathrm{e}+002$

$1.3124534 \mathrm{e}+0 \odot 1 \quad 1.5500 \odot \odot \odot \mathrm{e}+\odot \odot 2$

$1.3185295 \mathrm{e}+001 \quad 1.5500000 \mathrm{e}+0 \odot 2$

$1.3246057 \mathrm{e}+001 \quad 1.550000 \odot \mathrm{e}+\odot \odot 2$

$1.3306819 \mathrm{e}+001 \quad 1.5500000 \mathrm{e}+002$

$1.3367581 \mathrm{e}+001 \quad 1.5500000 \mathrm{e}+0 \odot 2$

$1.3428342 \mathrm{e}+001 \quad 1.5500000 \mathrm{e}+002$

$1.3489104 \mathrm{e}+001 \quad 1.5500000 \mathrm{e}+002$

$1.3549866 \mathrm{e}+0 \odot 1 \quad 1.55000 \odot \odot \mathrm{e}+\odot \odot 2$

$1.3610627 \mathrm{e}+001 \quad 1.5500000 \mathrm{e}+002$

$\begin{array}{lll}1.3671389 \mathrm{e}+\odot \odot 1 & 1.55000 \odot \odot \mathrm{e}+\odot \odot 2\end{array}$

$1.3732151 \mathrm{e}+001 \quad 1.5500000 \mathrm{e}+0 \odot 2$

$1.3792913 \mathrm{e}+001 \quad 1.550000 \odot \mathrm{e}+\odot \odot 2$

$1.3853674 \mathrm{e}+001 \quad 1.5500000 \mathrm{e}+0 \odot 2$

$1.3914436 \mathrm{e}+001 \quad 1.5500000 \mathrm{e}+002$

$1.3975198 \mathrm{e}+\odot \odot 1 \quad 1.5500 \odot \odot \odot \mathrm{e}+\odot \odot 2$

$1.4035960 \mathrm{e}+001 \quad 1.5500000 \mathrm{e}+002$

$1.4096721 \mathrm{e}+001 \quad 1.5500000 \mathrm{e}+002$

$1.4157483 \mathrm{e}+001 \quad 1.5500000 \mathrm{e}+002$

$1.4218245 \mathrm{e}+001 \quad 1.5500000 \mathrm{e}+002$

$1.4279007 \mathrm{e}+001 \quad 1.5500000 \mathrm{e}+002$

$1.4339768 \mathrm{e}+\odot \odot 1 \quad 1.5500 \odot \odot \odot \mathrm{e}+\odot \odot 2$

$1.4400530 \mathrm{e}+001 \quad 1.5500000 \mathrm{e}+002$

$1.4461292 \mathrm{e}+001 \quad 1.5510919 \mathrm{e}+0 \odot 2$

$1.4522054 \mathrm{e}+0 \odot 1 \quad 1.5533661 \mathrm{e}+\odot \odot 2$

$1.4582815 \mathrm{e}+001 \quad 1.5533592 \mathrm{e}+002$

$1.4643577 \mathrm{e}+0 \odot 1 \quad 1.5532745 \mathrm{e}+\odot \odot 2$

$1.4704339 \mathrm{e}+001 \quad 1.5502576 \mathrm{e}+002$

$1.4765100 \mathrm{e}+001 \quad 1.5500000 \mathrm{e}+002$

$1.4825862 \mathrm{e}+001 \quad 1.5500000 \mathrm{e}+002$

$1.4886624 \mathrm{e}+001 \quad 1.5500000 \mathrm{e}+002$

$1.4947385 \mathrm{e}+001 \quad 1.5500000 \mathrm{e}+\odot \odot 2$

$1.5008147 \mathrm{e}+001 \quad 1.5500000 \mathrm{e}+002$

$1.5068909 \mathrm{e}+001 \quad 1.5500000 \mathrm{e}+002$

$1.5129671 \mathrm{e}+001 \quad 1.5500000 \mathrm{e}+002$

$1.5190432 \mathrm{e}+001 \quad 1.5552616 \mathrm{e}+002$

$1.5251194 \mathrm{e}+001 \quad 1.5942040 \mathrm{e}+002$

$1.5311956 \mathrm{e}+0 \odot 1 \quad 1.6331327 \mathrm{e}+\odot \odot 2$

$1.5372718 \mathrm{e}+001 \quad 1.6666884 \mathrm{e}+002$

$1.5433479 \mathrm{e}+001 \quad 1.6948810 \mathrm{e}+\odot \odot 2$

$1.5494241 \mathrm{e}+001 \quad 1.7238837 \mathrm{e}+002$

$1.5555003 \mathrm{e}+001 \quad 1.7468900 \mathrm{e}+002$

$1.5615765 \mathrm{e}+001 \quad 1.7677150 \mathrm{e}+002$

$1.5676526 \mathrm{e}+001 \quad 1.7864255 \mathrm{e}+002$

$1.5737288 \mathrm{e}+0 \odot 1 \quad 1.7969076 \mathrm{e}+\odot \odot 2$

$1.5798050 \mathrm{e}+0 \odot 1 \quad 1.8111975 \mathrm{e}+\odot \odot 2$

$1.5858812 \mathrm{e}+001 \quad 1.8200000 \mathrm{e}+002$

$1.5919573 \mathrm{e}+001 \quad 1.8200000 \mathrm{e}+002$

$1.5980335 \mathrm{e}+001 \quad 1.8200000 \mathrm{e}+002$

$1.6041097 \mathrm{e}+001 \quad 1.8200000 \mathrm{e}+002$

$1.6101859 \mathrm{e}+001 \quad 1.8200000 \mathrm{e}+002$

$1.6162620 \mathrm{e}+001 \quad 1.8200000 \mathrm{e}+002$

$1.6223382 \mathrm{e}+001 \quad 1.8200000 \mathrm{e}+002$

$1.6284144 \mathrm{e}+001 \quad 1.8200000 \mathrm{e}+002$

$1.6344906 \mathrm{e}+001 \quad 1.8200000 \mathrm{e}+002$

$1.6405668 \mathrm{e}+001 \quad 1.8200000 \mathrm{e}+002$

$1.6466430 \mathrm{e}+001 \quad 1.8200000 \mathrm{e}+002$

$1.6527191 \mathrm{e}+001 \quad 1.8200000 \mathrm{e}+002$

$1.6587953 \mathrm{e}+001 \quad 1.8200000 \mathrm{e}+002$

$1.6648715 \mathrm{e}+001 \quad 1.8200000 \mathrm{e}+002$

$1.6709476 \mathrm{e}+001 \quad 1.8200000 \mathrm{e}+0 \odot 2$

$1.6770238 \mathrm{e}+0 \odot 1 \quad 1.8200 \odot \odot \odot \mathrm{e}+\odot \odot 2$

$1.6831000 \mathrm{e}+001 \quad 1.8200000 \mathrm{e}+002$

$1.6891762 \mathrm{e}+001 \quad 1.8200000 \mathrm{e}+002$

$1.6952524 \mathrm{e}+\odot \odot 1 \quad 1.82000 \odot \odot \mathrm{e}+\odot \odot 2$

$1.7013286 \mathrm{e}+001 \quad 1.8200000 \mathrm{e}+002$

$1.7074047 \mathrm{e}+001 \quad 1.820000 \odot \mathrm{e}+\odot \odot 2$

$1.7134809 \mathrm{e}+001 \quad 1.8200000 \mathrm{e}+002$

$1.7195571 \mathrm{e}+001 \quad 1.8200000 \mathrm{e}+002$

$1.7256332 \mathrm{e}+001 \quad 1.8200000 \mathrm{e}+002$

$1.7317094 \mathrm{e}+001 \quad 1.8655453 \mathrm{e}+\odot \odot 2$

$1.7377856 \mathrm{e}+0 \odot 1 \quad 1.9146493 \mathrm{e}+0 \odot 2$ 

$1.7438618 \mathrm{e}+0 \odot 1$
1. $9382147 \mathrm{e}+\odot \odot 2$
$1.7499379 \mathrm{e}+001$
$1.9409382 \mathrm{e}+\odot \odot 2$
$1.7560141 \mathrm{e}+001$
1. $9341049 \mathrm{e}+0 \odot 2$
$1.7620903 \mathrm{e}+001$
$1.9333171 \mathrm{e}+002$
$1.7681665 \mathrm{e}+001$
$1.9270308 \mathrm{e}+002$
$1.7742426 \mathrm{e}+001$
1. $9244155 \mathrm{e}+002$
$1.7803188 \mathrm{e}+001$
1. $9184128 \mathrm{e}+002$
1. $9150346 \mathrm{e}+002$
1. $7924712 \mathrm{e}+0 \odot 1$
1. $9142251 \mathrm{e}+\odot \odot 2$
$1.7985473 \mathrm{e}+0 \odot 1 \quad 1.9142456 \mathrm{e}+\odot \odot 2$
$1.8046235 \mathrm{e}+0 \odot 1 \quad 1.9101353 \mathrm{e}+\odot \odot 2$
$1.8106997 \mathrm{e}+001 \quad 1.9011578 \mathrm{e}+002$
$1.8167759 \mathrm{e}+001 \quad 1.8943076 \mathrm{e}+002$
$1.8228520 \mathrm{e}+\odot \odot 1 \quad 1.8954540 \mathrm{e}+\odot \odot 2$
$1.8289282 \mathrm{e}+001 \quad 1.9045081 \mathrm{e}+002$
$1.8350044 \mathrm{e}+001 \quad 1.9160600 \mathrm{e}+0 \odot 2$
$1.8410806 \mathrm{e}+001 \quad 1.9311592 \mathrm{e}+002$

\subsection{HF10TER}

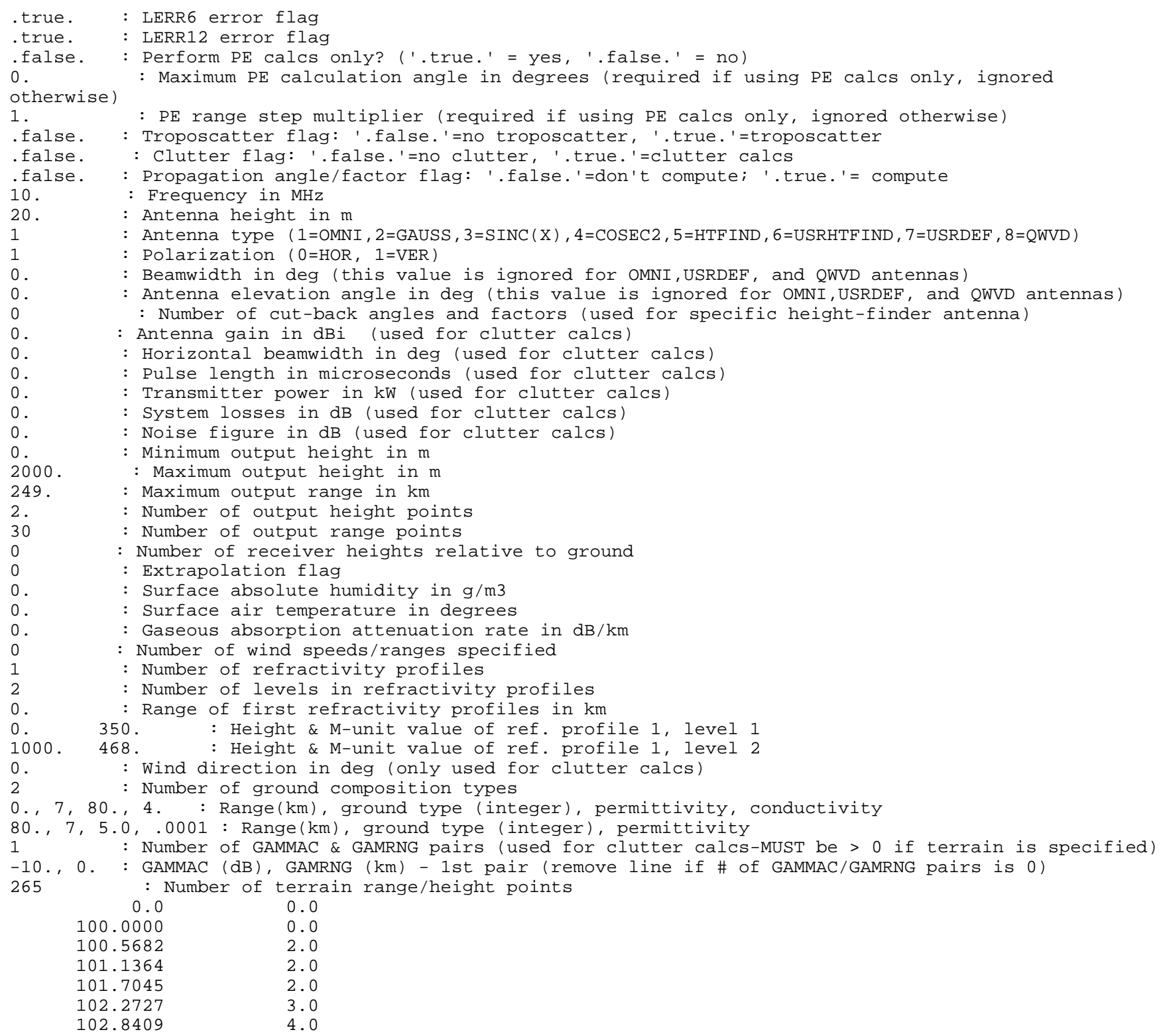




\begin{tabular}{|c|c|}
\hline 103.4091 & 5.0 \\
\hline 103.9773 & 4.0 \\
\hline 104.5455 & 7.0 \\
\hline 105.1136 & 6.0 \\
\hline 105.6818 & 9.0 \\
\hline 106.2500 & 12.0 \\
\hline 106.8182 & 9.0 \\
\hline 107.3864 & 9.0 \\
\hline 107.9545 & 8.0 \\
\hline 108.5227 & 10.0 \\
\hline 109.0909 & 19.0 \\
\hline 109.6591 & 21.0 \\
\hline 110.2273 & 27.0 \\
\hline 110.7955 & 32.0 \\
\hline 111.3636 & 32.0 \\
\hline 111.9318 & 47.0 \\
\hline 112.5000 & 43.0 \\
\hline 113.0682 & 58.0 \\
\hline 113.6364 & 82.0 \\
\hline 114.2045 & 75.0 \\
\hline 114.7727 & 96.0 \\
\hline 115.3409 & 63.0 \\
\hline 115.9091 & 100.0 \\
\hline 116.4773 & 123.0 \\
\hline 117.0455 & 98.0 \\
\hline 117.6136 & 95.0 \\
\hline 118.1818 & 100.0 \\
\hline 118.7500 & 106.0 \\
\hline 119.3182 & 100.0 \\
\hline 119.8864 & 108.0 \\
\hline 120.4545 & 89.0 \\
\hline 121.0227 & 90.0 \\
\hline 121.5909 & 95.0 \\
\hline 122.1591 & 89.0 \\
\hline 122.7273 & 107.0 \\
\hline 123.2955 & 97.0 \\
\hline 123.8636 & 108.0 \\
\hline 124.4318 & 87.0 \\
\hline 125.0000 & 76.0 \\
\hline 125.5682 & 73.0 \\
\hline 126.1364 & 88.0 \\
\hline 126.7045 & 86.0 \\
\hline 127.2727 & 101.0 \\
\hline 127.8409 & 101.0 \\
\hline 128.4091 & 92.0 \\
\hline 128.9773 & 65.0 \\
\hline 129.5455 & 62.0 \\
\hline 130.1136 & 47.0 \\
\hline 130.6818 & 59.0 \\
\hline 131.2500 & 44.0 \\
\hline 131.8182 & 33.0 \\
\hline 132.3864 & 21.0 \\
\hline 132.9545 & 20.0 \\
\hline 133.5227 & 21.0 \\
\hline 134.0909 & 11.0 \\
\hline 134.6591 & 7.0 \\
\hline 135.2273 & 7.0 \\
\hline 135.7955 & 4.0 \\
\hline 136.3636 & 12.0 \\
\hline 136.9318 & 9.0 \\
\hline 137.5000 & 6.0 \\
\hline 138.0682 & 5.0 \\
\hline 138.6364 & 7.0 \\
\hline 139.2045 & 5.0 \\
\hline 139.7727 & 8.0 \\
\hline 140.3409 & 14.0 \\
\hline 140.9091 & 7.0 \\
\hline 141.4773 & 12.0 \\
\hline 142.0455 & 10.0 \\
\hline 142.6136 & 8.0 \\
\hline 143.1818 & 14.0 \\
\hline 143.7500 & 15.0 \\
\hline 144.3182 & 18.0 \\
\hline 144.8864 & 29.0 \\
\hline 145.4545 & 78.0 \\
\hline
\end{tabular}




\begin{tabular}{|c|c|}
\hline 146.0227 & 76.0 \\
\hline 146.5909 & 89.0 \\
\hline 147.1591 & 139.0 \\
\hline 147.7273 & 168.0 \\
\hline 148.2955 & 173.0 \\
\hline 148.8636 & 184.0 \\
\hline 149.4318 & 193.0 \\
\hline 150.0000 & 232.0 \\
\hline 150.5682 & 227.0 \\
\hline 151.1364 & 264.0 \\
\hline 151.7045 & 222.0 \\
\hline 152.2727 & 267.0 \\
\hline 152.8409 & 247.0 \\
\hline 153.4091 & 287.0 \\
\hline 153.9773 & 363.0 \\
\hline 154.5455 & 427.0 \\
\hline 155.1136 & 399.0 \\
\hline 155.6818 & 344.0 \\
\hline 156.2500 & 258.0 \\
\hline 156.8182 & 188.0 \\
\hline 157.3864 & 182.0 \\
\hline 157.9545 & 94.0 \\
\hline 158.5227 & 85.0 \\
\hline 159.0909 & 63.0 \\
\hline 159.6591 & 43.0 \\
\hline 160.2273 & 18.0 \\
\hline 160.7955 & 16.0 \\
\hline 161.3636 & 16.0 \\
\hline 161.9318 & 13.0 \\
\hline 162.5000 & 21.0 \\
\hline 163.0682 & 20.0 \\
\hline 163.6364 & 22.0 \\
\hline 164.2045 & 26.0 \\
\hline 164.7727 & 27.0 \\
\hline 165.3409 & 31.0 \\
\hline 165.9091 & 45.0 \\
\hline 166.4773 & 58.0 \\
\hline 167.0455 & 64.0 \\
\hline 167.6136 & 87.0 \\
\hline 168.1818 & 92.0 \\
\hline 168.7500 & 112.0 \\
\hline 169.3182 & 124.0 \\
\hline 169.8864 & 144.0 \\
\hline 170.4545 & 178.0 \\
\hline 171.0227 & 154.0 \\
\hline 171.5909 & 172.0 \\
\hline 172.1591 & 192.0 \\
\hline 172.7273 & 192.0 \\
\hline 173.2955 & 196.0 \\
\hline 173.8636 & 216.0 \\
\hline 174.4318 & 222.0 \\
\hline $175.000 \odot$ & 234.0 \\
\hline 175.5682 & 236.0 \\
\hline 176.1364 & 262.0 \\
\hline 176.7045 & 287.0 \\
\hline 177.2727 & 372.0 \\
\hline 177.8409 & 546.0 \\
\hline 178.4091 & 699.0 \\
\hline 178.9773 & 821.0 \\
\hline 179.5455 & 682.0 \\
\hline 180.1136 & 544.0 \\
\hline 180.6818 & 477.0 \\
\hline 181.2500 & 509.0 \\
\hline 181.8182 & 510.0 \\
\hline 182.3864 & 546.0 \\
\hline 182.9545 & 582.0 \\
\hline 183.5227 & 844.0 \\
\hline 184.0909 & 873.0 \\
\hline 184.6591 & 776.0 \\
\hline 185.2273 & 819.0 \\
\hline 185.7955 & 830.0 \\
\hline 186.3636 & 814.0 \\
\hline 186.9318 & 860.0 \\
\hline 187.5000 & 870.0 \\
\hline 188.0682 & 993.0 \\
\hline
\end{tabular}




\begin{tabular}{|c|c|}
\hline 188.6364 & 901.0 \\
\hline 189.2045 & 886.0 \\
\hline 189.7727 & 946.0 \\
\hline 190.3409 & 911.0 \\
\hline 190.9091 & 1025.0 \\
\hline 191.4773 & 1123.0 \\
\hline 192.0455 & 1262.0 \\
\hline 192.6136 & 1424.0 \\
\hline 193.1818 & 1460.0 \\
\hline 193.7500 & 1442.0 \\
\hline 194.3182 & 1348.0 \\
\hline 194.8864 & 1152.0 \\
\hline 195.4545 & 940.0 \\
\hline 196.0227 & 1256.0 \\
\hline 196.5909 & 1111.0 \\
\hline 197.1591 & 943.0 \\
\hline 197.7273 & 1037.0 \\
\hline 198.2955 & 931.0 \\
\hline 198.8636 & 759.0 \\
\hline 199.4318 & 673.0 \\
\hline 200.0000 & 702.0 \\
\hline 200.5682 & 607.0 \\
\hline 201.1364 & 649.0 \\
\hline 201.7045 & 576.0 \\
\hline 202.2727 & 551.0 \\
\hline 202.8409 & 548.0 \\
\hline 203.4091 & 548.0 \\
\hline 203.9773 & 551.0 \\
\hline 204.5455 & 546.0 \\
\hline 205.1136 & 545.0 \\
\hline 205.6818 & 547.0 \\
\hline 206.2500 & 556.0 \\
\hline 206.8182 & 569.0 \\
\hline 207.3864 & 576.0 \\
\hline 207.9545 & 610.0 \\
\hline 208.5227 & 636.0 \\
\hline 209.0909 & 634.0 \\
\hline 209.6591 & 704.0 \\
\hline 210.2273 & 736.0 \\
\hline 210.7955 & 719.0 \\
\hline 211.3636 & 702.0 \\
\hline 211.9318 & 714.0 \\
\hline 212.5000 & 691.0 \\
\hline 213.0682 & 676.0 \\
\hline 213.6364 & 671.0 \\
\hline 214.2045 & 671.0 \\
\hline 214.7727 & 708.0 \\
\hline 215.3409 & 668.0 \\
\hline 215.9091 & 674.0 \\
\hline 216.4773 & 688.0 \\
\hline 217.0455 & 638.0 \\
\hline 217.6136 & 661.0 \\
\hline 218.1818 & 652.0 \\
\hline 218.7500 & 673.0 \\
\hline 219.3182 & 673.0 \\
\hline 219.8864 & 665.0 \\
\hline 220.4545 & 703.0 \\
\hline 221.0227 & 671.0 \\
\hline 221.5909 & 685.0 \\
\hline 222.1591 & 730.0 \\
\hline 222.7273 & 722.0 \\
\hline 223.2955 & 737.0 \\
\hline 223.8636 & 709.0 \\
\hline 224.4318 & 752.0 \\
\hline 225.0000 & 767.0 \\
\hline 225.5682 & 774.0 \\
\hline 226.1364 & 728.0 \\
\hline 226.7045 & 749.0 \\
\hline 227.2727 & 761.0 \\
\hline 227.8409 & 759.0 \\
\hline 228.4091 & 815.0 \\
\hline 228.9773 & 836.0 \\
\hline 229.5455 & 896.0 \\
\hline 230.1136 & 924.0 \\
\hline 230.6818 & 956.0 \\
\hline
\end{tabular}




$\begin{array}{lr}231.2500 & 1136.0 \\ 231.8182 & 1187.0 \\ 232.3864 & 1353.0 \\ 232.9545 & 1313.0 \\ 233.5227 & 1153.0 \\ 234.0909 & 1111.0 \\ 234.6591 & 1095.0 \\ 235.2273 & 1094.0 \\ 235.7955 & 1249.0 \\ 236.3636 & 1334.0 \\ 236.9318 & 1286.0 \\ 237.5000 & 1235.0 \\ 238.0682 & 1181.0 \\ 238.6364 & 1165.0 \\ 239.2045 & 1196.0 \\ 239.7727 & 1207.0 \\ 240.3409 & 1257.0 \\ 240.9091 & 1177.0 \\ 241.4773 & 1237.0 \\ 242.0455 & 1186.0 \\ 242.6136 & 1085.0 \\ 243.1818 & 964.0 \\ 243.7500 & 897.0 \\ 244.3182 & 861.0 \\ 244.8864 & 806.0 \\ 245.4545 & 796.0 \\ 246.0227 & 780.0 \\ 246.5909 & 773.0 \\ 247.1591 & 767.0 \\ 247.7273 & 764.0 \\ 248.2955 & 761.0 \\ 248.8636 & 753.0 \\ 249.4318 & 750.0\end{array}$

\subsection{HF20QWVD}

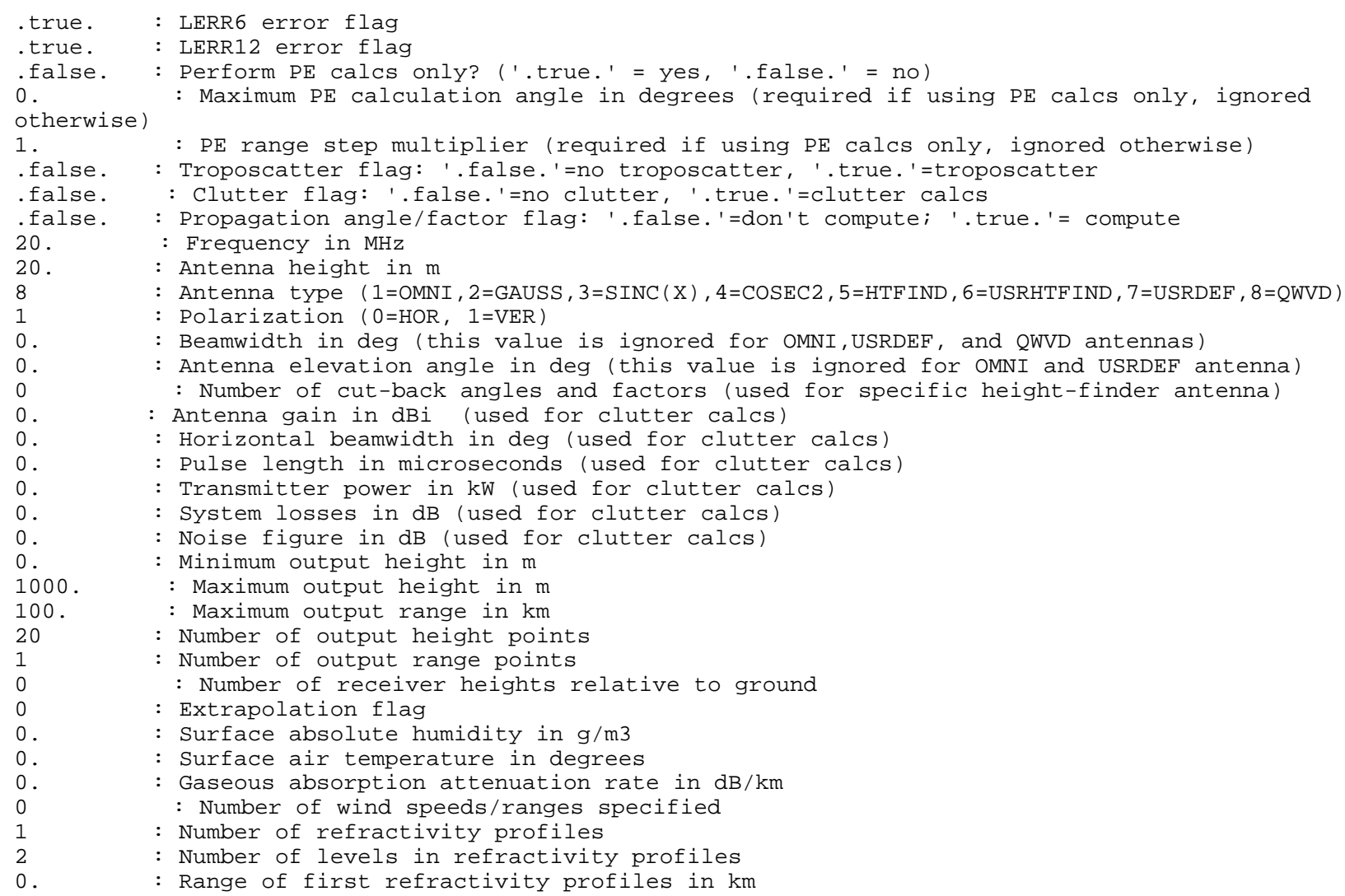




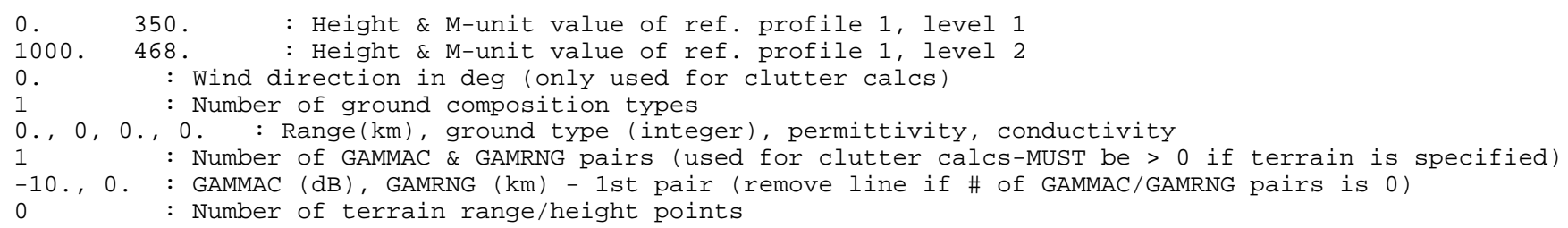

\subsection{HF20RF}

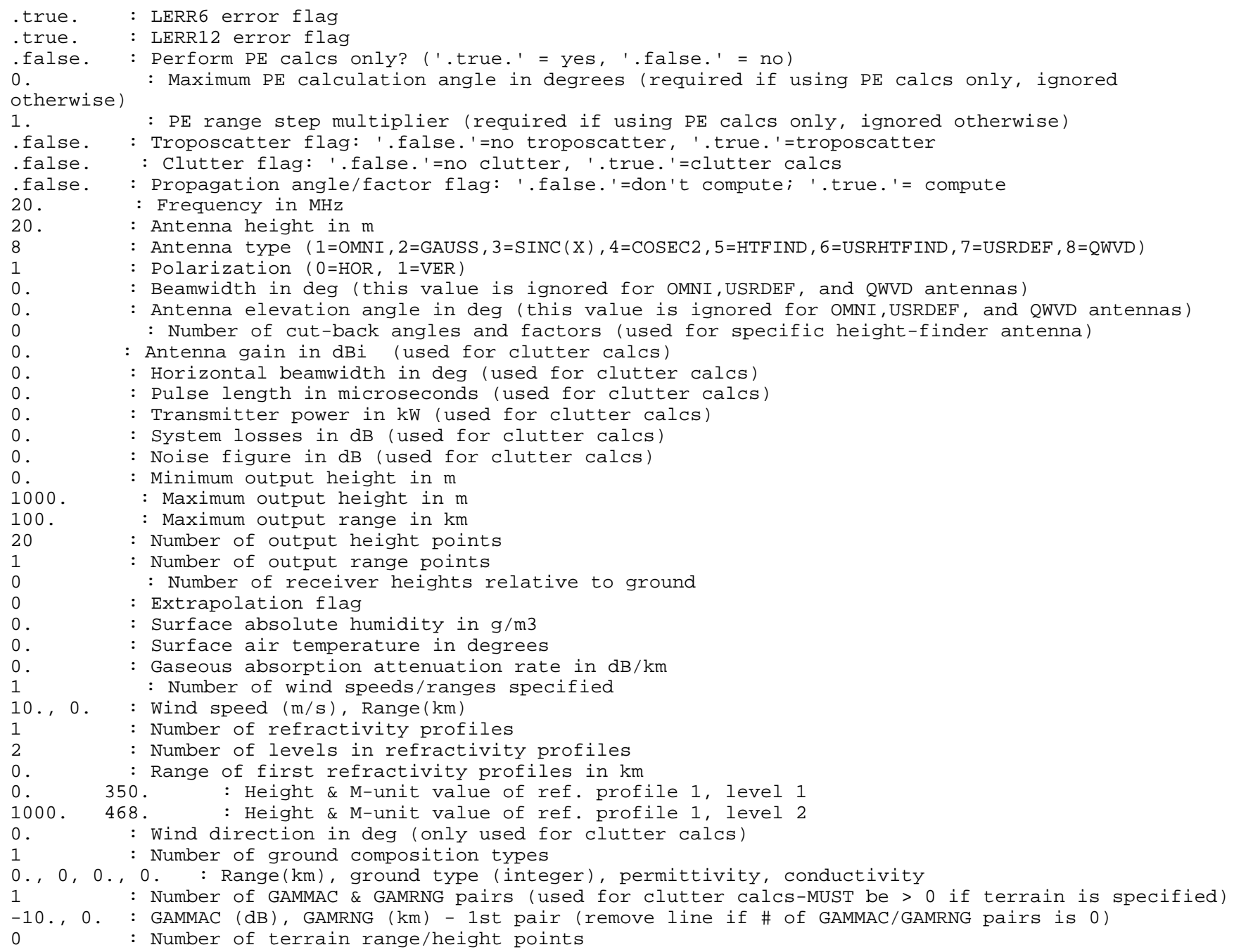

\subsection{HF30.IN}

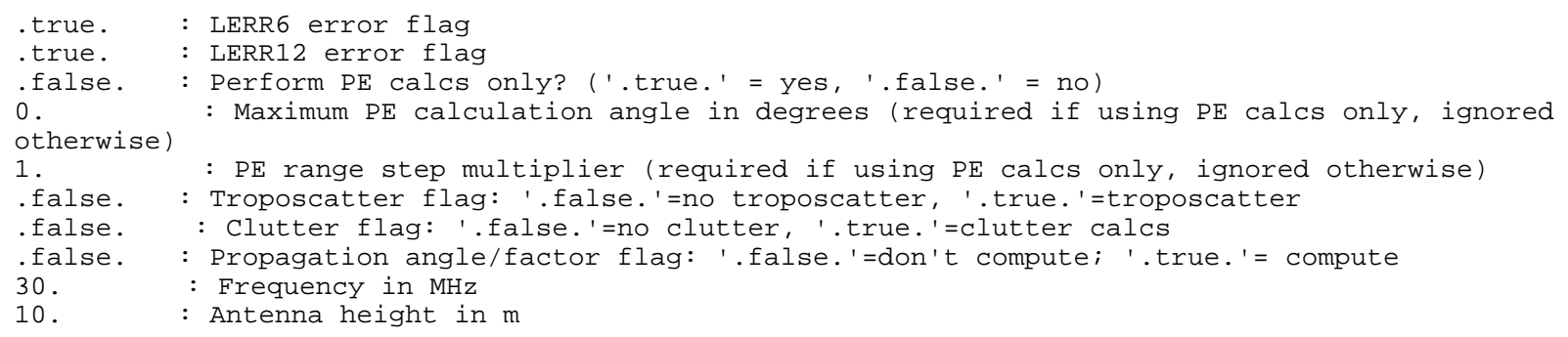




\subsection{HIBW.IN}

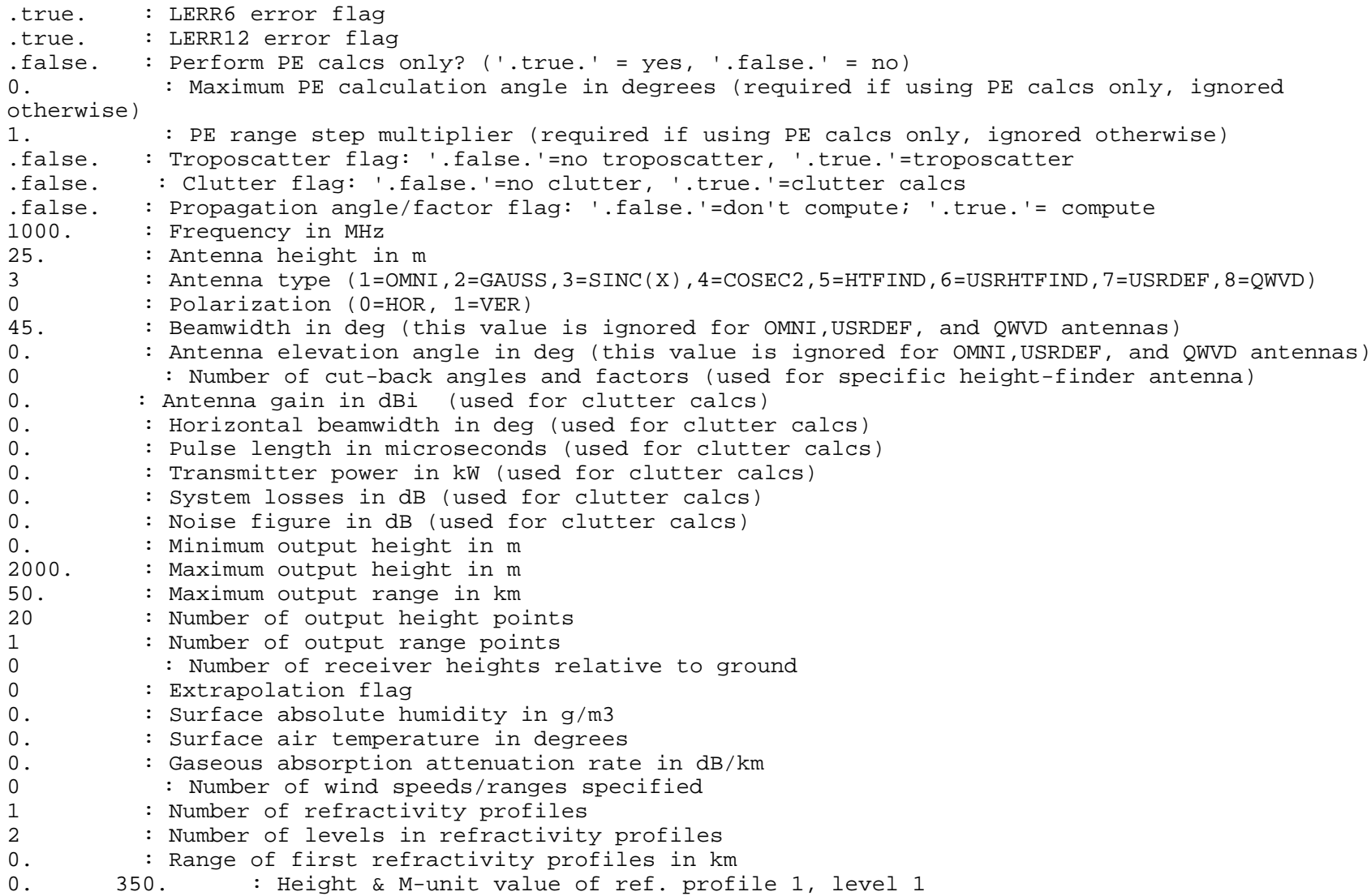




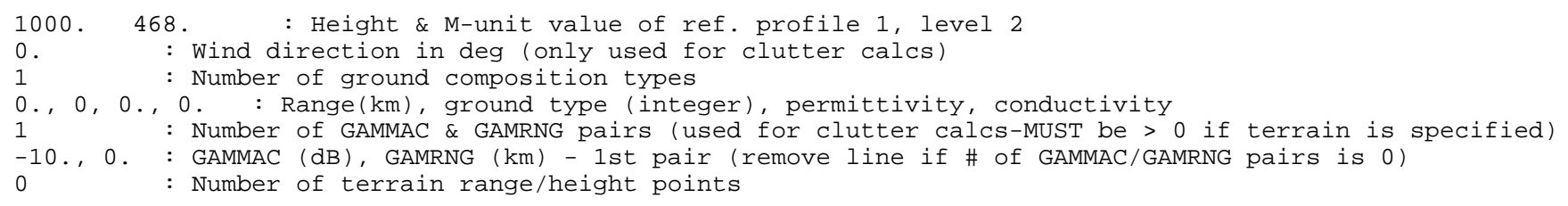

\subsection{HIEL.IN}

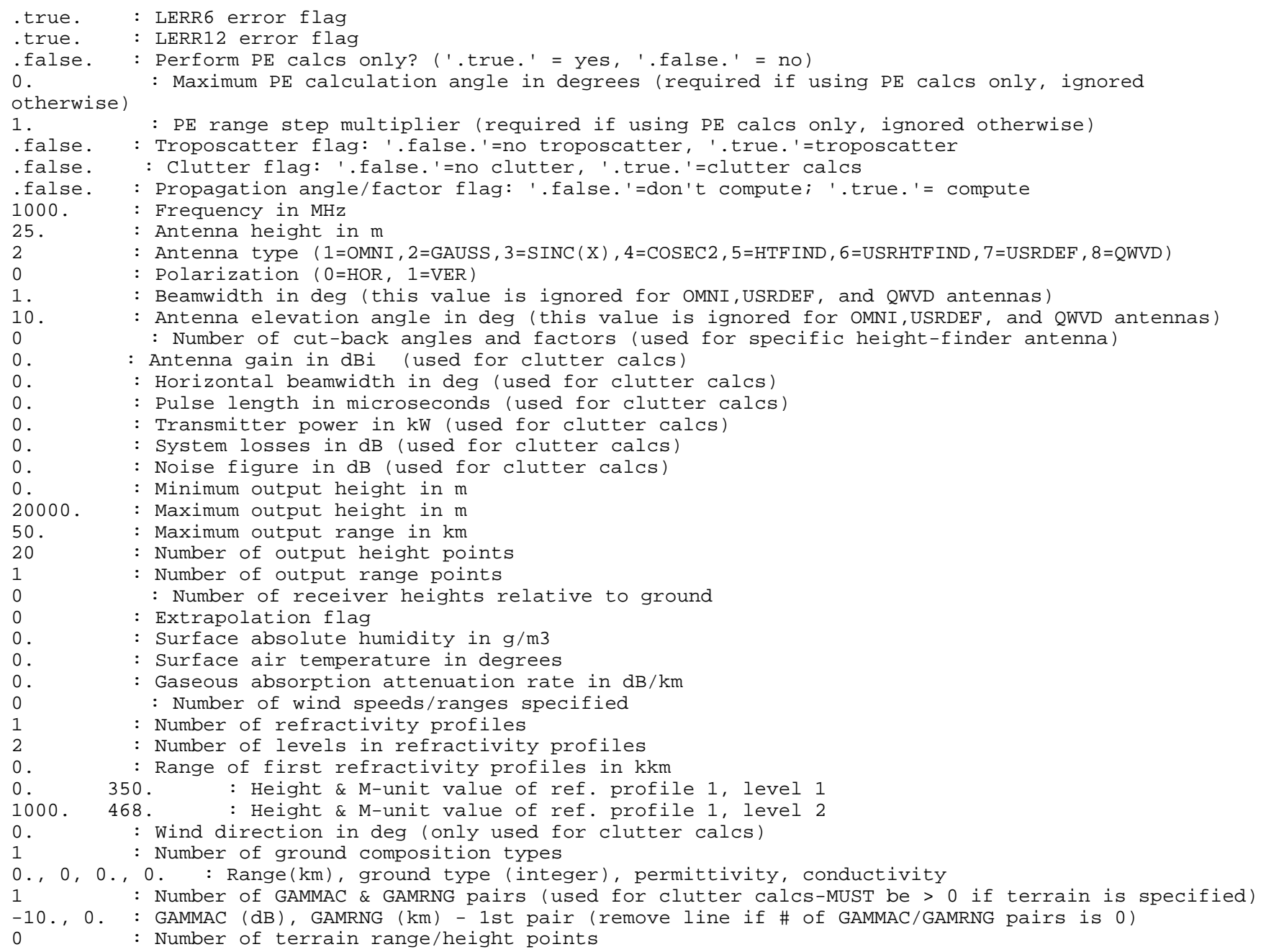

\subsection{HIFREQ.IN}

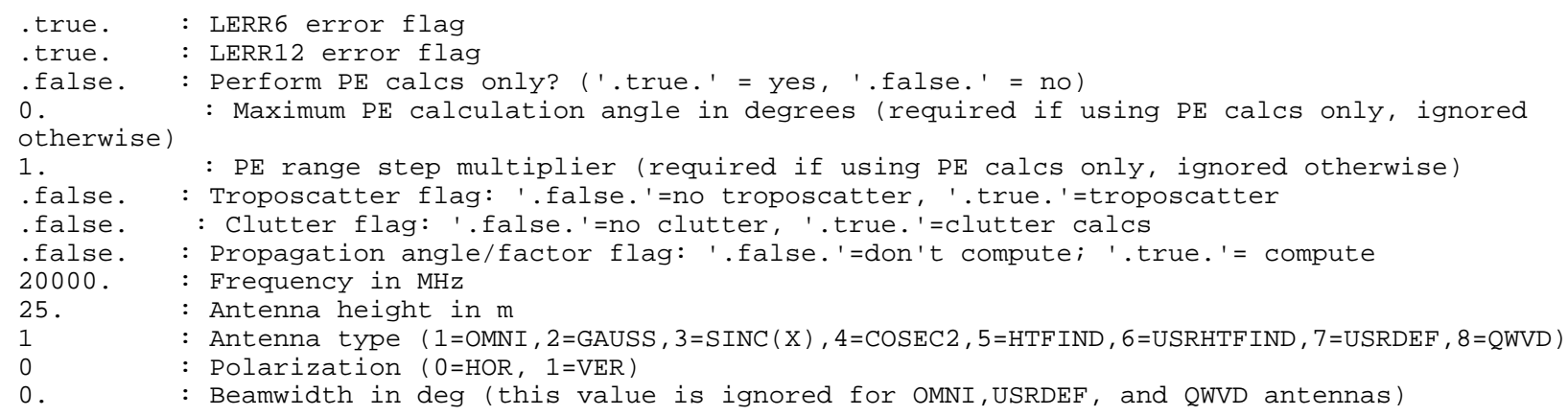


๑. : Antenna elevation angle in deg (this value is ignored for OMNI, USRDEF, and QWVD antennas)

$\odot \quad$ : Number of cut-back angles and factors (used for specific height-finder antenna)

$\odot$ : Antenna gain in dBi (used for clutter calcs)

๑. : Horizontal beamwidth in deg (used for clutter calcs)

$\odot$. : Pulse length in microseconds (used for clutter calcs)

$\odot$. : Transmitter power in $\mathrm{kW}$ (used for clutter calcs)

$\odot$. : System losses in $\mathrm{dB}$ (used for clutter calcs)

$\odot$. Noise figure in $\mathrm{dB}$ (used for clutter calcs)

$\odot$. : Minimum output height in $\mathrm{m}$

200. : Maximum output height in $\mathrm{m}$

50. : Maximum output range in $\mathrm{km}$

$20 \quad$ : Number of output height points

$1 \quad$ : Number of output range points

$\odot \quad$ : Number of receiver heights relative to ground

$\odot \quad$ : Extrapolation flag

๑. : Surface absolute humidity in $\mathrm{g} / \mathrm{m} 3$

$\odot$. : Surface air temperature in degrees

$\odot$ : Gaseous absorption attenuation rate in $\mathrm{dB} / \mathrm{km}$

$\odot \quad$ : Number of wind speeds/ranges specified

1 : Number of refractivity profiles

: Number of levels in refractivity profiles

0 . : Range of first refractivity profiles in $\mathrm{km}$

0 . 350. : Height \& M-unit value of ref. profile 1, level 1

1000. 468. : Height \& M-unit value of ref. profile 1, level 2

$\odot$. : Wind direction in deg (only used for clutter calcs)

$1 \quad$ : Number of ground composition types

$\odot ., \odot, \odot ., \odot . \quad$ : Range $(\mathrm{km})$, ground type (integer), permittivity, conductivity

1 : Number of GAMMAC \& GAMRNG pairs (used for clutter calcs-MUST be $>0$ if terrain is specified)

$-10 ., \odot . \quad$ : GAMMAC $(\mathrm{dB})$, GAMRNG $(\mathrm{km})$ - 1st pair (remove line if \# of GAMMAC/GAMRNG pairs is $\odot$ )

$\odot \quad$ : Number of terrain range/height points

\subsection{HITRAN.IN}

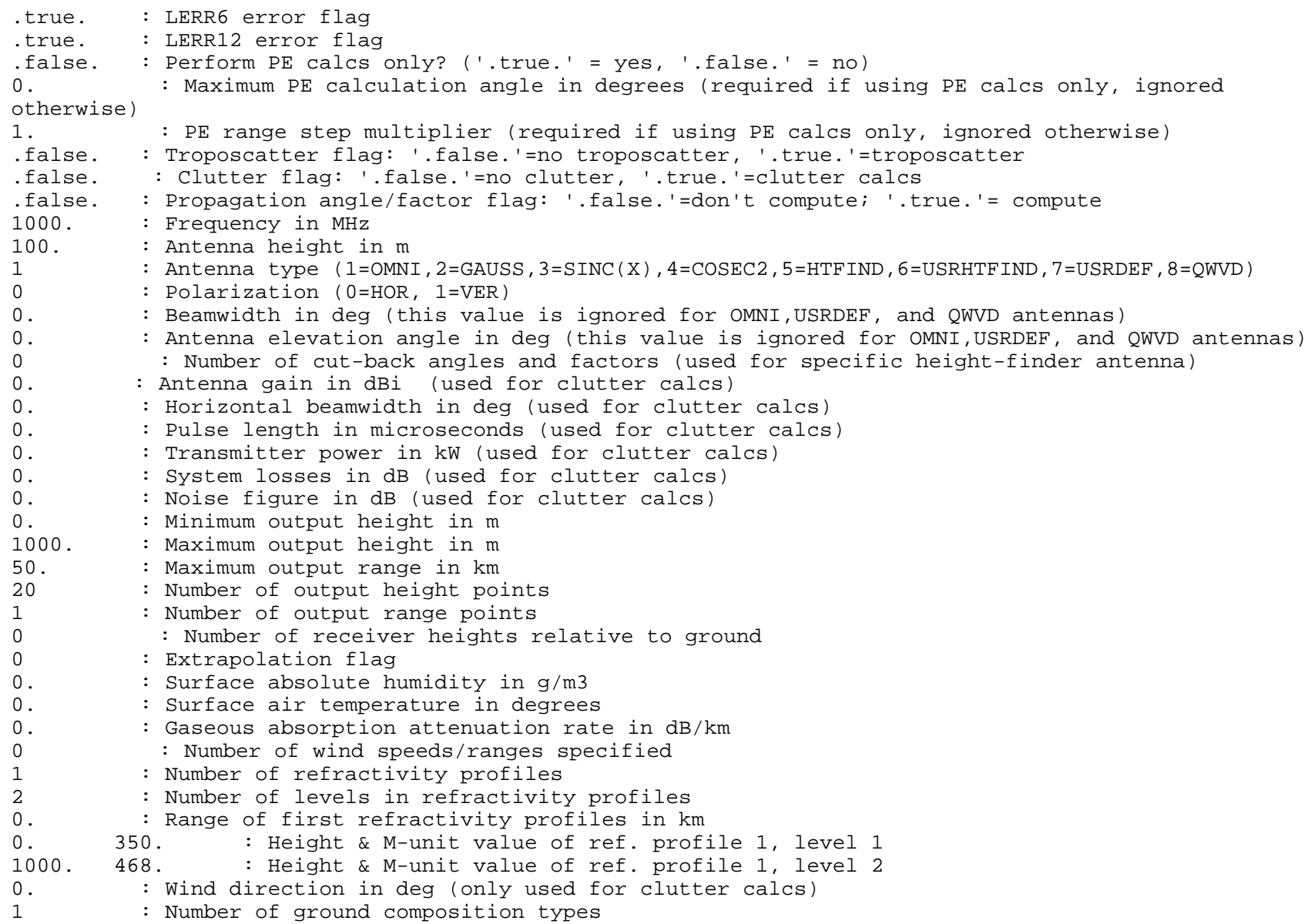


$\odot ., \odot, \odot ., \odot . \quad$ : Range $(\mathrm{km})$, ground type (integer), permittivity, conductivity

$1 \quad$ : Number of GAMMAC \& GAMRNG pairs (used for clutter calcs-MUST be > 0 if terrain is specified)

$-10 ., 0 . \quad$ : GAMMAC $(\mathrm{dB})$, GAMRNG $(\mathrm{km})$ - 1st pair (remove line if \# of GAMMAC/GAMRNG pairs is 0 )

$\odot \quad:$ Number of terrain range/height points

\subsection{HORZ.IN}

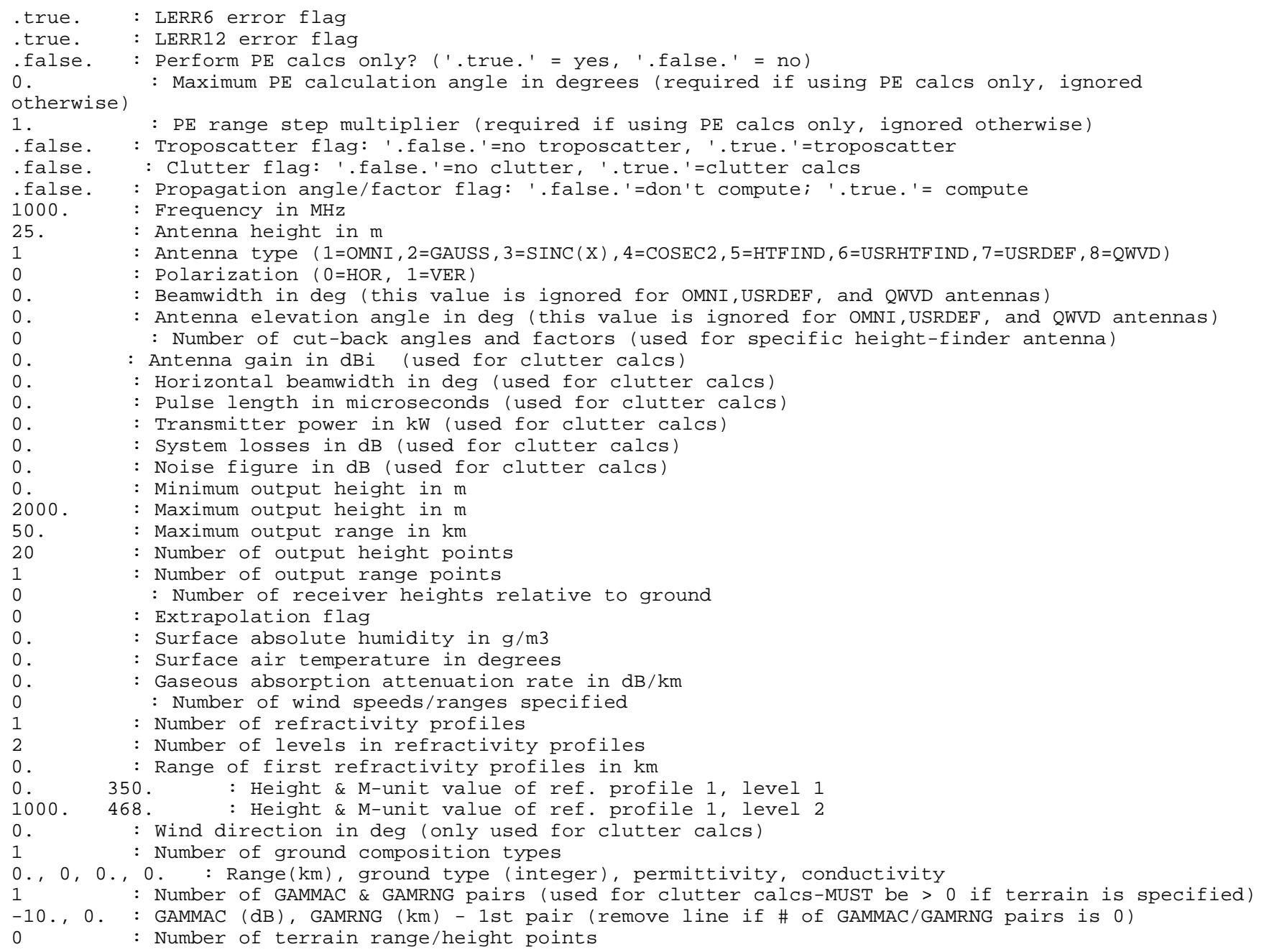

\subsection{HTFIND.IN}

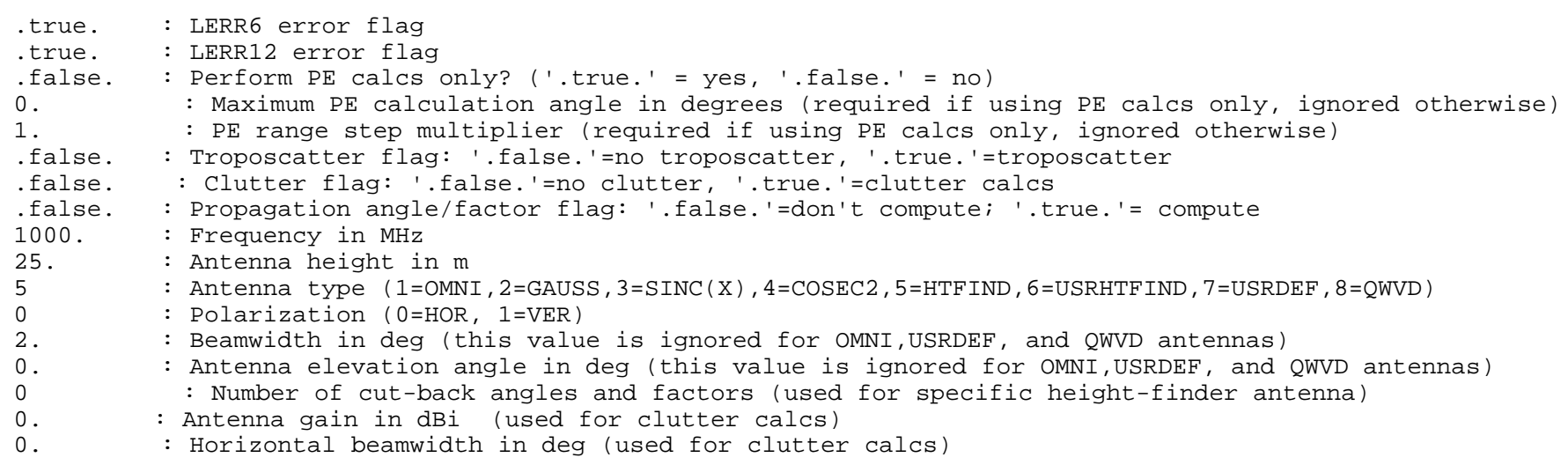




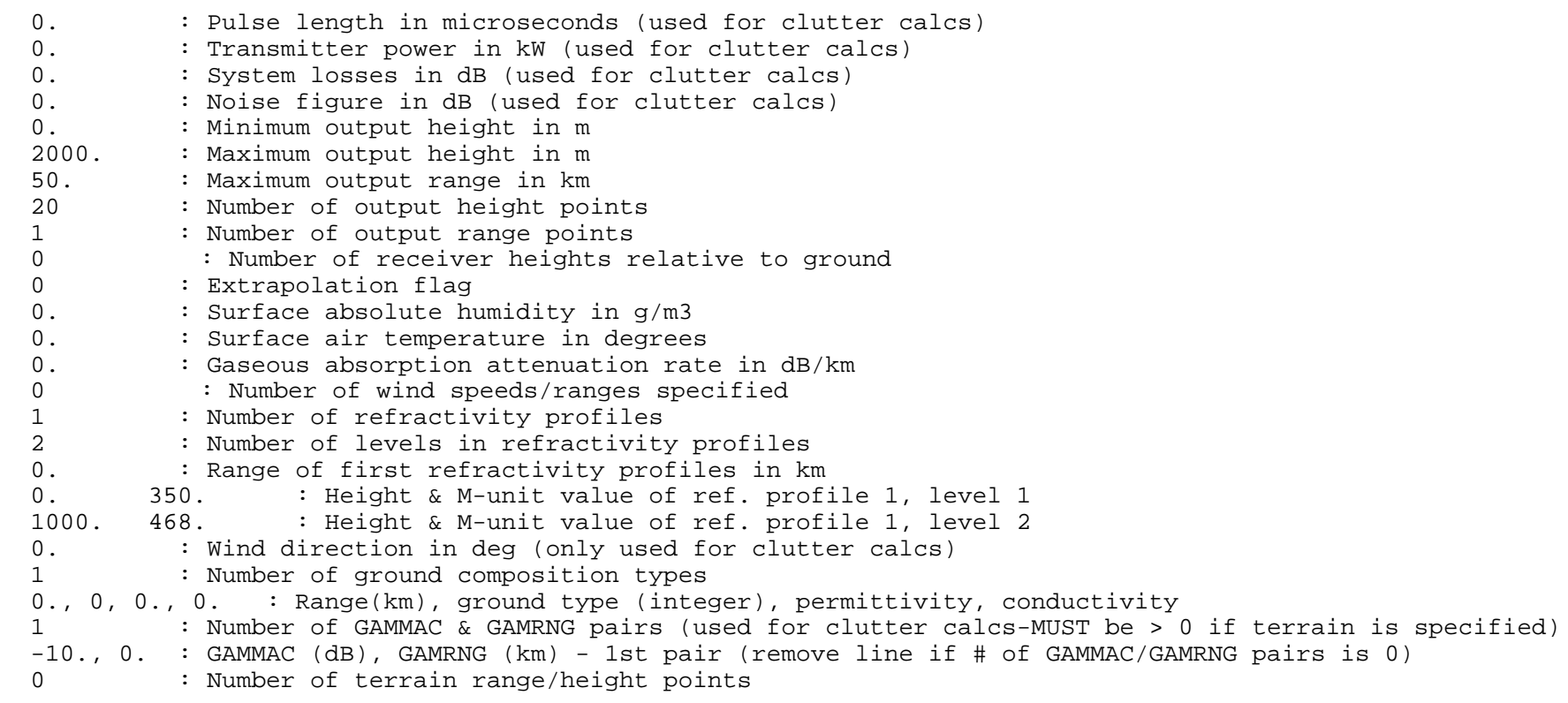

\subsection{LOBW.IN}

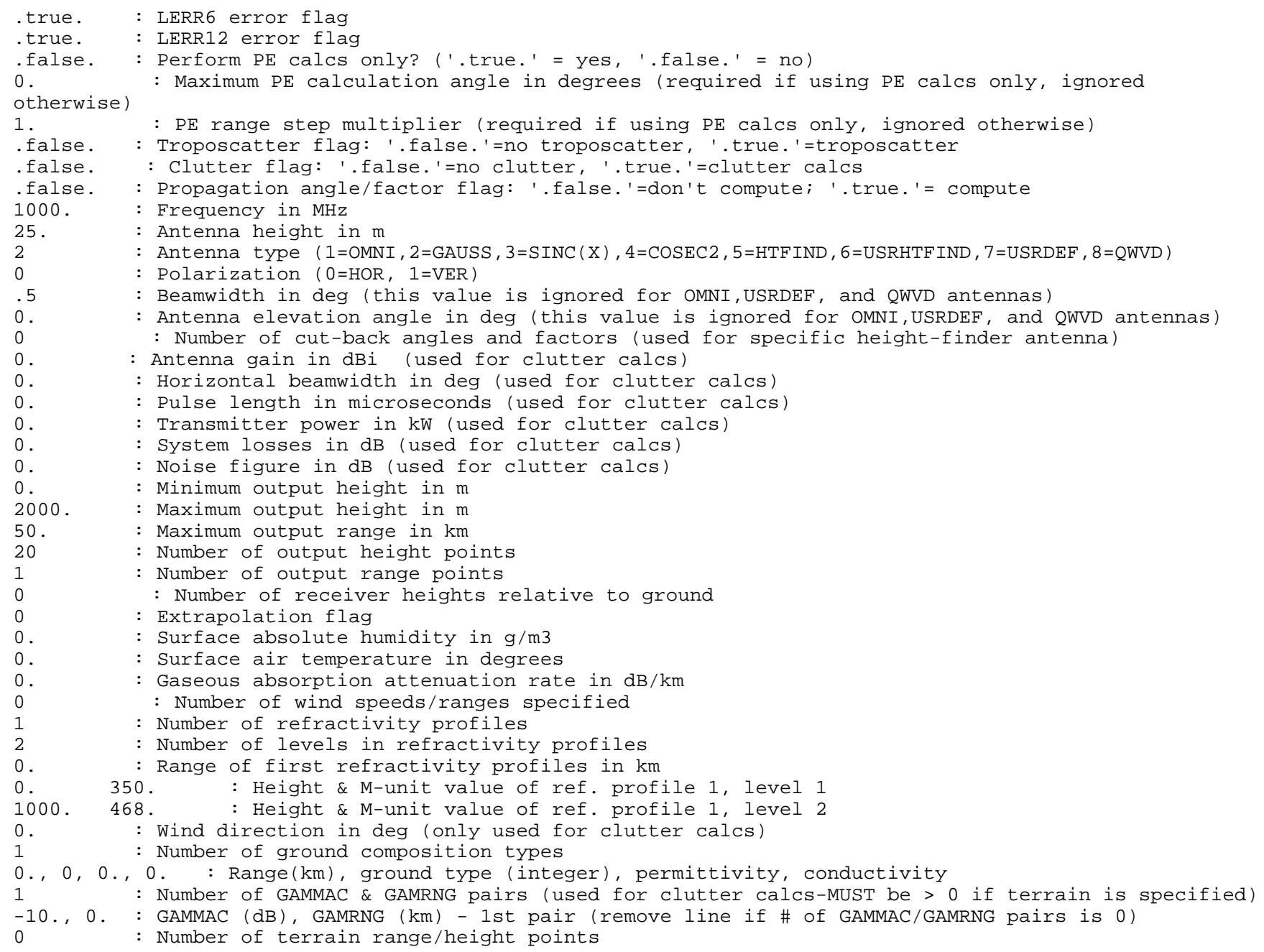




\subsection{LOEL.IN}

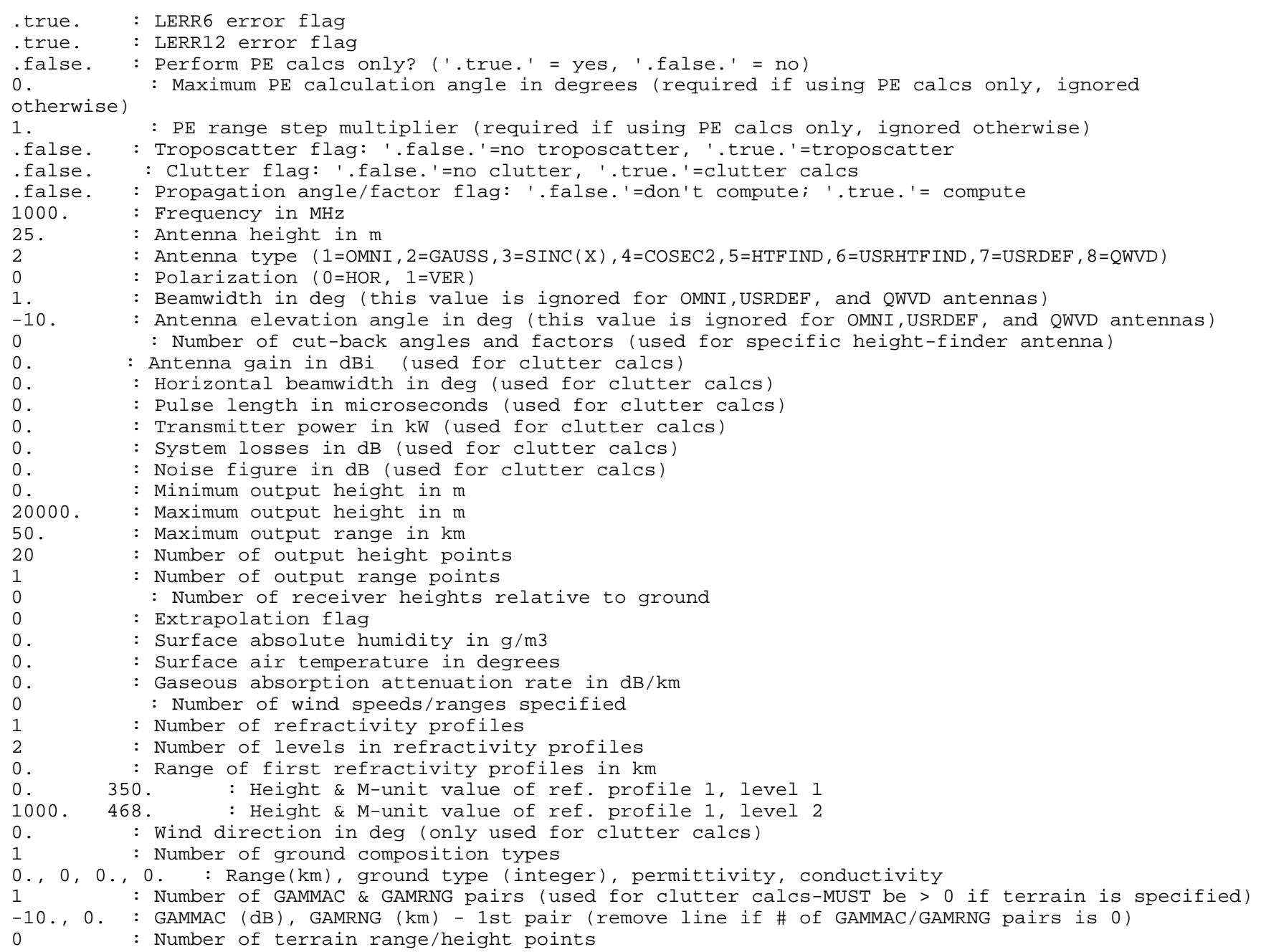

\subsection{LOFREQ.IN}

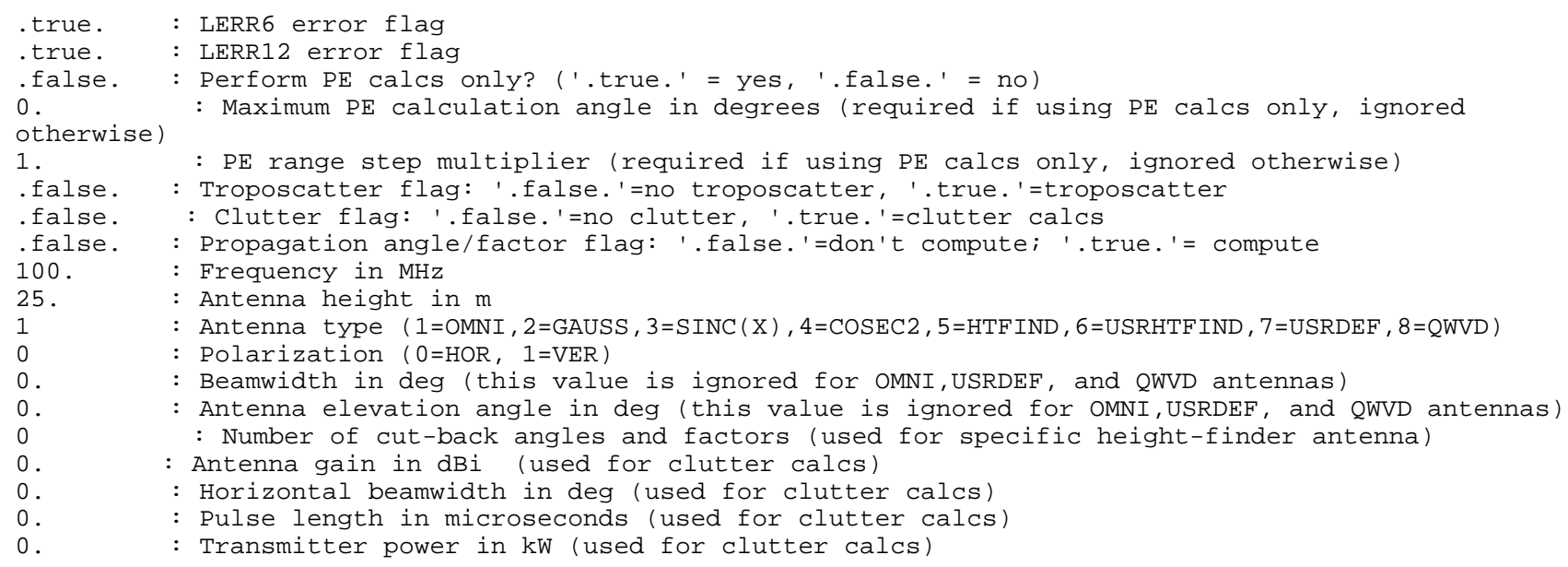




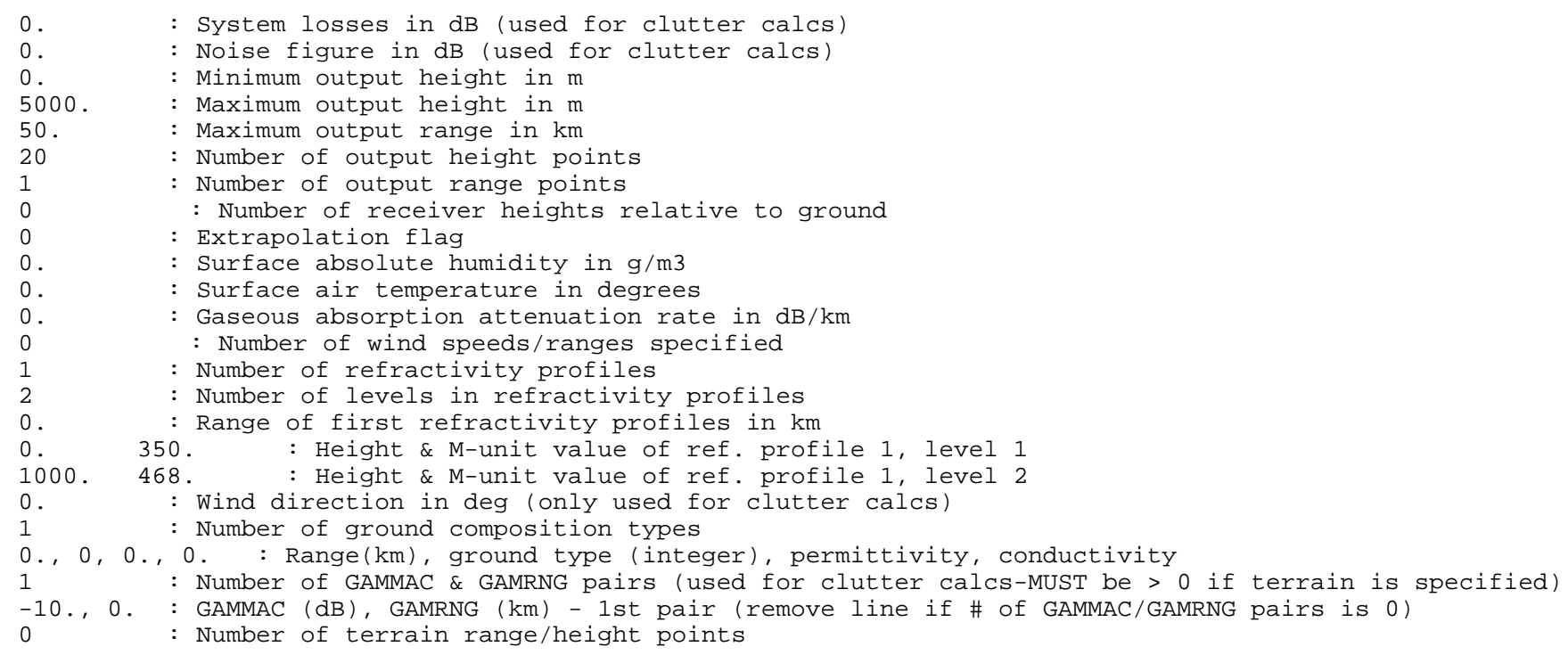

\subsection{LOTRAN.IN}

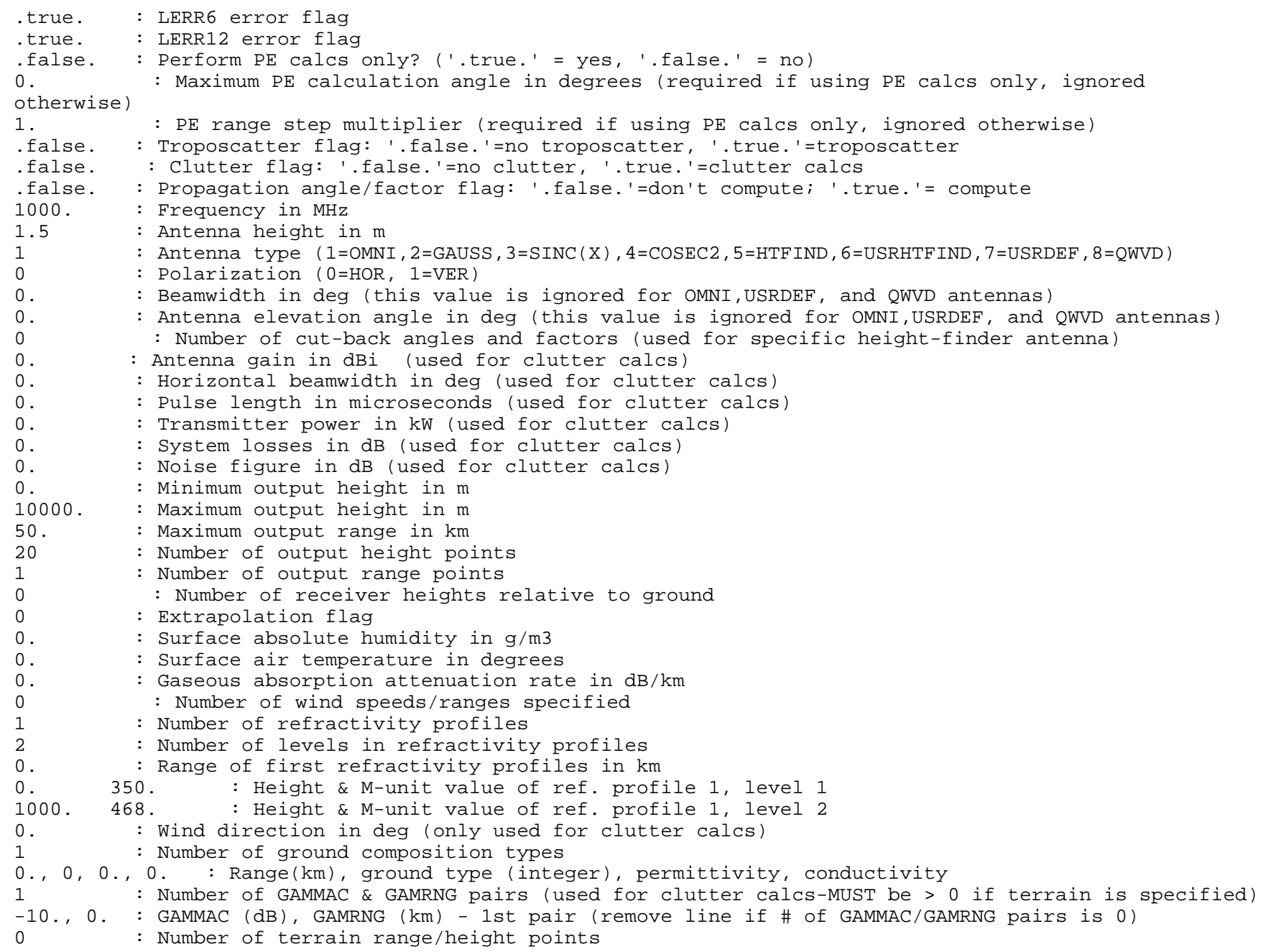




\subsection{MPRT.IN}

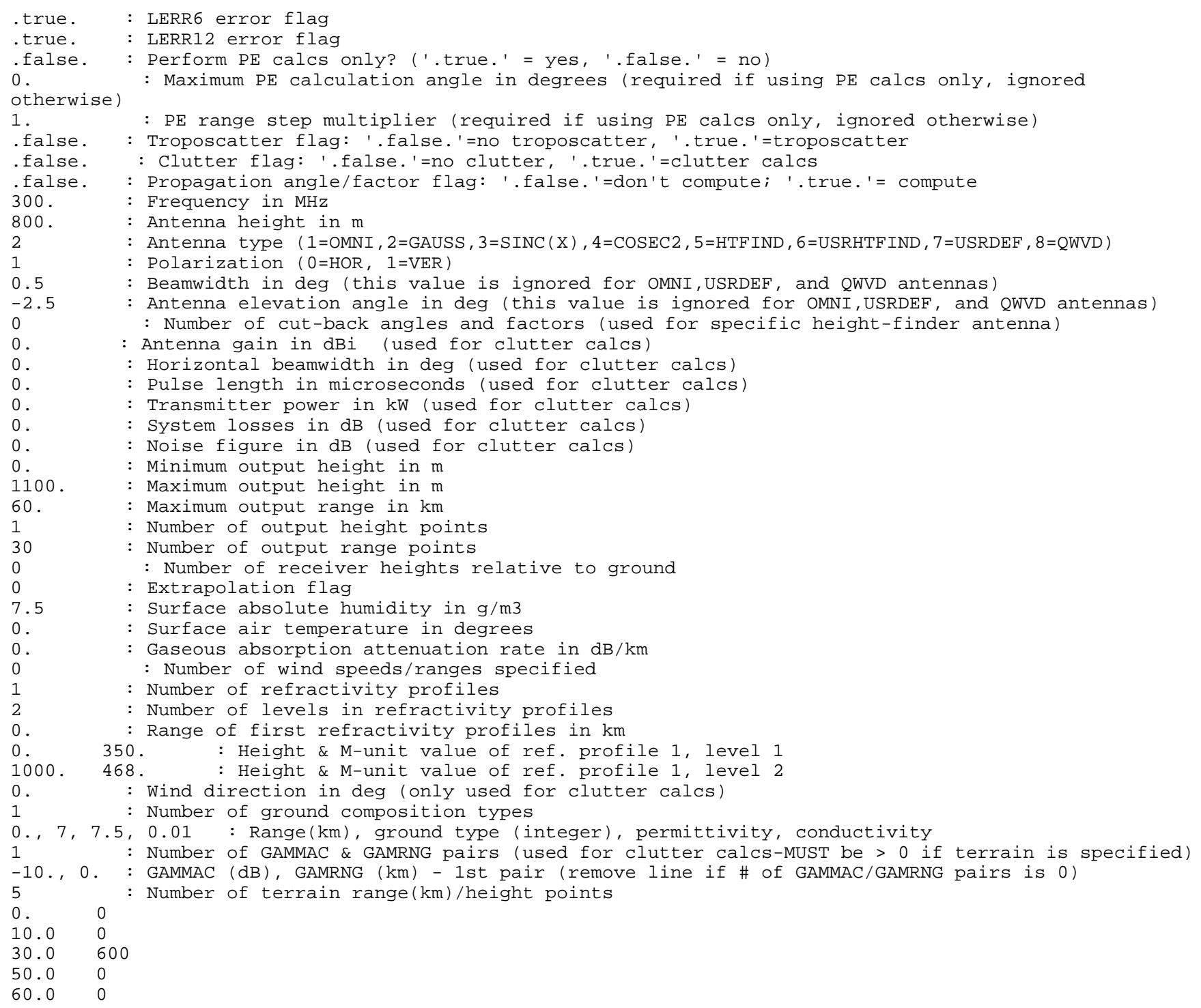

\subsection{PERW.IN}

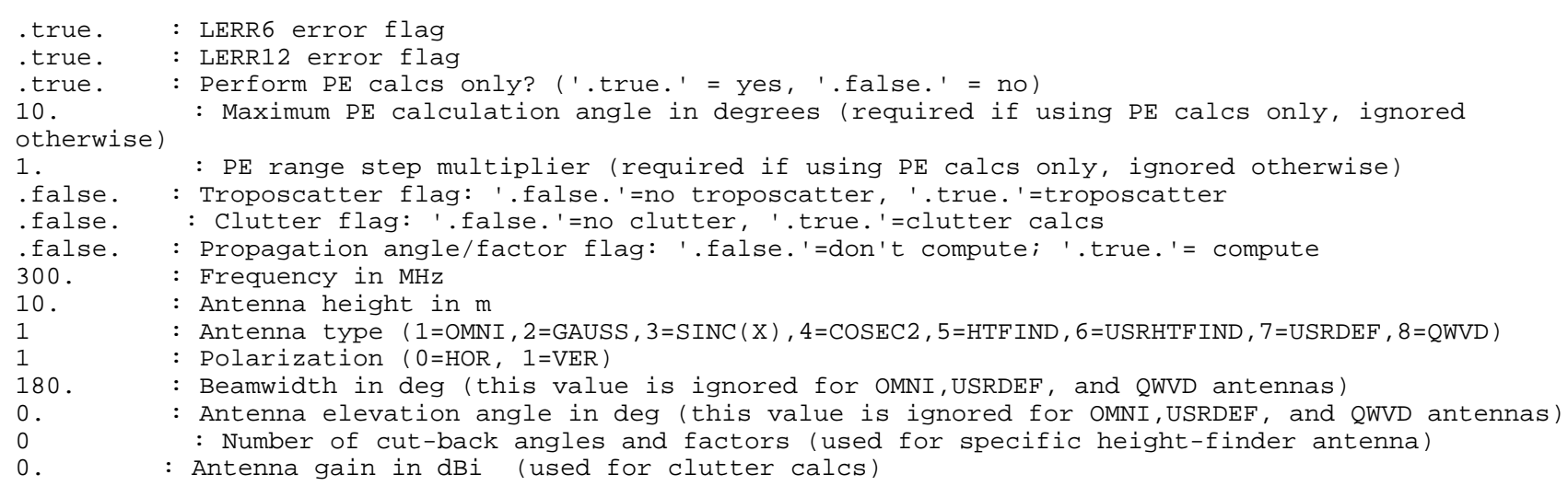




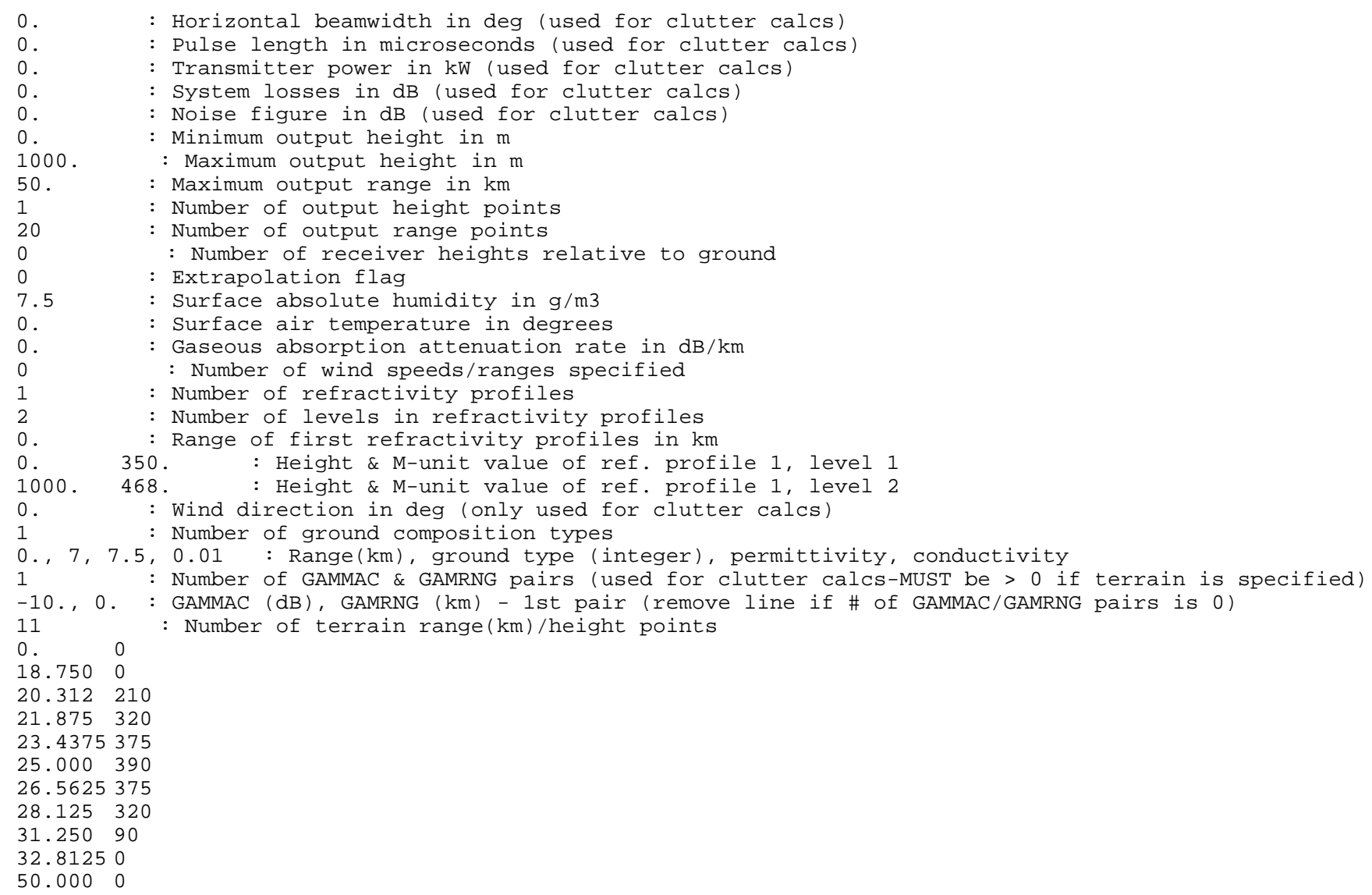

\subsection{PVT.IN}

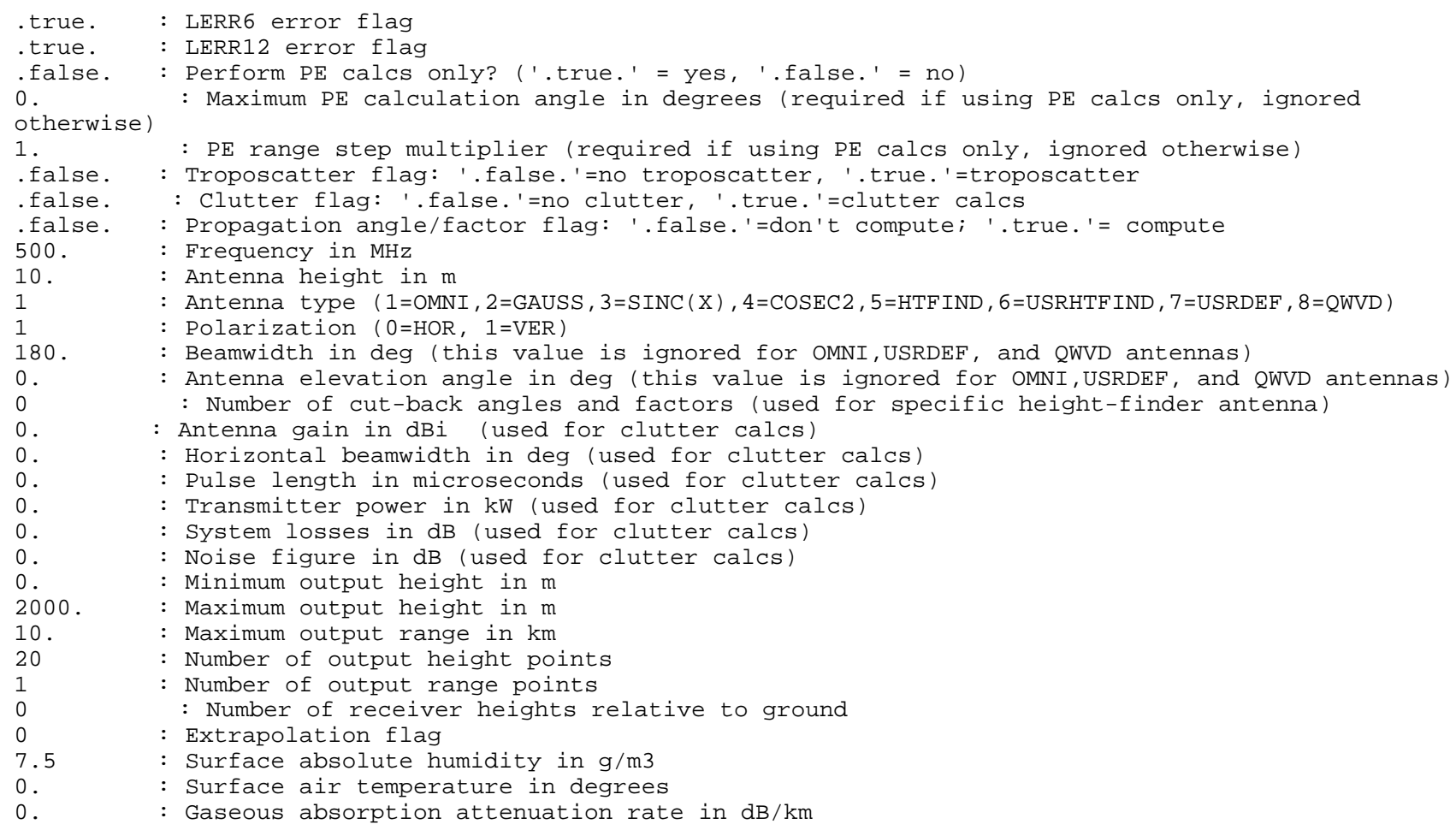




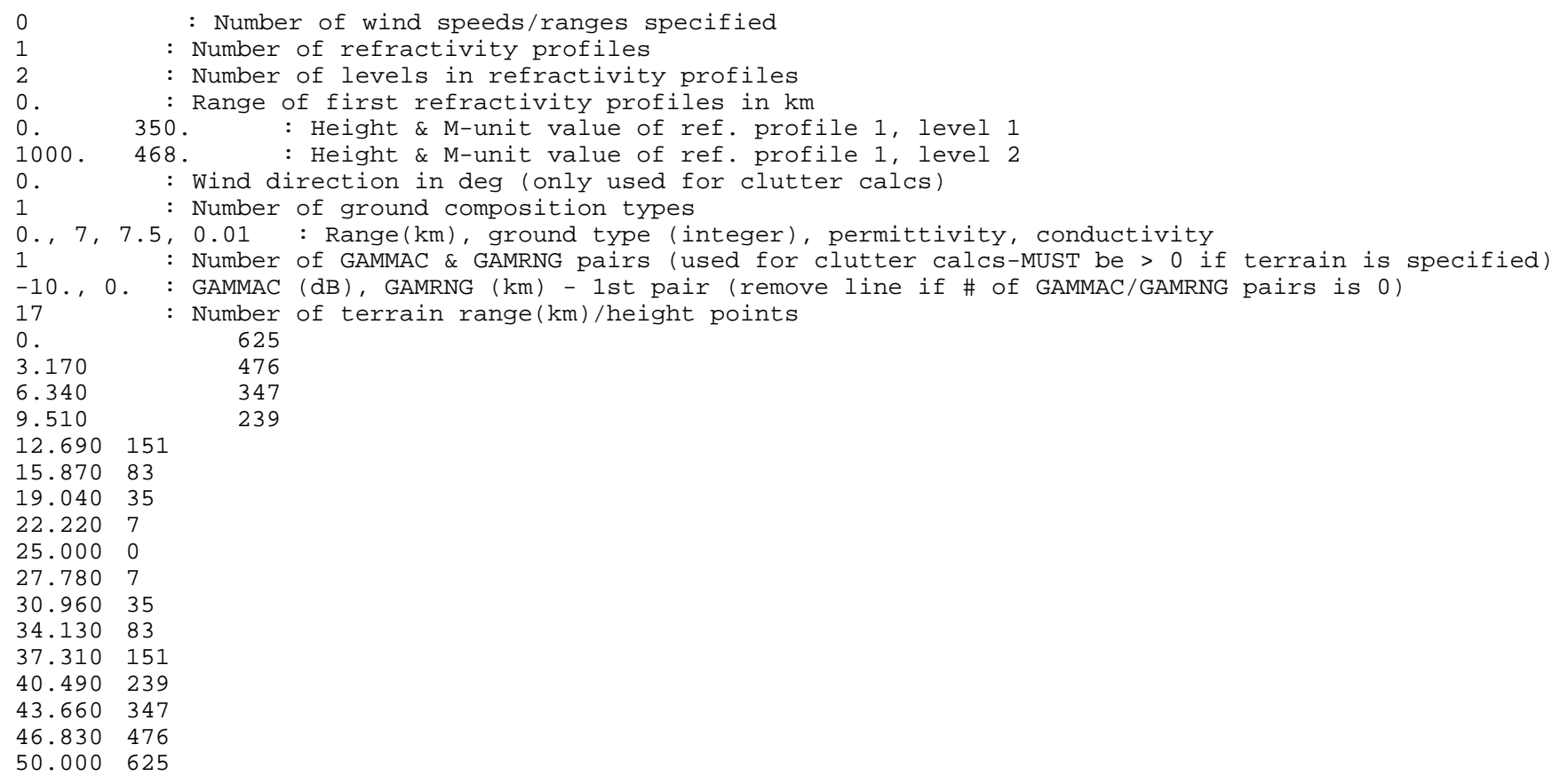

\subsection{RDLONGB.IN}

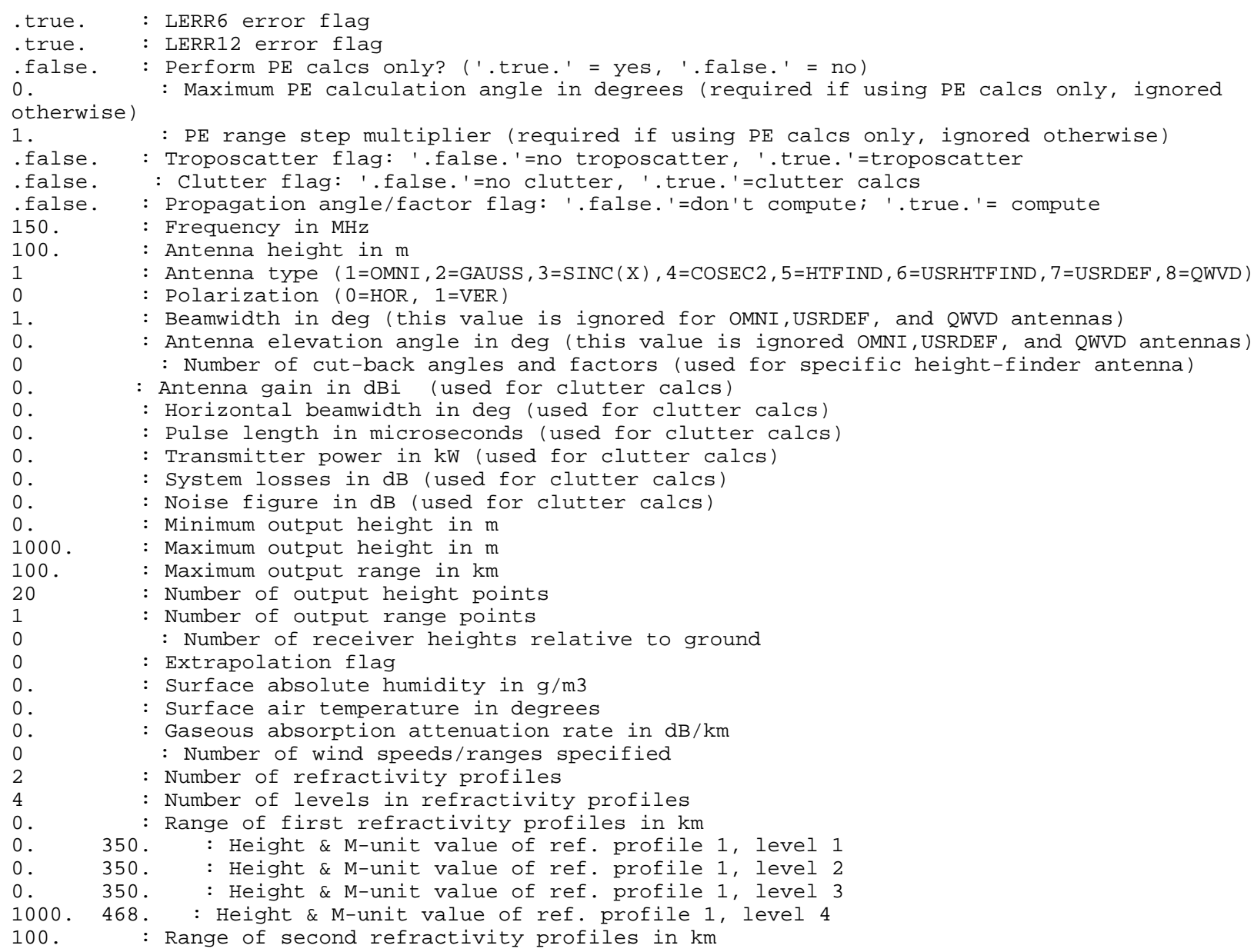




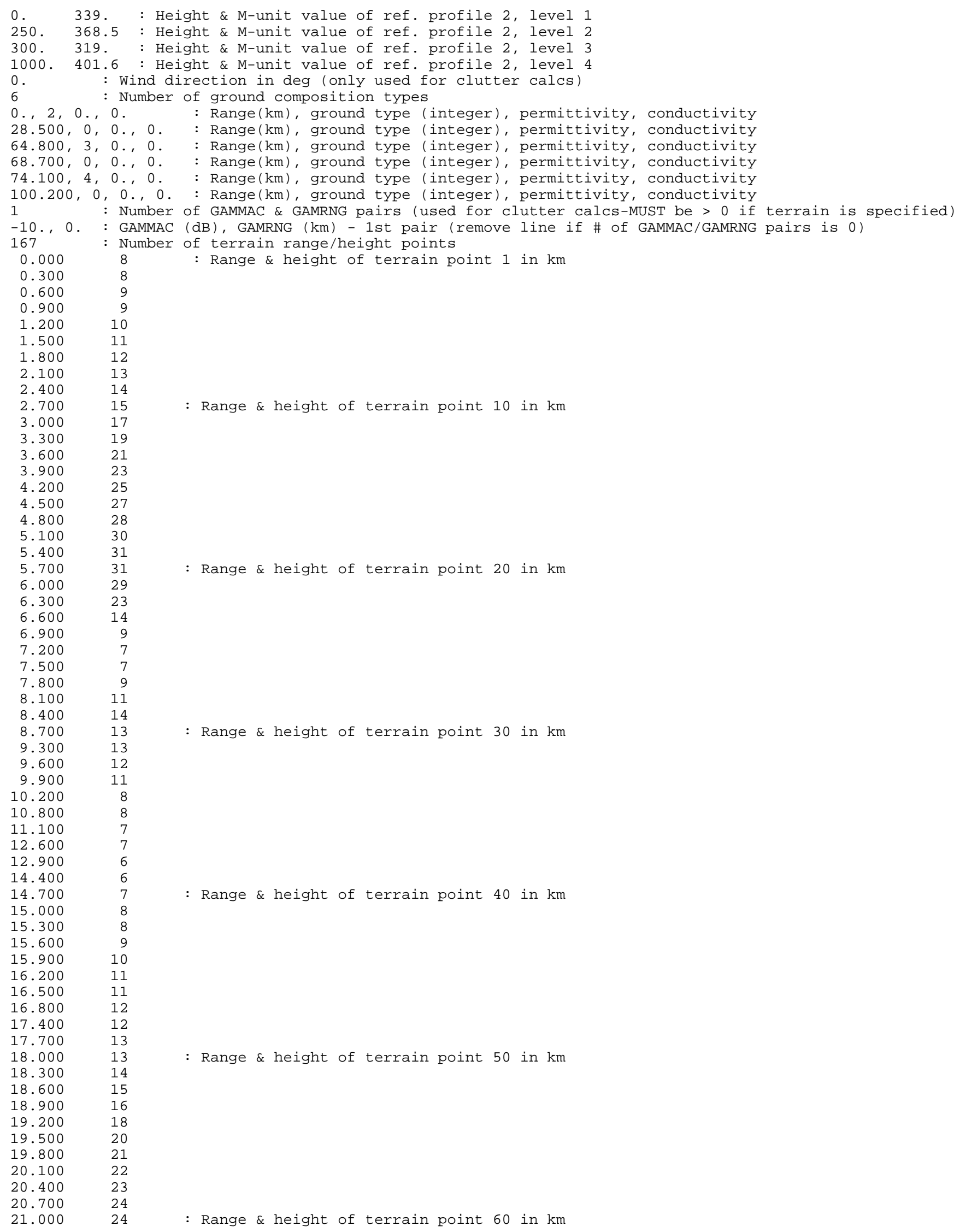




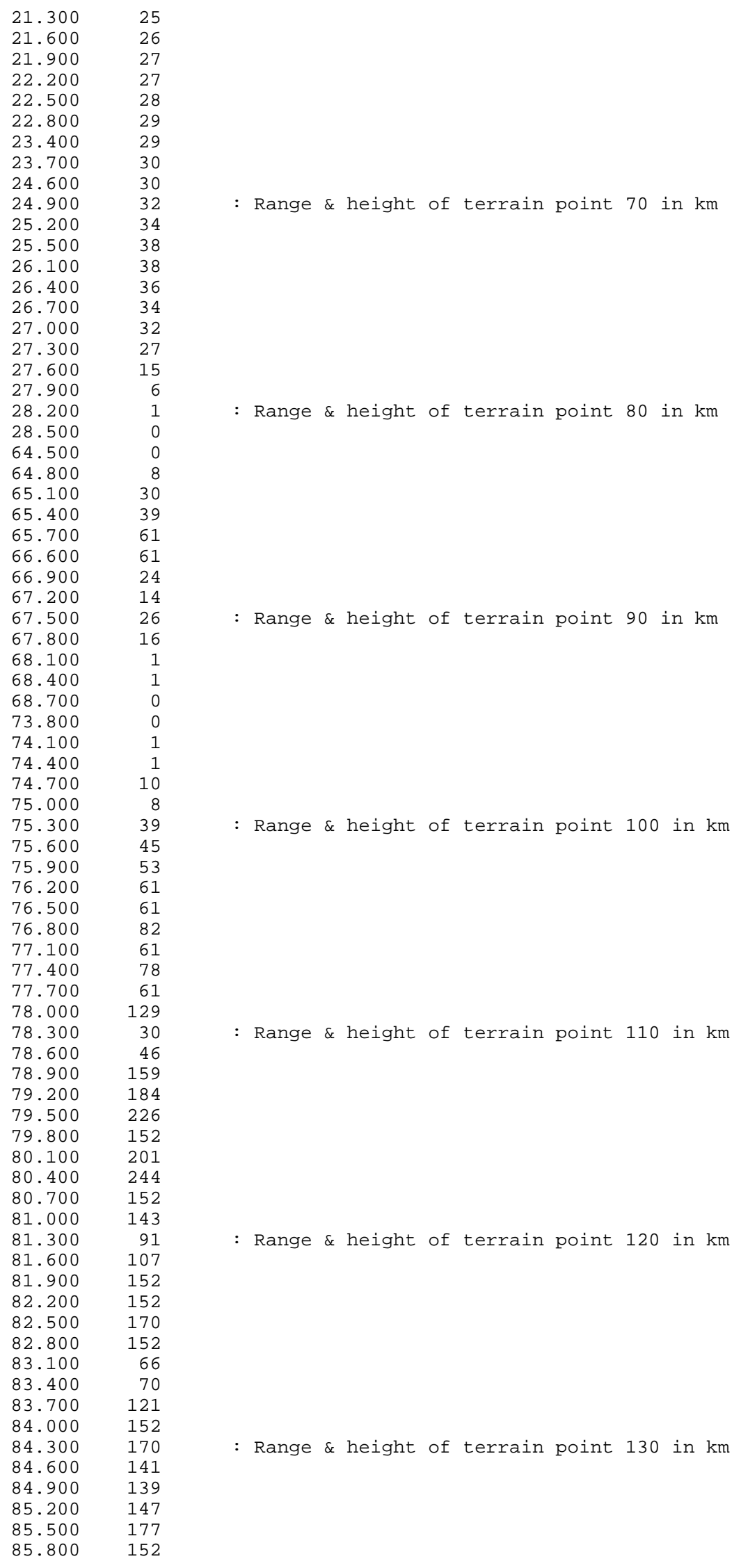




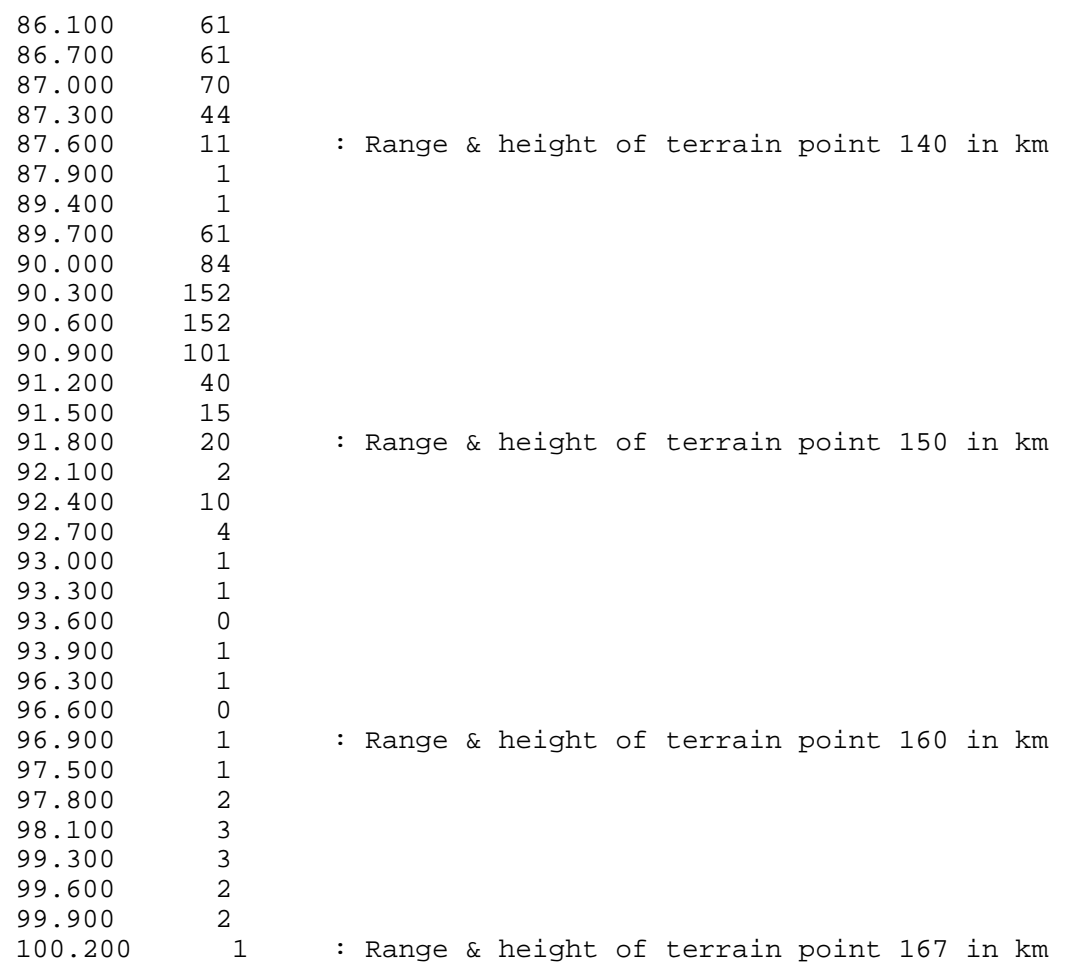

\subsection{RNGDEP.IN}

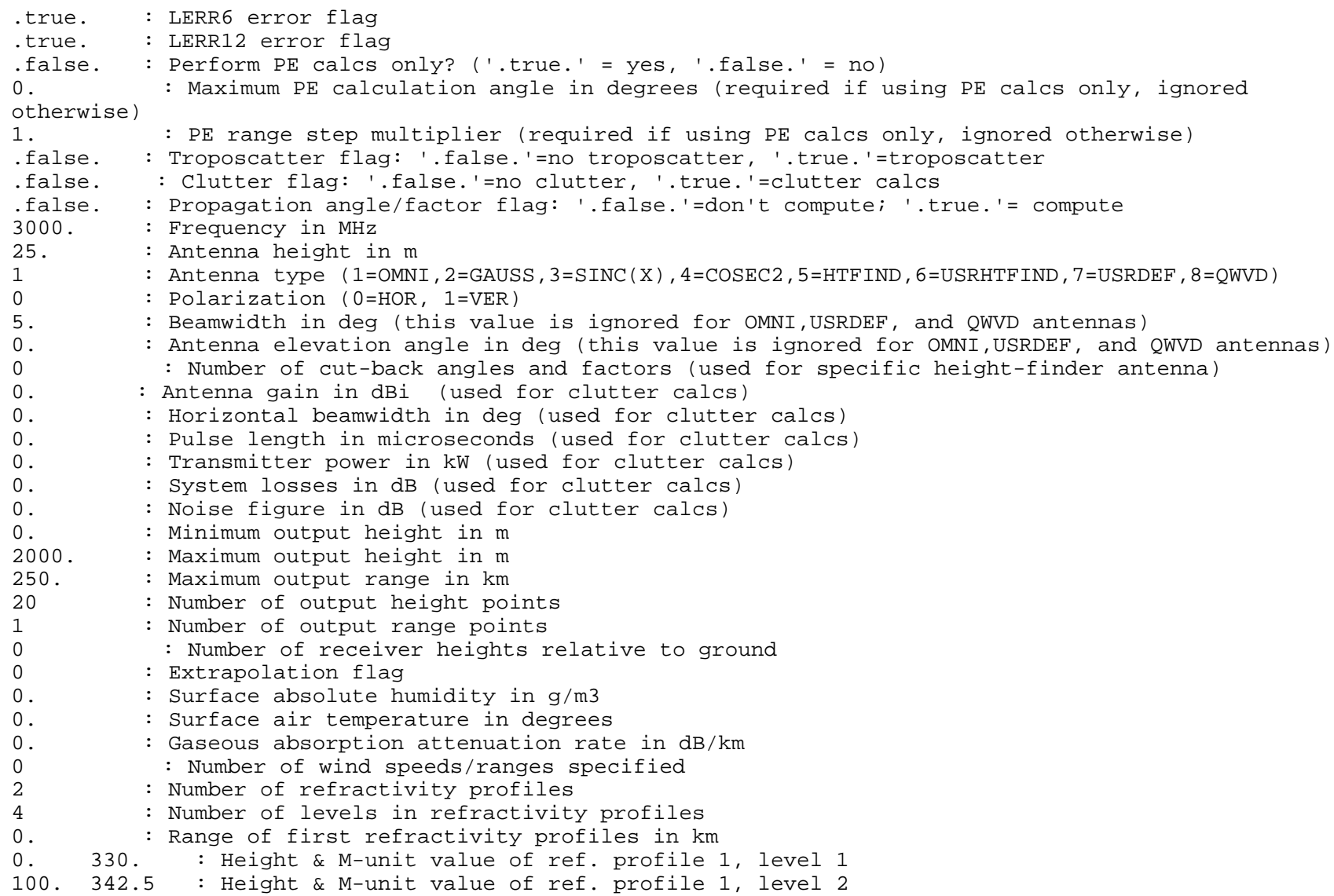


230. 312.5 : Height \& M-unit value of ref. profile 1 , level 3

2000. 517.8 : Height \& M-unit value of ref. profile 1, level 4

250. : Range of second refractivity profiles in $\mathrm{km}$

$\odot$. 330. : Height \& M-unit value of ref. profile 2, level 1

600. 405. : Height \& M-unit value of ref. profile 2, level 2

730. 375. : Height \& M-unit value of ref. profile 2, level 3

2000, 522.3 : Height \& M-unit value of ref, profile 2, level 4

$\odot$. Wind direction in deg (only used for clutter calcs)

$1 \quad$ : Number of ground composition types

$\odot ., 0,0 ., 0 . \quad$ : Range $(\mathrm{km})$, ground type (integer), permittivity, conductivity

1 : Number of GAMMAC \& GAMRNG pairs (used for clutter calcs-MUST be > 0 if terrain is specified)

-10., $\odot . \quad$ : GAMMAC $(\mathrm{dB})$, GAMRNG $(\mathrm{km})$ - 1st pair (remove line if \# of GAMMAC/GAMRNG pairs is 0 )

$\odot \quad$ : Number of terrain range $(\mathrm{km}) /$ height points

\subsection{SBDUCT.IN}

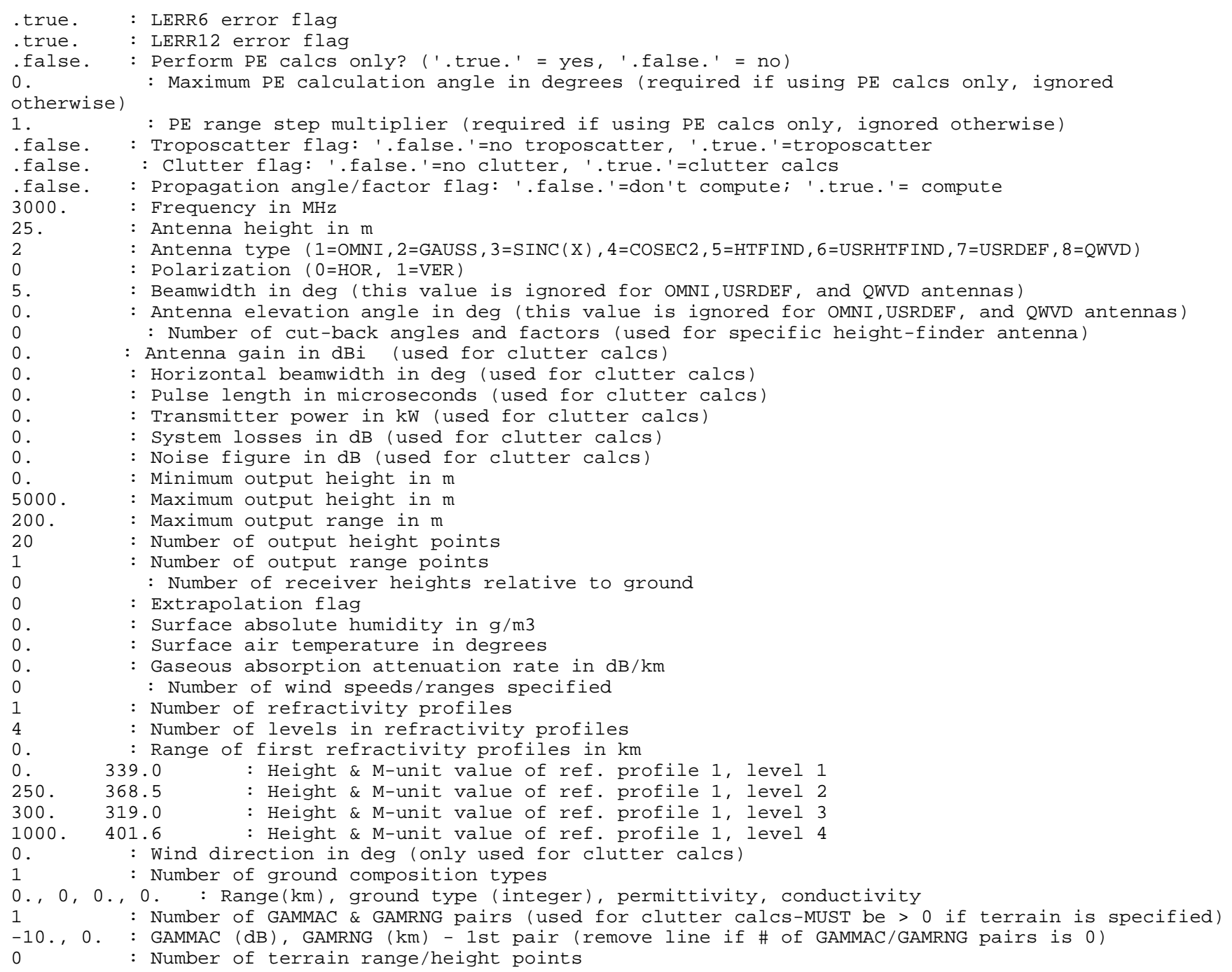

\subsection{SBDUCTRF.IN}

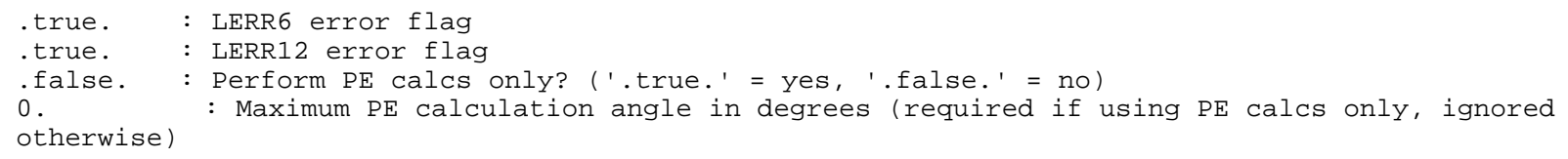


: Troposcatter flag: '.false.'=no troposcatter '.true.'=troposcatter

.false. : Clutter flag: '.false.'=no clutter, '.true.'=clutter calcs

.false. : Propagation angle/factor flag: '.false.'=don't compute; '.true.'= compute

3000. : Frequency in $\mathrm{MHz}$

25. : Antenna height in $\mathrm{m}$

$2 \quad:$ Antenna type (1=OMNI , 2=GAUSS, $3=\operatorname{SINC}(\mathrm{X}), 4=\mathrm{COSEC} 2,5=\mathrm{HTFIND}, 6=\mathrm{USRHTFIND}, 7=\mathrm{USRDEF}, 8=\mathrm{QWVD}$ )

$1 \quad$ : Polarization $(\theta=H O R, 1=V E R)$

5. : Beamwidth in deg (this value is ignored for OMNI, USRDEF, and QWVD antennas)

$\begin{array}{ll}\odot . & : \text { Antenna elevation angle in deg (this value is ignored for OMNI, USRDEF, and QWVD antennas) } \\ \odot & : \text { Number of cut-back angles and factors (used for specific height-finder antenna) }\end{array}$

$\odot$ : Antenna gain in $\mathrm{dBi}$ (used for clutter calcs)

$\odot$ : Horizontal beamwidth in deg (used for clutter calcs)

$\odot$ : Pulse length in microseconds (used for clutter calcs)

$0 . \quad$ : Transmitter power in $\mathrm{kW}$ (used for clutter calcs)

$0 . \quad$ : System losses in $\mathrm{dB}$ (used for clutter calcs)

$\odot$ : Noise figure in $\mathrm{dB}$ (used for clutter calcs)

$\odot$ : Minimum output height in $\mathrm{m}$

1000. : Maximum output height in $\mathrm{m}$

200. : Maximum output range in $\mathrm{km}$

$20 \quad$ : Number of output height points

$1 \quad$ : Number of output range points

$\odot \quad:$ Number of receiver heights relative to ground

$\odot \quad$ : Extrapolation flag

$\odot$ : Surface absolute humidity in $\mathrm{g} / \mathrm{m} 3$

$0 . \quad$ : Surface air temperature in degrees

$\odot$ : Gaseous absorption attenuation rate in $\mathrm{dB} / \mathrm{km}$

$1 \quad$ : Number of wind speeds/ranges specified

10., $\odot . \quad$ : Wind speed $(\mathrm{m} / \mathrm{s})$, Range $(\mathrm{km})$

$1 \quad$ : Number of refractivity profiles

$4 \quad$ : Number of levels in refractivity profiles

$0 . \quad$ : Range of first refractivity profiles in $\mathrm{km}$

$\odot$. $339.0 \quad$ : Height \& M-unit value of ref. profile 1 , level 1

250. 368.5 : Height \& M-unit value of ref. profile 1 , level 2

300. 319.0 : Height \& M-unit value of ref. profile 1, level 3

1000. 401.6 : Height \& M-unit value of ref. profile 1 , level 4

$\odot \quad \quad$ : Wind direction in deg (only used for clutter calcs)

$1 \quad$ : Number of ground composition types

$\odot ., \odot, \odot ., \odot . \quad$ : Range $(\mathrm{km})$, ground type (integer), permittivity, conductivity

1 : Number of GAMMAC \& GAMRNG pairs (used for clutter calcs-MUST be $>\odot$ if terrain is specified)

$-10 ., 0 . \quad$ : GAMMAC $(\mathrm{dB})$, GAMRNG $(\mathrm{km})$ - 1st pair (remove line if \# of GAMMAC/GAMRNG pairs is $\odot$ )

$\odot \quad:$ Number of terrain range/height points

\subsection{SINEX.IN}

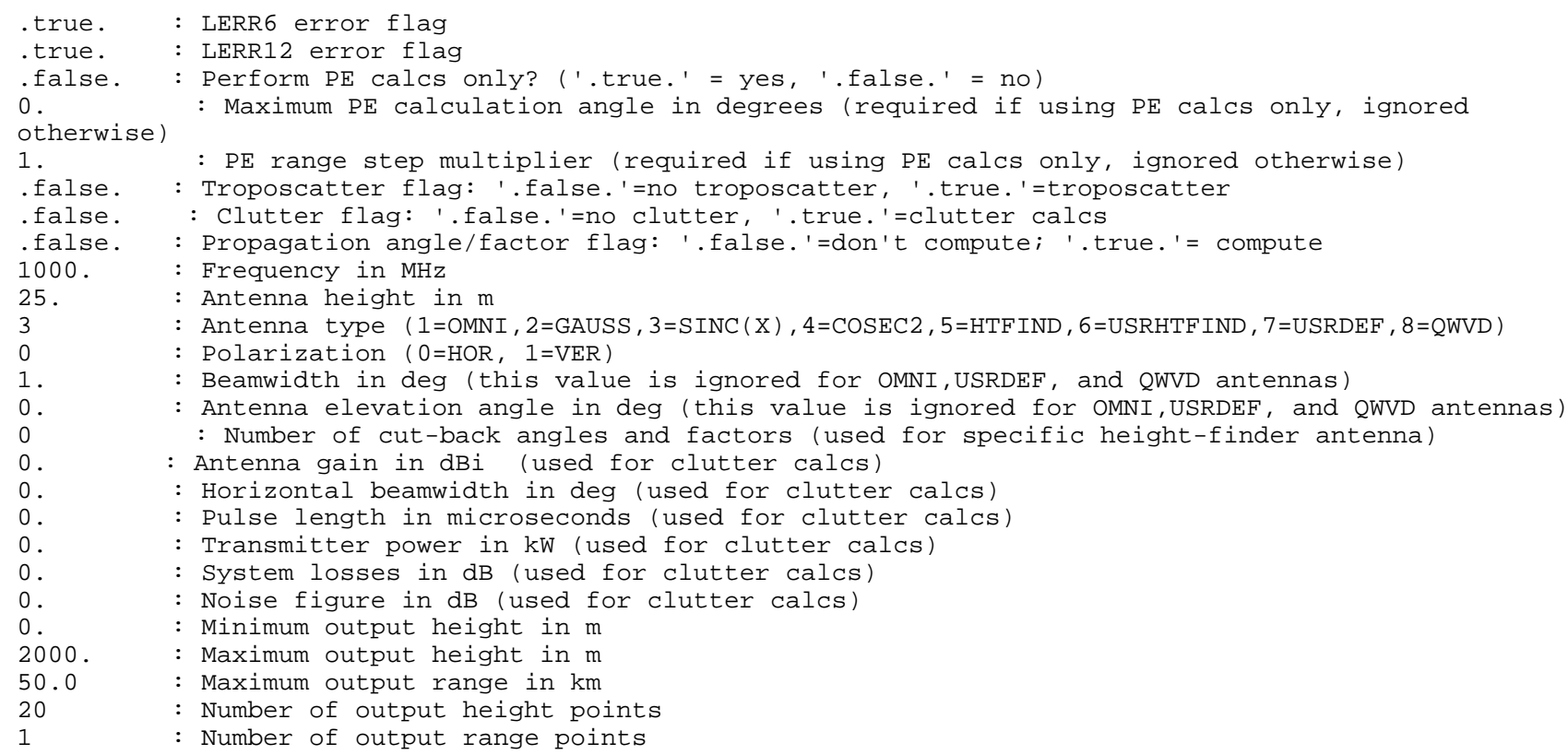




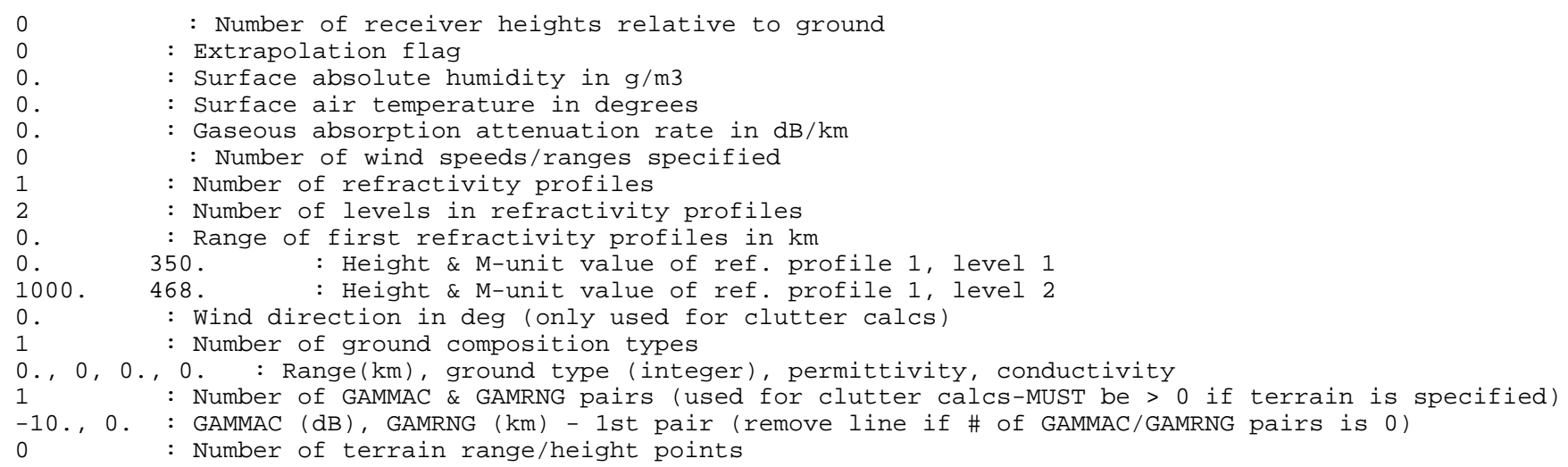

\subsection{TROPOS.IN}

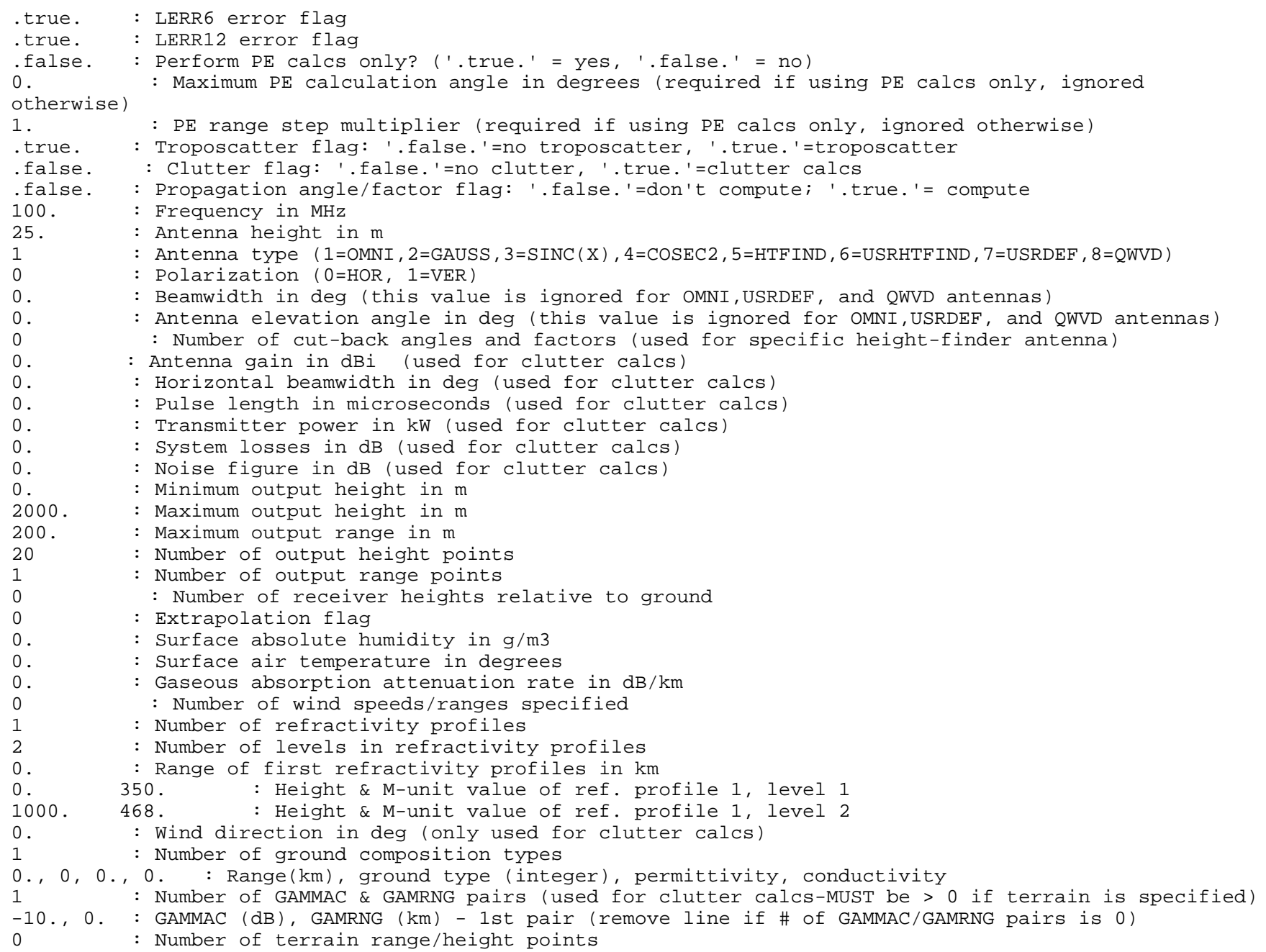

\subsection{TROPOT.IN}

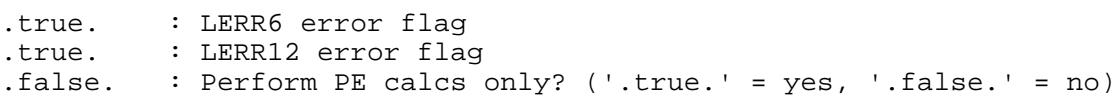




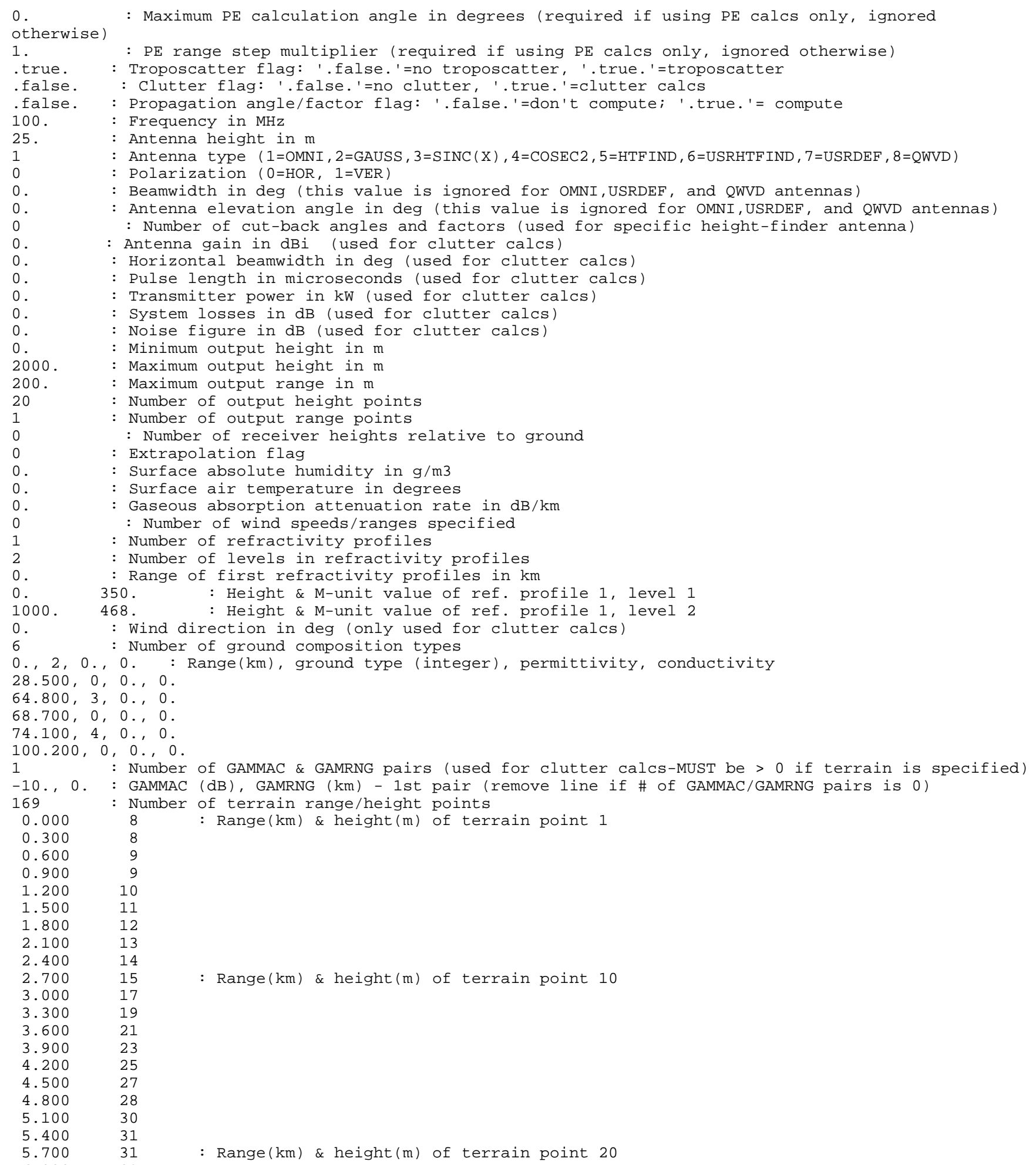




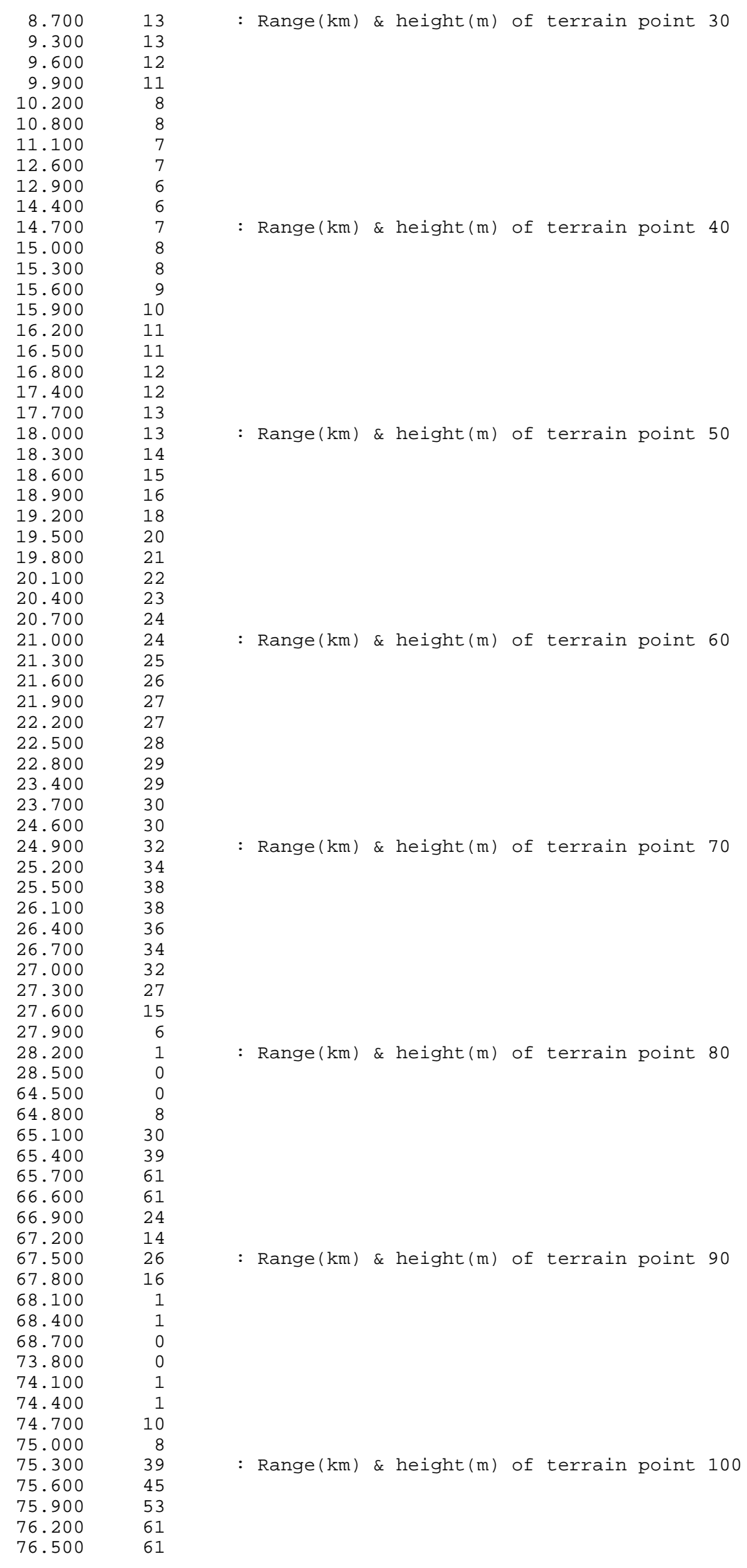




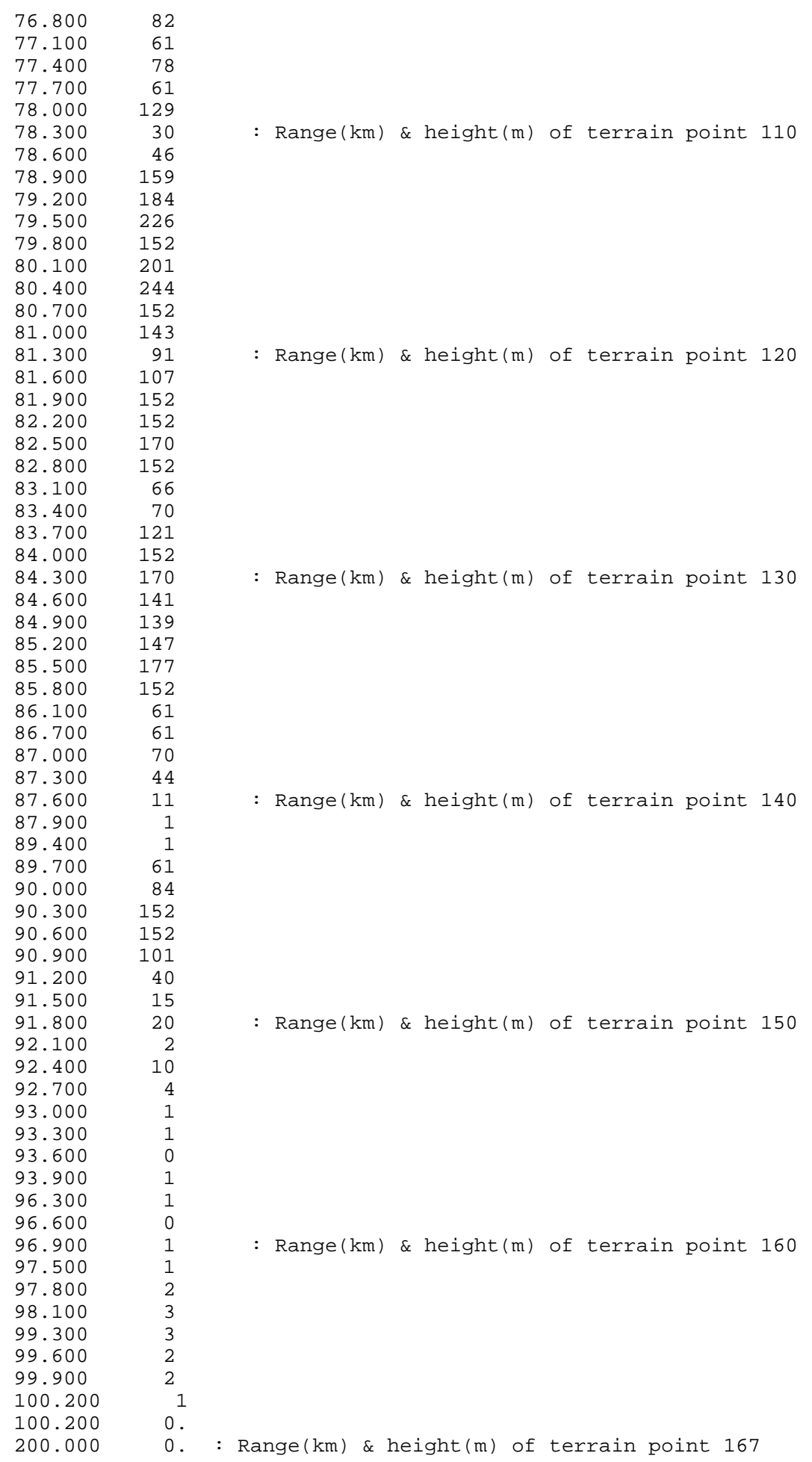

\subsection{USERDEFA}

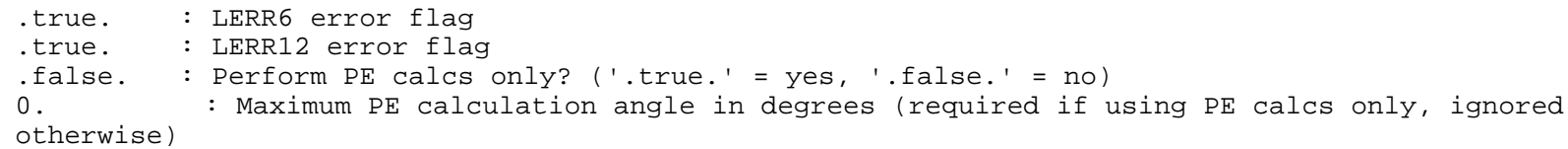


1

.false. : Troposcatter flag: '. false.'=no troposcatter, '. true.'=troposcatter
.false. $\quad$ : Clutter flag: '. false.'=no clutter, '. true.' $=$ clutter calcs

false. : Clutter flag: '.false.'=no clutter, '.true.'=clutter calcs

false. : Propagation angle/factor flag: '.false.'=don't compute; '.true.'= compute

: Frequency in $\mathrm{MHz}$

6. : Antenna height in $\mathrm{m}$

7 : Antenna type (1=OMNI , 2=GAUSS, $3=\mathrm{SINC}(\mathrm{X}), 4=\mathrm{COSEC} 2,5=\mathrm{HTFIND}, 6=\mathrm{USRHTFIND}, 7=\mathrm{USRDEF}, 8=\mathrm{QWVD}$ )

: Polarization ( $\theta=$ HOR, 1=VER)

$\odot$. : Beamwidth in deg (this value is ignored for OMNI, USRDEF, and QWVD antennas)

2. : Antenna elevation angle in deg (this value is ignored for OMNI, USRDEF, and QWVD antennas)

$54 \quad$ : Number of angle/factor pairs (used for antenna types 6 and 7)

0.182

0.245

0.316

$\odot .389$

0.479

0.556

0.631

0.716

0.785

0.861

0.912

$\odot .966$

$\odot .998$

1.000

1.000

0.966

0.902

0.822

0.742

0.646

0.569

0.501

0.452

0.422

0.402

0.389

0.375

$\odot .359$

0.339

0.305

0.276

0.245

0.221

0.210

$\odot .199$

0.190

0.180

0.164

0.148

0.130

0.110

0.095

0.077

0.070

0.065

0.058

0.050

0.039

0.031

0.025

: Antenna gain in dBi (used for clutter calcs)

: Horizontal beamwidth in deg (used for clutter calcs)

: Pulse length in microseconds (used for clutter calcs)

: Transmitter power in kW (used for clutter calcs)

: System losses in $\mathrm{dB}$ (used for clutter calcs)

: Noise figure in $d B$ (used for clutter calcs)

: Minimum output height in $\mathrm{m}$

: Maximum output height in $\mathrm{m}$

: Maximum output range in $\mathrm{km}$

: Number of output height points 


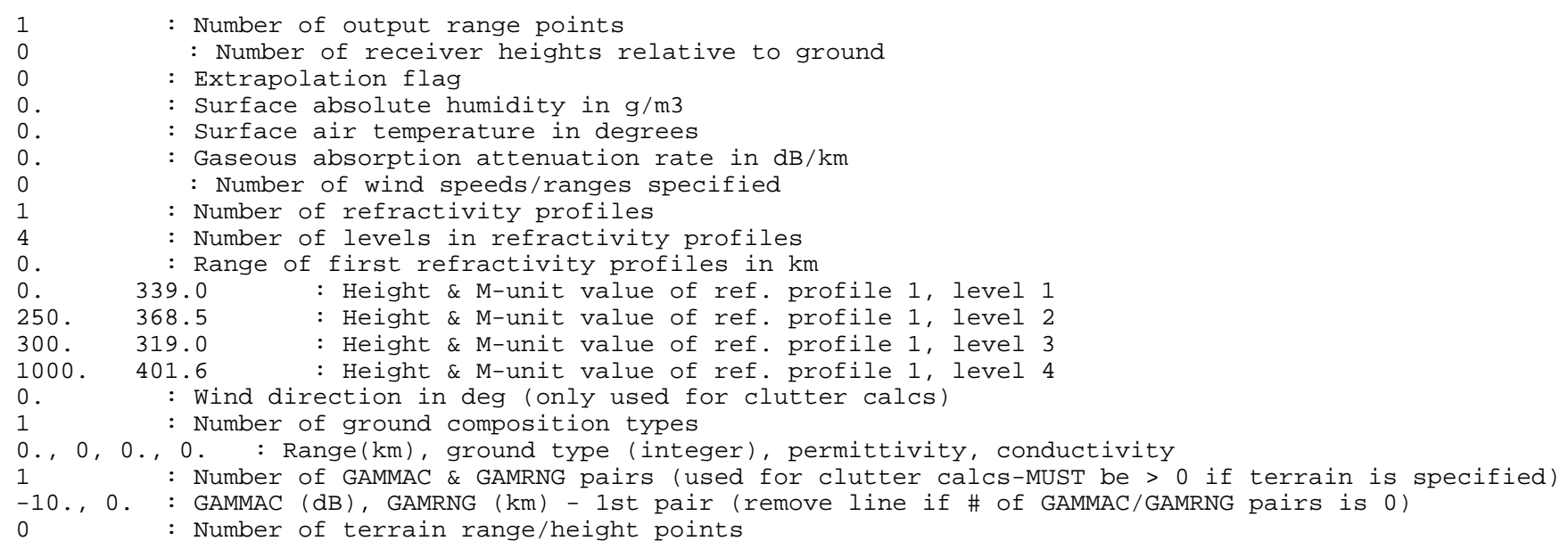

\subsection{USERHF.IN}

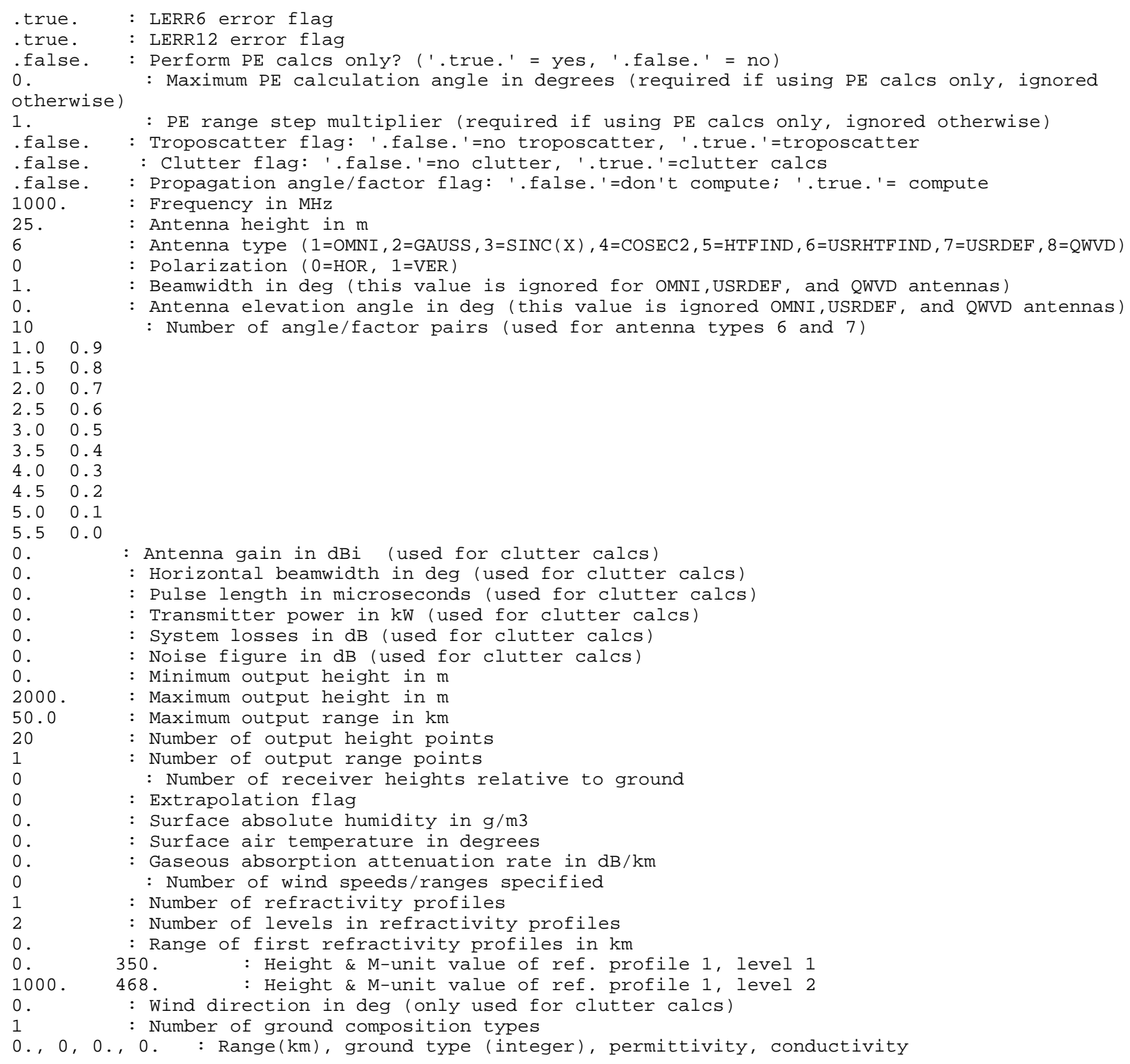


1 : Number of GAMMAC \& GAMRNG pairs (used for clutter calcs-MUST be > $\odot$ if terrain is specified) $-10 ., 0 . \quad$ : GAMMAC $(\mathrm{dB})$, GAMRNG $(\mathrm{km})$ - 1st pair (remove line if \# of GAMMAC/GAMRNG pairs is 0 )

\subsection{VERT.IN}

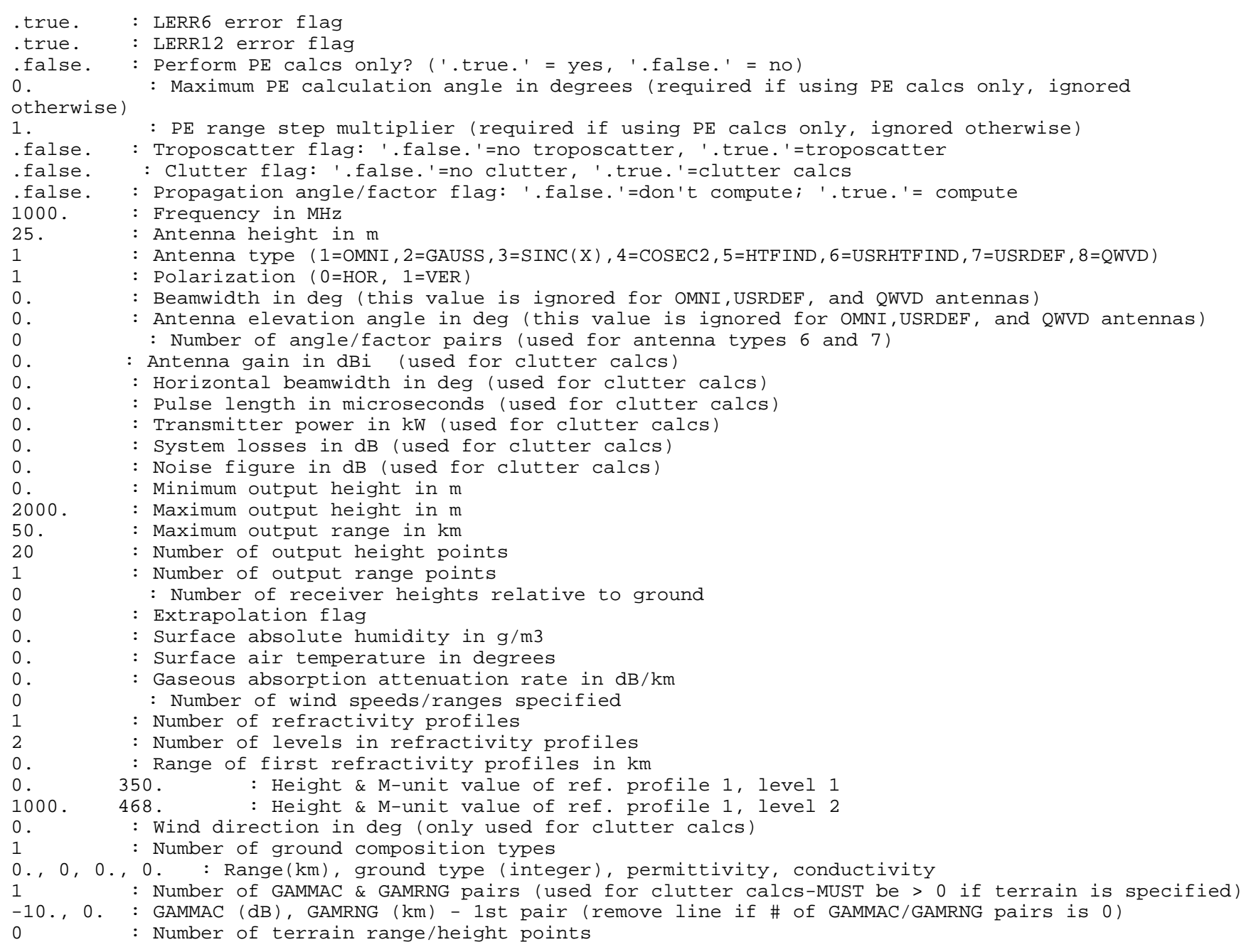

\subsection{VERTMIX.IN}

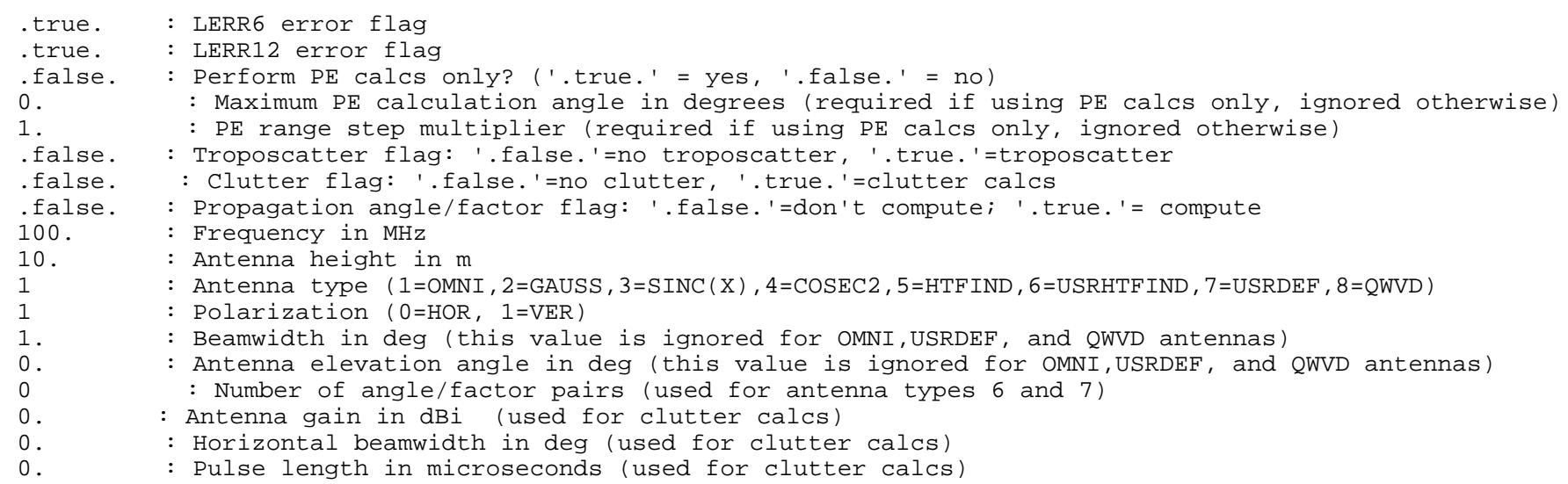




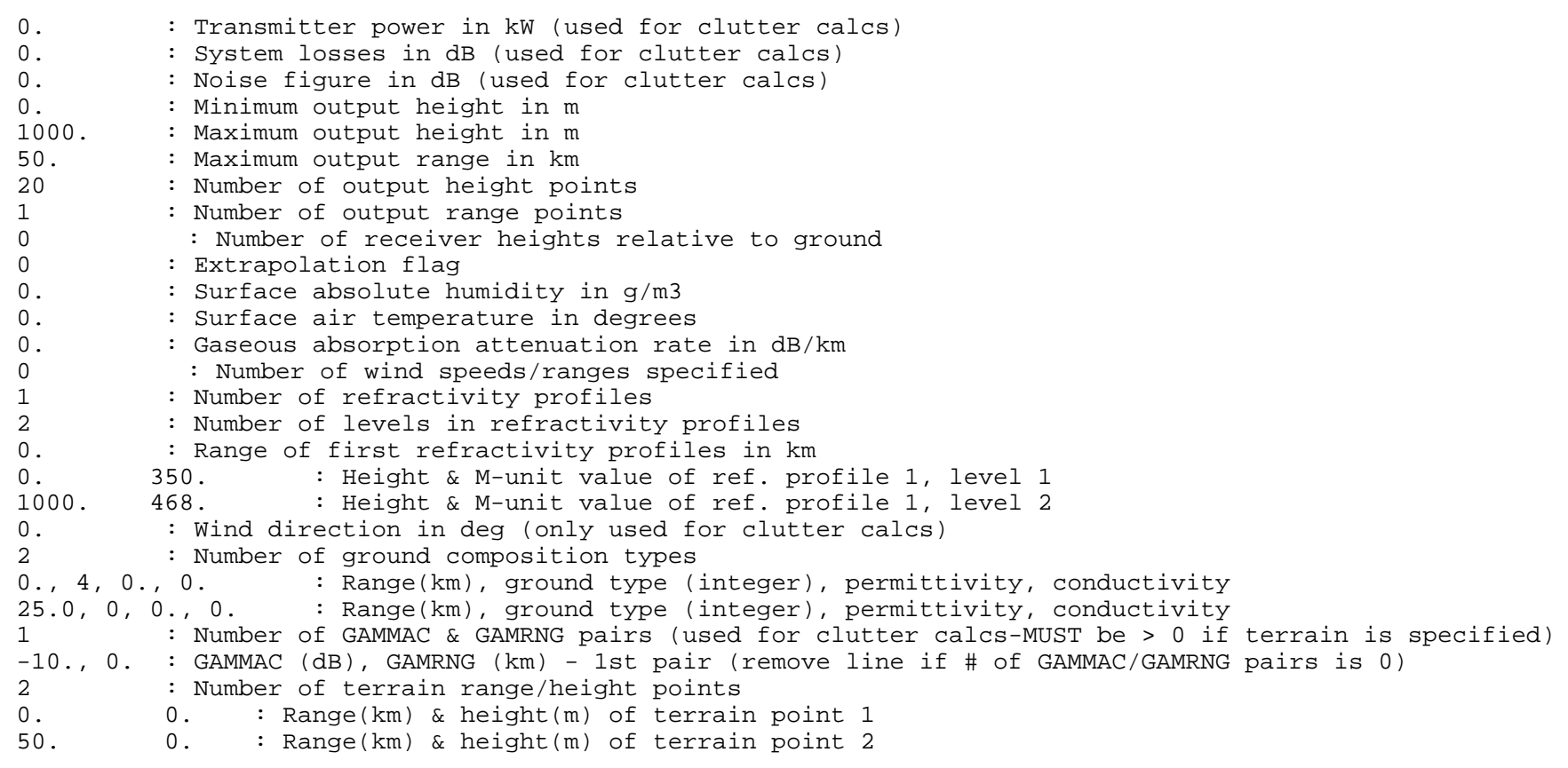

\subsection{VERTSEA.IN}

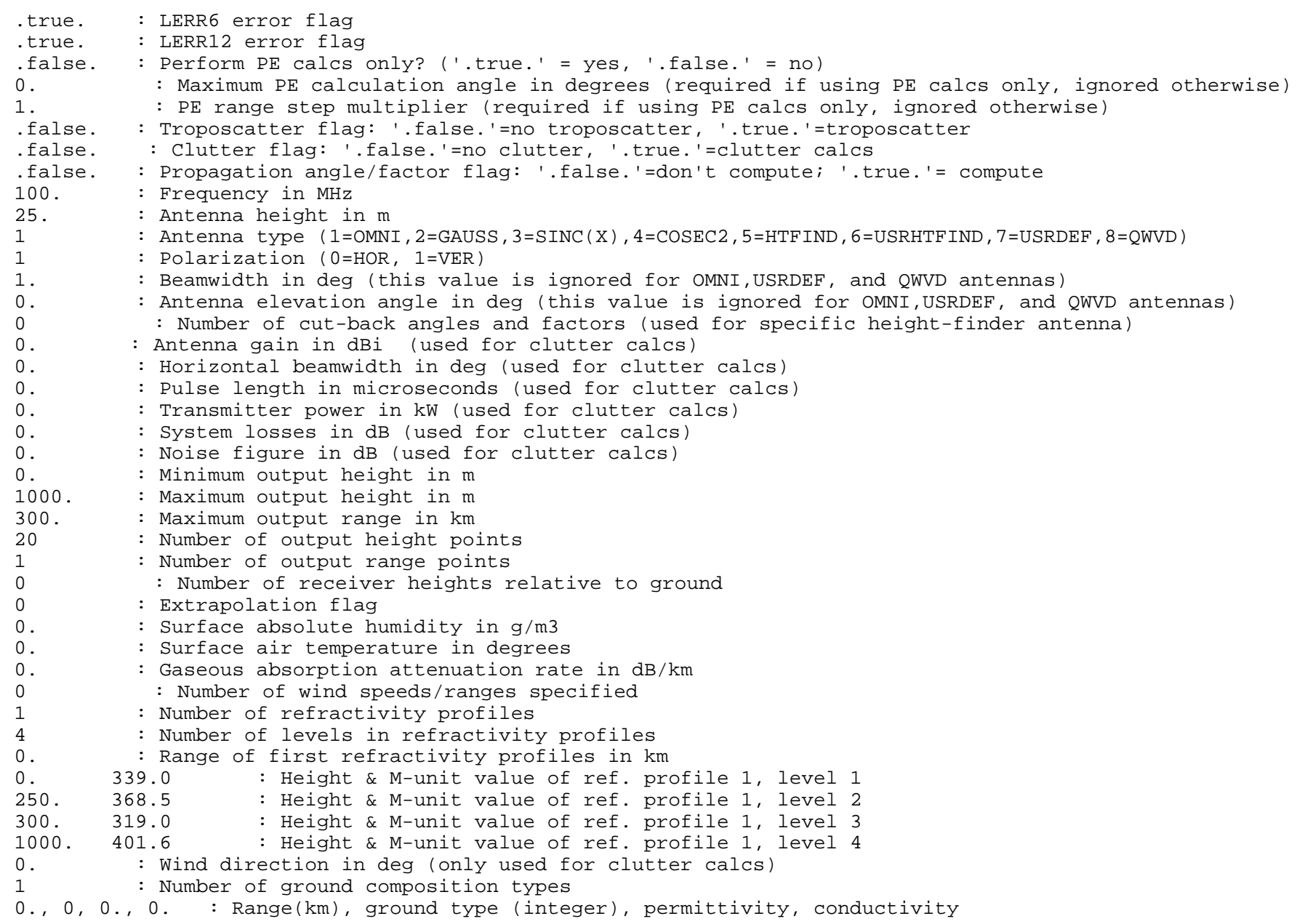


1 : Number of GAMMAC \& GAMRNG pairs (used for clutter calcs-MUST be > $\odot$ if terrain is specified) $-10 ., 0 . \quad$ : GAMMAC $(\mathrm{dB})$, GAMRNG $(\mathrm{km})$ - 1st pair (remove line if \# of GAMMAC/GAMRNG pairs is 0 )

\subsection{VERTUSRD.IN}

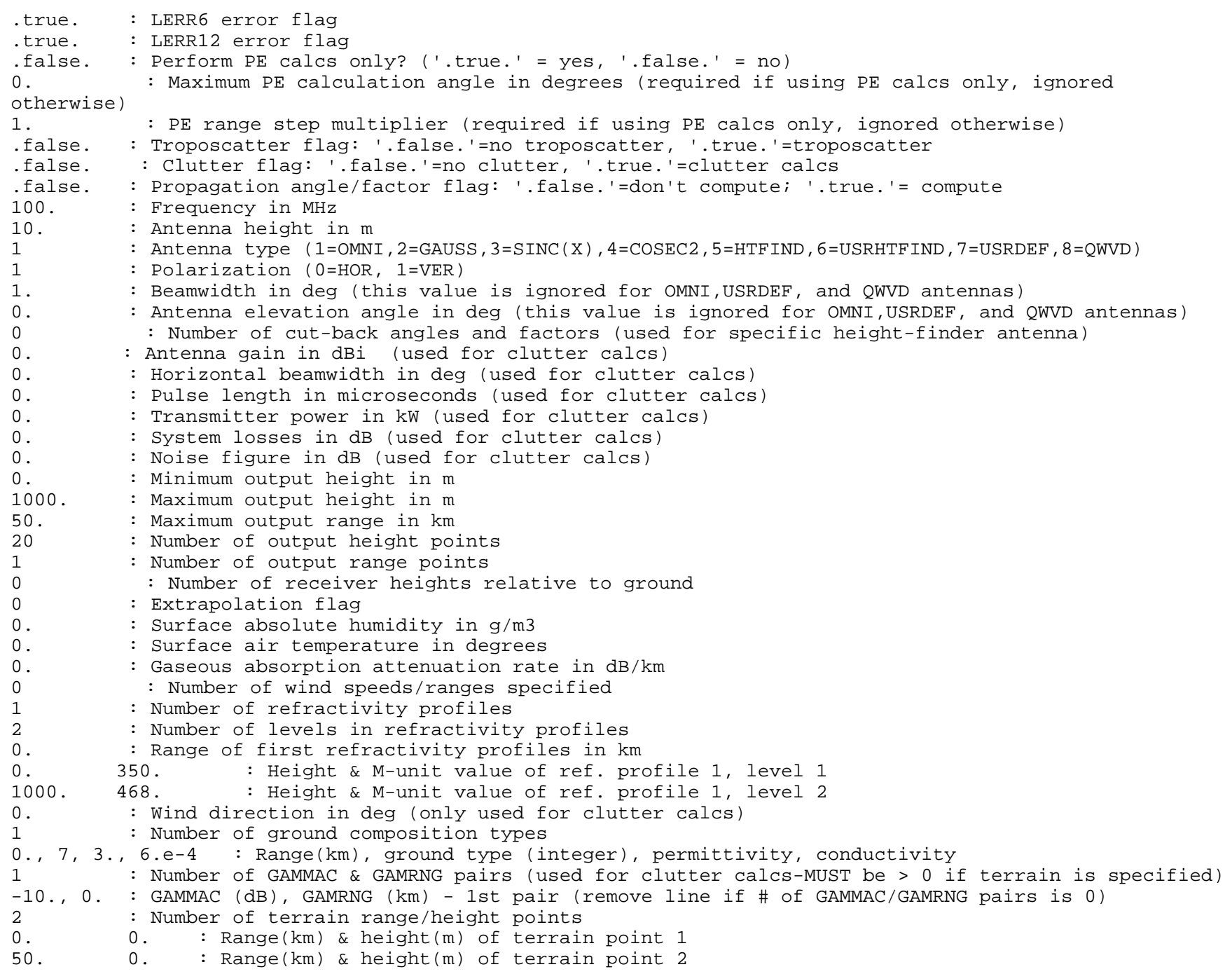

\subsection{WEDGE.IN}

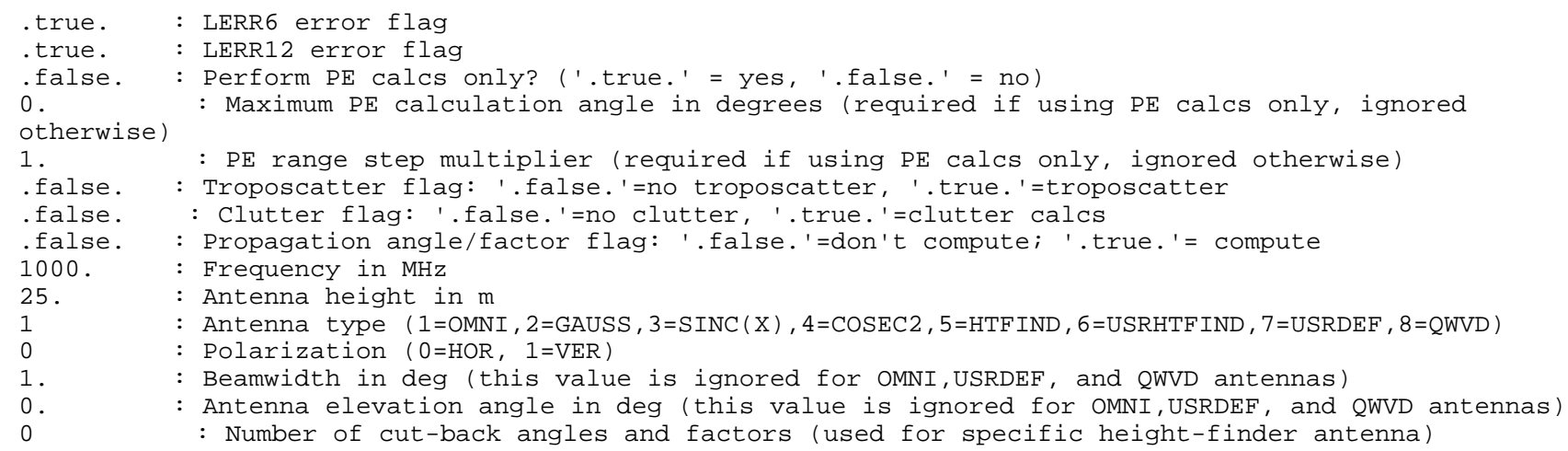




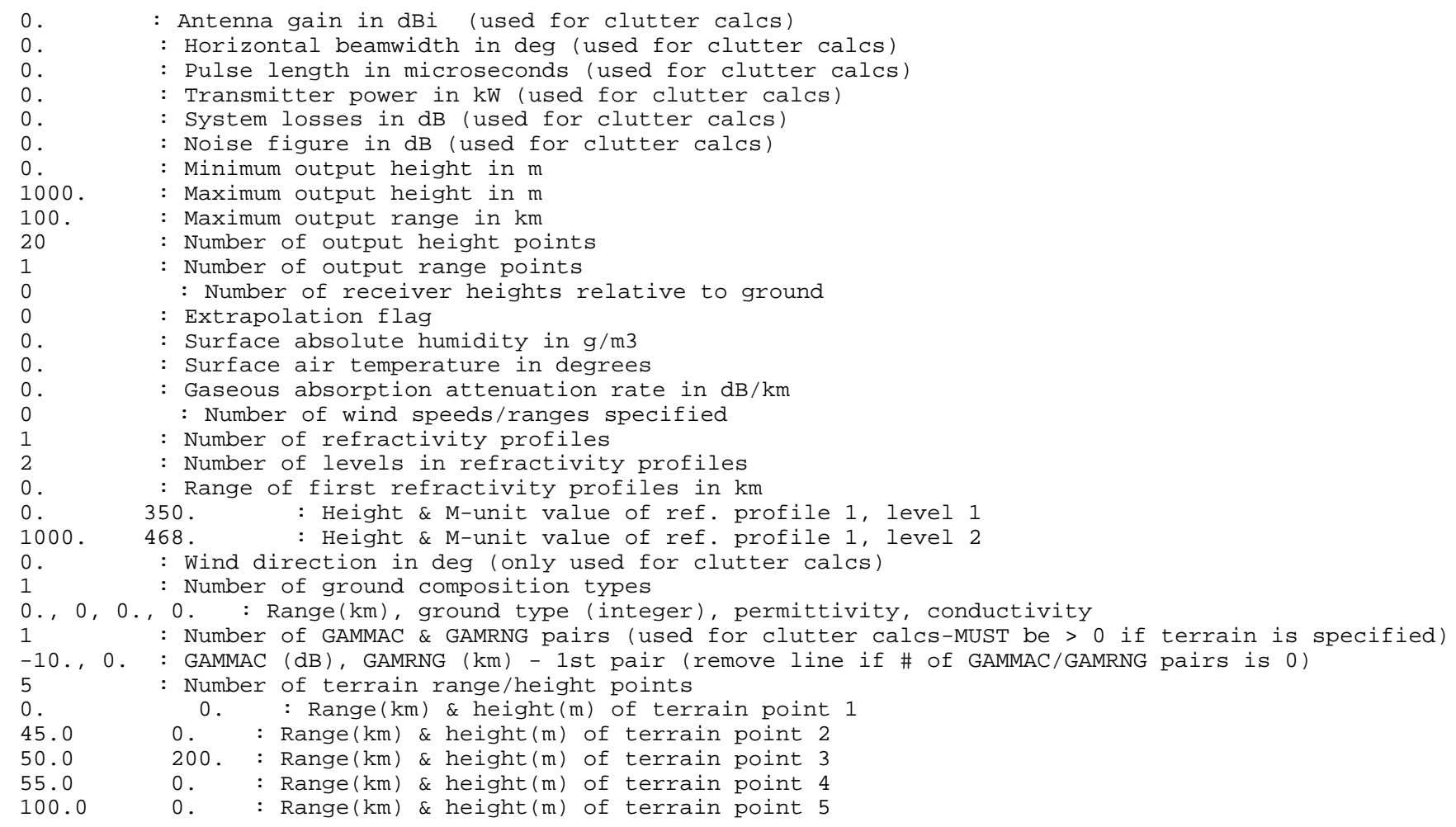


Approved for public release; distribution is unlimited. 